\title{
COMPLEX-WIDE WASTE FLOW ANALYSIS V1.0 VERIFICATION AND VALIDATION REPORT
}

\section{RECEIVED}

DEC 301997

O.STI

Karen M. Hsu

Allen S. Lundeen

Kyle B. Oswald

David E. Shropshire John M. Robinson

William H. West 


\section{DISCLAIMER}

This report was prepared as an account of work sponsored by an agency of the United States Government. Neither the United States Government nor any agency thereof, nor any of their employees, makes any warranty, express or implied, or assumes any legal liability or responsibility for the accuracy, completeness, or usefulness of any information, apparatus, product, or process disclosed, or represents that its use would not infringe privately owned rights. Reference herein to any specific commercial product, process, or service by trade name, trademark, manufacturer, or otherwise does not necessarily constitute or imply its endorsement, recommendation, or favoring by the United States Government or any agency thereof. The views and opinions of authors expressed herein do not necessarily state or reflect those of the United States Government or any agency thereof. 


\title{
COMPLEX-WIDE WASTE FLOW ANALYSIS V1.0 VERIFICATION AND VALIDATION REPORT
}

\author{
Karen M. Hsu \\ Allen S. Lundeen \\ Kyle B. Oswald \\ David E. Shropshire \\ John M. Robinson \\ William H. West
}

Published 11/21/97

Idaho National Engineering and Environmental Laboratory

Waste Management Technologies Department

Lockheed Martin Idaho Technologies Company

Idaho Falls, Idaho 83415

\author{
Prepared for the \\ U.S. Department of Energy \\ Assistant Secretary for Environmental Management \\ Under DOE Idaho Operations Office \\ Contract DE-AC07-94ID13223
}

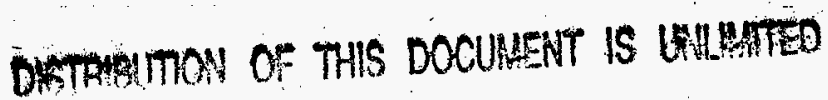

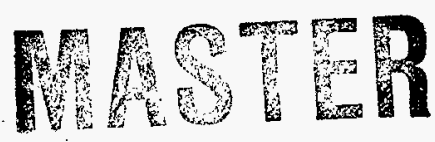




\section{CONTENTS}

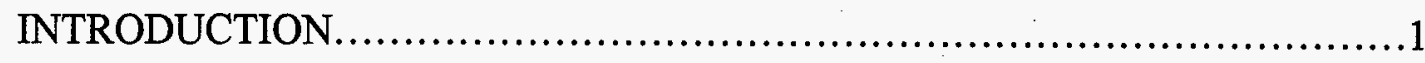

SYSTEMS ENGINEERING APPROACH......................................2

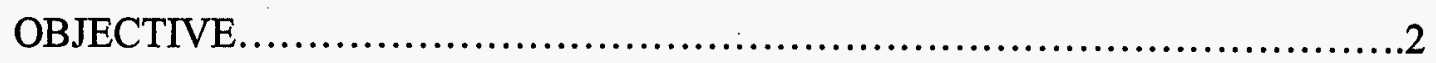

TEST PLANNING................................................................

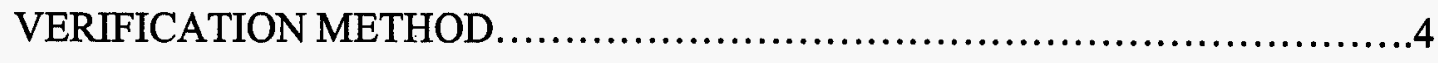

SUMMARY OF RESULTS ................................................4

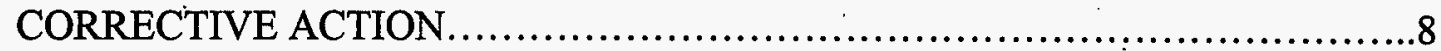

APPENDIX A..................................................................... 10

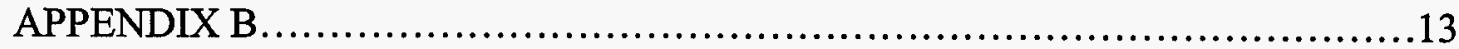




\section{CWWFA VERIFICATION \& VALIDATION REPORT}

\section{Introduction}

The Complex-Wide Waste Flow Analysis model (CWWFA) was developed to assist the Department of Energy (DOE) Environmental Management (EM) Office of Science and Technology (EM-50) to evaluate waste management scenarios with emphasis on identifying and prioritizing technology development opportunities to reduce waste flows and public risk. In addition, the model was intended to support the needs of the Complex-Wide Environmental Integration (EMI) team supporting the DOE's Accelerating Cleanup: 2006 Plan.

CWWFA represents an integrated environmental modeling system that covers the life cycle of waste management activities including waste generation, interim process storage, retrieval, characterization and sorting, waste preparation and processing, packaging, final interim storage, transport, and disposal at a final repository. The CWWFA shows waste flows through actual site-specific and facility-specific conditions. The system requirements for CWWFA are documented in the Technical Requirements Document (TRD) ${ }^{1}$. The TRD is intended to be a "living document" that will be modified over the course of the execution of CWWFA development. Thus, it is anticipated that CWWFA will continue to evolve as new requirements are identified (i.e., transportation, small sites, new streams, etc.).

This report provides a documented basis for system verification of CWWFA requirements. System verification is accomplished through formal testing and evaluation to ensure that all performance requirements as specified in the TRD have been satisfied. A Requiremènt Verification Matrix (RVM) was used to map the technical requirements to the test procedures. The RVM is attached as Appendix A.

Since February of 1997, substantial progress has been made toward development of the CWWFA to meet the system requirements. This system verification activity provides a baseline on system compliance to requirements and also an opportunity to reevaluate what requirements need to be satisfied in FY-98.

\footnotetext{
${ }^{1}$ Technical Requirements Document Complex-Wide Waste Flow Analysis (INEL/95-00340, March 1997)
} 


\section{System Engineering Approach}

A proven Lockheed Martin Corporation systems engineering approach was used in the development of the CWWFA model. This approach included the development of the technical requirements and decomposition into design specifications ${ }^{2}$ and prototyping activities. This approach is now applied to CWWFA verification and validation activities. As illustrated in Figure 1, the general approach consists of defining the requirements, identifying alternatives, analyzing the alternatives, verifying, and implementing the results. Additionally, the verification approach is consistent with IEEE Standard 730-1989 and with LMITCO Quality Level 2 standards.

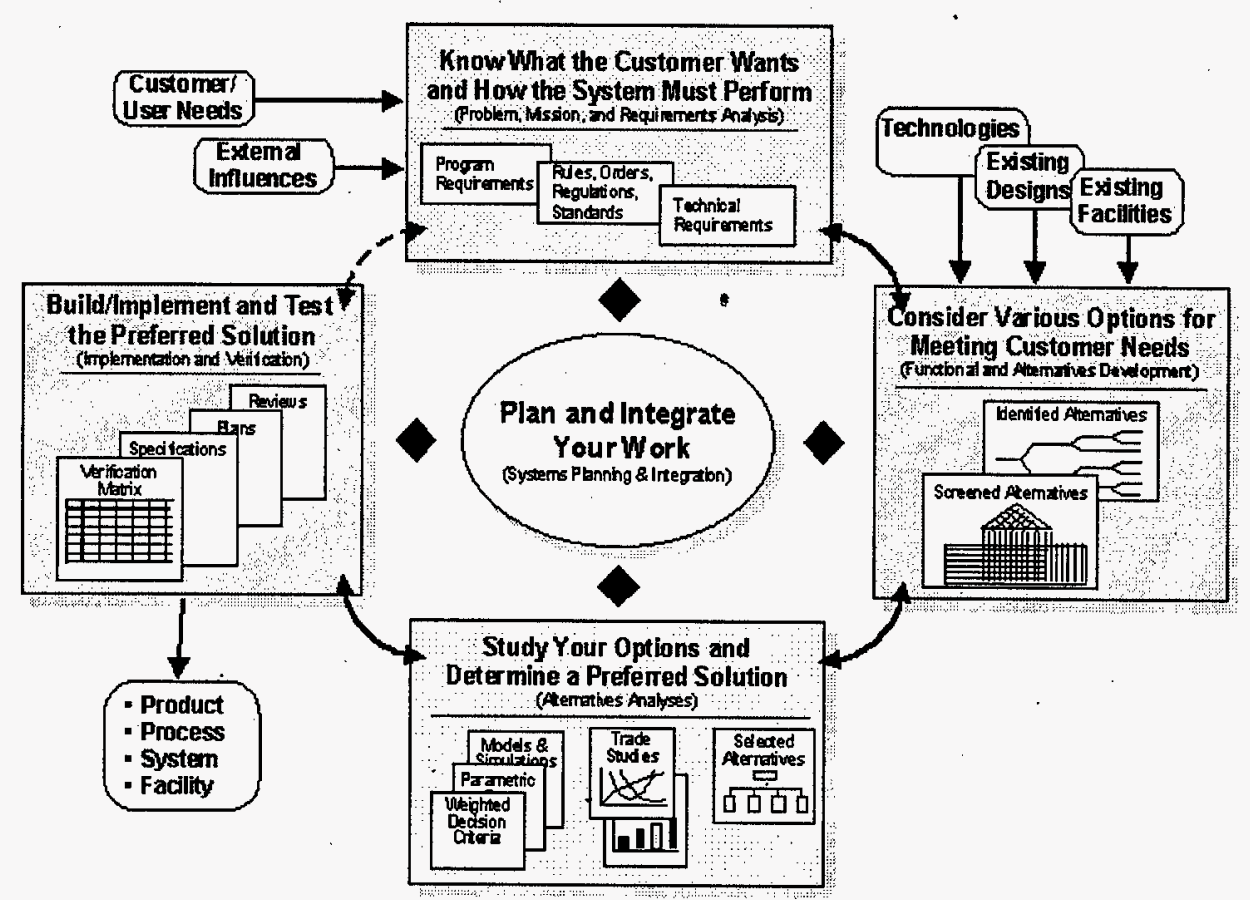

Figure 1. Systems engineering process

\section{Objective}

The objective of this report is to describe the test planning, verification method, summary of results, and corrective actions that emerge from the test and evaluation activities. The focus of the testing is meeting performance requirements (e.g., model must be capable of performing analyses on waste

${ }^{2}$ Complex-Wide Waste Flow Analysis v1.0 Design Specification Document (INEL/97-00339, March 1997) 
flow, capacity planning, cost, risk, stakeholder perception, transportation, and other factors) as defined in the RVM.

\section{Test Planning}

The testing program evaluates compliance to requirements with regard to multiple planning factors: waste flow, cost, risk, facility operational performance, decision analysis, equity (stakeholder position), transportation, and technology performance. These factors represent an integrated environmental management system that covers waste stream disposition from current state to final disposal.

The EMI team has developed waste disposition maps that are graphical depictions of waste streams moving through the system from the current state to final disposition. These disposition maps detail the waste disposition at 11 major DOE sites. Figure 2 shows an example of a waste disposition map for the Hanford site.

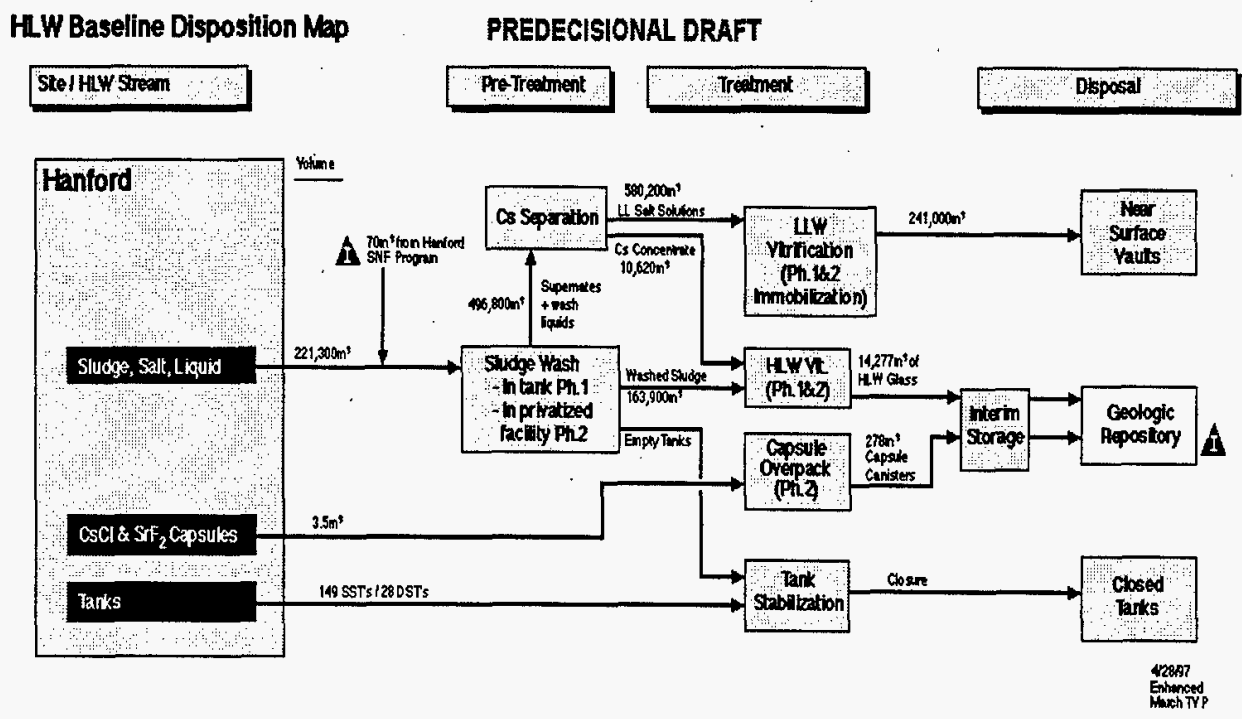

Figure 2. Waste Disposition Map

The CWWFA was developed to provide dynamic assessments of waste flow as shown in the disposition maps. Thus, test plans were needed to demonstrate that CWWFA results show reasonable behaviors that accurately depict the waste flow of various waste streams.

Test planning consisted of establishing test procedures (as described below) that satisfied verification of each requirement listed in the RVM. There were test procedures developed to address seven major performance areas. Each performance area includes one or more technical requirements. A total of 22 
requirements were tested for compliance. Details of the test procedures and test results can be found in Appendix B. Test procedures are listed as follows:

1. TP3.1.1 - Waste Flow Analysis and Scheduling

2. TP3.1.2 - Mortgage Reduction Performance

3. TP3.1.3 - Health and Safety Risk

4. TP3.1.4 - Facility Operational Performance

5. TP3.1.5 - Decision Analysis

6. TP3.1.6 - Equity Performance

7. TP3.1.7 - Technology Performance

Each test procedure provides a description of the test methods (or inspection), waste stream configuration (e.g., site, waste types, quantities, etc.), and appropriate test data . A typical profile includes facility, waste stream, waste type, shipping schedule, conversion profile, risk, and cost. A stakeholder profile is used for specific equity analysis testing.

Since CWWFA is capable of modeling multiple sites, waste types, waste streams, and routes (e.g., hundreds of configurations), specific test cases and test data were used to provide representation of CWWFA waste streams. The test cases were chosen to support rigorous testing of CWWFA processes.

After the testing and evaluation was completed, the results were analyzed for compliance and mapped to the RVM for completeness. Results of pass and failure were documented and corrective actions were defined to resolve the deficiencies.

\section{Verification Method}

Two methods will be used to verify requirements during test and evaluation of CWWFA.

- Inspection - an observation or examination to verify conformity with requirements.

- Test - an exercise wherein quantitative performance is compared to requirements and is documented.

\section{Summary of Results}

Below is a summary of the testing results presented in a table format. The test results are verified against all requirements and acknowledged as either pass or fail. Failed items are addressed in the corrective action section. 


\section{Summary of Results}

\begin{tabular}{|c|c|c|c|}
\hline Requirement & $\begin{array}{l}\text { Test } \\
\text { Procedure }\end{array}$ & $\begin{array}{l}\text { Pass / } \\
\text { Fail } \\
\end{array}$ & Comment \\
\hline $\begin{array}{l}\text { 3.1.1.1 - Provide quick interactive analysis of the effects of } \\
\text { waste flow over time to analyze storage requirements, } \\
\text { transportation, and disposal bottlenecks. }\end{array}$ & TP3.1.1 & Pass & $\begin{array}{l}\text { For every unit of waste, the model tracks a volume in cubic meters and } \\
\text { a density in kilograms per cubic meter. With both of these numbers the } \\
\text { model can convert between volume and mass as necessary. } \\
\text { Note: the dynamic scheduling capability of CWWFA needs to be } \\
\text { explained in the user's manual and specification document. }\end{array}$ \\
\hline $\begin{array}{l}\text { 3.1.1.2 - Summarize impacts of acceleration and delays } \\
\text { associated with the management of waste (reduction of } \\
\text { untreated waste inventories, reclassification of wastes, } \\
\text { accumulation in storage, buildup of waste in disposal). }\end{array}$ & TP3.1.1 & Pass & Model behaves as expected. \\
\hline $\begin{array}{l}\text { 3.1.1.3 - Identify need for new (or underutilized) facilities in } \\
\text { the DOE complex that could be used to process bottlenecks. }\end{array}$ & TP3.1.1 & Pass & $\begin{array}{l}\text { The facility capacity detail chart identifies bottlenecks due to } \\
\text { insufficient processing capacity or a timing conflict at the next facility } \\
\text { in the routing. }\end{array}$ \\
\hline $\begin{array}{l}\text { 3.1.1.4 - Identify impacts from transportation bottlenecks and } \\
\text { alternatives. Include alternative packaging and transport } \\
\text { mode options. }\end{array}$ & TP3.1.1 & Fail & $\begin{array}{l}\text { The model does not constrain the number of transport or the number of } \\
\text { packages. Thus no bottlenecks will occur due to transportation } \\
\text { limitations. The model does not allow alternative packages to be used } \\
\text { for transportation. The model only has transport mode by road.. }\end{array}$ \\
\hline $\begin{array}{l}\text { 3.1.1.5 - Summarize system impacts from future ER and D\&D } \\
\text { waste sources (show when/how much the current maximum } \\
\text { disposal receipt rate }\left(\mathrm{m}^{3} / \mathrm{yr}\right) \text { is impacted by input from waste } \\
\text { sources) }\end{array}$ & TP3.1.1 & Pass & $\begin{array}{l}\text { The model supports addition of new generation volumes for a waste } \\
\text { stream. The new generation volumes can be interpreted as incoming } \\
\text { waste from environmental restoration or decontamination and } \\
\text { decommissioning activities. }\end{array}$ \\
\hline $\begin{array}{l}\text { 3.1.1.6 - Method 1 } \\
\text { Show processing flow effects from varying split of waste } \\
\text { processed by alternative processes (e.g., WIPP Waste } \\
\text { Acceptance Criteria, versus treatment to land disposal } \\
\text { restricted, volume reduction, waste form density changes, and } \\
\text { secondary waste production. }\end{array}$ & TP3.1.1 & Pass & $\begin{array}{l}\text { The model allows the volume change factor for a facility to be changed } \\
\text { by the user. }\end{array}$ \\
\hline $\begin{array}{l}\text { 3.1.1.6 - Method } 2 \\
\text { Show processing flow effects for secondary waste generation }\end{array}$ & TP3.1.1 & Fail & $\begin{array}{l}\text { The model does not support secondary waste generation from } \\
\text { processing. However, the user could specify an additional shipping } \\
\text { route to duplicate the results of a secondary waste stream due to } \\
\text { processing. But this would have to be done on a stream by stream basis } \\
\text { as opposed to a single facility producing secondary waste regardless of } \\
\text { the characteristics of the waste being processed. }\end{array}$ \\
\hline
\end{tabular}




\begin{tabular}{|c|c|c|c|}
\hline $\begin{array}{l}\text { 3.1.1.7 - Allow separation of wastes after processing into } \\
\text { separate fractions (e.g., HLW is split into high and low } \\
\text { activity wastes). }\end{array}$ & TP3.1.1 & Pass & $\begin{array}{l}\text { The model supports multiple shipping routes between facilities. Each } \\
\text { shipping route can represent a fraction of waste generated from a } \\
\text { processing facility. }\end{array}$ \\
\hline $\begin{array}{l}\text { 3.1.1.8 - Show impacts from consolidated treatment, storage, } \\
\text { and disposal of similar wastes (e.g., aMLLW and mixed } \\
\text { TRU). }\end{array}$ & TP3.1.1 & Pass & $\begin{array}{l}\text { All charts within CWWFA matched those produced by the Excel } \\
\text { spreadsheet. }\end{array}$ \\
\hline $\begin{array}{l}\text { 3.1.1.9 - Allow new system capacity expansion to support } \\
\text { processing volume increases resulting from increased waste } \\
\text { generation or changes in the waste fraction of the waste } \\
\text { stream. Incorporate capacities at nominal and maximum } \\
\text { conditions. }\end{array}$ & TP3.1.1 & Fail & The model only supports operating capacities at nominal. \\
\hline $\begin{array}{l}\text { 3.1.1.10 - Identify orphan wastes that do not have a } \\
\text { disposition path. }\end{array}$ & TP3.1.1 & Pass & Model behaves as expected. \\
\hline $\begin{array}{l}\text { 3.1.2.1 - Alternatives shall be compared against the baseline } \\
\text { (Ten Year Plan) with delta costs identified. }\end{array}$ & TP3.1.2 & Pass & Minor correction to the LLW storage unit cost value. \\
\hline $\begin{array}{l}\text { 3.1.2.2 - Allow input of applicable cost information (when } \\
\text { cost data are available) }\end{array}$ & TP3.1.2 & Fail & A user input screen to enter cost data is not available. \\
\hline $\begin{array}{l}\text { 3.1.3.1 - Provide limited Health and Safety risk to workers } \\
\text { and the public. Utilize waste flow results to illustrate } \\
\text { alternatives with potential risk reduction potential (e.g., } \\
\text { reduced material handling, less storage, less transportation }\end{array}$ & TP3.1.3 & Fail & $\begin{array}{l}\text { There is a major conflict between the static risk calculation used by EMI } \\
\text { and the dynamic approach used in CWWFA, which can cause } \\
\text { inconsistent risk results. A review and validation of the method is } \\
\text { needed. }\end{array}$ \\
\hline $\begin{array}{l}\text { 3.1.3.2 - Indicate risk reduction as a function of the volume of } \\
\text { waste removed from sites with high populations. }\end{array}$ & TP3.1.3 & Fail & Same issue as above. \\
\hline $\begin{array}{l}\text { 3.1.3.3 - Indicate transportation risk reduction as a function of } \\
\text { number of shipments and number of transport miles }\end{array}$ & TP3.1.3 & Fail & Same issue as above. \\
\hline $\begin{array}{l}\text { 3.1.4.1 - Measure utilization of treatment/processing and } \\
\text { disposal facilities. } \\
\text { Compare maximum total processing/ disposal needed to } \\
\text { capacities actually utilized. } \\
\text { Provide identification of redundant capabilities, including } \\
\text { existing DOE facilities and private sector capabilities. } \\
\text { Identify applications for use of excess capacity }\end{array}$ & TP3.1.4 & $\begin{array}{l}\text { Pass } \\
\text { Fail } \\
\text { Fail } \\
\text { Fail }\end{array}$ & $\begin{array}{l}\text { There are four sub-requirements that were evaluated. } \\
\text { 1. Measure utilization at facilities - pass } \\
\text { 2. Compare maximum capacities - current output charts are not scaled } \\
\text { to maximum capacity. } \\
\text { 3. Provide identification of redundant capabilities - model is } \\
\text { inadequate to meet this requirement. } \\
\text { 4. Identify application for use of excess capacity - model is } \\
\text { inadequate to meet this requirement. }\end{array}$ \\
\hline 3.1.5.1 - Optimize systems based on performance criteria. & TP3.1.5 & Fail & No optimization capability is present. \\
\hline
\end{tabular}




\begin{tabular}{|c|c|c|c|}
\hline $\begin{array}{l}\text { 3.1.5.2 - Ability to address variable uncertainty and sensitivity } \\
\text { to the expected values and system choices. }\end{array}$ & TP3.1.5 & Pass & $\begin{array}{l}\text { Variable uncertainty is demonstrated with the drivers diagram on } \\
\text { specific variables. Inspection of the program code reveals a limited } \\
\text { sensitivity analysis capability in the model. Sensitivity is conducted on } \\
\text { a set list of variables. The user cannot choose individual variable to } \\
\text { perform sensitivity analysis. This requirement will be reevaluated in } \\
\text { the FY-98 technical requirements document. }\end{array}$ \\
\hline $\begin{array}{l}\text { 3.1.6.1 - (Phase 2, FY-97) Incorporate generic equity analysis } \\
\text { capability using existing stakeholder data. Develop capability } \\
\text { for analysts to weight positive and negative influences. } \\
\text { Provide graphical representation of projected state equity } \\
\text { positions based on modeled results from the CWWFA. } \\
\text { Incorporate input/output diagrams as relevant for equity } \\
\text { analysis. }\end{array}$ & TP3.1.6 & Pass & Minor corrections on stakeholder threshold values. \\
\hline $\begin{array}{l}\text { 3.1.6.2 - Incorporate actual stakeholder equity analysis by } \\
\text { identifying (weighting) stakeholder (including DOE site } \\
\text { management, tribal governments, state and local governments, } \\
\text { special interest groups) criteria on a site-to-site, state-to-state, } \\
\text { and EM complex basis. Provide for stakeholder input from } \\
\text { geographically diverse locations (regionally or nationally). } \\
\text { Provide for open and unrestricted stakeholder involvement. } \\
\text { Aggregate and summarize stakeholder comments into a } \\
\text { manageable number of criteria (ten or less). } \\
\text { Provide stakeholder involvement from a generic waste } \\
\text { management perspective without specific knowledge of } \\
\text { CWWFA model results }\end{array}$ & TP3.1.6 & N/A & Capability is planned for inclusion into the model in 1998. \\
\hline $\begin{array}{l}\text { 3.1.7.1 - Analyze new technology systems (e.g., mobile } \\
\text { characterization is substituted for small volumes of waste } \\
\text { instead of using fixed facilities. New systems would be } \\
\text { substituted for existing capabilities, and wastes would be } \\
\text { rerouted for processing. }\end{array}$ & TP3.1.7 & Pass & Minor correction on route and shipping schedule input screen. \\
\hline $\begin{array}{l}\text { 3.1.7.2 - Define technology performance specifications (e.g., } \\
\text { volume reduction) required to achieve required outputs or } \\
\text { goals }\end{array}$ & TP3.1.7 & Pass & Model behaves as expected. \\
\hline
\end{tabular}




\section{Corrective Actions}

Based on the test results above, deficiencies are segregated depending on the level of effort that will take to correct the deficiencies. The deficiencies are divided into category 1 and 2 .

Category 1 includes all items that require minor corrections. The definition of a minor correction is one that has not failed a requirement and can be accomplished by the end of this calendar year (1997). From the test results, most of the minor deficiencies are related to the graphical user interface (GUI) applications. For the minor corrections, a list has been prepared and a dedicated GUI designer has been tasked to correct the deficiency. The CWWFA lead software designer will be responsible for ensuring completion and signed off of the work.

Category 2 includes all items that require significant corrections. The definition of a significant correction is one that has not satisfied a requirement and requires effort beyond 1997. In general, many of the category 2 items have not satisfied their respective requirements due to the following reasons:

- Some modeling features were intentionally deferred until FY-98 when key data and algorithms describing the system processes (required to support the model features) are expected to be available from the DOE 2006 Plan. The completion of the model function in FY-97 would not be useful without supporting data/algorithms. This applies to several requirements that were not met (e.g., number of transports, packages, secondary waste generation, facility operating capacities).

- Funding was not available in FY-97 to support development of all modeling functions and requirements. For example, model enhancements for transportation were deferred to begin until September 1997 when National Transportation Program funding was received.

- Some requirements were reprioritized as a result of the review with the EMI Core Team on July 29, 1997. Additional model development on total life cycle cost, health and safety risk, and decision analysis (optimization) was deferred as a result of this review.

The category 2 corrective activities will be addressed as part of CWWFA maintenance task in FY-98. The TRD will be updated to reflect added, modified, or deleted requirements as a result of the CWWFA verification. The CWWFA project manager is responsible for ensuring the disposition of the corrective actions. 
Below is a list of the category 2 items and the corrective actions for gaining compliance.

\begin{tabular}{|l|l|}
\hline Requirement & Corrective Action \\
\hline 3.1 .1 .4 & $\begin{array}{l}\text { Programming change - Modify algorithm to provide maximum number } \\
\text { of transports or packages; providing for alternative packages; and } \\
\text { providing for transport via road or rail. }\end{array}$ \\
\hline Reevaluate current EMI need for requirement \\
\hline 3.1 .1 .9 & $\begin{array}{l}\text { Reevaluate requirement and obtain data on maximum capacity if } \\
\text { required. }\end{array}$ \\
\hline 3.1 .2 .2 & $\begin{array}{l}\text { Programming change - develop a user input screen to allow direct input } \\
\text { of cost data into the model. }\end{array}$ \\
\hline $\begin{array}{l}3.1 .3 .1, \\
3.1 .3 .3\end{array}$ & $\begin{array}{l}\text { Phase 1 - simplify the method to provide relative risk. } \\
\text { Phase 2 - reevaluate the risk method to support a dynamic risk } \\
\text { calculation. }\end{array}$ \\
\hline 3.1 .4 .1 & $\begin{array}{l}\text { Obtain facility capacity from DOE sites, subject matter experts, or } \\
\text { industrial sources. } \\
\text { Reevaluate requirement to provide identification of redundant } \\
\text { capabilities. } \\
\text { Reevaluate requirement to provide identification of excess capacities. }\end{array}$ \\
\hline 3.1 .5 .1 & $\begin{array}{l}\text { This requirement will be changed to remove optimization based on } \\
\text { DOE direction. }\end{array}$ \\
\hline 3.1 .5 .2 & $\begin{array}{l}\text { Programming change - provide for sensitivity analysis on full set of } \\
\text { variables. }\end{array}$ \\
\hline & \\
\hline
\end{tabular}




\section{Appendix A - Requirements Verification Matrix}

\begin{tabular}{|c|c|}
\hline Requirement & $\begin{array}{l}\text { Verification } \\
\text { Method }\end{array}$ \\
\hline \multicolumn{2}{|l|}{ 3.1.1 WFA/Scheduling } \\
\hline $\begin{array}{l}\text { 3.1.1.1 } \\
\text { Provide quick interactive analysis of the effects of waste flow over time to } \\
\text { analyze storage requirements, transportation, and disposal bottlenecks. } \\
\text { Mass and volume flows will be developed as a function of time. } \\
\text { Constraints associated with the waste streams will be identified }\end{array}$ & $\begin{array}{l}\text { Test-TP3.1.1 } \\
\text { Inspection-TP3.1.1 } \\
\text { Test- TP3.1.1 }\end{array}$ \\
\hline $\begin{array}{l}\text { Summarize impacts of acceleration and delays associated with the } \\
\text { management of waste (reduction of untreated waste inventories, } \\
\text { reclassification of wastes, accumulation in storage, buildup of waste in } \\
\text { disposal). }\end{array}$ & Test-TP3.1.1 \\
\hline $\begin{array}{l}\text { 3.1.1.3 } \\
\text { Identify need for new (or underutilized) facilities in the DOE complex that } \\
\text { could be used to process bottlenecks. } \\
\text { Allow facility use preference to facilitate analysis of capacity utilization }\end{array}$ & $\begin{array}{l}\text { Test-TP3.1.1 } \\
\text { Inspection- TP3.1.1 }\end{array}$ \\
\hline $\begin{array}{l}\text { 3.1.1.4 } \\
\text { Identify impacts from transportation bottlenecks and alternatives. } \\
\text { Include alternative packaging and transport mode options. }\end{array}$ & Inspection- TP3.1.1 \\
\hline $\begin{array}{l}\text { 3.1.1.5 } \\
\text { Summarize system impacts from future ER and D\&D waste sources (show } \\
\text { when/how much the current maximum disposal receipt rate }\left(\mathrm{m}^{3} / \mathrm{yr}\right) \text { is } \\
\text { impacted by input from waste sources) }\end{array}$ & $\begin{array}{l}\text { Test \& inspection } \\
\text { TP3.1.1 }\end{array}$ \\
\hline $\begin{array}{l}\text { 3.1.1.6 } \\
\text { Show processing flow effects from varying split of waste processed by } \\
\text { alternative processes (e.g., WIPP Waste Acceptance Criteria, versus treatment } \\
\text { to land disposal restricted, volume reduction, waste form density changes, and } \\
\text { secondary waste production. }\end{array}$ & $\begin{array}{l}\text { Test \& Inspection } \\
\text { TP3.1.1 }\end{array}$ \\
\hline $\begin{array}{l}\text { A.1.1.7 } \\
\text { Allow separation of wastes after processing into separate fractions (e.g., HLW } \\
\text { is split into high and low activity wastes). Separated waste fractions can be } \\
\text { directed to alternative management processes }\end{array}$ & Inspection -TP3.1.1 \\
\hline $\begin{array}{l}\text { 3.1.1.8 } \\
\text { Show impacts from consolidated treatment, storage, and disposal of similar } \\
\text { wastes (e.g., aMLLW and mixed TRU). }\end{array}$ & Test-TP3.1.1 \\
\hline $\begin{array}{l}3.1 .1 .9 \\
\text { Allow new system capacity expansion to support processing volume increases } \\
\text { resulting from increased waste generation or changes in the waste fraction of } \\
\text { the waste stream. }\end{array}$ & Inspection-TP3.1.1 \\
\hline
\end{tabular}




\begin{tabular}{|c|c|}
\hline \multicolumn{2}{|l|}{ Incorporate capacities at nominal and maximum conditions. } \\
\hline $\begin{array}{l}\text { 3.1.1.10 } \\
\text { Identify orphan wastes that do not have a disposition path. }\end{array}$ & Test-TP3.1.1 \\
\hline . & \\
\hline \multicolumn{2}{|l|}{ 3.1.2 Mortgage Reduction Performance } \\
\hline $\begin{array}{l}3.1 .2 .1 \\
\text { Alternatives shall be compared against the baseline (Ten Year Plan) with delta } \\
\text { costs identified. }\end{array}$ & Test-TP3.1.2 \\
\hline $\begin{array}{l}3.1 .2 .2 \\
\text { Allow input of applicable cost information (when cost data are available) }\end{array}$ & Test-TP3.1.2 \\
\hline & \\
\hline \multicolumn{2}{|l|}{ 3.1.3 Risk Reduction } \\
\hline $\begin{array}{l}\text { 3.1.3.1 } \\
\text { Provide limited Health and Safety risk to workers and the public. } \\
\text { Utilize waste flow results to illustrate alternatives with potential risk reduction } \\
\text { potential (e.g., reduced material handling, less storage, less transportation }\end{array}$ & Test-TP3.1.3 \\
\hline $\begin{array}{l}\text { Indicate risk reduction as a function of the volume of waste removed from } \\
\text { sites with high populations. Risk is assumed to be reduced due to offsite } \\
\text { population no longer exposed to the potential hazards of the wastes. }\end{array}$ & Test-TP3.1.3 \\
\hline $\begin{array}{l}\text { Indicate transportation risk reduction as a function of number of shipments } \\
\text { and number of transport miles }\end{array}$ & Test-TP3.1.3 \\
\hline \multicolumn{2}{|l|}{ Facility Operational Performance } \\
\hline $\begin{array}{l}3.1 .4 .1 \\
\text { Measure utilization of treatment/processing and disposal facilities. } \\
\text { Compare maximum total processing/ disposal needed to capacities actually } \\
\text { utilized. } \\
\text { Provide identification of redundant capabilities, including existing DOE } \\
\text { facilities and private sector capabilities. } \\
\text { Identify applications for use of excess capacity }\end{array}$ & Test-TP3.1.4 \\
\hline$\cdot$ & \\
\hline \multicolumn{2}{|l|}{ 3.1.5 Decision Analysis } \\
\hline $\begin{array}{l}3.1 .5 .1 \\
\text { Optimize systems based on performance criteria (e.g., identify alternatives } \\
\text { that maximize waste moved to final disposition during the next ten years. }\end{array}$ & Test-TP3.1.5 \\
\hline $\begin{array}{l}3.1 .5 .2 \\
\text { Ability to address variable uncertainty and sensitivity to the expected values } \\
\text { and system choices. }\end{array}$ & Test-TP3.1.5 \\
\hline \multicolumn{2}{|l|}{ 3.1.6 Other Performance Measures (Equity) } \\
\hline $\begin{array}{l}3.1 .6 .1 \\
\text { (Phase 2, FY-97) Incorporate generic equity analysis capability using existing } \\
\text { stakeholder data. }\end{array}$ & \begin{tabular}{|l} 
Test-TP3.1.6 \\
\end{tabular} \\
\hline Develop capability for analysts to weight positive and negative influences. & \\
\hline Provide graphical representation of projected state equity positions based on & \\
\hline
\end{tabular}


modeled results from the CWWFA.

Incorporate input/output diagrams as relevant for equity analysis. 3.1.6.2 (FY-98)

Incorporate actual stakeholder equity analysis by identifying (weighting)

Test-TP3.1.6 stakeholder (including DOE site management, tribal governments, state and local governments, special interest groups) criteria on a site-to-site, state-tostate, and EM complex basis.

Provide for stakeholder input from geographically diverse locations (regionally or nationally).

Provide for open and unrestricted stakeholder involvement.

Aggregate and summarize stakeholder comments into a manageable number of criteria (ten or less).

Provide stakeholder involvement from a generic waste management perspective without specific knowledge of CWWFA model results

\subsubsection{Technology Performance}

3.1.7.1

Analyze new technology systems (e.g., mobile characterization is substituted

Test-TP3.1.7

for small volumes of waste instead of using fixed facilities. New systems would be substituted for existing capabilities, and wastes would be rerouted for processing.

3.1.7.2

Define technology performance specifications (e.g., volume reduction) required to achieve required outputs or goals 
APPENDIX B

TEST PROCEDURES 


\section{TEST PROCEDURE - TP 3.1.1 \\ Waste Flow and Scheduling}

\section{Purpose}

This test procedure has been designed to test the waste flow aspects of the CWWFA. The ability to track the movement or flow of waste between facilities provides the basis for all other functions of CWWFA (i.e., risk, cost, equity analysis). Waste flow is the scheduling of waste between facilities for processing, storage, or disposal. This procedure will is designed to verify requirements 3.1.1 through 3.1.10 in the Requirements Verification Matrix in Appendix A.

\section{Objective}

This test procedure is designed to exercise the waste flow aspect of the CWWFA. The requirements for the waste flow functionality will be verified by inspection and testing. When the requirement is verified by testing, the CWWFA output charts will be compared against a spreadsheet.

\section{Test Method}

Method 1 - Verification of output charts related to waste flow. This method is comprised of several cases. Each case increases in complexity and is intended to verify the requirements over the full spectrum of potential use. General guidelines for case methodology are described in the Case Discussion below. The tester will use these guidelines to define the case. Input Parameter Recording Sheets will be used to document the conditions under which the test is executed. After running the model, the tester will compare the results with a spreadsheet. If the model results are within $\pm 10 \%$, the test passes. If the model results are outside the acceptable range, corrective action will be identified and the model will be re-tested. After the corrective action has been implemented, earlier tests will be rerun to ensure that there were no unexpected effects of the action. Rerunning the previous tests is at the discretion of the Software Lead.

\section{Case 1}

- Transfer of waste from legacy storage to treatment

- New generation schedule and transfer of combined legacy and newGen to treatment

- Treatment facility volume changes

- Orphan waste due to conversion profiles and shipping route conflict

- Back-end storage on treatment facility when next facility. is unavailable

Case 2

- Transportation of waste from one site's legacy storage to another site's treatment for three different waste types (HLW, TRU, and MLLW).

- Transportation of one site's combined legacy and new generation of waste to another site's treatment. Effect of multiple treatment facility volume changes and density changes (TRUW) on transportation shipments and packages. 
Case 3

- Multiple shipping routes from legacy storage

- Multiple shipping schedules per shipping route

\section{Case 4}

- Multiple shipping routes from legacy storage and treatment

- Multiple shipping schedules per shipping route

- Effects of facility conversion profiles

Case 5

- Multiple waste streams requiring processing or disposal at a single facility

Method 2 - Units of waste flow. Verify via discussions with programmers the units in which flows are tracked.

Method 3-Bottleneck processing. Verify via inspection the ability to identify under or over utilized facilities within the system that could impact possible bottlenecks. Verify via inspection the ability to impact bottlenecks by re-routing waste volumes to a different facility.

Method 4 - Transportation bottlenecks. Verify via discussions with programmers the system constraints that are imposed on transportation.

Method 5 - Alternative packaging and transport mode options. Verify via discussions with programmers the available transportation packaging and mode options.

Method 6-Addition of new volumes. Verify via inspection the ability to input new volumes to accommodate future environmental restoration or decontamination and decommissioning waste volumes.

Method 7 - Treatment facility characteristics. Verify via inspection, the ability to change treatment facility characteristics.

Method 8-Secondary waste production. Verify via inspection, the capability to incorporate secondary waste production.

Method 9 - Fractionate waste. Verify via inspection the ability to separate a single waste stream after processing into fractions and the ability to have each fraction further processed in a different manner.

Method 10 - Nominal and maximum operating capacities of facilities. Verify via discussions with programmers the ability to have more than one operating capacity for a facility. 


\section{Reporting}

TP - Test Procedure

REQD - Requirement

P/F - Pass/Fail

\begin{tabular}{|c|c|c|c|}
\hline TP & REQD & Result/Comment & $\mathrm{P} / \mathrm{F}$ \\
\hline $\begin{array}{l}\text { TP3.1.1 } \\
\text { Method } 1\end{array}$ & $\begin{array}{l}3.1 .1 .1 \text {, } \\
3.1 .1 .2, \\
3.1 .1 .5 \\
3.1 .1 .6 \\
3.1 .1 .8 \\
3.1 .1 .10\end{array}$ & $\begin{array}{l}\text { All charts within CWWFA matched those produced by the } \\
\text { Excel spreadsheet with the following exception: CASE2E } \\
\text { the disposal facility's facility capacity detail chart did not } \\
\text { show the receipt of waste. However, the underlying data for } \\
\text { the chart is correct. } \\
\text { Additional comment: the dynamic scheduling capability of } \\
\text { the CWWFA needs to be explained in the user's manual and } \\
\text { the specification document. This capability worked } \\
\text { correctly, but was not incorporated in to the test procedure. }\end{array}$ & $\begin{array}{l}\text { Pass - } \\
\text { need } \\
\text { minor } \\
\text { correction }\end{array}$ \\
\hline $\begin{array}{l}\text { TP3.1.1 } \\
\text { Method } 2\end{array}$ & 3.1 .1 .1 & $\begin{array}{l}\text { For every unit of waste, the model tracks a volume in cubic } \\
\text { meters and a density in kilograms per cubic meter. With } \\
\text { both of these numbers the model can convert between } \\
\text { volume and mass as necessary. }\end{array}$ & Pass \\
\hline $\begin{array}{l}\text { TP3.1.1 } \\
\text { Method } 3\end{array}$ & 3.1 .1 .3 & $\begin{array}{l}\text { The facility capacity detail chart identifies bottlenecks due to } \\
\text { insufficient processing capacity or a timing conflict at the } \\
\text { next facility in the routing. } \\
\text { In the shipping route window for a waste stream, the user } \\
\text { can change the destination facilities according to their } \\
\text { preference or add a new routing if a bottleneck is } \\
\text { encountered. }\end{array}$ & Pass \\
\hline $\begin{array}{l}\text { TP3.1.1 } \\
\text { Method } 4\end{array}$ & 3.1 .1 .4 & $\begin{array}{l}\text { The model does not constrain the number of transports or the } \\
\text { number of packages. Thus no bottlenecks will occur due to } \\
\text { transportation limitations. }\end{array}$ & Fail \\
\hline $\begin{array}{l}\text { TP3.1.1 } \\
\text { Method } 5\end{array}$ & 3.1 .1 .4 & $\begin{array}{l}\text { The model does not allow alternative packages to be used for } \\
\text { transportation. It also only transports waste via road. }\end{array}$ & Fail \\
\hline $\begin{array}{l}\text { TP3.1.1 } \\
\text { Method } 6\end{array}$ & 3.1 .1 .5 & $\begin{array}{l}\text { The model supports addition of new generation volumes for } \\
\text { a waste stream. The new generation volumes can be } \\
\text { interpreted as incoming waste from environmental } \\
\text { restoration or decontamination and decommissioning } \\
\text { activities. }\end{array}$ & Pass \\
\hline $\begin{array}{l}\text { TP3.1.1 } \\
\text { Method } 7\end{array}$ & 3.1 .1 .6 & $\begin{array}{l}\text { The model allows the volume change factor for a facility to } \\
\text { be changed by the user. The user may also change the } \\
\text { conversion profile for a processing facility to impact } \\
\text { physical characteristics of the waste exiting the facility. }\end{array}$ & Pass \\
\hline
\end{tabular}




\begin{tabular}{|l|l|l|l|}
\hline $\begin{array}{l}\text { TP3.1.1 } \\
\text { Method 8 }\end{array}$ & 3.1 .1 .6 & $\begin{array}{l}\text { The model does not support secondary waste generation } \\
\text { from processing. However, the user could specify an } \\
\text { additional shipping route to duplicate the results of a } \\
\text { secondary waste stream due to processing. But this would } \\
\text { have to be done on a stream by stream basis as opposed to a } \\
\text { single facility producing secondary waste regardless of the } \\
\text { characteristics of the waste being processed. }\end{array}$ & Fail \\
\hline $\begin{array}{l}\text { TP3.1.1 } \\
\text { Method 9 }\end{array}$ & 3.1 .1 .7 & $\begin{array}{l}\text { The model supports multiple shipping routes between } \\
\text { facilities. Each shipping route can represent a fraction of } \\
\text { waste generated from a processing facility. }\end{array}$ & Pass \\
\hline $\begin{array}{l}\text { TP3.1.1 } \\
\text { Method } \\
10\end{array}$ & 3.1 .19 & The model only supports operating capacities at nominal. & Fail \\
\hline
\end{tabular}




\section{Case Discussion}

The fundamental basis of the CWWFA is the ability to track waste as it "flows" between facilities for processing, storage, or disposal. Five test cases have been established to examine how the CWWFA behaves under certain conditions. The test cases increase in level of complexity and the tests are targeted toward a specific functionality with CWWFA.

Functionality that has been previously tested is not re-tested in subsequent cases. Specific details regarding the initial parameters and settings for each case can be found in Appendix A.

\begin{tabular}{|c|c|c|c|c|c|}
\hline Case & $\begin{array}{c}\text { Target } \\
\text { Functionality }\end{array}$ & \multicolumn{4}{|c|}{ Description } \\
\hline 1 & Scheduling & $\begin{array}{l}\text { Whate strean } \\
\text { This case in } \\
\text { at the same } \\
\text { Variations } \\
\text { changing th } \\
\text { volume cha } \\
\text { conversion }\end{array}$ & $\begin{array}{l}\text { Treatrent } \\
\text { Facity } \\
\text { lves a si } \\
\text { e. The } \\
\text { hin this } \\
\text { hipping } \\
\text { e factor } \\
\text { lit with }\end{array}$ & $\begin{array}{l}\text { Dispossit } \\
\text { Facility } \\
\text { Naste str } \\
\text { f waste i } \\
\text { nclude a } \\
\text { lules bet } \\
\text { treatme } \\
\text { e type ch }\end{array}$ & $\begin{array}{l}\text { eing processed and disposed } \\
\text { significant for this test. } \\
\text { a new generation volume, } \\
\text { facilities, changing the } \\
\text { lity, and adding a }\end{array}$ \\
\hline 2 & $\begin{array}{l}\text { Transportation } \\
\text { and Conversion } \\
\text { Profile }\end{array}$ & $\begin{array}{l}\text { Asaste streant } \\
\text { As with Ca } \\
\text { functionalit } \\
\text { site transpo } \\
\text { case there a } \\
\text { number of } \\
\text { type of was } \\
\text { changing th } \\
\text { profile. }\end{array}$ & $\begin{array}{l}\text { Treatment } \\
\text { Facility } \\
11 \text { this ca } \\
\text { of this te } \\
\text { Each cr } \\
\text { two site } \\
\text { ckages, } \mathrm{r} \\
\text { being tr } \\
\text { density a }\end{array}$ & $\begin{array}{l}\text { Treatrent } \\
\text { agcility } \\
\text { olves a s } \\
\text { ansporta } \\
\text { box rep } \\
\text { ved. Th } \\
\text { of ship } \\
\text { ed. Va } \\
\text { ste type }\end{array}$ & $\begin{array}{l}\text { Disposal } \\
\text { Faciity } \\
\text { waste stream. As the target } \\
\text { it is necessary to incur off- } \\
\text { ts a different site. In this } \\
\text { nsportation variables (i.e., } \\
\text { s) are dependent upon the } \\
\text { as within this case include } \\
\text { treatment conversion }\end{array}$ \\
\hline 3 & $\begin{array}{l}\text { Split from } \\
\text { legacy storage }\end{array}$ & $\begin{array}{l}\text { Waste strearn } \\
\text { For a singl } \\
\text { shipping ro } \\
\text { within this } \\
\text { shipping ro }\end{array}$ & \begin{tabular}{|c}
$\begin{array}{c}\text { Treatment } \\
\text { Facility }\end{array}$ \\
Treatment \\
Facility \\
aste stre \\
es betwe \\
se incluc \\
e and mu
\end{tabular} & $\begin{array}{l}\text { Disposal } \\
\text { Facility } \\
\text { se } 3 \text { inv } \\
\text { acy stor } \\
\text { ging th } \\
\text { shipping }\end{array}$ & $\begin{array}{l}\text { two sites and multiple } \\
\text { d treatment. Variations } \\
\text { tion of waste for each } \\
\text { dules per shipping route. }\end{array}$ \\
\hline
\end{tabular}




\begin{tabular}{|c|c|c|}
\hline Case & $\begin{array}{c}\text { Target } \\
\text { Functionality }\end{array}$ & Description \\
\hline 4 & $\begin{array}{l}\text { Split from } \\
\text { primary } \\
\text { treatment }\end{array}$ & $\begin{array}{l}\text { Case } 4 \text { is similar to case three in that it focuses on multiple shipping } \\
\text { routes departing from a single facility. Case } 4 \text { involves } 3 \text { sites. } \\
\text { Variations within this case include multiple shipping routes from } \\
\text { legacy storage and from each treatment facility. This case will also } \\
\text { involve a fairly complex conversion profile for each treatment facility. }\end{array}$ \\
\hline 5 & $\begin{array}{l}\text { Multiple waste } \\
\text { stream feeds to } \\
\text { a single facility }\end{array}$ & $\begin{array}{l}\text { waste Stream } \\
\text { Waste Stream } \\
\text { Waste Stream } 5 \text { involves multiple waste streams from different sites feeding } \\
\text { into a single facility. Variations within this case include varying the } \\
\text { volumes and dates of waste transferred between facilities. }\end{array}$ \\
\hline
\end{tabular}


CASE: Case 1a

INITIAL PARAMETERS \& RESULTS REPORT

DATE: $11 / 12 / 97$

Evaluator: K B Oswald

1.0 Waste Stream Information

\begin{tabular}{|l|l|}
\hline Originating Site & MOPL \\
\hline Waste Stream ID \& Name & MOPLWS1-Case1 \\
\hline Legacy volume (m3) & 10,000 \\
\hline
\end{tabular}

1.1 New Generation

\begin{tabular}{|l|l|l|}
\hline \multicolumn{1}{|c|}{ Start Year } & \multicolumn{1}{|c|}{ End Year } & Annual Volume (m3) \\
\hline NA & NA & NA \\
\hline & & \\
\hline & & \\
\hline & & \\
\hline
\end{tabular}

1.2 Shipping Route(s)

\begin{tabular}{|l|l|l|}
\hline \multicolumn{1}{|c|}{ Route ID } & \multicolumn{1}{|c|}{ Source Facility } & Destination Facility \\
\hline C1a-1 & Legacy-MOPL & MOPLFAC1-CASE1 \\
\hline C1a-2 & MOPLFAC1-CASE1 & MOPLFAC2-CASE1 \\
\hline & & \\
\hline & & \\
\hline & & \\
\hline & & \\
\hline
\end{tabular}

1.3 Shipping Schedule(s)

\begin{tabular}{|c|c|c|c|}
\hline Route ID: Cla-1 & & & \\
\hline Start Year & End Year & Waste Type & Annual Volume (m3) \\
\hline 1997 & 2070 & ILW & 135.14 \\
\hline & & & \\
\hline & & & \\
\hline
\end{tabular}

1.3 Shipping Schedule(s) (continued)

\begin{tabular}{|c|c|c|c|}
\hline Route ID: C1a-2 & \multicolumn{2}{|c|}{ Waste Type } & Annual Volume (m3) \\
\hline Start Year & \multicolumn{1}{|c|}{ End Year } & Wh & 135.14 \\
\hline & 2070 & & \\
\hline & & & \\
\hline & & & \\
\hline
\end{tabular}

2.0 Facility Information

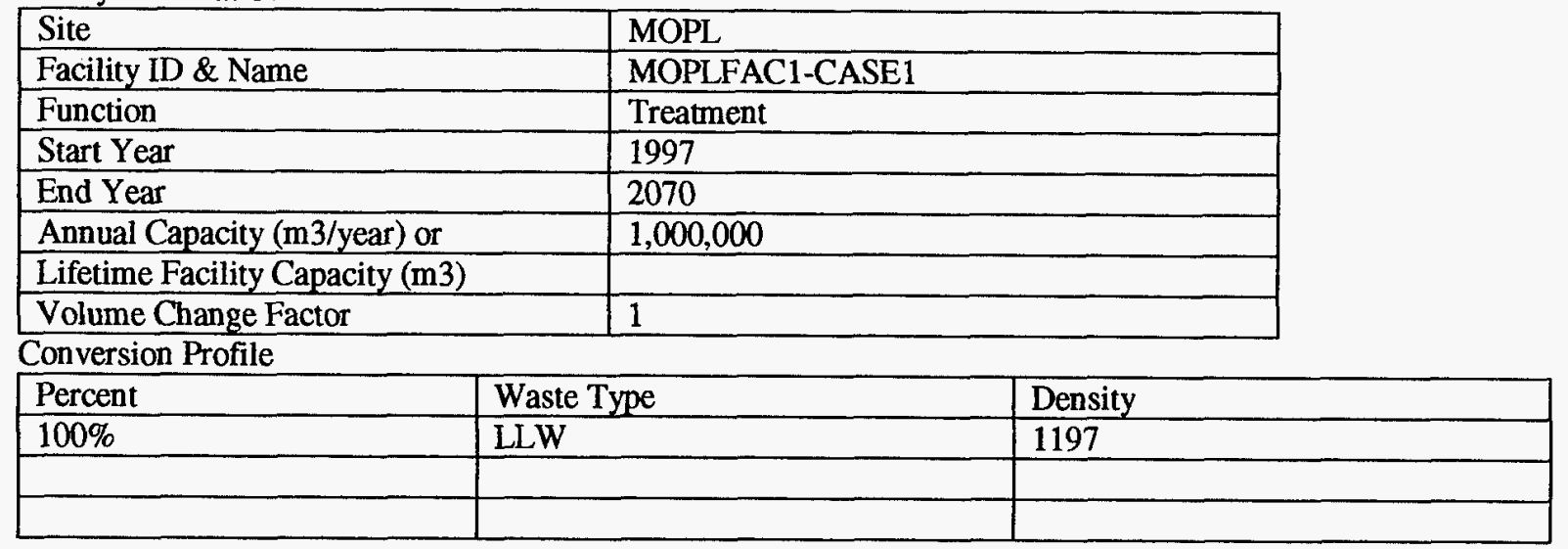


INITIAL PARAMETERS \& RESULTS REPORT

CASE: Case 1a

DATE: $11 / 12 / 97$

Evaluator: K B Oswald

2.0 Facility Information

\begin{tabular}{|l|l|}
\hline Site & MOPL \\
\hline Facility ID \& Name & MOPLFAC2-CASE1 \\
\hline Function & Disposal \\
\hline Start Year & 1997 \\
\hline End Year & 2070 \\
\hline Annual Capacity (m3/year) or & $1,000,000$ \\
\hline Lifetime Facility Capacity (m3) & \\
\hline Volume Change Factor & 1 \\
\hline
\end{tabular}

Conversion Profile

\begin{tabular}{|l|l|l|}
\hline Percent & Waste Type & Density \\
\hline NA & NA & NA \\
\hline & & \\
\hline
\end{tabular}




\begin{tabular}{lll} 
CASE: Case 1a & DATE: $11 / 12 / 97$ & Evaluator: K B Oswald \\
\hline
\end{tabular}

EXCEL CHART:

\section{Waste Disposition}

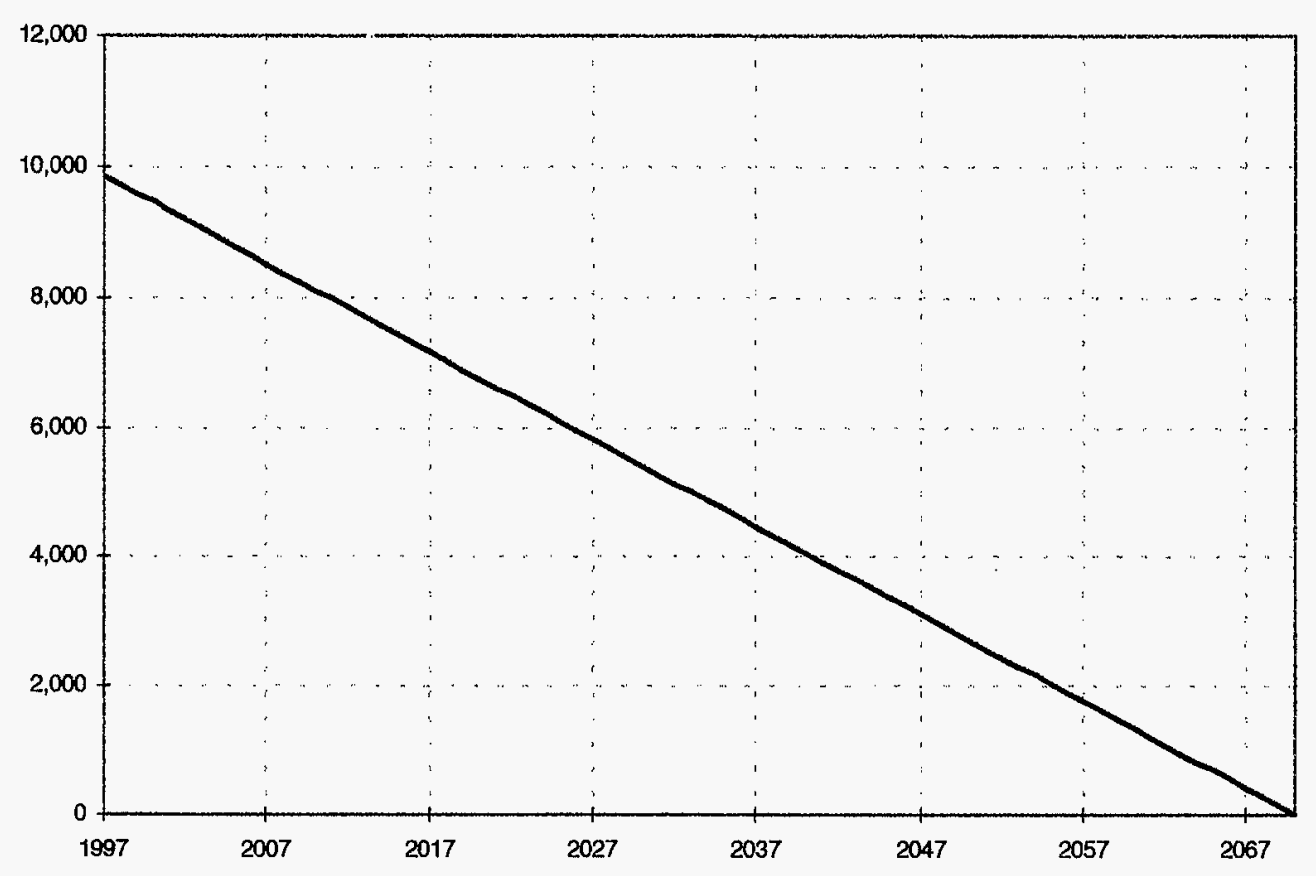

\section{CWWFA CHART:}

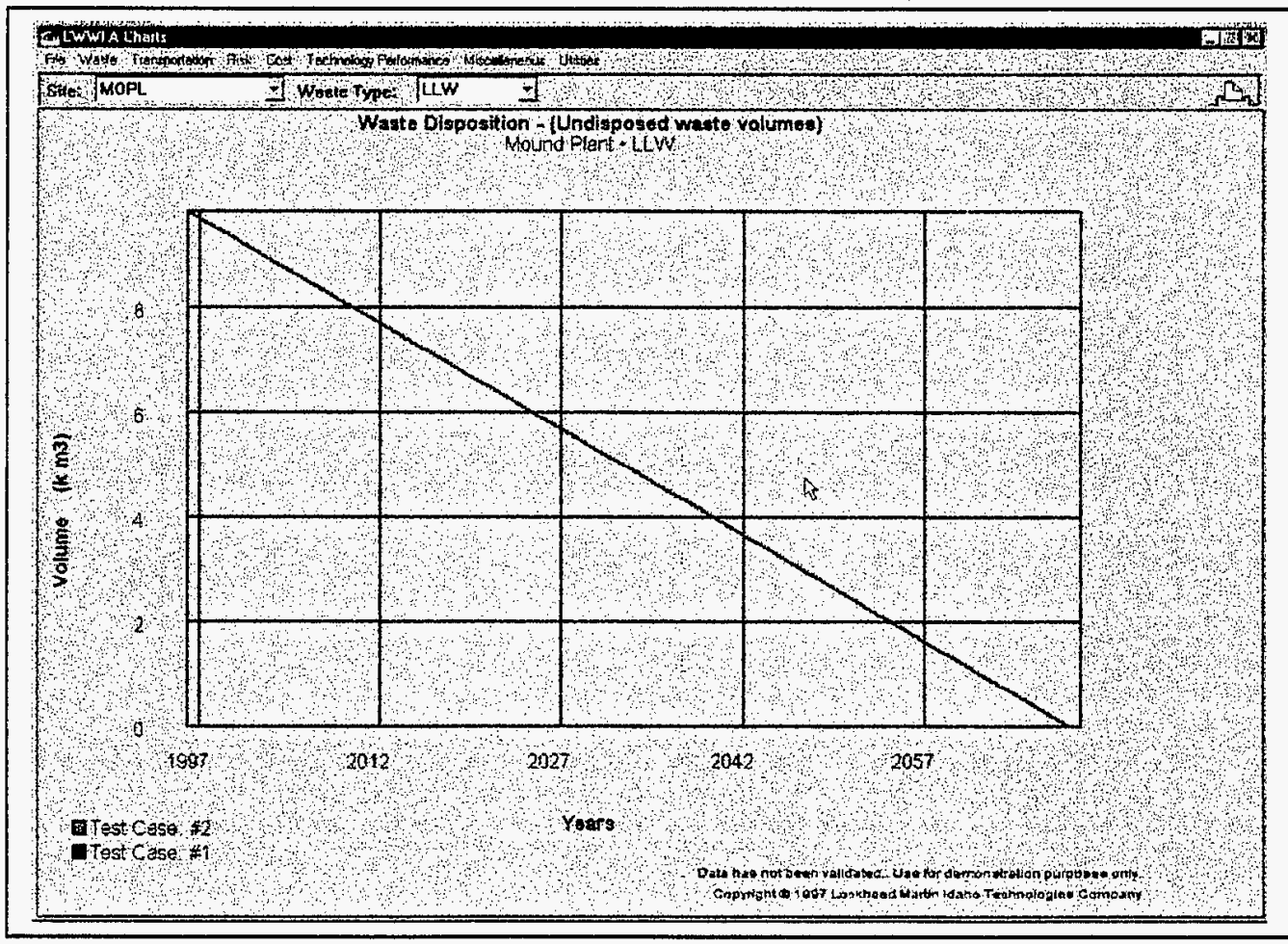


INITIAL PARAMETERS \& RESULTS REPORT

CASE: Case 1a

DATE: $11 / 12 / 97$

Evaluator: K B Oswald

\section{EXCEL CHART (MOPLFAC1-CASE1):}

\section{Facility Capacity Detail}

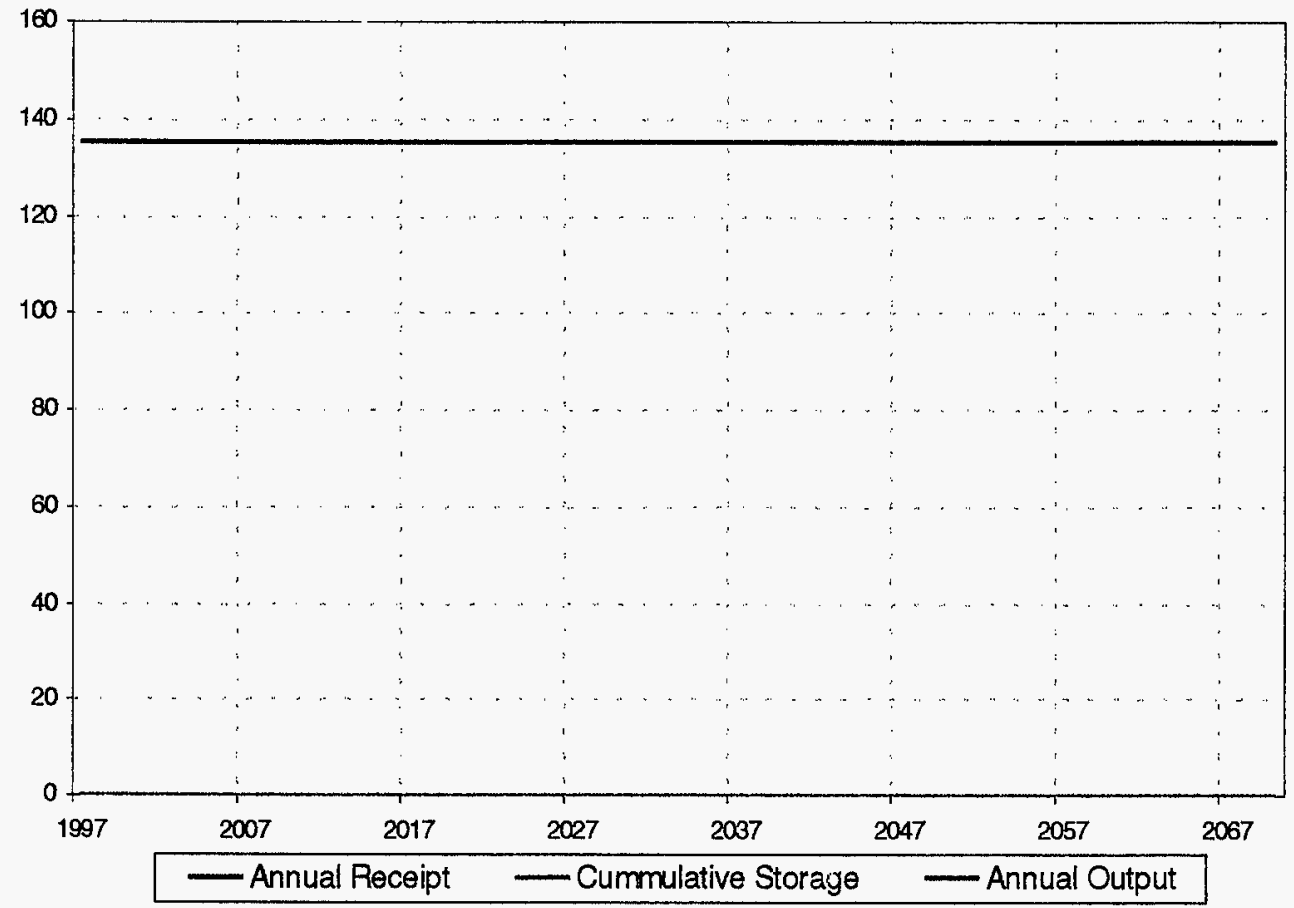

\section{CWWFA CHART:}

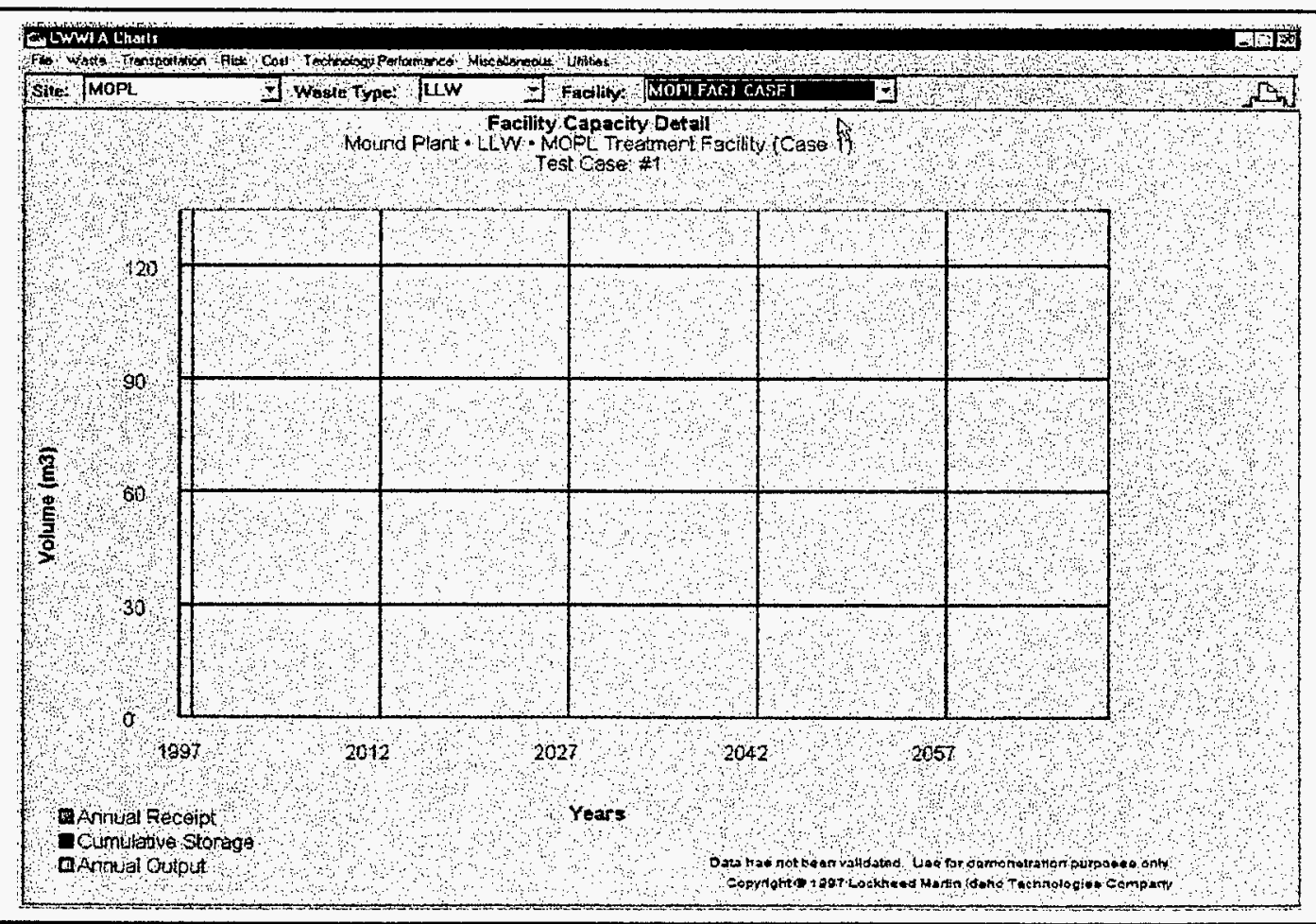


CASE: Case $1 b$

INITIAL PARAMETERS \& RESULTS REPORT

DATE: $11 / 12 / 97$

Evaluator: K B Oswald

1.0 Waste Stream Information

\begin{tabular}{|l|l|}
\hline Originating Site & MOPL \\
\hline Waste Stream ID \& Name & MOPLWS1-Case1 \\
\hline Legacy volume (m3) & 10,000 \\
\hline
\end{tabular}

1.1 New Generation

\begin{tabular}{|l|l|l|}
\hline \multicolumn{1}{|c|}{ Start Year } & \multicolumn{1}{|c|}{ End Year } & \multicolumn{1}{c|}{ Annual Volume (m3) } \\
\hline NA & NA & NA \\
\hline & & \\
\hline & & \\
\hline & & \\
\hline
\end{tabular}

1.2 Shipping Route(s)

\begin{tabular}{|l|l|l|}
\hline \multicolumn{1}{|c|}{ Route ID } & \multicolumn{1}{|c|}{ Source Facility } & Destination Facility \\
\hline Clb-1 & Legacy-MOPL & MOPLFAC1-CASE1 \\
\hline C1b-2 & MOPLFAC1-CASE1 & MOPLFAC2-CASE1 \\
\hline & & \\
\hline & & \\
\hline & & \\
\hline & & \\
\hline
\end{tabular}

1.3 Shipping Schedule(s)

\begin{tabular}{|c|c|c|c|}
\hline \multicolumn{4}{|l|}{ Route ID: C1b-1 } \\
\hline Start Year & End Year & Waste Type & Annual Volume (m3) \\
\hline 1997 & 2020 & LLW & 417 \\
\hline & & & \\
\hline & & & \\
\hline & & & \\
\hline
\end{tabular}

1.3 Shipping Schedule(s) (continued)

\begin{tabular}{|c|l|l|l|}
\hline Route ID: C1b-2 & \multicolumn{2}{|c|}{ Waste Type } & Annual Volume (m3) \\
\hline Start Year & \multicolumn{1}{|c|}{ End Year } & LLW & 152 \\
\hline & 2070 & & \\
\hline & & & \\
\hline
\end{tabular}

2.0 Facility Information

\begin{tabular}{|l|l|}
\hline Site & MOPL \\
\hline Facility ID \& Name & MOPLFAC1-CASE1 \\
\hline Function & Treatment \\
\hline Start Year & 1997 \\
\hline End Year & 2070 \\
\hline Annual Capacity (m3/year) or & $1,000,000$ \\
\hline Lifetime Facility Capacity (m3) & \\
\hline Volume Change Factor & 2 \\
\hline
\end{tabular}

Conversion Profile

\begin{tabular}{|l|l|l|}
\hline Percent & Waste Type & Density \\
\hline $100 \%$ & LLW & 1197 \\
\hline & & \\
\hline & & \\
\hline
\end{tabular}



CASE: Case $1 b$
DATE: $11 / 12 / 97$
Evaluator: K B Oswald

EXCEL CHART:

Waste Disposition

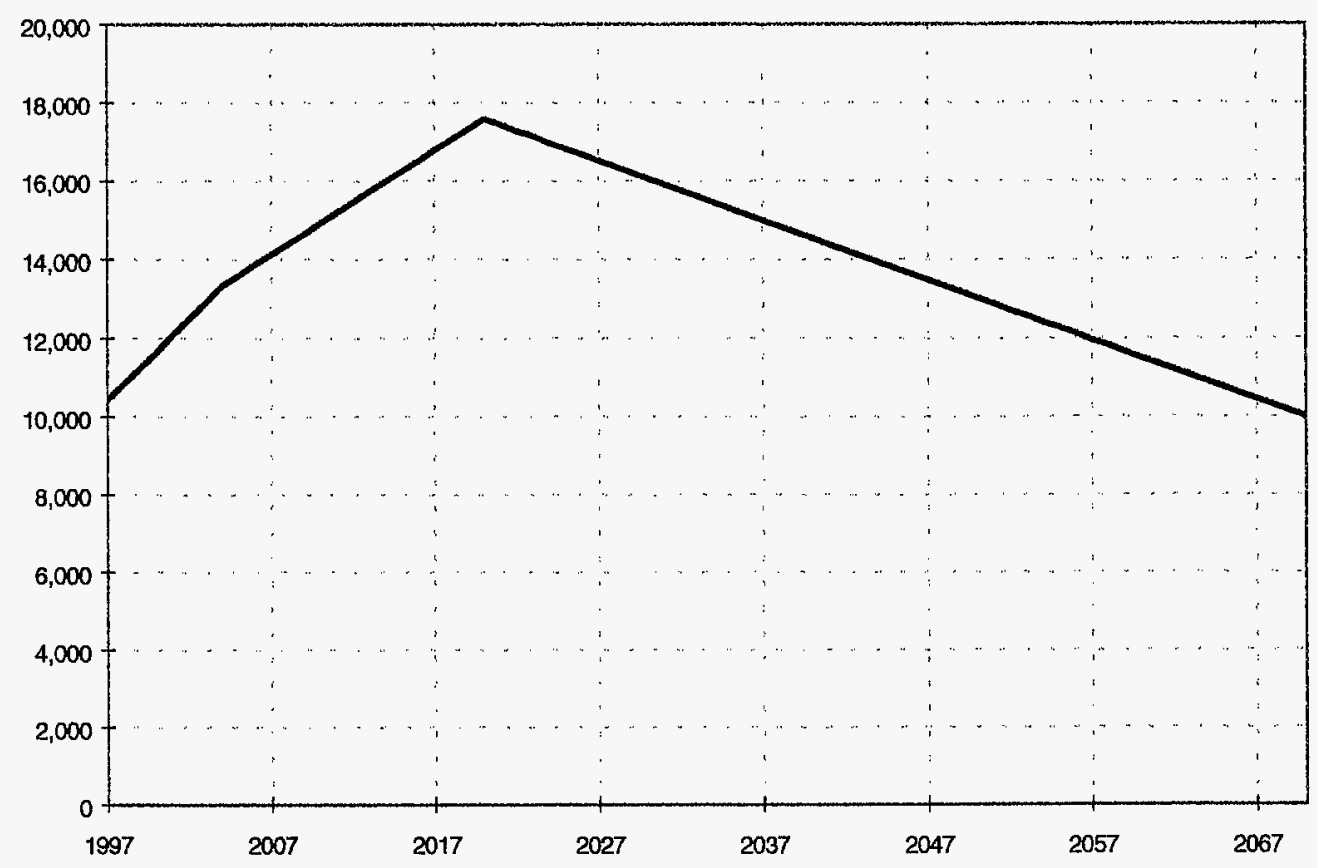

CWWFA CHART:

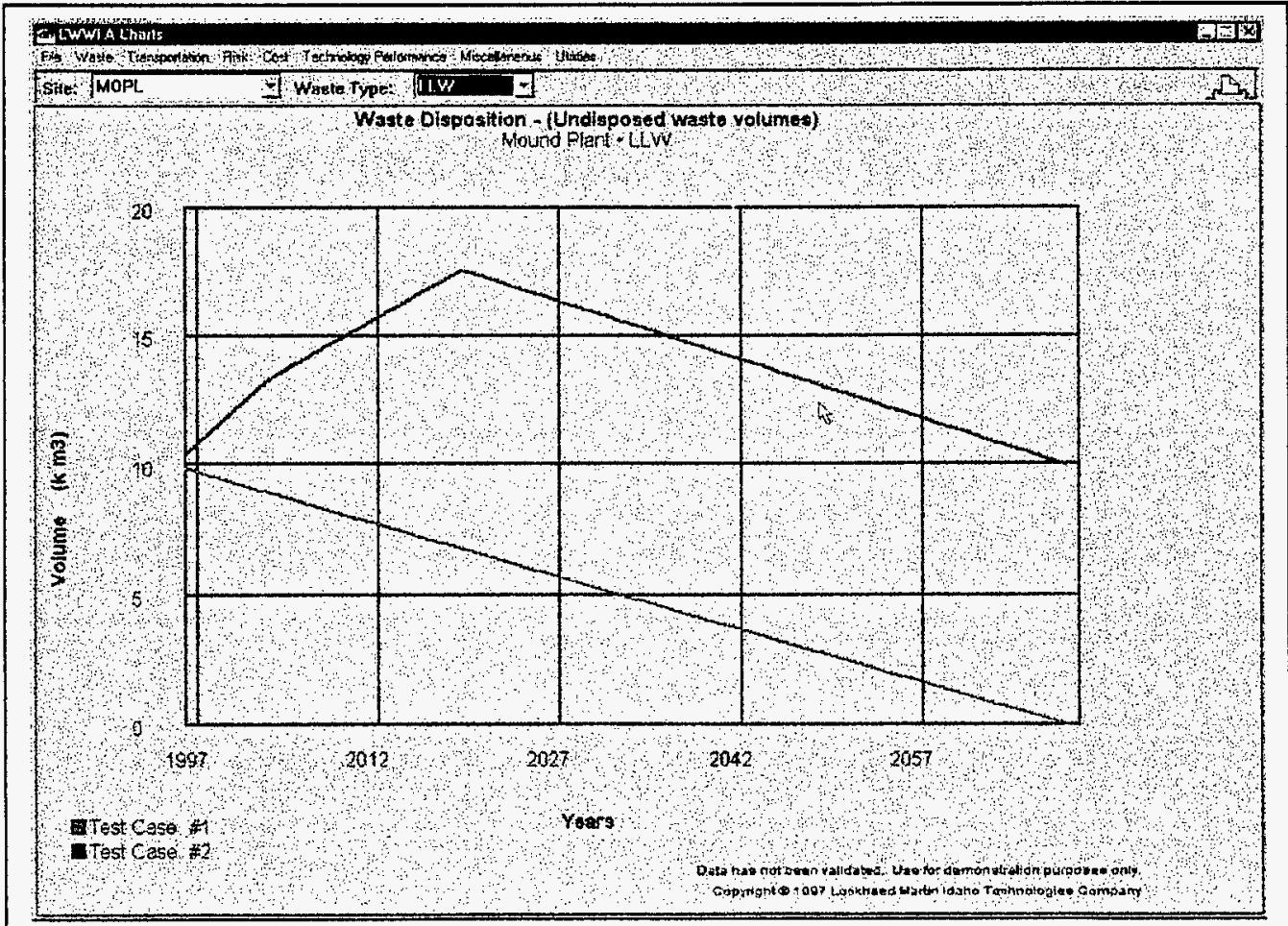


EXCEL CHART (MOPLFAC1-CASE1):

\section{Eacility Capacity Detail}

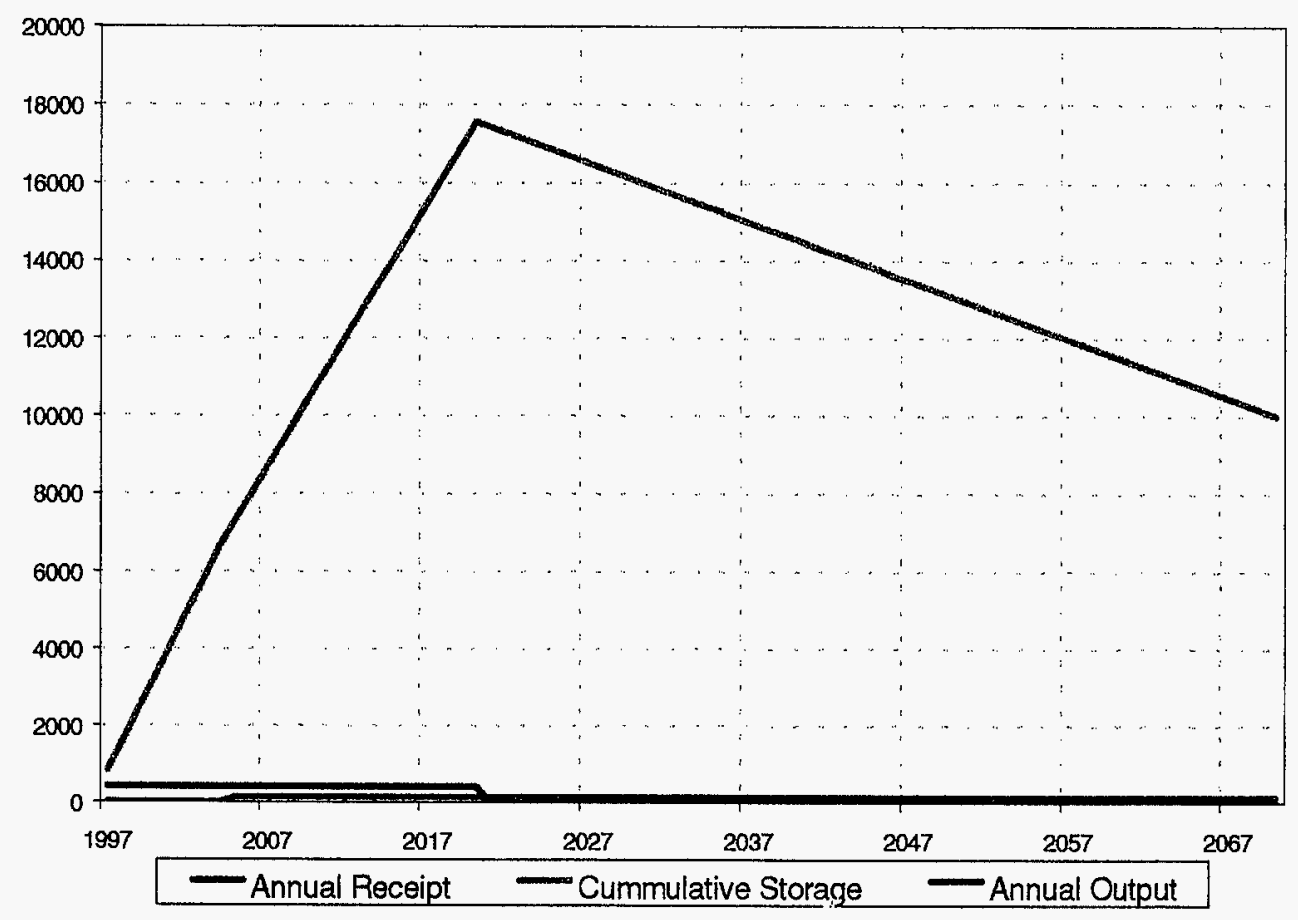

CWWFA CHART:

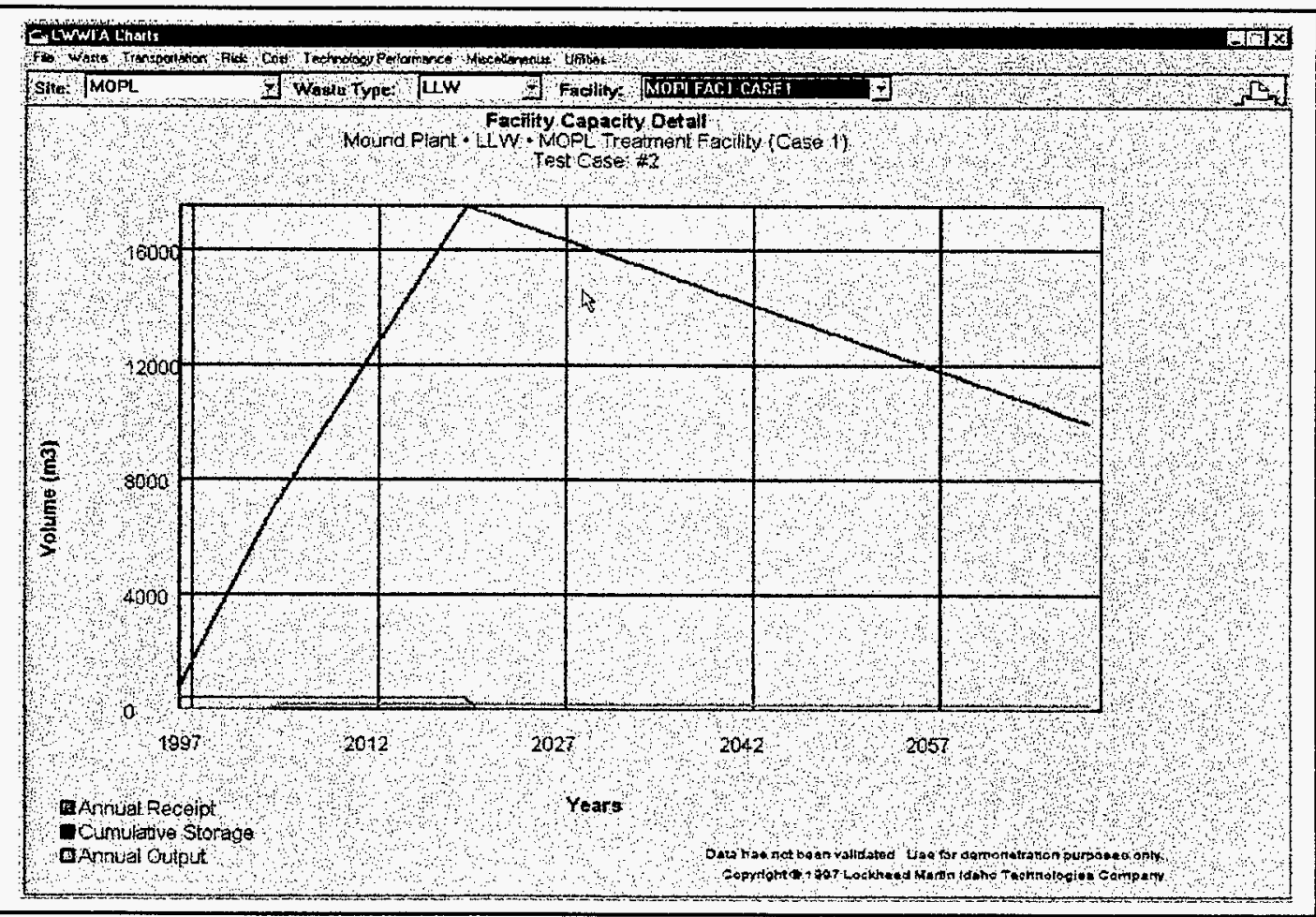


INITIAL PARAMETERS \& RESULTS REPORT

CASE: Case 1c

DATE: $11 / 12 / 97$

Evaluator: K B Oswald

1.0 Waste Stream Information

\begin{tabular}{|l|l|}
\hline Originating Site & MOPL \\
\hline Waste Stream ID \& Name & MOPLWS1-Case1 \\
\hline Legacy volume (m3) & 10,000 \\
\hline
\end{tabular}

1.1 New Generation

\begin{tabular}{|l|l|l|}
\hline \multicolumn{1}{|c|}{ Start Year } & \multicolumn{1}{|c|}{ End Year } & Annual Volume (m3) \\
\hline NA & NA & NA \\
\hline & & \\
\hline & & \\
\hline & & \\
\hline
\end{tabular}

1.2 Shipping Route(s)

\begin{tabular}{|l|l|c|}
\hline \multicolumn{1}{|c|}{ Route ID } & \multicolumn{1}{|c|}{ Source Facility } & Destination Facility \\
\hline C1c-1 & Legacy-MOPL & MOPLFAC1-CASE1 \\
\hline C1c-2 & MOPLFAC1-CASE1 & MOPLFAC2-CASE1 \\
\hline & & \\
\hline & & \\
\hline & & \\
\hline & & \\
\hline
\end{tabular}

1.3 Shipping Schedule(s)

\begin{tabular}{|c|c|c|c|}
\hline Route ID: C1c-1 & \multicolumn{2}{|c|}{ Waste Type } & \multicolumn{1}{c|}{ Annual Volume (m3) } \\
\hline Start Year & \multicolumn{1}{|c|}{ End Year } & \multicolumn{1}{|c|}{ W17 } \\
\hline 1997 & 2020 & LLW & \\
\hline & & & \\
\hline & & & \\
\hline
\end{tabular}

1.3 Shipping Schedule(s) (continued)

\begin{tabular}{|c|c|c|c|}
\hline Route ID: C1c-2 & \multicolumn{2}{|c|}{ Waste Type } & \multicolumn{1}{|c|}{ Annual Volume (m3) } \\
\hline Start Year & \multicolumn{1}{|c|}{ End Year } & 100 \\
\hline & 2070 & LLW & \\
\hline & & & \\
\hline & & & \\
\hline
\end{tabular}

2.0 Facility Information

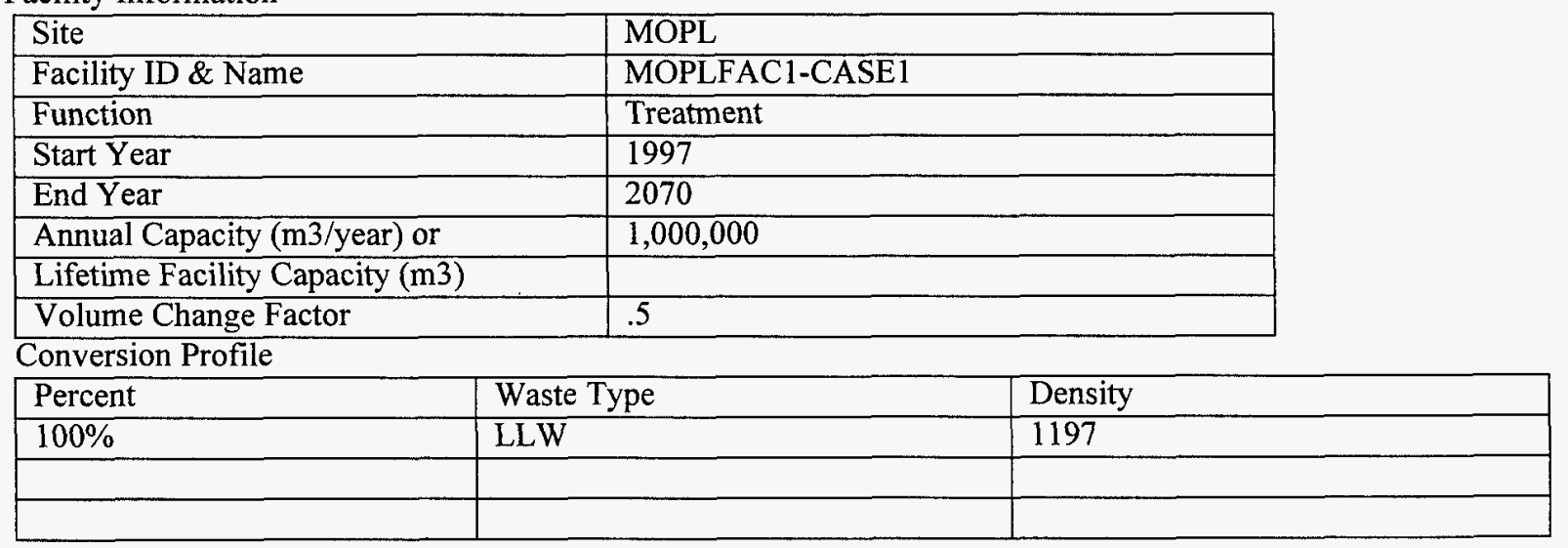


EXCEL CHART:

\section{Waste Disposition}

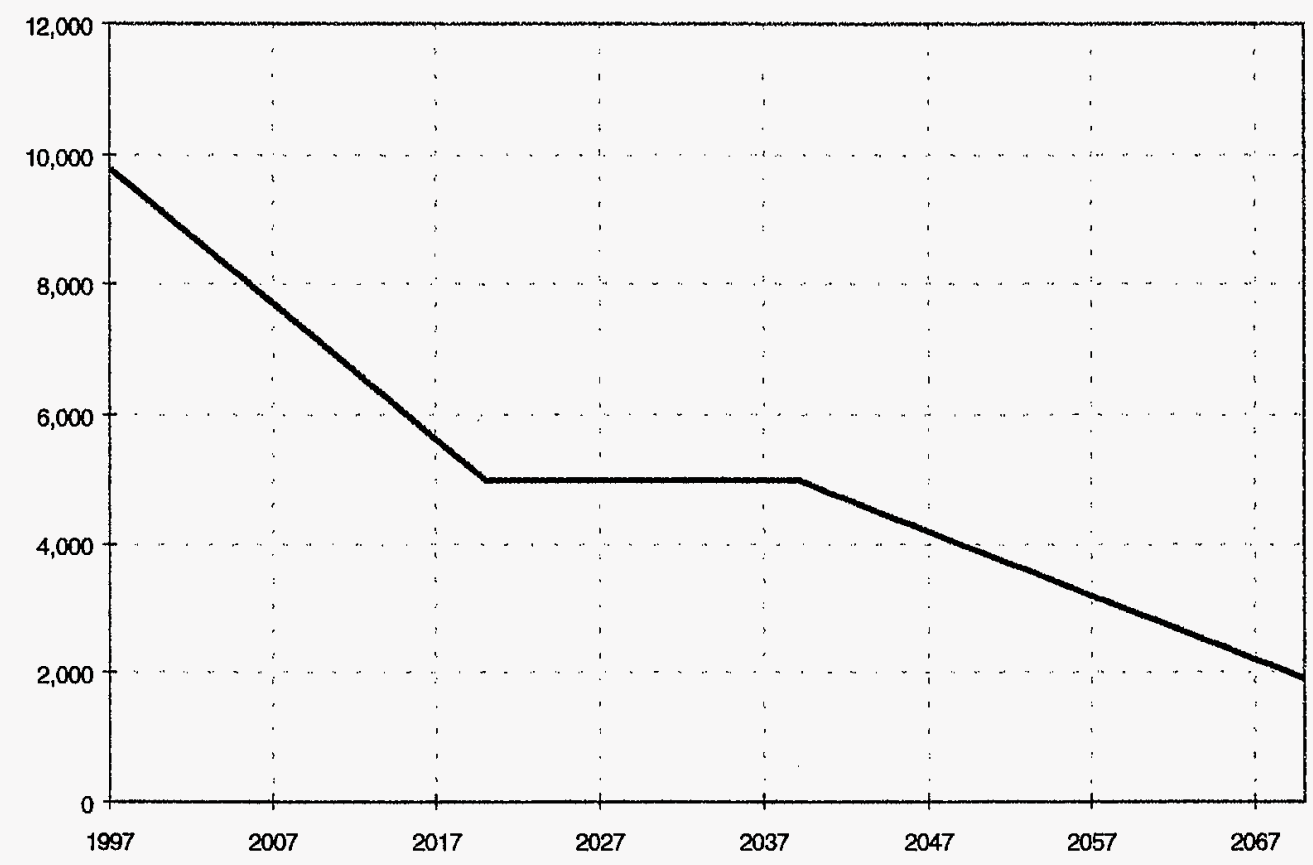

\section{CWWFA CHART:}

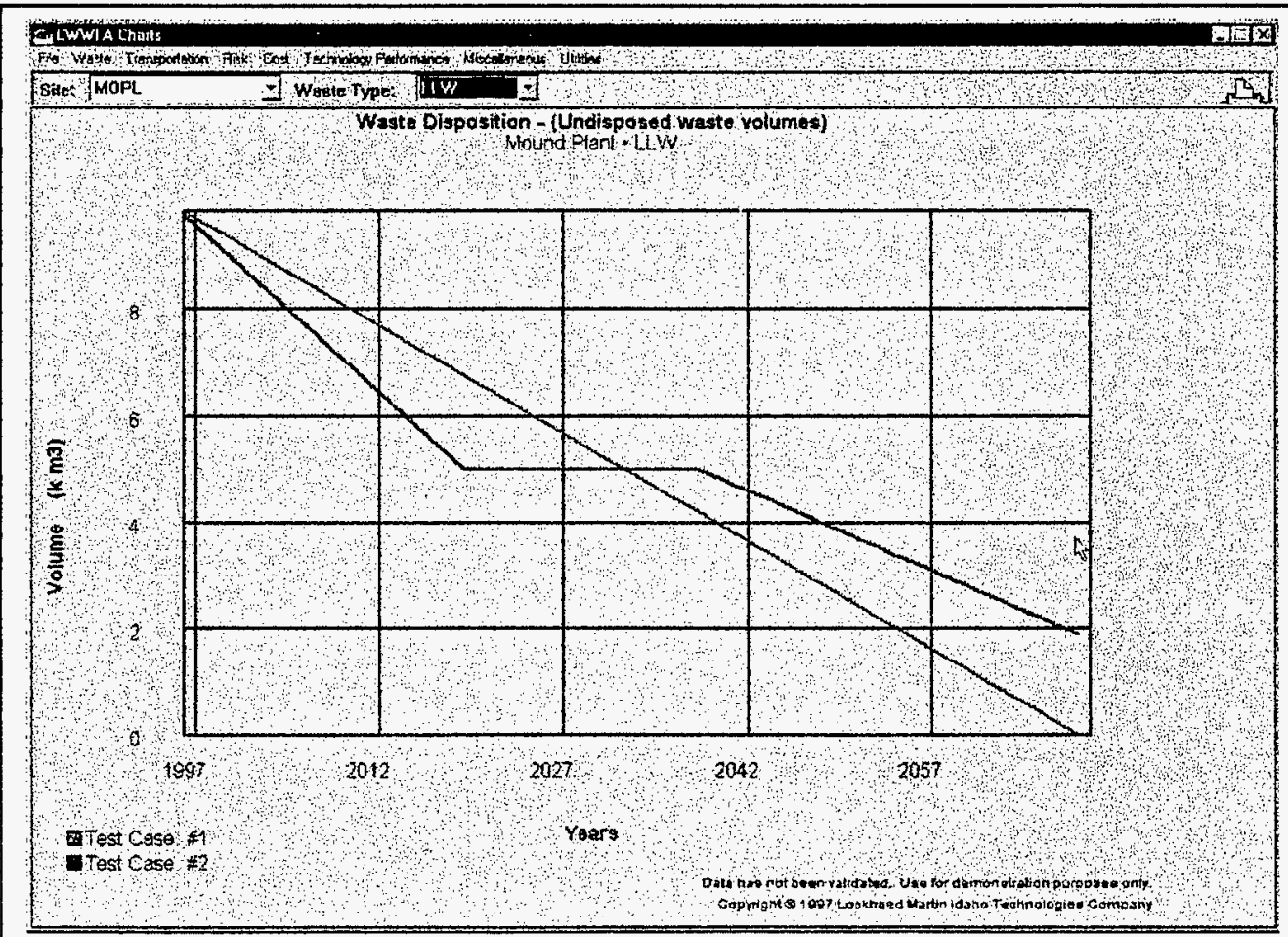




\section{EXCEL CHART (MOPLFAC1-CASE1):}

\section{Eacility Capacity Detail}

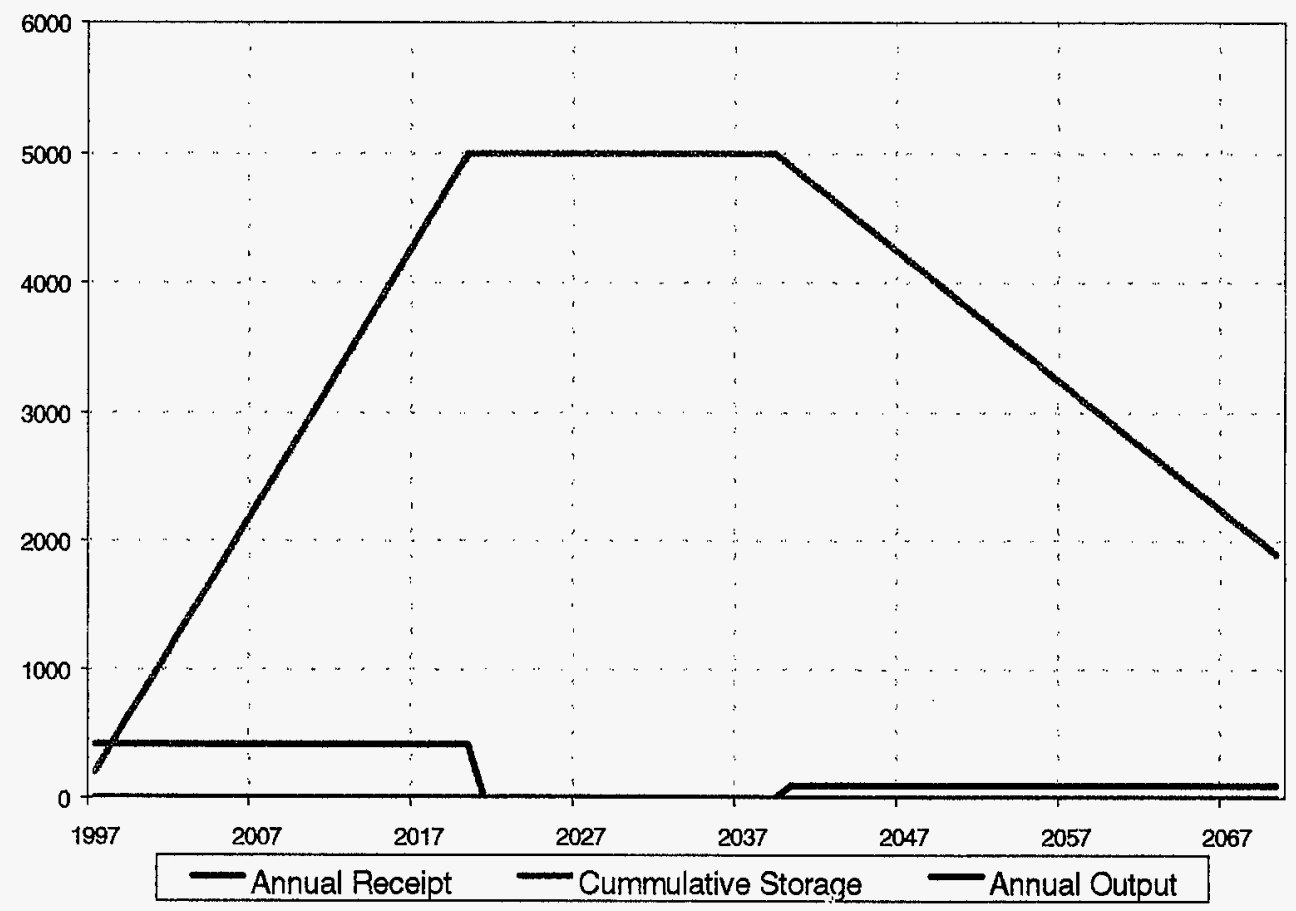

CWWFA CHART:

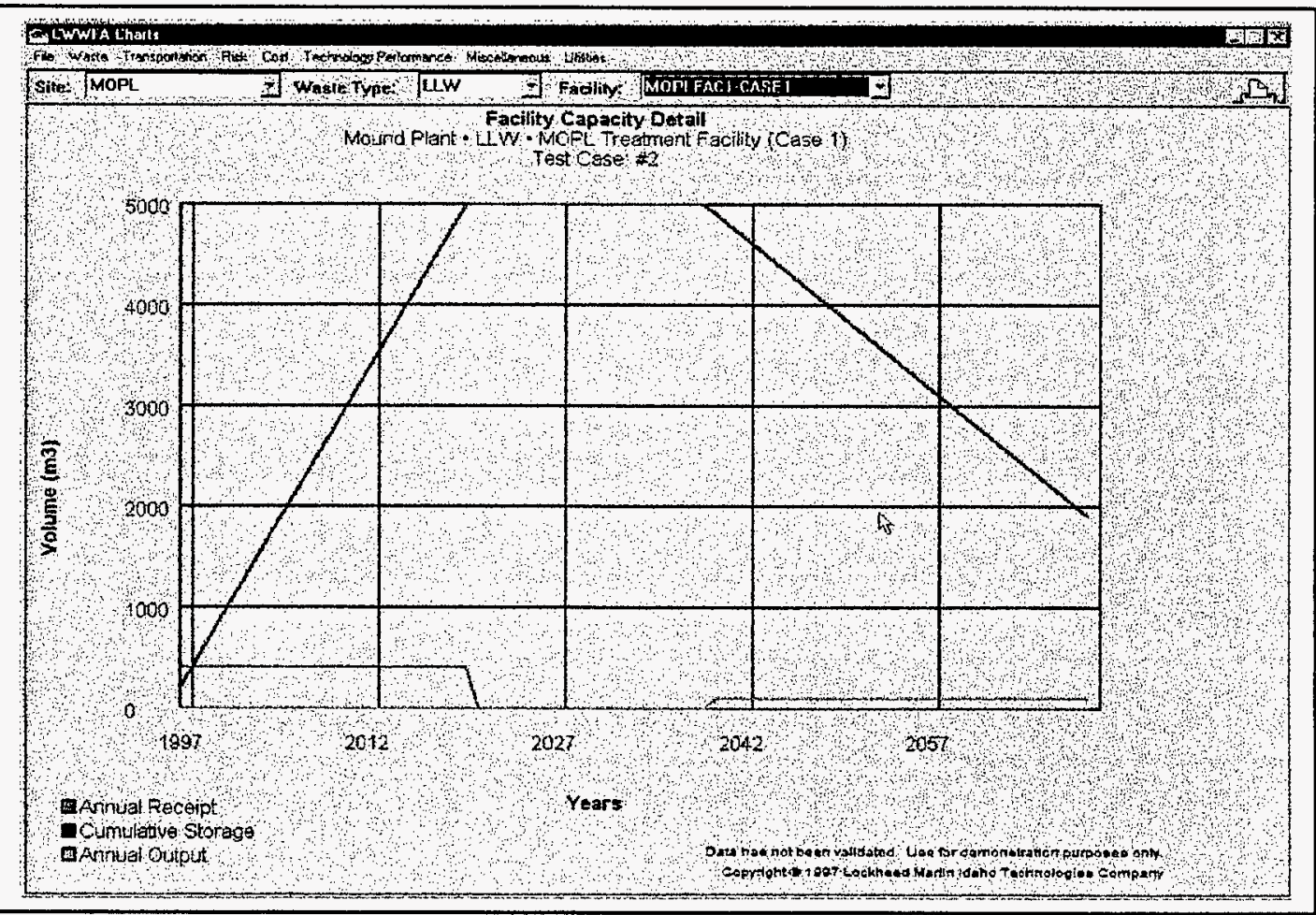


1.0 Waste Stream Information

\begin{tabular}{|l|l|}
\hline Originating Site & MOPL \\
\hline Waste Stream ID \& Name & MOPLWS1-Case1 \\
\hline Legacy volume (m3) & 10,000 \\
\hline
\end{tabular}

1.1 New Generation

\begin{tabular}{|l|l|l|}
\hline \multicolumn{1}{|c|}{ Start Year } & \multicolumn{1}{|c|}{ End Year } & Annual Volume (m3) \\
\hline NA & NA & NA \\
\hline & & \\
\hline & & \\
\hline & & \\
\hline
\end{tabular}

1.2 Shipping Route(s)

\begin{tabular}{|l|l|c|}
\hline \multicolumn{1}{|c|}{ Route ID } & \multicolumn{1}{|c|}{ Source Facility } & Destination Facility \\
\hline Cld-1 & Legacy-MOPL & MOPLFAC1-CASE1 \\
\hline Cld-2 & MOPLFAC1-CASE1 & MOPLFAC2-CASE1 \\
\hline & & \\
\hline & & \\
\hline & & \\
\hline & & \\
\hline
\end{tabular}

1.3 Shipping Schedule(s)

\begin{tabular}{|c|c|c|c|}
\hline \multicolumn{4}{|l|}{ Route ID: C1d-1 } \\
\hline Start Year & End Year & Waste Type & Annual Volume (m3) \\
\hline 1997 & 2010 & LLW & 135.14 \\
\hline 2011 & 2020 & LLW & 811 \\
\hline & & & \\
\hline
\end{tabular}

1.3 Shipping Schedule(s) (continued)

\begin{tabular}{|c|c|c|c|}
\hline Route ID: Cld-2 & \multicolumn{2}{|c|}{} \\
\hline Start Year & \multicolumn{1}{|c|}{ End Year } & Waste Type & Annual Volume (m3) \\
\hline 1997 & 2070 & LLW & 135.14 \\
\hline & & & \\
\hline & & & \\
\hline & & & \\
\hline
\end{tabular}

2.0 Facility Information

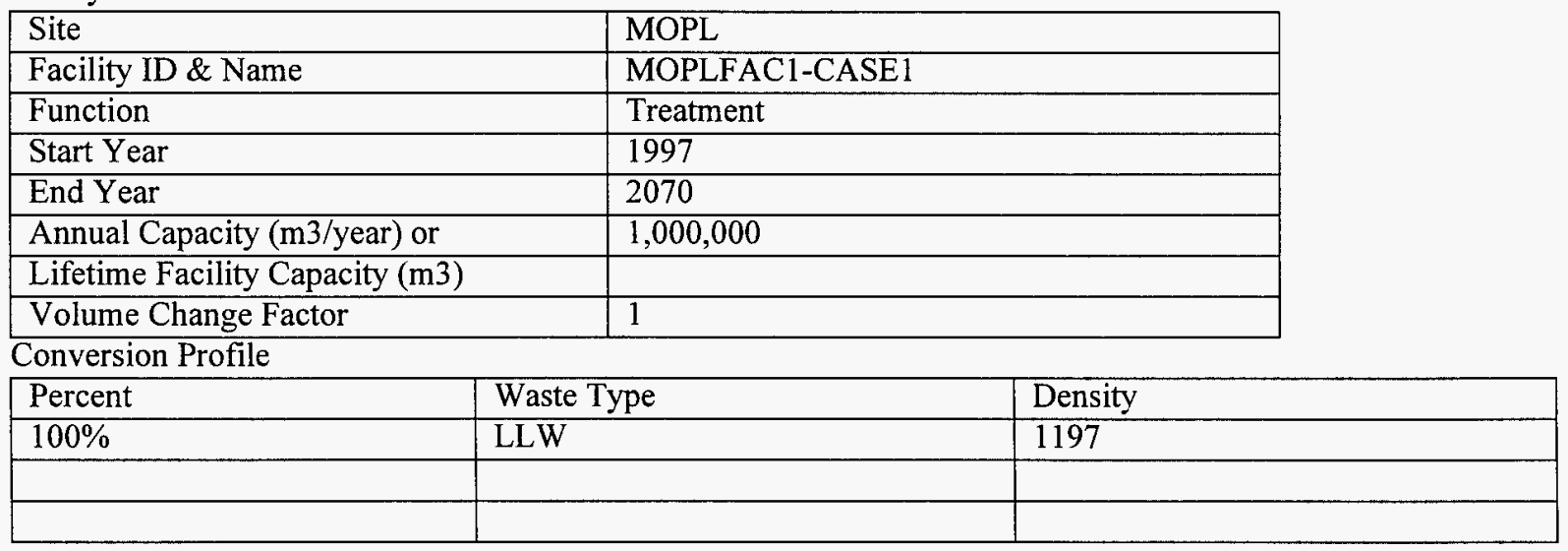




\section{EXCEL CHART:}

\section{Waste-Disposition}

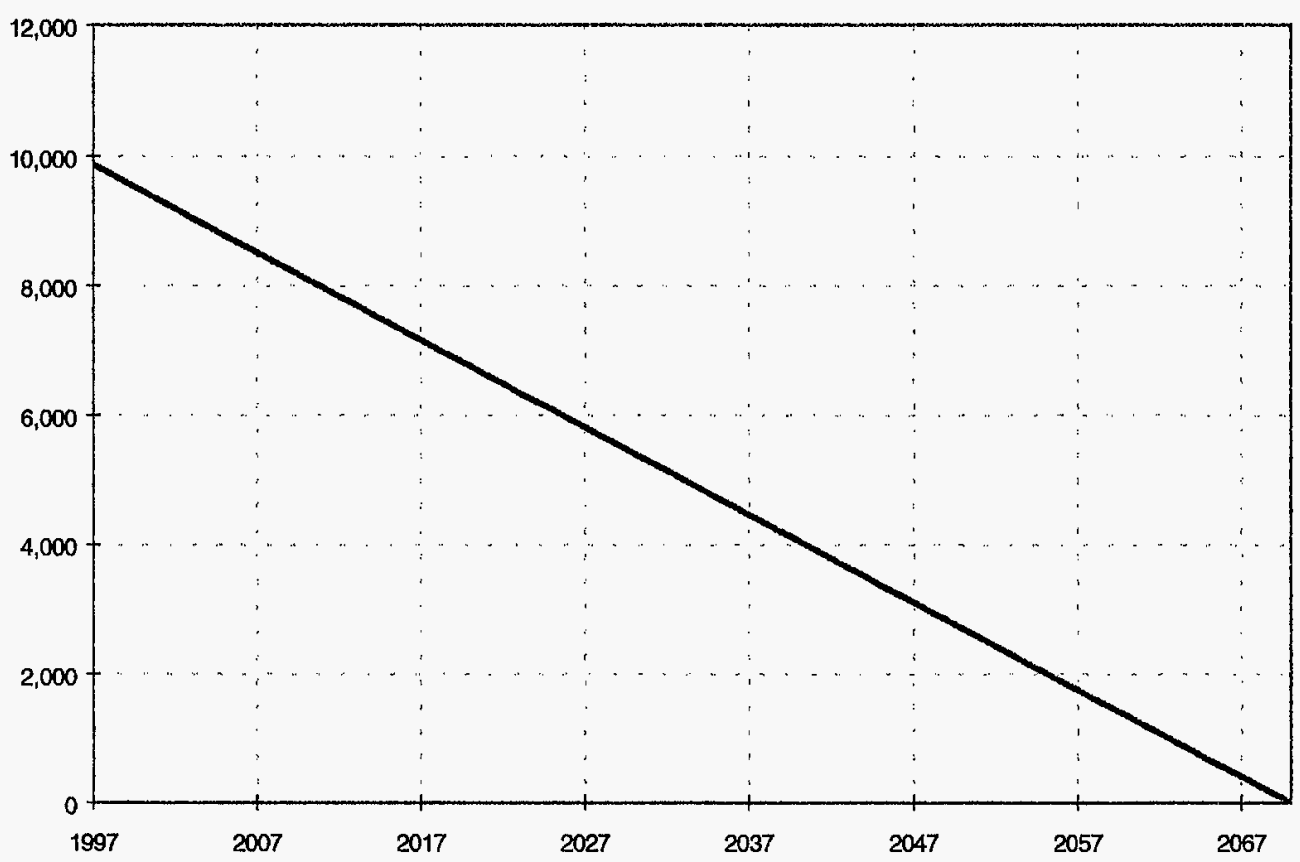

\section{CWWFA CHART:}

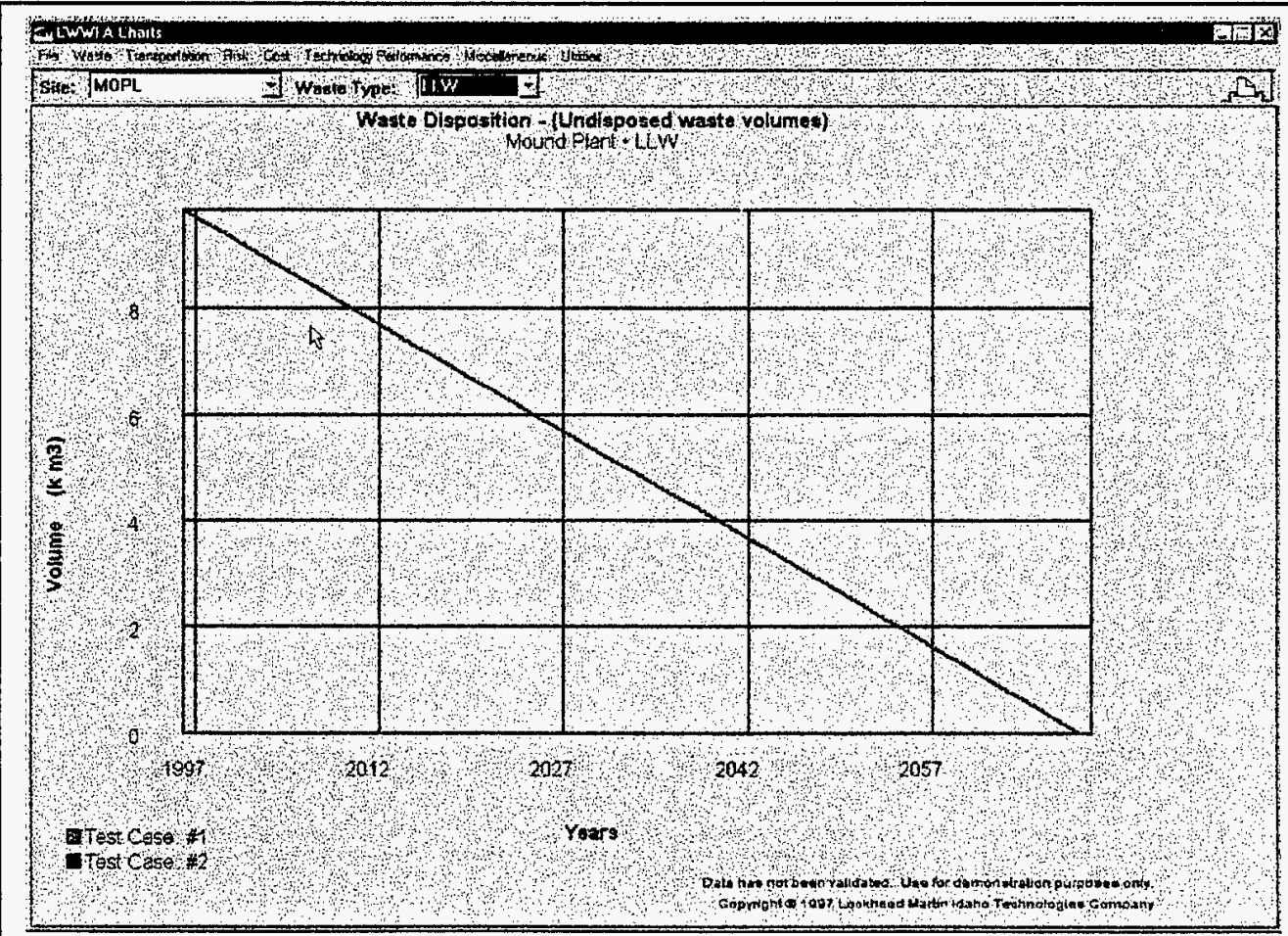




\section{Eacility Capacity Detail}

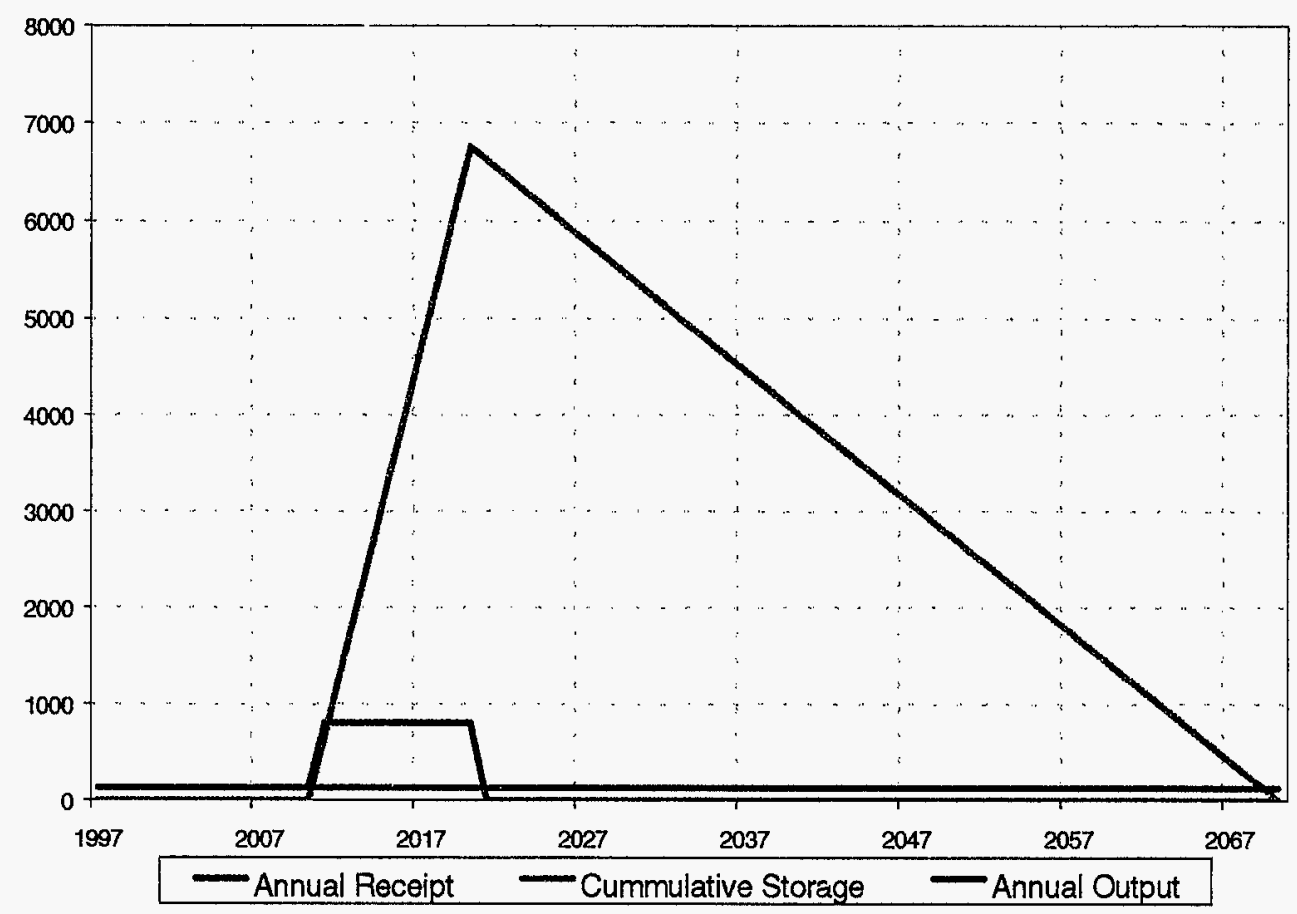

CWWFA CHART:

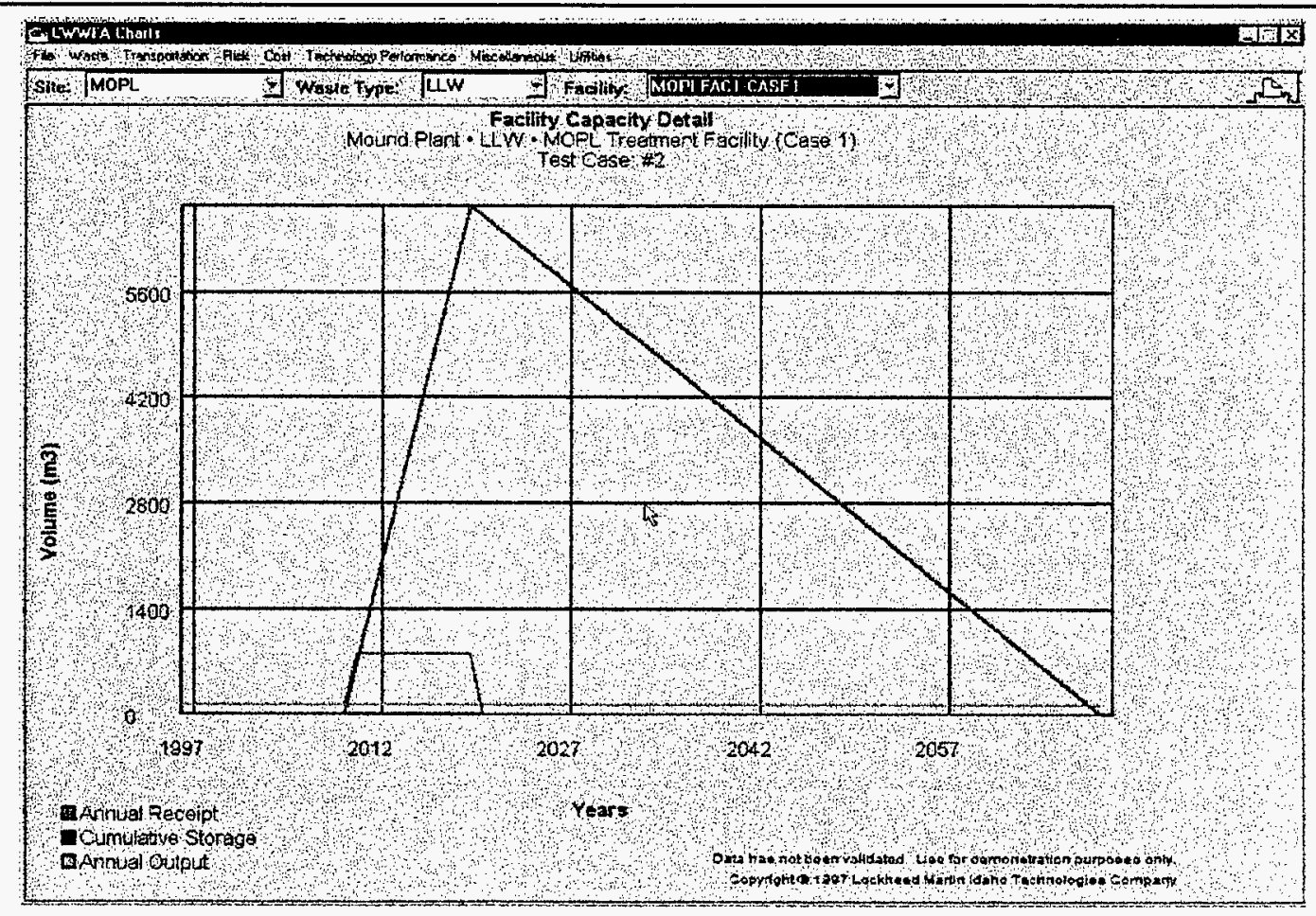


1.0 Waste Stream Information

\begin{tabular}{|l|l|}
\hline Originating Site & MOPL \\
\hline Waste Stream ID \& Name & MOPLWS1-Case 1 \\
\hline Legacy volume (m3) & 10,000 \\
\hline
\end{tabular}

1.1 New Generation

\begin{tabular}{|l|l|l|}
\hline \multicolumn{1}{|c|}{ Start Year } & \multicolumn{1}{|c|}{ End Year } & Annual Volume (m3) \\
\hline NA & NA & NA \\
\hline & & \\
\hline & & \\
\hline & & \\
\hline
\end{tabular}

\subsection{Shipping Route(s)}

\begin{tabular}{|l|l|l|}
\hline \multicolumn{1}{|c|}{ Route ID } & \multicolumn{1}{|c|}{ Source Facility } & Destination Facility \\
\hline Cle-1 & Legacy-MOPL & MOPLFAC1-CASE1 \\
\hline C1e-2 & MOPLFAC1-CASE1 & MOPLFAC2-CASE1 \\
\hline & & \\
\hline & & \\
\hline & & \\
\hline & & \\
\hline
\end{tabular}

1.3 Shipping Schedule(s)

\begin{tabular}{|c|c|c|c|}
\hline Route ID: Cle-1 & \multicolumn{2}{|c|}{} & \multicolumn{1}{|c|}{ Waste Type } \\
\hline Start Year & \multicolumn{1}{|c|}{ End Year } & Annual Volume (m3) \\
\hline 1997 & 2070 & LLW & 135 \\
\hline & & & \\
\hline & & & \\
\hline & & & \\
\hline
\end{tabular}

1.3 Shipping Schedule(s) (continued)

\begin{tabular}{|c|l|l|l|}
\hline Route ID: C1e-2 & \multicolumn{2}{|c|}{ Waste Type } & \multicolumn{1}{|c|}{ Annual Volume (m3) } \\
\hline \multicolumn{1}{|c|}{ Start Year } & \multicolumn{1}{|c|}{ End Year } & \multicolumn{1}{|c|}{ WLW } & 135 \\
\hline 1997 & 2005 & LLW & \\
\hline & 2070 & & \\
\hline & & & \\
\hline
\end{tabular}

2.0 Facility Information

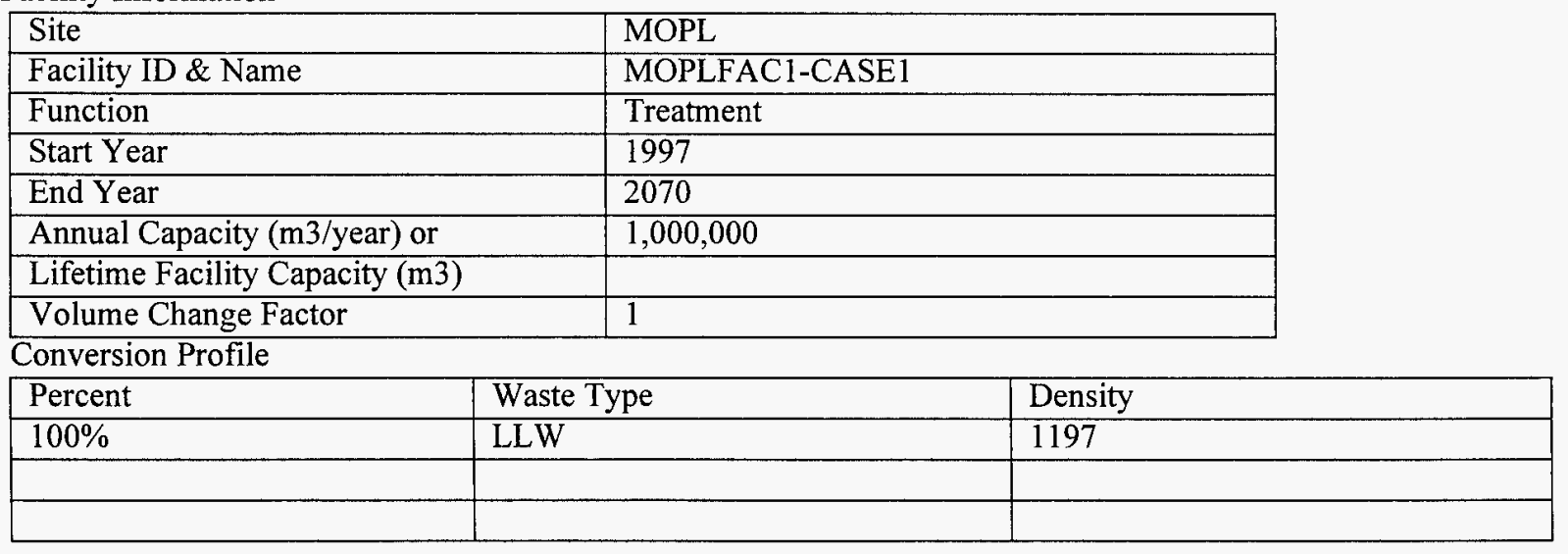




\section{EXCEL CHART:}

\section{Waste Disposition}

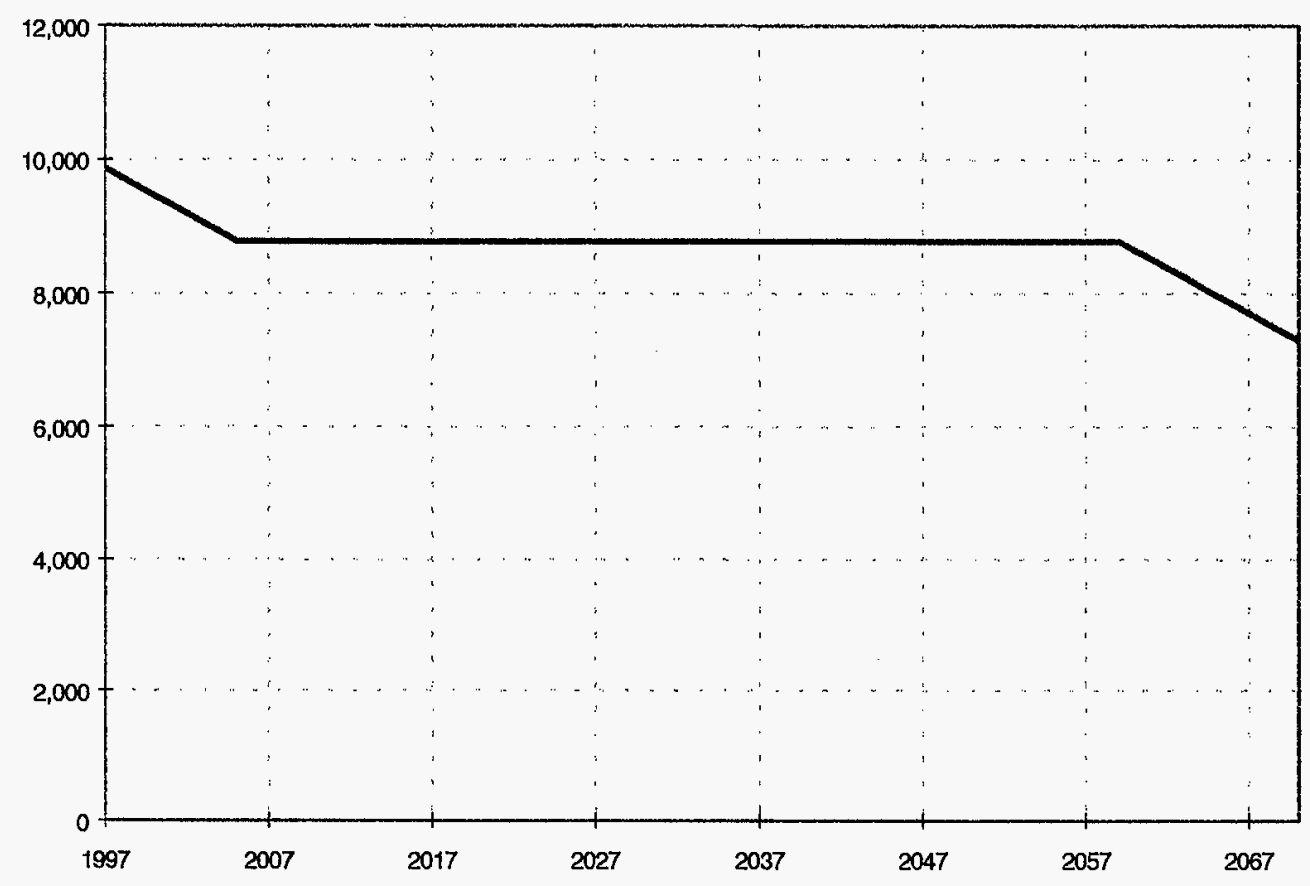

\section{CWWFA CHART:}

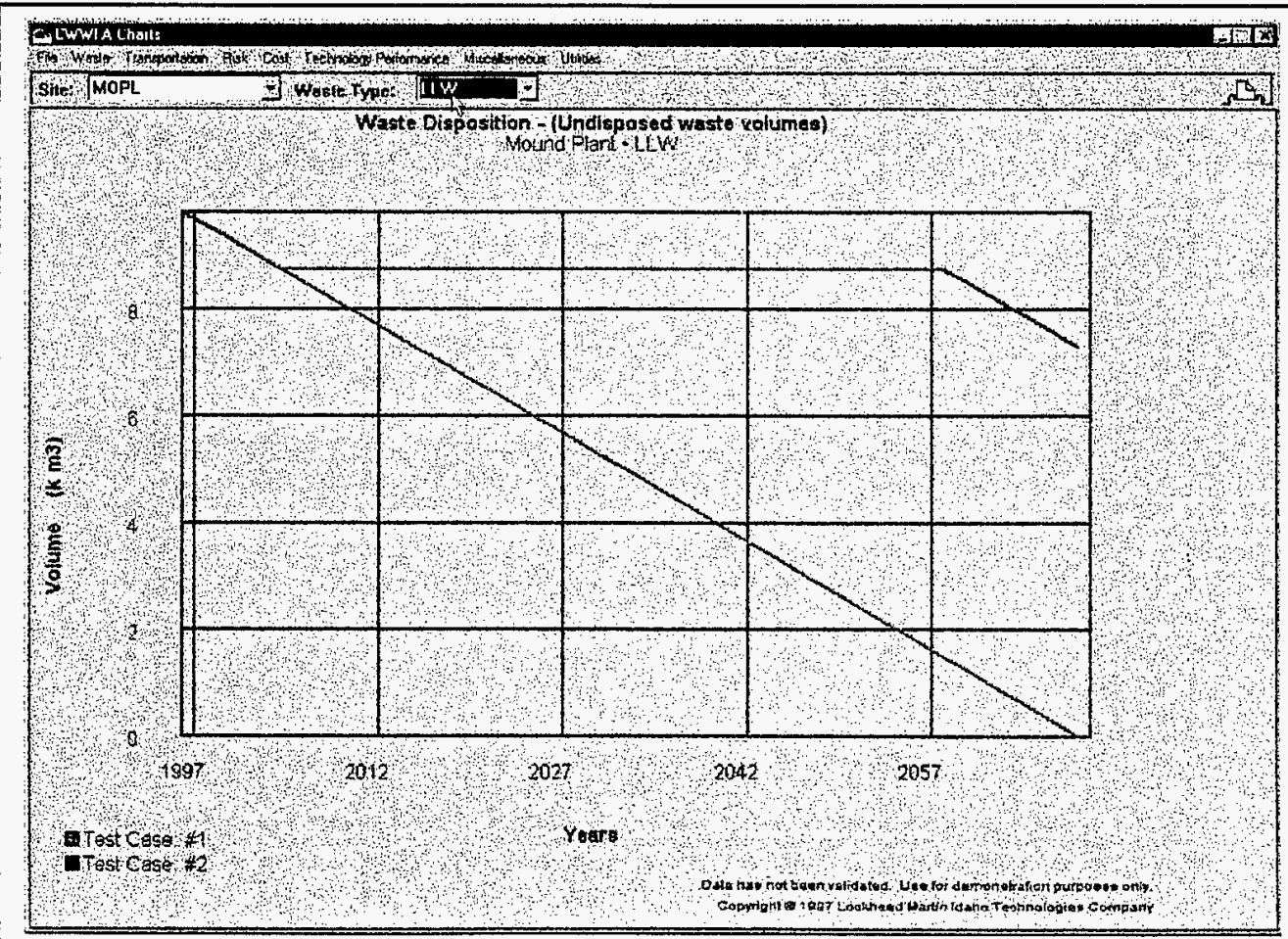


INITIAL PARAMETERS \& RESULTS REPORT

CASE: Case 1e

DATE: $11 / 12 / 97$

Evaluator: K B Oswald

EXCEL CHART (MOPLFAC2-CASE1):

Eacility Capacity Detail

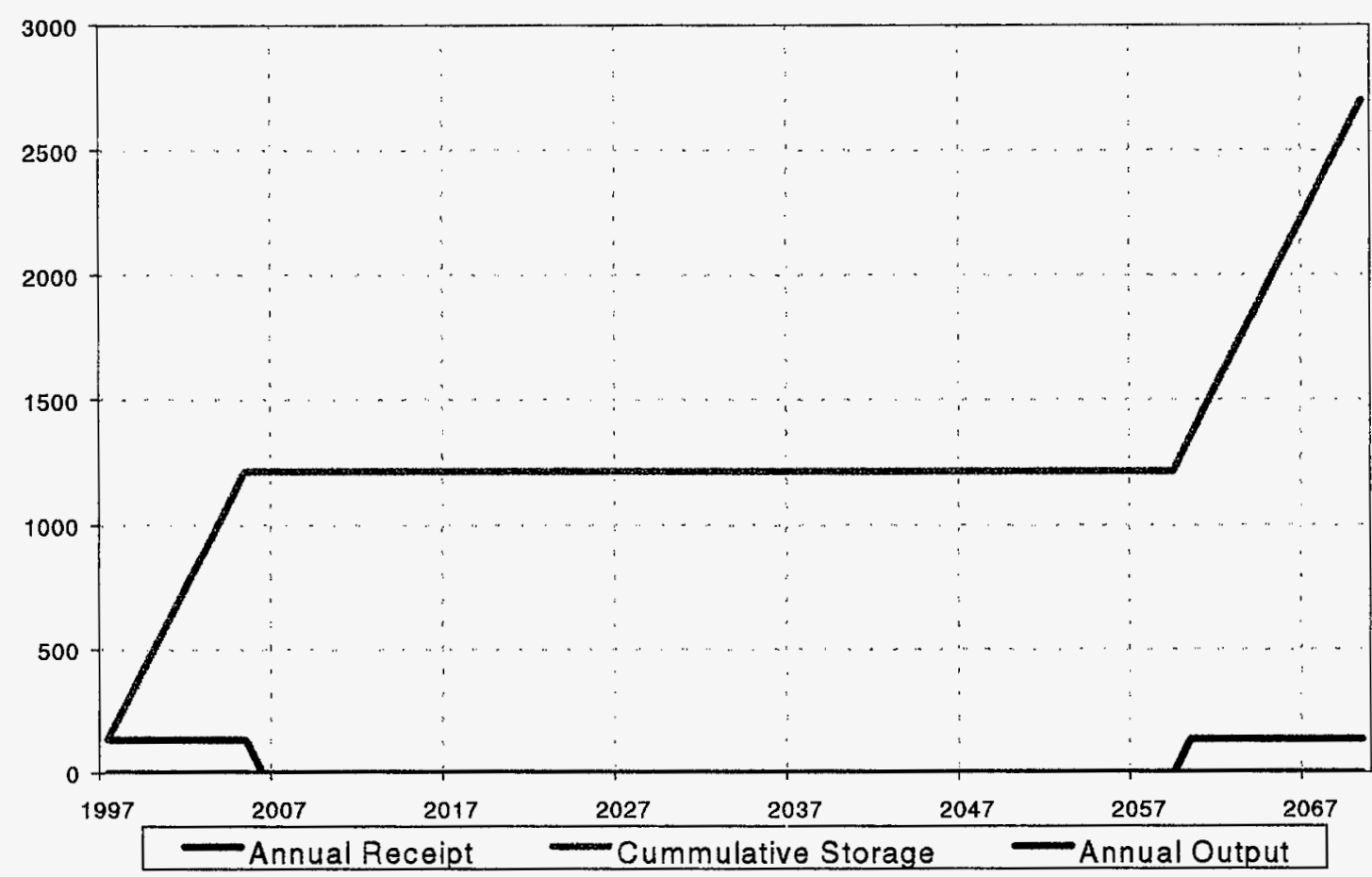

CWWFA CHART:

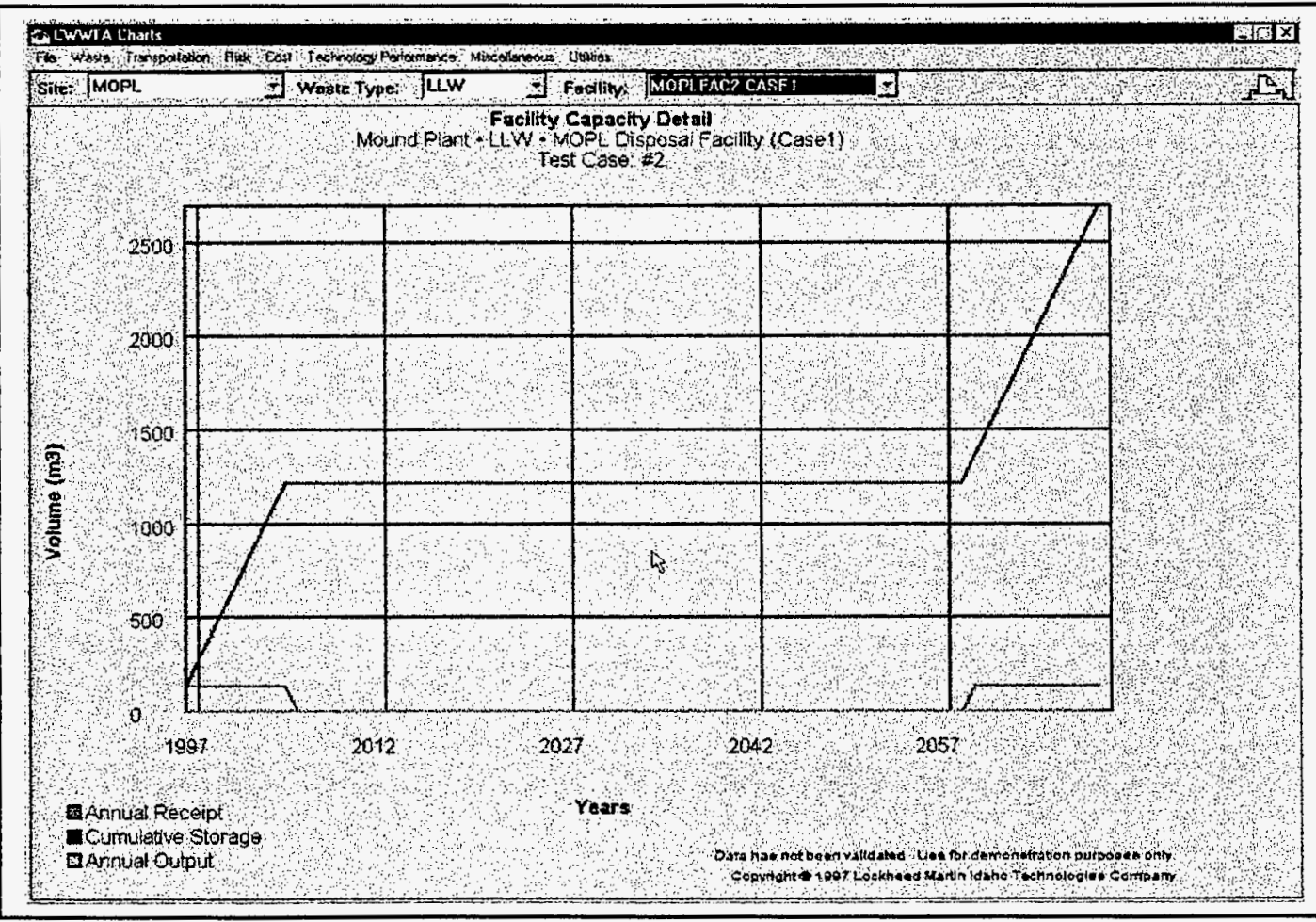


INITIAL PARAMETERS \& RESULTS REPORT

CASE: Case $1 \mathrm{f}$

DATE: $11 / 13 / 97$

Evaluator: K B Oswald

1.0 Waste Stream Information

\begin{tabular}{|l|l|}
\hline Originating Site & MOPL \\
\hline Waste Stream ID \& Name & MOPLWS1-Case 1 \\
\hline Legacy volume (m3) & 10,000 \\
\hline
\end{tabular}

1.1 New Generation

\begin{tabular}{|l|l|l|}
\hline \multicolumn{1}{|c|}{ Start Year } & \multicolumn{1}{|c|}{ End Year } & Annual Volume (m3) \\
\hline NA & NA & NA \\
\hline & & \\
\hline & & \\
\hline & & \\
\hline
\end{tabular}

1.2 Shipping Route(s)

\begin{tabular}{|l|l|l|}
\hline \multicolumn{1}{|c|}{ Route ID } & \multicolumn{1}{|c|}{ Source Facility } & Destination Facility \\
\hline C1f-1 & Legacy-MOPL & MOPLFAC1-CASE1 \\
\hline C1f-2 & MOPLFAC1-CASE1 & MOPLFAC2-CASE1 \\
\hline & & \\
\hline & & \\
\hline & & \\
\hline & & \\
\hline
\end{tabular}

1.3 Shipping Schedule(s)

\begin{tabular}{|l|l|l|l|}
\hline Route ID: C1f-1 & \multicolumn{1}{|c|}{ Waste Type } & \multicolumn{1}{c|}{ Annual Volume (m3) } \\
\hline \multicolumn{1}{|c|}{ Start Year } & \multicolumn{1}{|c|}{ End Year } & \multicolumn{1}{|c|}{ - } & 135 \\
\hline 1997 & 2010 & LLW & 300 \\
\hline 2011 & 2020 & LLW & 500 \\
\hline 2040 & 2050 & LLW & \\
\hline & & & \\
\hline
\end{tabular}

1.3 Shipping Schedule(s) (continued)

\begin{tabular}{|c|c|c|c|}
\hline Route ID: C1f-2 & \multicolumn{2}{|c|}{ Waste Type } & \multicolumn{1}{|c|}{ Annual Volume (m3) } \\
\hline \multicolumn{1}{|c|}{ Start Year } & End Year & 135 \\
\hline 1997 & 2005 & LLW & 100 \\
\hline 2020 & 2035 & LLW & 500 \\
\hline 2060 & 2070 & LLW & \\
\hline & & & \multicolumn{2}{|c|}{} \\
\hline
\end{tabular}

2.0 Facility Information

\begin{tabular}{|l|l|}
\hline Site & MOPL \\
\hline Facility ID \& Name & MOPLFAC1-CASE1 \\
\hline Function & Treatment \\
\hline Start Year & 1997 \\
\hline End Year & 2070 \\
\hline Annual Capacity (m3/year) or & $1,000,000$ \\
\hline Lifetime Facility Capacity (m3) & \\
\hline Volume Change Factor & 1 \\
\hline \\
\begin{tabular}{|l|l|l|}
\hline Conversion Profile & Waste Type \\
\hline $100 \%$ & LLW & Density \\
\hline & & 1197 \\
\hline & & \\
\hline
\end{tabular} \\
\hline
\end{tabular}


WASTE DISPOSITION - EXCEL CHART (MOPL, LLW):

\section{Waste Disposition}

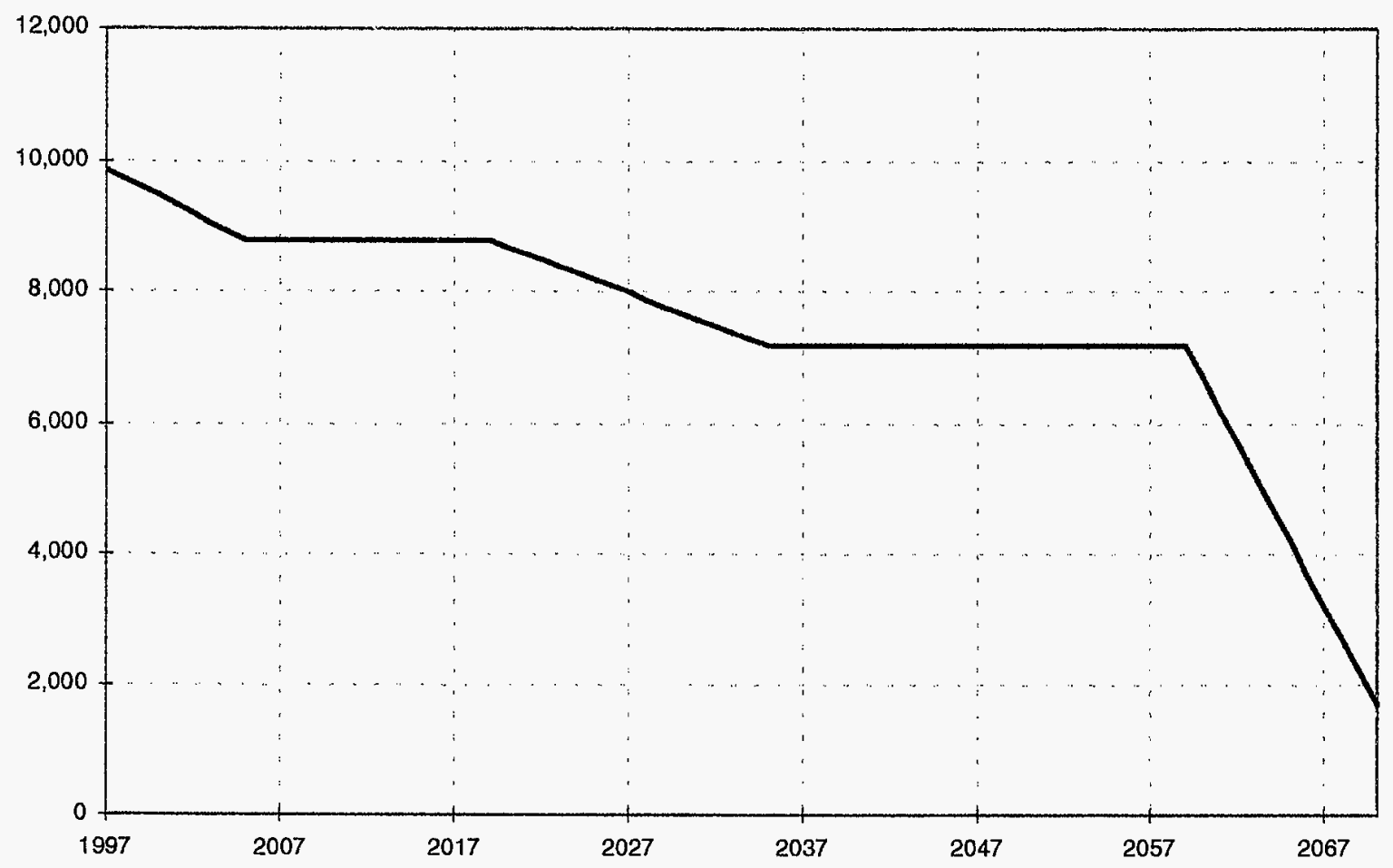

WASTE DISPOSITION - CWWFA CHART:

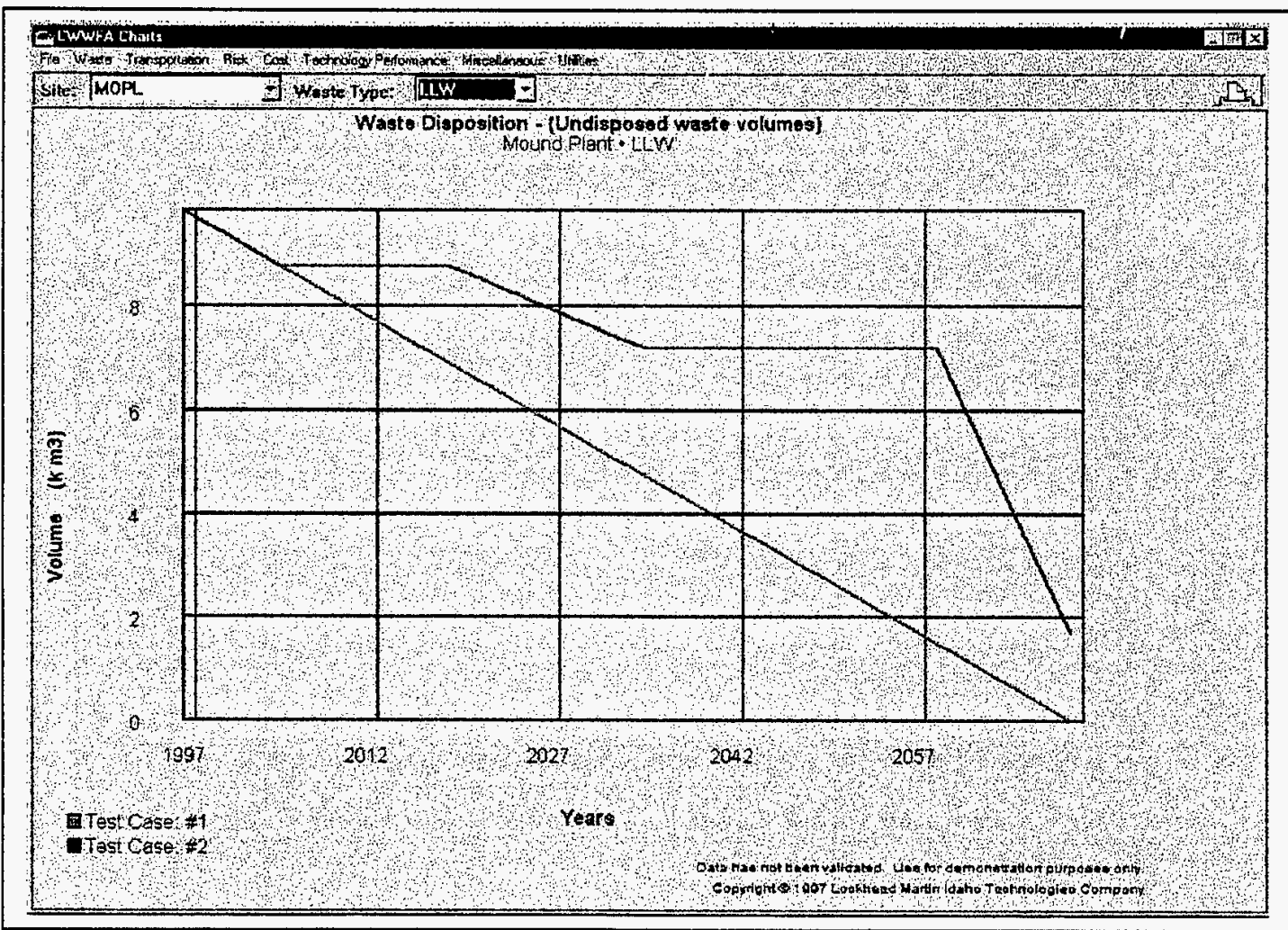




\section{INITIAL PARAMETERS \& RESULTS REPORT}

FACILITY CAPACITY EXCEL CHART (MOPL, MOPLFAC1-CASE1):

\section{Facility Capacity Detail}

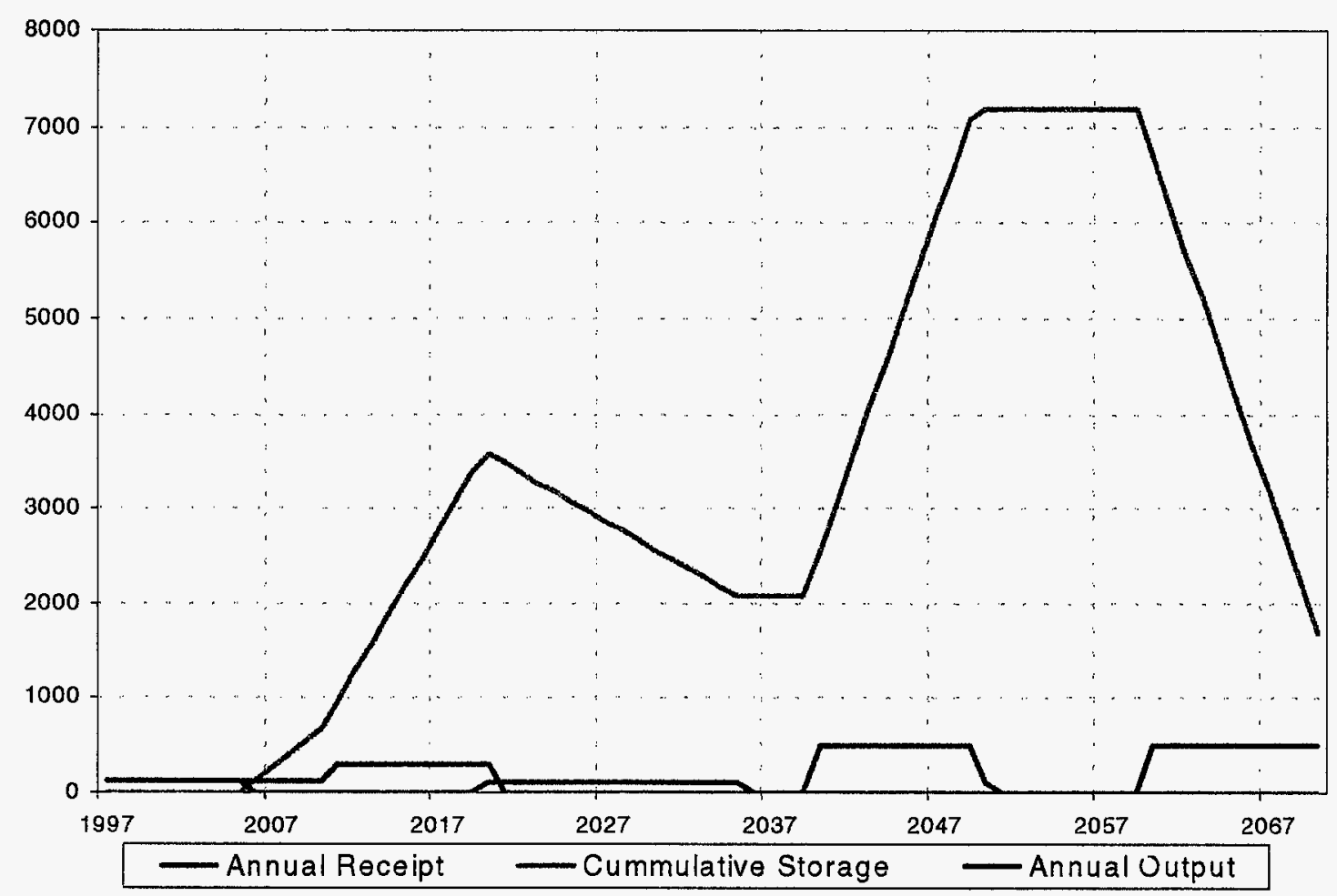

FACILITY CAPACITY CWWFA CHART:

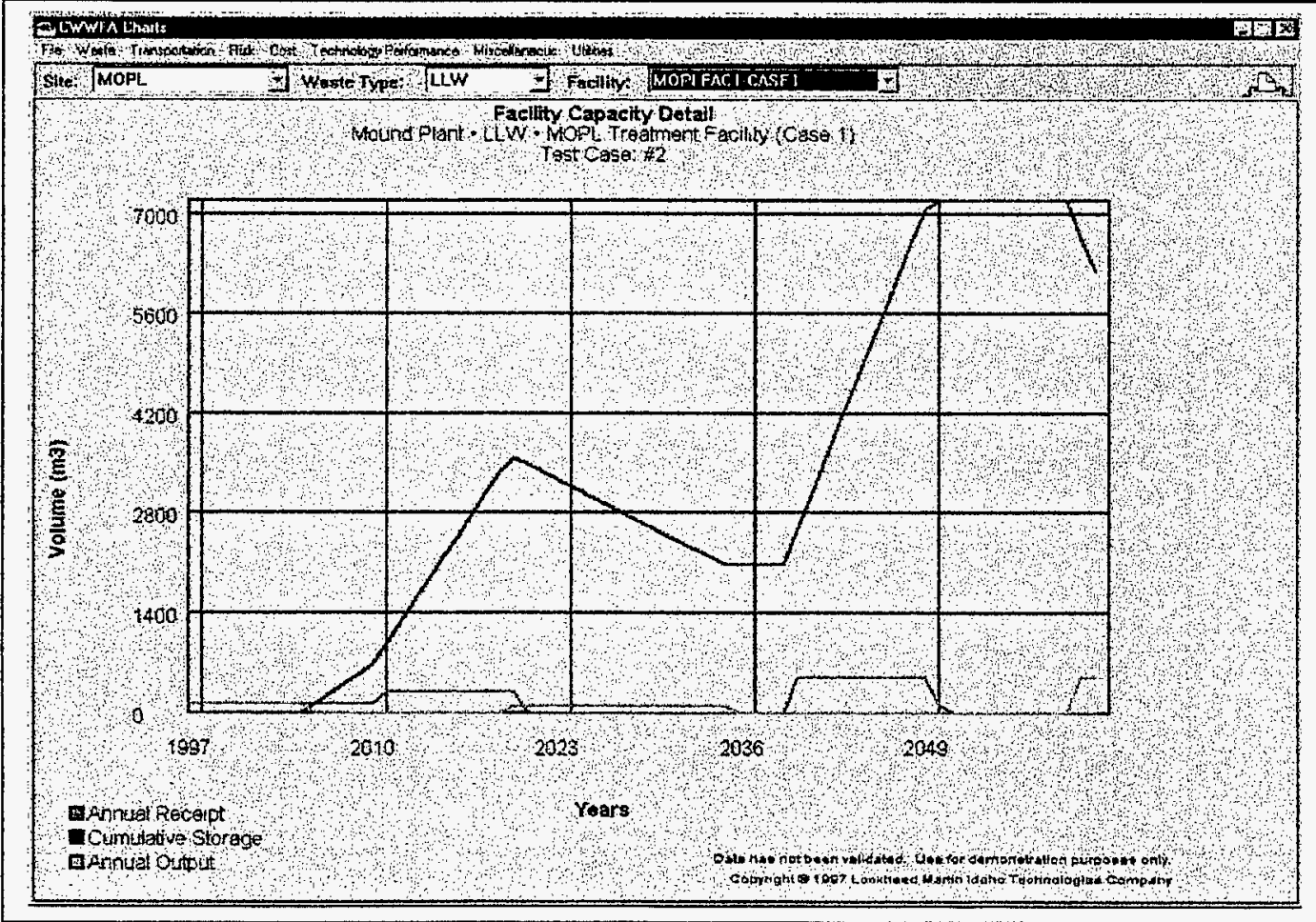


1.0 Waste Stream Information

\begin{tabular}{|l|l|}
\hline Originating Site & MOPL \\
\hline Waste Stream ID \& Name & MOPLWS1-Case 1 \\
\hline Legacy volume (m3) & 10,000 \\
\hline
\end{tabular}

1.1 New Generation

\begin{tabular}{|c|c|c|}
\hline \multicolumn{1}{|c|}{ Start Year } & \multicolumn{1}{|c|}{ End Year } & Annual Volume (m3) \\
\hline 1997 & 2010 & 1000 \\
\hline & & \\
\hline & & \\
\hline & & \\
\hline
\end{tabular}

1.2 Shipping Route(s)

\begin{tabular}{|l|l|l|}
\hline \multicolumn{1}{|c|}{ Route ID } & \multicolumn{1}{|c|}{ Source Facility } & Destination Facility \\
\hline C1g-1 & Legacy-MOPL & MOPLFAC1-CASE1 \\
\hline C1g-2 & MOPLFAC1-CASE1 & MOPLFAC2-CASE1 \\
\hline & & \\
\hline & & \\
\hline & & \\
\hline & & \\
\hline
\end{tabular}

1.3 Shipping Schedule(s)

\begin{tabular}{|c|c|c|c|}
\hline Route ID: Clg-1 & \multicolumn{2}{|c|}{ Waste Type } & \multicolumn{1}{|c|}{ Annual Volume (m3) } \\
\hline Start Year & \multicolumn{1}{|c|}{ End Year } & 135.14 \\
\hline & 2070 & LLW & \\
\hline & & & \\
\hline & & & \\
\hline & & & \\
\hline
\end{tabular}

1.3 Shipping Schedule(s) (continued)

\begin{tabular}{|c|c|c|c|}
\hline Route ID: C1g-2 & \multicolumn{2}{|c|}{ Waste Type } & \multicolumn{1}{|c|}{ Annual Volume (m3) } \\
\hline Start Year & \multicolumn{1}{|c|}{ End Year } & LLW & 135.14 \\
\hline 1997 & 2070 & & \\
\hline & & & \\
\hline & & & \\
\hline
\end{tabular}

2.0 Facility Information

\begin{tabular}{|l|l|}
\hline Site & MOPL \\
\hline Facility ID \& Name & MOPLFAC1-CASE1 \\
\hline Function & Treatment \\
\hline Start Year & 1997 \\
\hline End Year & 2070 \\
\hline Annual Capacity (m3/year) or & $1,000,000$ \\
\hline Lifetime Facility Capacity (m3) & \\
\hline Volume Change Factor & 1 \\
\hline
\end{tabular}

Conversion Profile

\begin{tabular}{|l|l|l|}
\hline Percent & Waste Type & Density \\
\hline $100 \%$ & LLW & 1197 \\
\hline & & \\
\hline & & \\
\hline
\end{tabular}




\section{Waste Disposition}

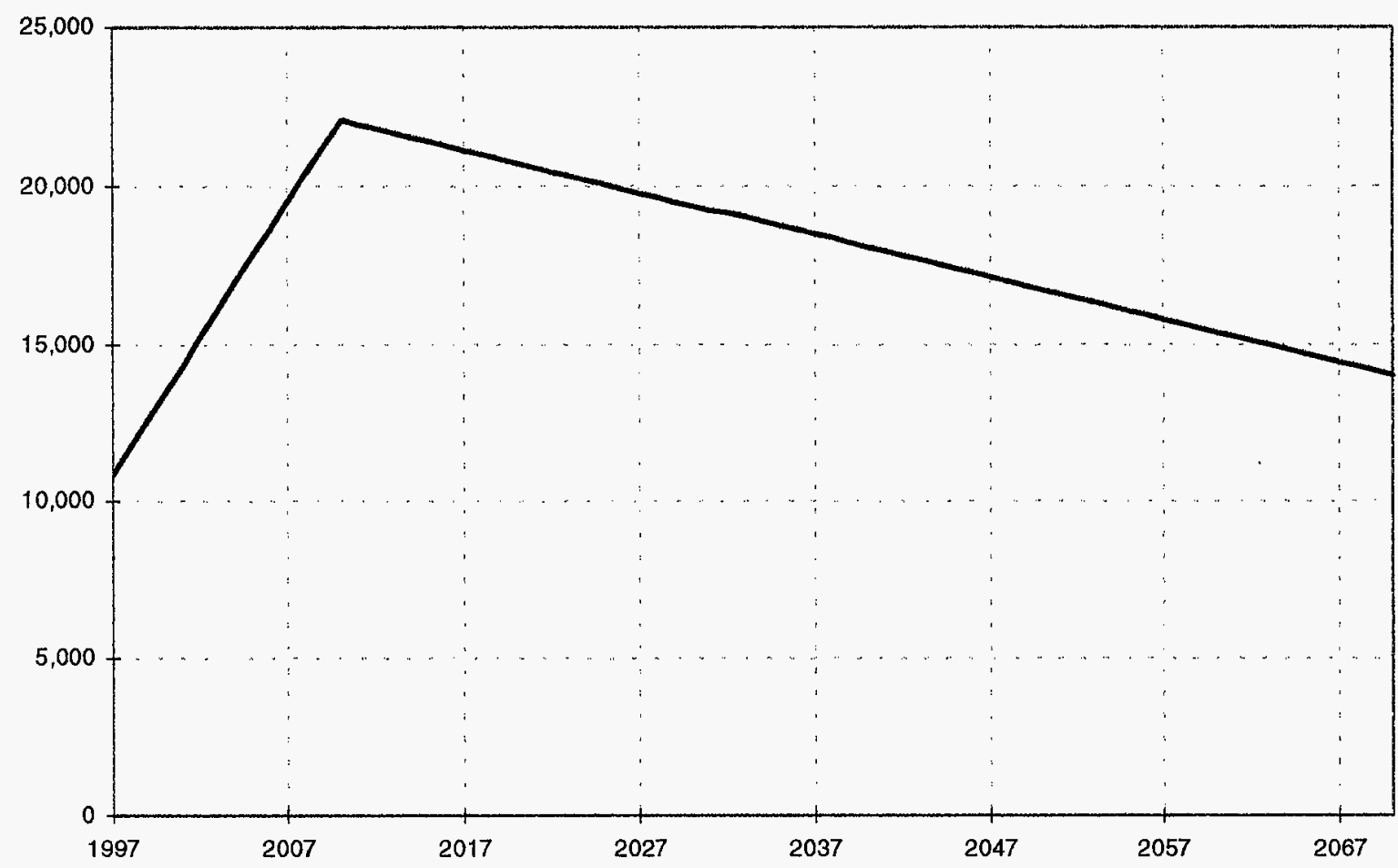

\section{WASTE DISPOSITION - CWWFA CHART:}

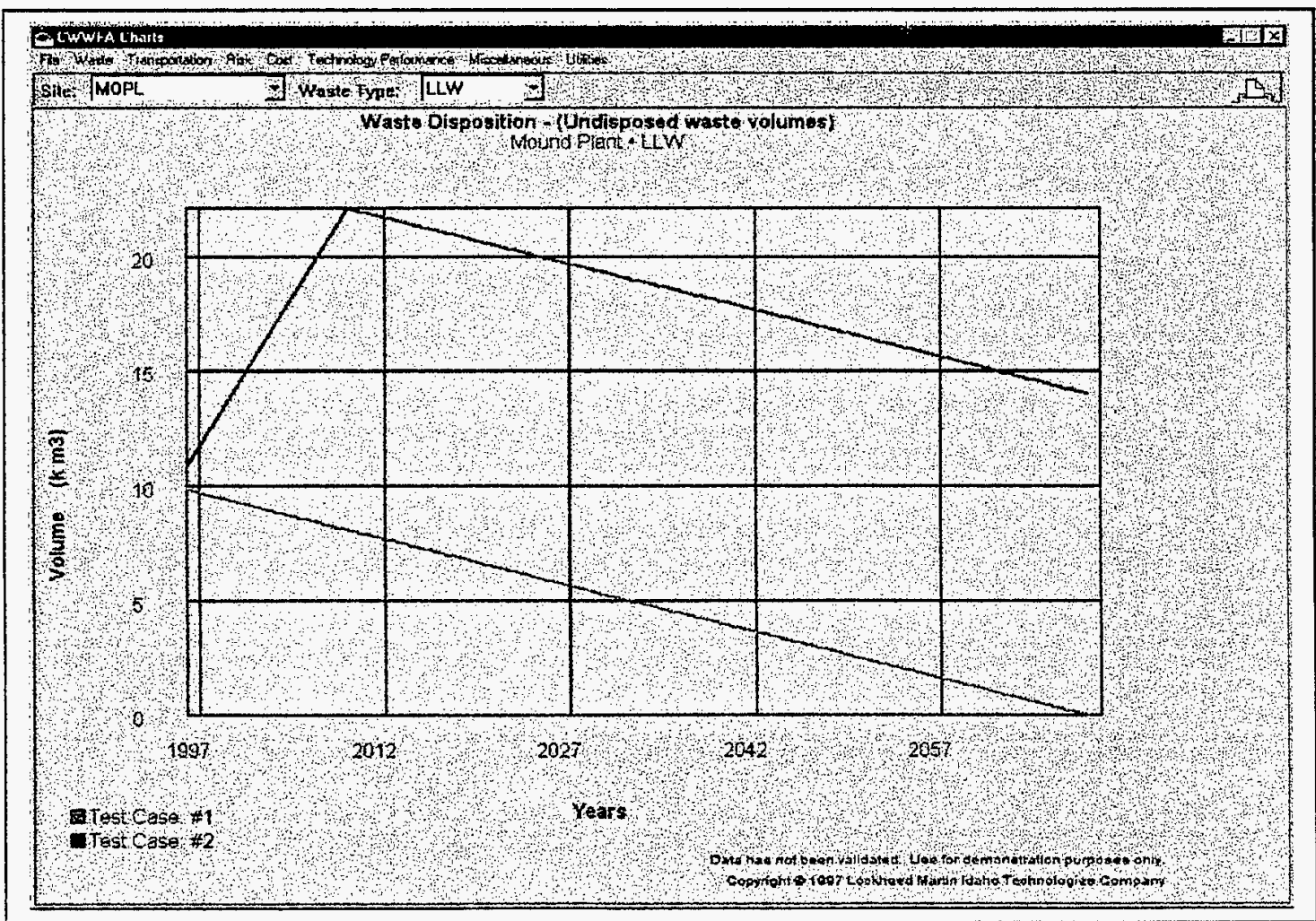


FACILITY CAPACITY EXCEL CHART (MOPL, MOPLFAC2-CASE1):

Facility Capacity Detail

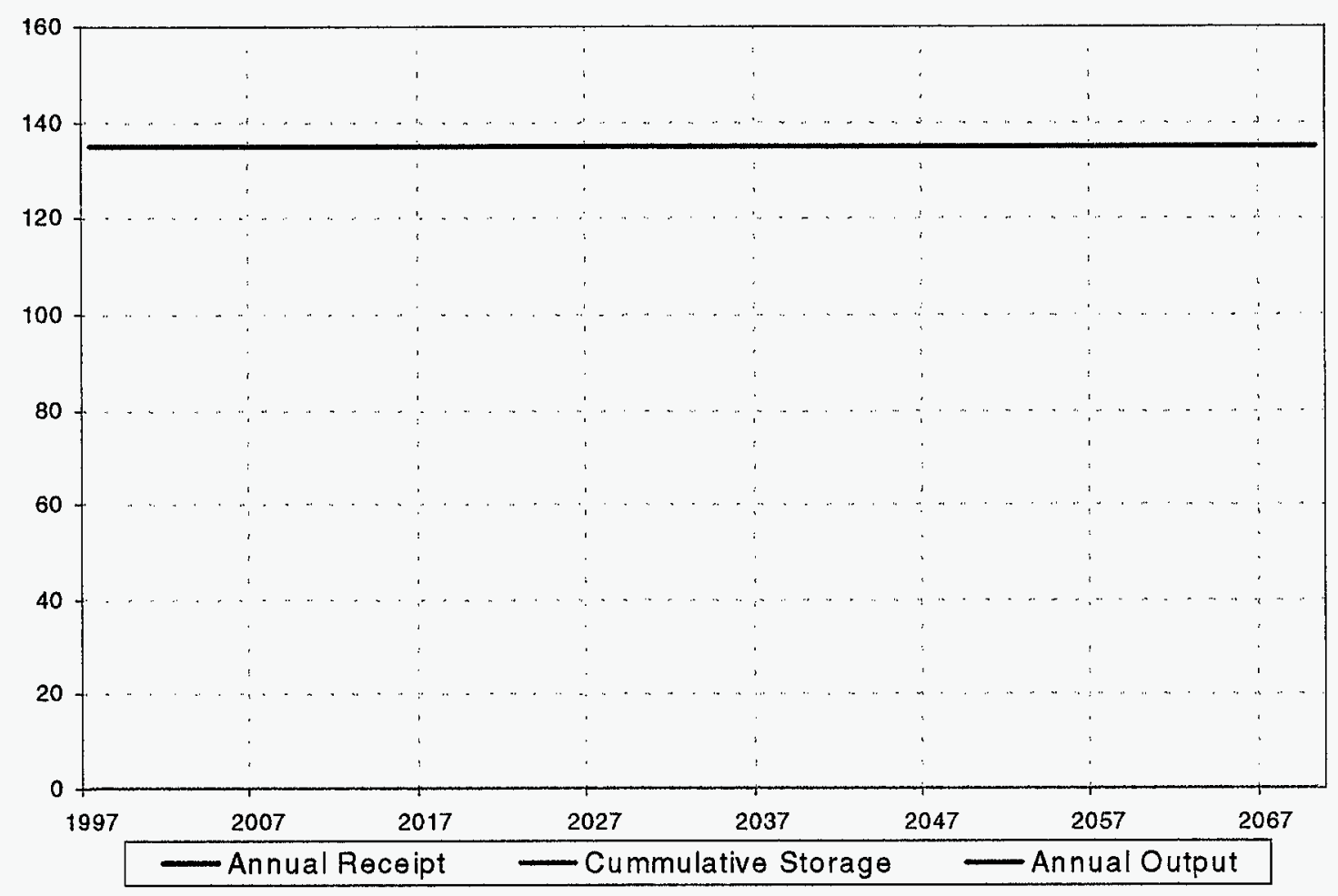

FACILITY CAPACITY CWWFA CHART:

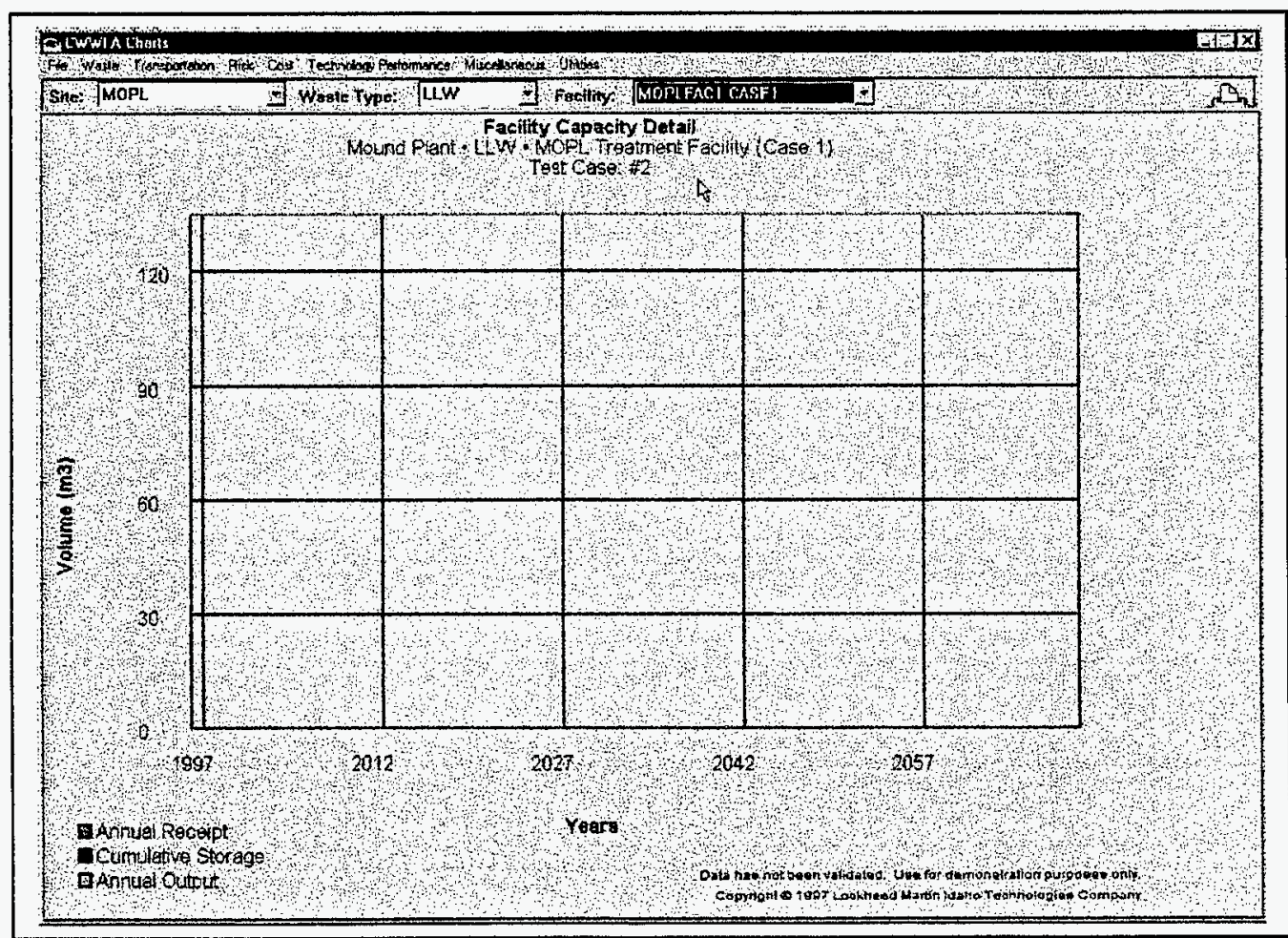


INITIAL PARAMETERS \& RESULTS REPORT

CASE: Case $1 \mathrm{~h}$

DATE: $11 / 13 / 97$

Evaluator: K B Oswald

1.0 Waste Stream Information

\begin{tabular}{|l|l|}
\hline Originating Site & MOPL \\
\hline Waste Stream ID \& Name & MOPLWS1-Case1 \\
\hline Legacy volume (m3) & 10,000 \\
\hline
\end{tabular}

1.1 New Generation

\begin{tabular}{|l|l|l|}
\hline \multicolumn{1}{|c|}{ Start Year } & \multicolumn{1}{|c|}{ End Year } & Annual Volume (m3) \\
\hline 1997 & 2010 & 1000 \\
\hline 2040 & 2050 & 1500 \\
\hline & & \\
\hline & & \\
\hline
\end{tabular}

1.2 Shipping Route(s)

\begin{tabular}{|l|l|c|}
\hline \multicolumn{1}{|c|}{ Route ID } & \multicolumn{1}{|c|}{ Source Facility } & Destination Facility \\
\hline C1h-1 & Legacy-MOPL & MOPLFAC1-CASE1 \\
\hline C1h-2 & MOPLFAC1-CASE1 & MOPLFAC2-CASE1 \\
\hline & & \\
\hline & & \\
\hline & & \\
\hline & & \\
\hline
\end{tabular}

1.3 Shipping Schedule(s)

\begin{tabular}{|c|l|l|l|}
\hline Route ID: C1h-1 & \multicolumn{2}{|c|}{ Waste Type } & Annual Volume (m3) \\
\hline Start Year & \multicolumn{1}{|c|}{ End Year } & 135.14 \\
\hline & 2070 & LLW & \\
\hline & & & \\
\hline & & & \\
\hline
\end{tabular}

1.3 Shipping Schedule(s) (continued)

\begin{tabular}{|c|c|c|c|}
\hline Route ID: Clh-2 & \multicolumn{2}{|c|}{ Waste Type } & \multicolumn{1}{|c|}{ Annual Volume (m3) } \\
\hline Start Year & \multicolumn{1}{|c|}{ End Year } & 135.14 \\
\hline 1997 & 2070 & LLW & \\
\hline & & & \\
\hline & & & \\
\hline
\end{tabular}

2.0 Facility Information

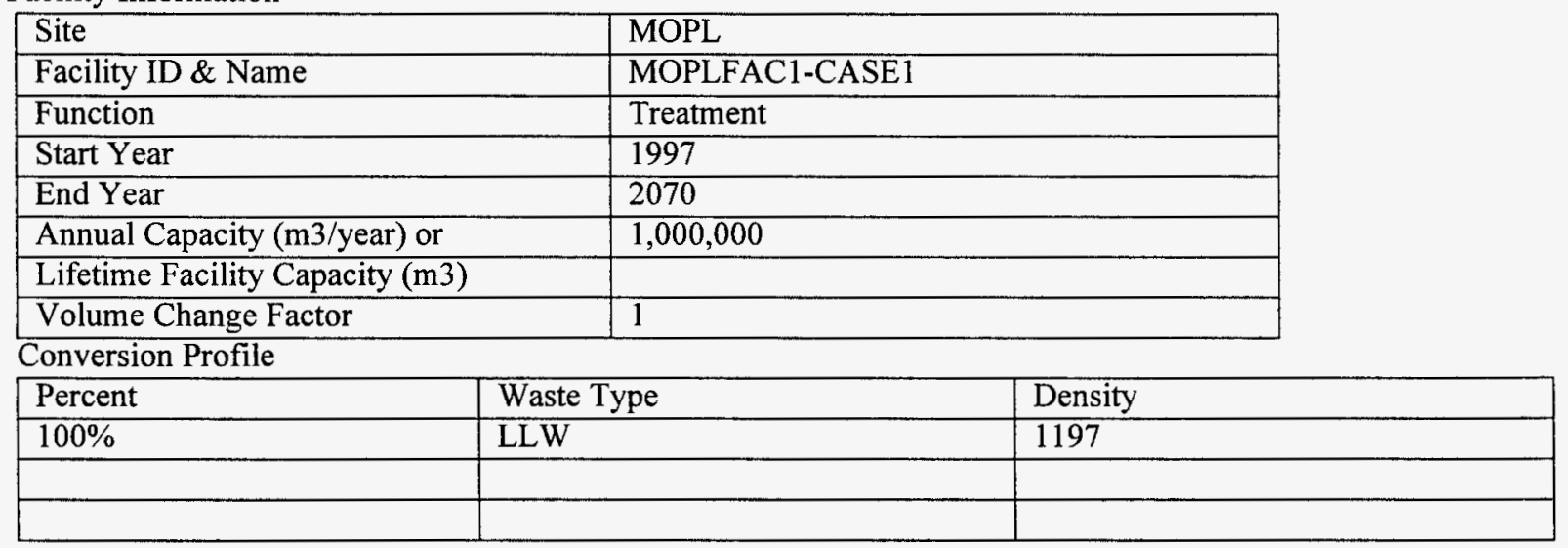


INITIAL PARAMETERS \& RESULTS REPORT

CASE: Case $1 \mathrm{~h}$

DATE: $11 / 13 / 97$

Evaluator: K B Oswald

2.0 Facility Information

\begin{tabular}{|l|l|}
\hline Site & MOPL \\
\hline Facility ID \& Name & MOPLFAC2-CASE1 \\
\hline Function & Disposal \\
\hline Start Year & 1997 \\
\hline End Year & 2070 \\
\hline Annual Capacity (m3/year) or & $1,000,000$ \\
\hline Lifetime Facility Capacity (m3) & \\
\hline Volume Change Factor & 1 \\
\hline
\end{tabular}

Conversion Profile

\begin{tabular}{|l|l|l|}
\hline Percent & Waste Type & Density \\
\hline NA & NA & NA \\
\hline & & \\
\hline
\end{tabular}


WASTE DISPOSITION - EXCEL CHART (MOPL, LLW):

\section{Waste Disposition}

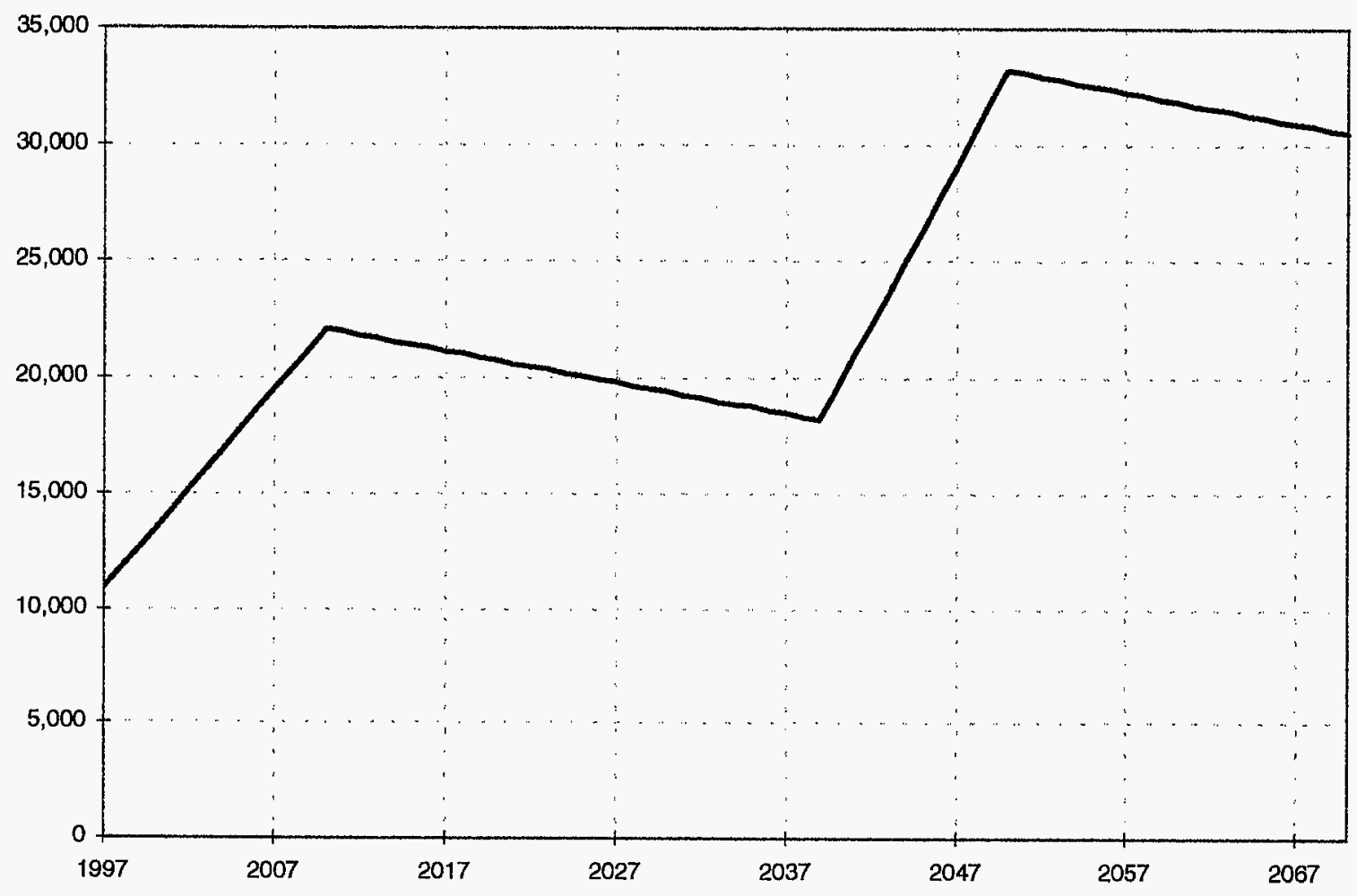

WASTE DISPOSITION - CWWFA CHART:

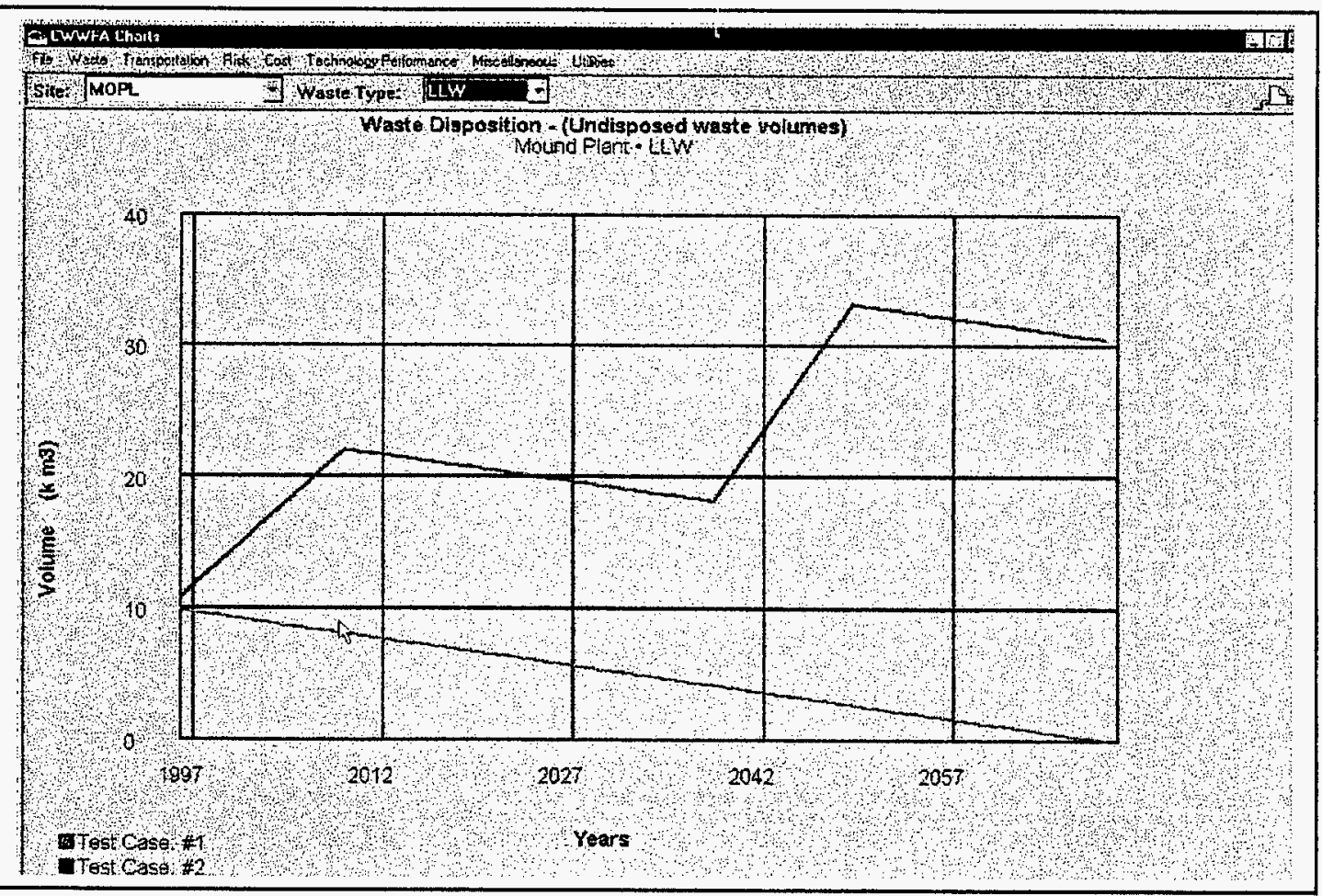


WASTE STREAM DETAIL EXCEL CHART (MOPLWS1-CASE1):

\section{Waste Stream Detail}

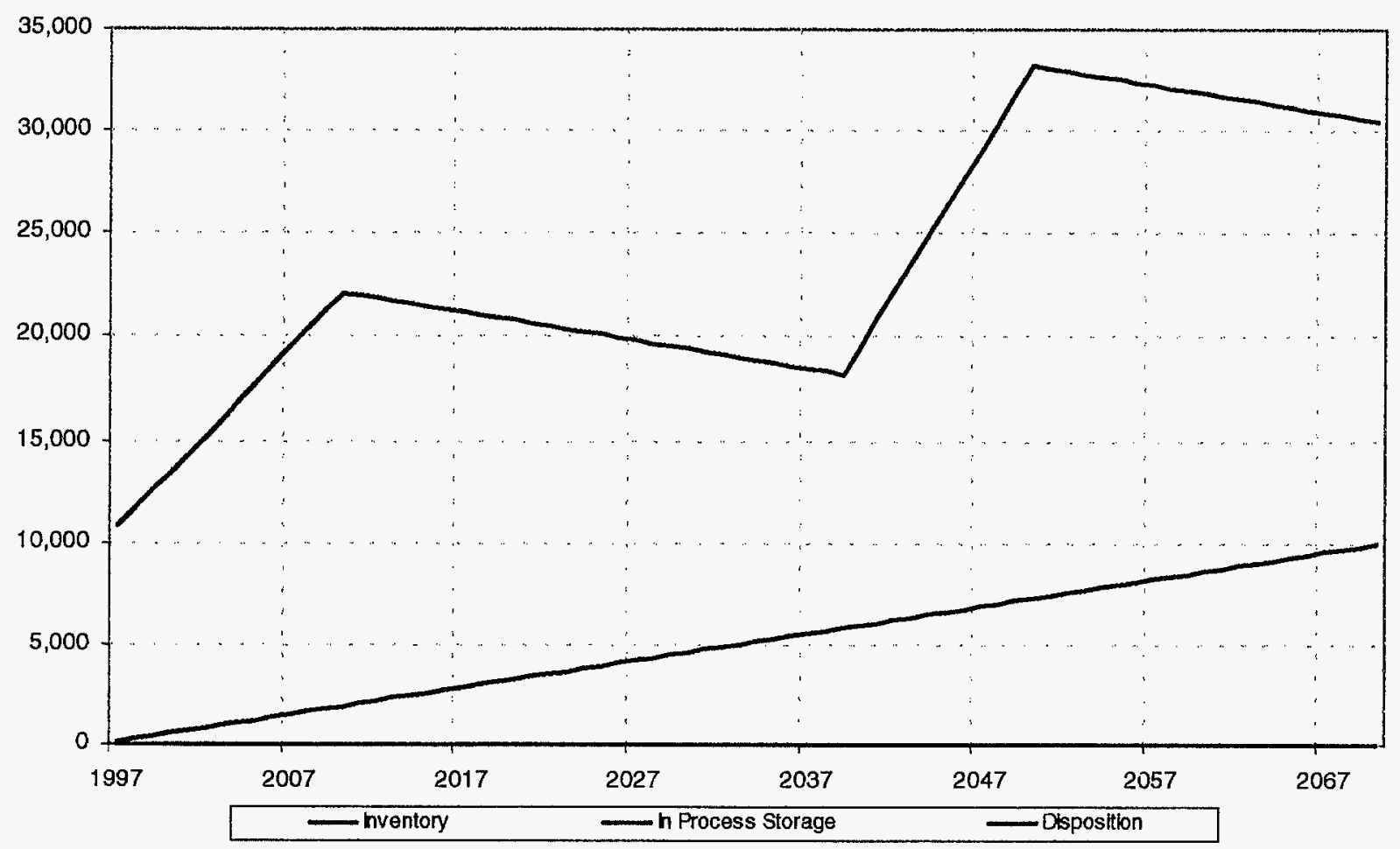

WASTE STREAM DETAIL CWWFA CHART:

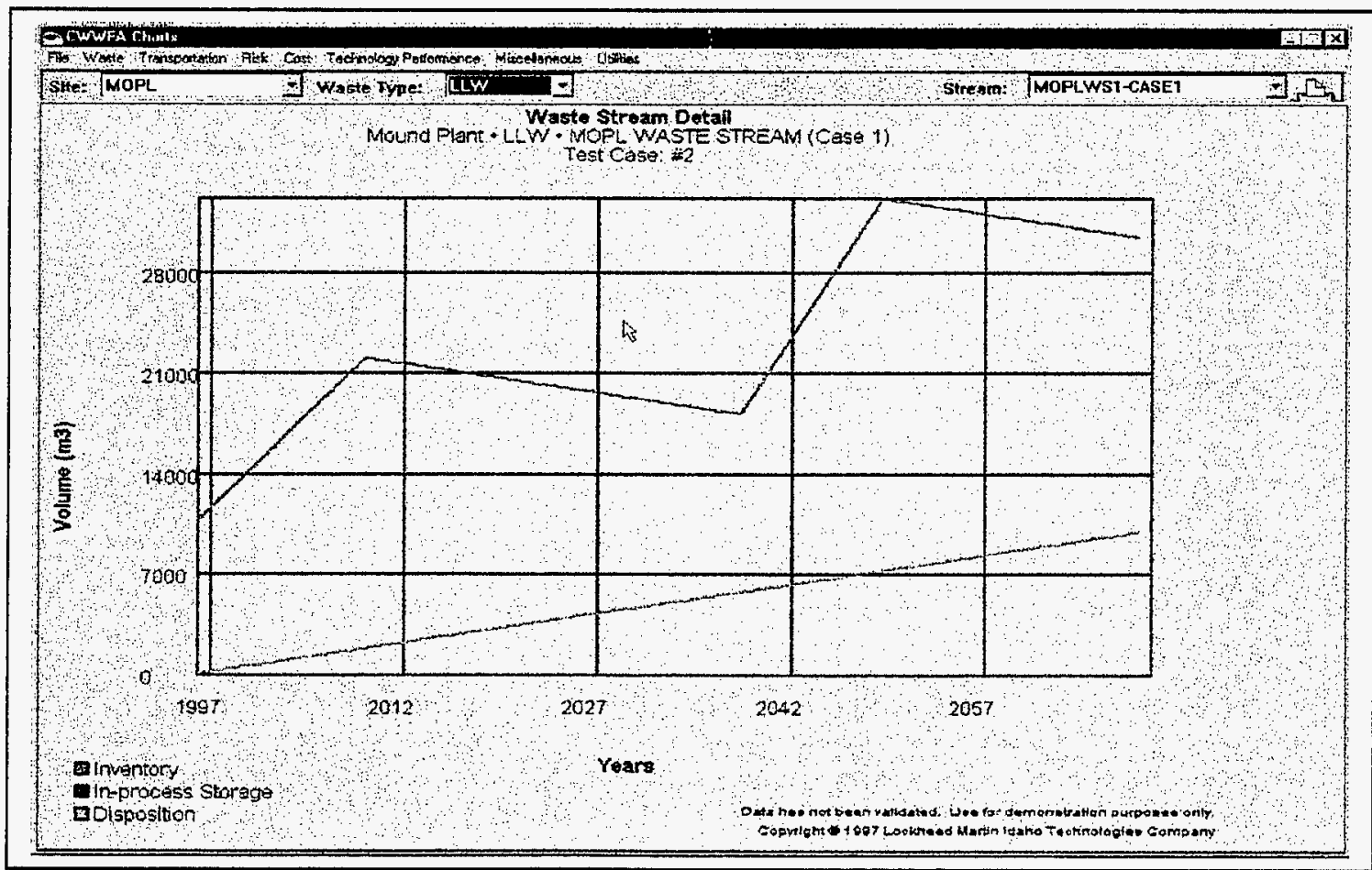


FACILITY CAPACITY EXCEL CHART (MOPL, MOPLFAC2-CASE1):

\section{Facility Capacity Detail}

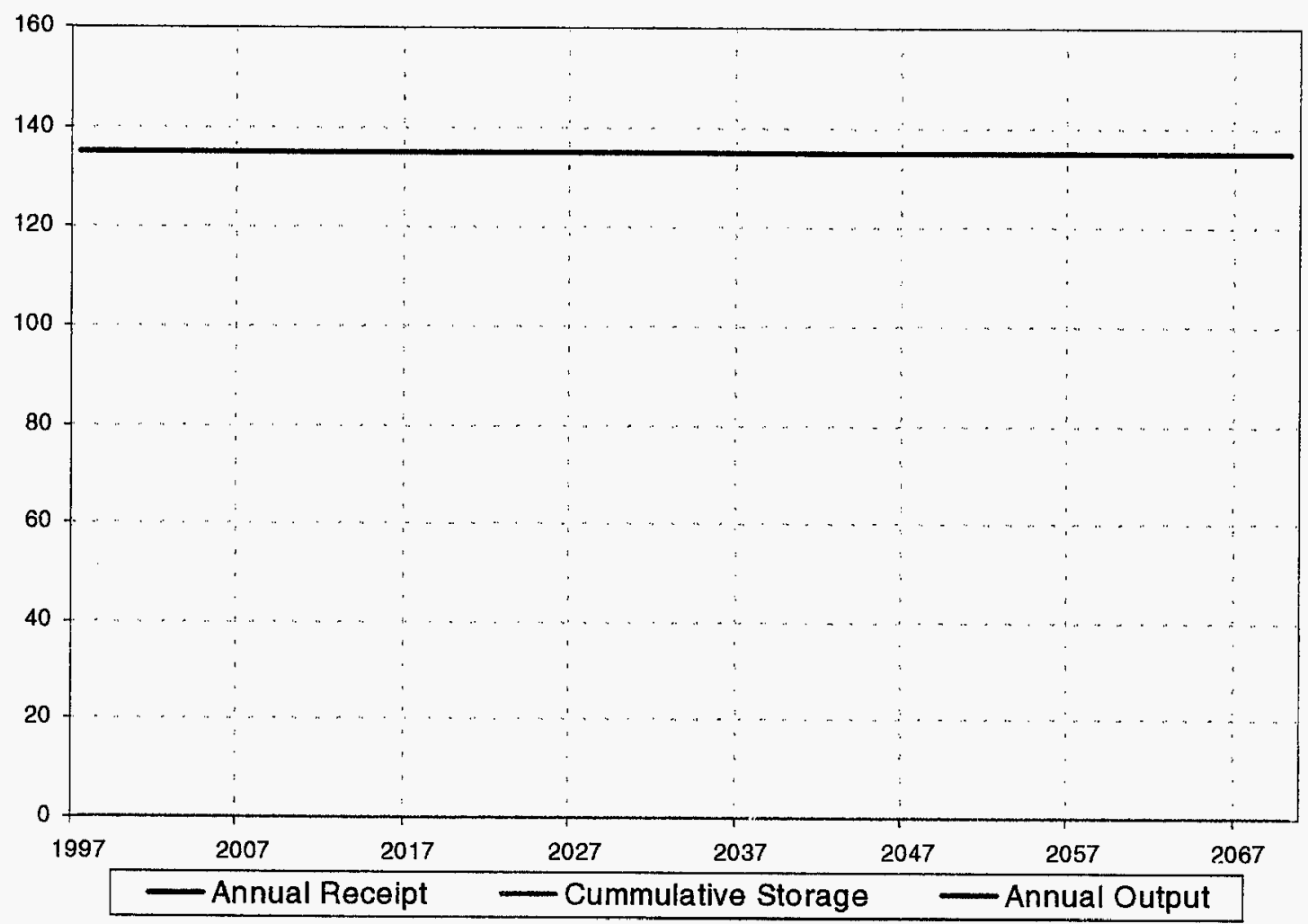

\section{FACILITY CAPACITY CWWFA CHART:}

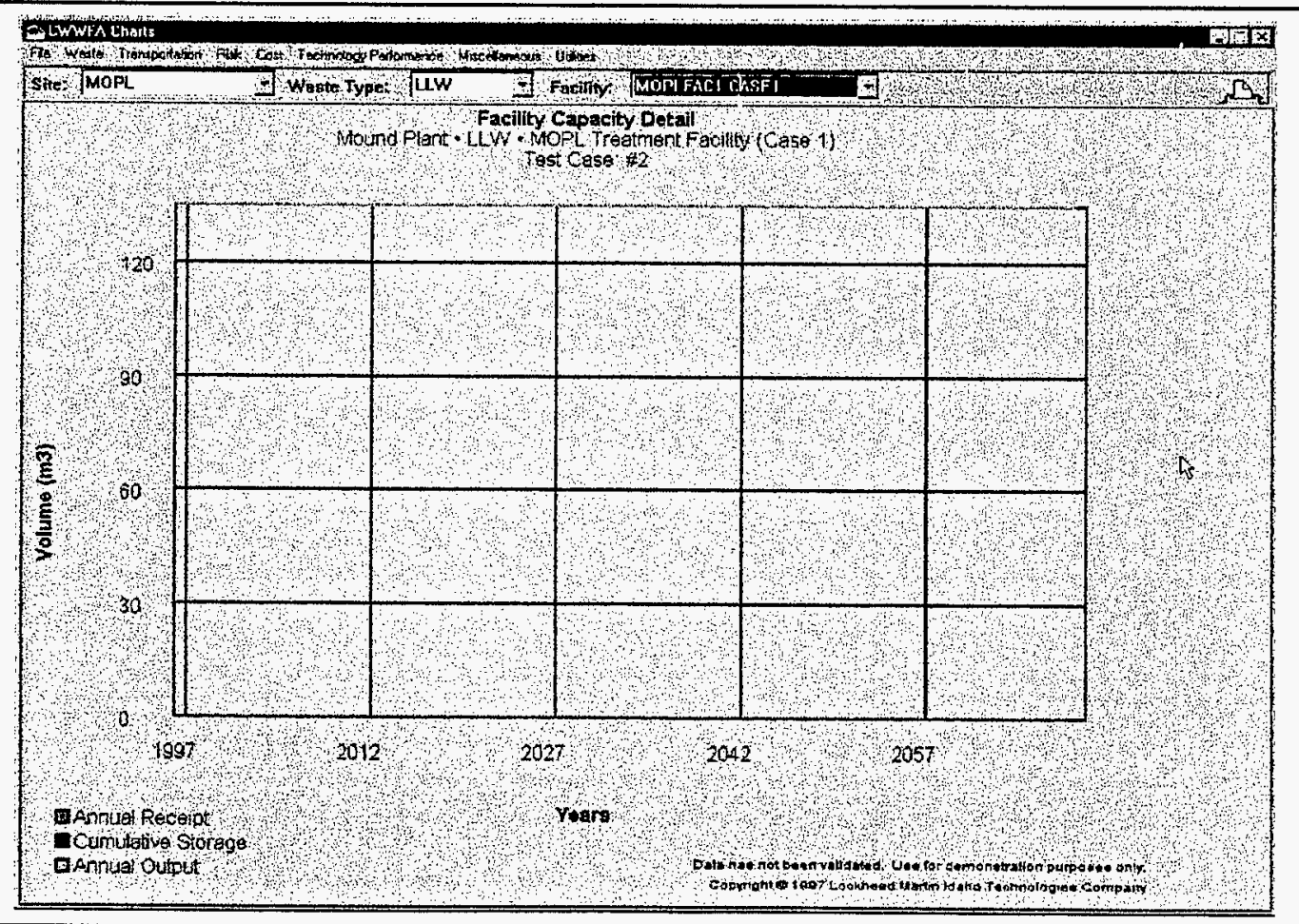


1.0 Waste Stream Information

\begin{tabular}{|l|l|}
\hline Originating Site & MOPL \\
\hline Waste Stream ID \& Name & MOPLWS1-Case1 \\
\hline Legacy volume (m3) & 10,000 \\
\hline
\end{tabular}

1.1 New Generation

\begin{tabular}{|l|l|l|}
\hline \multicolumn{1}{|c|}{ Start Year } & End Year & Annual Volume (m3) \\
\hline $1997 \quad 2020$ & 1000 \\
\hline 2040 & 2060 & 1500 \\
\hline & & \\
\hline
\end{tabular}

1.2 Shipping Route(s)

\begin{tabular}{|l|l|l|}
\hline \multicolumn{1}{|c|}{ Route ID } & \multicolumn{1}{|c|}{ Source Facility } & Destination Facility \\
\hline C1I -1 & Legacy-MOPL & MOPLFAC1-CASE1 \\
\hline C1I-2 & MOPLFAC1-CASE1 & MOPLFAC2-CASE1 \\
\hline & & \\
\hline & & \\
\hline & & \\
\hline & & \\
\hline
\end{tabular}

1.3 Shipping Schedule(s)

\begin{tabular}{|c|l|l|l|}
\hline Route ID: C1I-1 & \multicolumn{2}{|c|}{ Waste Type } & \multicolumn{1}{|c|}{ Annual Volume (m3) } \\
\hline Start Year & \multicolumn{1}{|c|}{ End Year } & \multicolumn{1}{|c|}{ - } & 200 \\
\hline 1997 & 2010 & LLW & 900 \\
\hline & 2060 & LLW & \\
\hline & & & \\
\hline
\end{tabular}

1.3 Shipping Schedule(s) (continued)

\begin{tabular}{|c|c|c|c|}
\hline Route ID: C1I-2 & \multicolumn{2}{|c|}{ Waste Type } & \multicolumn{1}{|c|}{ Annual Volume (m3) } \\
\hline Start Year & \multicolumn{1}{|c|}{ End Year } & \multicolumn{1}{|c|}{ W } & 500 \\
\hline 1997 & 2005 & LLW & 500 \\
\hline & 2070 & LLW & \\
\hline & & & \\
\hline
\end{tabular}

2.0 Facility Information

\begin{tabular}{|l|l|}
\hline Site & MOPL \\
\hline Facility ID \& Name & MOPLFAC1-CASE1 \\
\hline Function & Treatment \\
\hline Start Year & 1997 \\
\hline End Year & 2070 \\
\hline Annual Capacity (m3/year) or & $1,000,000$ \\
\hline Lifetime Facility Capacity (m3) & \\
\hline Volume Change Factor & 1 \\
\hline
\end{tabular}

Conversion Profile

\begin{tabular}{|l|l|l|}
\hline Percent & Waste Type & Density \\
\hline $80 \%$ & LLW & 1197 \\
\hline $20 \%$ & MLLW & 500 \\
\hline & & \\
\hline
\end{tabular}


2.0 Facility Information

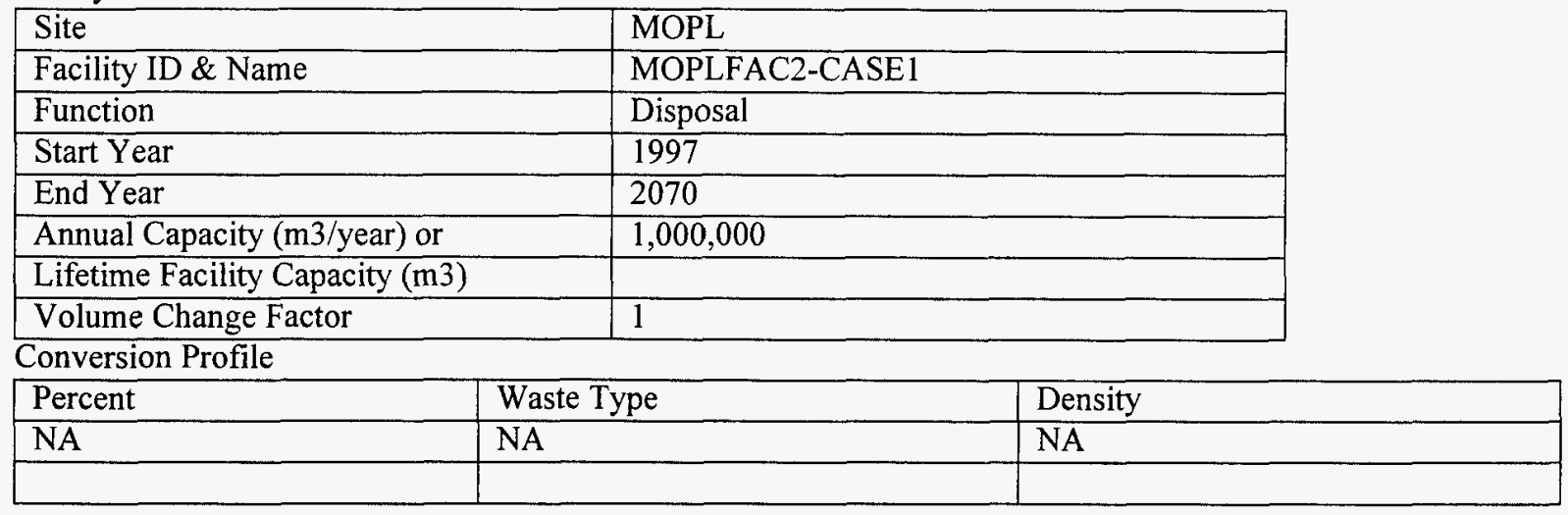


WASTE DISPOSITION - EXCEL CHART (MOPL, LLW):

\section{Waste Disposition}

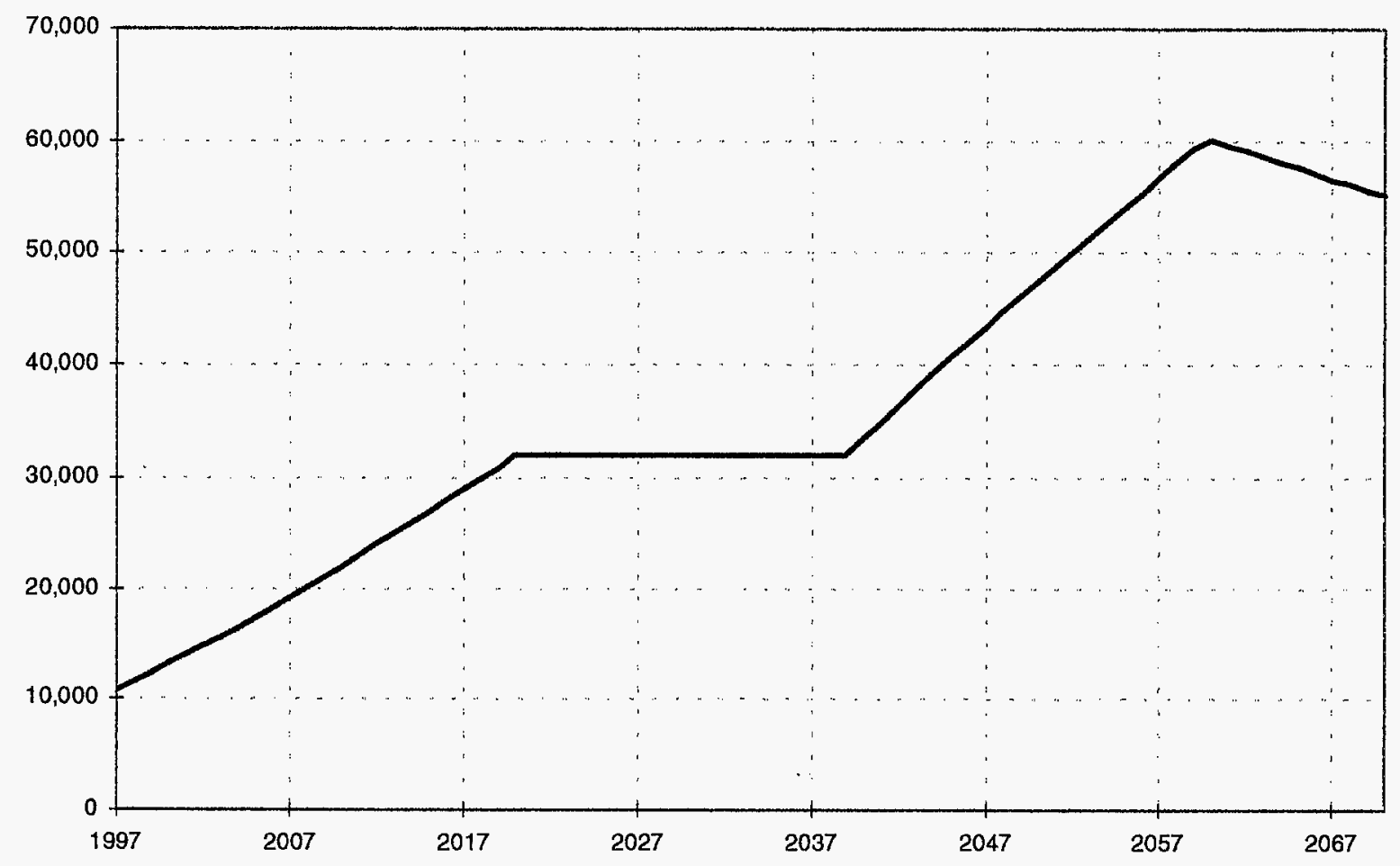

\section{WASTE DISPOSITION - CWWFA CHART:}

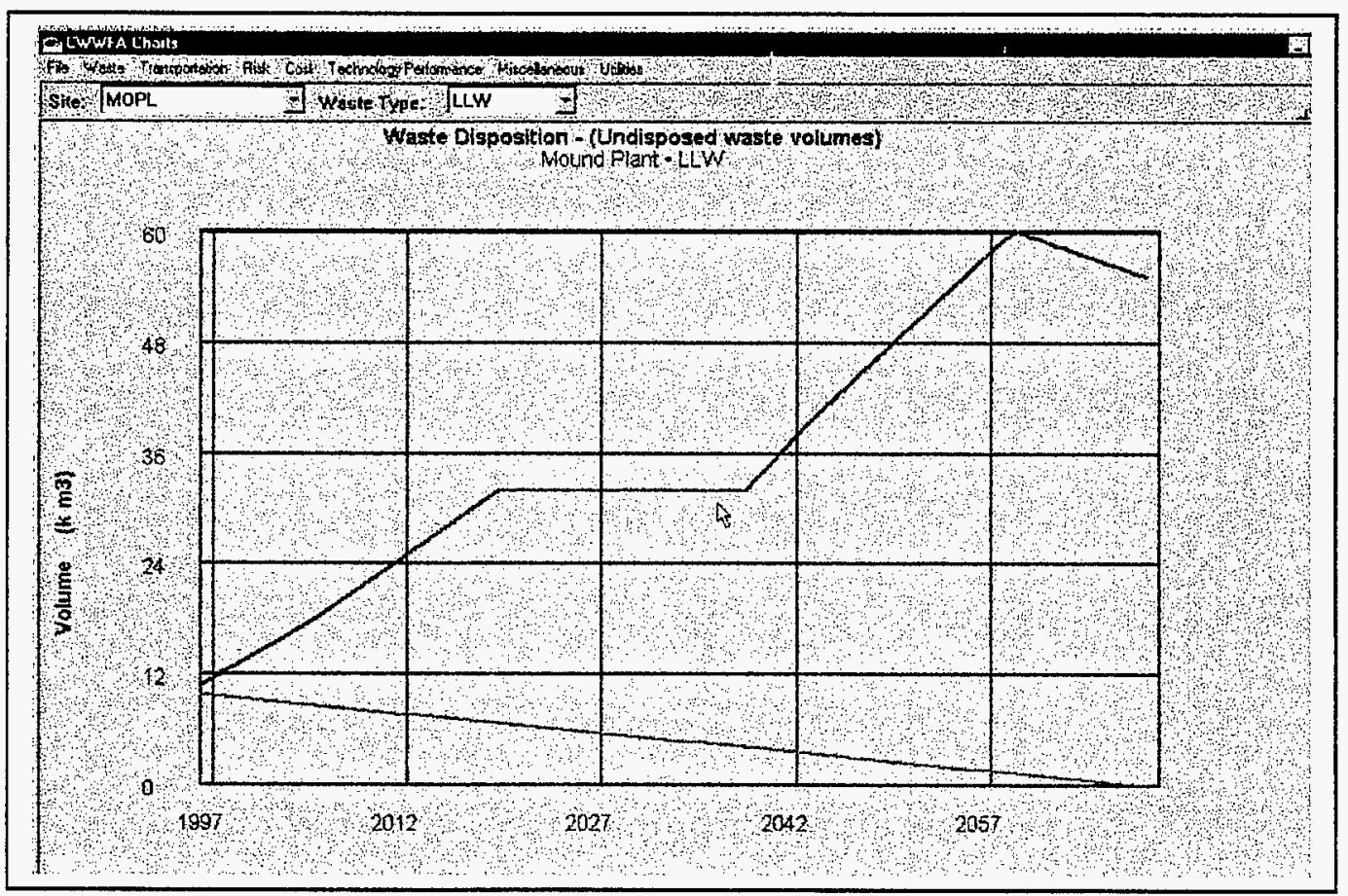




\section{WASTE STREAM DETAIL EXCEL CHART (MOPLWS1-CASE1):}

\section{Waste Stream Detail}

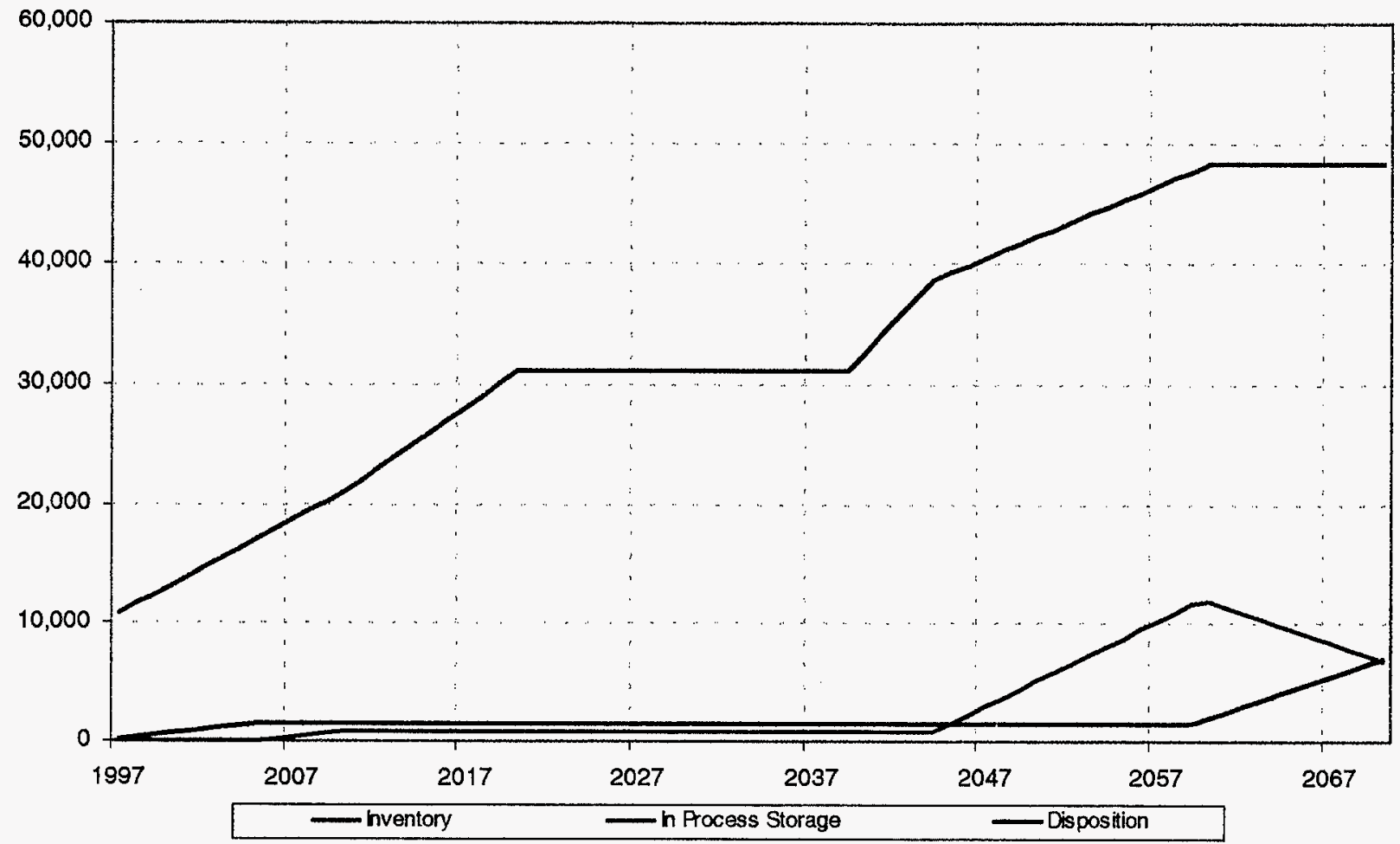

WASTE STREAM DETAIL CWWFA CHART:

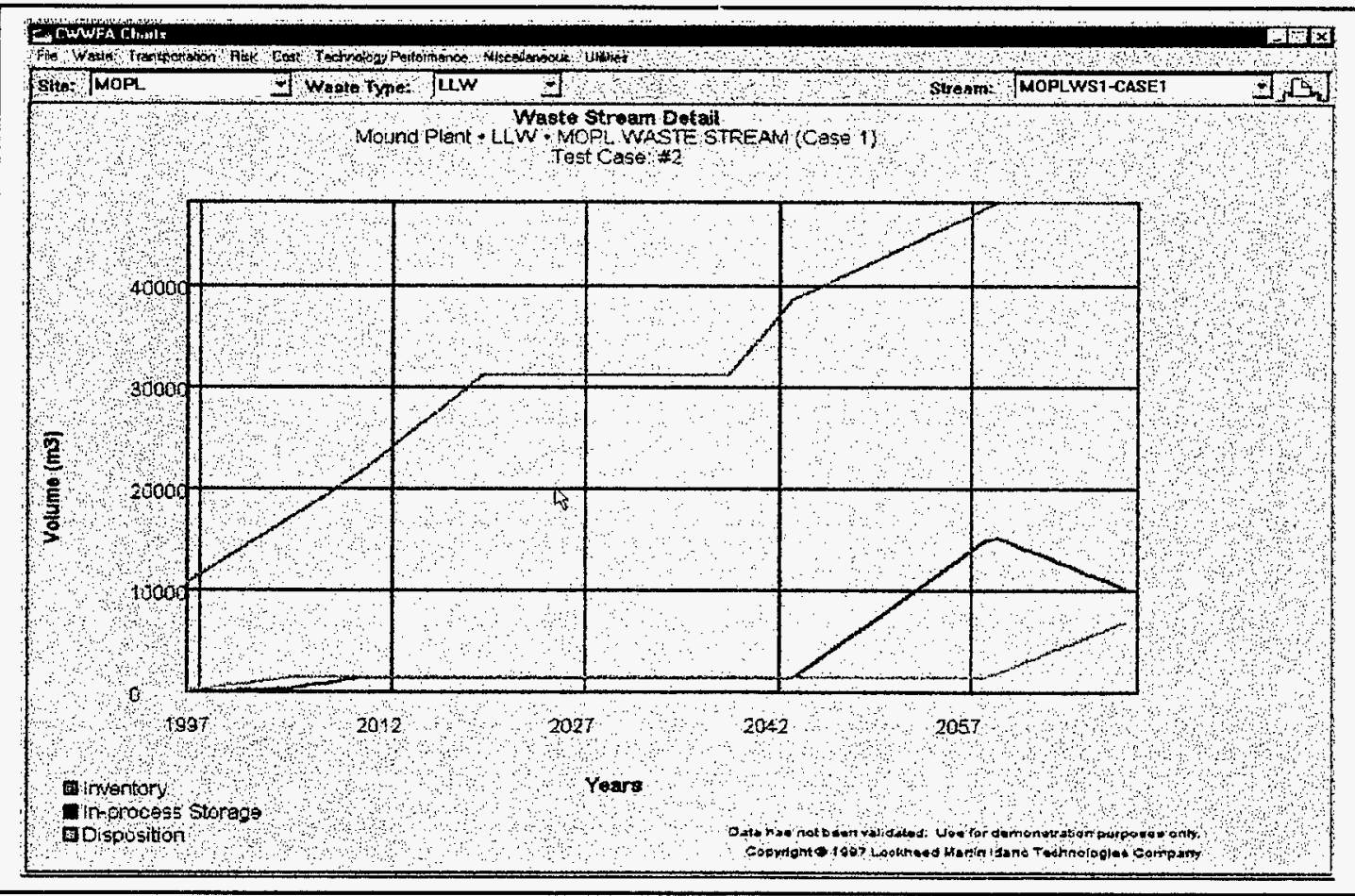


FACILITY CAPACITY EXCEL CHART (MOPL, MOPLFAC2-CASE1):

Facility Capacity Detail

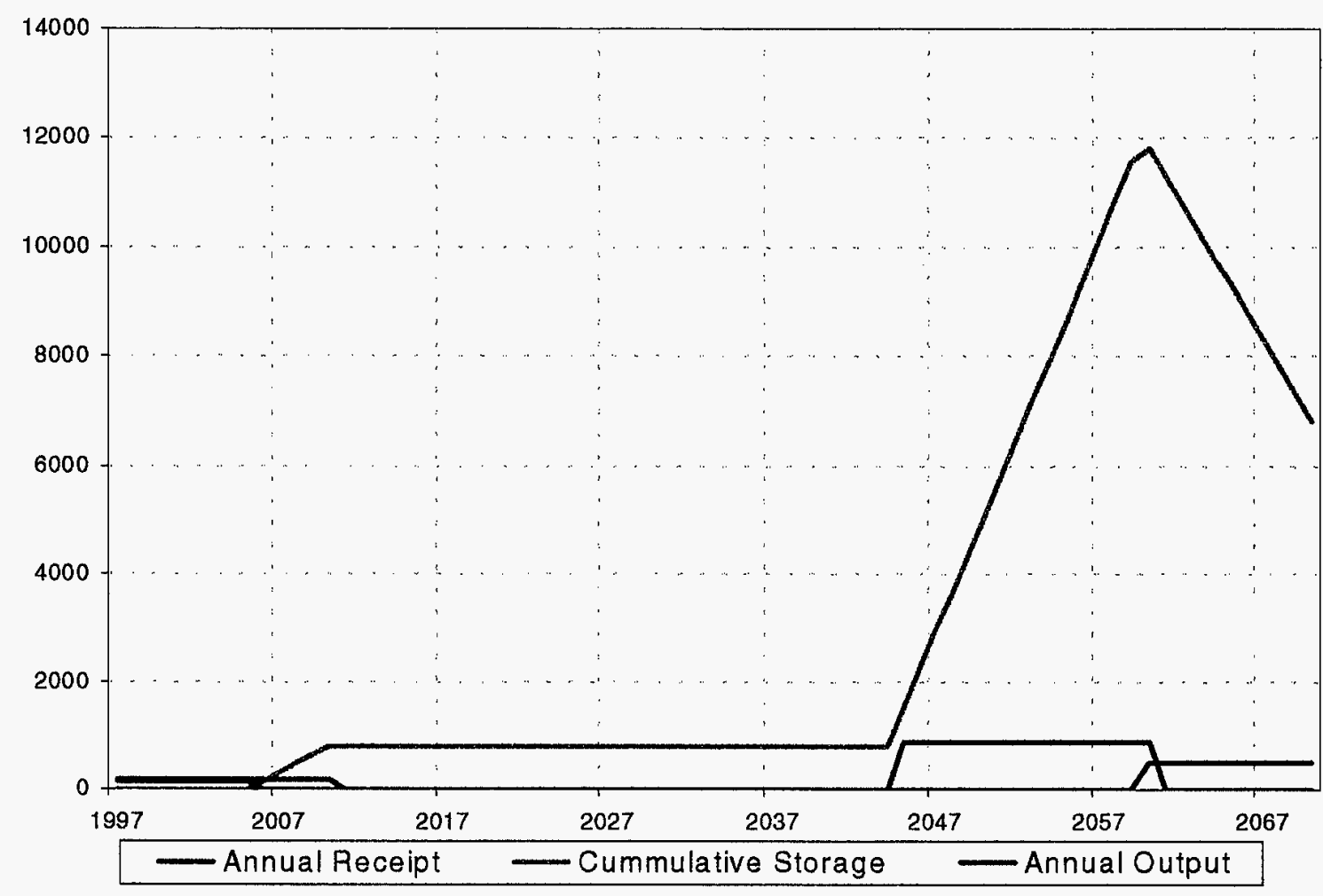

\section{FACILITY CAPACITY CWWFA CHART:}

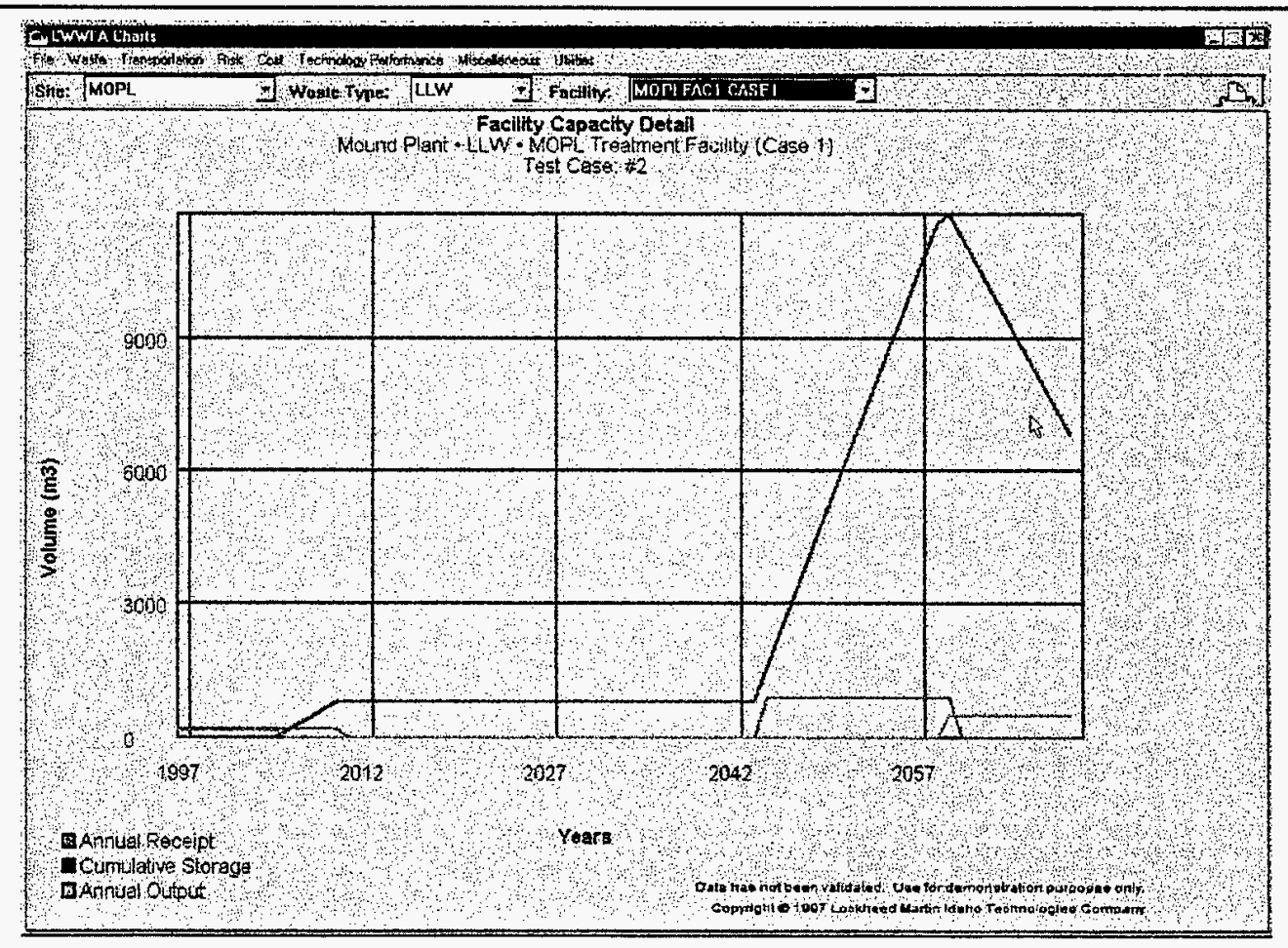


1.0 Waste Stream Information

\begin{tabular}{|l|l|}
\hline Originating Site & WVDP \\
\hline Waste Stream ID \& Name & WVDPWS1-CASE2a \\
\hline Originating Type & HLW \\
\hline Legacy volume (m3) & 15,000 \\
\hline
\end{tabular}

1.1 New Generation

\begin{tabular}{|l|l|l|}
\hline \multicolumn{1}{|c|}{ Start Year } & \multicolumn{1}{|c|}{ End Year } & Annual Volume (m3) \\
\hline NA & NA & NA \\
\hline & & \\
\hline & & \\
\hline & & \\
\hline
\end{tabular}

1.2 Shipping Route(s)

\begin{tabular}{|l|l|l|}
\hline \multicolumn{1}{|c|}{ Route ID } & \multicolumn{1}{|c|}{ Source Facility } & \multicolumn{1}{c|}{ Destination Facility } \\
\hline C2a-1 & Legacy-WW & SARSFAC1-CASE2 \\
\hline C2a-2 & SARSFAC1-CASE2 & WVDPFAC1-CASE2 \\
\hline C2a-3 & WWDPFAC1-CASE2 & SARSFAC2-CASE2 \\
\hline & & \\
\hline & & \\
\hline & & \\
\hline
\end{tabular}

1.3 Shipping Schedule(s)

\begin{tabular}{|c|c|c|c|}
\hline Route ID: C2a-1 & \multicolumn{2}{|c|}{} & \multicolumn{1}{|c|}{ Waste Type } \\
\hline Start Year & \multicolumn{1}{|c|}{ End Year } & Annual Volume (m3) \\
\hline 1997 & 2070 & HLW & 203 \\
\hline & & & \\
\hline & & & \\
\hline
\end{tabular}

1.3 Shipping Schedule(s) (continued)

\begin{tabular}{|c|l|l|l|}
\hline Route ID: C2a-2 & \multicolumn{2}{|c|}{ Ennual Volume (m3) } \\
\hline Start Year & \multicolumn{1}{|c|}{ End Year } & \multicolumn{1}{|c|}{ Waste Type } & Ann \\
\hline 1997 & 2070 & HLW & \\
\hline & & & \\
\hline & & & \\
\hline
\end{tabular}

1.3 Shipping Schedule(s) (continued)

\begin{tabular}{|c|c|c|c|}
\hline Route ID: C2a-3 & \multicolumn{2}{|c|}{ Waste Type } & Annual Volume (m3) \\
\hline Start Year & \multicolumn{1}{|c|}{ End Year } & HLW & 203 \\
\hline & 2070 & & \\
\hline & & & \\
\hline
\end{tabular}


2.0 Facility Information

\begin{tabular}{|l|l|}
\hline Site & SARS \\
\hline Facility ID \& Name & SARSFAC1-CASE2 \\
\hline Function & Treatment \\
\hline Start Year & 1997 \\
\hline End Year & 2070 \\
\hline Annual Capacity (m3/year) or & $1,000,000$ \\
\hline Lifetime Facility Capacity (m3) & \\
\hline Volume Change Factor & 1 \\
\hline
\end{tabular}

Conversion Profile

\begin{tabular}{|l|l|l|}
\hline Percent & Waste Type & Density \\
\hline $100 \%$ & HLW & 650 \\
\hline & & \\
\hline & & \\
\hline
\end{tabular}

2.0 Facility Information

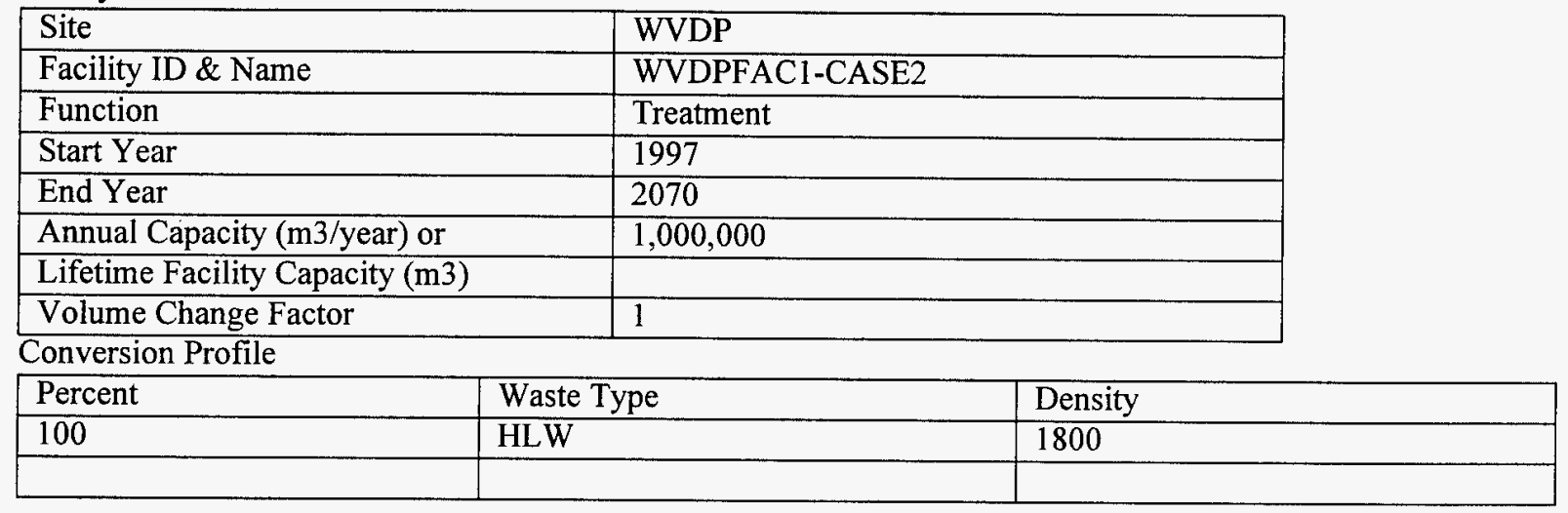

2.0 Facility Information

\begin{tabular}{|l|l|}
\hline Site & SARS \\
\hline Facility ID \& Name & SARSFAC1-CASE2 \\
\hline Function & Disposal \\
\hline Start Year & 1997 \\
\hline End Year & 2070 \\
\hline Annual Capacity (m3/year) or & $1,000,000$ \\
\hline Lifetime Facility Capacity (m3) & \\
\hline Volume Change Factor & 1 \\
\hline
\end{tabular}

Conversion Profile

\begin{tabular}{|l|l|l|}
\hline Percent & Waste Type & Density \\
\hline NA & NA & NA \\
\hline & & \\
\hline
\end{tabular}


WASTE DISPOSITION - EXCEL CHART (WVDP, HLW):

\section{Waste Disposition}

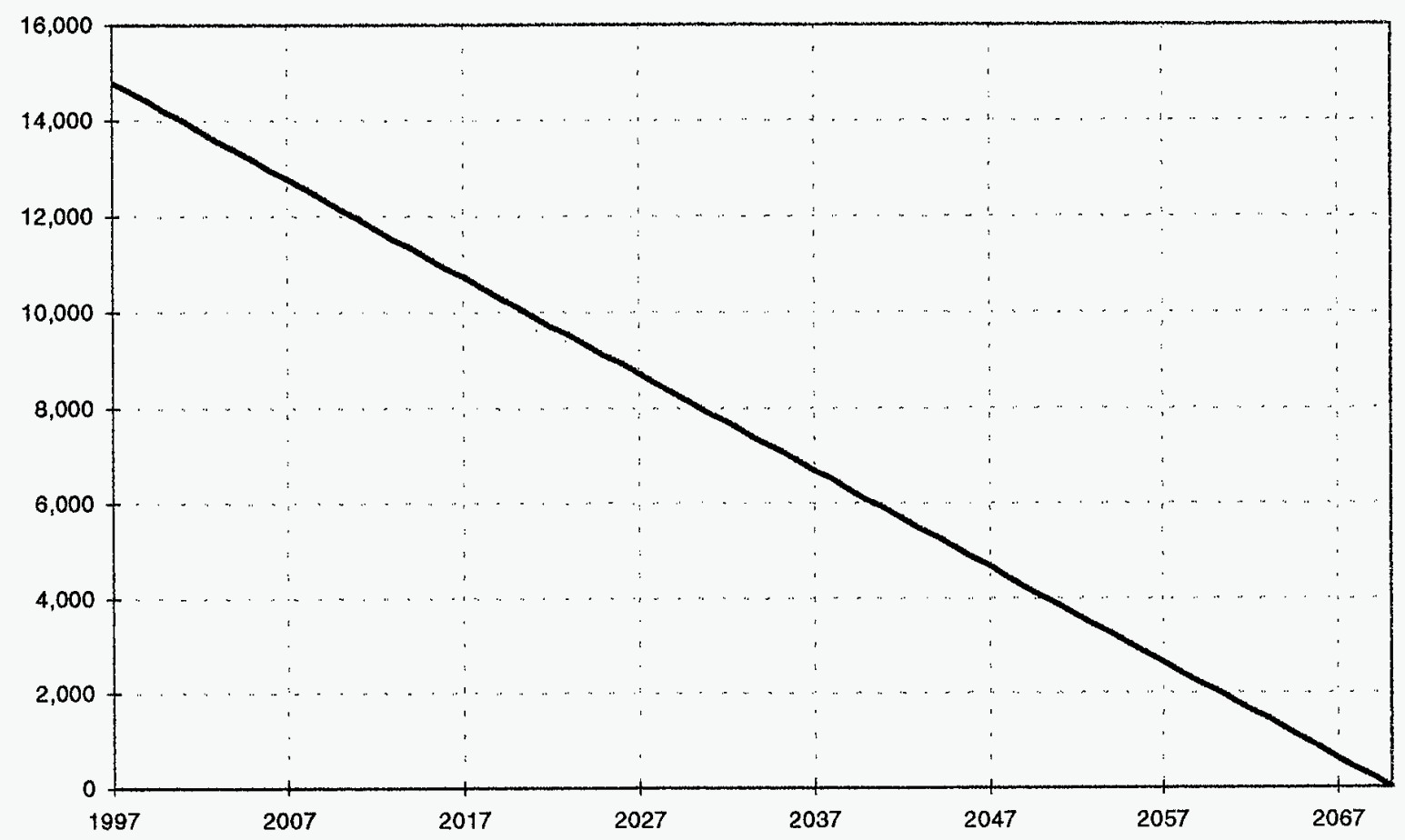

\section{WASTE DISPOSITION - CWWFA CHART:}

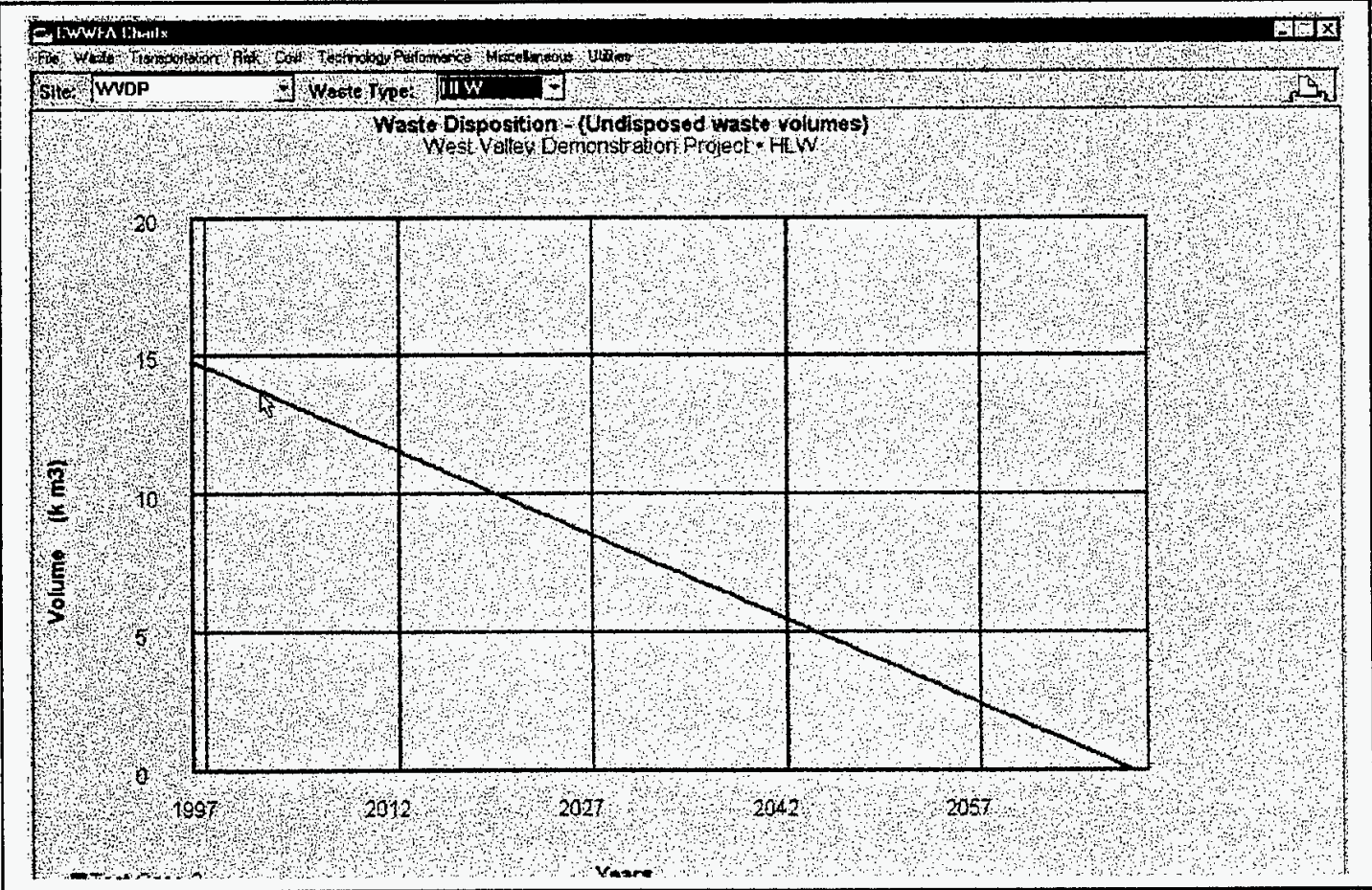


WASTE STREAM DETAIL EXCEL CHART (WVDPWS1-CASE2a):

\section{Waste Stream Detail}

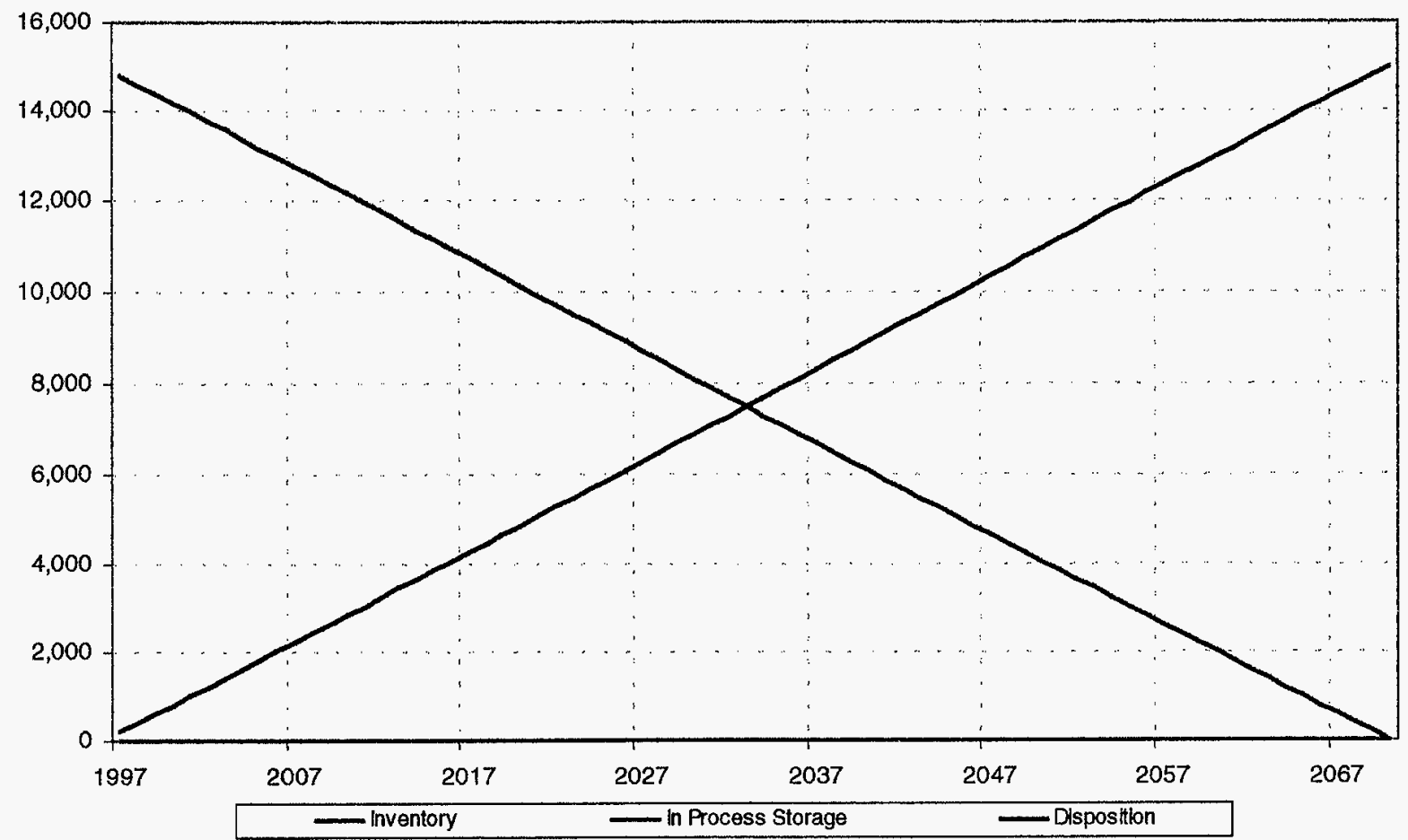

WASTE STREAM DETAIL CWWFA CHART:

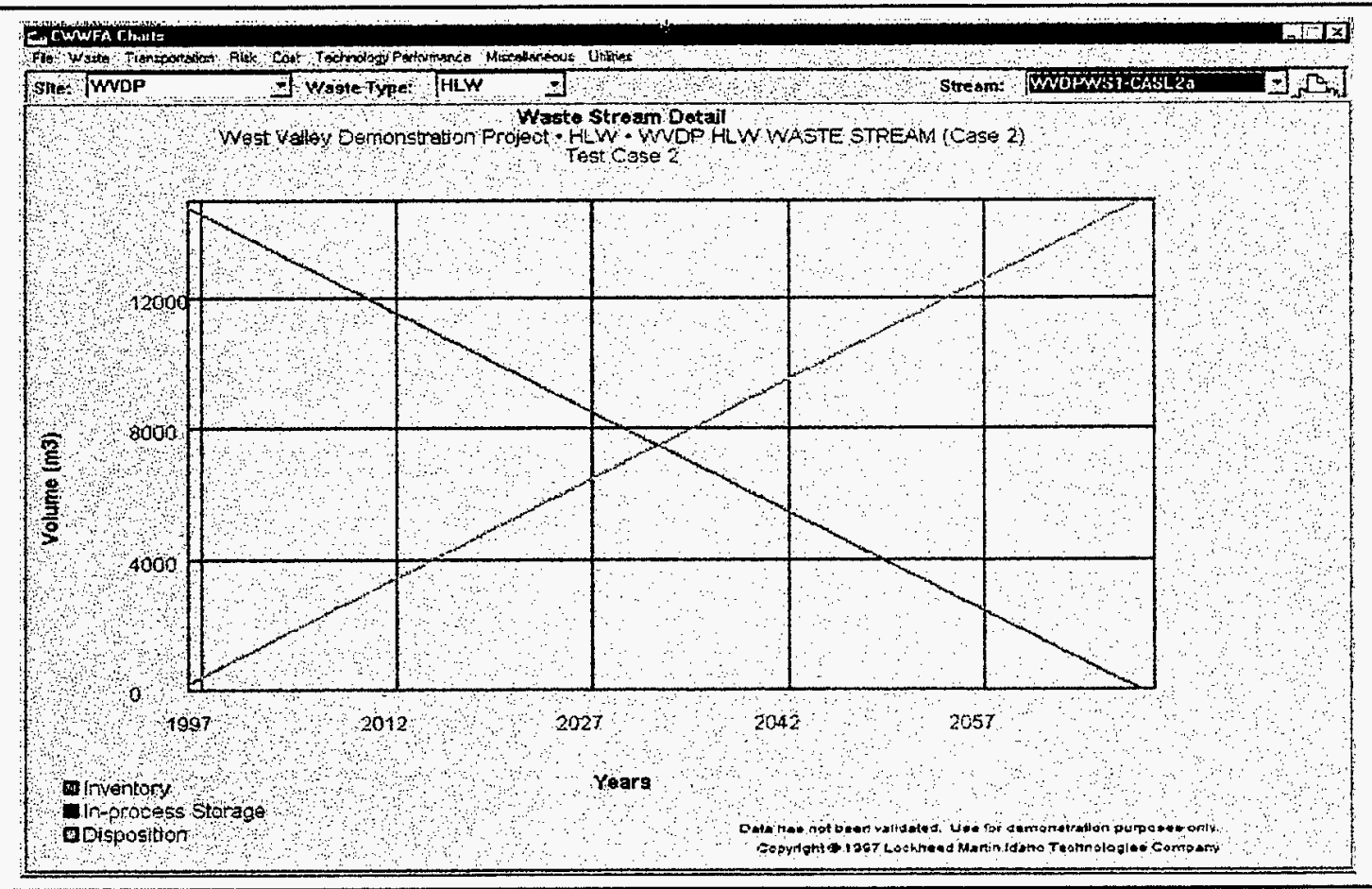


FACILITY CAPACITY EXCEL CHART (WVDP, MOPLFAC2-CASE1):

\section{Facility Capacity Detail}

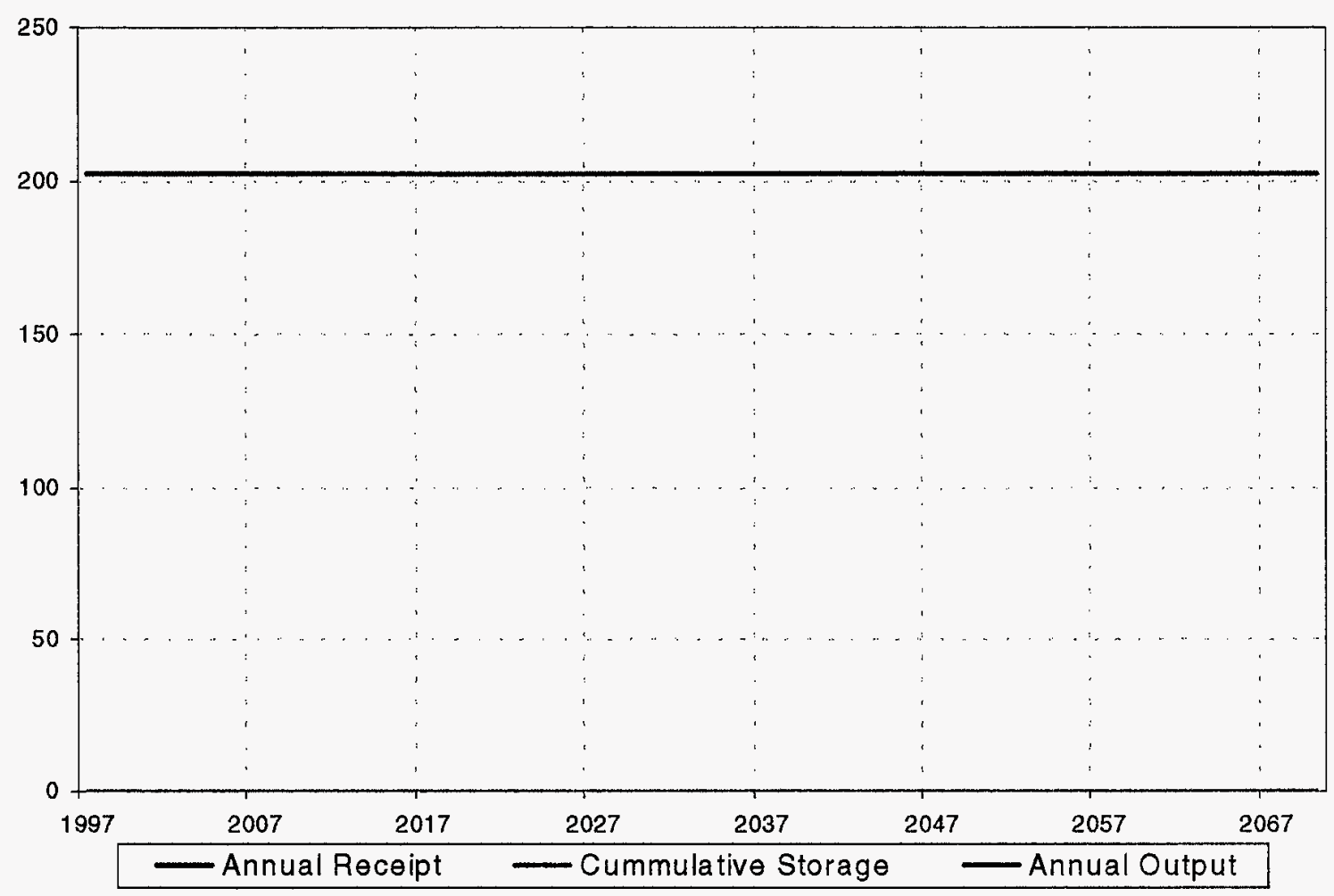

\section{FACILITY CAPACITY CWWFA CHART:}

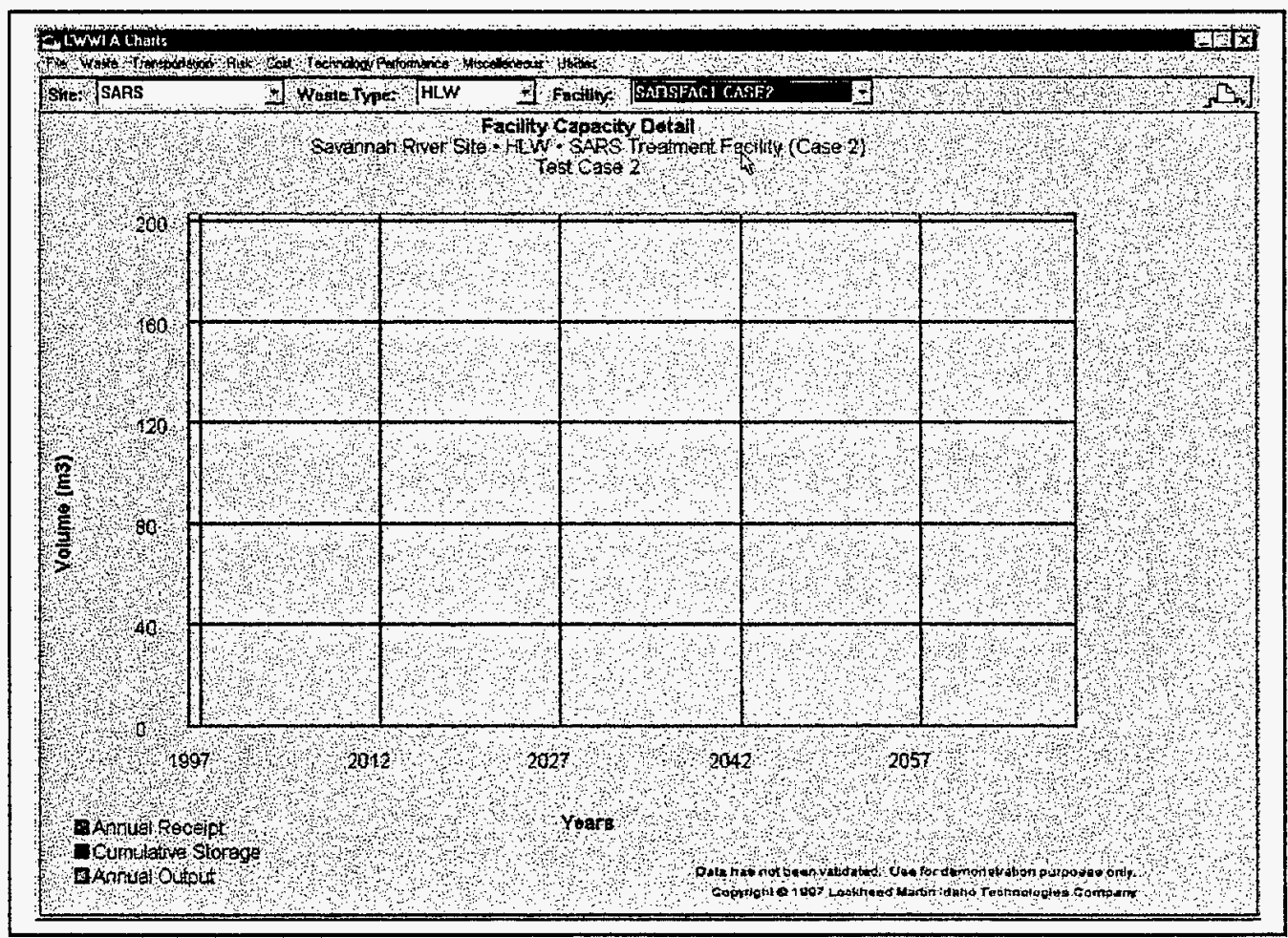


INITIAL PARAMETERS \& RESULTS REPORT
CASE: Case 2a
DATE: $11 / 13 / 97$
Evaluator: K B Oswald

SHIPMENTS RECEIVED EXCEL CHART (MOPL):

Shipments Received

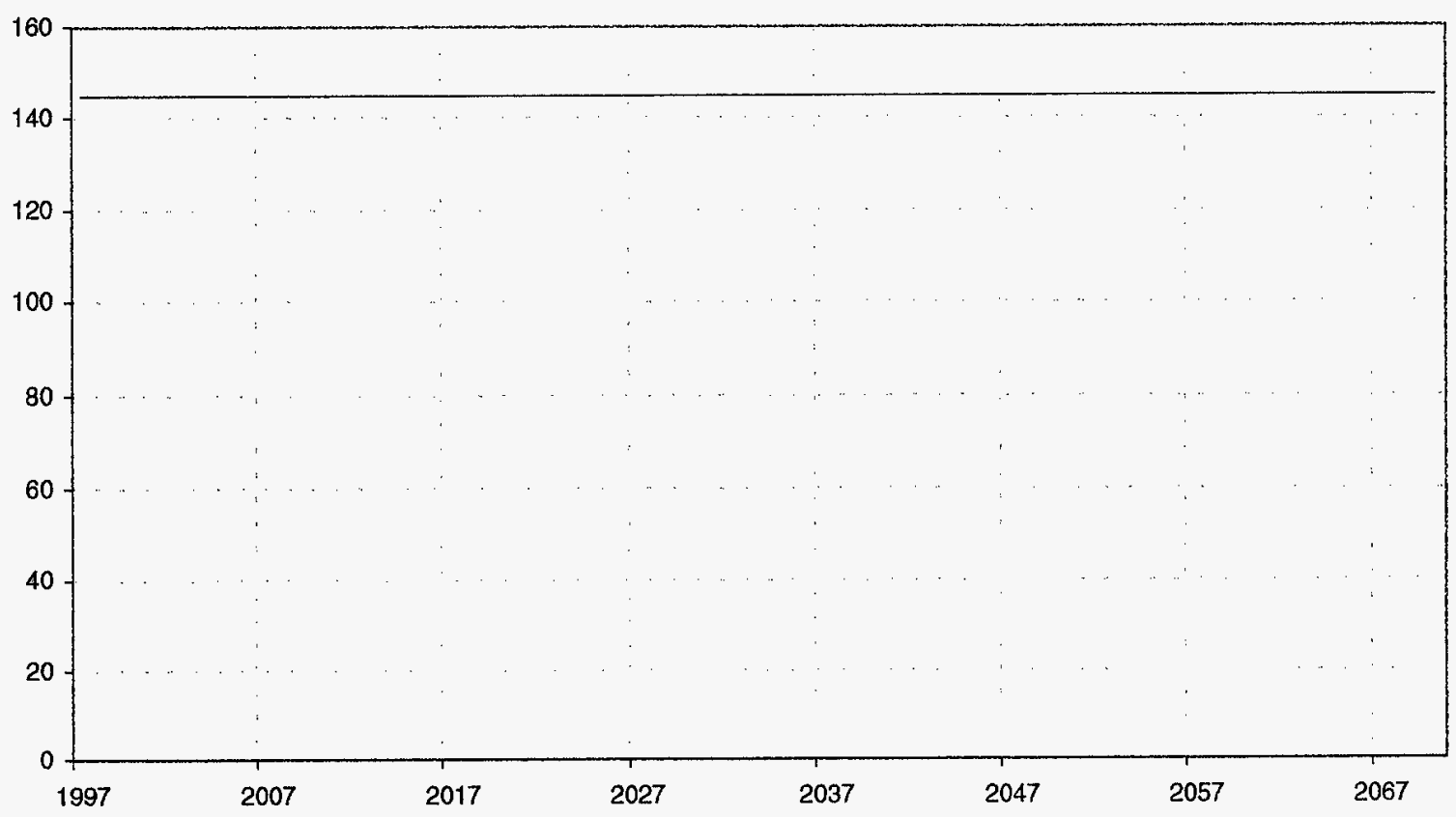

SHIPMENTS RECEIVED CWWFA CHART:

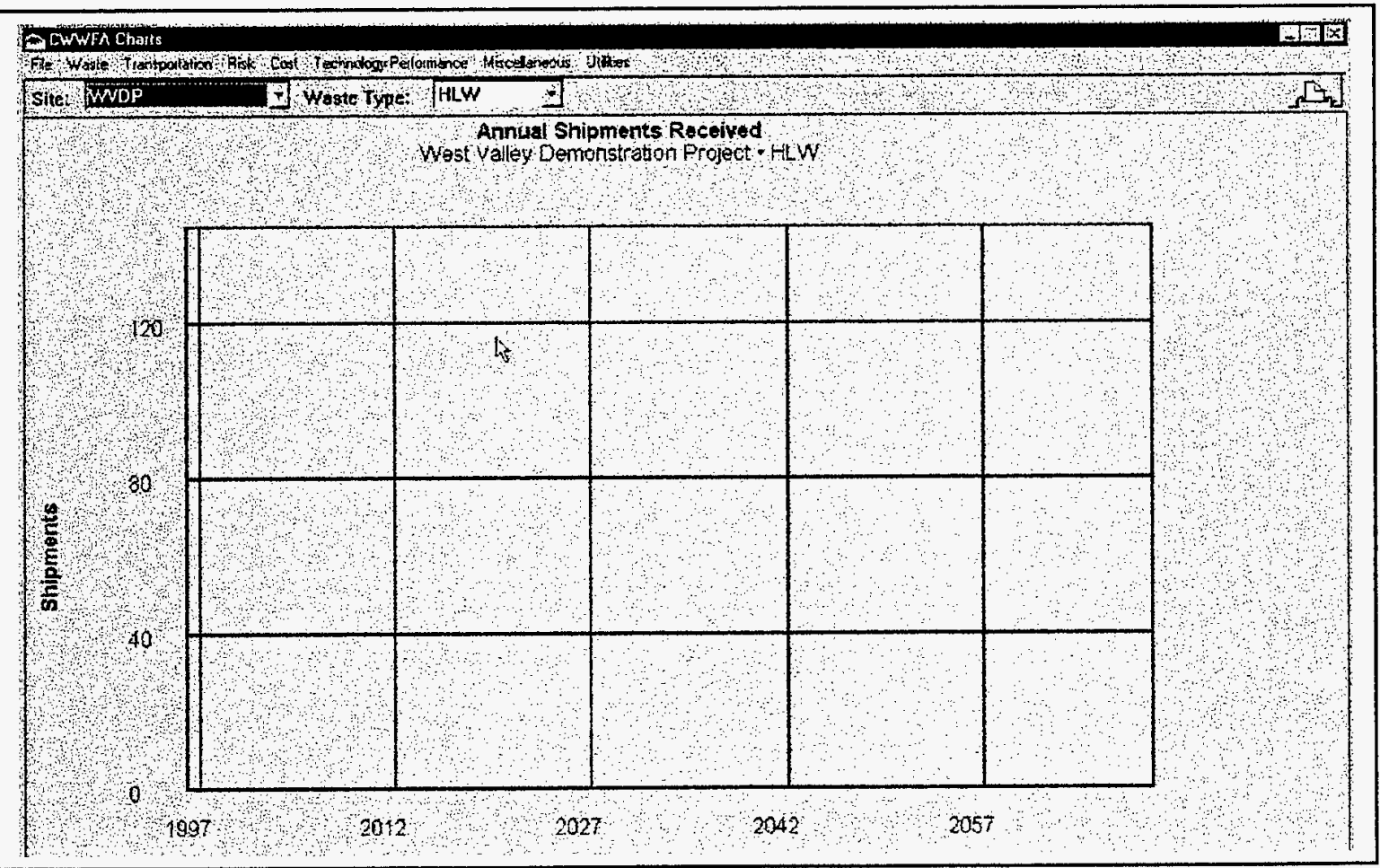


CASE: Case 2a

SHIPMENTS SENT EXCEL CHART (WVDP):

Evaluator: K B Oswald

\section{Shipments Sent}

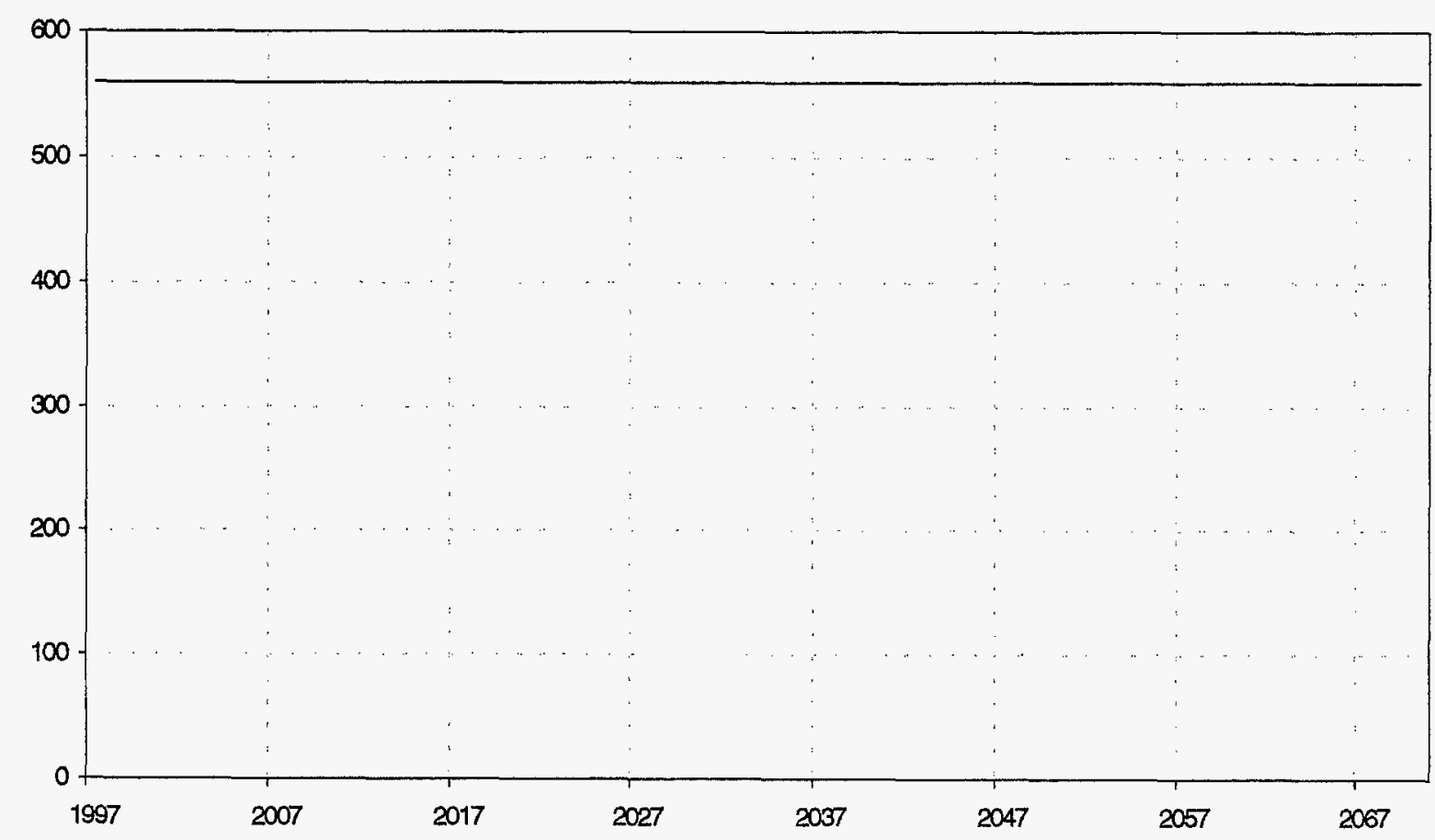

\section{SHIPMENTS SENT CWWFA CHART:}

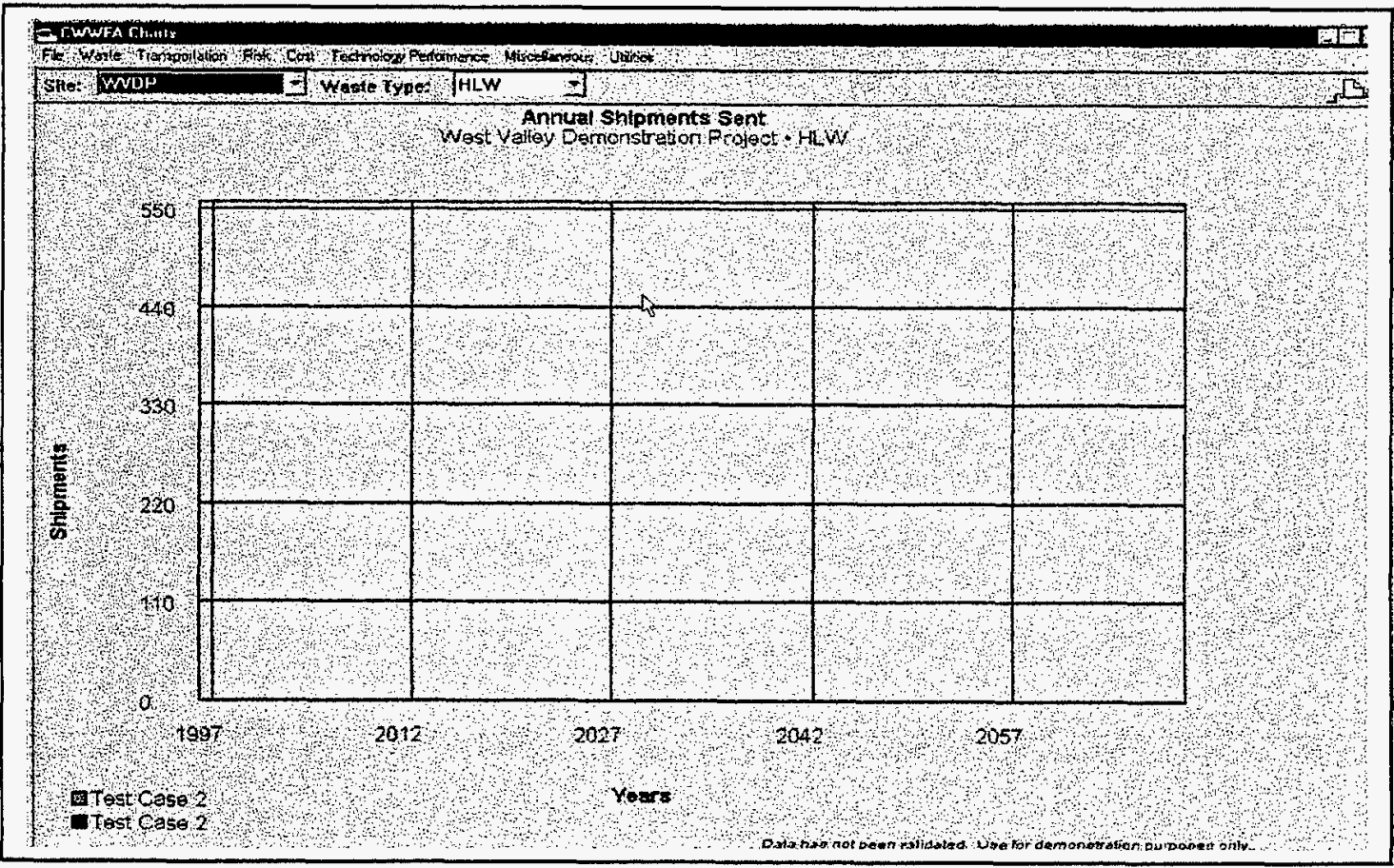


INITIAL PARAMETERS \& RESULTS REPORT

CASE: Case $2 \mathrm{a}$

DATE: $11 / 13 / 97$

Evaluator: K B Oswald

TRANSPORTATION COMPARISON EXCEL CHART:

TRANSPORTAION COMPARISON

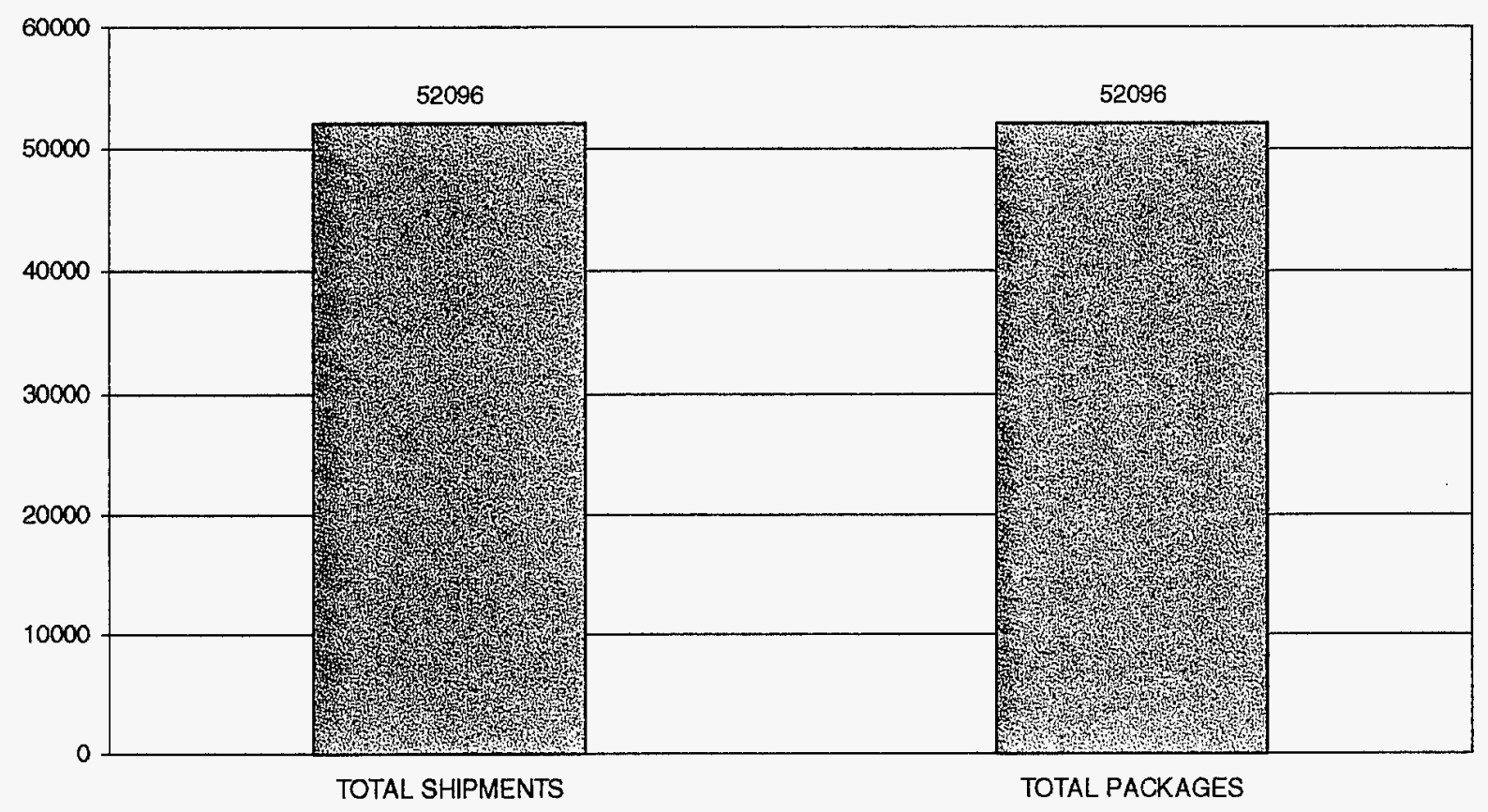

TRANSPORTATION COMPARISON CWWFA CHART:

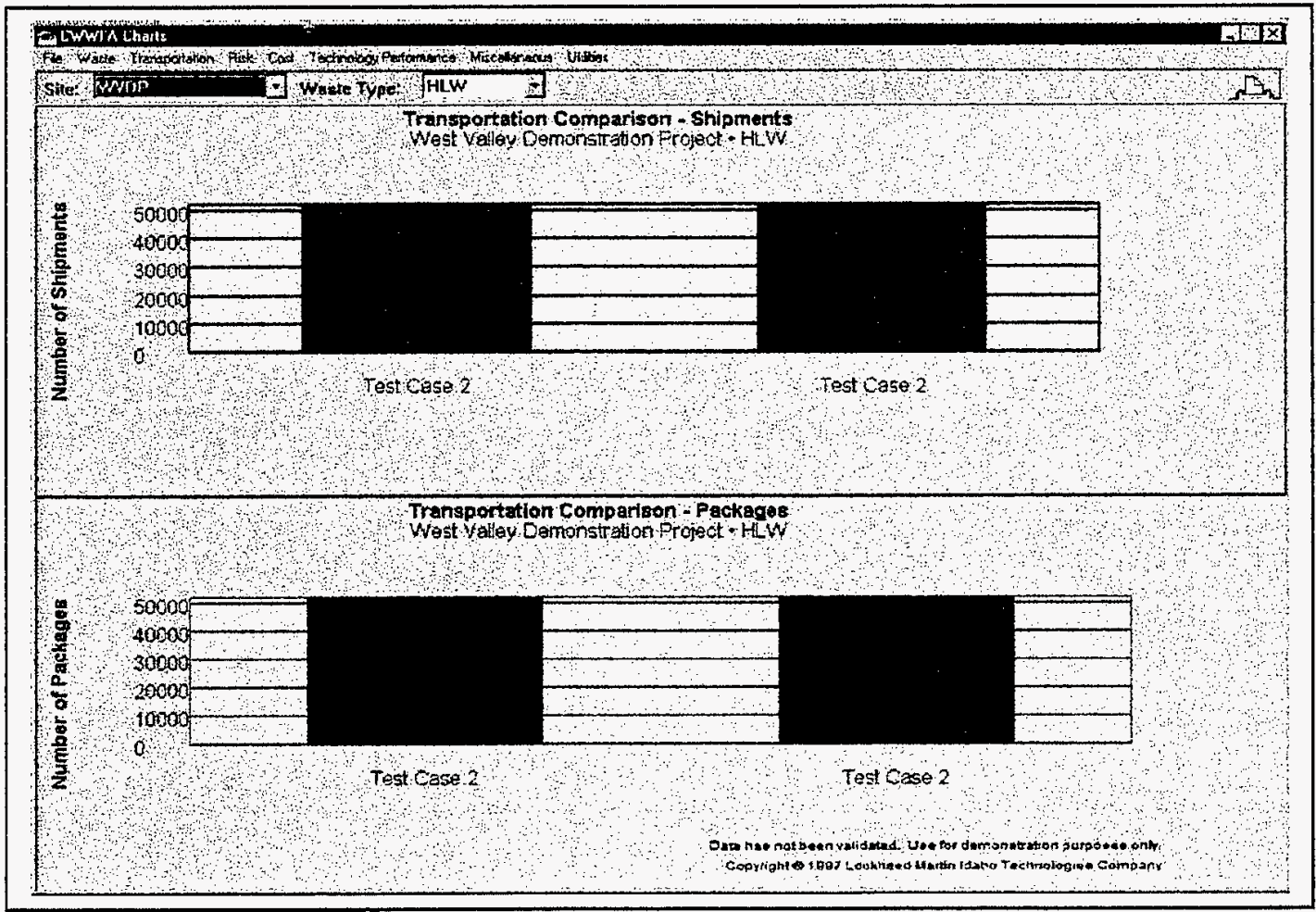


CASE: Case $2 b$

INITIAL PARAMETERS \& RESULTS REPORT

1.0 Waste Stream Information

\begin{tabular}{|l|l|}
\hline Originating Site & WVDP \\
\hline Waste Stream ID \& Name & WVDPWS1-CASE2a \\
\hline Originating Type & HLW \\
\hline Legacy volume (m3) & 15,000 \\
\hline
\end{tabular}

1.1 New Generation

\begin{tabular}{|l|l|l|}
\hline \multicolumn{1}{|c|}{ Start Year } & \multicolumn{1}{|c|}{ End Year } & \multicolumn{1}{c|}{ Annual Volume (m3) } \\
\hline NA & NA & NA \\
\hline & & \\
\hline & & \\
\hline & & \\
\hline
\end{tabular}

1.2 Shipping Route(s)

\begin{tabular}{|l|l|l|}
\hline \multicolumn{1}{|c|}{ Route ID } & \multicolumn{1}{|c|}{ Source Facility } & \multicolumn{1}{c|}{ Destination Facility } \\
\hline $\mathrm{C} 2 \mathrm{~b}-1$ & Legacy-WV & SARSFAC1-CASE2 \\
\hline $\mathrm{C} 2 \mathrm{~b}-2$ & SARSFAC1-CASE2 & WWDPFAC1-CASE2 \\
\hline $\mathrm{C} 2 \mathrm{~b}-3$ & WDDPFAC1-CASE2 & SARSFAC2-CASE2 \\
\hline & & \\
\hline & & \\
\hline & & \\
\hline
\end{tabular}

1.3 Shipping Schedule(s)

\begin{tabular}{|c|c|c|c|}
\hline Route ID: $\mathrm{C} 2 \mathrm{~b}-1$ & & & \\
\hline Start Year & End Year & Waste Type & Annual Volume (m3) \\
\hline 1997 & 2020 & HLW & 100 \\
\hline 2021 & 2030 & HLW & 100 \\
\hline 2031 & 2040 & HLW & 1160 \\
\hline
\end{tabular}

1.3 Shipping Schedule(s) (continued)

\begin{tabular}{|c|c|c|c|}
\hline Route ID: C2b-2 & & & \\
\hline Start Year & End Year & Waste Type & Annual Volume (m3) \\
\hline 1997 & 2005 & HLW & 1000 \\
\hline 2046 & 2055 & HLW & 600 \\
\hline & & & \\
\hline
\end{tabular}

1.3 Shipping Schedule(s) (continued)

\begin{tabular}{|l|l|l|l|}
\hline Route ID: C2b-3 & \multicolumn{2}{|c|}{ Waste Type } & Annual Volume (m3) \\
\hline \multicolumn{1}{|c|}{ Start Year } & \multicolumn{1}{|c|}{ End Year } & HLW & 1400 \\
\hline 2056 & 2065 & HLW & 1000 \\
\hline & 2066 & & \\
\hline & & & \\
\hline
\end{tabular}


2.0 Facility Information

\begin{tabular}{|l|l|}
\hline Site & SARS \\
\hline Facility ID \& Name & SARSFAC1-CASE2 \\
\hline Function & Treatment \\
\hline Start Year & 1997 \\
\hline End Year & 2070 \\
\hline Annual Capacity (m3/year) or & $1,000,000$ \\
\hline Lifetime Facility Capacity (m3) & \\
\hline Volume Change Factor & 1 \\
\hline
\end{tabular}

Conversion Profile

\begin{tabular}{|l|l|l|}
\hline Percent & Waste Type & Density \\
\hline $100 \%$ & HLW & 650 \\
\hline & & \\
\hline & & \\
\hline
\end{tabular}

2.0 Facility Information

\begin{tabular}{|l|l|}
\hline Site & WVDP \\
\hline Facility ID \& Name & WVDPFAC1-CASE2 \\
\hline Function & Treatment \\
\hline Start Year & 1997 \\
\hline End Year & 2070 \\
\hline Annual Capacity (m3/year) or & $1,000,000$ \\
\hline Lifetime Facility Capacity (m3) & \\
\hline Volume Change Factor & 1 \\
\hline
\end{tabular}

Conversion Profile

\begin{tabular}{|l|l|l|}
\hline Percent & Waste Type & Density \\
\hline 100 & HLW & 1800 \\
\hline & & \\
\hline
\end{tabular}

2.0 Facility Information

\begin{tabular}{|l|l|}
\hline Site & SARS \\
\hline Facility ID \& Name & SARSFAC1-CASE2 \\
\hline Function & Disposal \\
\hline Start Year & 1997 \\
\hline End Year & 2070 \\
\hline Annual Capacity (m3/year) or & $1,000,000$ \\
\hline Lifetime Facility Capacity (m3) & \\
\hline Volume Change Factor & 1 \\
\hline
\end{tabular}

Conversion Profile

\begin{tabular}{|l|l|l|}
\hline Percent & Waste Type & Density \\
\hline NA & NA & NA \\
\hline & & \\
\hline
\end{tabular}


WASTE DISPOSITION - EXCEL CHART (WVDP, HLW):

\section{Waste Disposition}

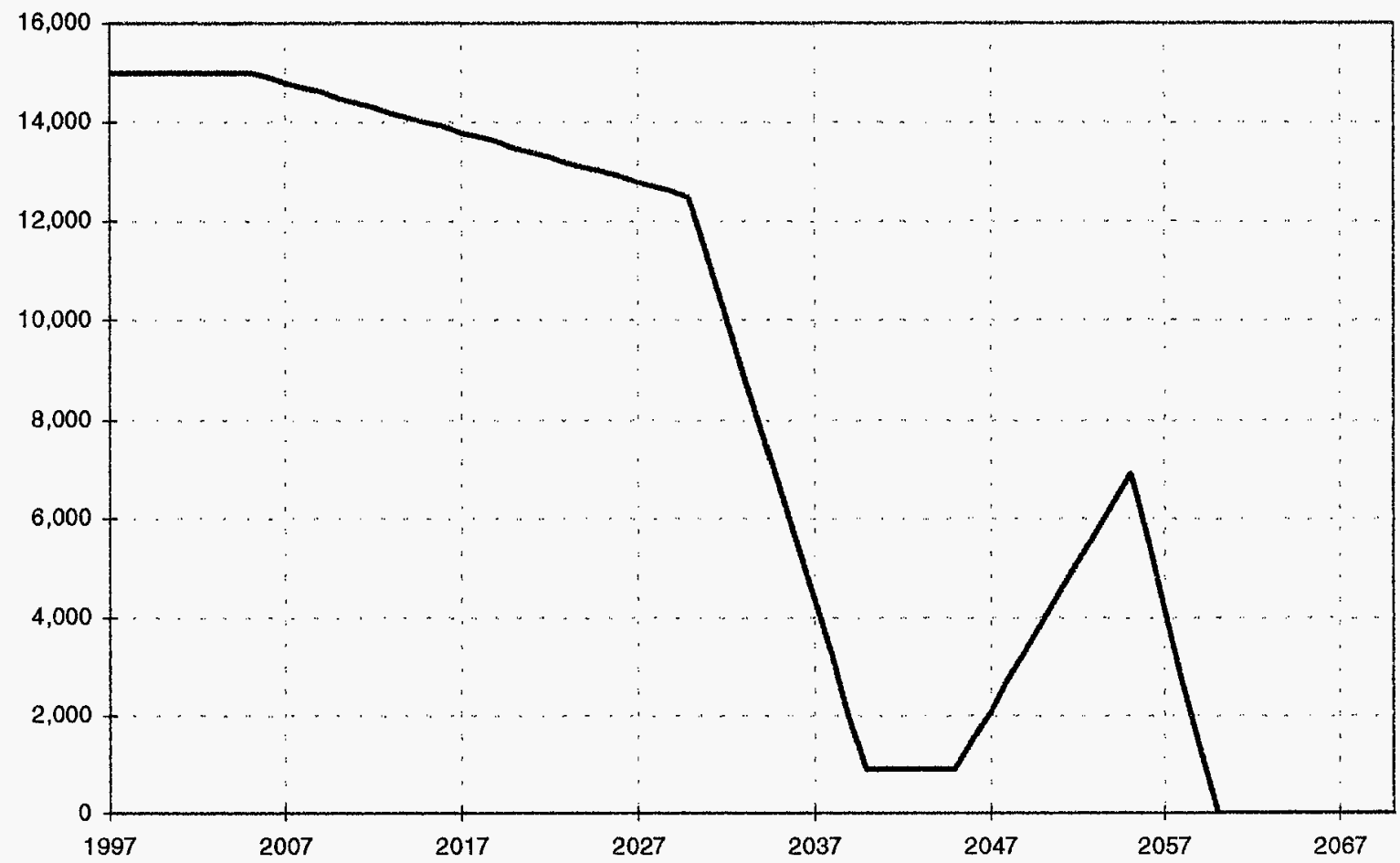

\section{WASTE DISPOSITION - CWWFA CHART:}

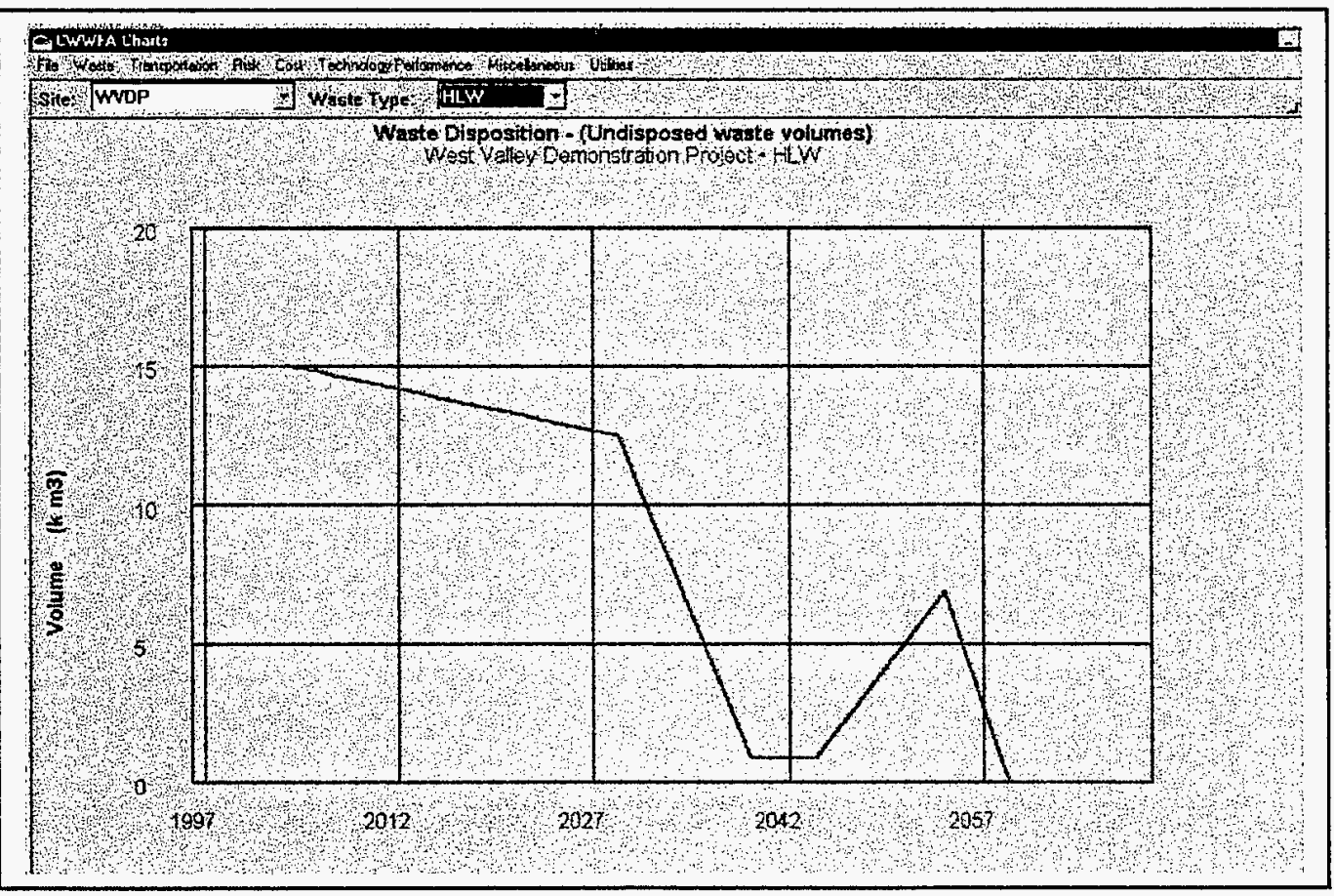


FACILITY CAPACITY EXCEL CHART (WVDP, MOPLFAC2-CASE1):

\section{Facility Capacity Detail}

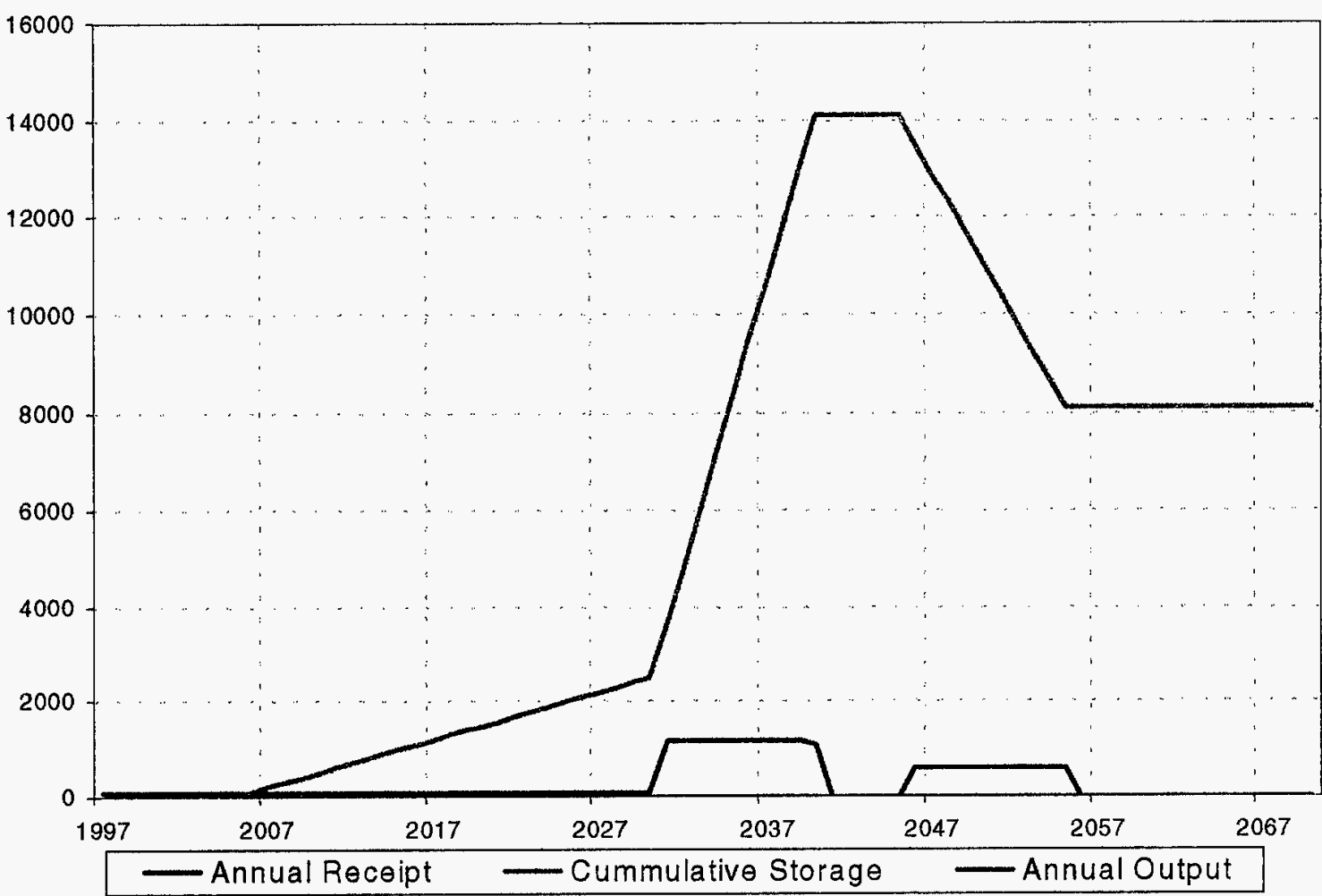

\section{FACILITY CAPACITY CWWFA CHART:}

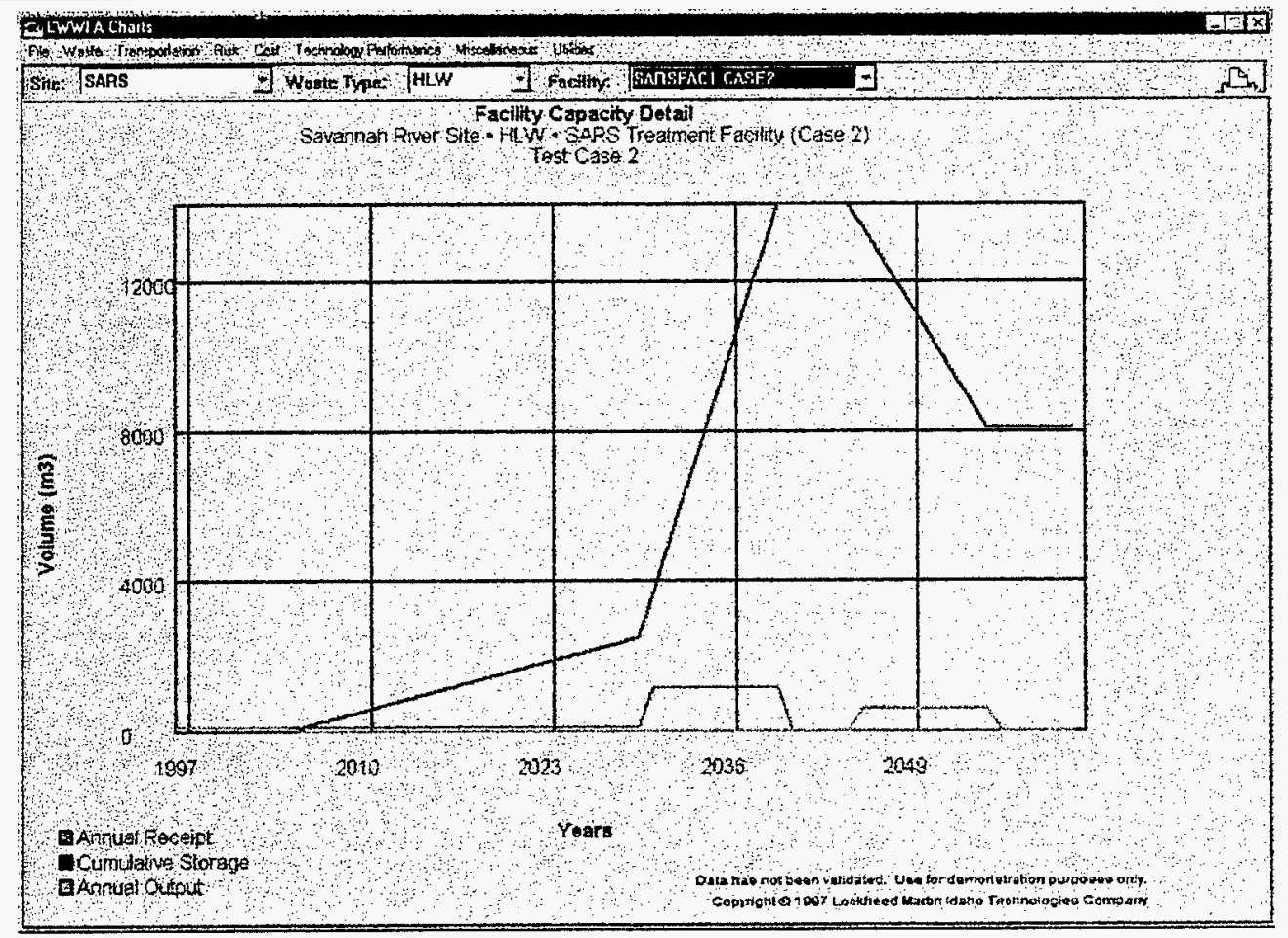


WASTE STREAM DETAIL EXCEL CHART (WVDPWS1-CASE2a):

\section{Waste Stream Detail}

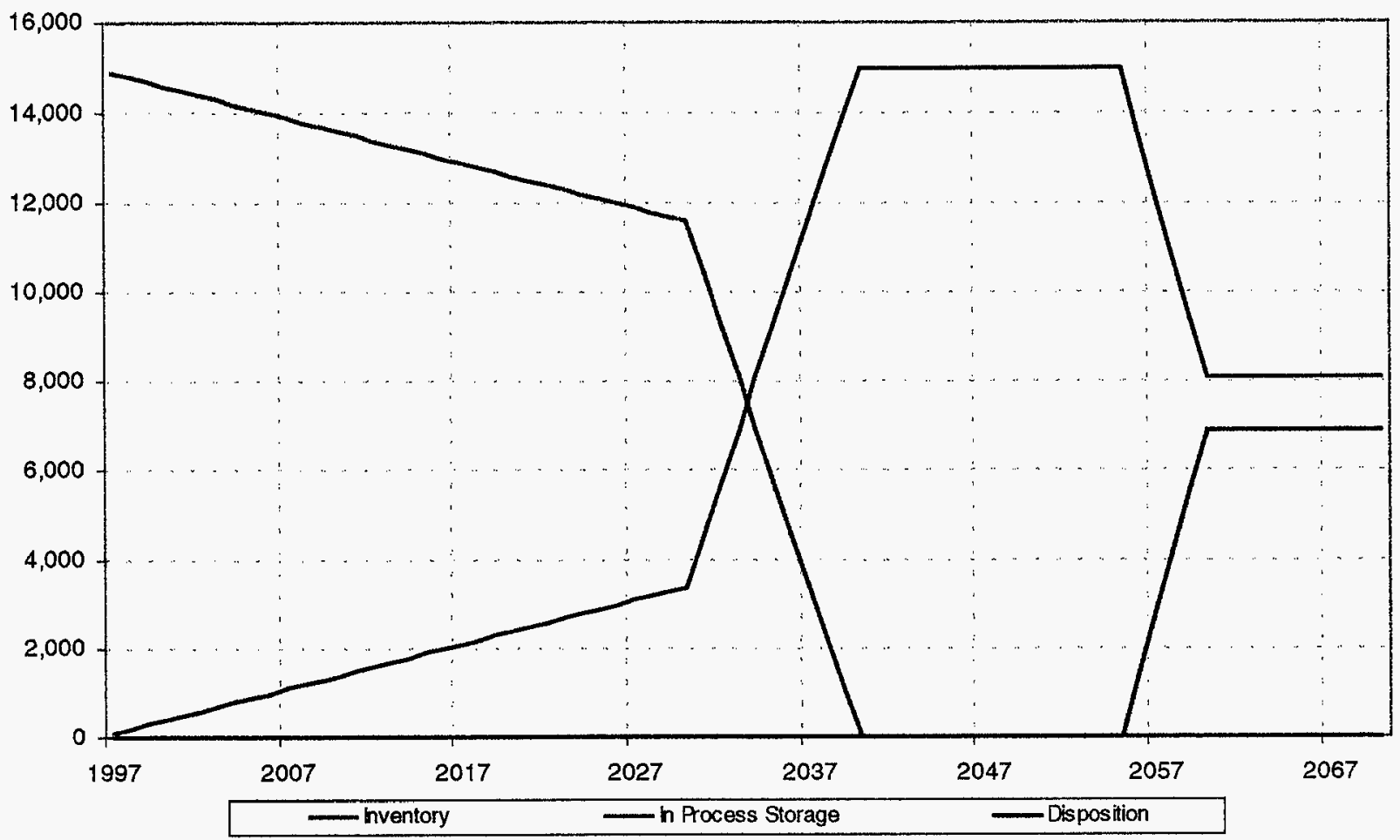

WASTE STREAM DETAIL CWWFA CHART:

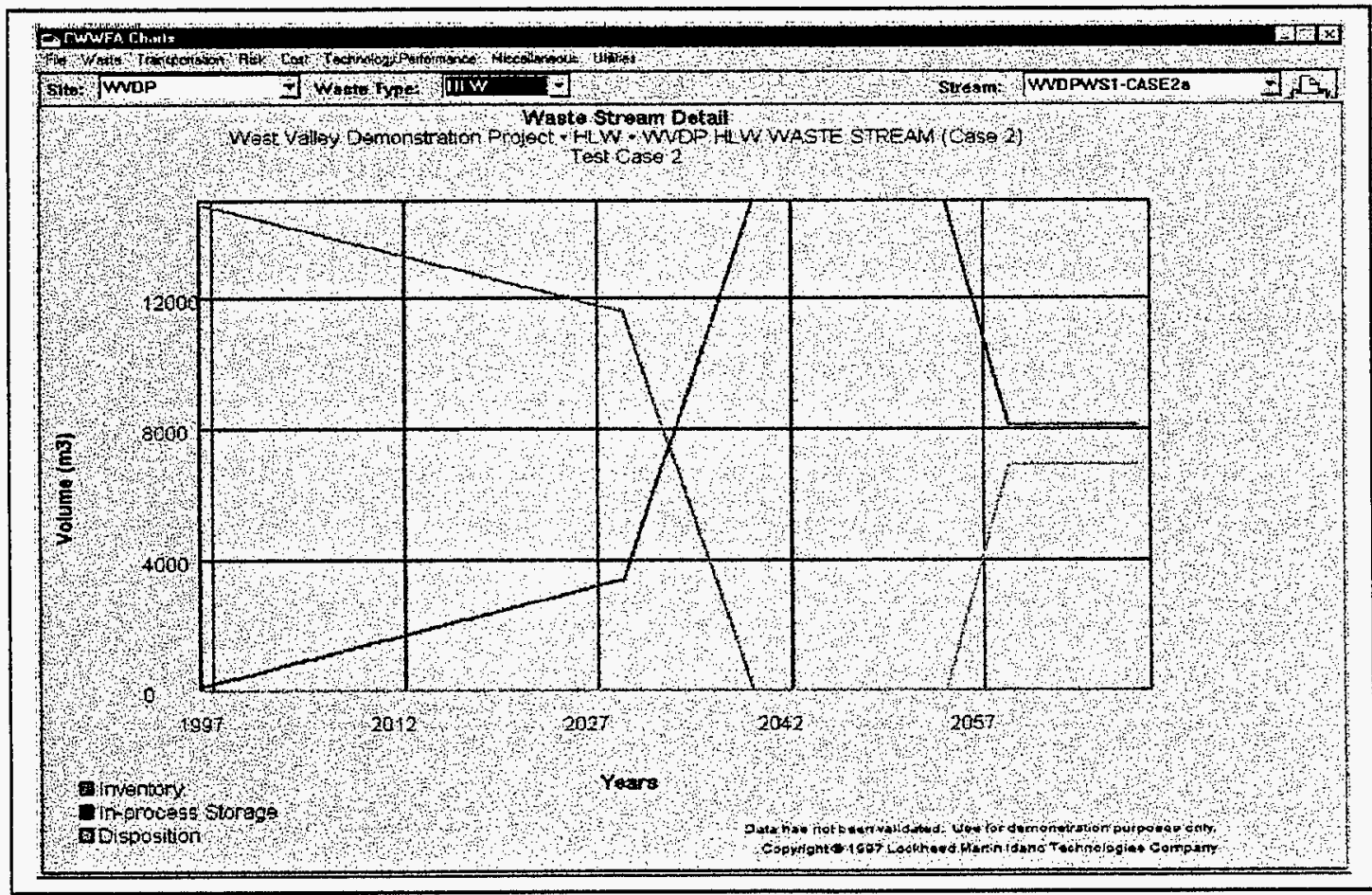


INITIAL PARAMETERS \& RESULTS REPORT

CASE: Case $2 b$

DATE: $11 / 13 / 97$

Evaluator: K B Oswald

\section{SHIPMENTS RECEIVED EXCEL CHART (MOPL):}

\section{Shipments Received}

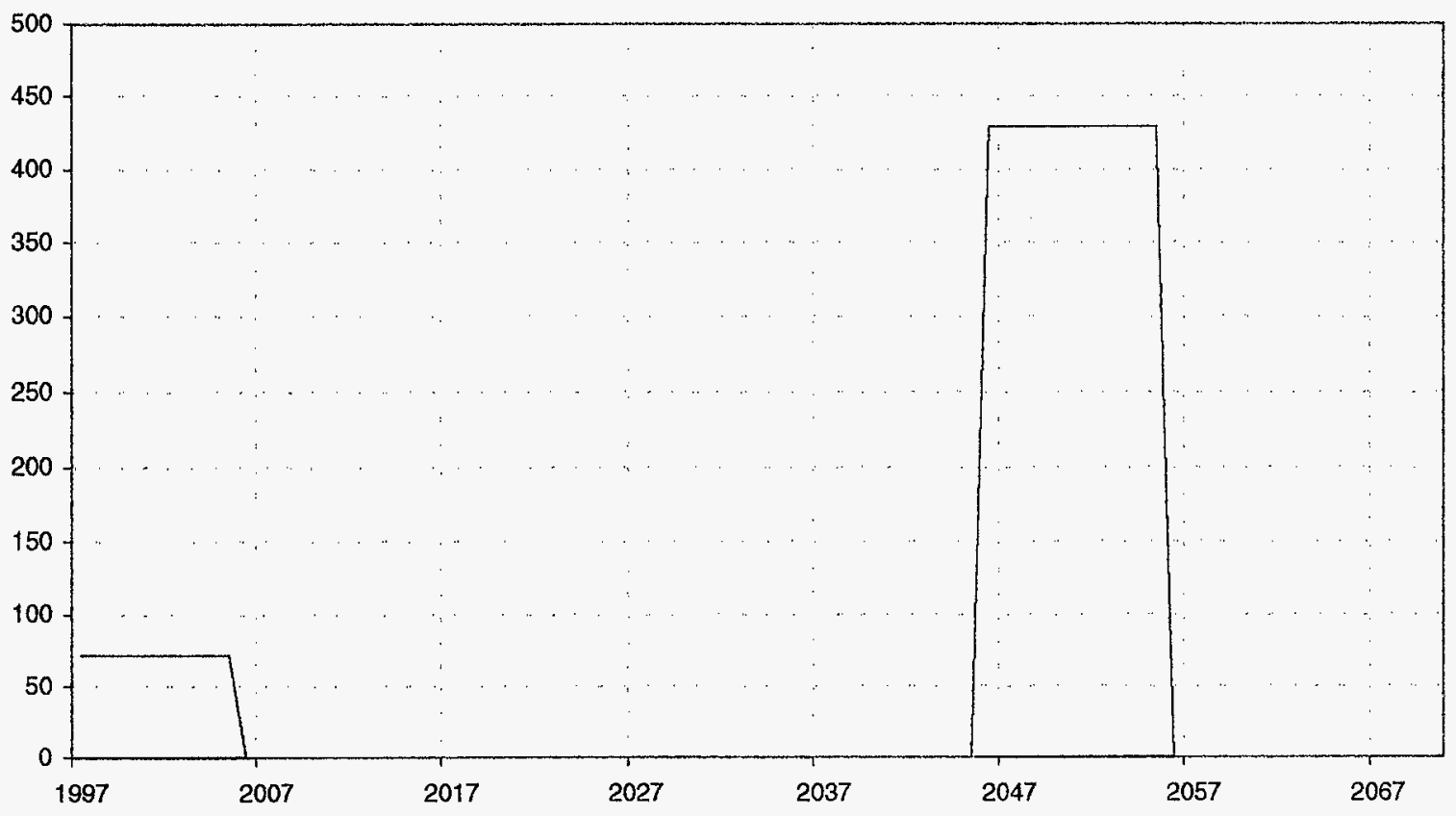

\section{SHIPMENTS RECEIVED CWWFA CHART:}

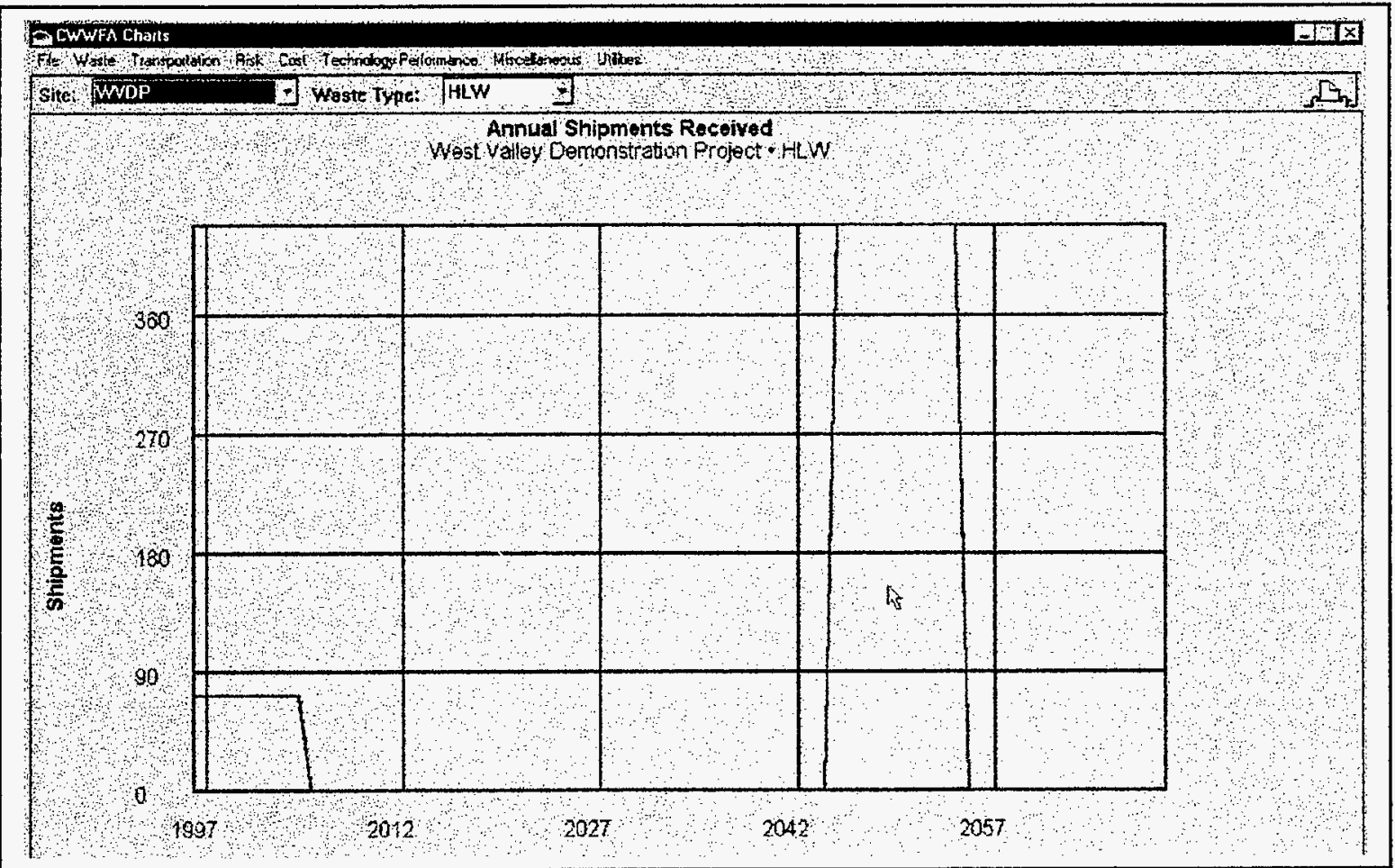


SHIPMENTS SENT EXCEL CHART (WVDP):

\section{Shipments Sent}

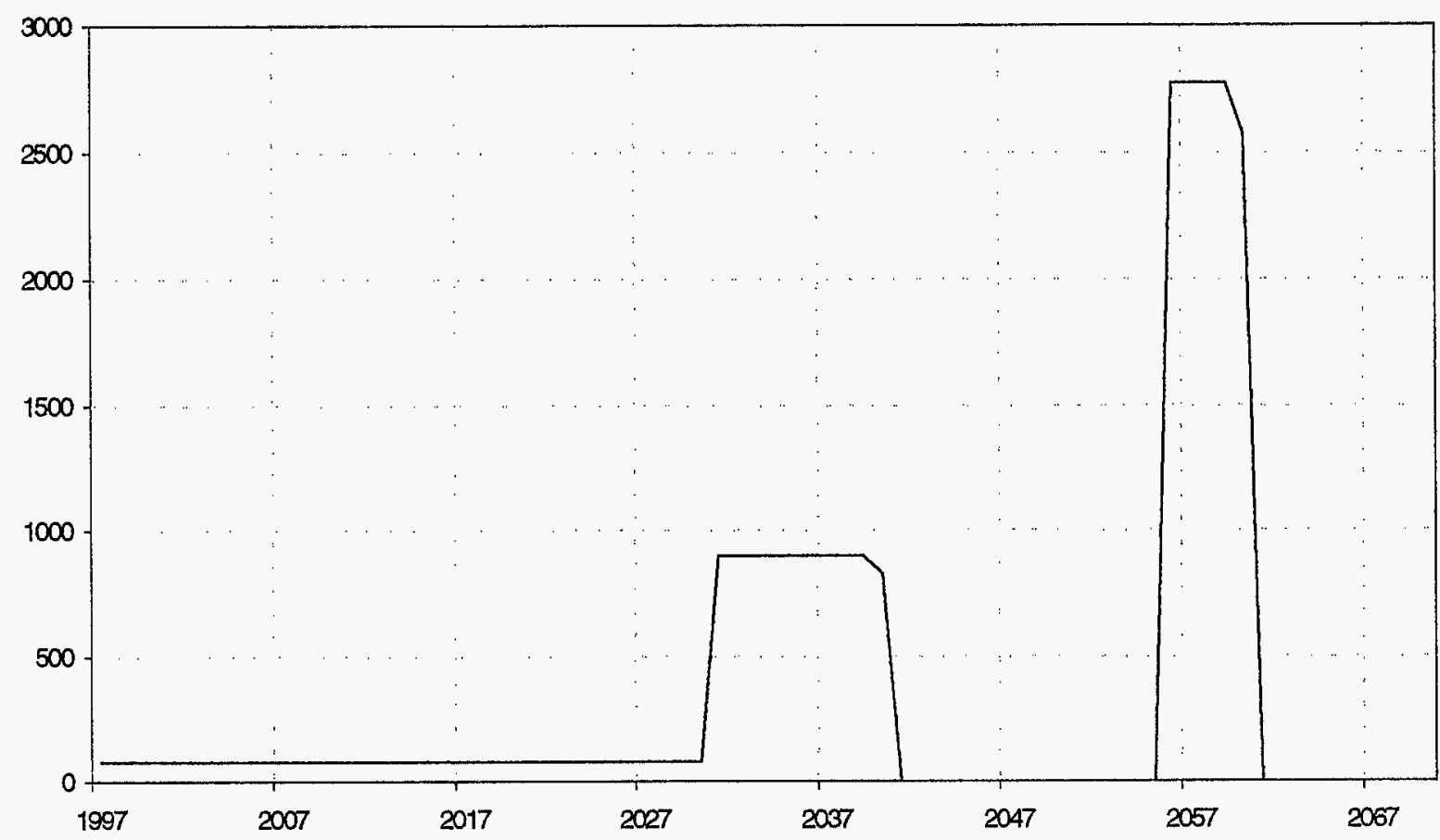

\section{SHIPMENTS SENT CWWFA CHART:}

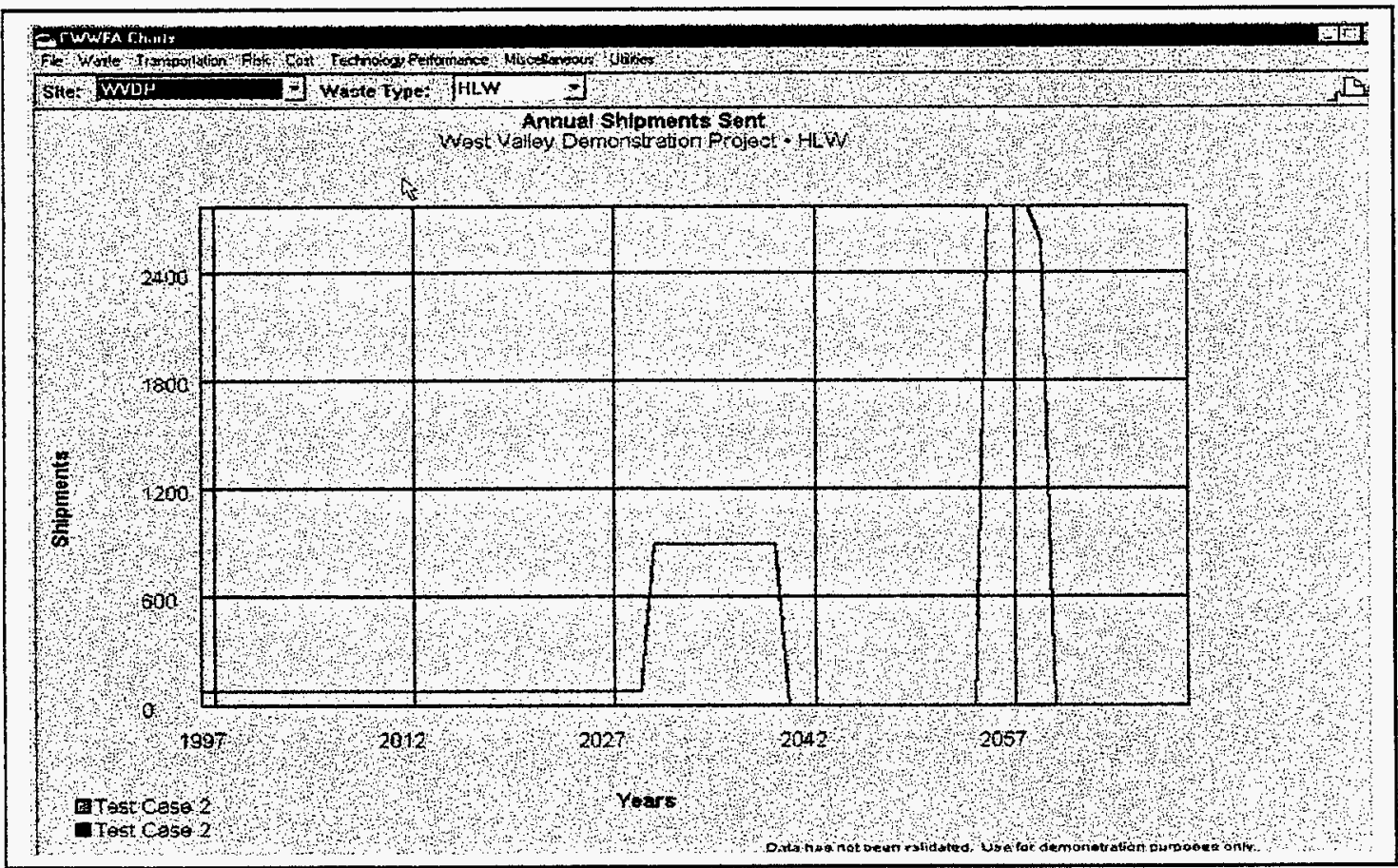




\section{TRANSPORTAION COMPARISON}

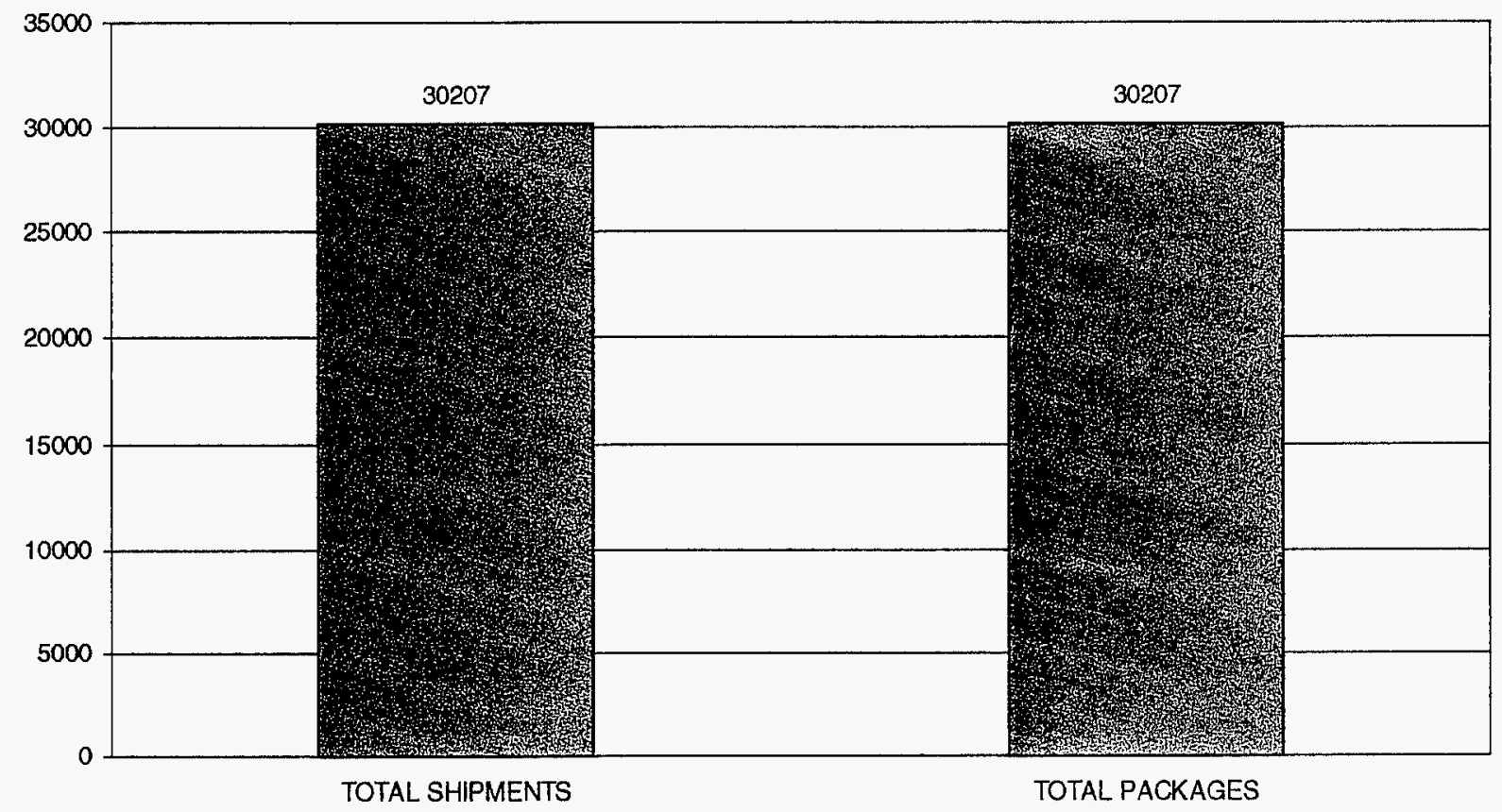

TRANSPORTATION COMPARISON CWWFA CHART:

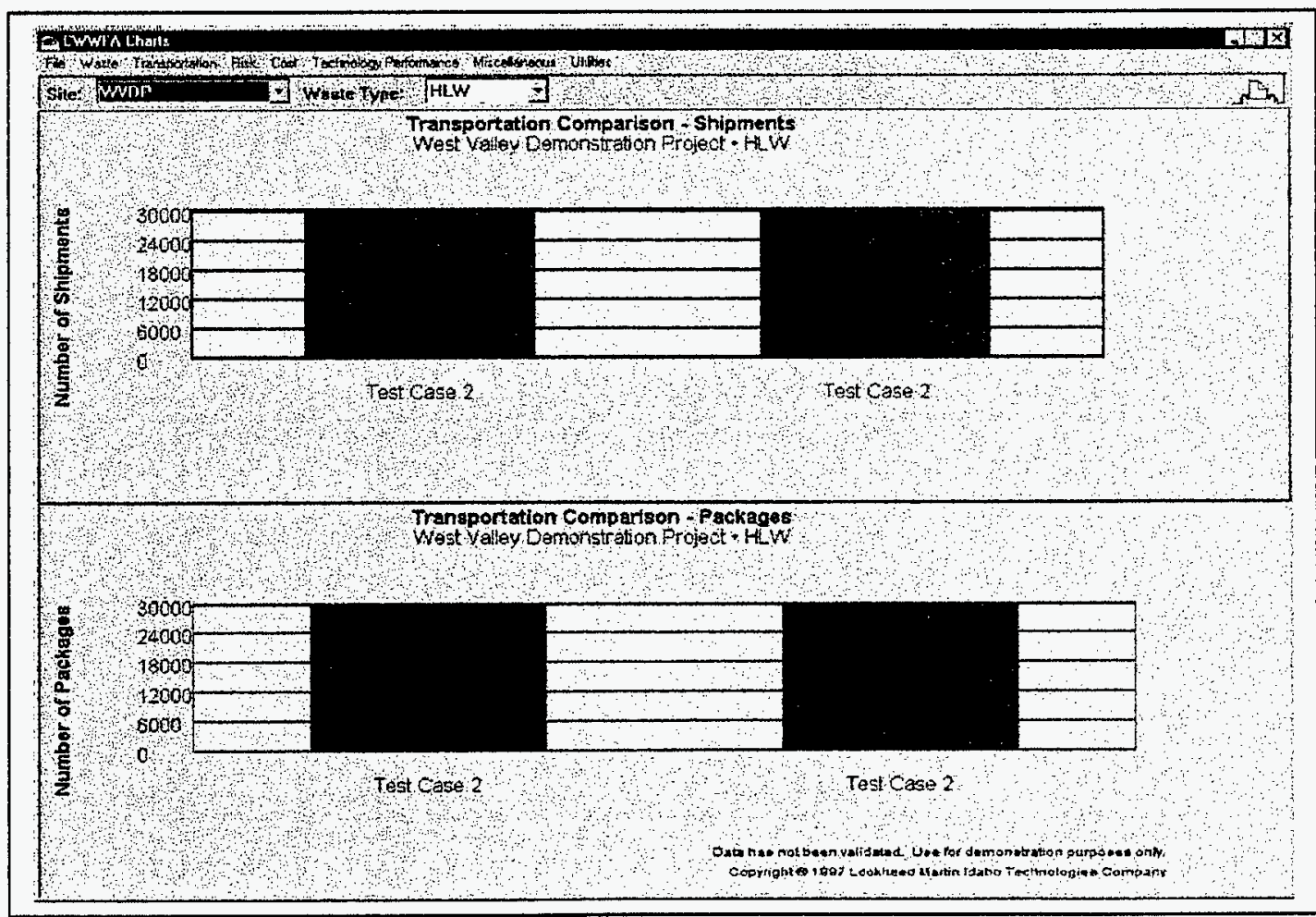


1.0 Waste Stream Information

\begin{tabular}{|l|l|}
\hline Originating Site & WVDP \\
\hline Waste Stream ID \& Name & WVDPWS1-CASE2a \\
\hline Originating Type & HLW \\
\hline Legacy volume $(\mathrm{m} 3)$ & 15,000 \\
\hline
\end{tabular}

1.1 New Generation

\begin{tabular}{|c|c|c|}
\hline \multicolumn{1}{|c|}{ Start Year } & End Year & Annual Volume (m3) \\
\hline 2031 & 2040 & 1000 \\
\hline & & \\
\hline & & \\
\hline & & \\
\hline
\end{tabular}

1.2 Shipping Route(s)

\begin{tabular}{|l|l|l|}
\hline \multicolumn{1}{|c|}{ Route ID } & \multicolumn{1}{|c|}{ Source Facility } & \multicolumn{1}{c|}{ Destination Facility } \\
\hline C2c-1 & Legacy-WW & SARSFAC1-CASE2 \\
\hline C2c-2 & SARSFAC1-CASE2 & WWDPFAC1-CASE2 \\
\hline C2c-3 & WWDPFAC1-CASE2 & SARSFAC2-CASE2 \\
\hline & & \\
\hline & & \\
\hline & & \\
\hline
\end{tabular}

1.3 Shipping Schedule(s)

\begin{tabular}{|c|c|c|c|}
\hline Route ID: C2c-1 & \multicolumn{2}{|c|}{ Waste Type } & \multicolumn{1}{|c|}{ Annual Volume (m3) } \\
\hline Start Year & \multicolumn{1}{|c|}{ End Year } & \multicolumn{1}{|c|}{ WLW } & \\
\hline 1997 & 2070 & & \\
\hline & & & \\
\hline & & & \\
\hline
\end{tabular}

1.3 Shipping Schedule(s) (continued)

\begin{tabular}{|c|c|c|c|}
\hline Route ID: C2c-2 & \multicolumn{2}{|c|}{ Waste Type } & \multicolumn{1}{|c|}{ Annual Volume (m3) } \\
\hline Start Year & \multicolumn{1}{|c|}{ End Year } & HLW & 203 \\
\hline 1997 & 2070 & & \\
\hline & & & \\
\hline & & & \\
\hline
\end{tabular}

1.3 Shipping Schedule(s) (continued)

\begin{tabular}{|c|c|c|c|}
\hline Route ID: C2c-3 & \multicolumn{2}{|c|}{ Waste Type } & \multicolumn{1}{|c|}{ Annual Volume (m3) } \\
\hline Start Year & \multicolumn{1}{|c|}{ End Year } & HLW & \\
\hline 1997 & 2070 & & \\
\hline & & & \\
\hline & & & \\
\hline & & & \\
\hline
\end{tabular}


2.0 Facility Information

\begin{tabular}{|l|l|}
\hline Site & SARS \\
\hline Facility ID \& Name & SARSFAC1-CASE2 \\
\hline Function & Treatment \\
\hline Start Year & 1997 \\
\hline End Year & 2070 \\
\hline Annual Capacity (m3/year) or & $1,000,000$ \\
\hline Lifetime Facility Capacity (m3) & \\
\hline Volume Change Factor & 1 \\
\hline
\end{tabular}

Conversion Profile

\begin{tabular}{|l|l|l|}
\hline Percent & Waste Type & Density \\
\hline $100 \%$ & HLW & 650 \\
\hline & & \\
\hline & & \\
\hline
\end{tabular}

2.0 Facility Information

\begin{tabular}{|c|c|c|c|}
\hline \multicolumn{2}{|c|}{ Site } & \multicolumn{2}{|l|}{ WVDP } \\
\hline \multicolumn{2}{|c|}{ Facility ID \& Name } & \multicolumn{2}{|l|}{ WVDPFAC1-CASE2 } \\
\hline \multicolumn{2}{|l|}{ Function } & \multicolumn{2}{|l|}{ Treatment } \\
\hline \multicolumn{2}{|l|}{ Start Year } & \multicolumn{2}{|l|}{1997} \\
\hline \multicolumn{2}{|l|}{ End Year } & \multicolumn{2}{|c|}{2070} \\
\hline \multicolumn{2}{|l|}{ Annual Capacity (m3/year) or } & \multicolumn{2}{|l|}{$1,000,000$} \\
\hline \multicolumn{4}{|l|}{ Lifetime Facility Capacity (m3) } \\
\hline Volume Change Factor & & \multicolumn{2}{|c|}{1} \\
\hline \multicolumn{4}{|l|}{ Conversion Profile } \\
\hline Percent & Waste Typ & & Density \\
\hline 100 & LLW & & 1800 \\
\hline
\end{tabular}

2.0 Facility Information

\begin{tabular}{|l|l|}
\hline Site & SARS \\
\hline Facility ID \& Name & SARSFAC1-CASE2 \\
\hline Function & Disposal \\
\hline Start Year & 1997 \\
\hline End Year & 2070 \\
\hline Annual Capacity (m3/year) or & $1,000,000$ \\
\hline Lifetime Facility Capacity (m3) & \\
\hline Volume Change Factor & 1 \\
\hline
\end{tabular}

Conversion Profile

\begin{tabular}{|l|l|l|}
\hline Percent & Waste Type & Density \\
\hline NA & NA & NA \\
\hline & & \\
\hline
\end{tabular}


WASTE DISPOSITION - EXCEL CHART (WVDP, HLW):

\section{Waste Disposition}

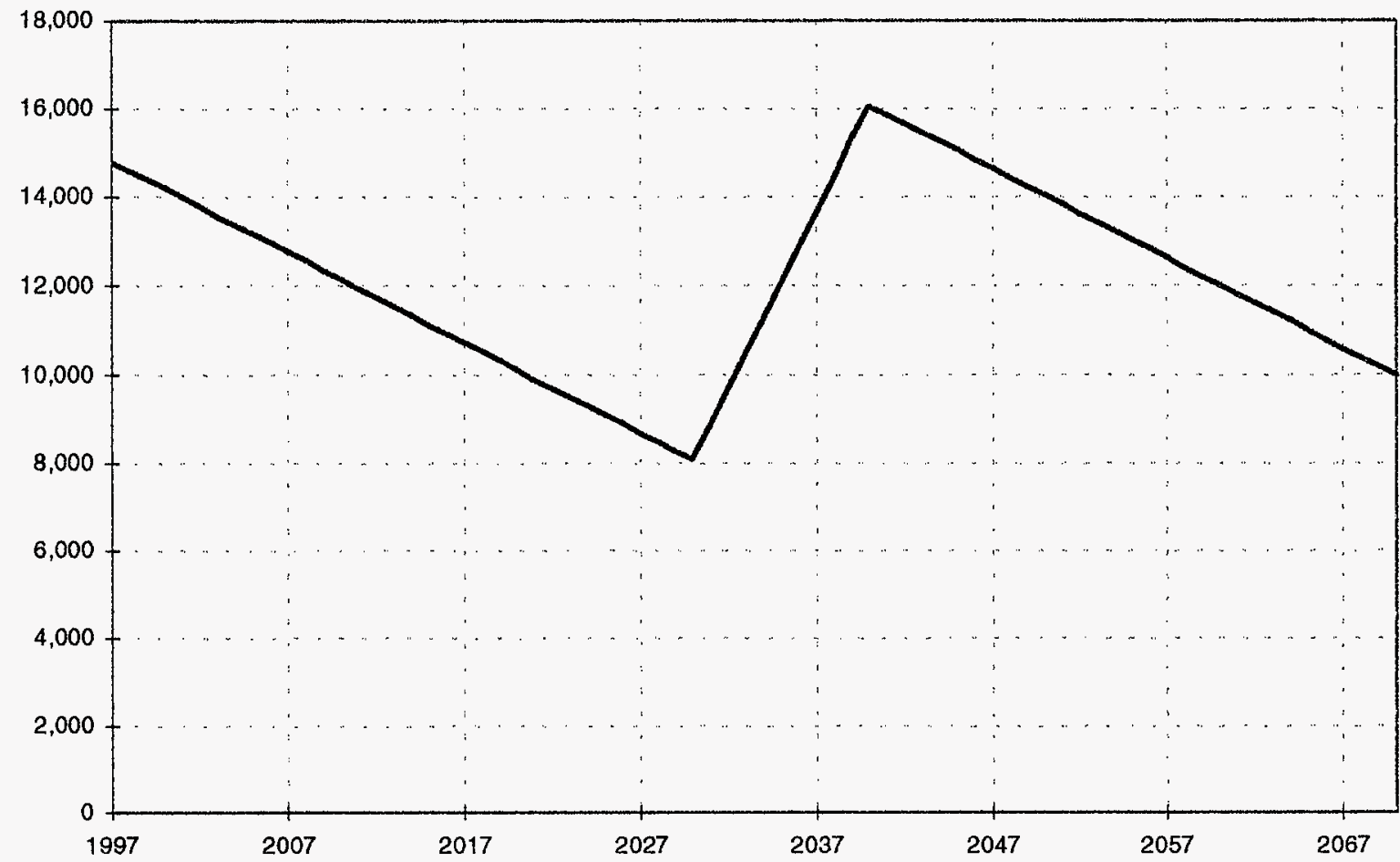

WASTE DISPOSITION - CWWFA CHART:

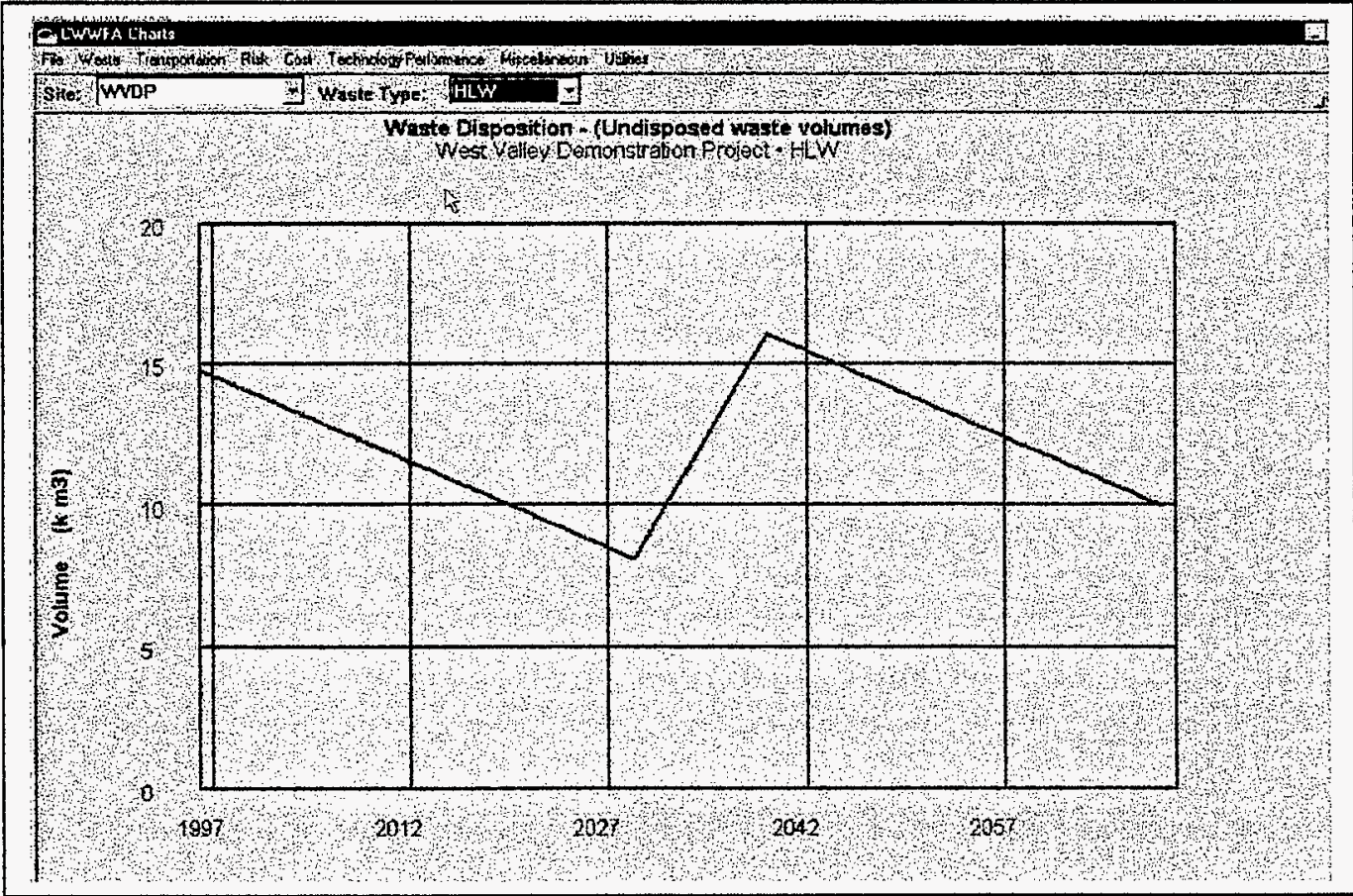


FACILITY CAPACITY EXCEL CHART (SARS, SARSFAC1-CASE2):

\section{Facility Capacity Detail}

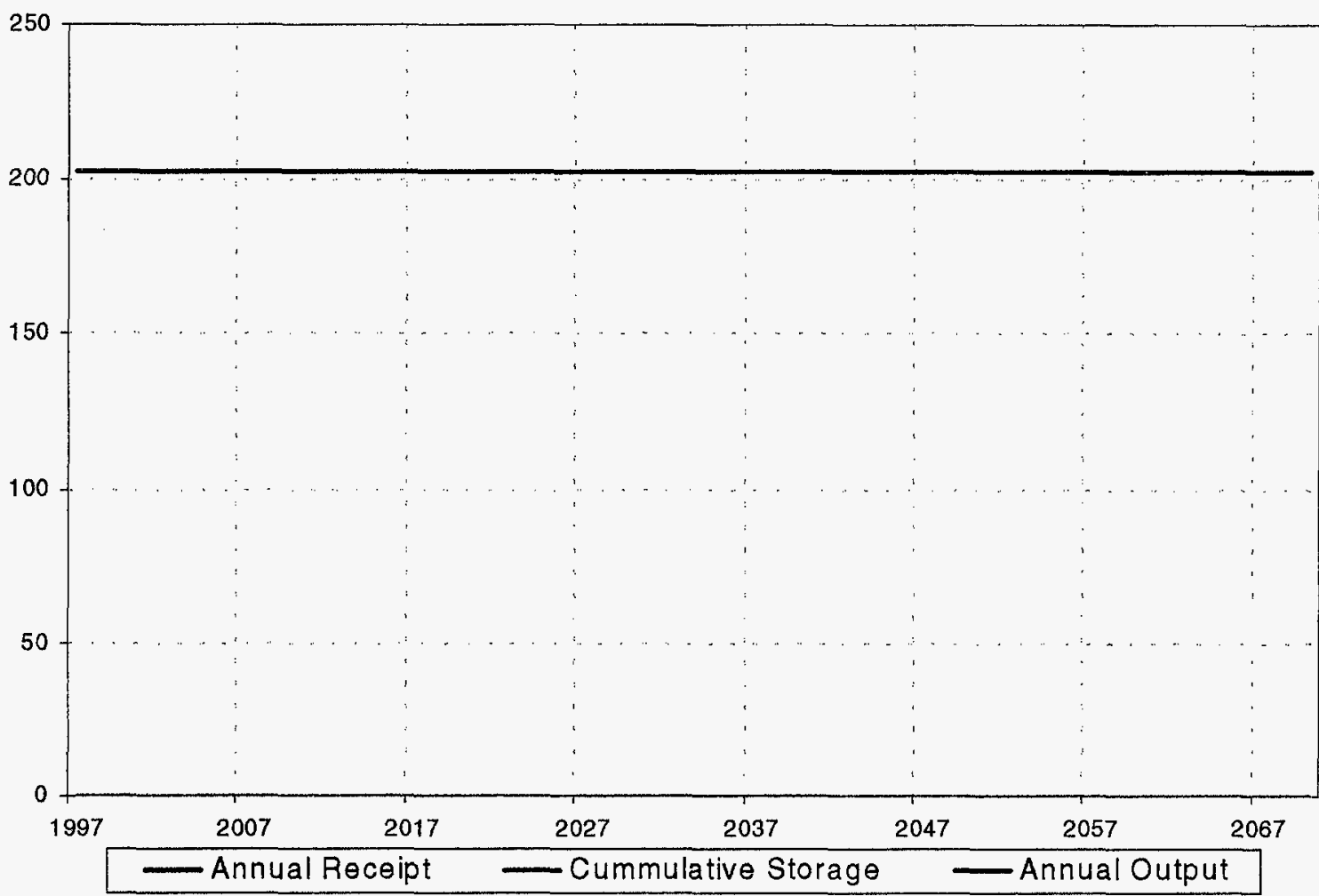

FACILITY CAPACITY CWWFA CHART:

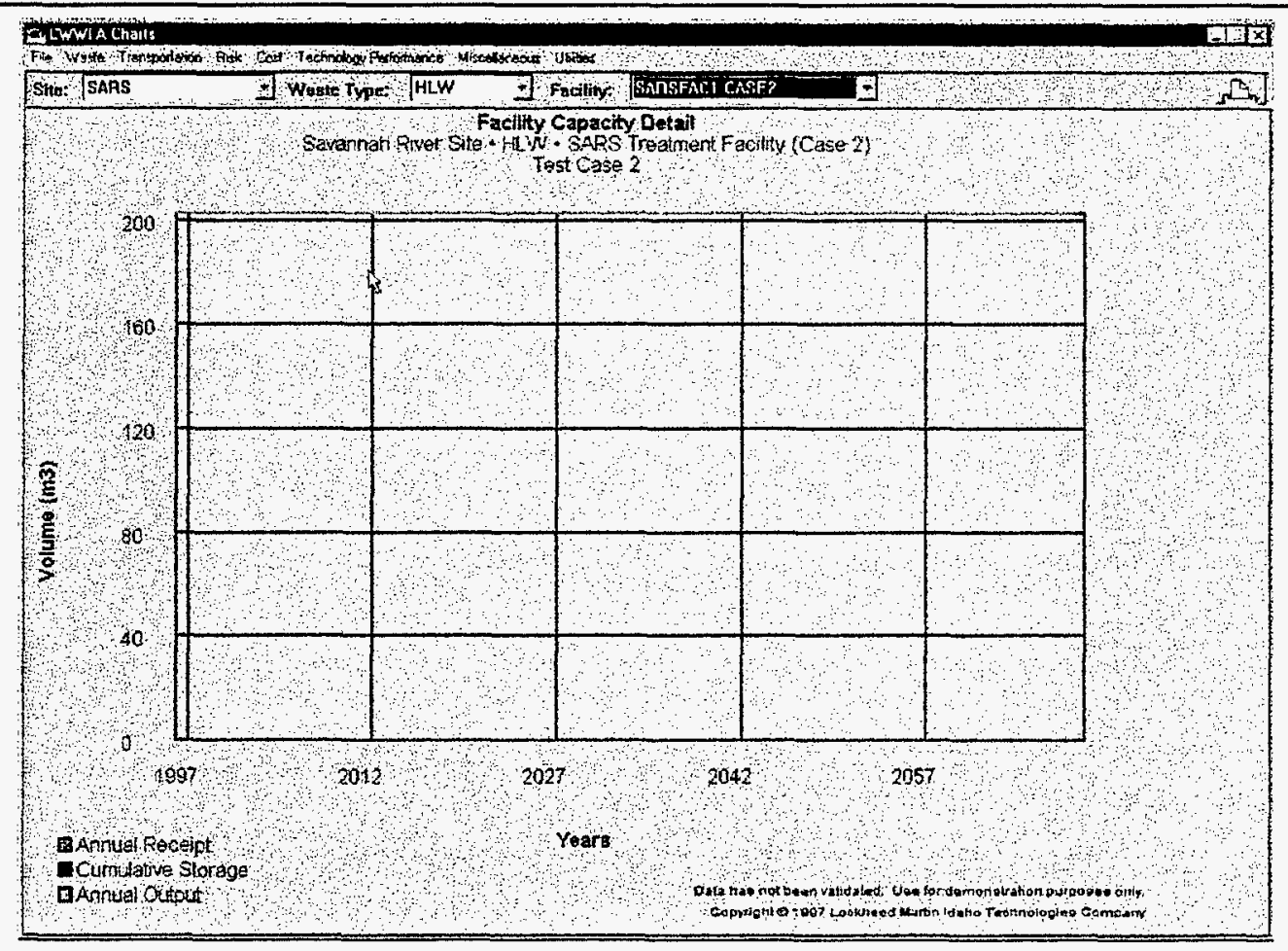


WASTE STREAM DETAIL EXCEL CHART (WVDPWS1-CASE2a):

\section{Waste Stream Detail}

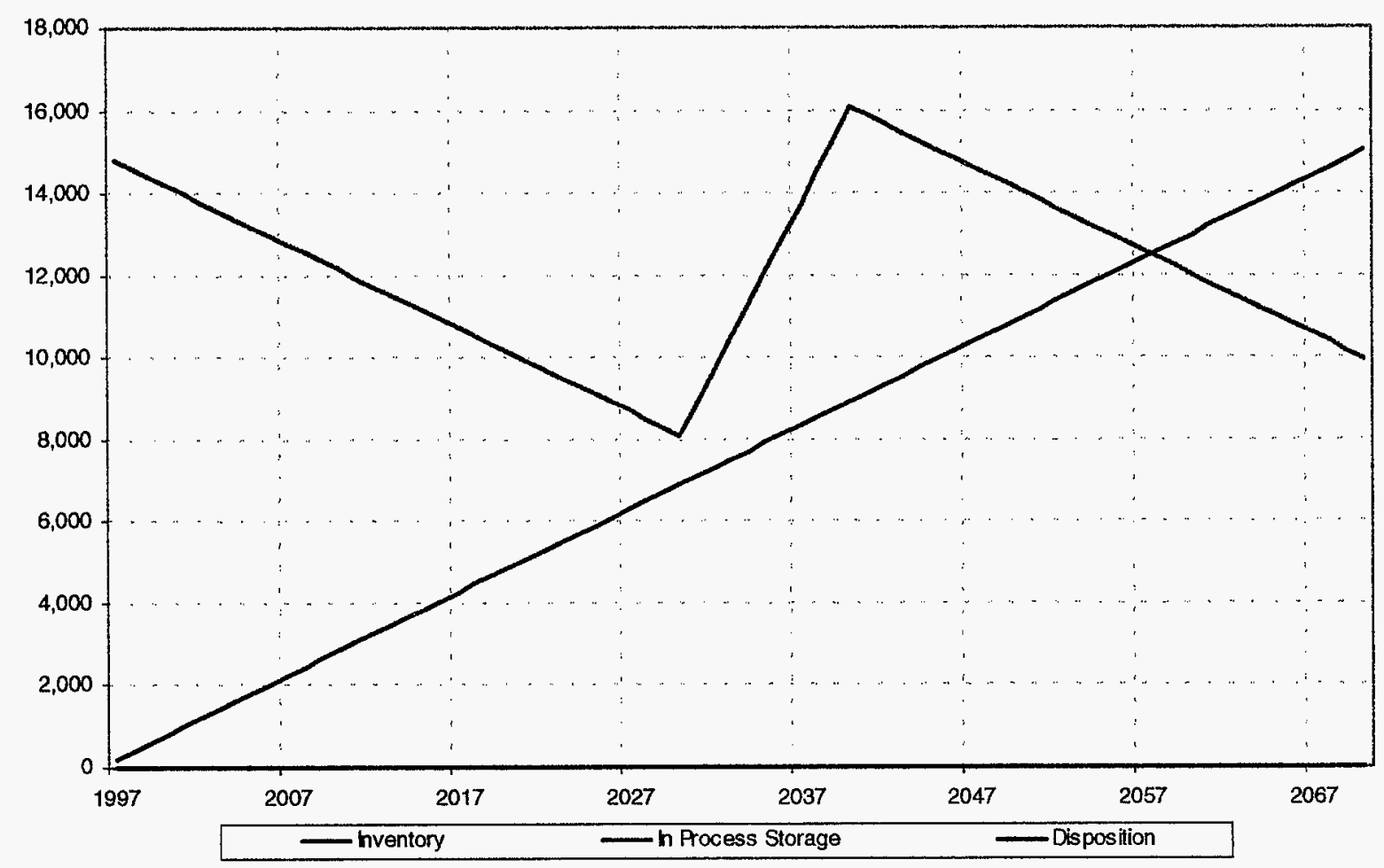

WASTE STREAM DETAIL CWWFA CHART:

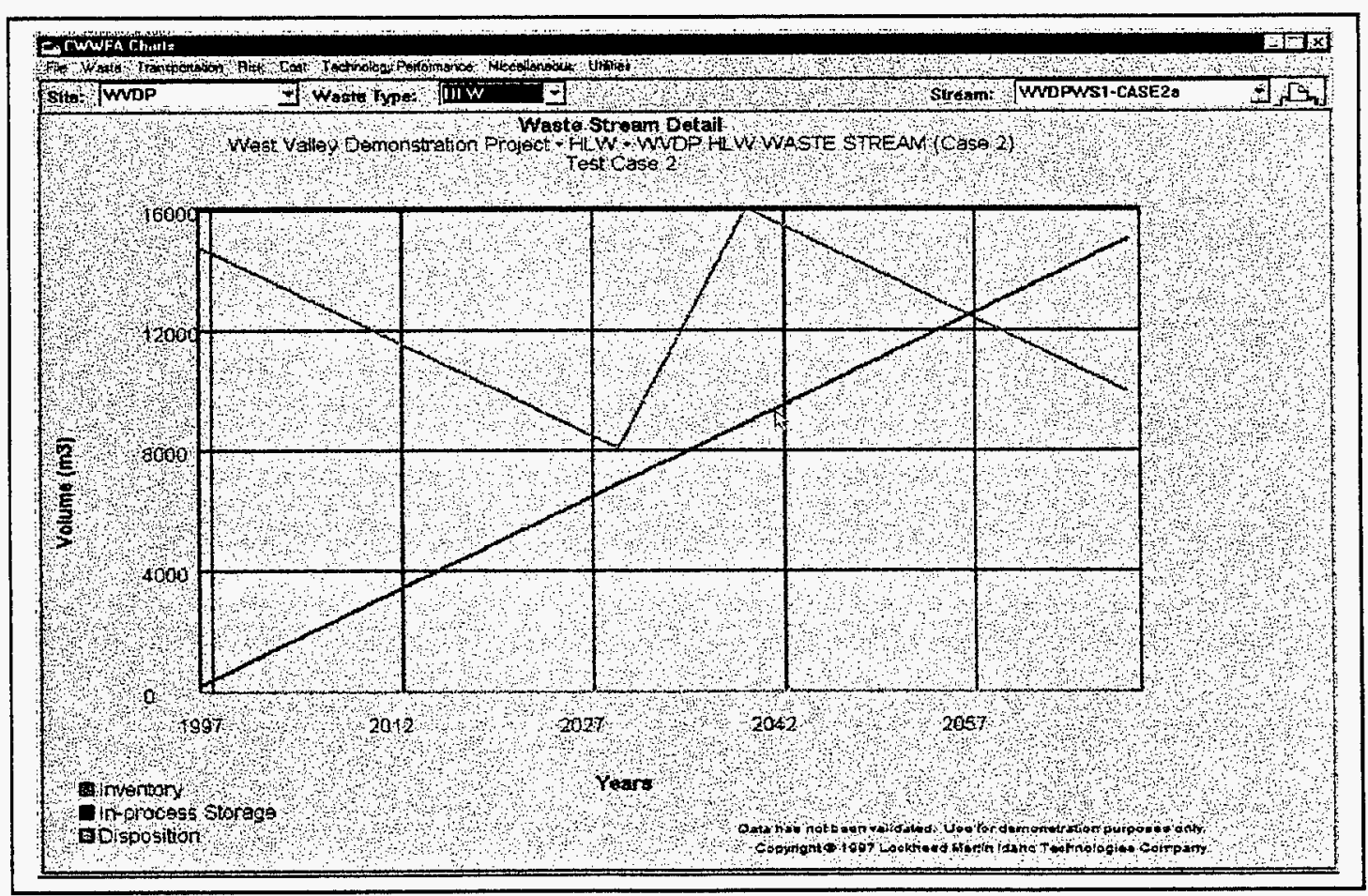


CASE: Case $2 c$

DATE: $11 / 13 / 97$

Evaluator: K B Oswald

SHIPMENTS RECEIVED EXCEL CHART (MOPL):

Shipments Received

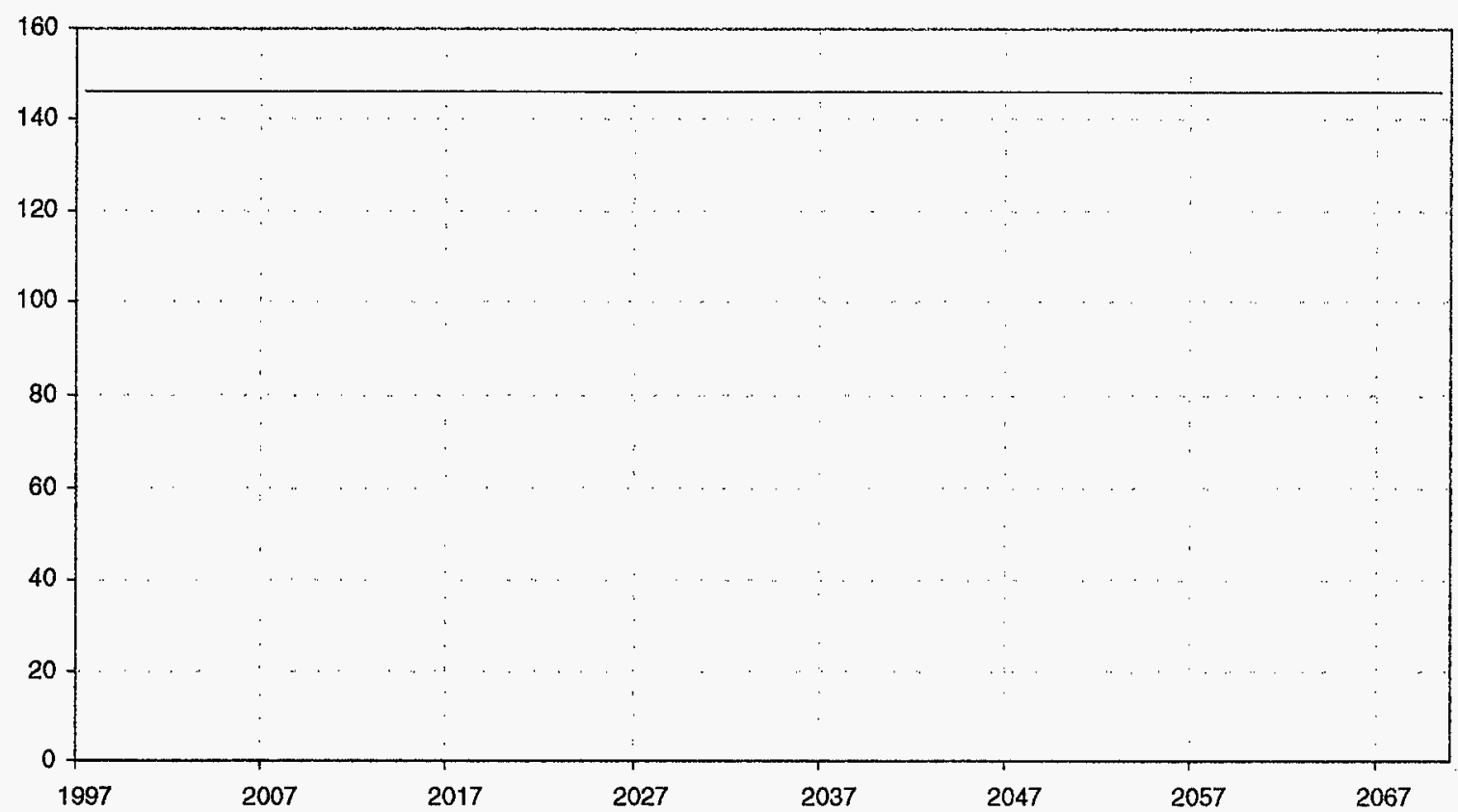

SHIPMENTS RECEIVED CWWFA CHART:

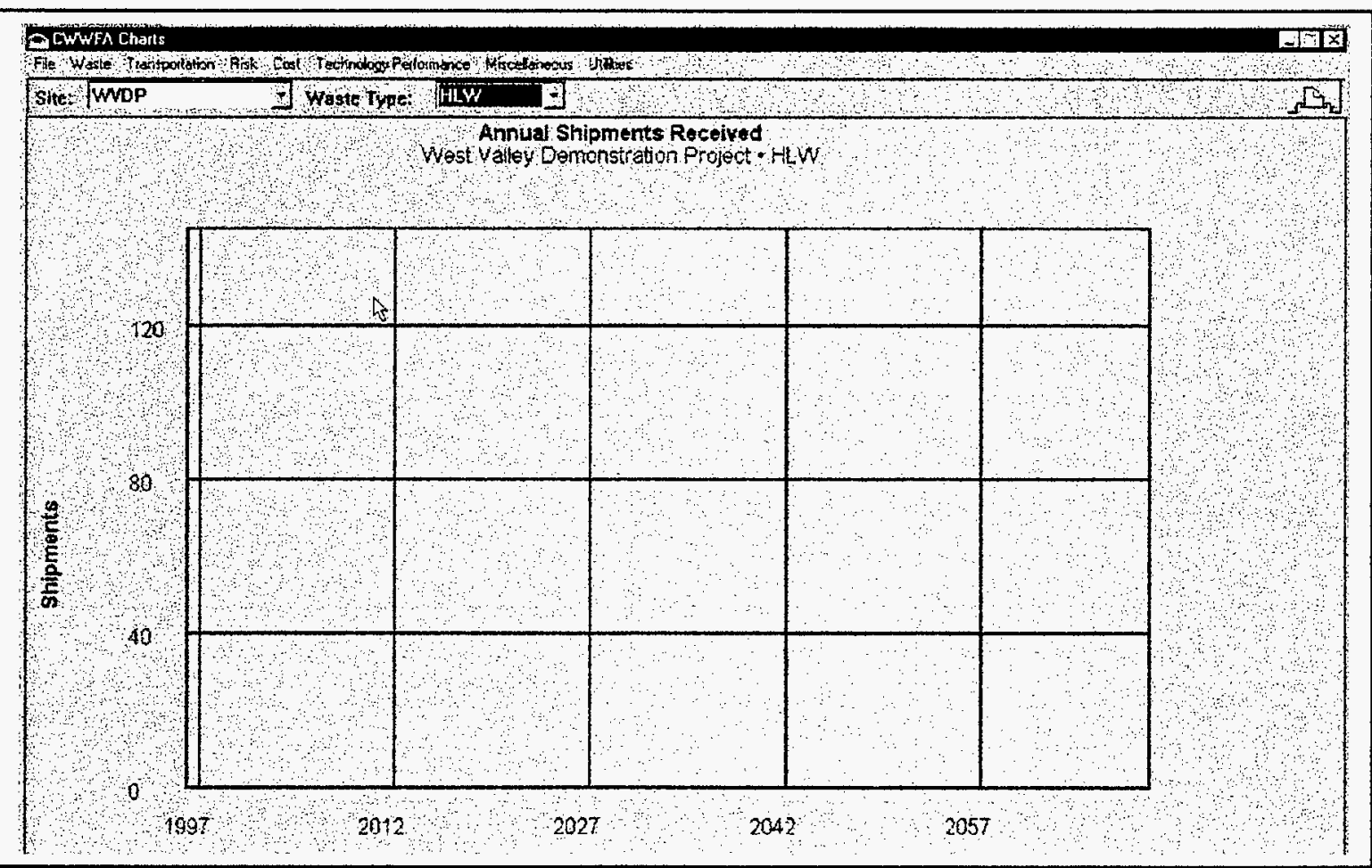




\section{Shipments Sent}

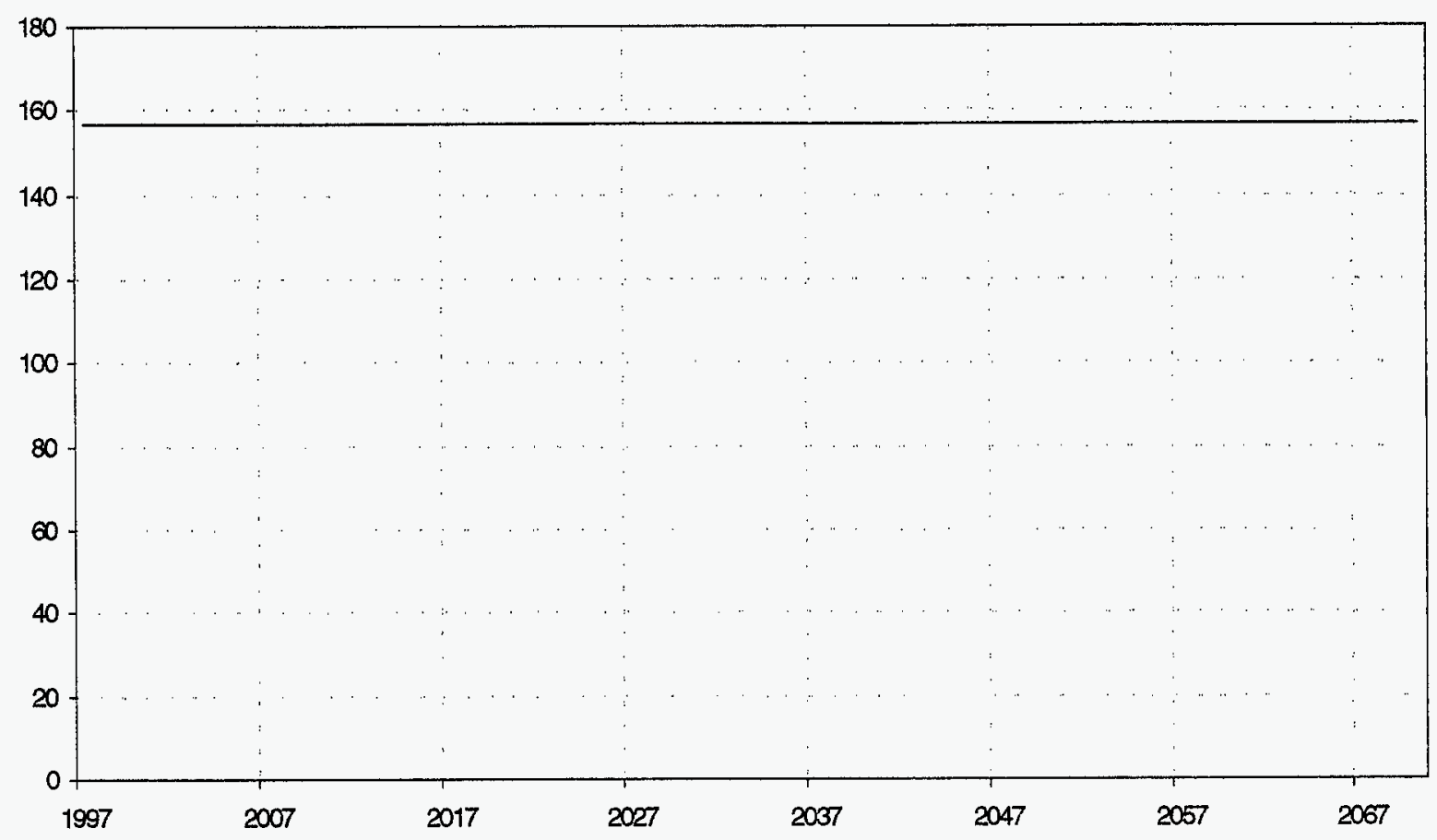

\section{SHIPMENTS SENT CWWFA CHART:}

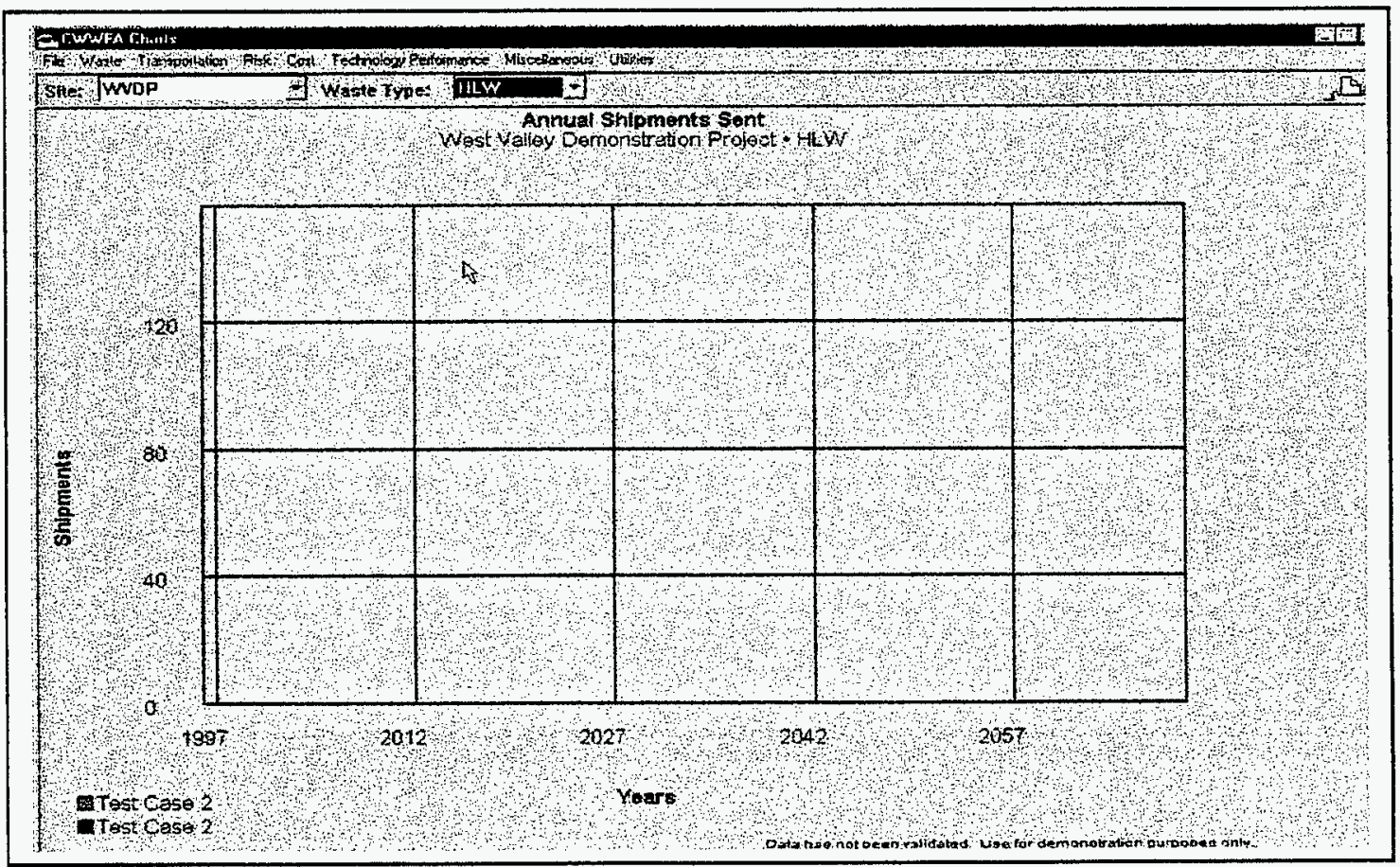


INITIAL PARAMETERS \& RESULTS REPORT

CASE: Case $2 c$

DATE: $11 / 13 / 97$

Evaluator: K B Oswald

TRANSPORTATION COMPARISON EXCEL CHART:

TRANSPORTAION COMPARISON

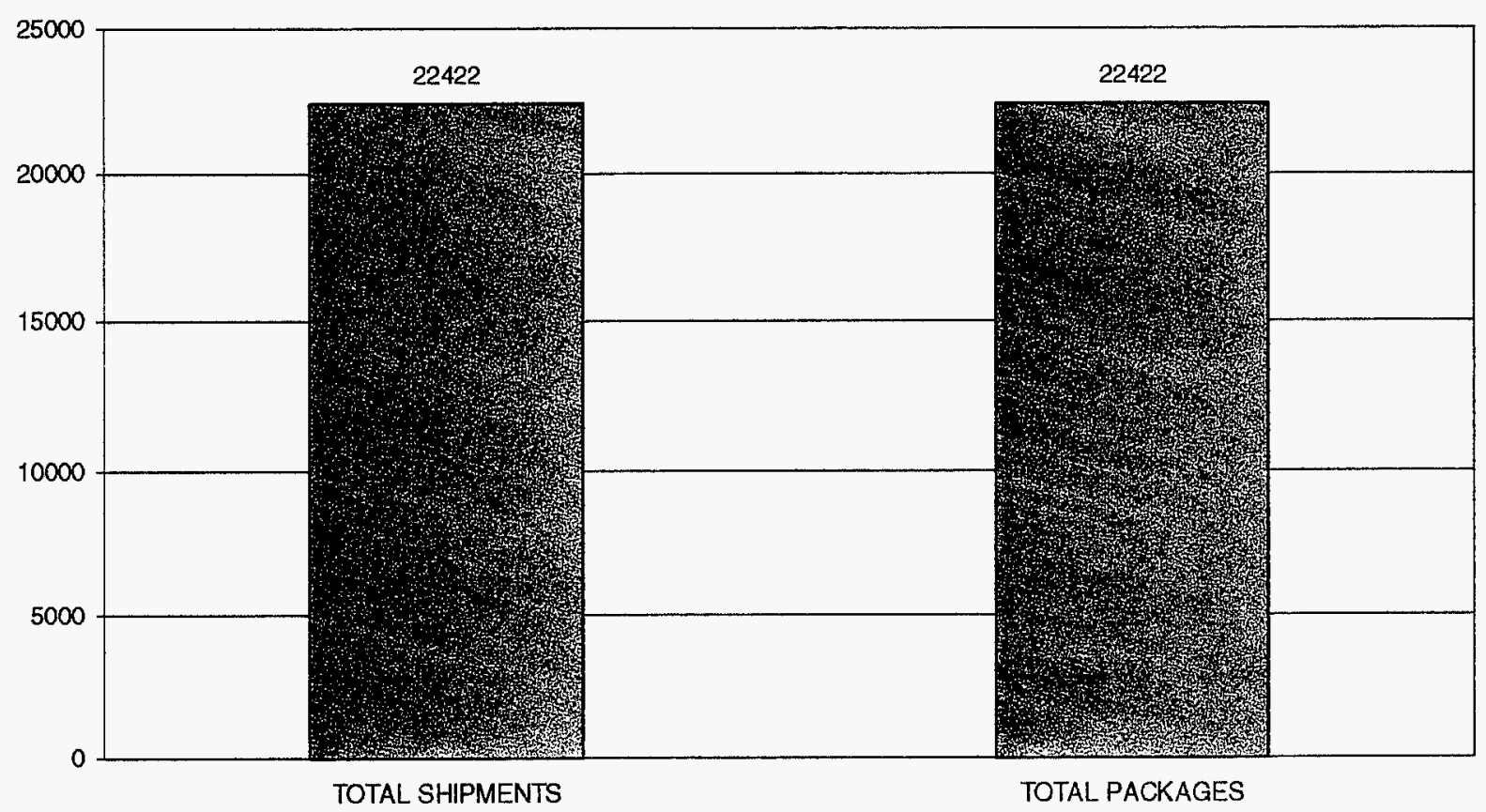

TRANSPORTATION COMPARISON CWWFA CHART:

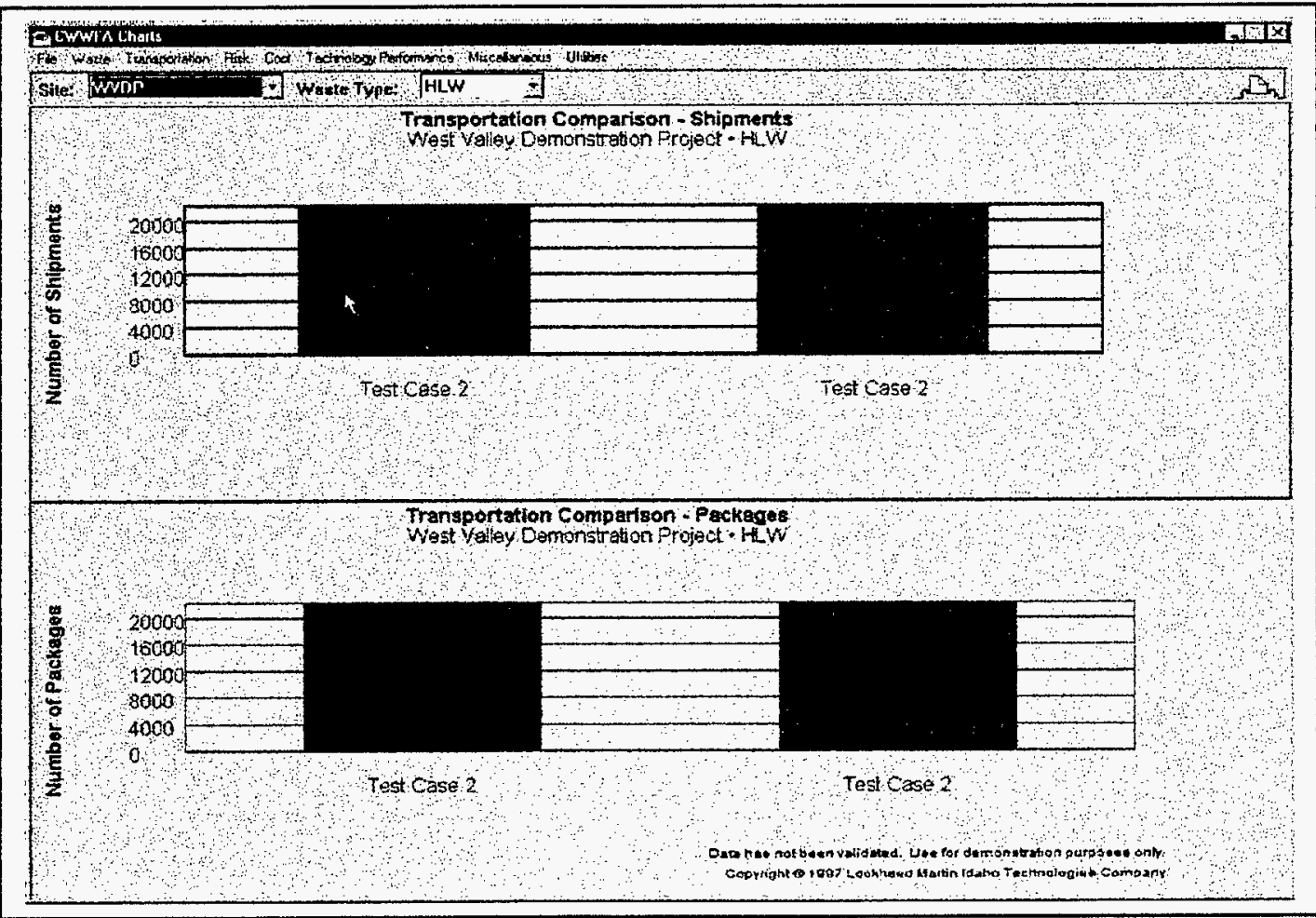


1.0 Waste Stream Information

\begin{tabular}{|l|l|}
\hline Originating Site & WVDP \\
\hline Waste Stream ID \& Name & WVDPWS1-CASE2a \\
\hline Originating Type & HLW \\
\hline Legacy volume (m3) & 15,000 \\
\hline
\end{tabular}

1.1 New Generation

\begin{tabular}{|c|c|c|}
\hline \multicolumn{1}{|c|}{ Start Year } & End Year & Annual Volume (m3) \\
\hline 2031 & 2040 & 1000 \\
\hline & & \\
\hline & & \\
\hline & & \\
\hline
\end{tabular}

1.2 Shipping Route(s)

\begin{tabular}{|l|l|l|}
\hline \multicolumn{1}{|c|}{ Route ID } & \multicolumn{1}{|c|}{ Source Facility } & \multicolumn{1}{c|}{ Destination Facility } \\
\hline $\mathrm{C} 2 \mathrm{~d}-1$ & Legacy-WN & SARSFAC1-CASE2 \\
\hline $\mathrm{C} 2 \mathrm{~d}-2$ & SARSFAC1-CASE2 & WVDPFAC1-CASE2 \\
\hline $\mathrm{C} 2 \mathrm{~d}-3$ & WVDPFAC1-CASE2 & SARSFAC2-CASE2 \\
\hline & & \\
\hline & & \\
\hline & & \\
\hline
\end{tabular}

1.3 Shipping Schedule(s)

\begin{tabular}{|c|c|c|c|}
\hline Route ID: C2d-1 & \multicolumn{2}{|c|}{} & \multicolumn{1}{|c|}{ Waste Type } \\
\hline Start Year & \multicolumn{1}{|c|}{ End Year } & Annual Volume (m3) \\
\hline 1997 & 2070 & HLW & 203 \\
\hline & & & \\
\hline & & & \\
\hline & & & \\
\hline
\end{tabular}

1.3 Shipping Schedule(s) (continued)

\begin{tabular}{|c|l|l|l|}
\hline Route ID: C2d-2 & \multicolumn{2}{|c|}{ Waste Type } & \multicolumn{1}{|c|}{ Annual Volume (m3) } \\
\hline Start Year & \multicolumn{1}{|c|}{ End Year } & HLW & \\
\hline 1997 & 2070 & & \\
\hline & & & \\
\hline & & & \\
\hline
\end{tabular}

1.3 Shipping Schedule(s) (continued)

\begin{tabular}{|c|c|c|c|}
\hline Route ID: C2d-3 & \multicolumn{2}{|c|}{} & \multicolumn{1}{|c|}{ Waste Type } \\
\hline Start Year & \multicolumn{1}{|c|}{ End Year } & Annual Volume (m3) \\
\hline 1997 & 2070 & HLW & 203 \\
\hline & & & \\
\hline & & & \\
\hline & & & \\
\hline
\end{tabular}


INITIAL PARAMETERS \& RESULTS REPORT

CASE: Case $2 \mathrm{~d}$

DATE: $11 / 13 / 97$

Evaluator: K B Oswald

2.0 Facility Information

\begin{tabular}{|l|l|}
\hline Site & SARS \\
\hline Facility ID \& Name & SARSFAC1-CASE2 \\
\hline Function & Treatment \\
\hline Start Year & 1997 \\
\hline End Year & 2070 \\
\hline Annual Capacity (m3/year) or & $1,000,000$ \\
\hline Lifetime Facility Capacity (m3) & \\
\hline Volume Change Factor & 1 \\
\hline
\end{tabular}

Conversion Profile

\begin{tabular}{|l|l|l|}
\hline Percent & Waste Type & Density \\
\hline $100 \%$ & HLW & 650 \\
\hline & & \\
\hline & & \\
\hline
\end{tabular}

2.0 Facility Information

\begin{tabular}{|l|l|}
\hline Site & WVDP \\
\hline Facility ID \& Name & WVDPFAC2-CASE2 \\
\hline Function & Treatment \\
\hline Start Year & 1997 \\
\hline End Year & 2070 \\
\hline Annual Capacity (m3/year) or & $1,000,000$ \\
\hline Lifetime Facility Capacity (m3) & \\
\hline Volume Change Factor & 1 \\
\hline
\end{tabular}

Conversion Profile

\begin{tabular}{|l|l|l|}
\hline Percent & Waste Type & Density \\
\hline 100 & HLW & 1800 \\
\hline & & \\
\hline
\end{tabular}

2.0 Facility Information

\begin{tabular}{|l|l|}
\hline Site & SARS \\
\hline Facility ID \& Name & SARSFAC1-CASE2 \\
\hline Function & Disposal \\
\hline Start Year & 1997 \\
\hline End Year & 2070 \\
\hline Annual Capacity (m3/year) or & $1,000,000$ \\
\hline Lifetime Facility Capacity (m3) & \multicolumn{2}{|}{} \\
\hline Volume Change Factor & 1 \\
\hline \\
\begin{tabular}{|l|l|l|}
\hline Ponversion Profile & Waste Type & Density \\
\hline NA & NA & NA \\
\hline & & \\
\hline
\end{tabular}
\end{tabular}


WASTE DISPOSITION - EXCEL CHART (WVDP, HLW):

\section{Waste Disposition}

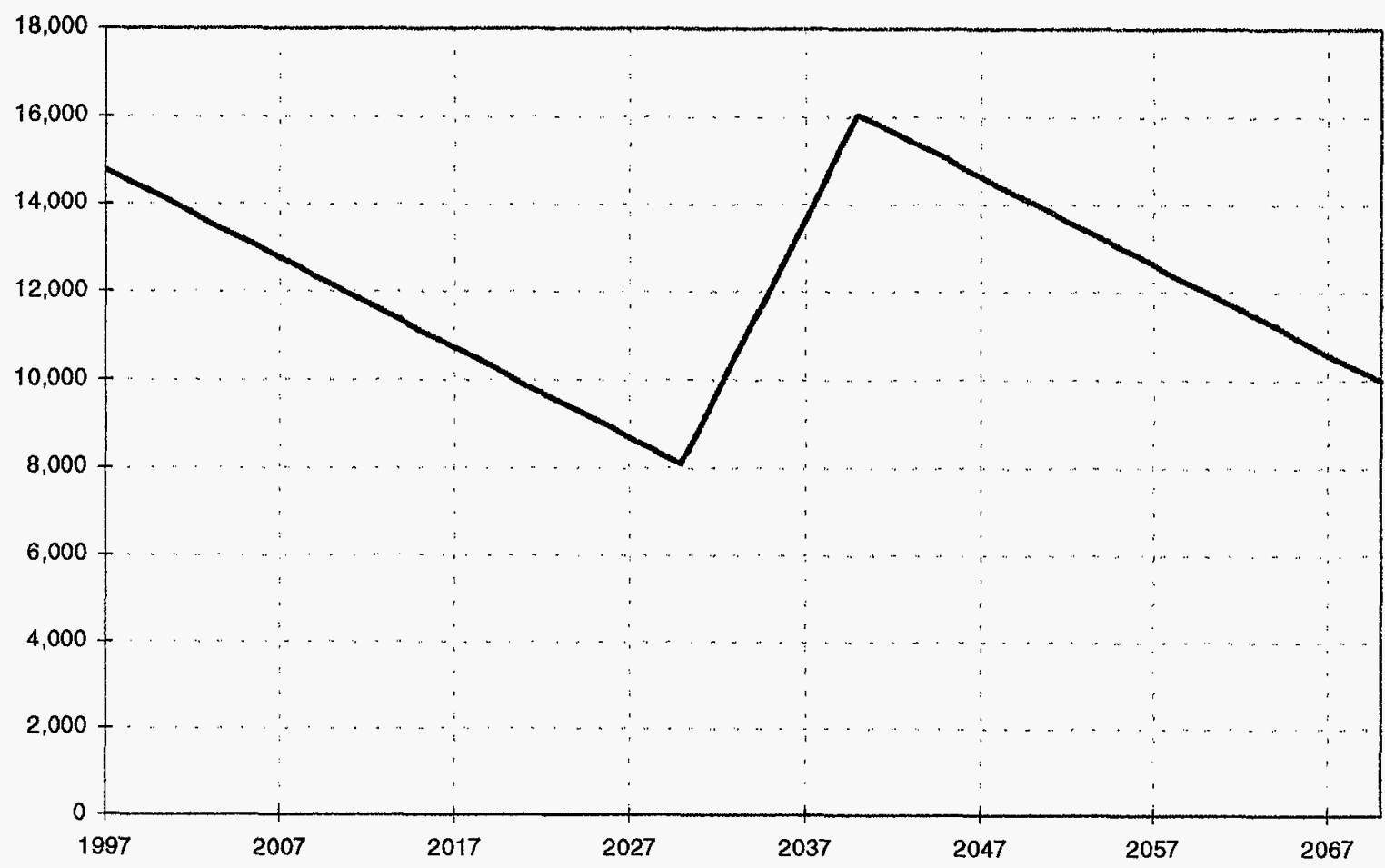

\section{WASTE DISPOSITION - CWWFA CHART:}

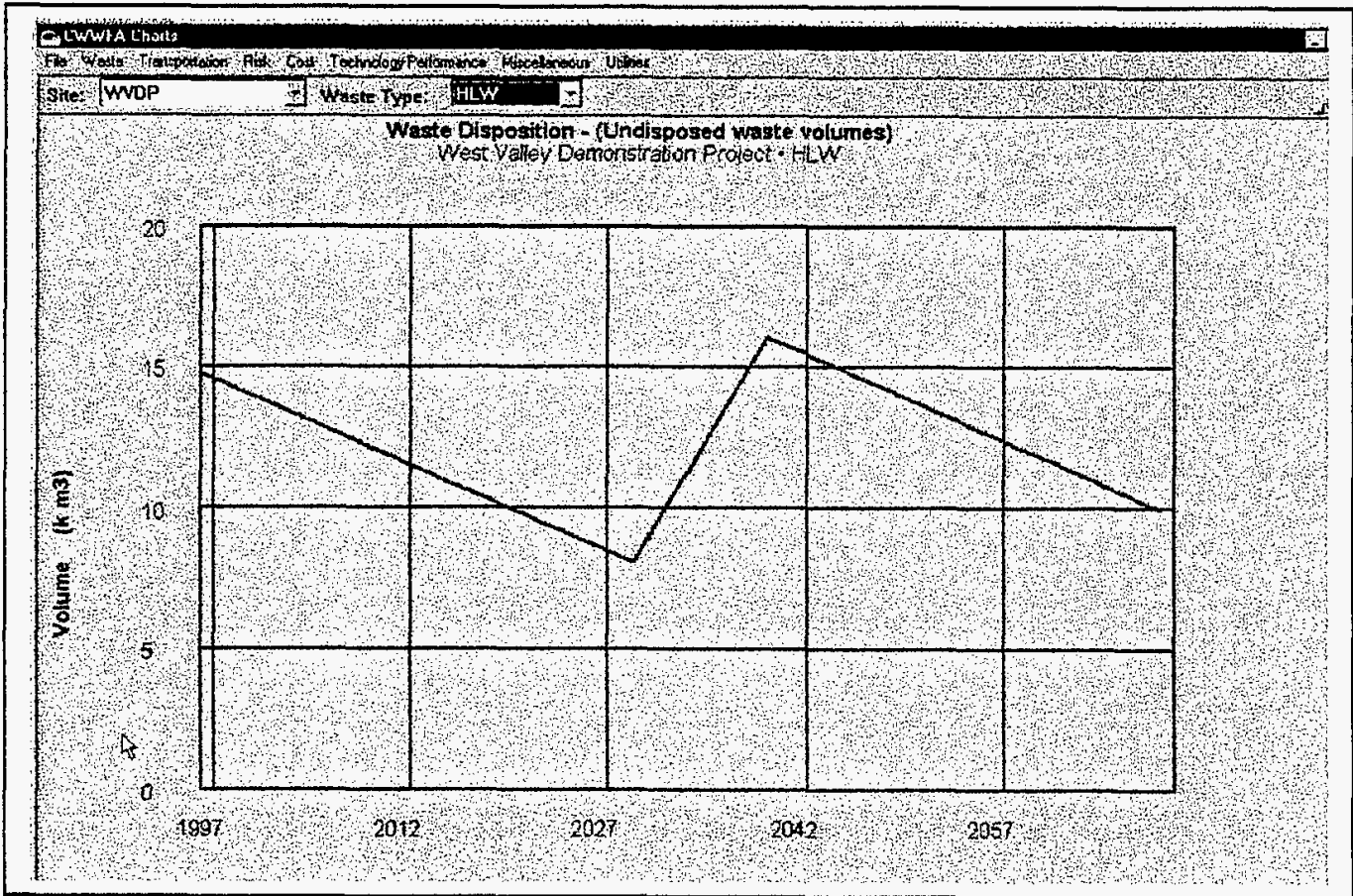


INITIAL PARAMETERS \& RESULTS REPORT

CASE: Case $2 \mathrm{~d}$

DATE: $11 / 13 / 97$

Evaluator: K B Oswald

\section{FACILITY CAPACITY EXCEL CHART (WVDP, SARSFAC2-CASE2):}

\section{Facility Capacity Detail}

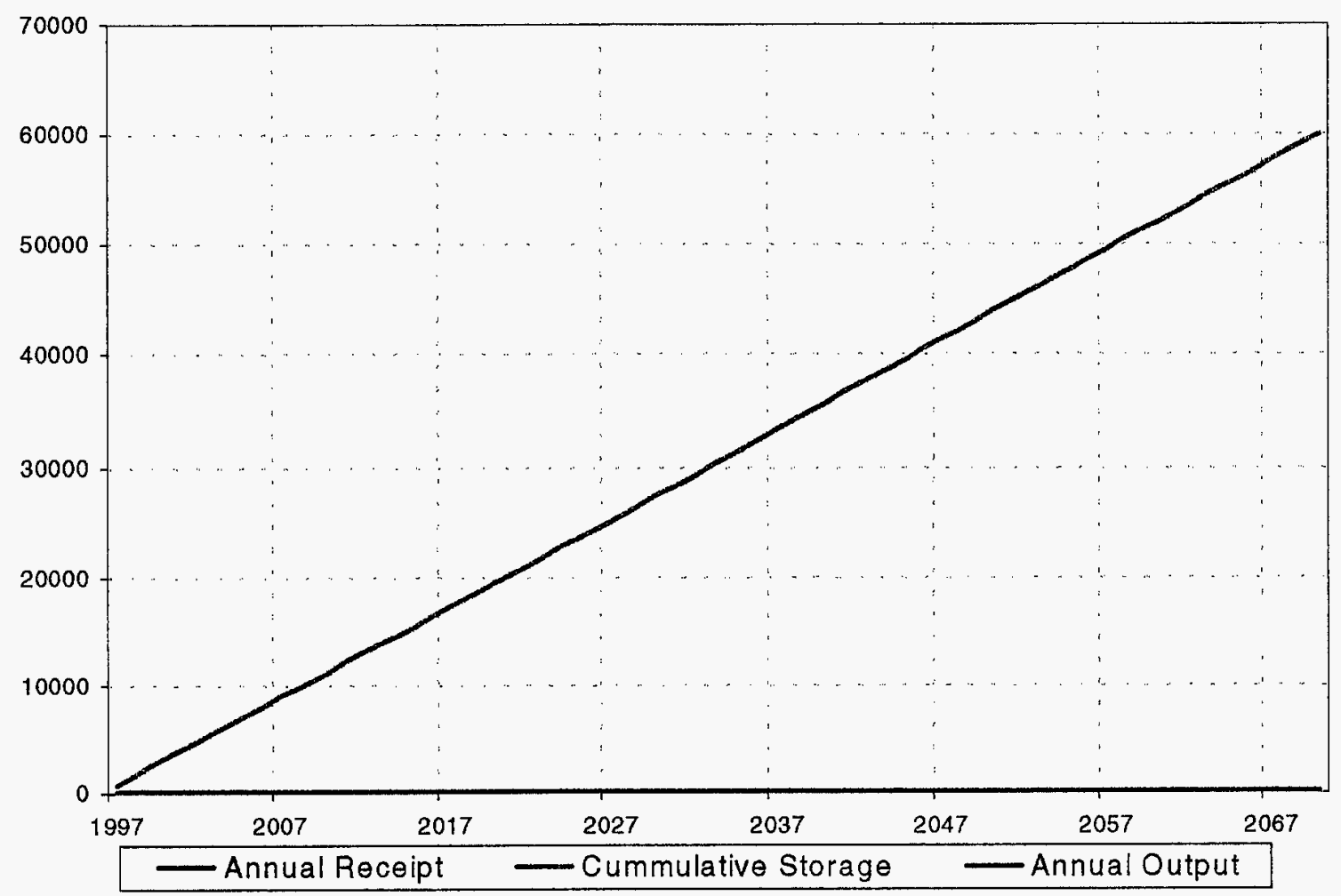

\section{FACILITY CAPACITY CWWFA CHART:}

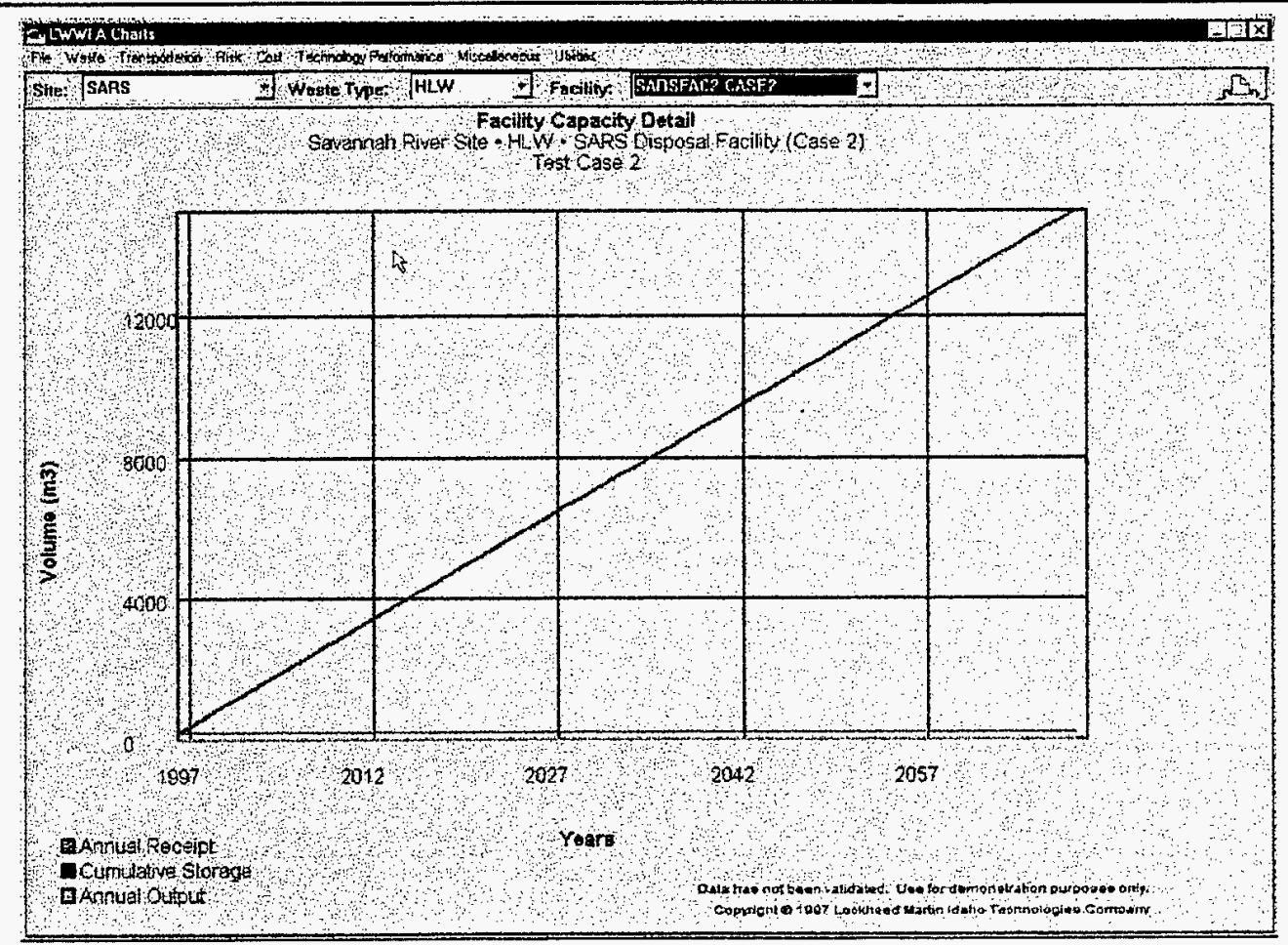


WASTE STREAM DETAIL EXCEL CHART (WVDPWS1-CASE2a):

\section{Waste Stream Detail}

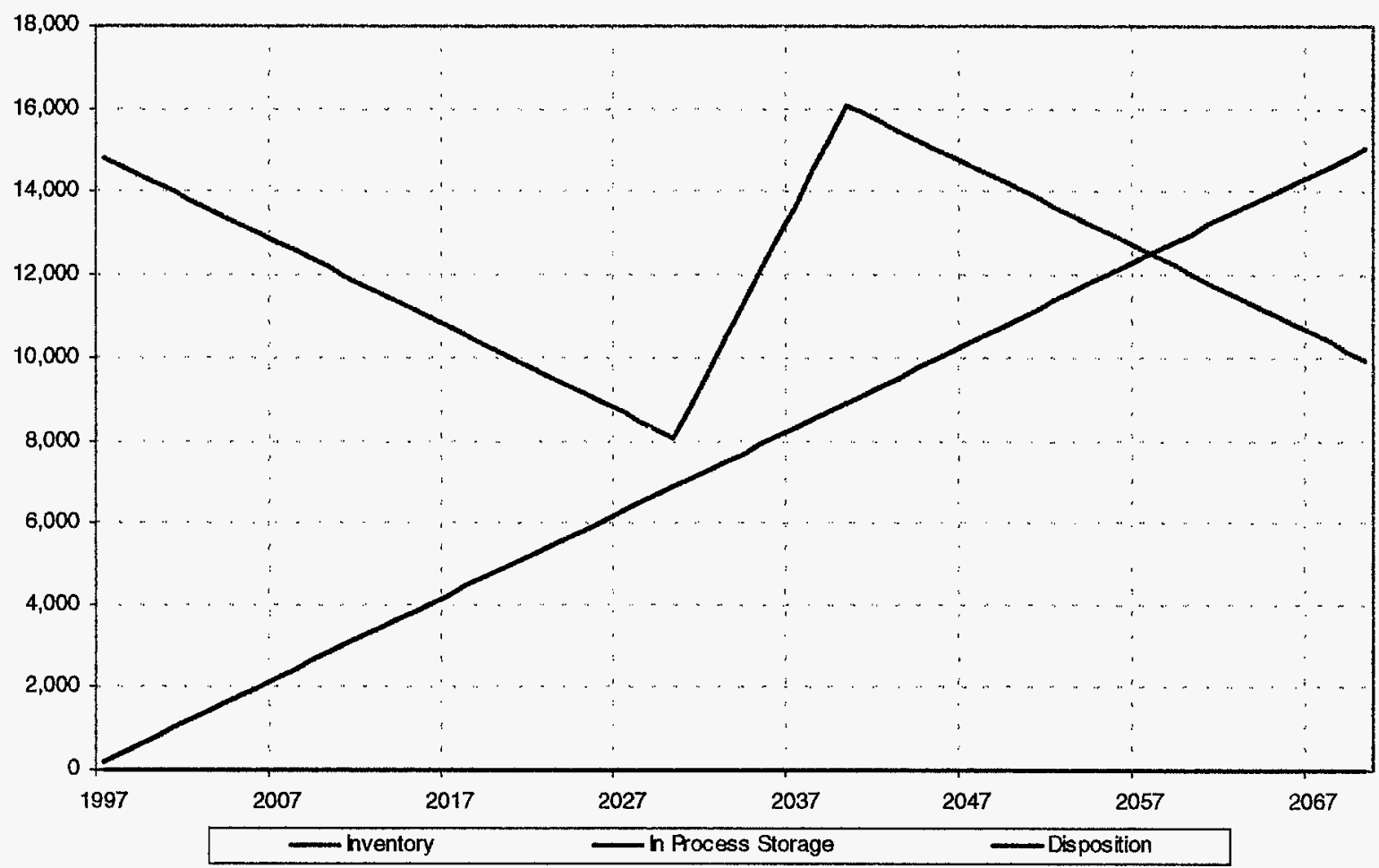

WASTE STREAM DETAIL CWWFA CHART:

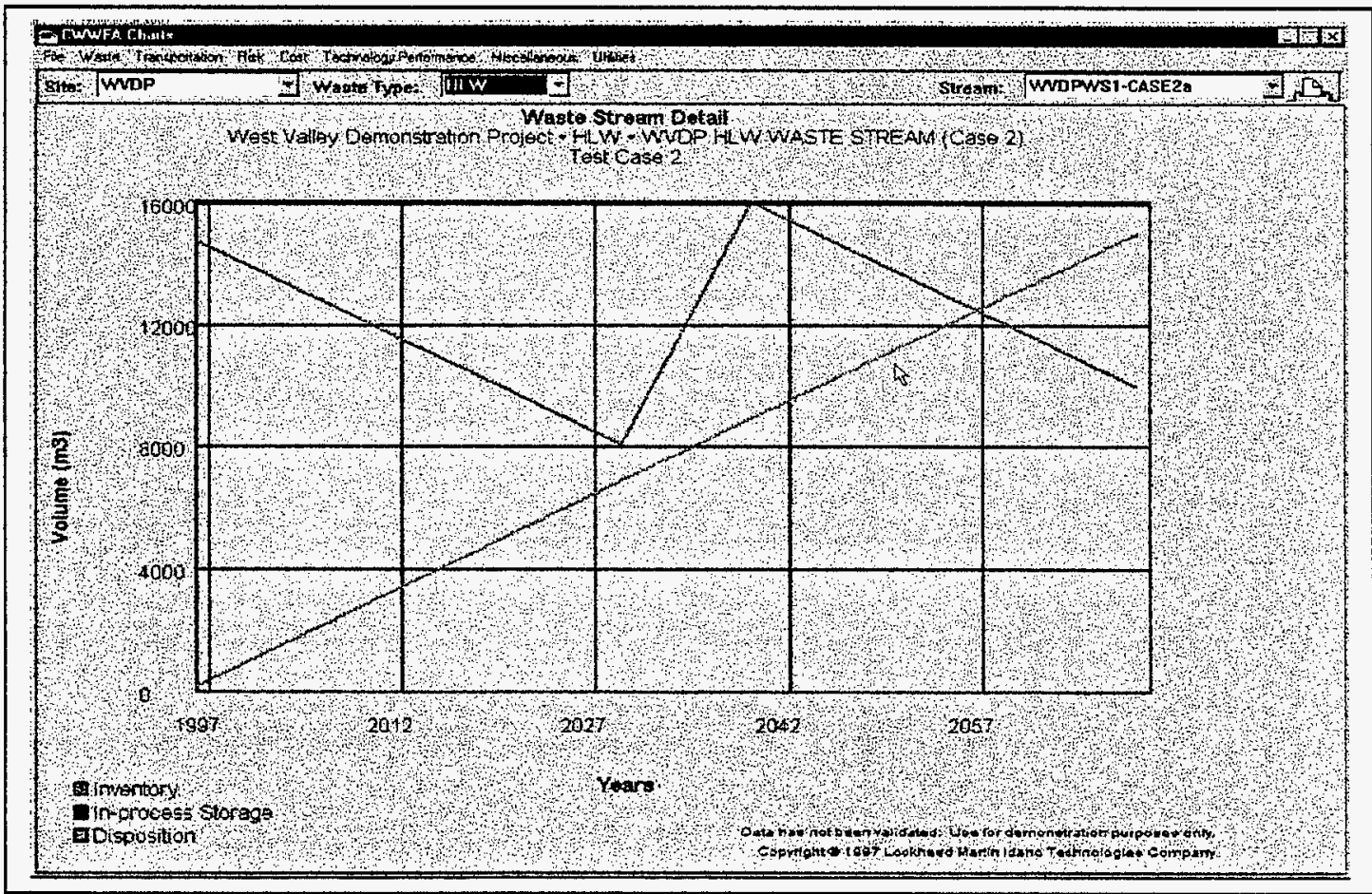


INITIAL PARAMETERS \& RESULTS REPORT

CASE: Case $2 d$

DATE: $11 / 13 / 97$

Evaluator: K B Oswald

SHIPMENTS RECEIVED EXCEL CHART (WVDP):

Shipments Received

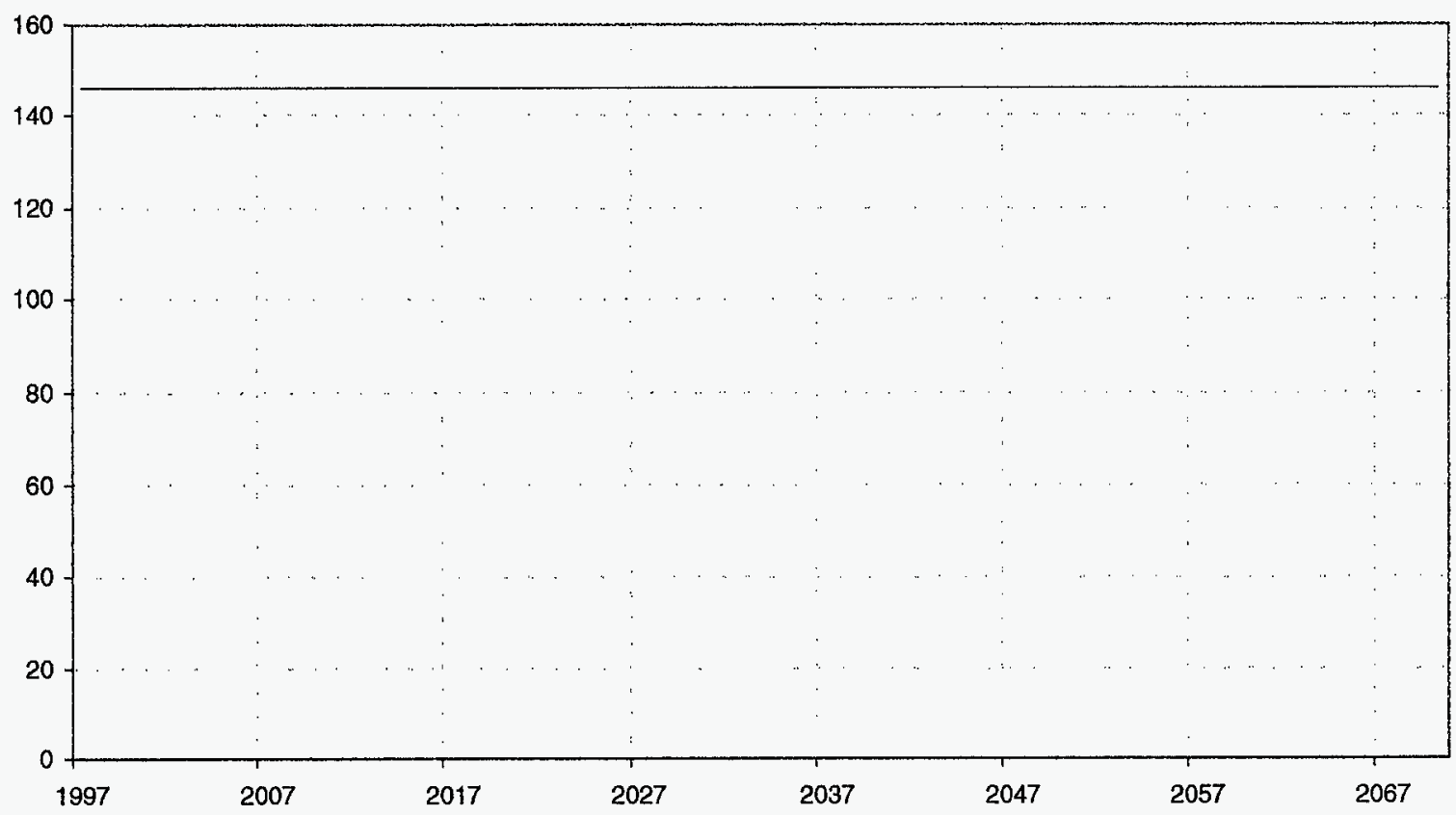

SHIPMENTS RECEIVED CWWFA CHART:

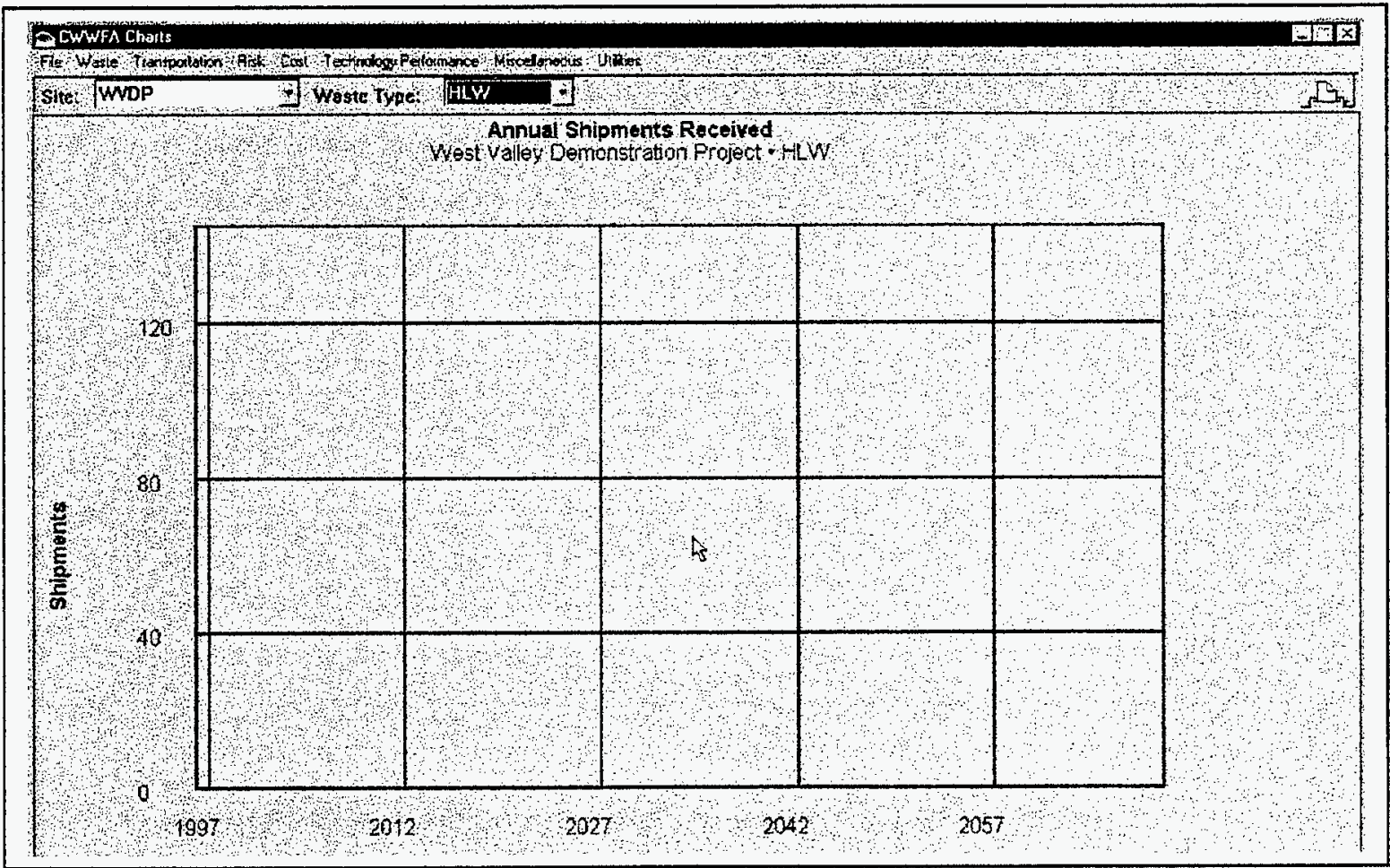




\section{SHIPMENTS SENT EXCEL CHART (WVDP):}

\section{Shipments Sent}

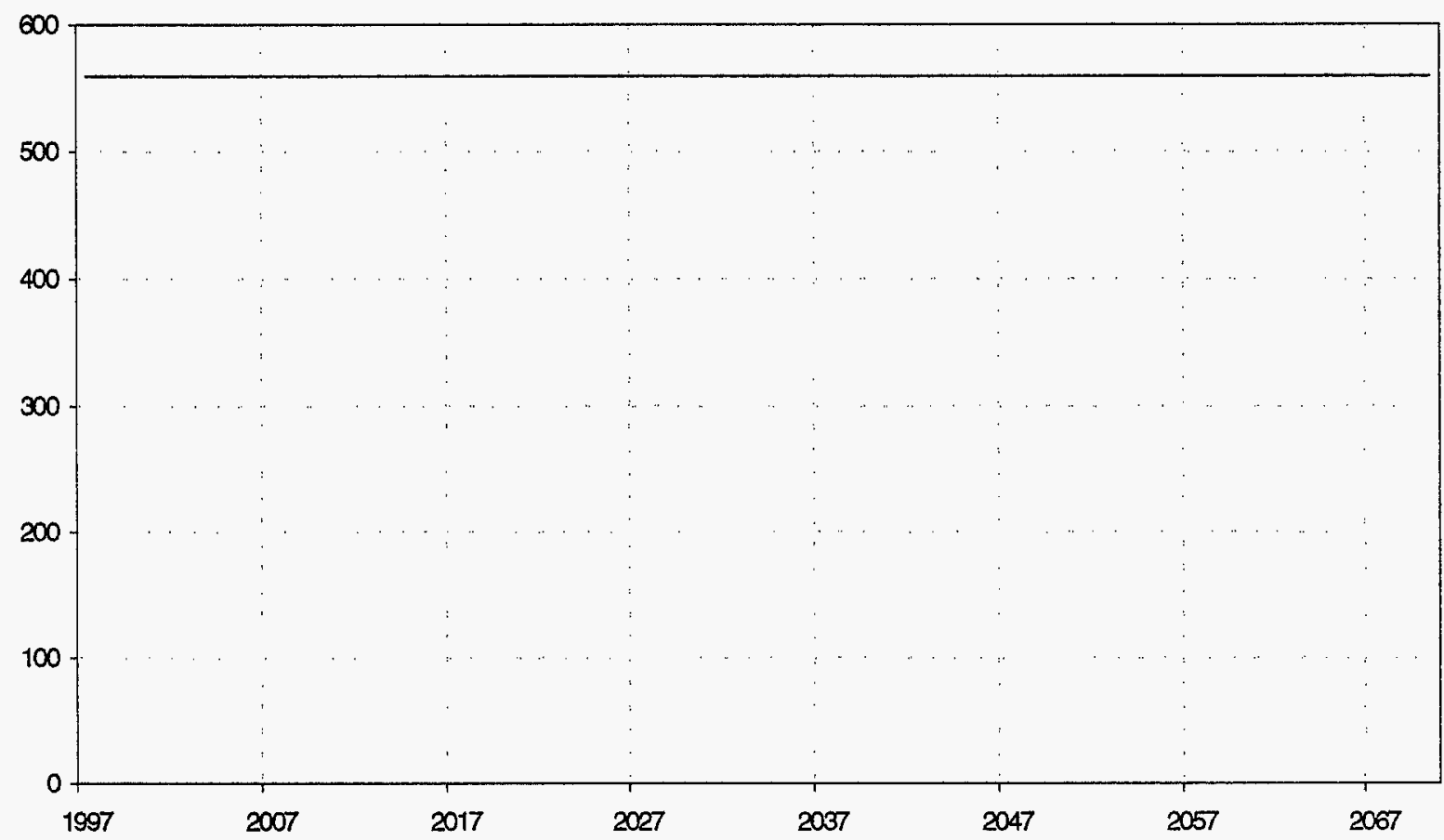

\section{SHIPMENTS SENT CWWFA CHART:}

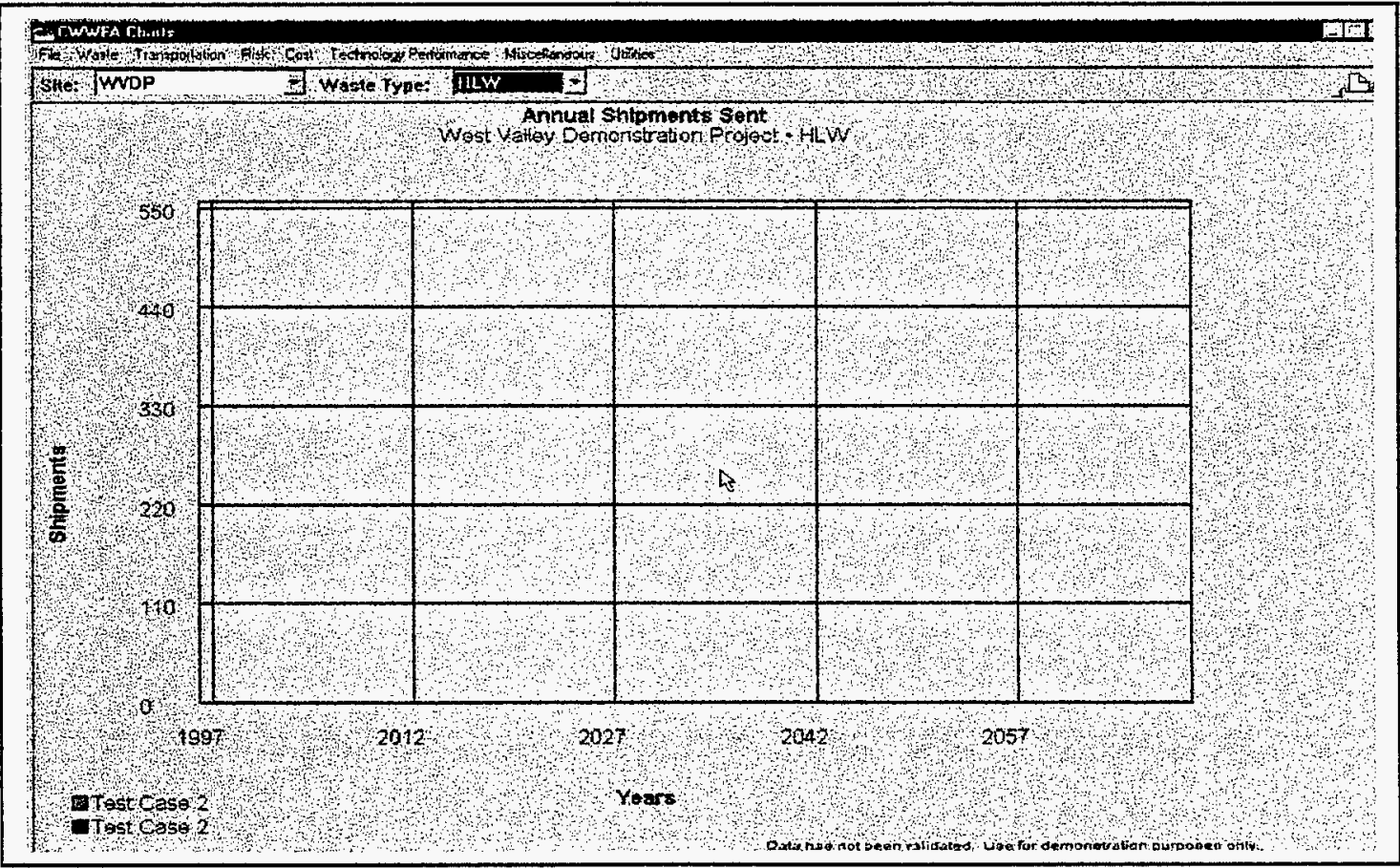




\section{TRANSPORTAION COMPARISON}

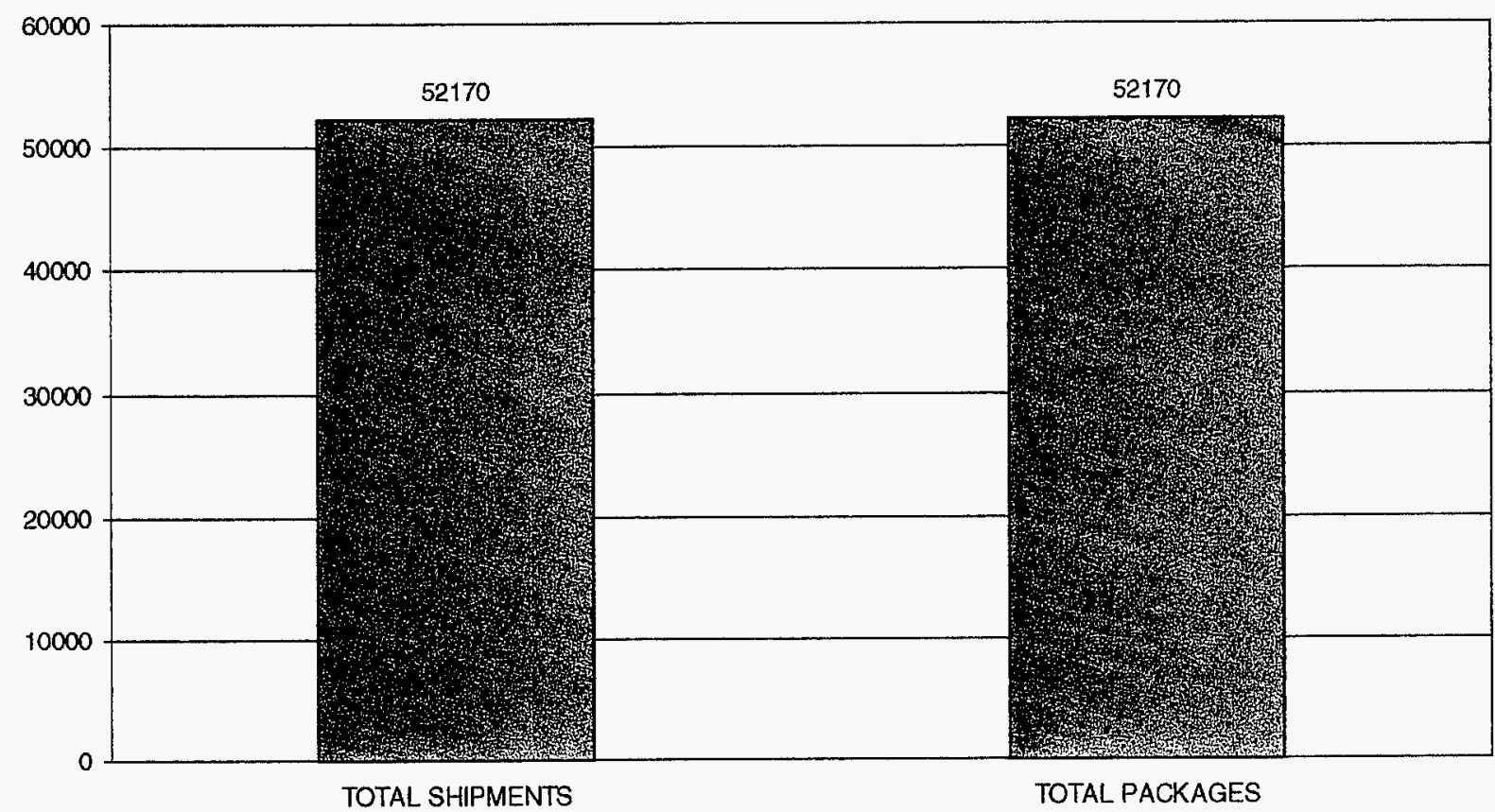

TRANSPORTATION COMPARISON CWWFA CHART:

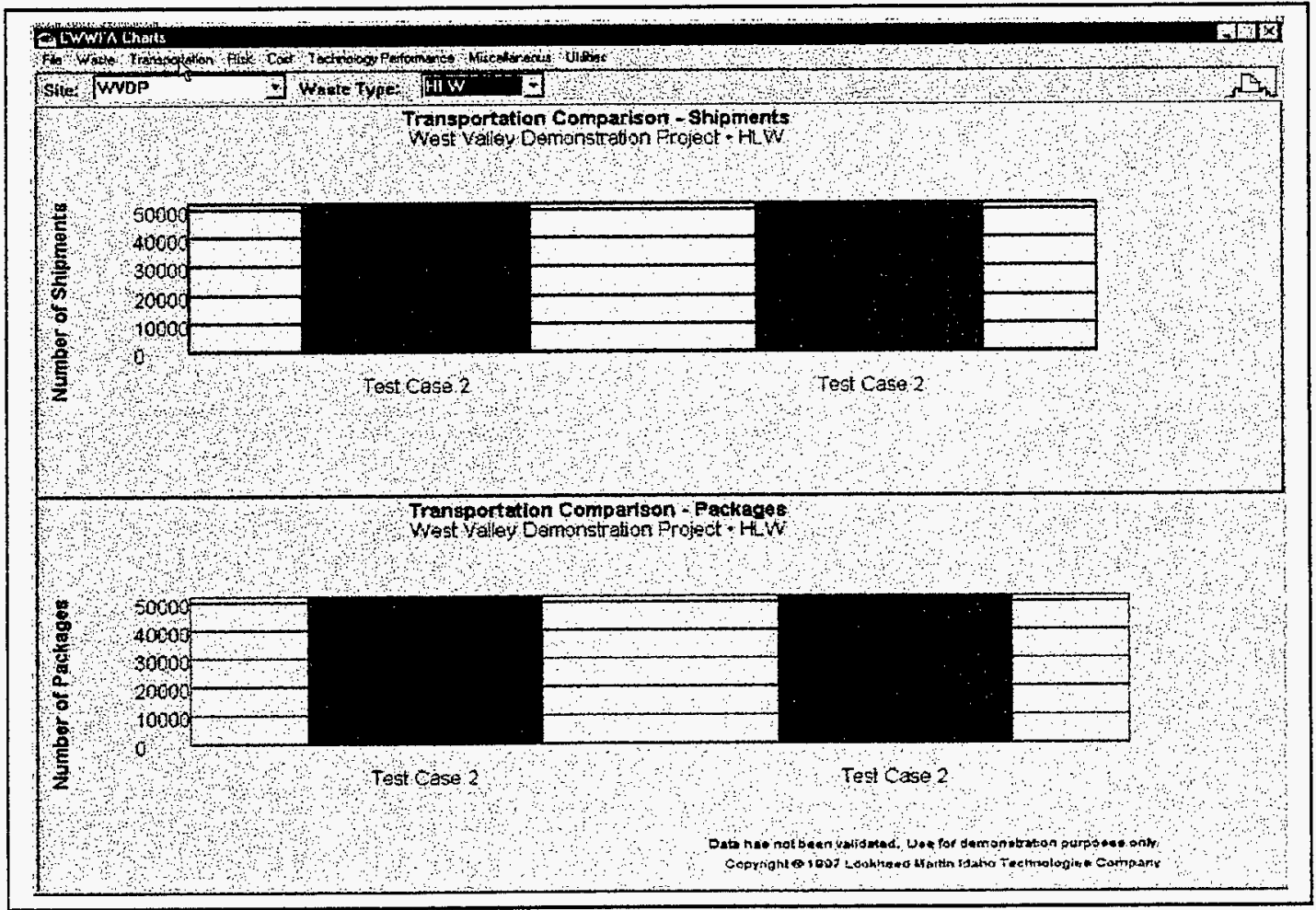


CASE: Case 2e

INITIAL PARAMETERS \& RESULTS REPORT

Oswald

DATE: $11 / 13 / 97$

Evaluator: K B

1.0 Waste Stream Information

\begin{tabular}{|l|l|}
\hline Originating Site & WVDP \\
\hline Waste Stream ID \& Name & WVDPWS1-CASE2a \\
\hline Originating Type & HLW \\
\hline Legacy volume (m3) & 15,000 \\
\hline
\end{tabular}

1.1 New Generation

\begin{tabular}{|l|l|l|}
\hline \multicolumn{1}{|c|}{ Start Year } & End Year & Annual Volume (m3) \\
\hline 2031 & 2040 & 1000 \\
\hline & & \\
\hline & & \\
\hline & & \\
\hline
\end{tabular}

1.2 Shipping Route(s)

\begin{tabular}{|l|l|l|}
\hline \multicolumn{1}{|c|}{ Route ID } & \multicolumn{1}{|c|}{ Source Facility } & \multicolumn{1}{c|}{ Destination Facility } \\
\hline C2e-1 & Legacy-WW & SARSFAC1-CASE2 \\
\hline C2e-2 & SARSFAC1-CASE2 & WWDPFAC1-CASE2 \\
\hline C2e-3 & WWDPFAC1-CASE2 & SARSFAC2-CASE2 \\
\hline & & \\
\hline & & \\
\hline & & \\
\hline
\end{tabular}

1.3 Shipping Schedule(s)

\begin{tabular}{|c|c|c|c|}
\hline \multicolumn{4}{|l|}{ Route ID: $\mathrm{C} 2 \mathrm{e}-1$} \\
\hline Start Year & End Year & Waste Type & Annual Volume (m3) \\
\hline 1997 & 2070 & HLW & 338 \\
\hline & & & \\
\hline 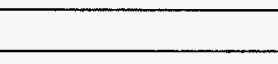 & & & \\
\hline
\end{tabular}

1.3 Shipping Schedule(s) (continued)

\begin{tabular}{|c|l|l|l|}
\hline Route ID: C2e-2 & \multicolumn{2}{|c|}{} & \multicolumn{1}{|c|}{ Waste Type } \\
\hline Start Year & \multicolumn{1}{|c|}{ End Year } & Annual Volume (m3) \\
\hline 1997 & 2020 & HLW & 338 \\
\hline 2061 & 2070 & HLW & 1689 \\
\hline & & & \\
\hline & & & \\
\hline
\end{tabular}

1.3 Shipping Schedule(s) (continued)

\begin{tabular}{|c|c|c|c|}
\hline Route ID: C2e-3 & \multicolumn{2}{|c|}{} & \multicolumn{1}{|c|}{ Waste Type } \\
\hline Start Year & \multicolumn{1}{|c|}{ End Year } & Annual Volume (m3) \\
\hline 2061 & 2070 & LLW & 2500 \\
\hline & & & \\
\hline & & & \\
\hline
\end{tabular}


CASE: Case $2 \mathrm{e}$

Oswald
INITIAL PARAMETERS \& RESULTS REPORT

DATE: $11 / 13 / 97$

Evaluator: K B

2.0 Facility Information

\begin{tabular}{|l|l|}
\hline Site & SARS \\
\hline Facility ID \& Name & SARSFAC1-CASE2 \\
\hline Function & Treatment \\
\hline Start Year & 1997 \\
\hline End Year & 2070 \\
\hline Annual Capacity (m3/year) or & $1,000,000$ \\
\hline Lifetime Facility Capacity (m3) & \\
\hline Volume Change Factor & 1 \\
\hline
\end{tabular}

Conversion Profile

\begin{tabular}{|l|l|l|}
\hline Percent & Waste Type & Density \\
\hline $100 \%$ & HLW & 650 \\
\hline & & \\
\hline & & \\
\hline
\end{tabular}

2.0 Facility Information

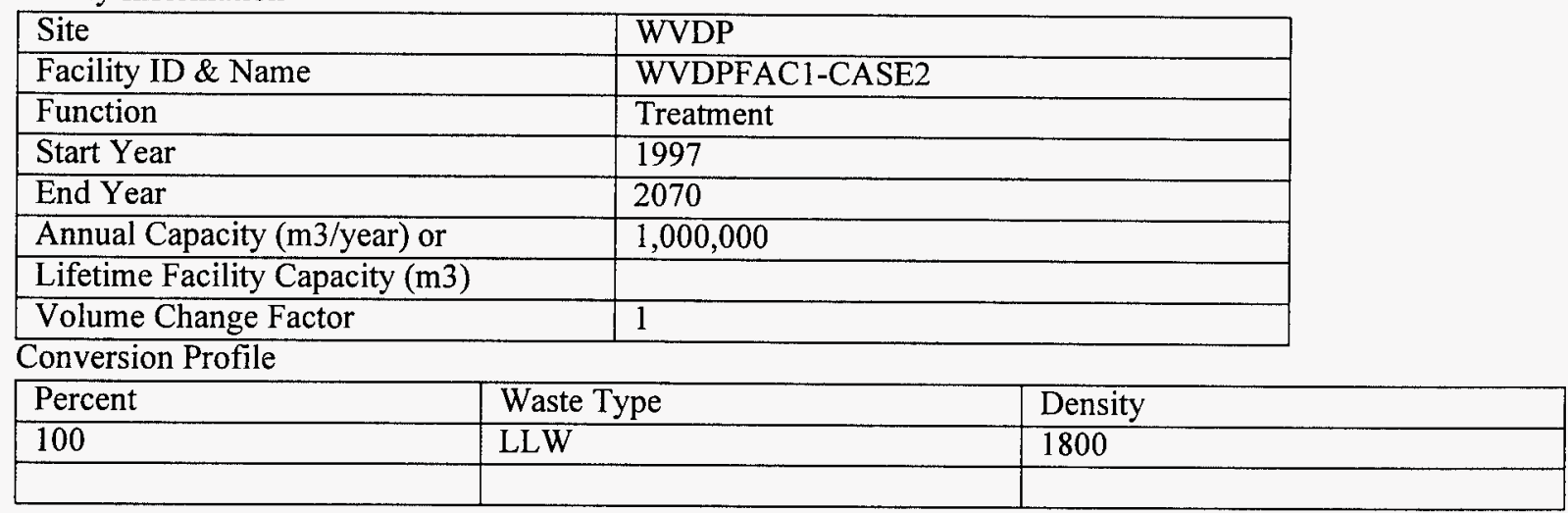

2.0 Facility Information

\begin{tabular}{|l|l|}
\hline Site & SARS \\
\hline Facility ID \& Name & SARSFAC1-CASE2 \\
\hline Function & Disposal \\
\hline Start Year & 1997 \\
\hline End Year & 2070 \\
\hline Annual Capacity (m3/year) or & $1,000,000$ \\
\hline Lifetime Facility Capacity (m3) & \\
\hline Volume Change Factor & 1 \\
\hline
\end{tabular}

Conversion Profile

\begin{tabular}{|l|l|l|}
\hline Percent & Waste Type & Density \\
\hline NA & NA & NA \\
\hline & & \\
\hline
\end{tabular}


CASE: Case 2e

DATE: $11 / 13 / 97$

Evaluator: K B

Oswald

WASTE DISPOSITION - EXCEL CHART (WVDP, HLW):

\section{Waste Disposition}

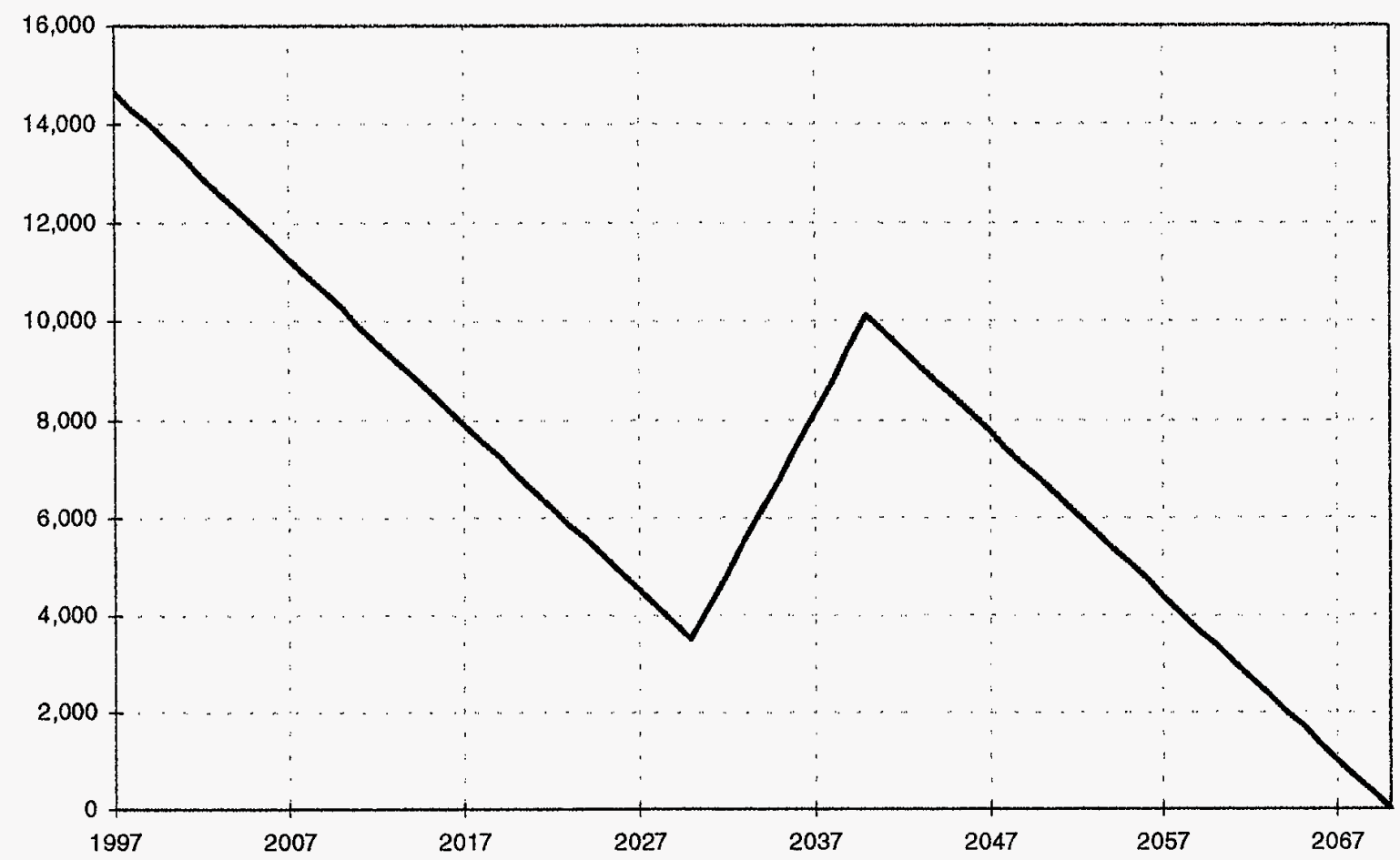

WASTE DISPOSITION - CWWFA CHART:

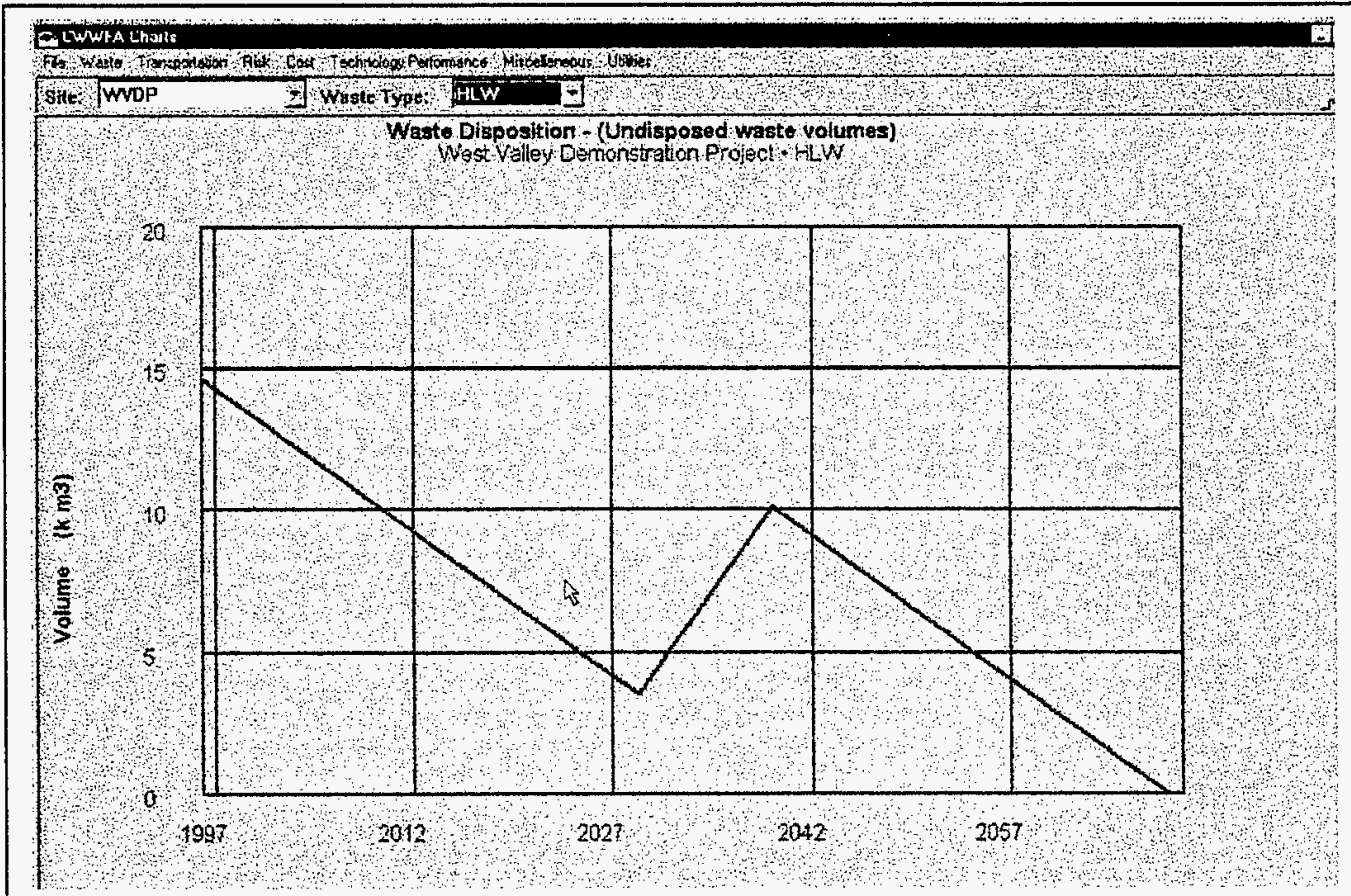


CASE: Case 2e

DATE: $11 / 13 / 97$

Evaluator: K B

Oswald

FACILITY CAPACITY EXCEL CHART (SARS, SARSFAC2-CASE2):

Facility Capacity Detail

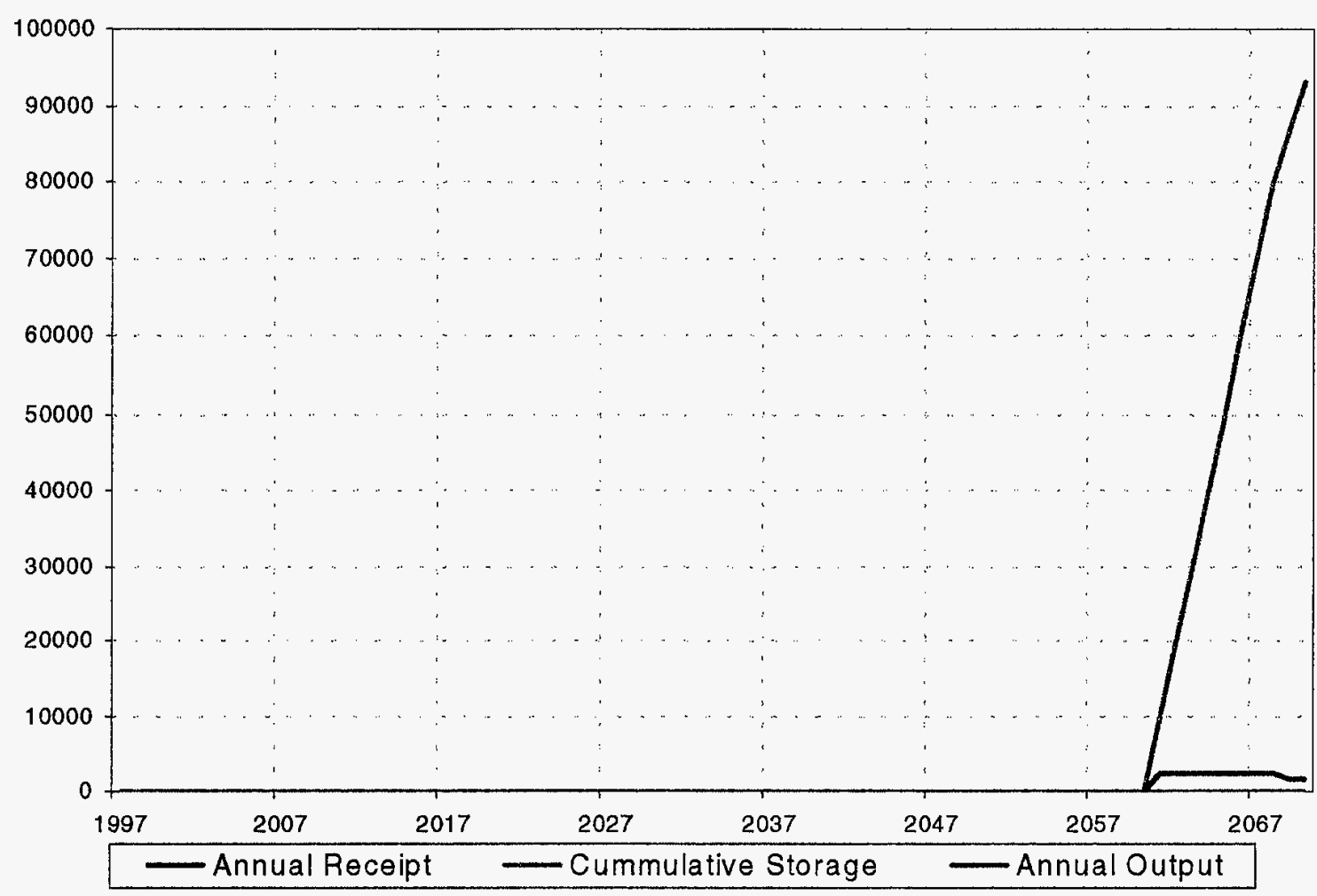

FACILITY CAPACITY CWWFA CHART:

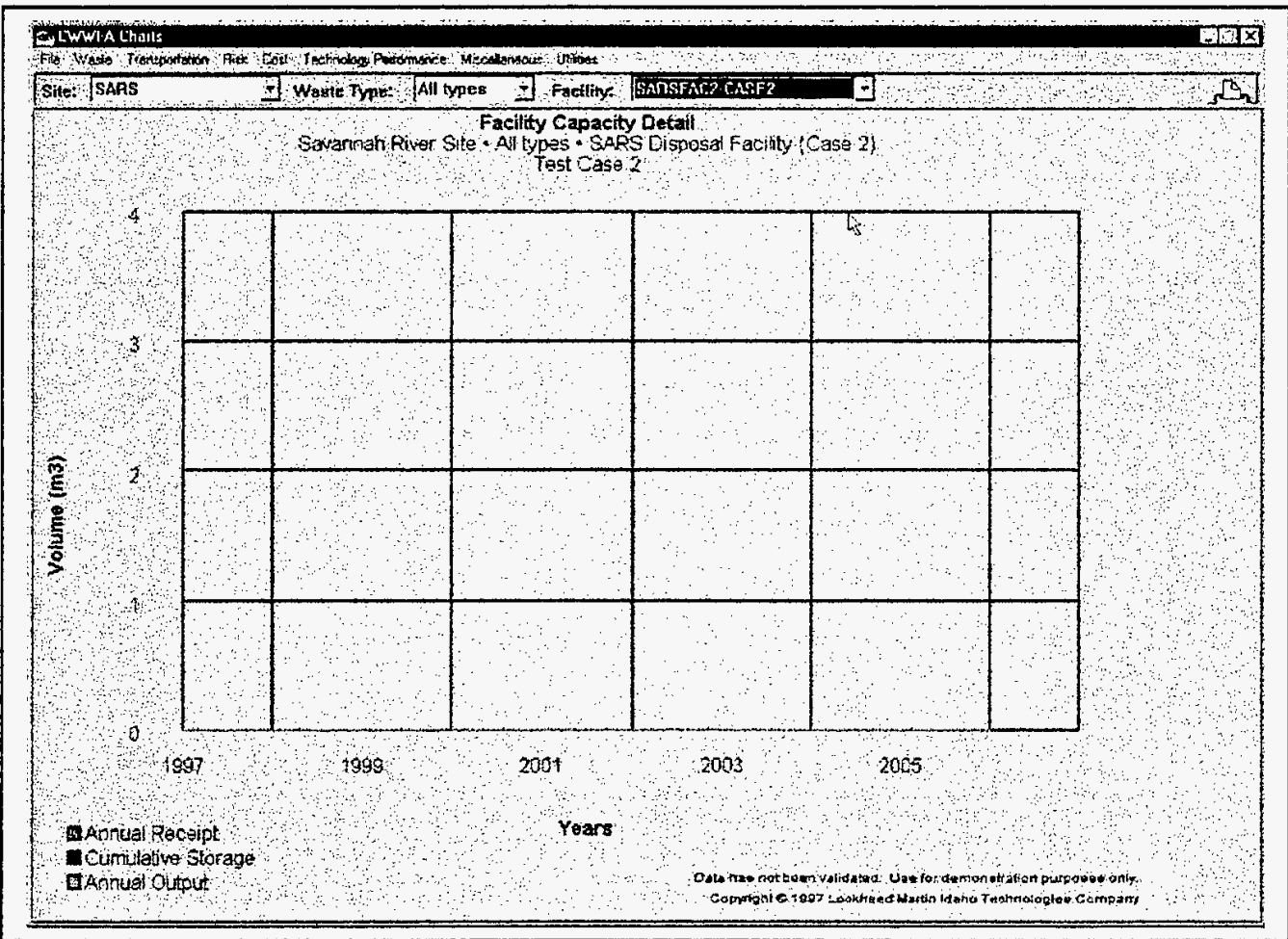


CASE: Case $2 \mathrm{e}$

DATE: $11 / 13 / 97$

Evaluator: K B

Oswald

WASTE STREAM DETAIL EXCEL CHART (WVDPWS1-CASE2a):

\section{Waste Stream Detail}

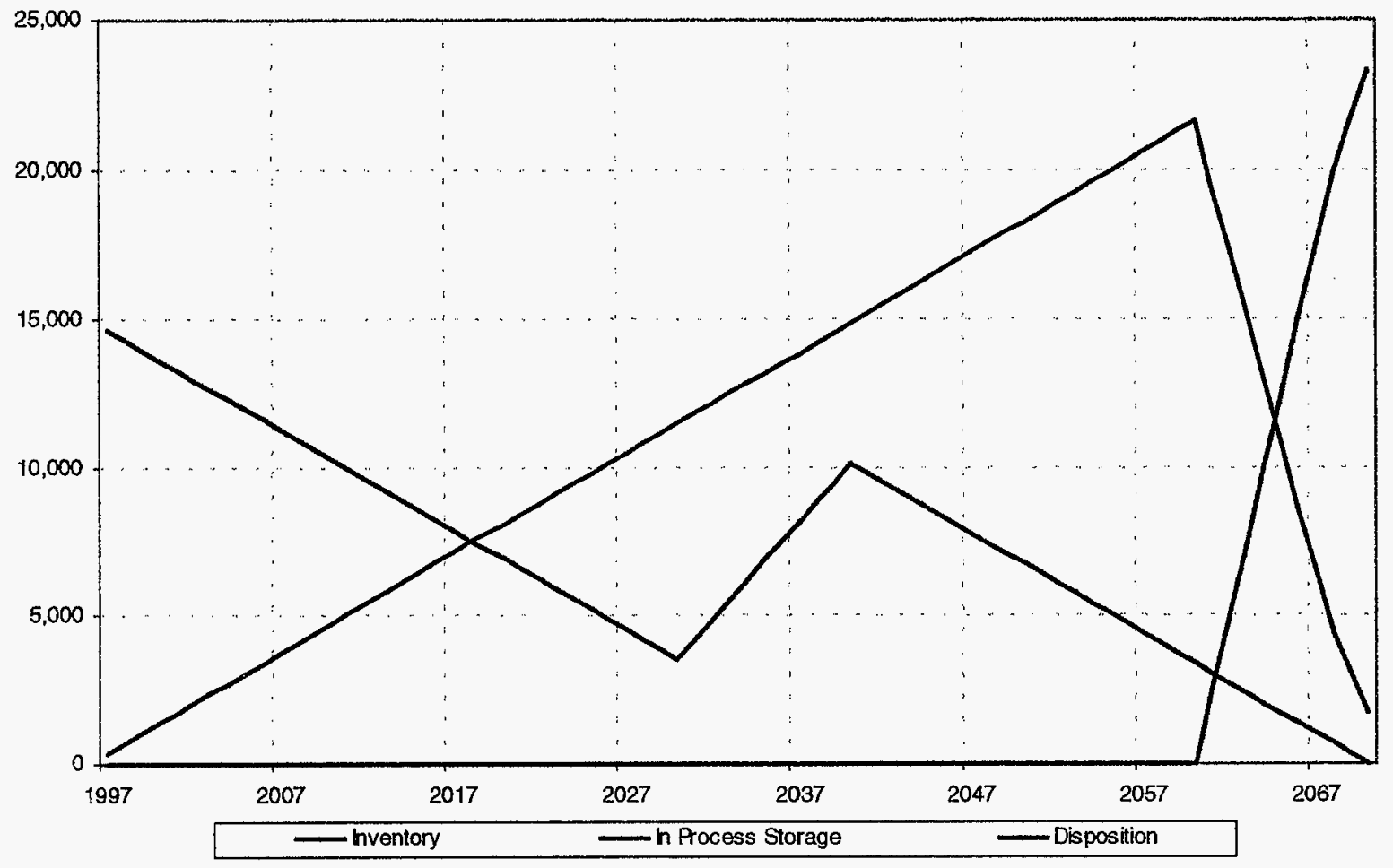

WASTE STREAM DETAIL CWWFA CHART:

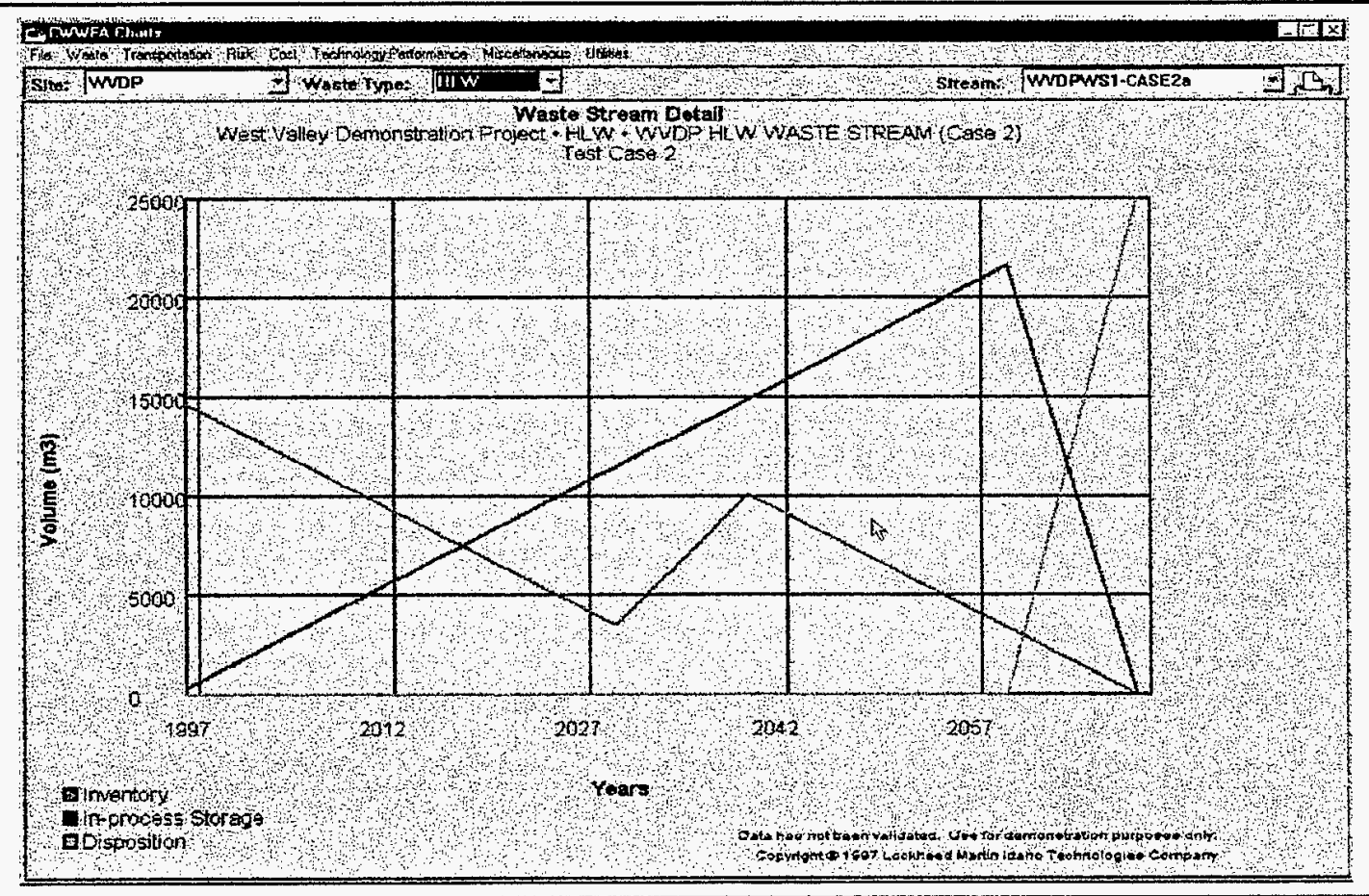


CASE: Case 2e

DATE: $11 / 13 / 97$

Evaluator: K B

Oswald

SHIPMENTS RECEIVED EXCEL CHART (WVDP):

\section{Shipments Received}

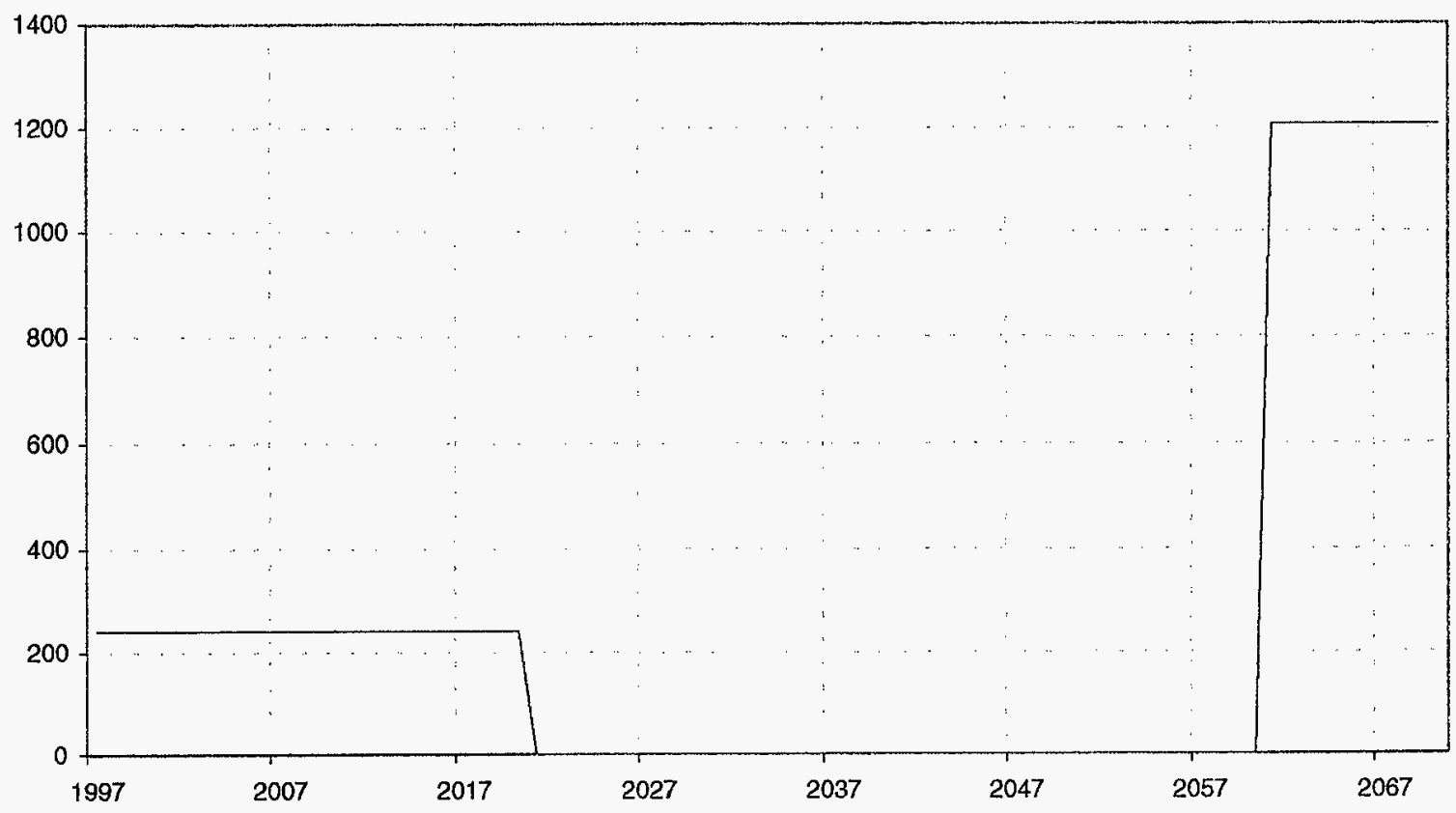

SHIPMENTS RECEIVED CWWFA CHART:

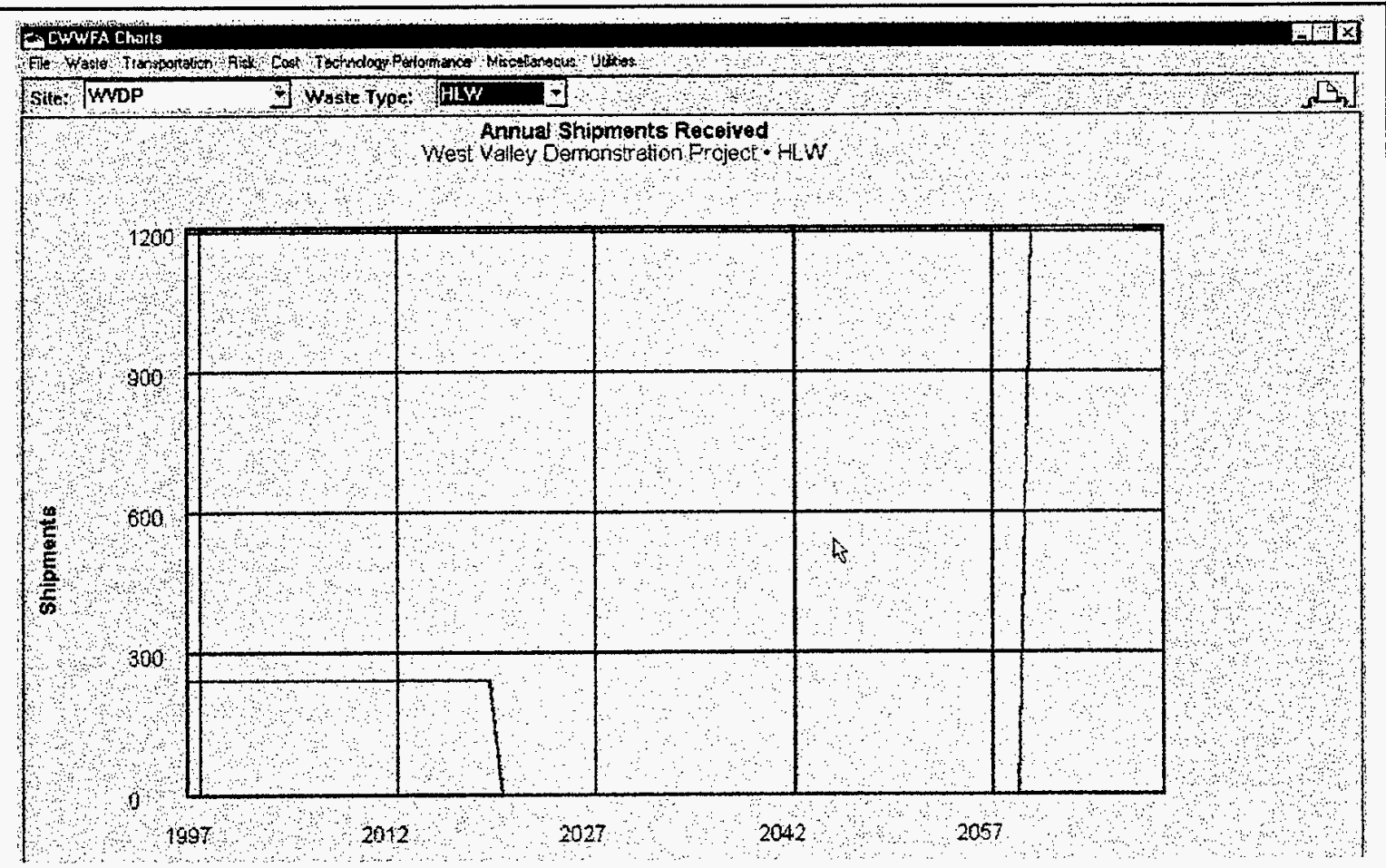


CASE: Case 2e

DATE: $11 / 13 / 97$

Evaluator: K B

Oswald

SHIPMENTS SENT EXCEL CHART (WVDP):

Shipments Sent

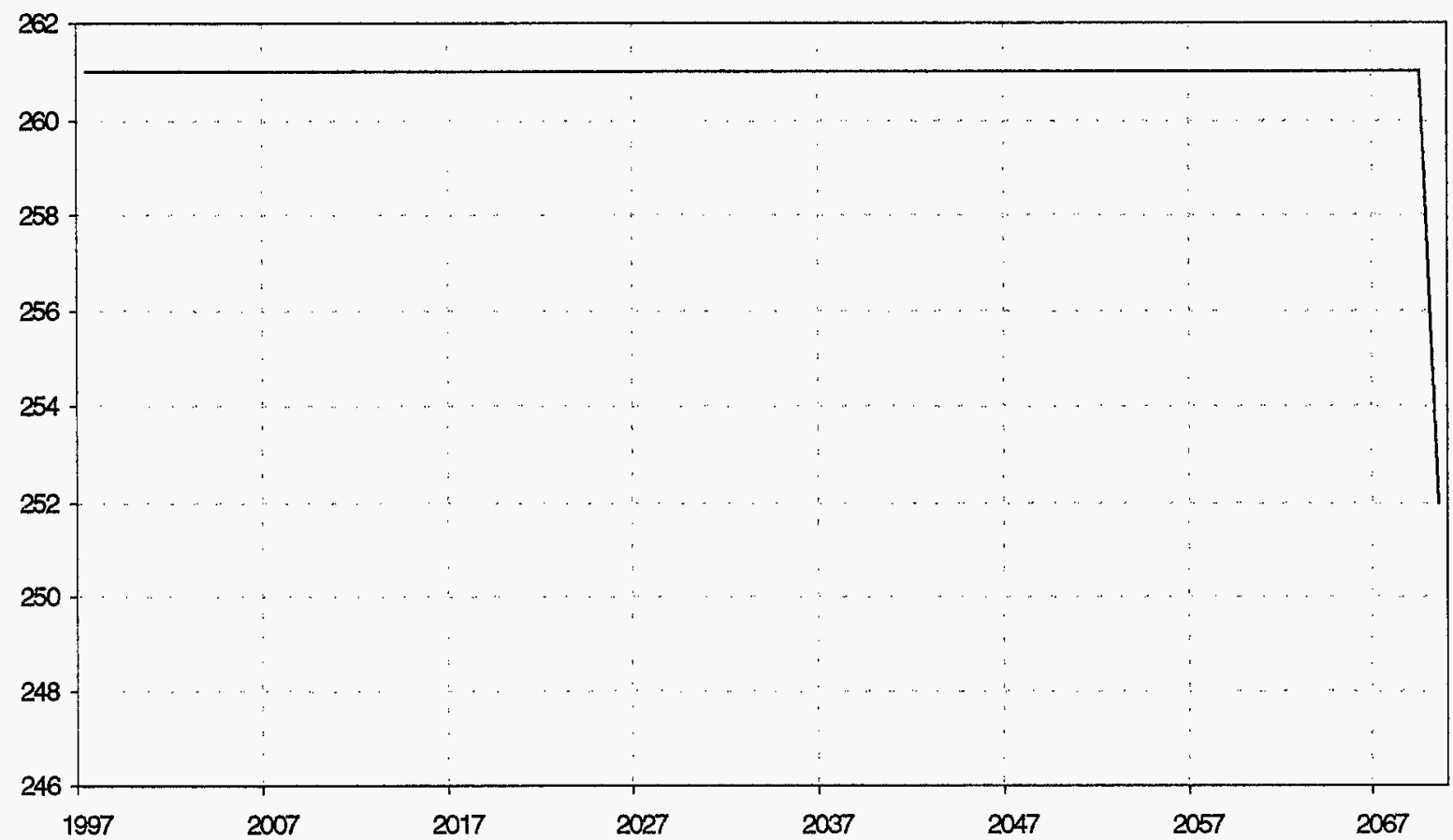

\section{SHIPMENTS SENT CWWFA CHART:}

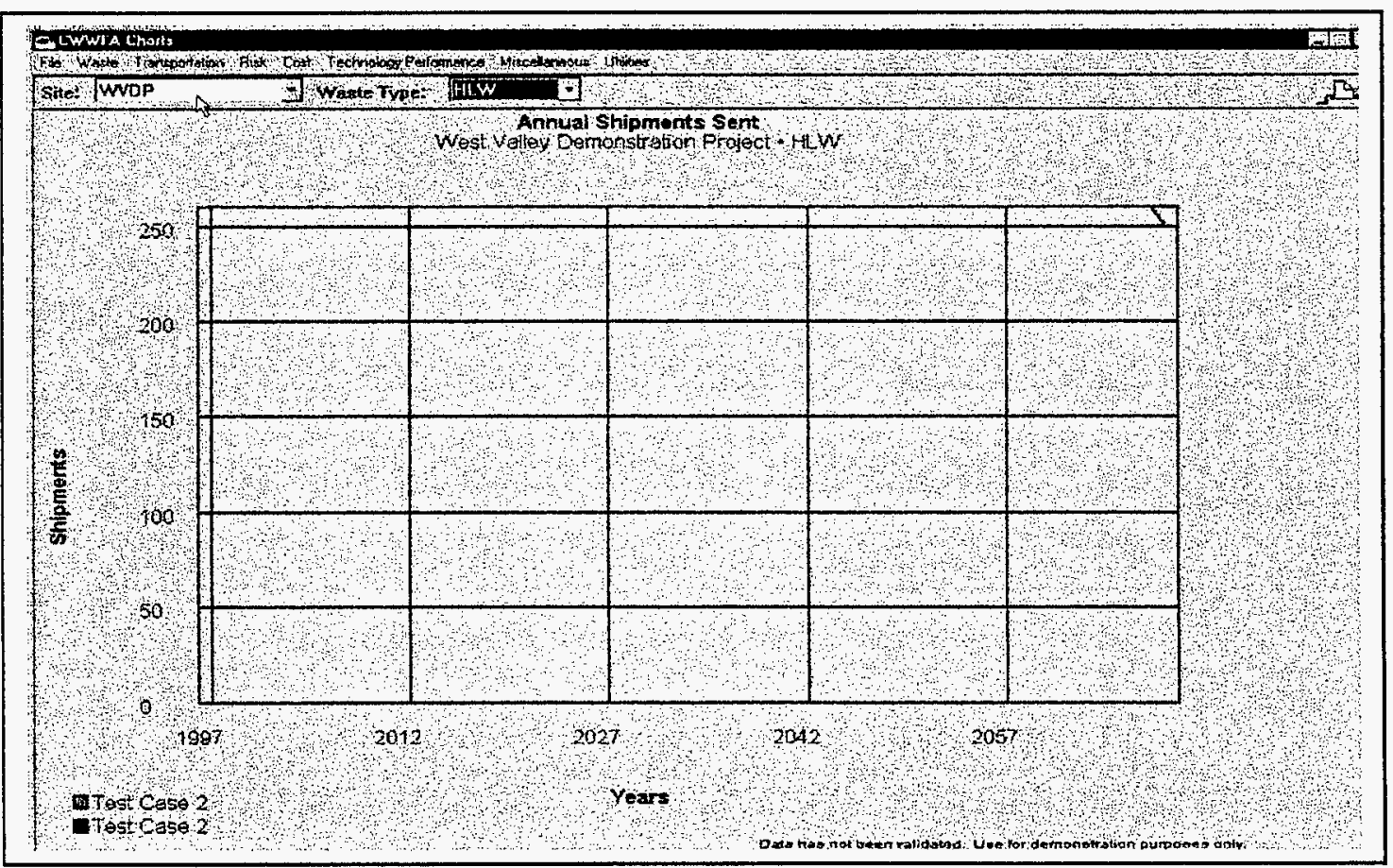


INITIAL PARAMETERS \& RESULTS REPORT
CASE: Case 2e
DATE: $11 / 13 / 97$
Evaluator: K B

Oswald

TRANSPORTATION COMPARISON EXCEL CHART (WVDP, LLW):

TRANSPORTAION COMPARISON

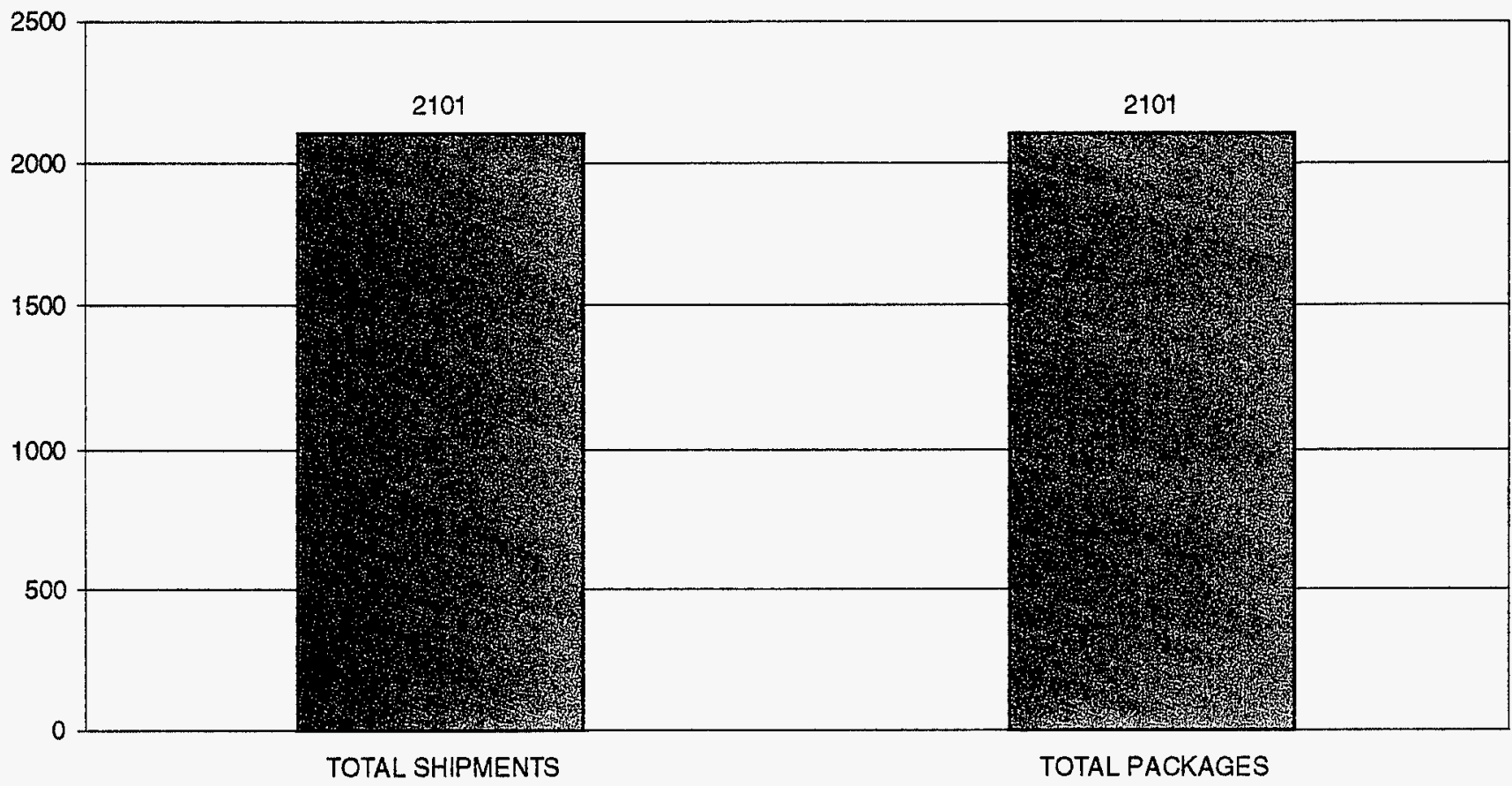

TRANSPORTATION COMPARISON CWWFA CHART:

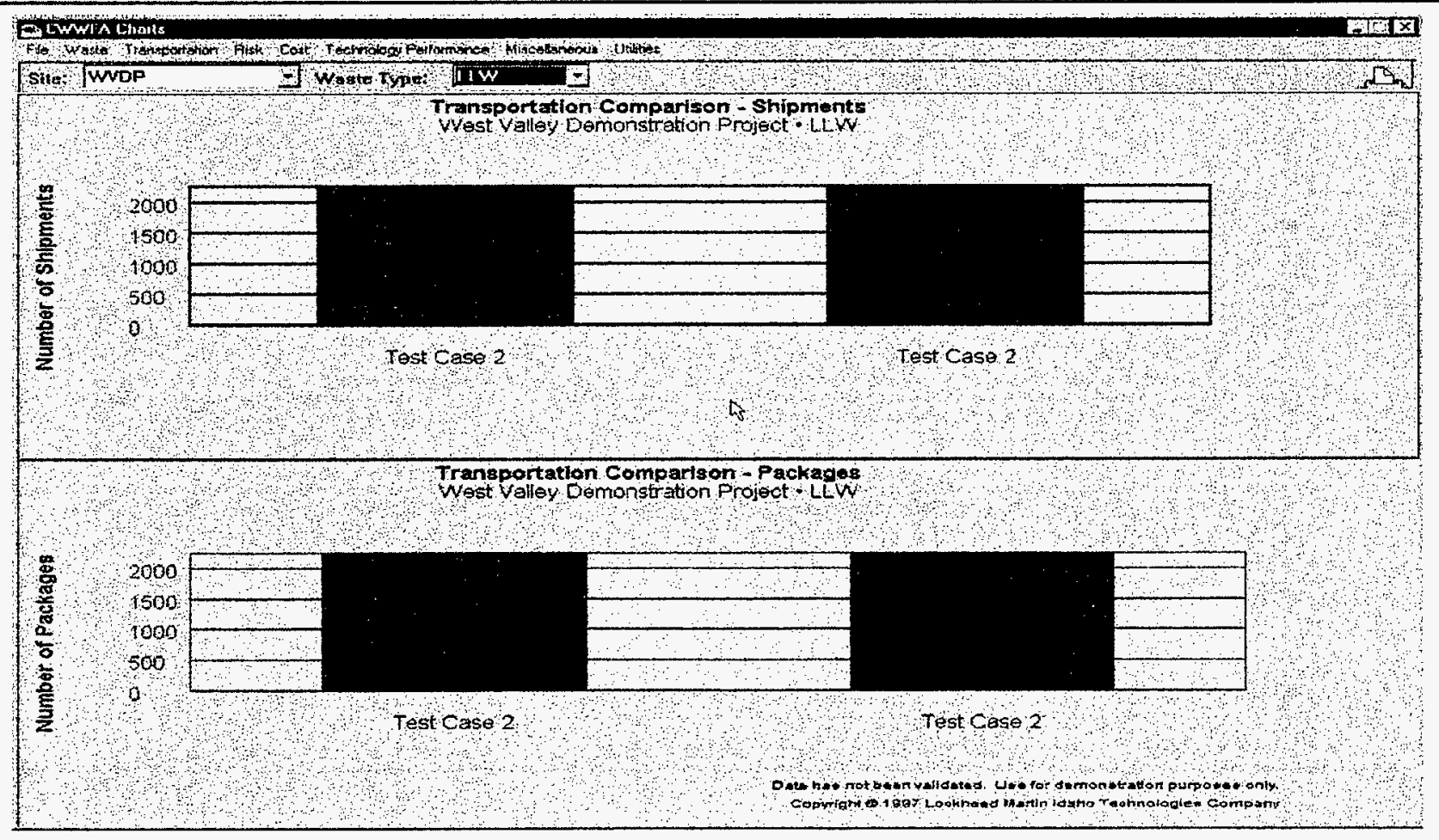


1.0 Waste Stream Information

\begin{tabular}{|l|l|}
\hline Originating Site & LANL \\
\hline Waste Stream ID \& Name & LANLWS1-CASE3 \\
\hline Originating Type & TRU \\
\hline Legacy volume $(\mathrm{m} 3)$ & 20,000 \\
\hline
\end{tabular}

1.1 New Generation

\begin{tabular}{|l|l|l|}
\hline \multicolumn{1}{|c|}{ Start Year } & \multicolumn{1}{|c|}{ End Year } & Annual Volume (m3) \\
\hline NA & NA & NA \\
\hline & & \\
\hline & & \\
\hline & & \\
\hline
\end{tabular}

1.2 Shipping Route(s)

\begin{tabular}{|l|l|l|}
\hline \multicolumn{1}{|c|}{ Route ID } & \multicolumn{1}{|c|}{ Source Facility } & \multicolumn{1}{c|}{ Destination Facility } \\
\hline C3a-1 & Legacy-LANL & RFTSFAC1-CASE3 \\
\hline C3a-2 & Legacy-LANL & LANLFAC1-CASE3 \\
\hline C3a-3 & RFTSFAC1-CASE3 & LANLFAC2-CASE3 \\
\hline C3a-4 & LANLFAC1-CASE3 & RFTSFAC2-CASE3 \\
\hline & & \\
\hline & & \\
\hline
\end{tabular}

1.3 Shipping Schedule(s)

\begin{tabular}{|c|c|c|c|}
\hline Route ID: C3a-1 & & & \\
\hline Start Year & End Year & Waste Type & Annual Volume (m3) \\
\hline 1997 & 2070 & TRU & 135 \\
\hline & & & \\
\hline & & & \\
\hline
\end{tabular}

1.3 Shipping Schedule(s) (continued)

\begin{tabular}{|c|l|l|l|}
\hline Route ID: C3a-2 & \multicolumn{1}{|c|}{ Waste Type } & Annual Volume (m3) \\
\hline Start Year & \multicolumn{1}{|c|}{ End Year } & 135 \\
\hline & 2070 & TRU & \\
\hline & & & \\
\hline & & & \\
\hline
\end{tabular}

1.3 Shipping Schedule(s) (continued)

\begin{tabular}{|c|l|l|l|}
\hline Route ID: C3a-3 & \multicolumn{2}{|c|}{ Waste Type } & \multicolumn{1}{c|}{ Annual Volume (m3) } \\
\hline Start Year & \multicolumn{1}{|c|}{ End Year } & 135 \\
\hline & 2070 & TRU & \\
\hline & & & \\
\hline & & & \\
\hline
\end{tabular}

1.3 Shipping Schedule(s) (continued)

\begin{tabular}{|c|c|c|c|}
\hline Route ID: C3a-4 & \multicolumn{2}{|c|}{} \\
\hline Start Year & \multicolumn{1}{|c|}{ End Year } & Waste Type & Annual Volume (m3) \\
\hline 1997 & 2070 & TRU & 135 \\
\hline & & & \\
\hline & & & \\
\hline & & & \\
\hline
\end{tabular}




\section{INITIAL PARAMETERS \& RESULTS REPORT}

CASE: Case 3a

DATE: $11 / 13 / 97$

Evaluator: K B Oswald

2.0 Facility Information

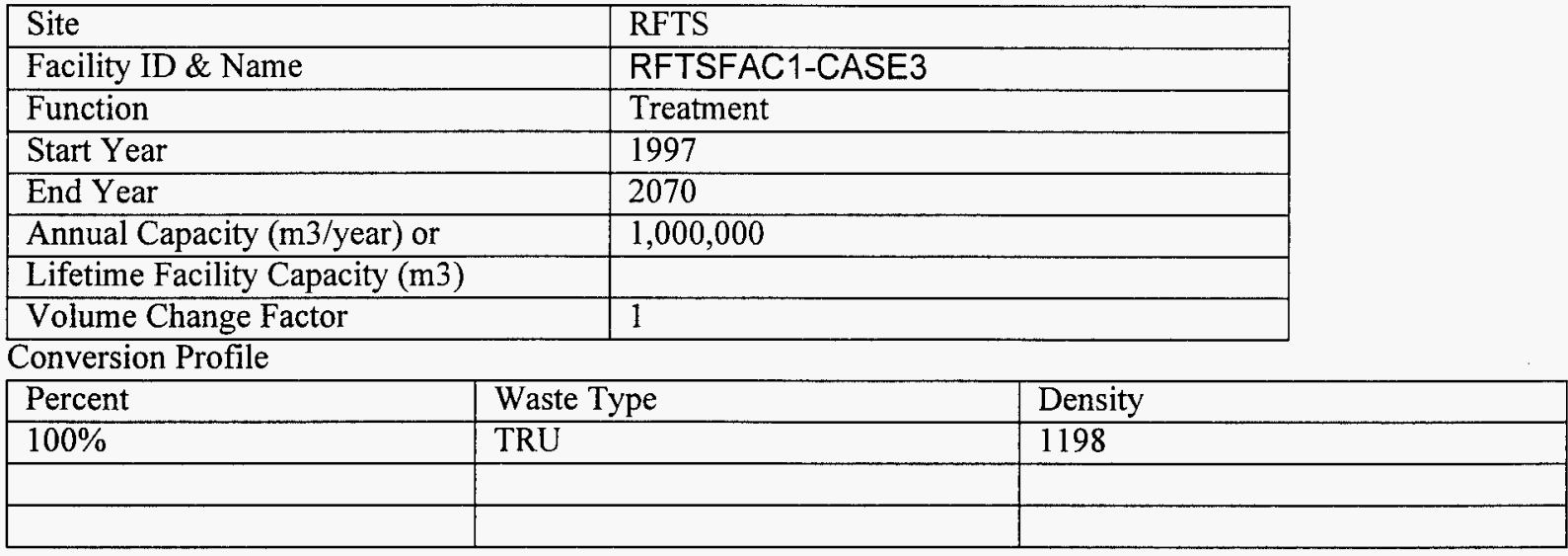

2.0 Facility Information

\begin{tabular}{|c|c|c|c|}
\hline \multicolumn{2}{|l|}{ Site } & \multicolumn{2}{|l|}{ RFTS } \\
\hline \multicolumn{2}{|l|}{ Facility ID \& Name } & \multicolumn{2}{|l|}{ RFTSFAC2-CASE3 } \\
\hline \multicolumn{2}{|l|}{ Function } & \multicolumn{2}{|l|}{ Disposal } \\
\hline \multicolumn{2}{|l|}{ Start Year } & \multicolumn{2}{|l|}{1997} \\
\hline \multicolumn{2}{|l|}{ End Year } & \multicolumn{2}{|c|}{2070} \\
\hline \multicolumn{2}{|l|}{ Annual Capacity (m3/year) or } & \multicolumn{2}{|l|}{$1,000,000$} \\
\hline \multicolumn{4}{|l|}{ Lifetime Facility Capacity (m3) } \\
\hline Volume Change Factor & & \multicolumn{2}{|c|}{1} \\
\hline \multicolumn{4}{|l|}{ Conversion Profile } \\
\hline Percent & Waste Typ & & Density \\
\hline NA & NA & & NA \\
\hline
\end{tabular}

2.0 Facility Information

\begin{tabular}{|l|l|}
\hline Site & LANL \\
\hline Facility ID \& Name & LANLFAC1-CASE3 \\
\hline Function & Treatment \\
\hline Start Year & 1997 \\
\hline End Year & 2070 \\
\hline Annual Capacity (m3/year) or & $1,000,000$ \\
\hline Lifetime Facility Capacity (m3) & \\
\hline Volume Change Factor & 1 \\
\hline \\
\begin{tabular}{|l|l|l|}
\hline Ponversion Profile & Waste Type & Density \\
\hline 100 & TRU & 1198 \\
\hline & & \\
\hline
\end{tabular}
\end{tabular}

2.0 Facility Information

\begin{tabular}{|l|l|}
\hline Site & LANL \\
\hline Facility ID \& Name & LANLFAC2-CASE3 \\
\hline Function & Disposal \\
\hline Start Year & 1997 \\
\hline End Year & 2070 \\
\hline Annual Capacity (m3/year) or & $1,000,000$ \\
\hline Lifetime Facility Capacity (m3) & \\
\hline Volume Change Factor & 1 \\
\hline \\
\begin{tabular}{|l|l|l|}
\hline Ponversion Profile & Waste Type & Density \\
\hline NA & NA & NA \\
\hline & & \\
\hline
\end{tabular}
\end{tabular}


WASTE DISPOSITION - EXCEL CHART (LANL, TRU):

\section{Waste Disposition}

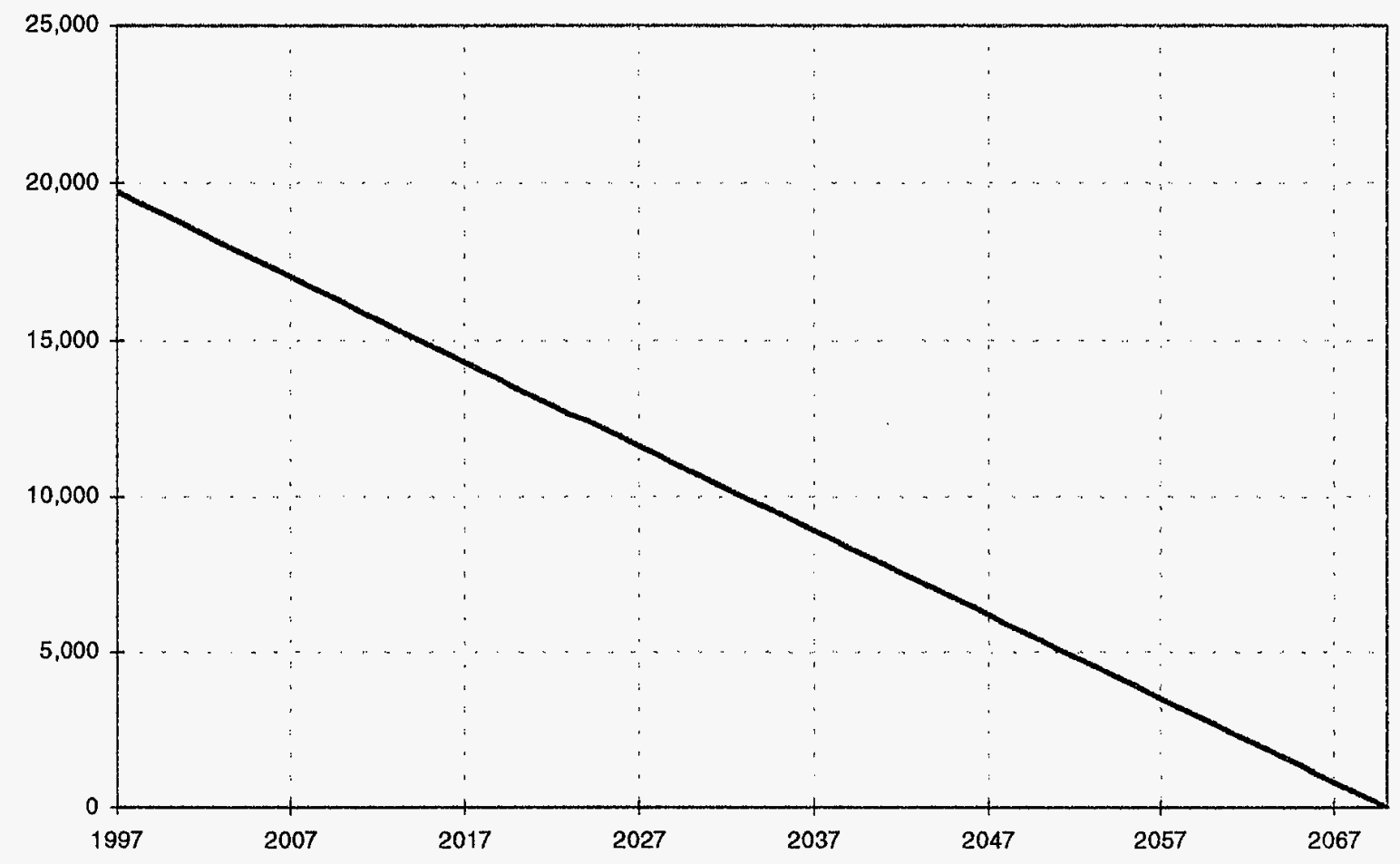

\section{WASTE DISPOSITION - CWWFA CHART:}

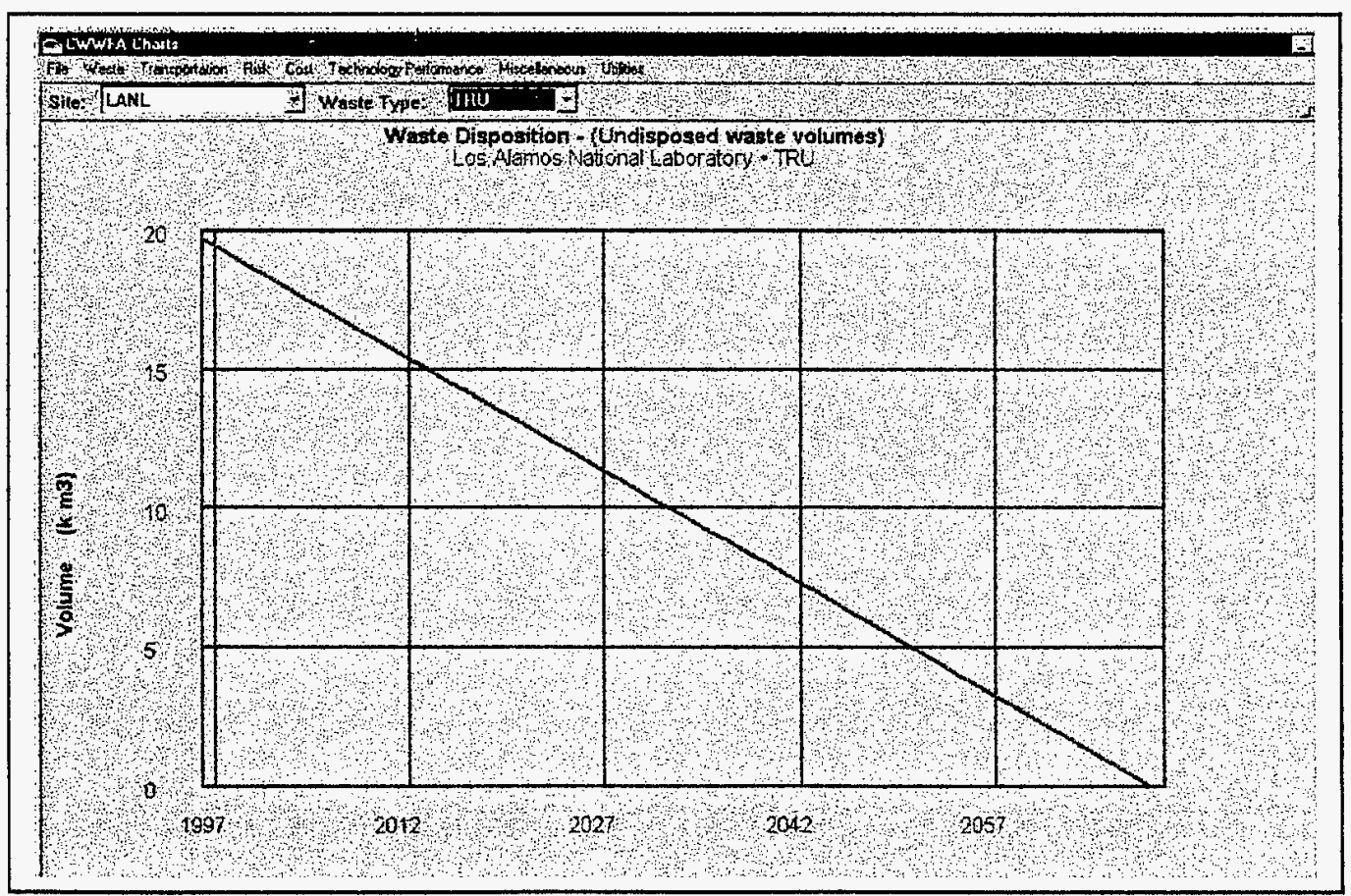




\section{FACILITY CAPACITY EXCEL CHART (RFTS, RFTSFAC2-CASE3):}

\section{Facility Capacity Detail}

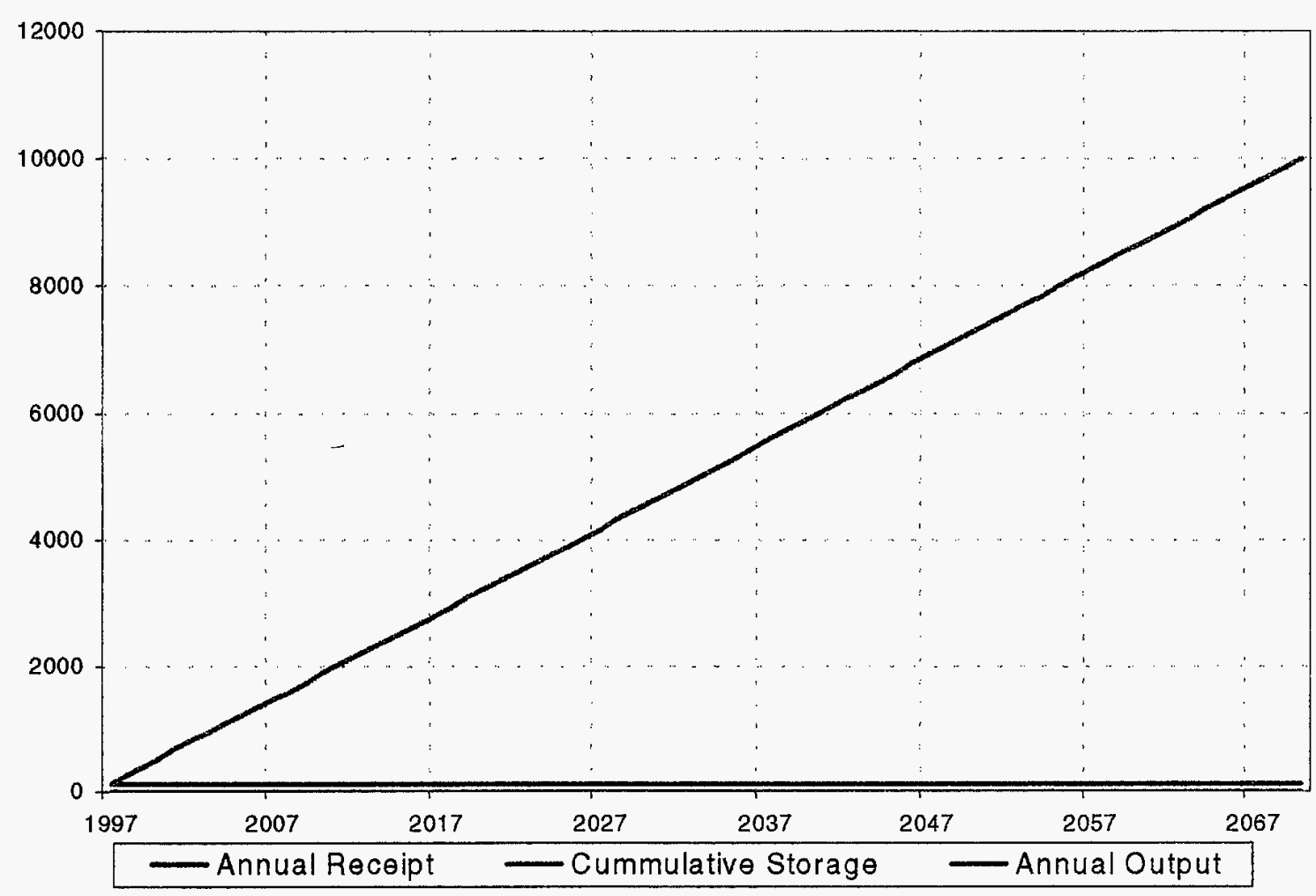

FACILITY CAPACITY CWWFA CHART:

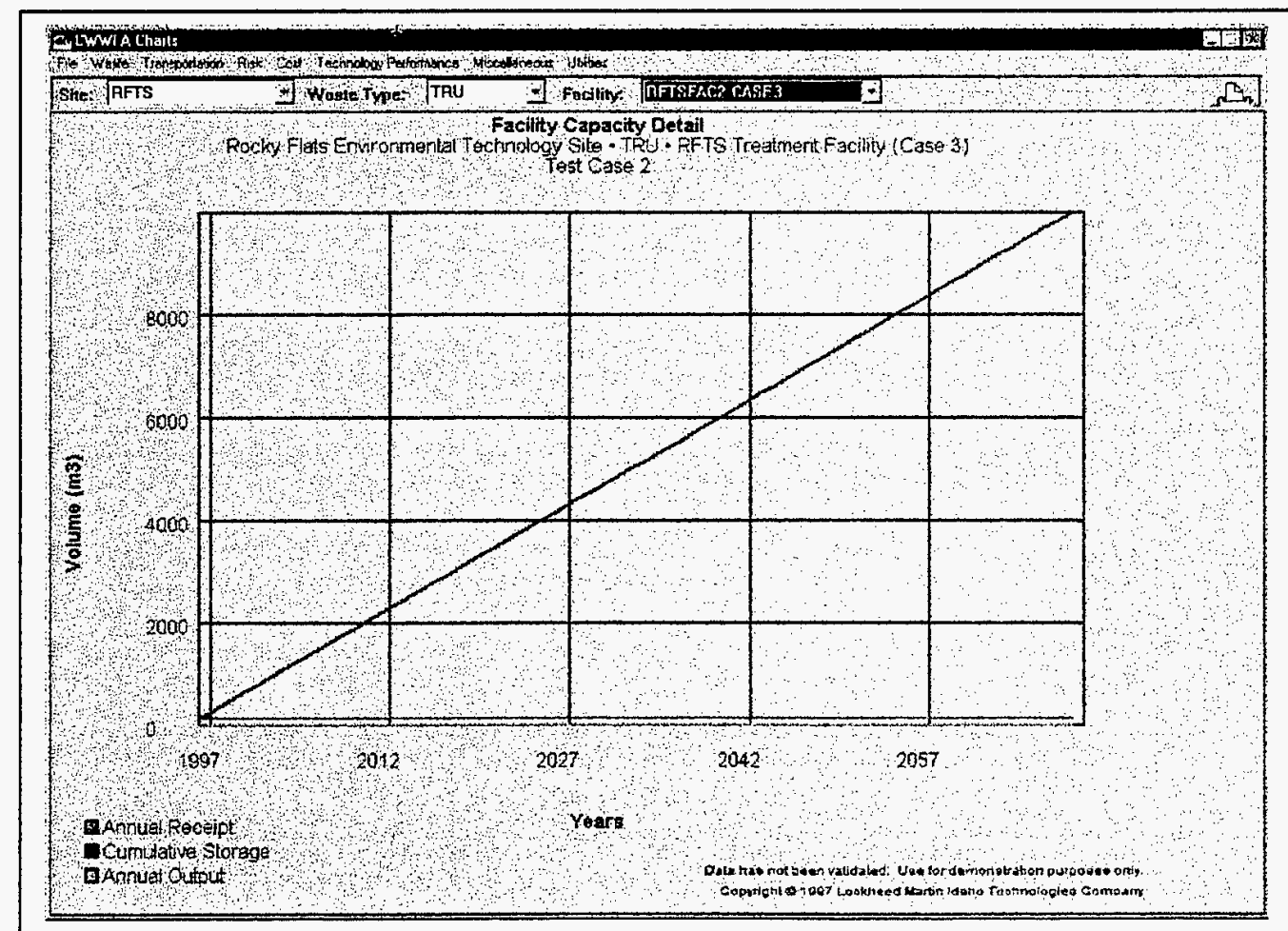


CASE: Case $3 \mathrm{a}$

DATE: $11 / 13 / 97$

Evaluator: K B Oswald

WASTE STREAM DETAIL EXCEL CHART (LANLWS1-CASE3):

\section{Waste Stream Detail}

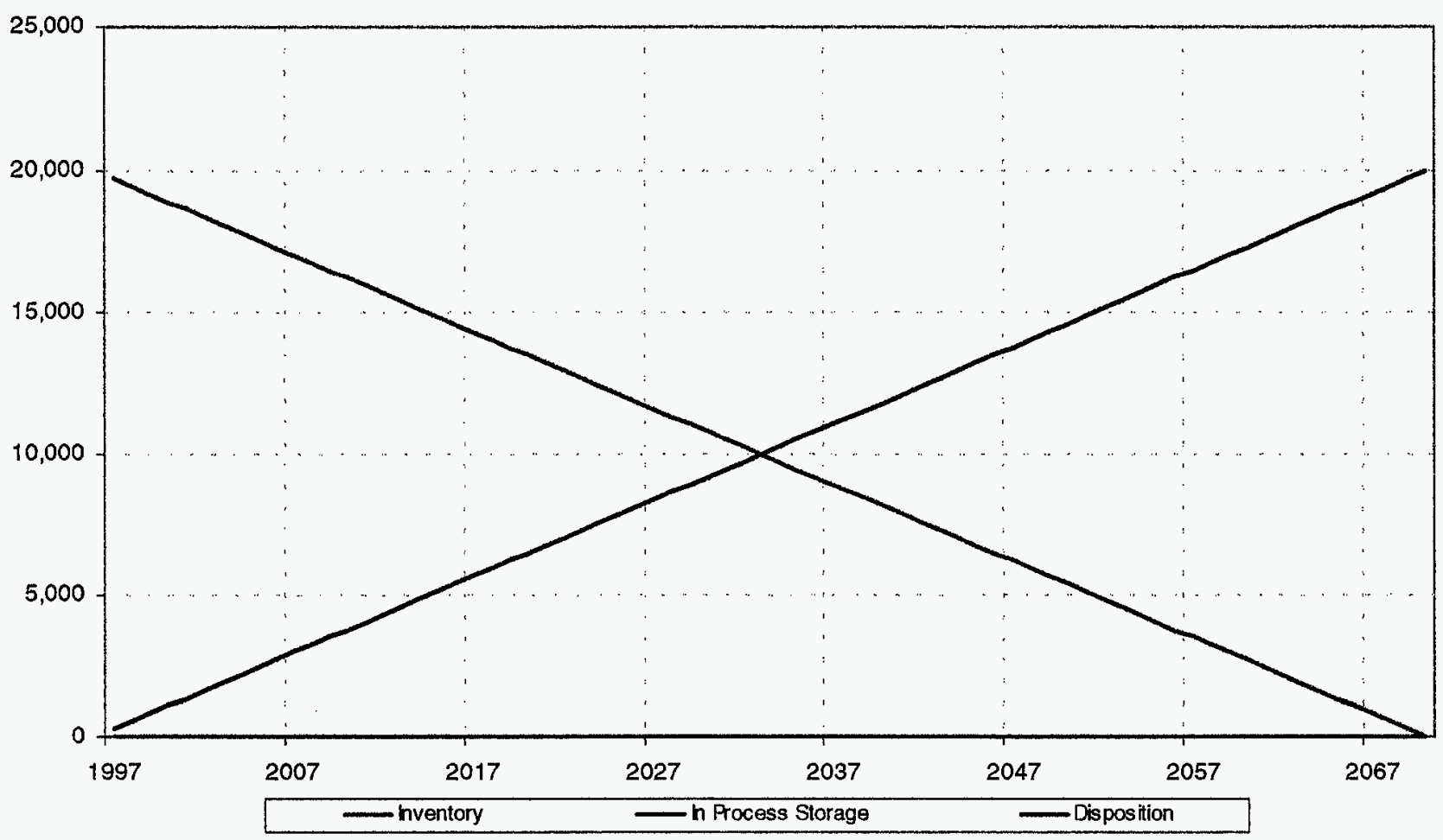

WASTE STREAM DETAIL CWWFA CHART:

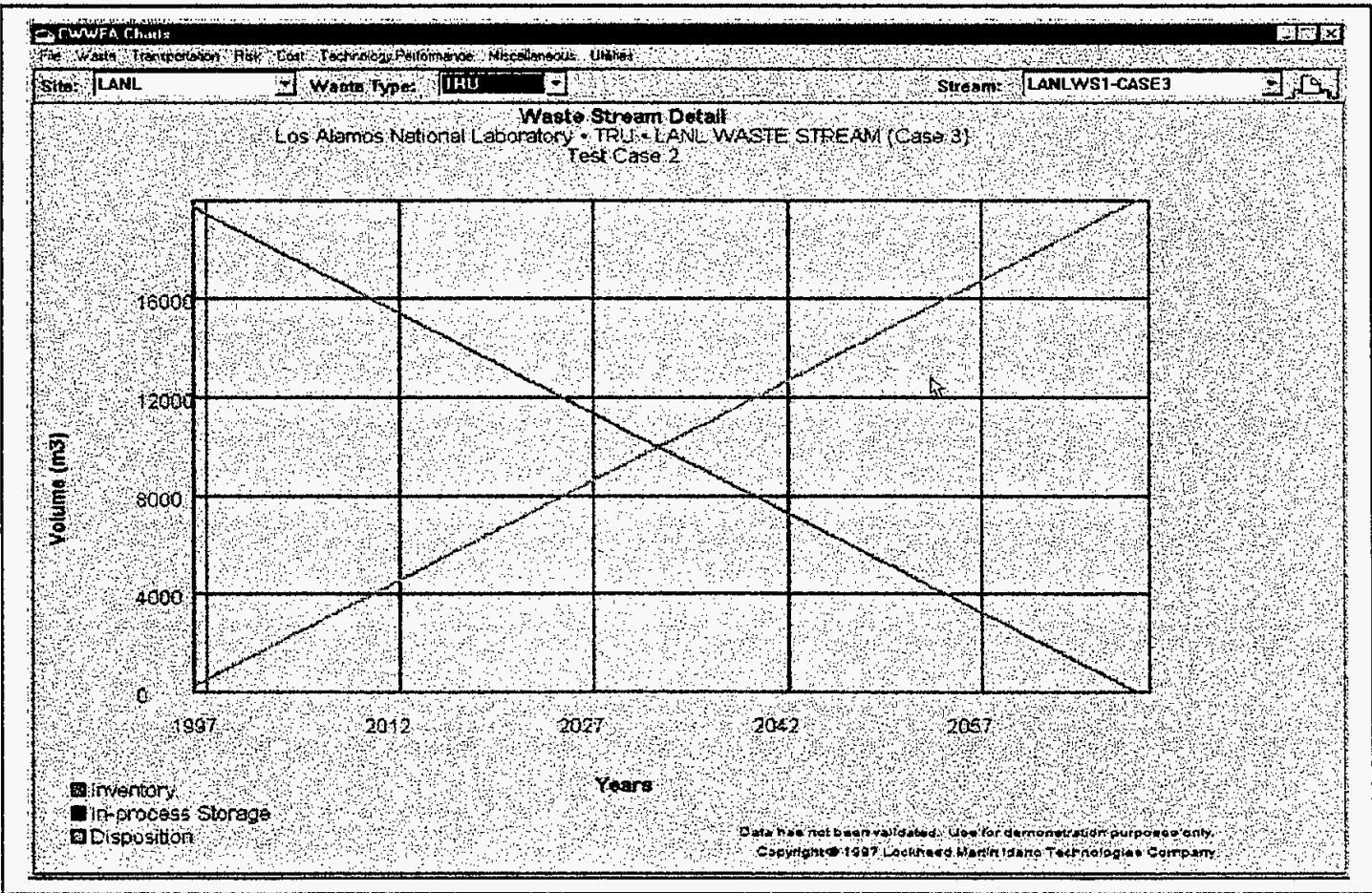


INITIAL PARAMETERS \& RESULTS REPORT
CASE: Case 3a
DATE: $11 / 13 / 97$
Evaluator: K B Oswald

SHIPMENTS RECEIVED EXCEL CHART (LANL):

\section{Shipments Received}

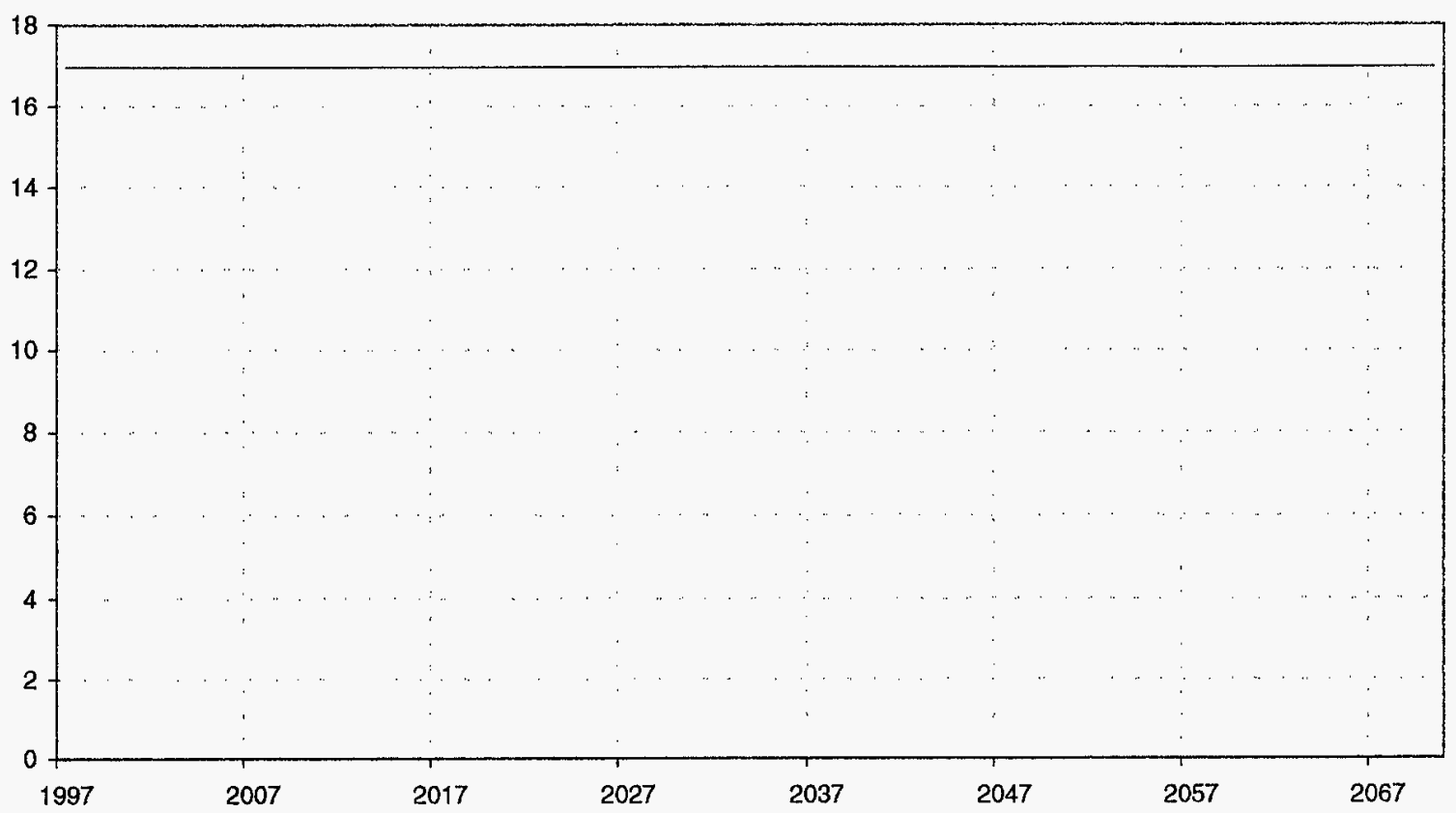

SHIPMENTS RECEIVED CWWFA CHART:

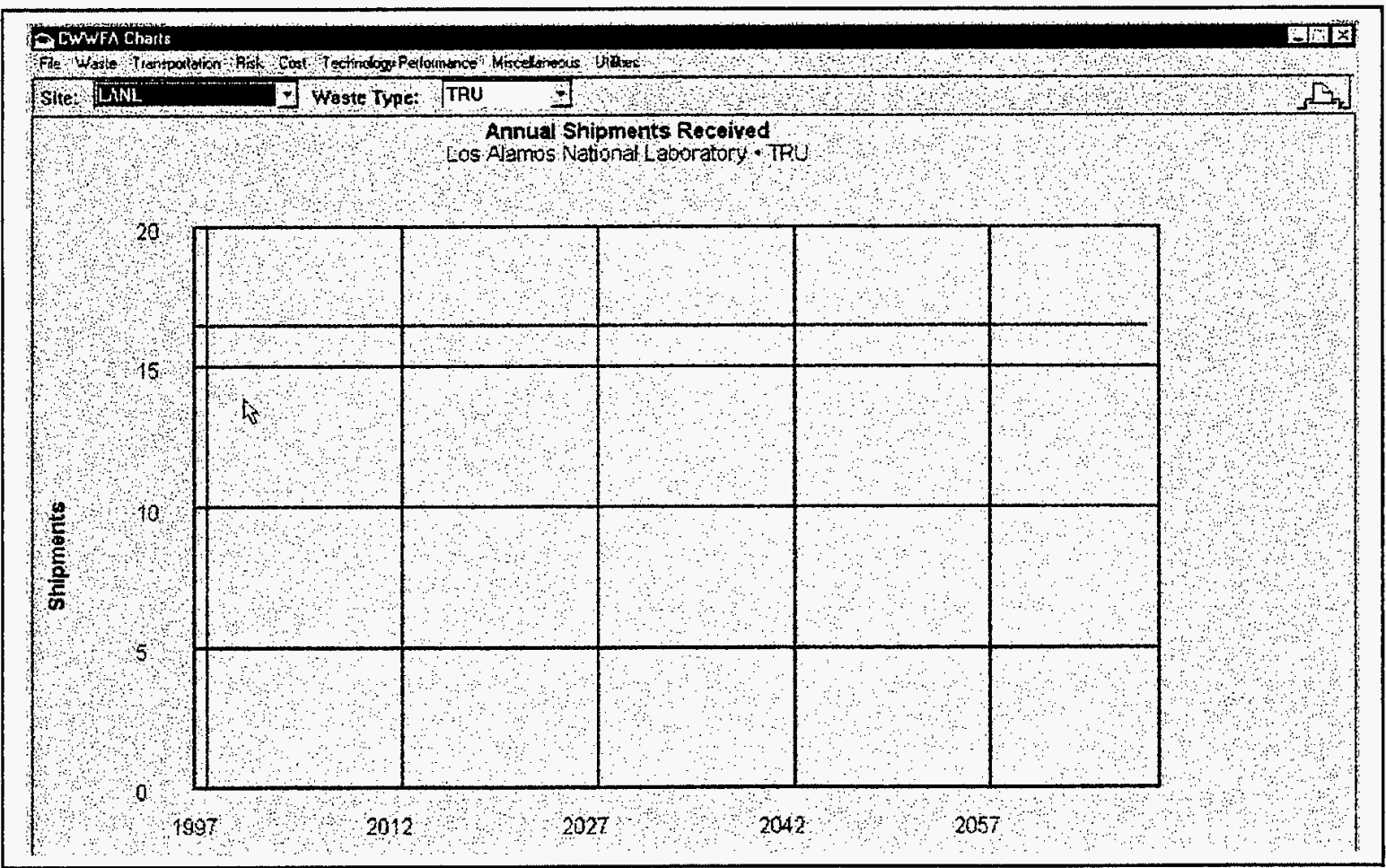


SHIPMENTS SENT EXCEL CHART (LANL):

\section{Shipments Sent}

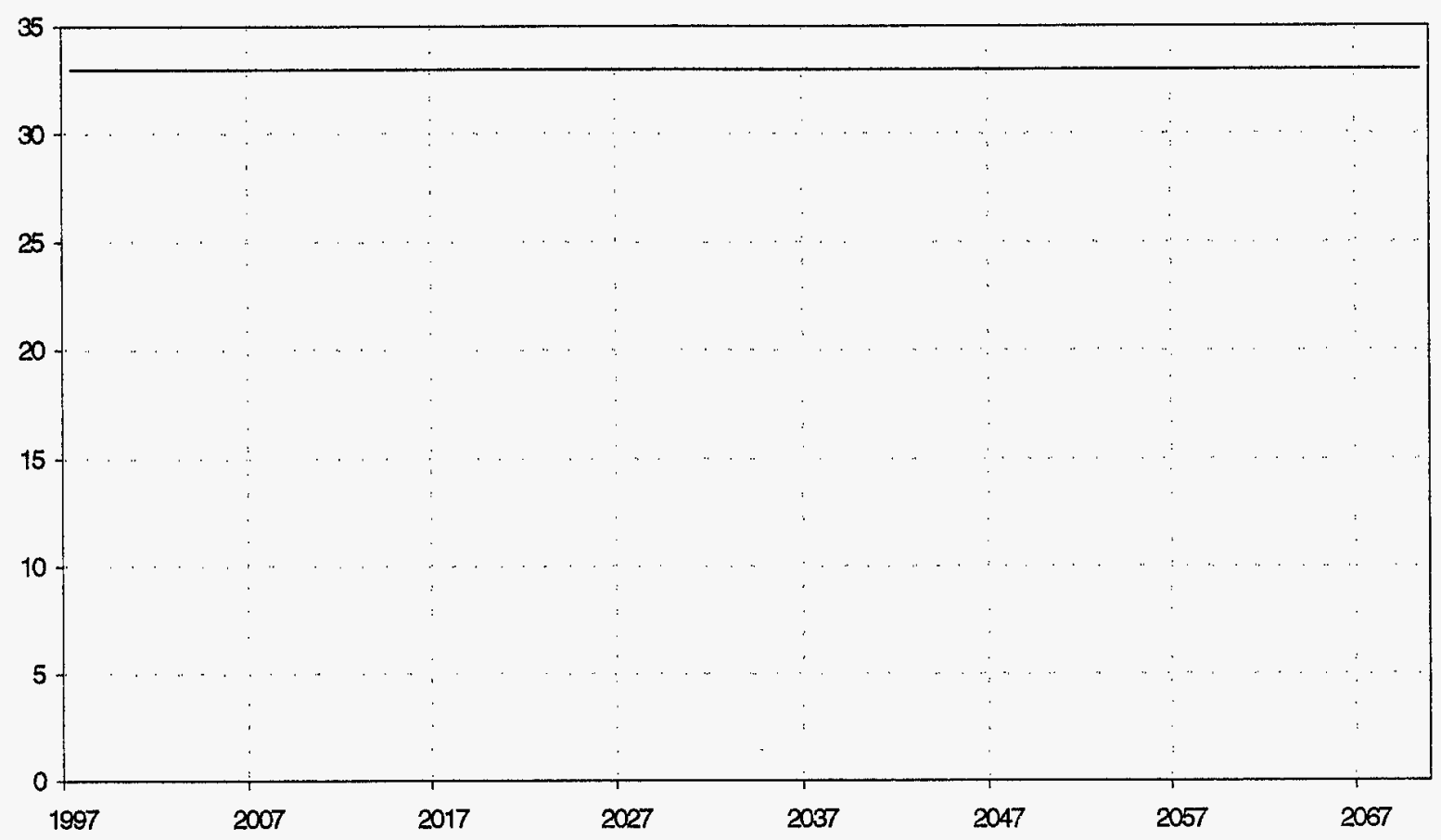

\section{SHIPMENTS SENT CWWFA CHART:}

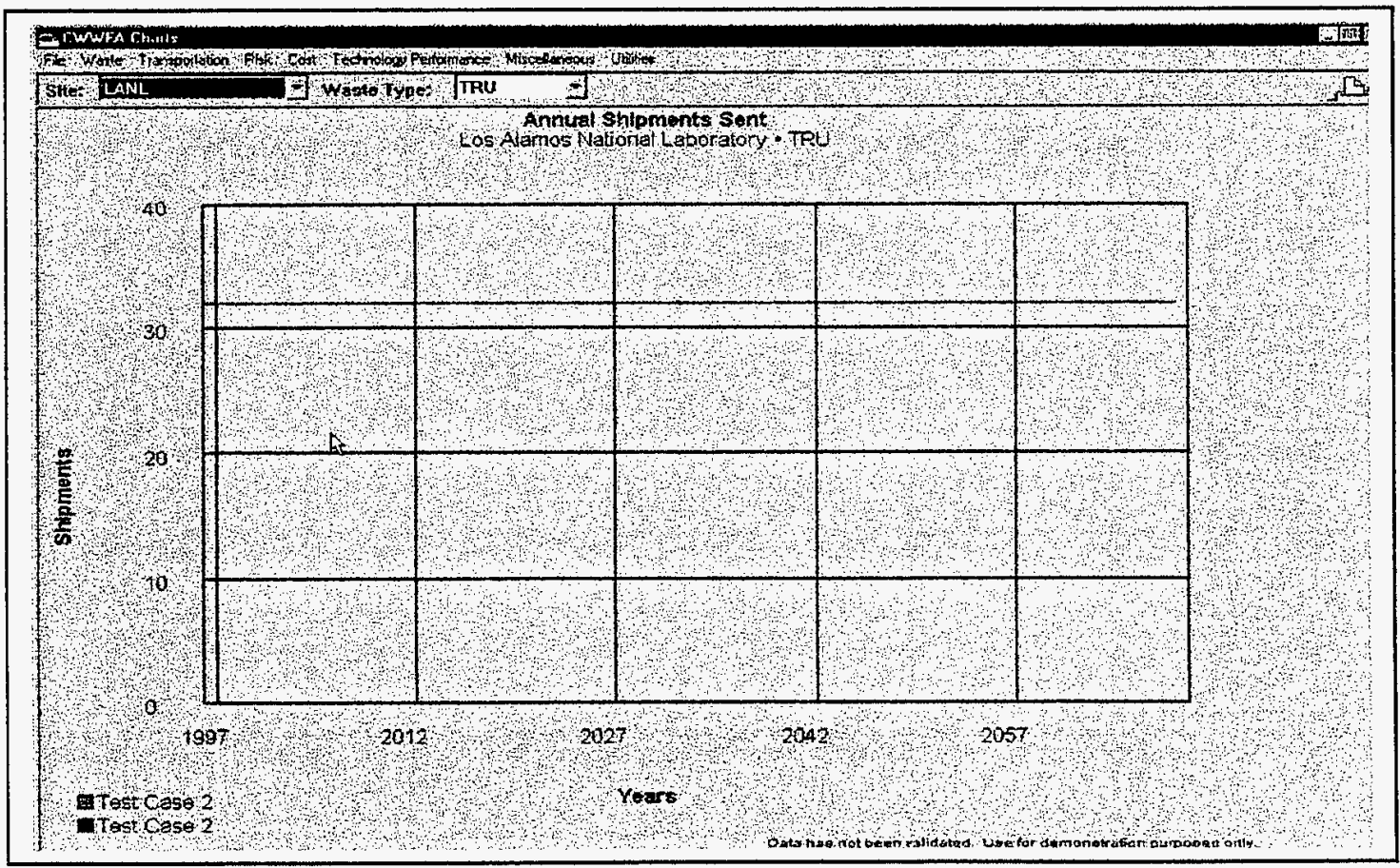


INITIAL PARAMETERS \& RESULTS REPORT

CASE: Case $3 \mathrm{a}$

DATE: $11 / 13 / 97$

Evaluator: K B Oswald

TRANSPORTATION COMPARISON EXCEL CHART (LANL):

TRANSPORTAION COMPARISON

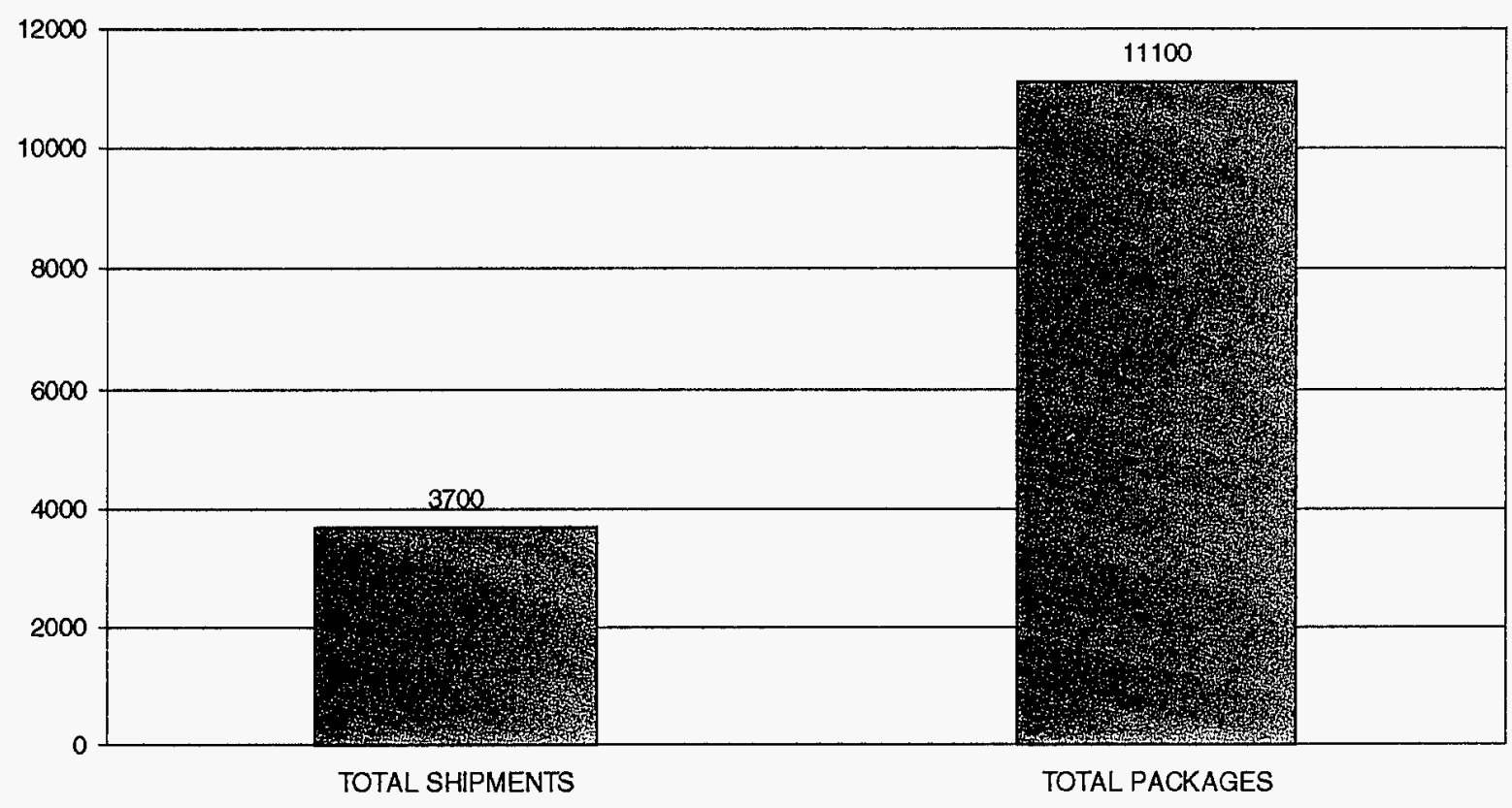

TRANSPORTATION COMPARISON CWWFA CHART:

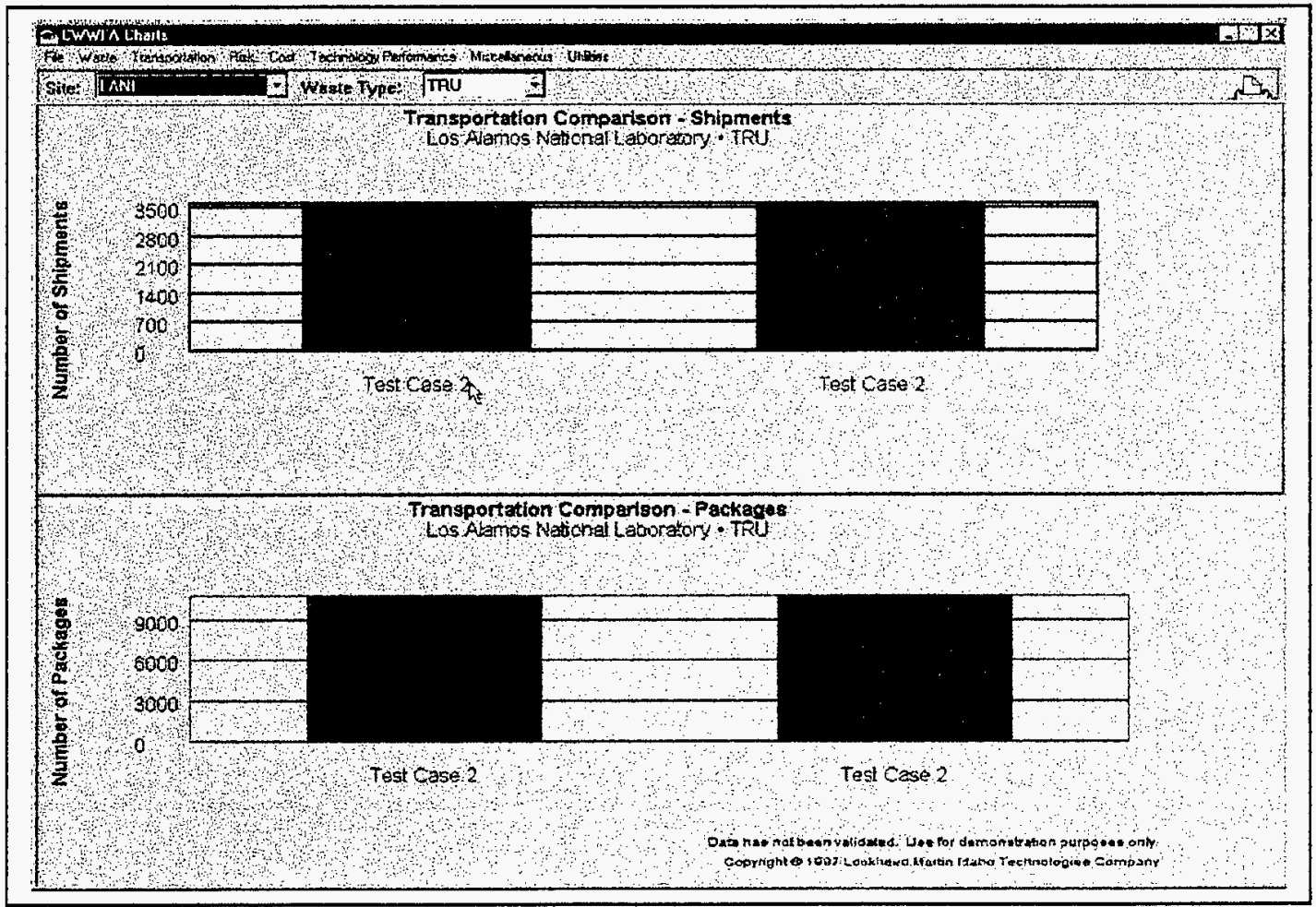




\section{CASE: Case $3 b$}

\section{INITIAL PARAMETERS \& RESULTS REPORT}

1.0 Waste Stream Information

\begin{tabular}{|l|l|}
\hline Originating Site & LANL \\
\hline Waste Stream ID \& Name & LANLWS1-CASE3 \\
\hline Originating Type & TRU \\
\hline Legacy volume (m3) & 20,000 \\
\hline
\end{tabular}

1.1 New Generation

\begin{tabular}{|l|l|l|}
\hline \multicolumn{1}{|c|}{ Start Year } & \multicolumn{1}{|c|}{ End Year } & Annual Volume (m3) \\
\hline NA & NA & NA \\
\hline & & \\
\hline & & \\
\hline & & \\
\hline
\end{tabular}

1.2 Shipping Route(s)

\begin{tabular}{|l|l|l|}
\hline \multicolumn{1}{|c|}{ Route ID } & \multicolumn{1}{|c|}{ Source Facility } & \multicolumn{1}{c|}{ Destination Facility } \\
\hline C3a-1 & Legacy-LANL & RFTSFAC1-CASE3 \\
\hline C3a-2 & Legacy-LANL & LANLFAC1-CASE3 \\
\hline C3a-3 & RFTSFAC1-CASE3 & LANLFAC2-CASE3 \\
\hline C3a-4 & LANLFAC1-CASE3 & RFTSFAC2-CASE3 \\
\hline & & \\
\hline & & \\
\hline
\end{tabular}

1.3 Shipping Schedule(s)

\begin{tabular}{|c|l|l|l|}
\hline Route ID: C3a-1 & \multicolumn{2}{|c|}{ Waste Type } & Annual Volume (m3) \\
\hline Start Year & \multicolumn{1}{|c|}{ End Year } & \multicolumn{1}{|c|}{ W } & 200 \\
\hline 1997 & $2026 \quad$ TRU & 136 \\
\hline 2027 & 2070 & & \\
\hline & & & \\
\hline
\end{tabular}

1.3 Shipping Schedule(s) (continued)

\begin{tabular}{|c|l|l|l|}
\hline Route ID: C3a-2 & \multicolumn{2}{|c|}{ Waste Type } & Annual Volume (m3) \\
\hline Start Year & \multicolumn{1}{|c|}{ End Year } & TRU & 400 \\
\hline 2017 & 2016 & TRU & 200 \\
\hline & 2026 & & \\
\hline & & & \\
\hline
\end{tabular}

1.3 Shipping Schedule(s) (continued)

\begin{tabular}{|c|c|c|c|}
\hline Route ID: C3a-3 & \multicolumn{2}{|c|}{ Waste Type } & Annual Volume (m3) \\
\hline Start Year & \multicolumn{1}{|c|}{ End Year } & TRU & 135 \\
\hline 1997 & 2070 & & \\
\hline & & & \\
\hline & & & \\
\hline
\end{tabular}

1.3 Shipping Schedule(s) (continued)

\begin{tabular}{|c|c|c|c|}
\hline Route ID: C3a-4 & \multicolumn{2}{|c|}{ Waste Type } & Annual Volume (m3) \\
\hline Start Year & \multicolumn{1}{|c|}{ End Year } & 135 \\
\hline 1997 & 2070 & TRU & \\
\hline & & & \\
\hline & & & \\
\hline
\end{tabular}


2.0 Facility Information

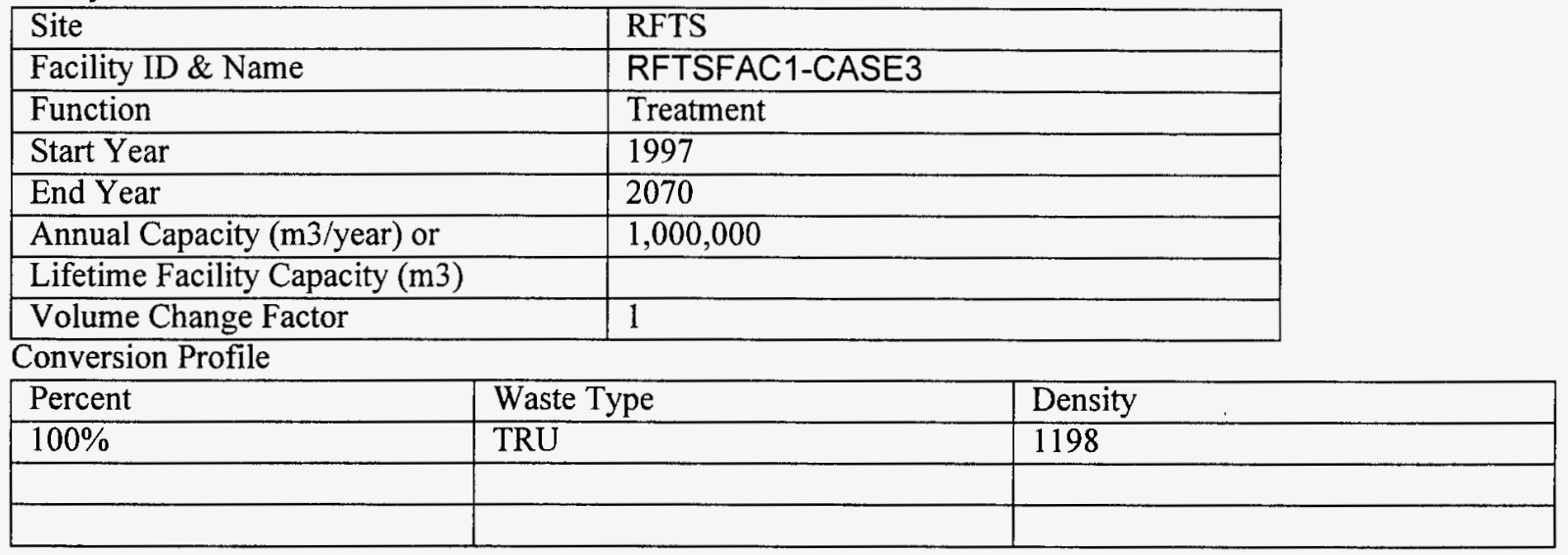

2.0 Facility Information

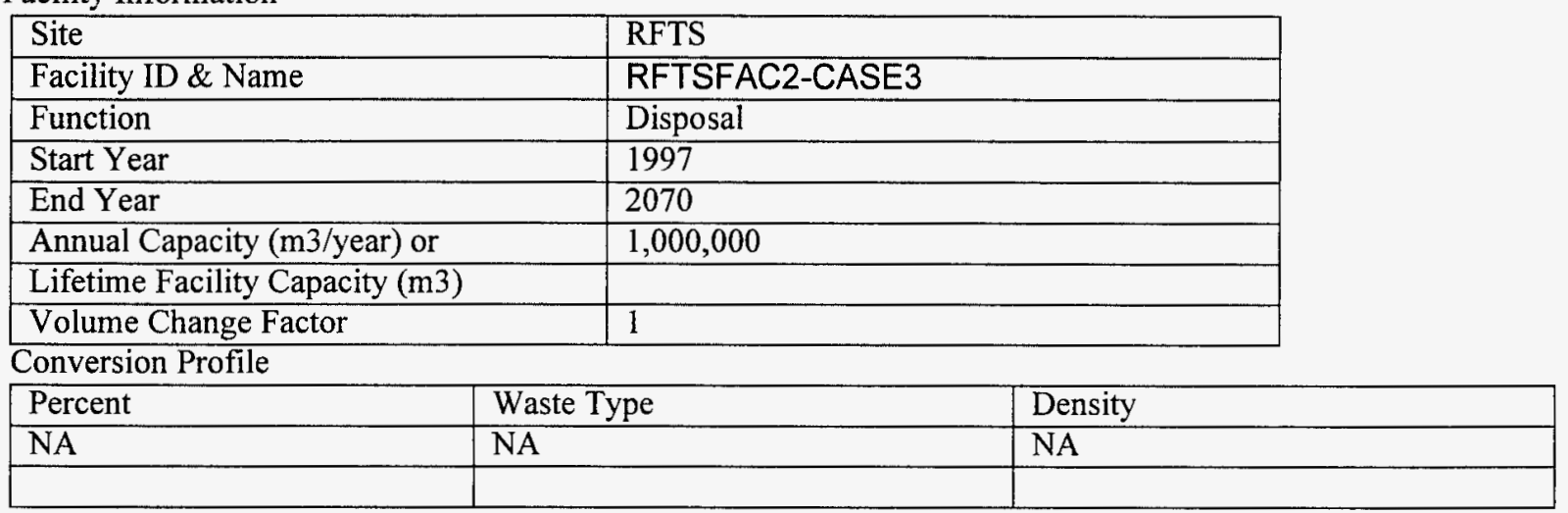

2.0 Facility Information

\begin{tabular}{|l|l|}
\hline Site & LANL \\
\hline Facility ID \& Name & LANLFAC1-CASE3 \\
\hline Function & Treatment \\
\hline Start Year & 1997 \\
\hline End Year & 2070 \\
\hline Annual Capacity (m3/year) or & $1,000,000$ \\
\hline Lifetime Facility Capacity (m3) & \\
\hline Volume Change Factor & 1 \\
\hline
\end{tabular}

Conversion Profile

\begin{tabular}{|l|l|l|}
\hline Percent & Waste Type & Density \\
\hline 100 & TRU & 1198 \\
\hline & & \\
\hline
\end{tabular}

2.0 Facility Information

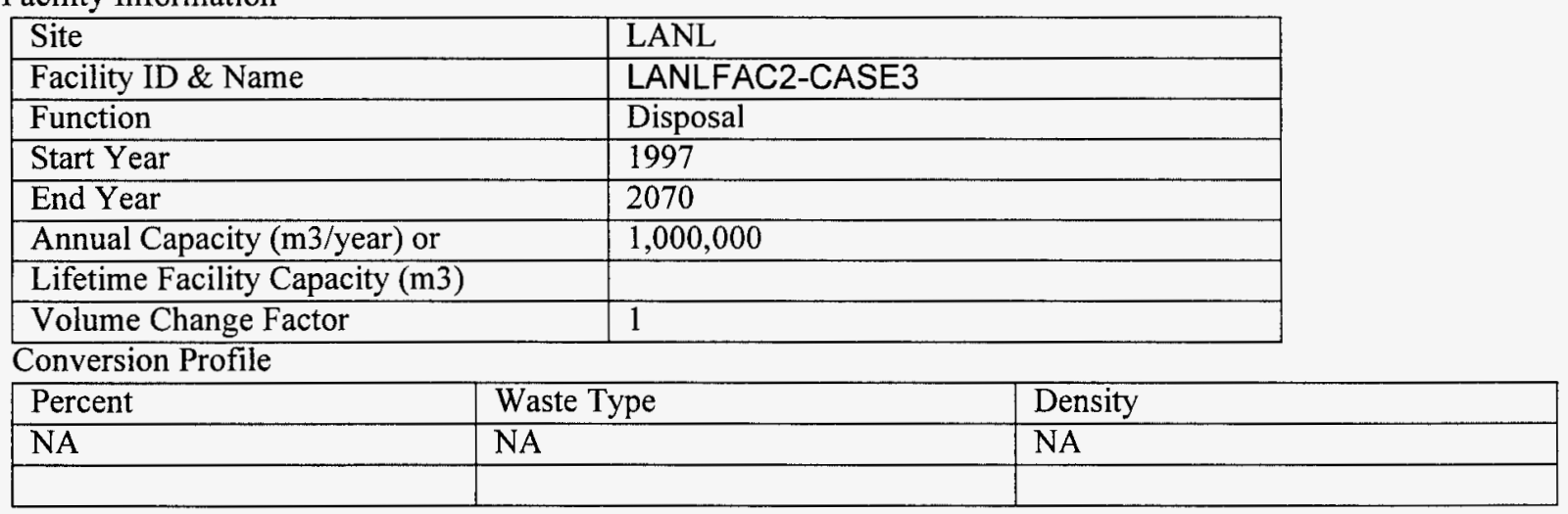


WASTE DISPOSITION - EXCEL CHART (LANL, TRU):

\section{Waste Disposition}

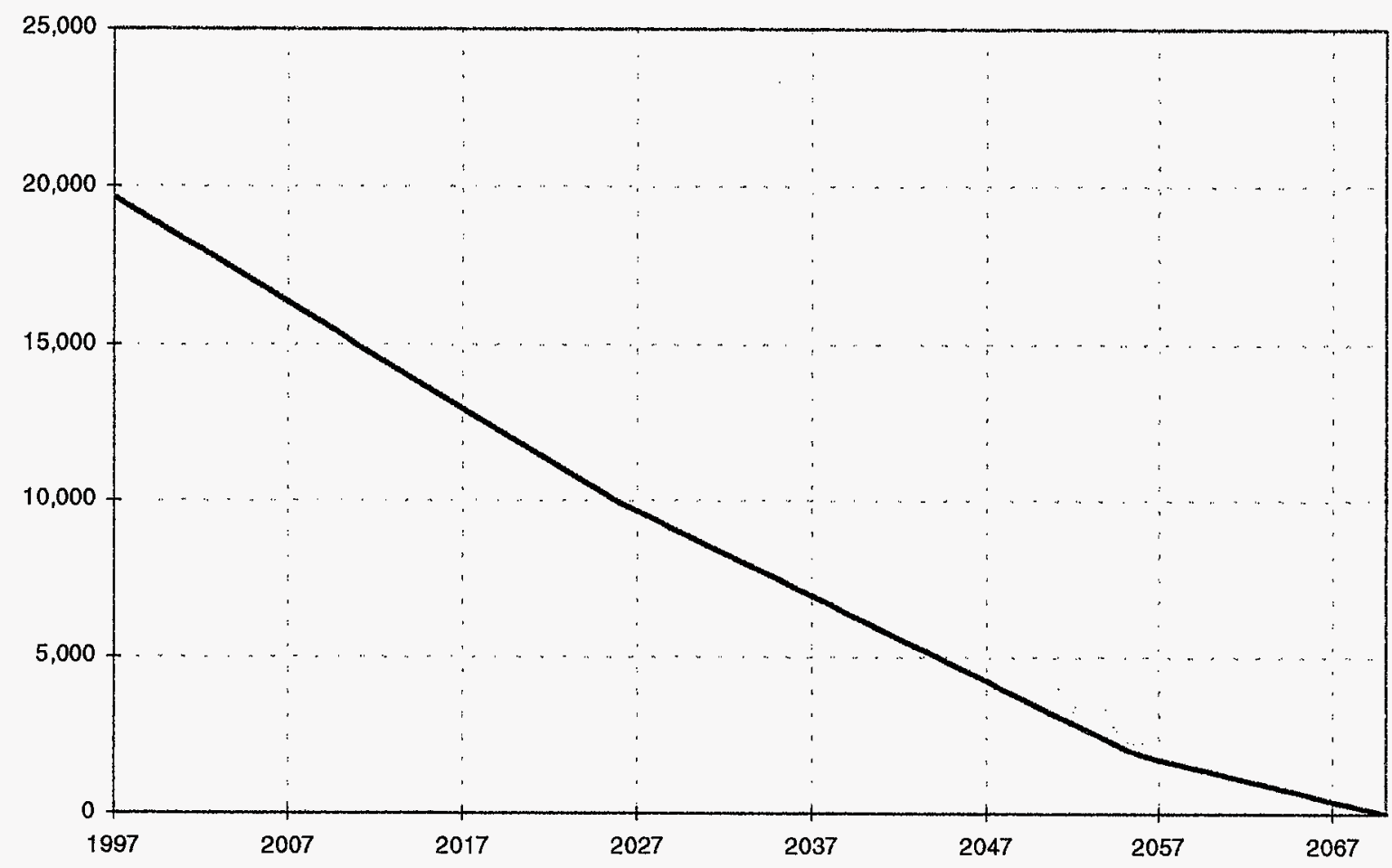

WASTE DISPOSITION - CWWFA CHART:

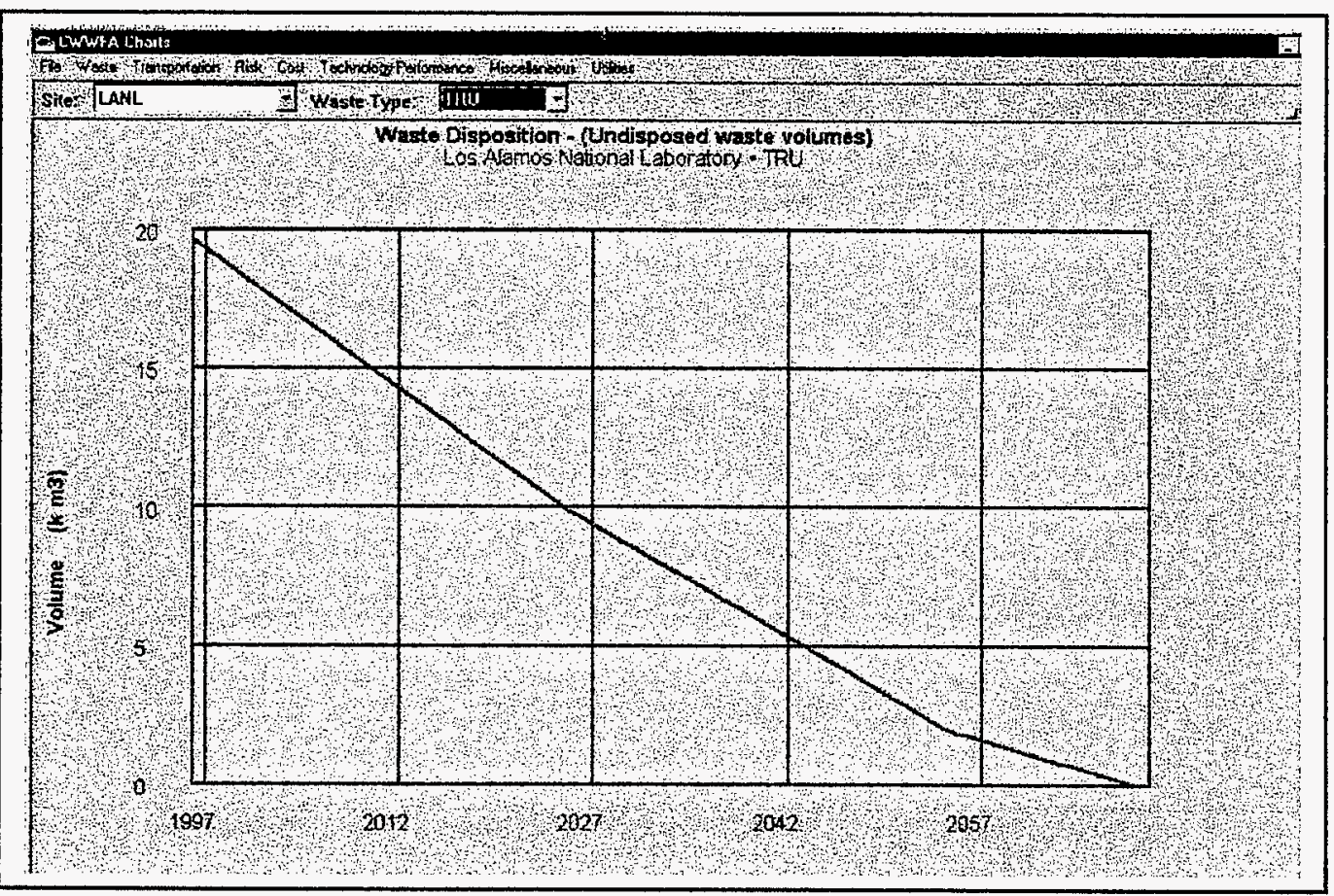




\section{FACILITY CAPACITY EXCEL CHART (RFTS, RFTSFAC2-CASE3):}

\section{Facility Capacity Detail}

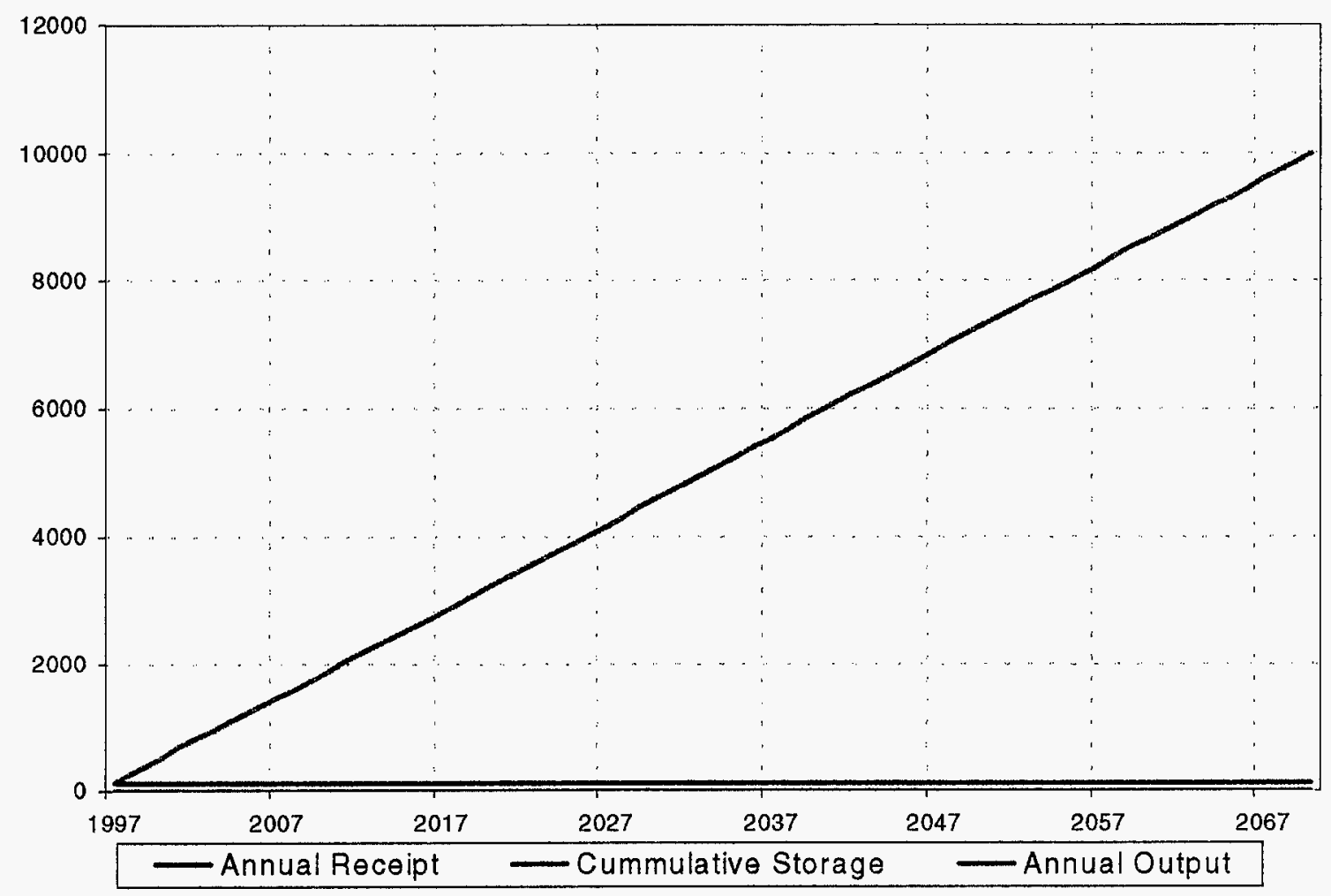

FACILITY CAPACITY CWWFA CHART:

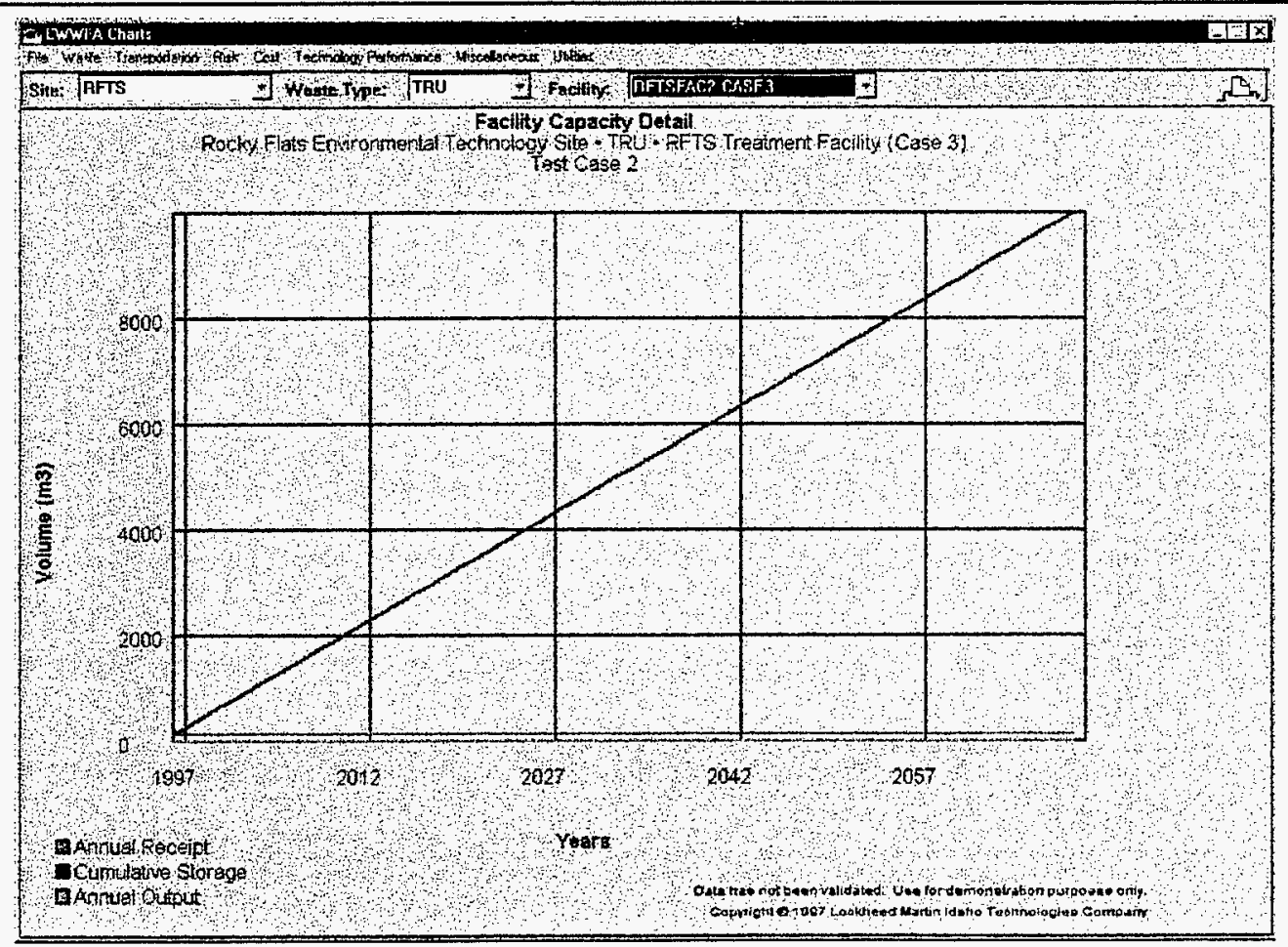


WASTE STREAM DETAIL EXCEL CHART (LANLWS1-CASE3):

\section{Waste Stream Detail}

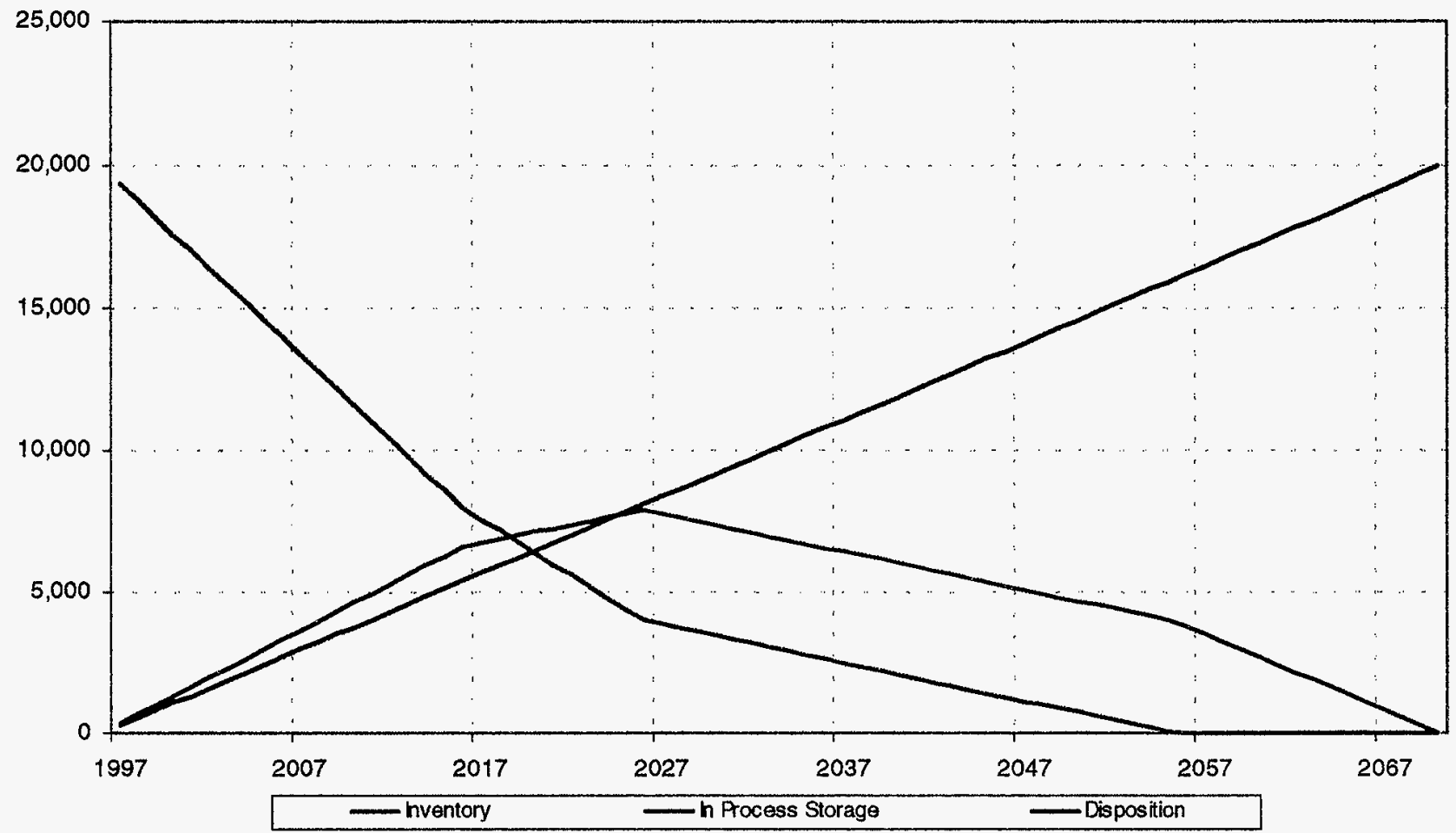

WASTE STREAM DETAIL CWWFA CHART:

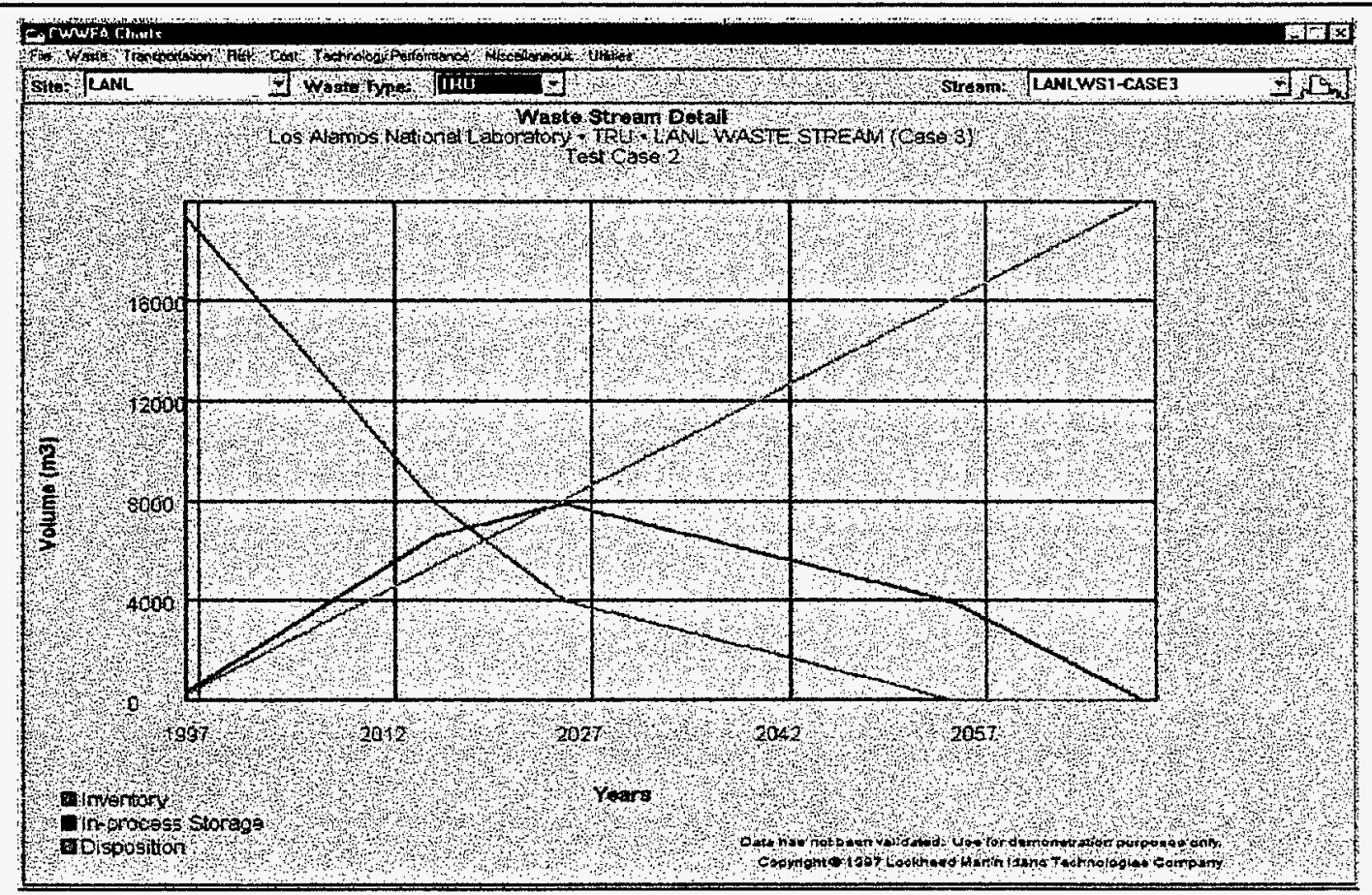


SHIPMENTS RECEIVED EXCEL CHART (LANL):

\section{Shipments Received}

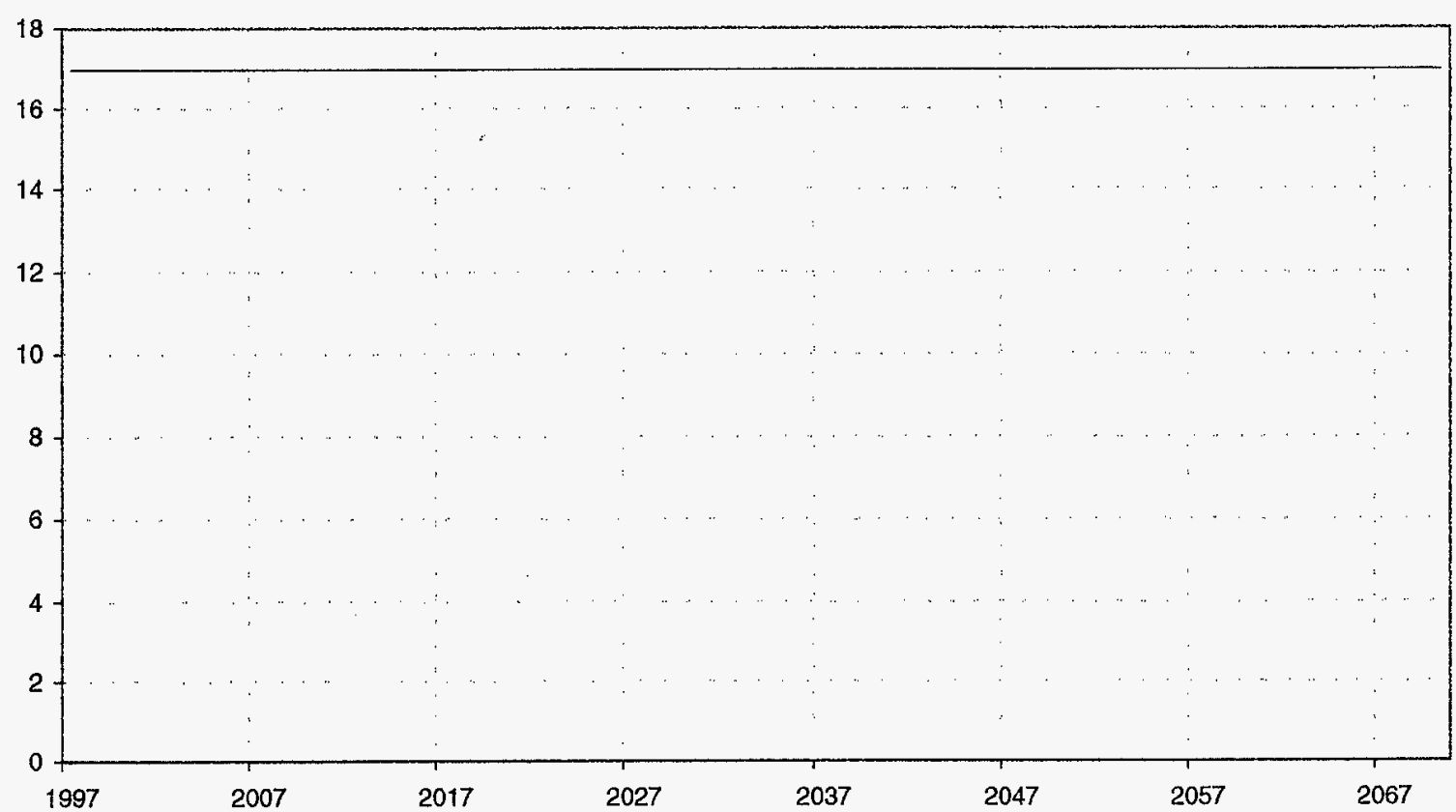

SHIPMENTS RECEIVED CWWFA CHART:

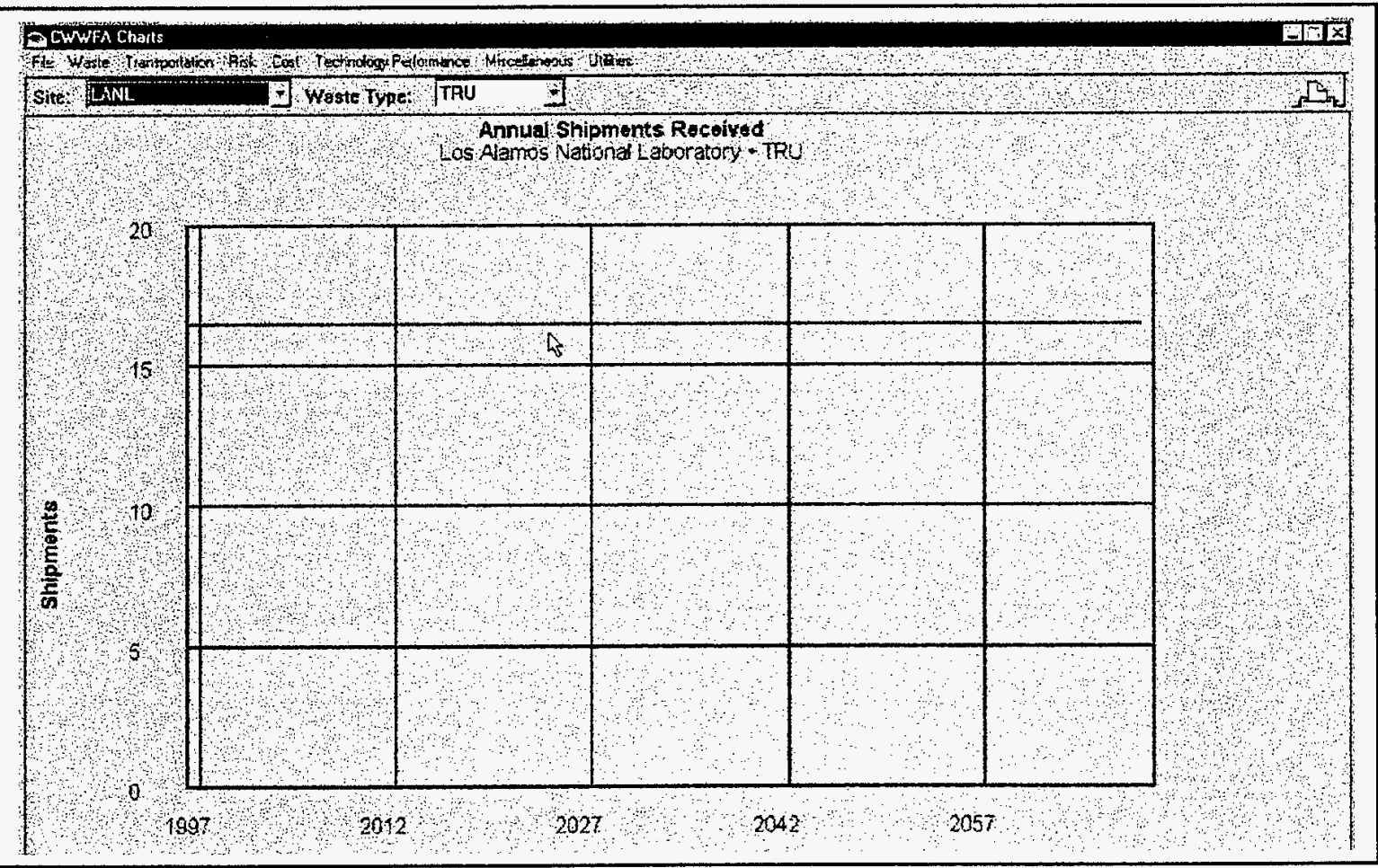


SHIPMENTS SENT EXCEL CHART (LANL):

\section{Shipments Sent}

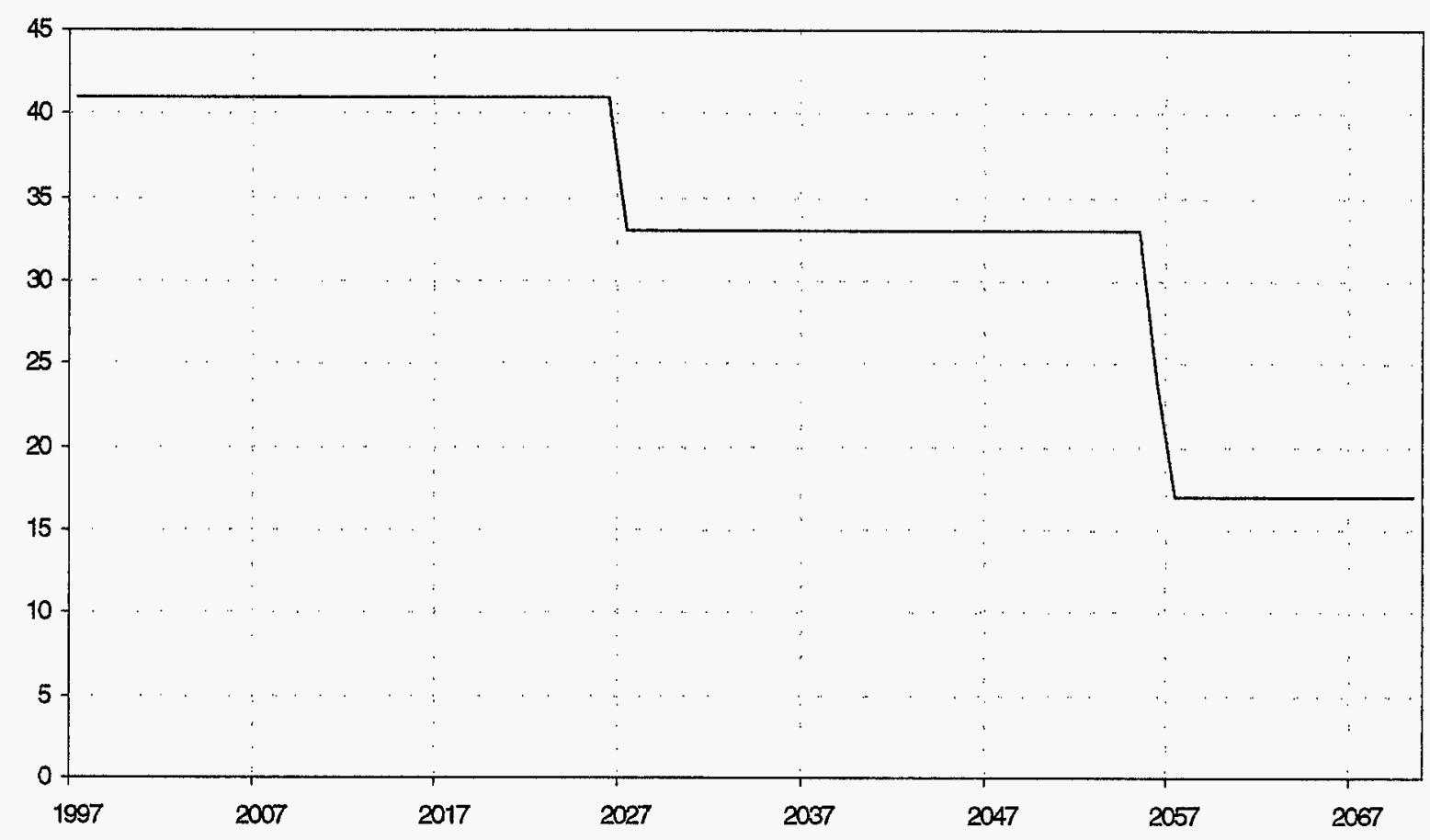

\section{SHIPMENTS SENT CWWFA CHART:}

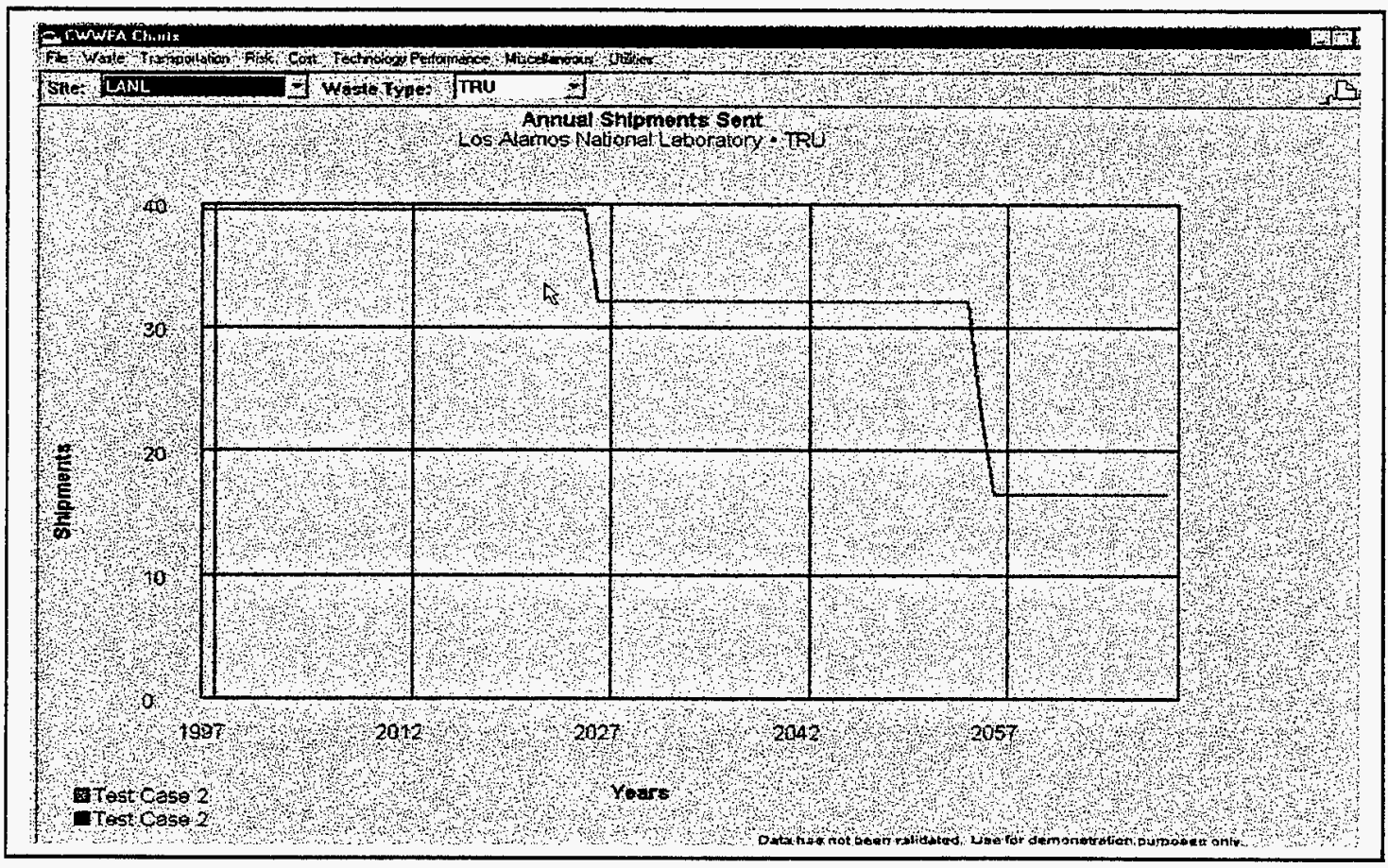


TRANSPORTAION COMPARISON

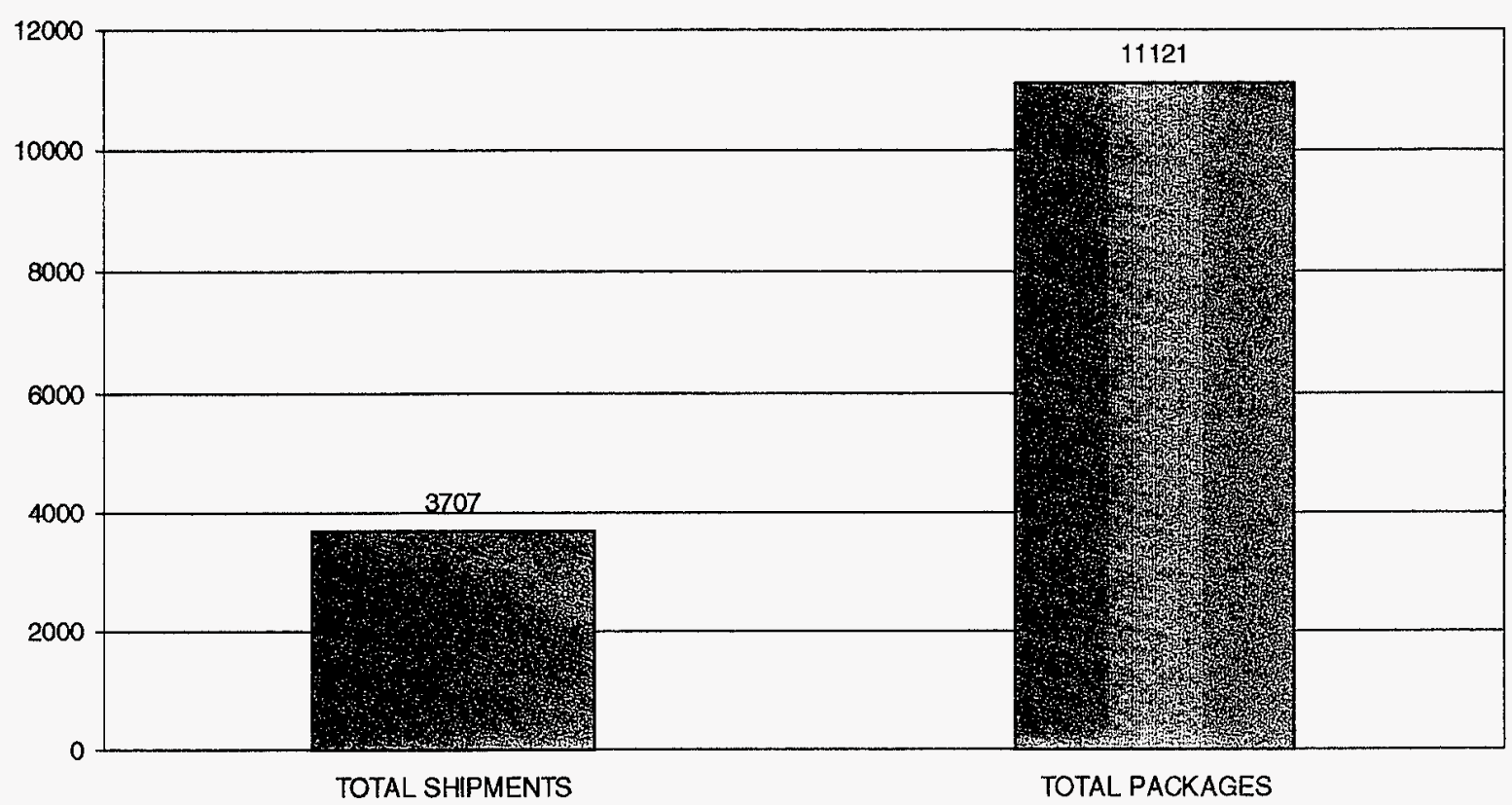

TRANSPORTATION COMPARISON CWWFA CHART:

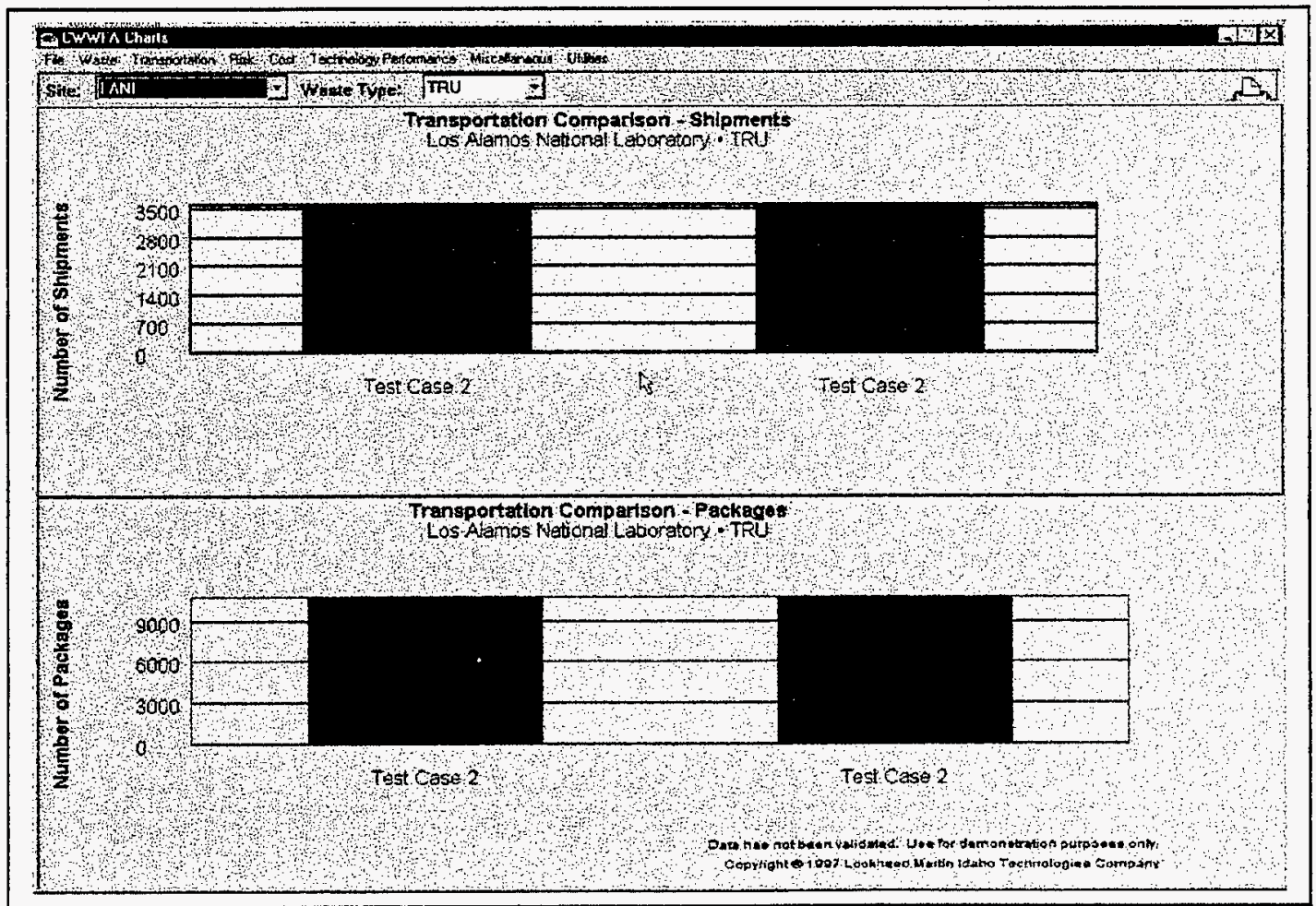




\section{CASE: Case $3 \mathrm{c}$}

INITIAL PARAMETERS \& RESULTS REPORT

1.0 Waste Stream Information

\begin{tabular}{|l|l|}
\hline Originating Site & LANL \\
\hline Waste Stream ID \& Name & LANLWS1-CASE3 \\
\hline Originating Type & TRU \\
\hline Legacy volume $(\mathrm{m} 3)$ & 20,000 \\
\hline
\end{tabular}

1.1 New Generation

\begin{tabular}{|l|l|l|}
\hline \multicolumn{1}{|c|}{ Start Year } & \multicolumn{1}{|c|}{ End Year } & Annual Volume (m3) \\
\hline NA & NA & NA \\
\hline & & \\
\hline & & \\
\hline & & \\
\hline
\end{tabular}

1.2 Shipping Route(s)

\begin{tabular}{|l|l|l|}
\hline \multicolumn{1}{|c|}{ Route ID } & \multicolumn{1}{|c|}{ Source Facility } & \multicolumn{1}{c|}{ Destination Facility } \\
\hline C3a-1 & Legacy-LANL & RFTSFAC1-CASE3 \\
\hline C3a-2 & Legacy-LANL & LANLFAC1-CASE3 \\
\hline C3a-3 & RFTSFAC1-CASE3 & LANLFAC2-CASE3 \\
\hline C3a-4 & LANLFAC1-CASE3 & RFTSFAC2-CASE3 \\
\hline & & \\
\hline & & \\
\hline
\end{tabular}

1.3 Shipping Schedule(s)

\begin{tabular}{|c|c|c|c|}
\hline Route ID: C3a-1 & \multicolumn{2}{|c|}{} & \multicolumn{1}{|c|}{ Waste Type } \\
\hline Start Year & \multicolumn{1}{|c|}{ End Year } & Annual Volume (m3) \\
\hline 1997 & 2070 & TRU & \\
\hline & & & \\
\hline & & & \\
\hline & & & \\
\hline
\end{tabular}

1.3 Shipping Schedule(s) (continued)

\begin{tabular}{|c|c|c|c|}
\hline Route ID: C3a-2 & \multicolumn{2}{|c|}{ Waste Type } & \multicolumn{1}{|c|}{ Annual Volume (m3) } \\
\hline Start Year & \multicolumn{1}{|c|}{ End Year } & 27 \\
\hline & $2070 \quad$ TRU & \\
\hline & & & \\
\hline & & & \\
\hline
\end{tabular}

1.3 Shipping Schedule(s) (continued)

\begin{tabular}{|c|c|c|c|}
\hline Route ID: C3a-3 & \multicolumn{2}{|c|}{ Waste Type } & \multicolumn{1}{|c|}{ Annual Volume (m3) } \\
\hline Start Year & \multicolumn{1}{|c|}{ End Year } & 243 \\
\hline & 2070 & TRU & \\
\hline & & & \\
\hline & & & \\
\hline
\end{tabular}

1.3 Shipping Schedule(s) (continued)

\begin{tabular}{|c|c|c|c|}
\hline \multicolumn{4}{|l|}{ Route ID: C3a-4 } \\
\hline Start Year & End Year & Waste Type & Annual Volume (m3) \\
\hline 1997 & 2070 & TRU & 27 \\
\hline & & & \\
\hline & & & \\
\hline
\end{tabular}




\section{INITIAL PARAMETERS \& RESULTS REPORT}

\section{CASE: Case $3 \mathrm{c}$}

2.0 Facility Information

\begin{tabular}{|l|l|}
\hline Site & RFTS \\
\hline Facility ID \& Name & RFTSFAC1-CASE3 \\
\hline Function & Treatment \\
\hline Start Year & 1997 \\
\hline End Year & 2070 \\
\hline Annual Capacity (m3/year) or & $1,000,000$ \\
\hline Lifetime Facility Capacity (m3) & \\
\hline Volume Change Factor & 1 \\
\hline
\end{tabular}

\section{Conversion Profile}

\begin{tabular}{|l|l|l|}
\hline Percent & Waste Type & Density \\
\hline $100 \%$ & TRU & 1198 \\
\hline & & \\
\hline & & \\
\hline
\end{tabular}

2.0 Facility Information

\begin{tabular}{|l|l|}
\hline Site & RFTS \\
\hline Facility ID \& Name & RFTSFAC2-CASE3 \\
\hline Function & Disposal \\
\hline Start Year & 1997 \\
\hline End Year & 2070 \\
\hline Annual Capacity (m3/year) or & $1,000,000$ \\
\hline Lifetime Facility Capacity (m3) & \\
\hline Volume Change Factor & 1 \\
\hline \\
\begin{tabular}{|l|l|l|}
\hline Penversion Profile & Waste Type & Density \\
\hline NA & NA & NA \\
\hline & & \\
\hline
\end{tabular}
\end{tabular}

2.0 Facility Information

\begin{tabular}{|c|c|c|}
\hline \multirow{2}{*}{$\begin{array}{l}\text { Site } \\
\text { Facility ID \& Name }\end{array}$} & \multicolumn{2}{|l|}{ LANL } \\
\hline & \multicolumn{2}{|l|}{ LANLFAC1-CASE3 } \\
\hline Function & \multicolumn{2}{|l|}{ Treatment } \\
\hline Start Year & \multicolumn{2}{|l|}{1997} \\
\hline End Year & \multicolumn{2}{|l|}{2070} \\
\hline Annual Capacity (m3/year) or & $1,000,000$ & \\
\hline \multicolumn{3}{|l|}{ Lifetime Facility Capacity (m3) } \\
\hline Volume Change Factor & 1 & \\
\hline \multicolumn{3}{|l|}{ Conversion Profile } \\
\hline Percent & Waste Type & Density \\
\hline 100 & TRU & 1198 \\
\hline
\end{tabular}

2.0 Facility Information

\begin{tabular}{|c|c|c|c|}
\hline \multirow{2}{*}{\multicolumn{2}{|c|}{$\begin{array}{l}\text { Site } \\
\text { Facility ID \& Name }\end{array}$}} & \multicolumn{2}{|l|}{ LANL } \\
\hline & & \multicolumn{2}{|l|}{ LANLFAC2-CASE3 } \\
\hline \multicolumn{2}{|l|}{ Function } & \multicolumn{2}{|l|}{ Disposal } \\
\hline \multicolumn{2}{|l|}{ Start Year } & \multicolumn{2}{|l|}{1997} \\
\hline \multicolumn{2}{|l|}{ End Year } & \multirow{2}{*}{\multicolumn{2}{|c|}{$\begin{array}{l}2070 \\
1,000,000\end{array}$}} \\
\hline \multicolumn{2}{|l|}{ Annual Capacity (m3/year) or } & & \\
\hline \multicolumn{4}{|l|}{ Lifetime Facility Capacity (m3) } \\
\hline Volume Change Factor & & 1 & \\
\hline \multicolumn{4}{|l|}{ Conversion Profile } \\
\hline Percent & Waste Typ & & Density \\
\hline NA & NA & & NA \\
\hline
\end{tabular}


WASTE DISPOSITION - EXCEL CHART (LANL, TRU):

\section{Waste Disposition}

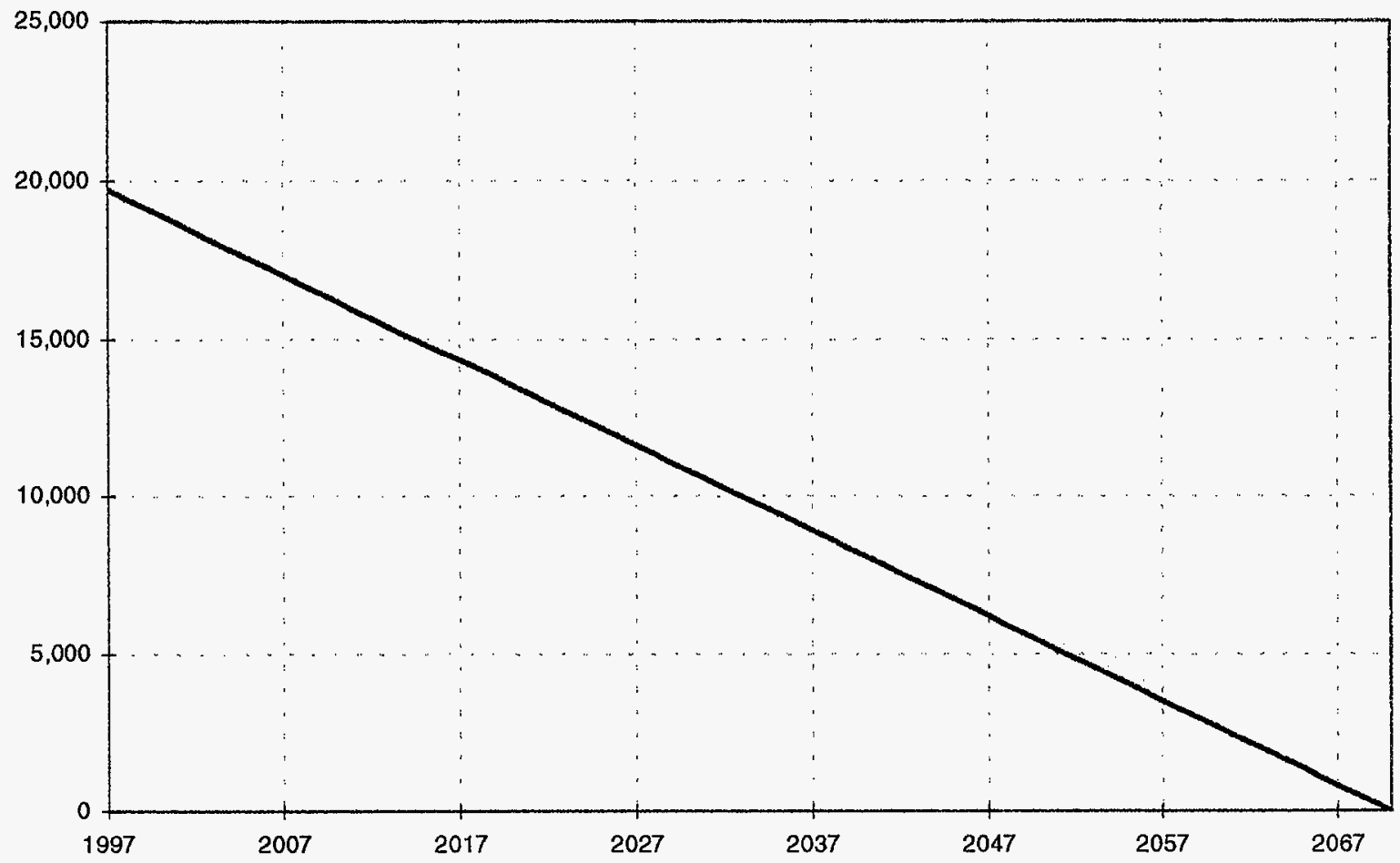

\section{WASTE DISPOSITION - CWWFA CHART:}

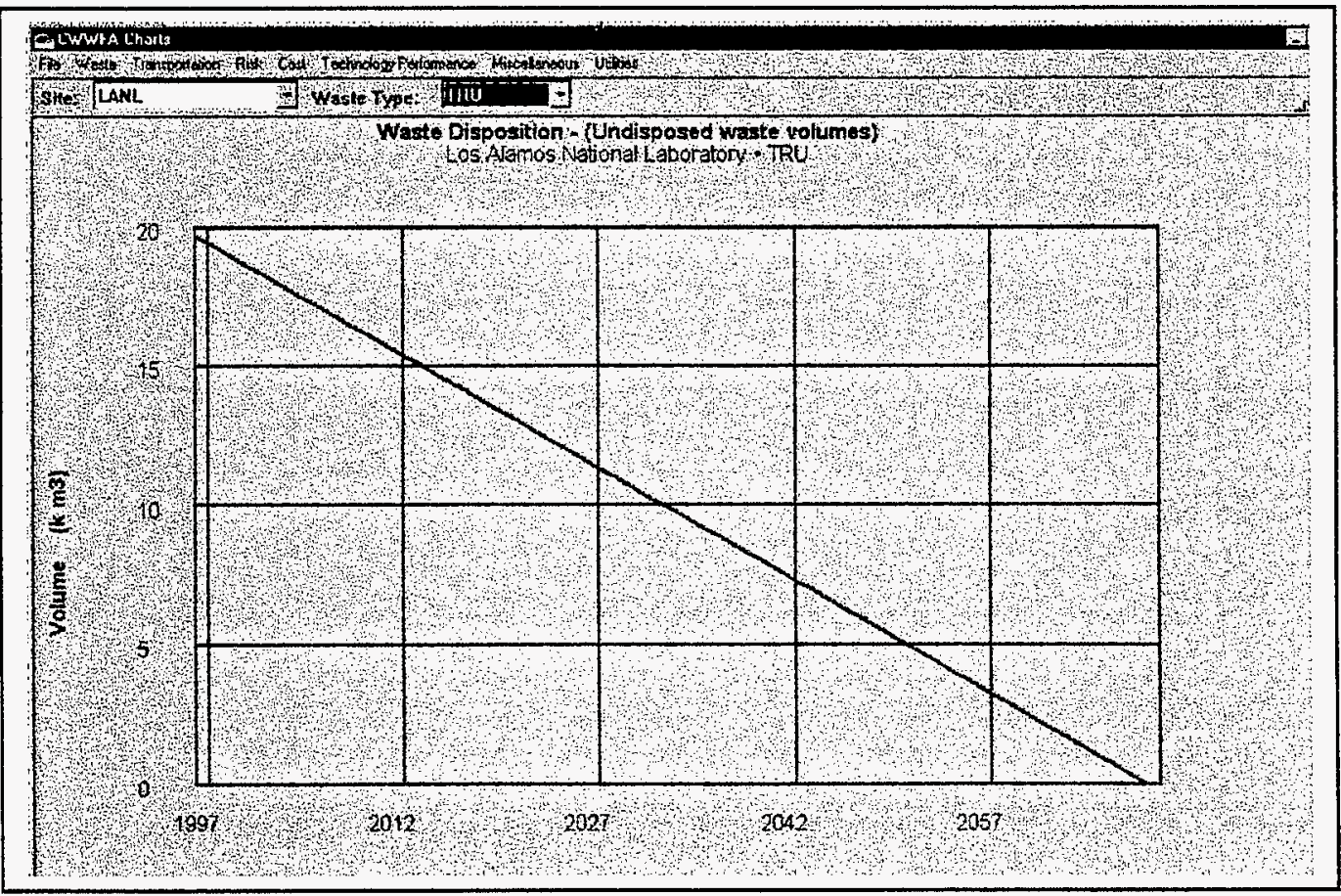


FACILITY CAPACITY EXCEL CHART (LANL, RFTSFAC2-CASE3):

\section{Facility Capacity Detail}

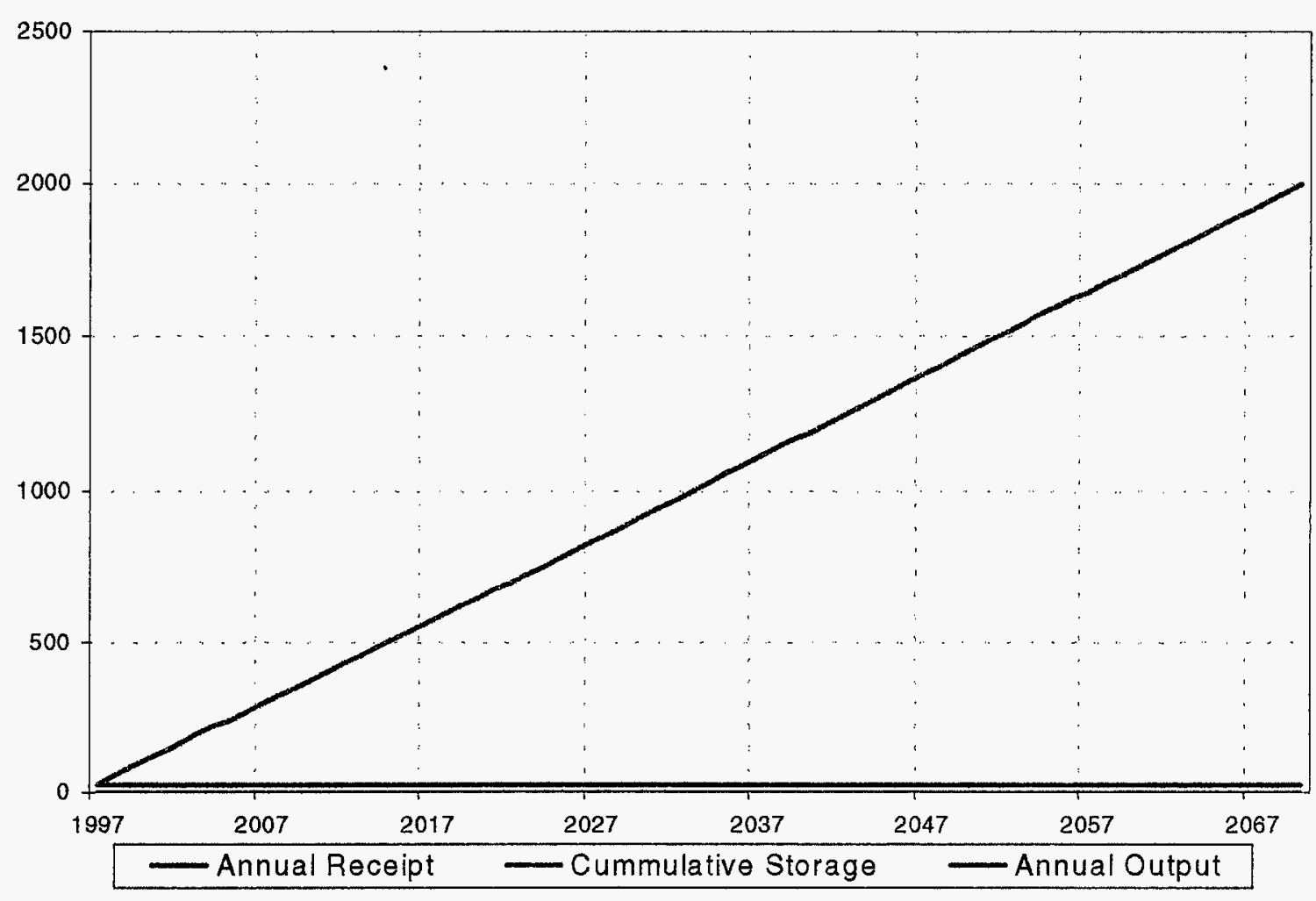

\section{FACILITY CAPACITY CWWFA CHART:}

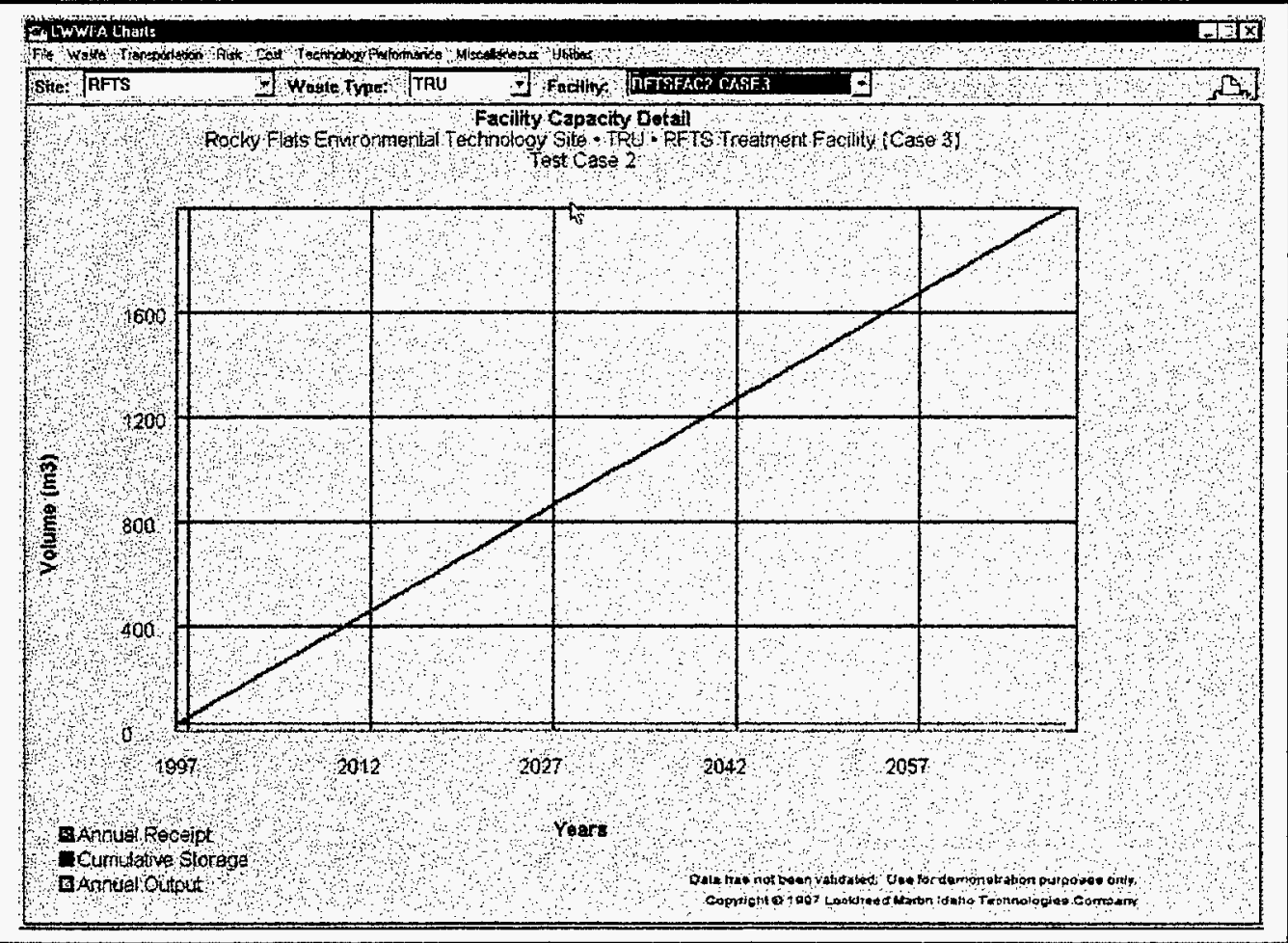


CASE: Case $3 \mathrm{c}$

DATE: $11 / 13 / 97$

Evaluator: K B Oswald

WASTE STREAM DETAIL EXCEL CHART (LANLWS1-CASE3):

\section{Waste Stream Detail}

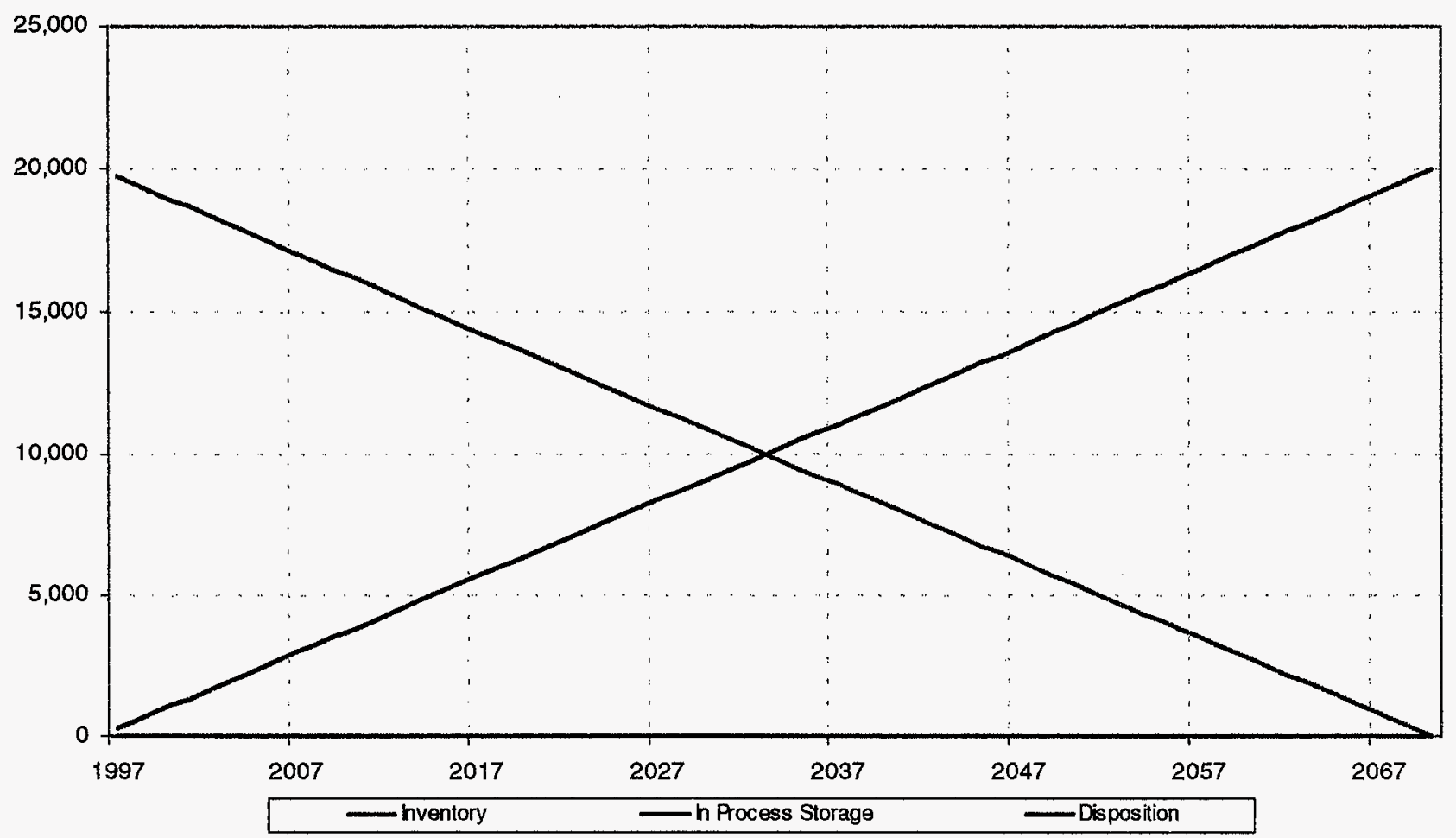

WASTE STREAM DETAIL CWWFA CHART:

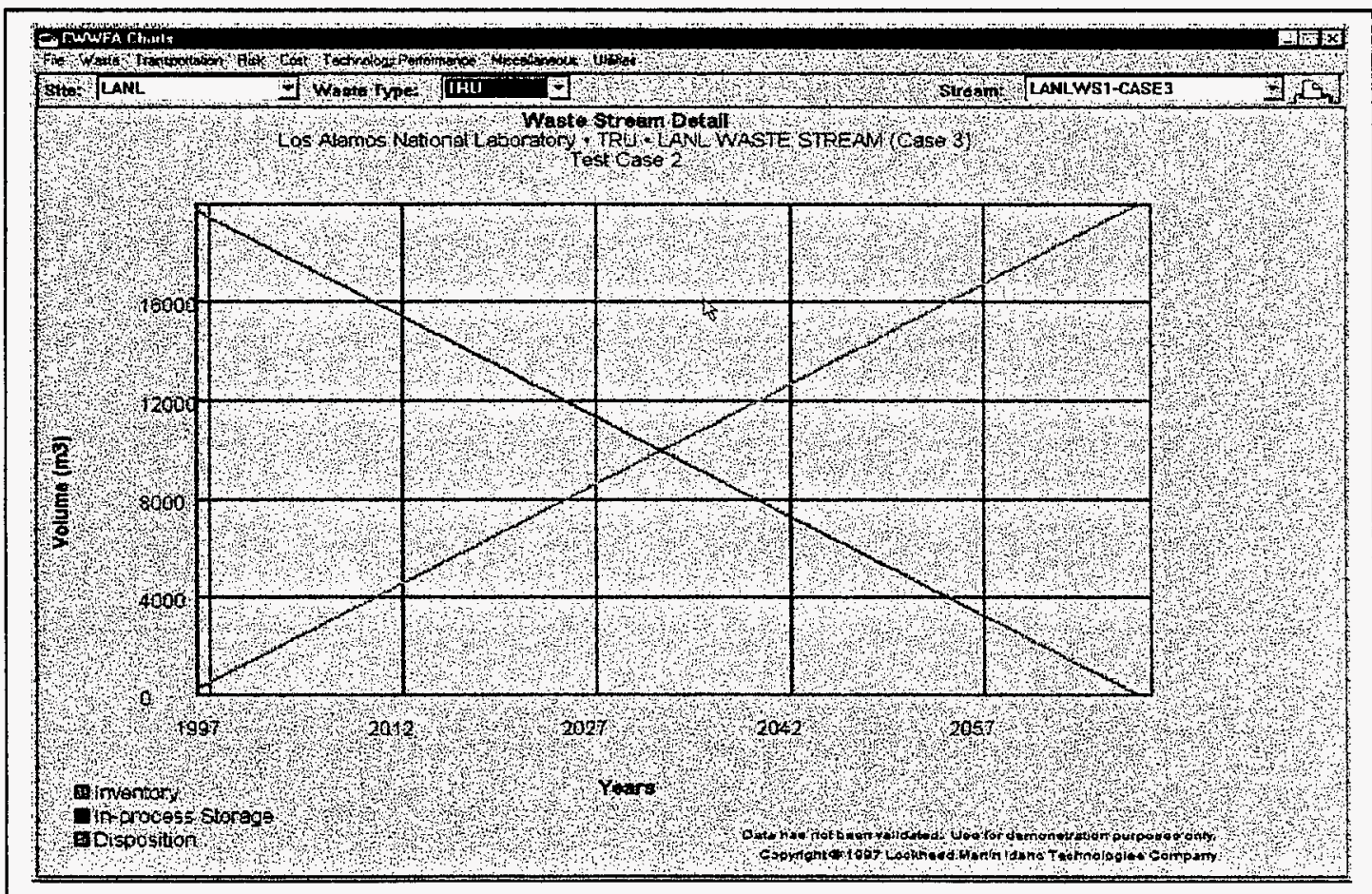


CASE: Case 3c

DATE: $11 / 13 / 97$

Evaluator: K B Oswald

SHIPMENTS RECEIVED EXCEL CHART (LANL):

Shipments Received

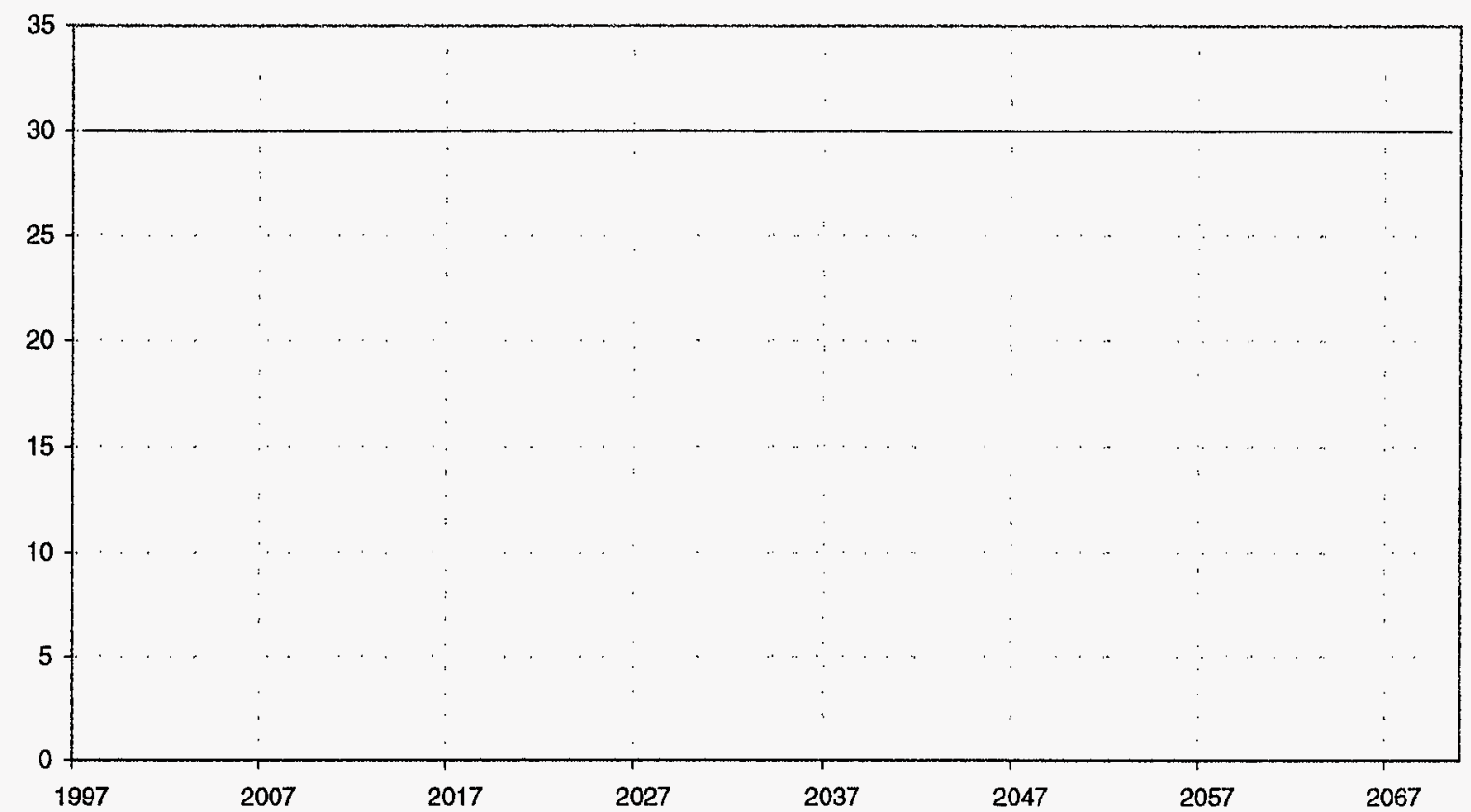

SHIPMENTS RECEIVED CWWFA CHART:

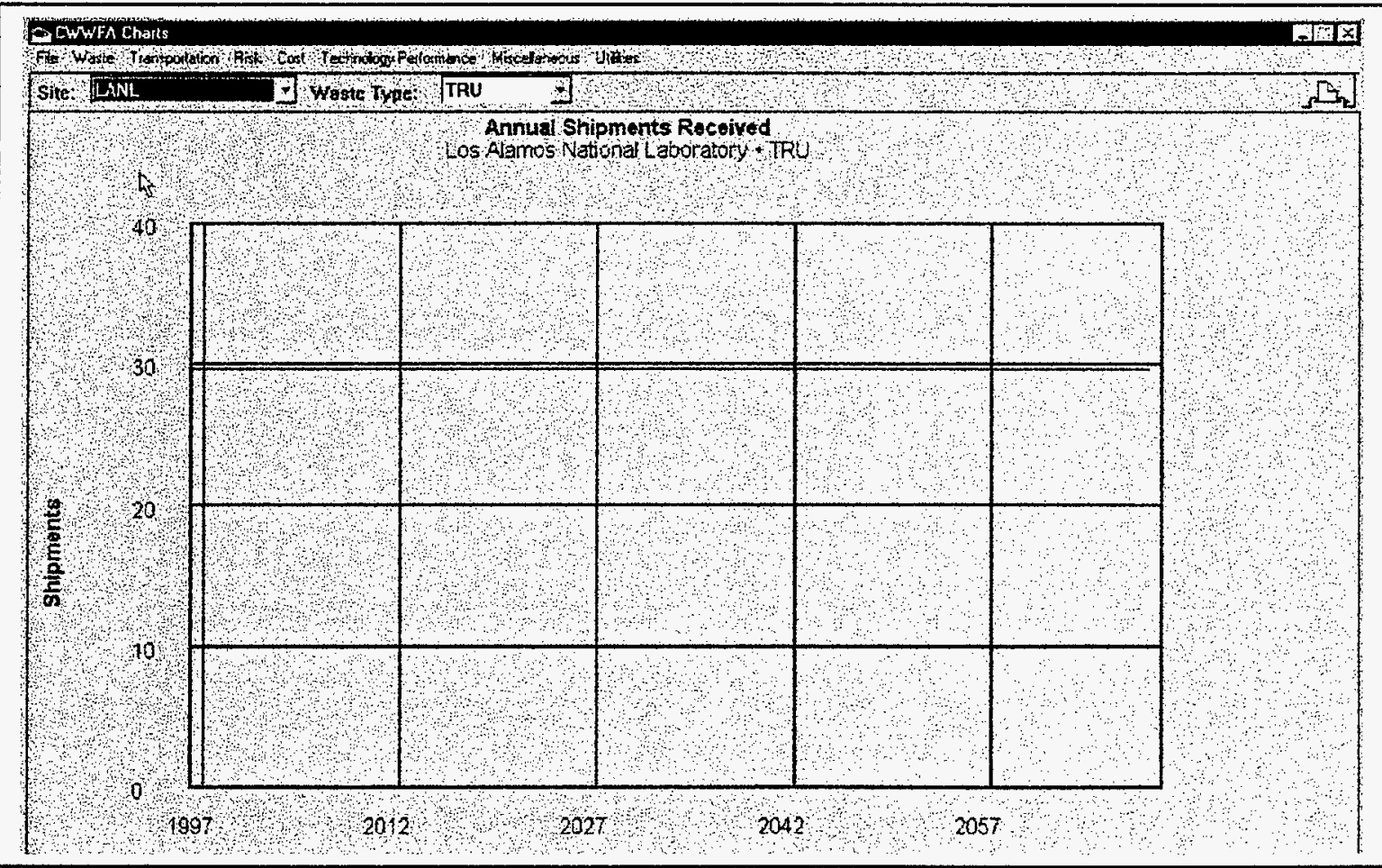


SHIPMENTS SENT EXCEL CHART (LANL):

\section{Shipments Sent}

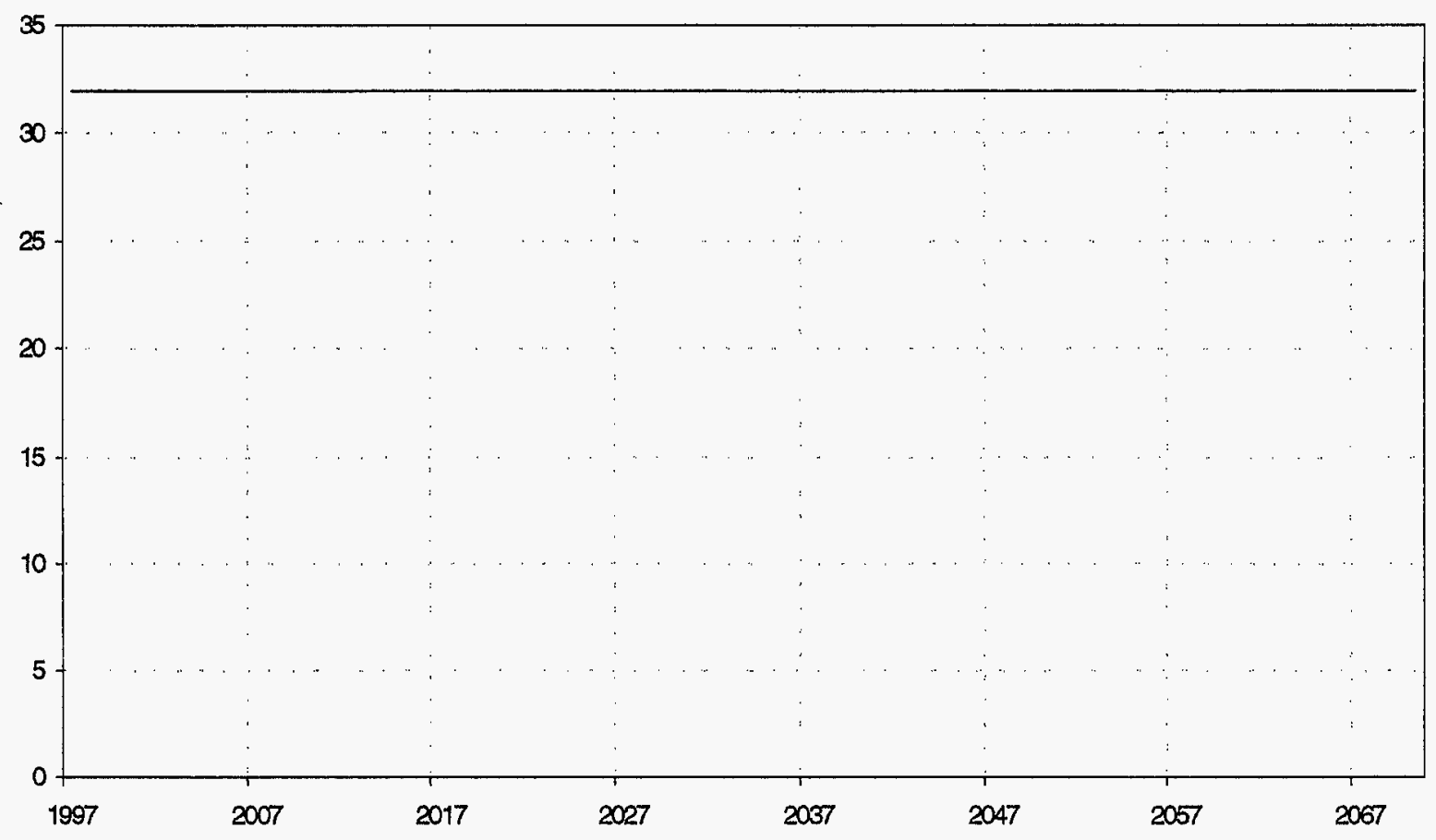

\section{SHIPMENTS SENT CWWFA CHART:}

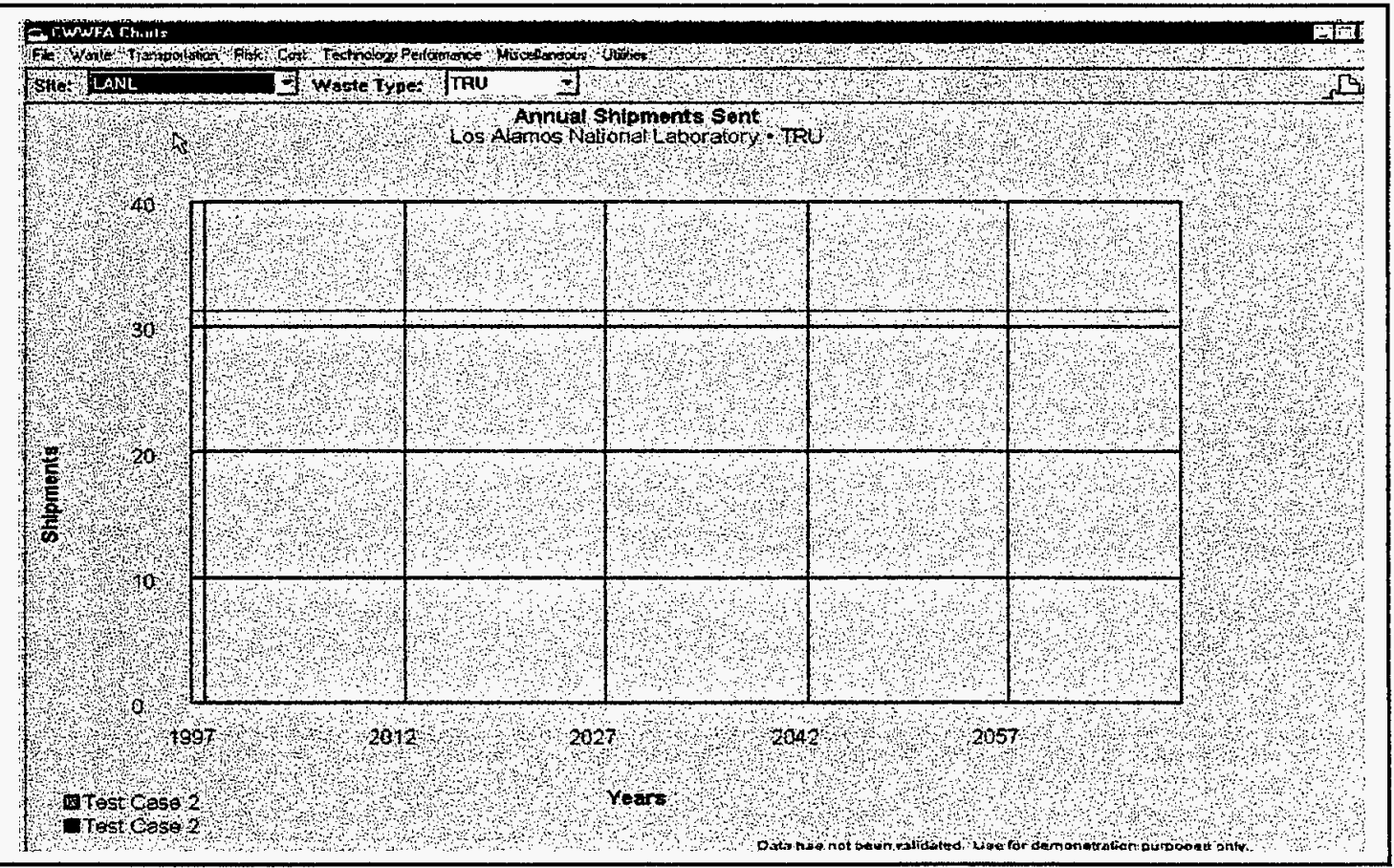


TRANSPORTATION COMPARISON EXCEL CHART (LANL):

TRANSPORTAION COMPARISON

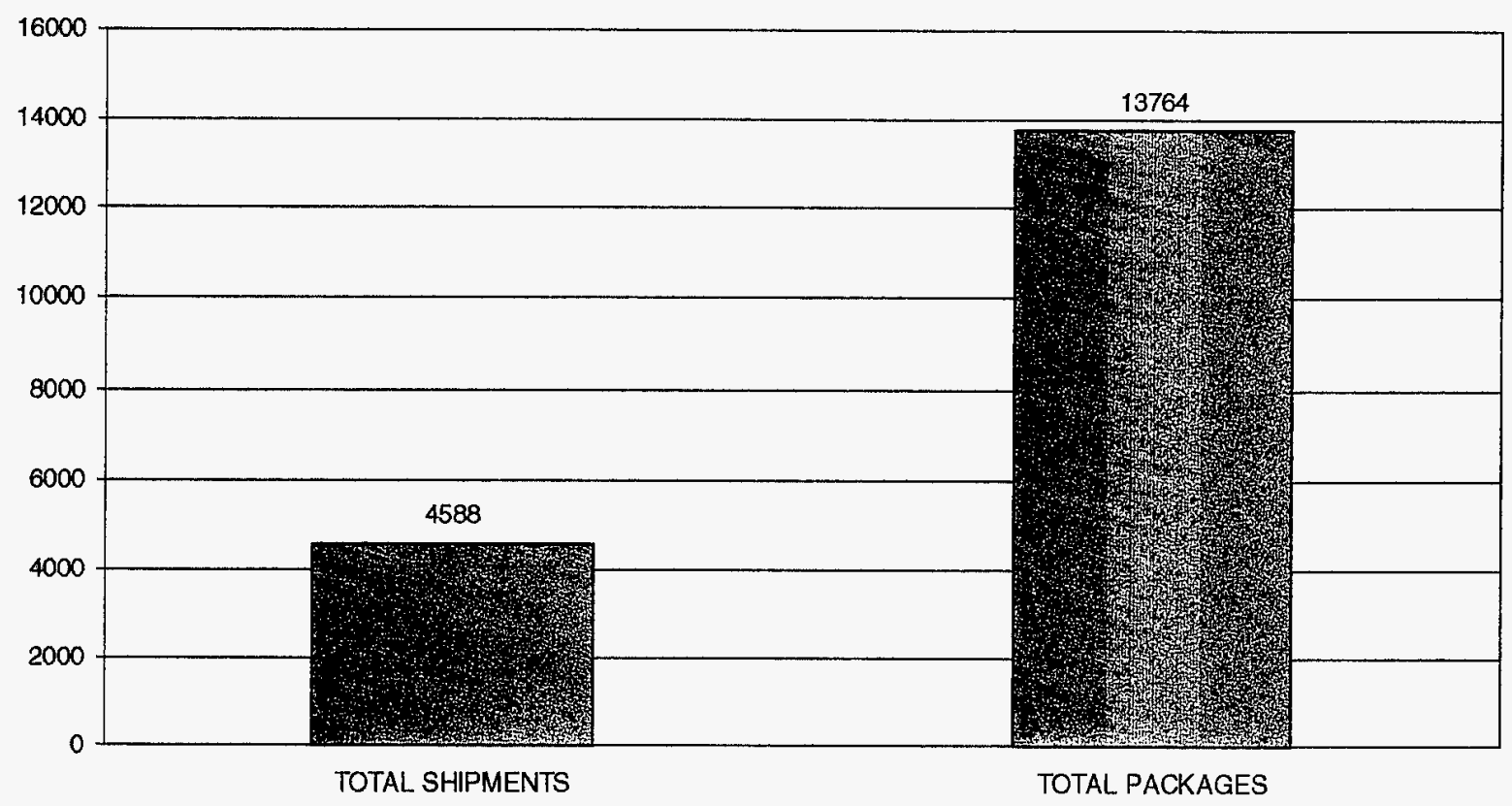

TRANSPORTATION COMPARISON CWWFA CHART:

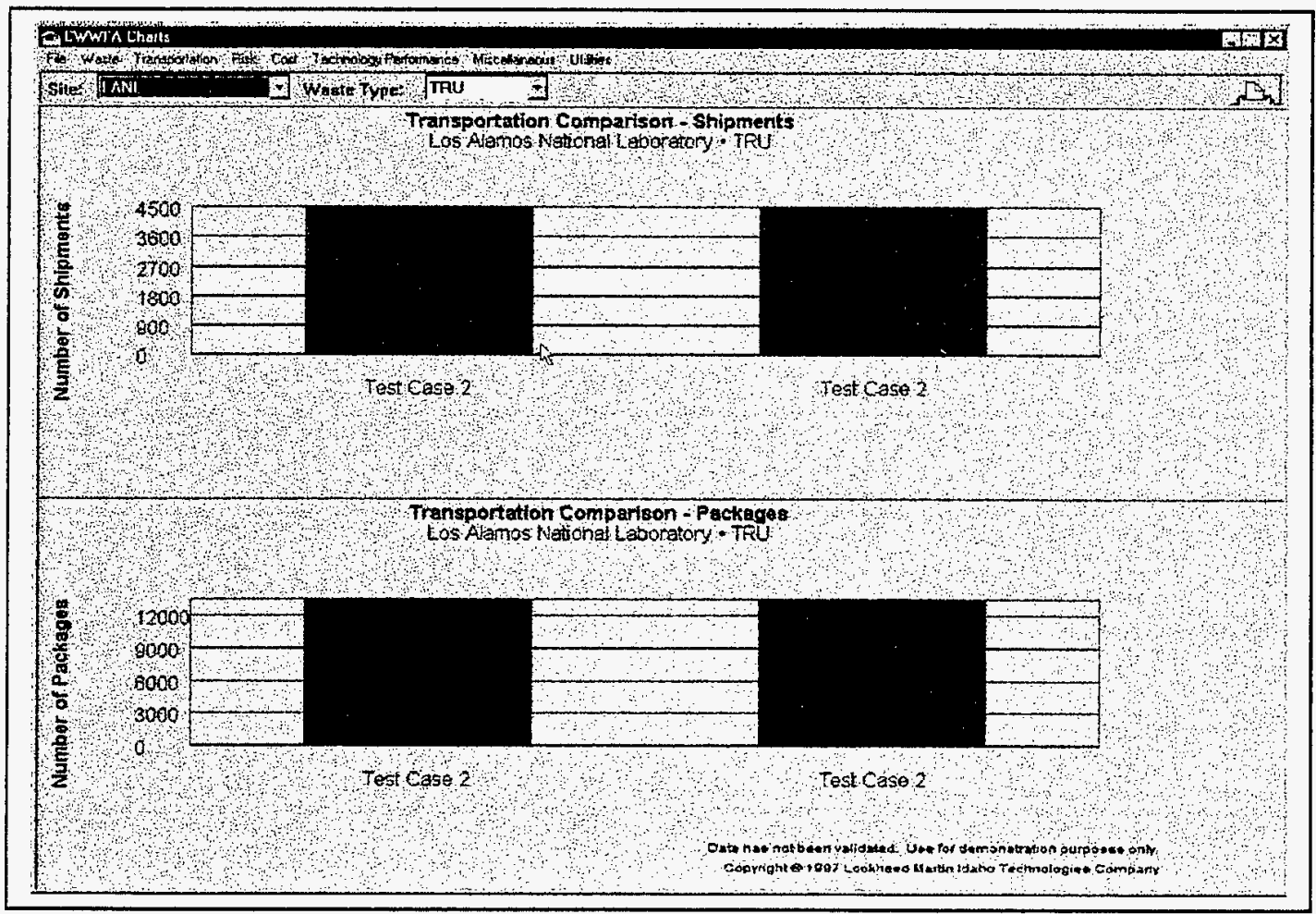


1.0 Waste Stream Information

\begin{tabular}{|l|l|}
\hline Originating Site & LANL \\
\hline Waste Stream ID \& Name & LANLWS1-CASE3 \\
\hline Originating Type & TRU \\
\hline Legacy volume $(\mathrm{m} 3)$ & 20,000 \\
\hline
\end{tabular}

1.1 New Generation

\begin{tabular}{|l|l|l|}
\hline \multicolumn{1}{|c|}{ Start Year } & \multicolumn{1}{|c|}{ End Year } & Annual Volume (m3) \\
\hline NA & NA & NA \\
\hline & & \\
\hline & & \\
\hline & & \\
\hline
\end{tabular}

1.2 Shipping Route(s)

\begin{tabular}{|l|l|l|}
\hline \multicolumn{1}{|c|}{ Route ID } & \multicolumn{1}{|c|}{ Source Facility } & \multicolumn{1}{c|}{ Destination Facility } \\
\hline C3d-1 & Legacy-LANL & RFTSFAC1-CASE3 \\
\hline C3d-2 & Legacy-LANL & LANLFAC1-CASE3 \\
\hline C3d-3 & RFTSFAC1-CASE3 & LANLFAC2-CASE3 \\
\hline C3d-4 & LANLFAC1-CASE3 & RFTSFAC2-CASE3 \\
\hline & & \\
\hline & & \\
\hline
\end{tabular}

1.3 Shipping Schedule(s)

\begin{tabular}{|l|l|l|l|}
\hline Route ID: C3d-1 & \multicolumn{2}{|c|}{ Waste Type } & \multicolumn{1}{|c|}{ Annual Volume (m3) } \\
\hline \multicolumn{1}{|c|}{ Start Year } & \multicolumn{1}{|c|}{ End Year } & \multicolumn{1}{|c|}{. } & 1000 \\
\hline 1997 & 2010 & TRU & 400 \\
\hline & 2029 & TRU & \\
\hline & & & \\
\hline
\end{tabular}

1.3 Shipping Schedule(s) (continued)

\begin{tabular}{|l|l|l|l|}
\hline Route ID: C3d-2 & \multicolumn{1}{|c|}{ Waste Type } & \multicolumn{1}{|c|}{ Annual Volume (m3) } \\
\hline \multicolumn{1}{|c|}{ Start Year } & \multicolumn{1}{|c|}{ End Year } & \multicolumn{1}{|c|}{. } & 250 \\
\hline 1997 & 2000 & TRU & 1000 \\
\hline 2001 & 2001 & & \\
\hline & & & \\
\hline & & TRU & \\
\hline
\end{tabular}

1.3 Shipping Schedule(s) (continued)

\begin{tabular}{|c|c|c|c|}
\hline Route ID: C3d-3 & \multicolumn{2}{|c|}{ Waste Type } & \multicolumn{1}{|c|}{ Annual Volume (m3) } \\
\hline Start Year & \multicolumn{1}{|c|}{ End Year } & 243 \\
\hline 1997 & 2070 & TRU & \\
\hline & & & \\
\hline & & & \\
\hline
\end{tabular}

1.3 Shipping Schedule(s) (continued)

\begin{tabular}{|c|c|c|c|}
\hline \multicolumn{4}{|l|}{ Route ID: C3d-4 } \\
\hline Start Year & End Year & Waste Type & Annual Volume (m3) \\
\hline 1997 & 2070 & TRU & 27 \\
\hline & & & \\
\hline & & & \\
\hline
\end{tabular}




\section{INITIAL PARAMETERS \& RESULTS REPORT}

CASE: Case 3d

DATE: $11 / 13 / 97$

Evaluator: K B Oswald

2.0 Facility Information

\begin{tabular}{|l|l|}
\hline Site & RFTS \\
\hline Facility ID \& Name & RFTSFAC1-CASE3 \\
\hline Function & Treatment \\
\hline Start Year & 1997 \\
\hline End Year & 2070 \\
\hline Annual Capacity (m3/year) or & $1,000,000$ \\
\hline Lifetime Facility Capacity (m3) & \\
\hline Volume Change Factor & 1 \\
\hline
\end{tabular}

Conversion Profile

\begin{tabular}{|l|l|l|}
\hline Percent & Waste Type & Density \\
\hline $100 \%$ & TRU & 1198 \\
\hline & & \\
\hline & & \\
\hline
\end{tabular}

2.0 Facility Information

\begin{tabular}{|l|l|}
\hline Site & RFTS \\
\hline Facility ID \& Name & RFTSFAC2-CASE3 \\
\hline Function & Disposal \\
\hline Start Year & 1997 \\
\hline End Year & 2070 \\
\hline Annual Capacity (m3/year) or & $1,000,000$ \\
\hline Lifetime Facility Capacity (m3) & \\
\hline Volume Change Factor & 1 \\
\hline \\
\begin{tabular}{|l|l|l|}
\hline Ponversion Profile & Waste Type & Density \\
\hline NA & NA & NA \\
\hline & & \\
\hline
\end{tabular}
\end{tabular}

2.0 Facility Information

\begin{tabular}{|c|c|c|c|}
\hline \multirow{2}{*}{\multicolumn{2}{|c|}{$\begin{array}{l}\text { Site } \\
\text { Facility ID \& Name }\end{array}$}} & \multicolumn{2}{|c|}{ LANL } \\
\hline & & \multicolumn{2}{|l|}{ LANLFAC1-CASE3 } \\
\hline \multicolumn{2}{|l|}{ Function } & \multicolumn{2}{|l|}{ Treatment } \\
\hline \multicolumn{2}{|l|}{ Start Year } & \multicolumn{2}{|l|}{$\frac{1997}{2070}$} \\
\hline \multicolumn{2}{|l|}{ End Year } & 2070 & \\
\hline \multicolumn{2}{|l|}{ Annual Capacity (m3/year) or } & \multicolumn{2}{|l|}{$1,000,000$} \\
\hline \multicolumn{4}{|l|}{ Lifetime Facility Capacity (m3) } \\
\hline \multirow{2}{*}{\multicolumn{4}{|c|}{$\begin{array}{l}\text { Volume Change Factor } \\
\text { Conversion Profile }\end{array}$}} \\
\hline & & & \\
\hline Percent & Waste Typ & & Density \\
\hline 100 & TRU & & 1198 \\
\hline
\end{tabular}

2.0 Facility Information

\begin{tabular}{|l|l|}
\hline Site & LANL \\
\hline Facility ID \& Name & LANLFAC2-CASE3 \\
\hline Function & Disposal \\
\hline Start Year & 1997 \\
\hline End Year & 2070 \\
\hline Annual Capacity (m3/year) or & $1,000,000$ \\
\hline Lifetime Facility Capacity (m3) & \\
\hline Volume Change Factor & 1 \\
\hline \\
\begin{tabular}{|l|l|l|}
\hline Penversion Profile & Waste Type & Density \\
\hline NA & NA & \\
\hline & & \\
\hline
\end{tabular}
\end{tabular}


WASTE DISPOSITION - EXCEL CHART (LANL, TRU):

\section{Waste Disposition}

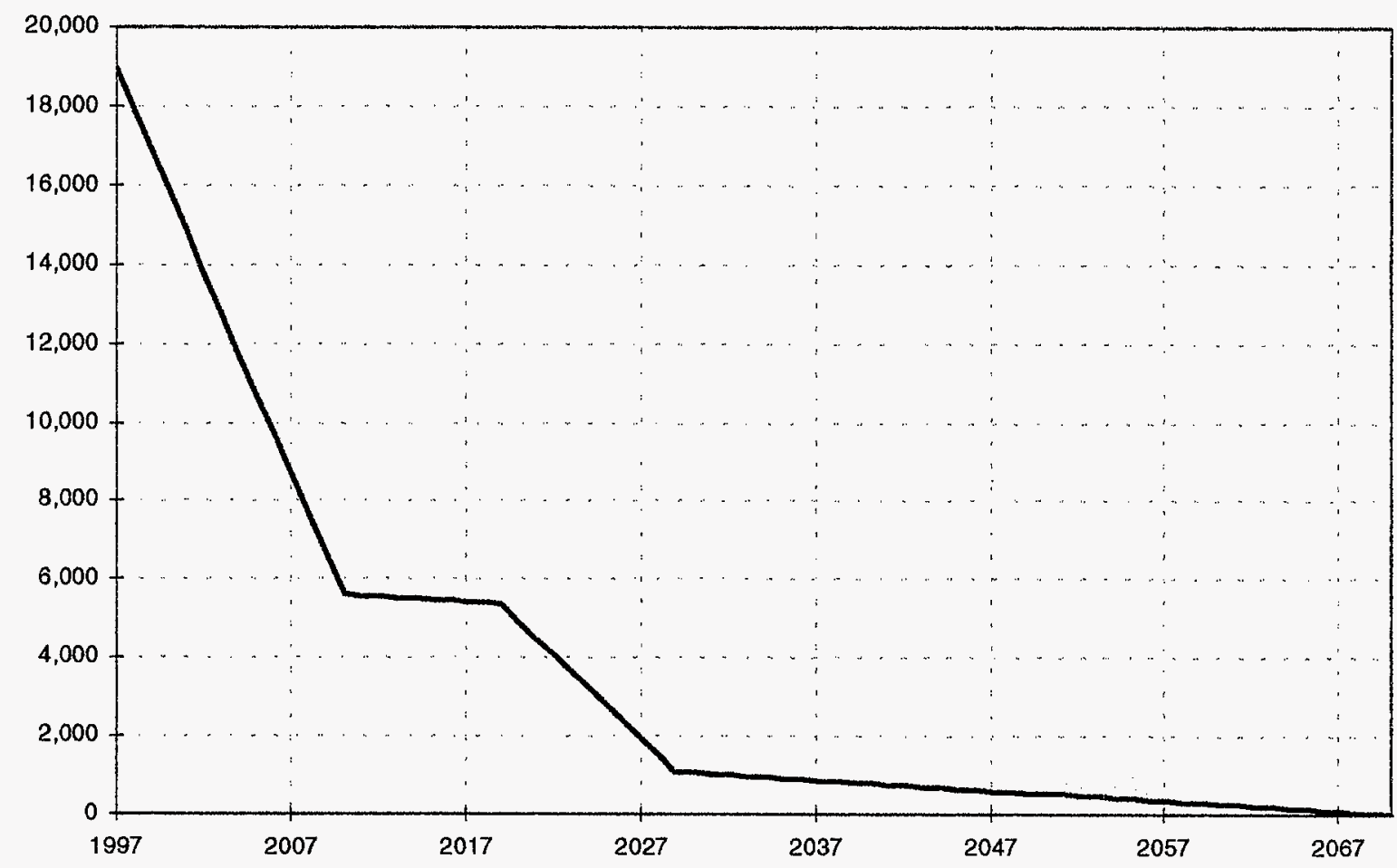

WASTE DISPOSITION - CWWFA CHART:

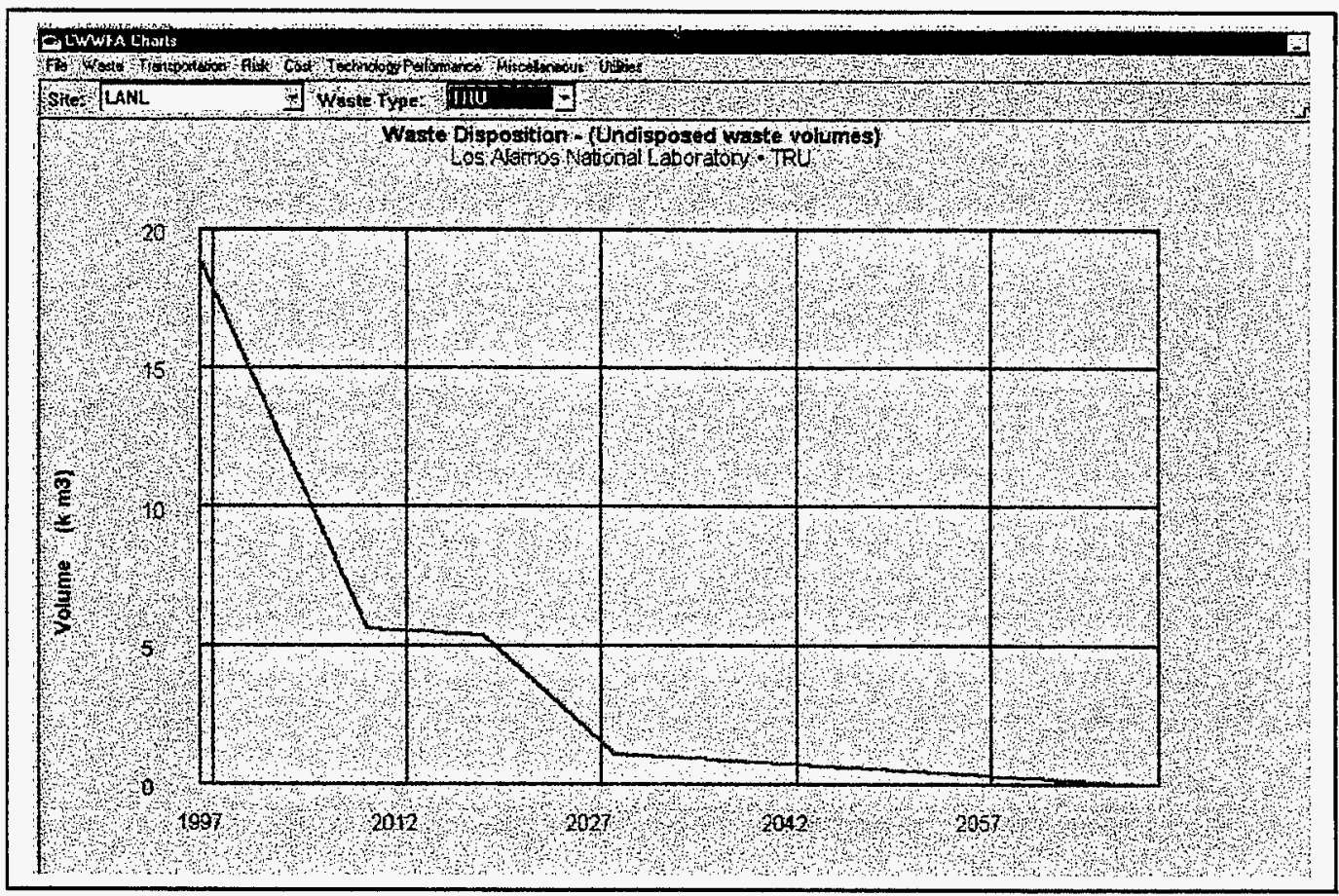


INITIAL PARAMETERS \& RESULTS REPORT

CASE: Case 3d

DATE: $11 / 13 / 97$

Evaluator: K B Oswald

FACILITY CAPACITY EXCEL CHART (LANL, RFTSFAC2-CASE3):

\section{Facility Capacity Detail}

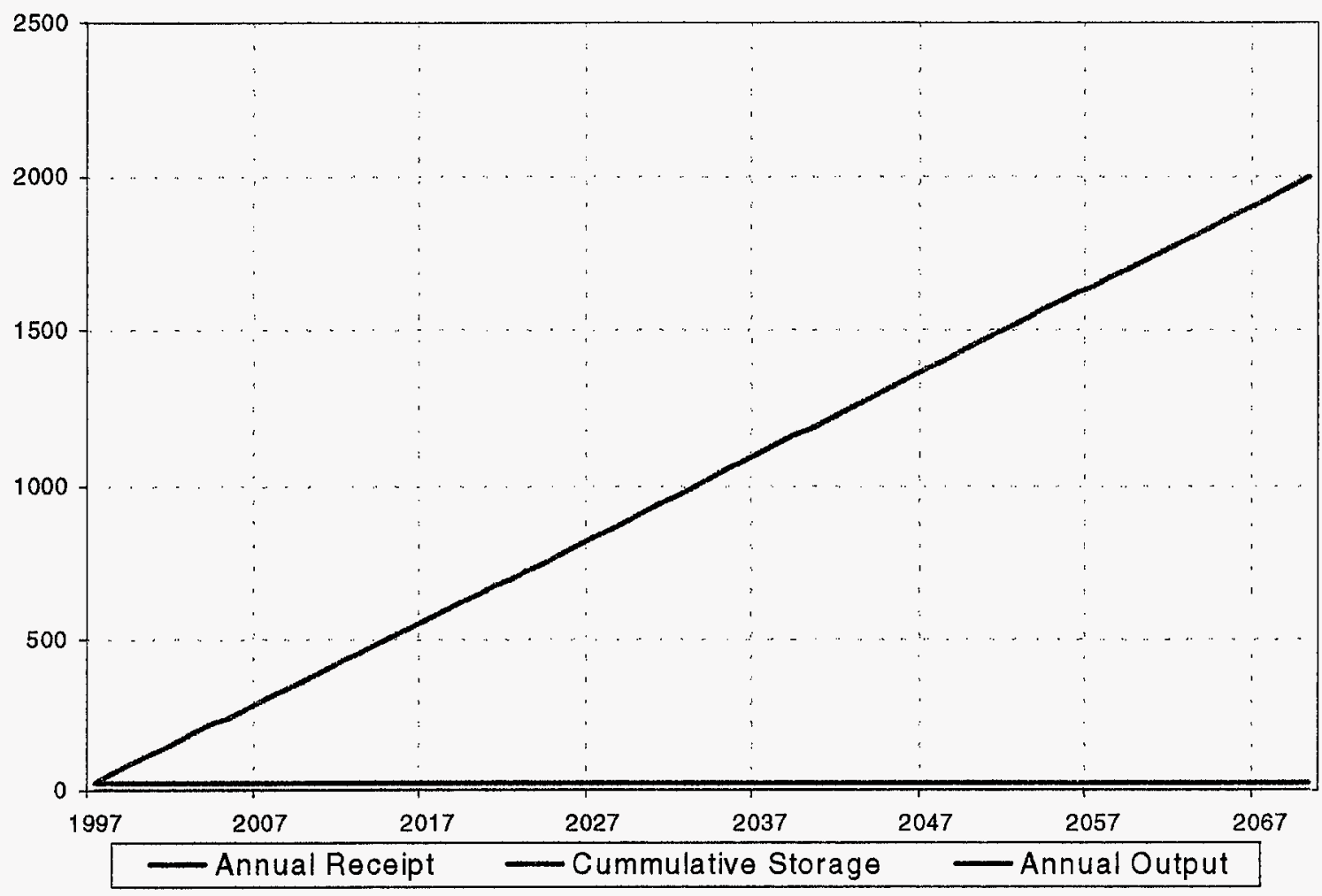

FACILITY CAPACITY CWWFA CHART:

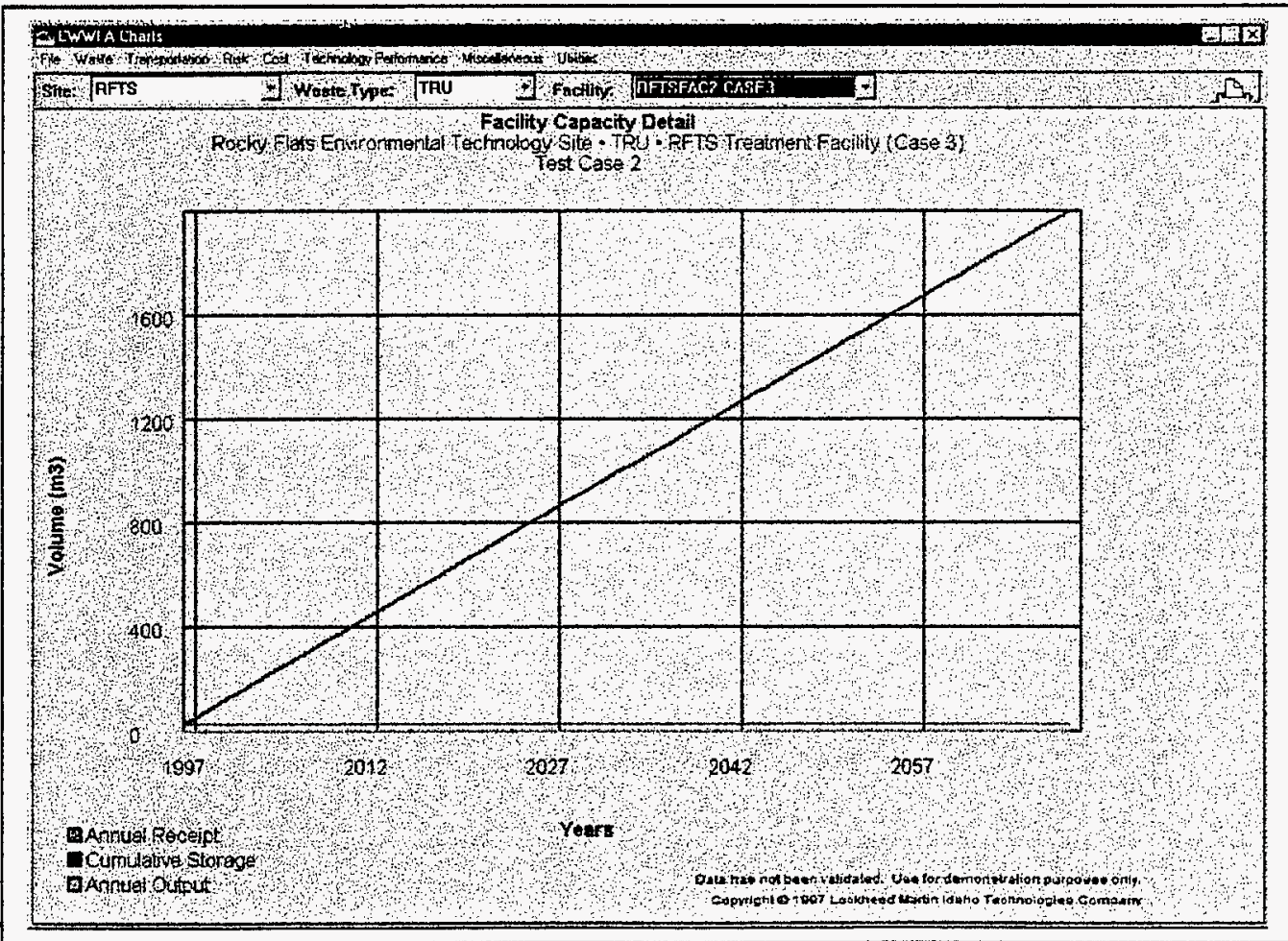


WASTE STREAM DETAIL EXCEL CHART (LANLWS1-CASE3):

\section{Waste Stream Detail}

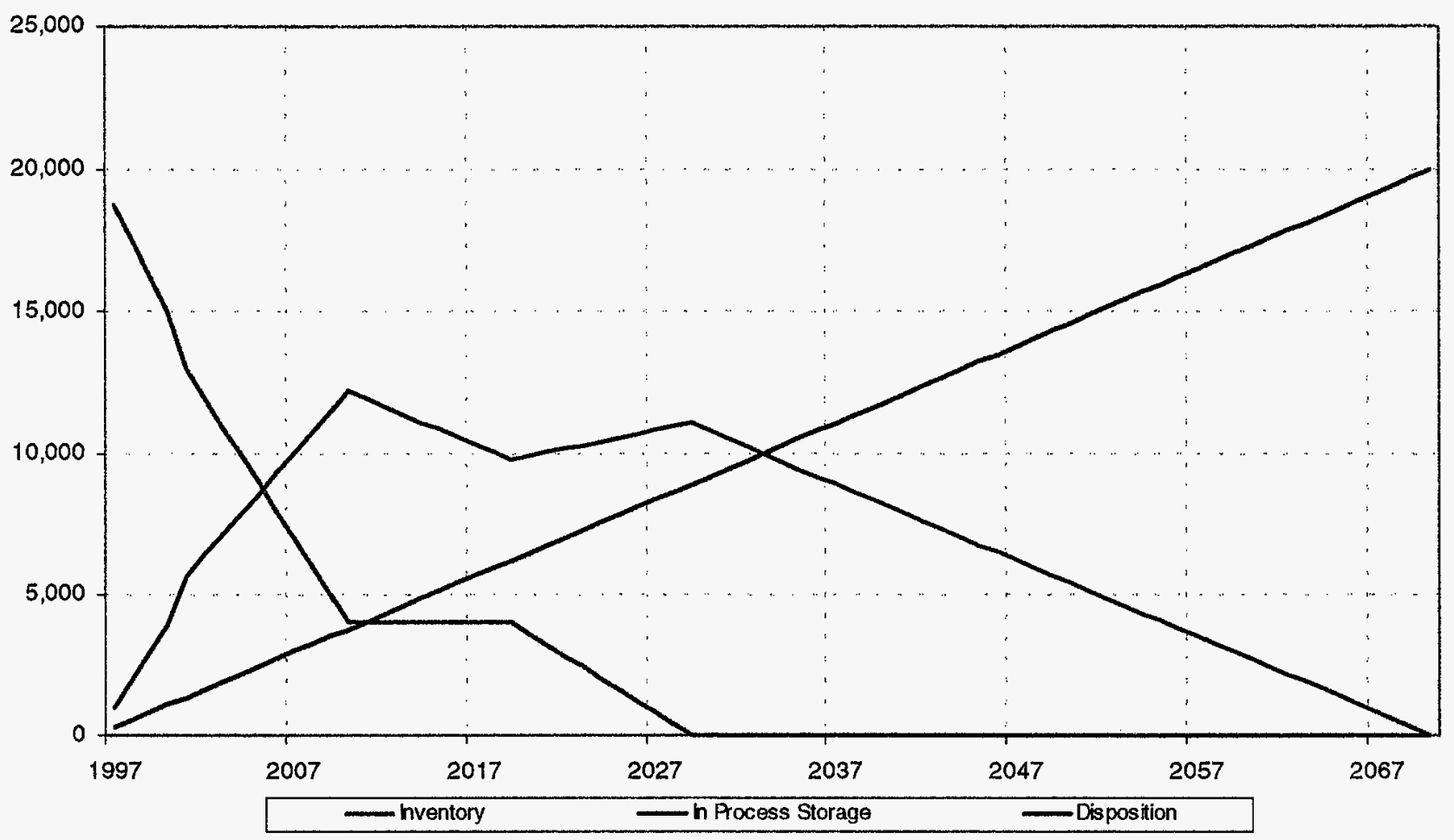

WASTE STREAM DETAIL CWWFA CHART:

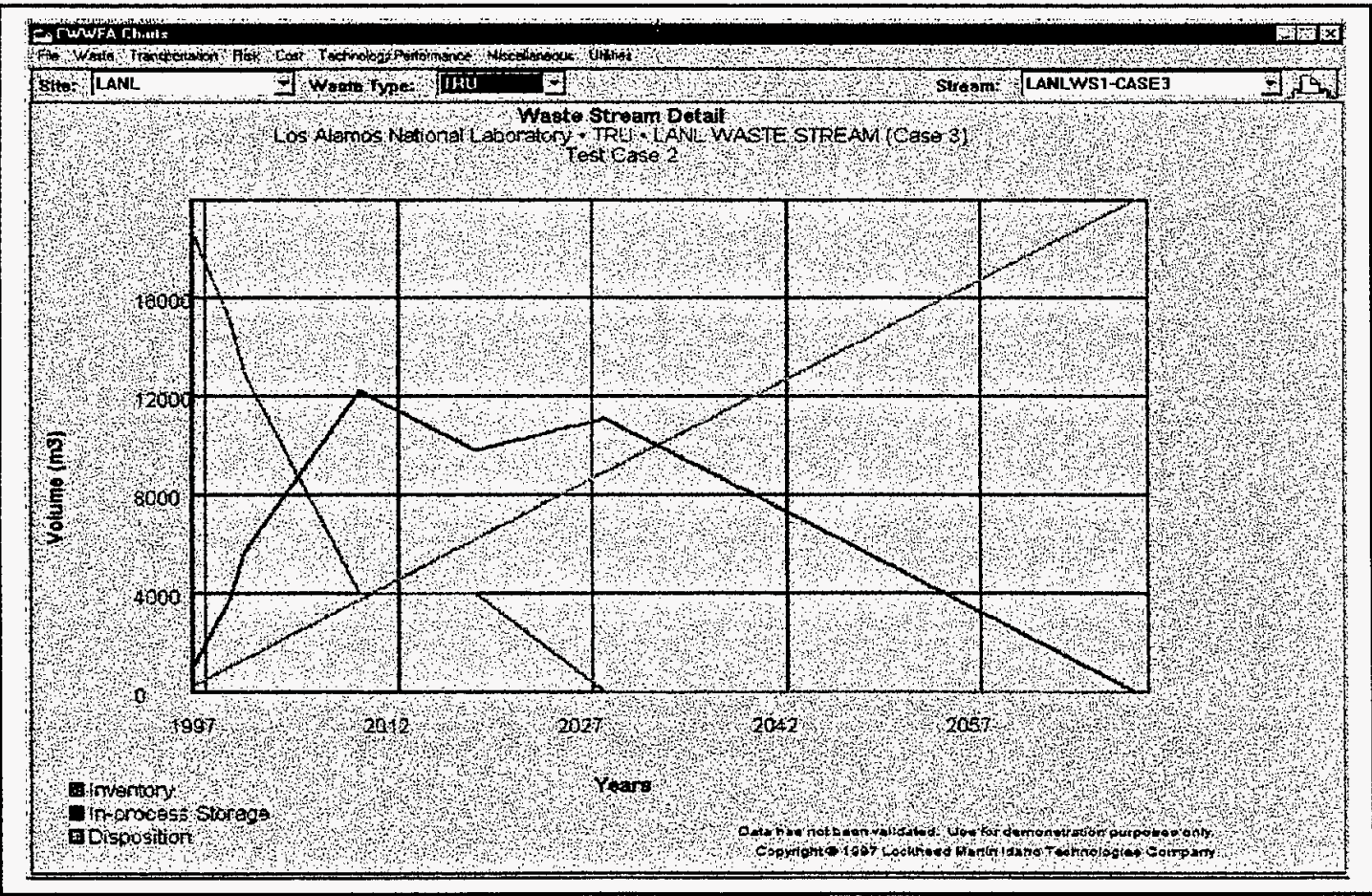


SHIPMENTS RECEIVED EXCEL CHART (LANL):

\section{Shipments Received}

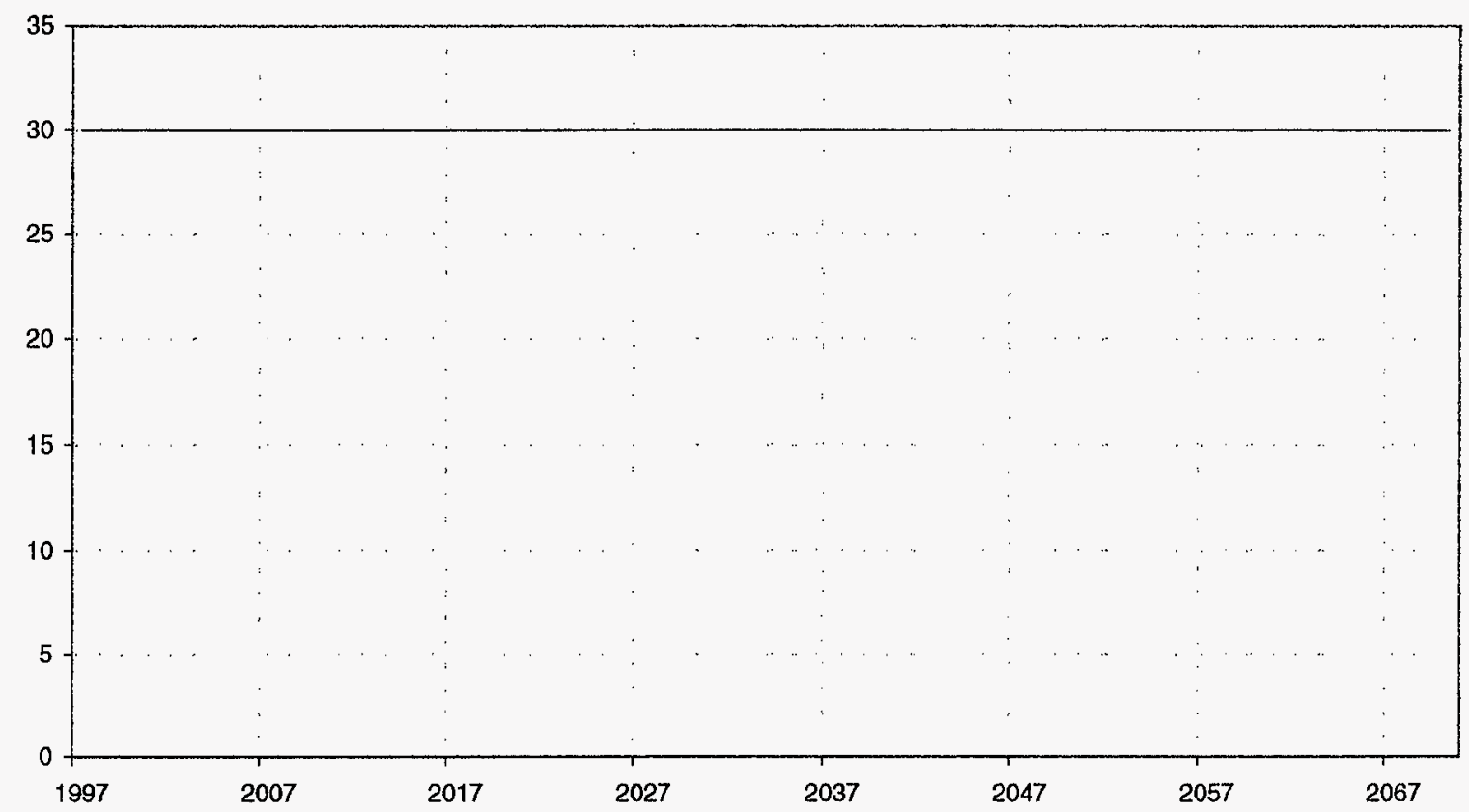

\section{SHIPMENTS RECEIVED CWWFA CHART:}

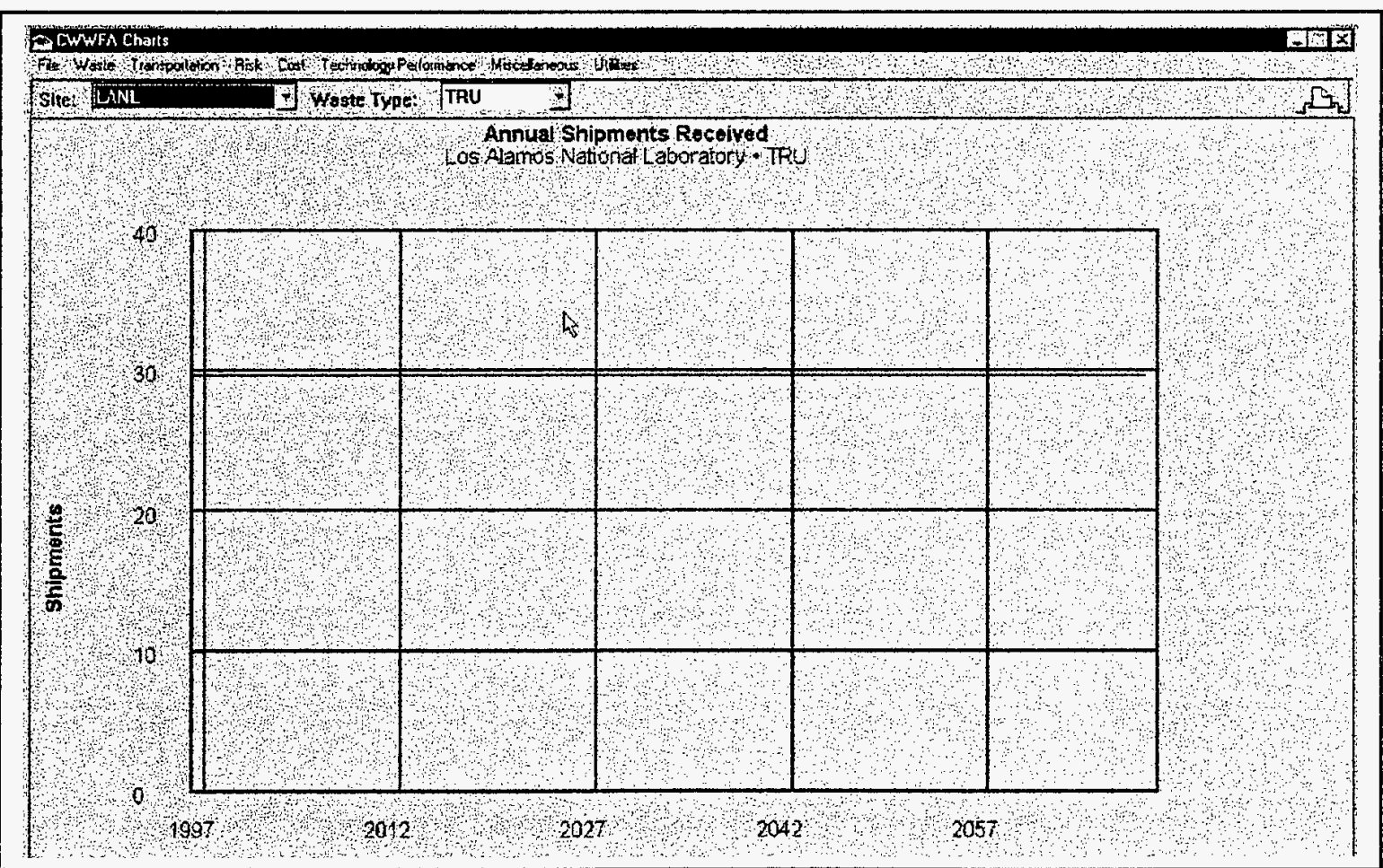


SHIPMENTS SENT EXCEL CHART (LANL):

\section{Shipments Sent}

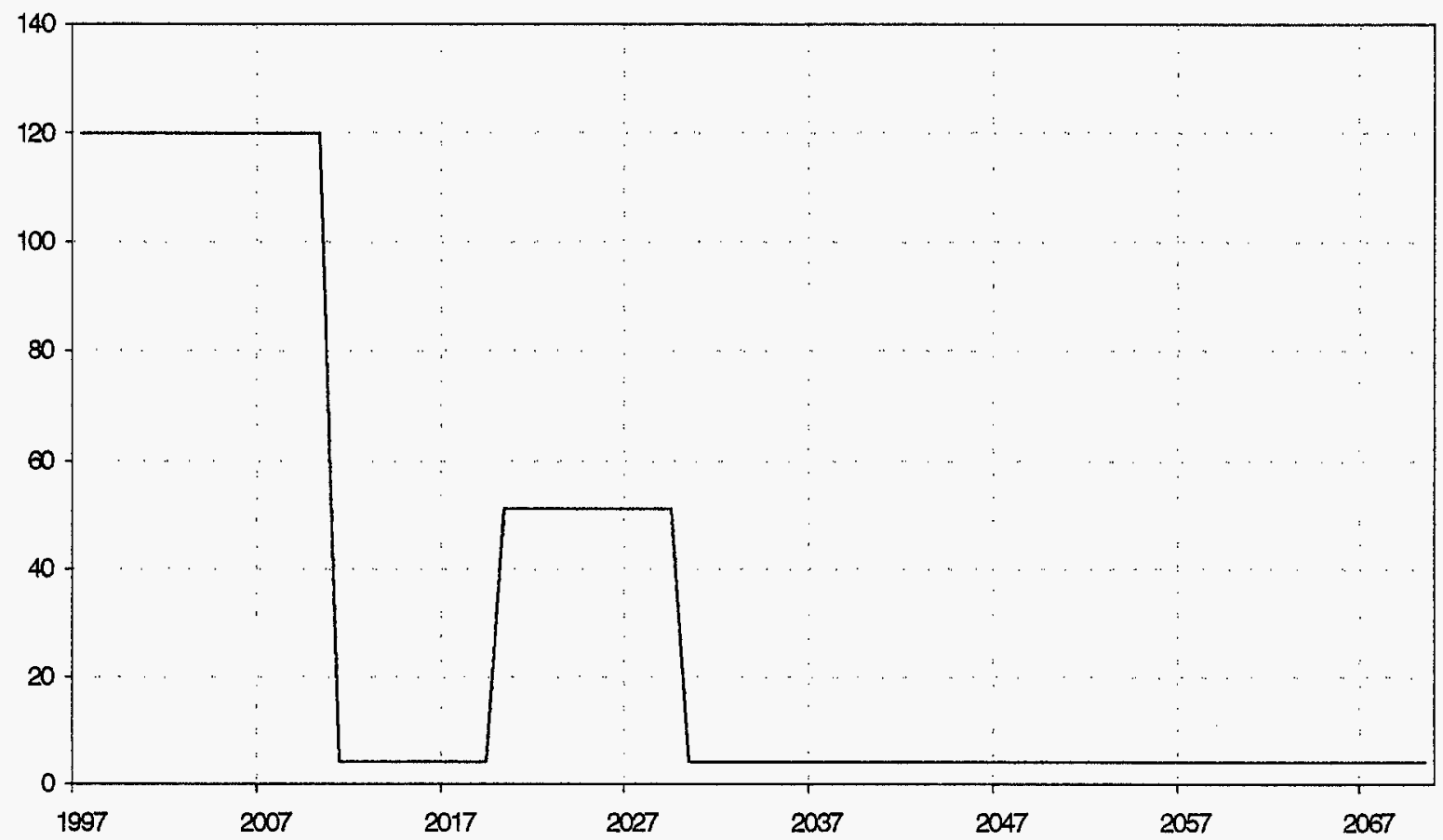

SHIPMENTS SENT CWWFA CHART:

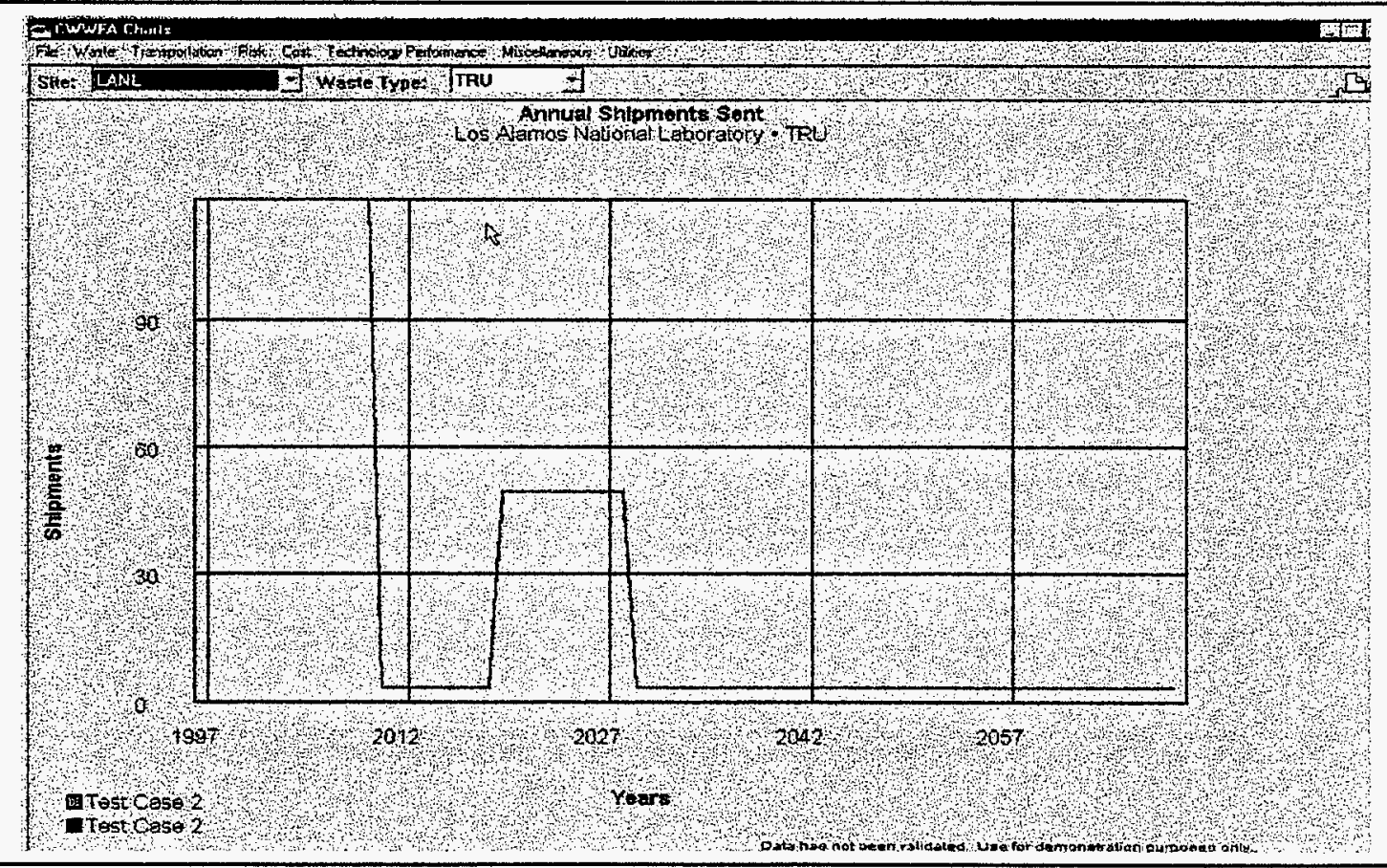


TRANSPORTAION COMPARISON

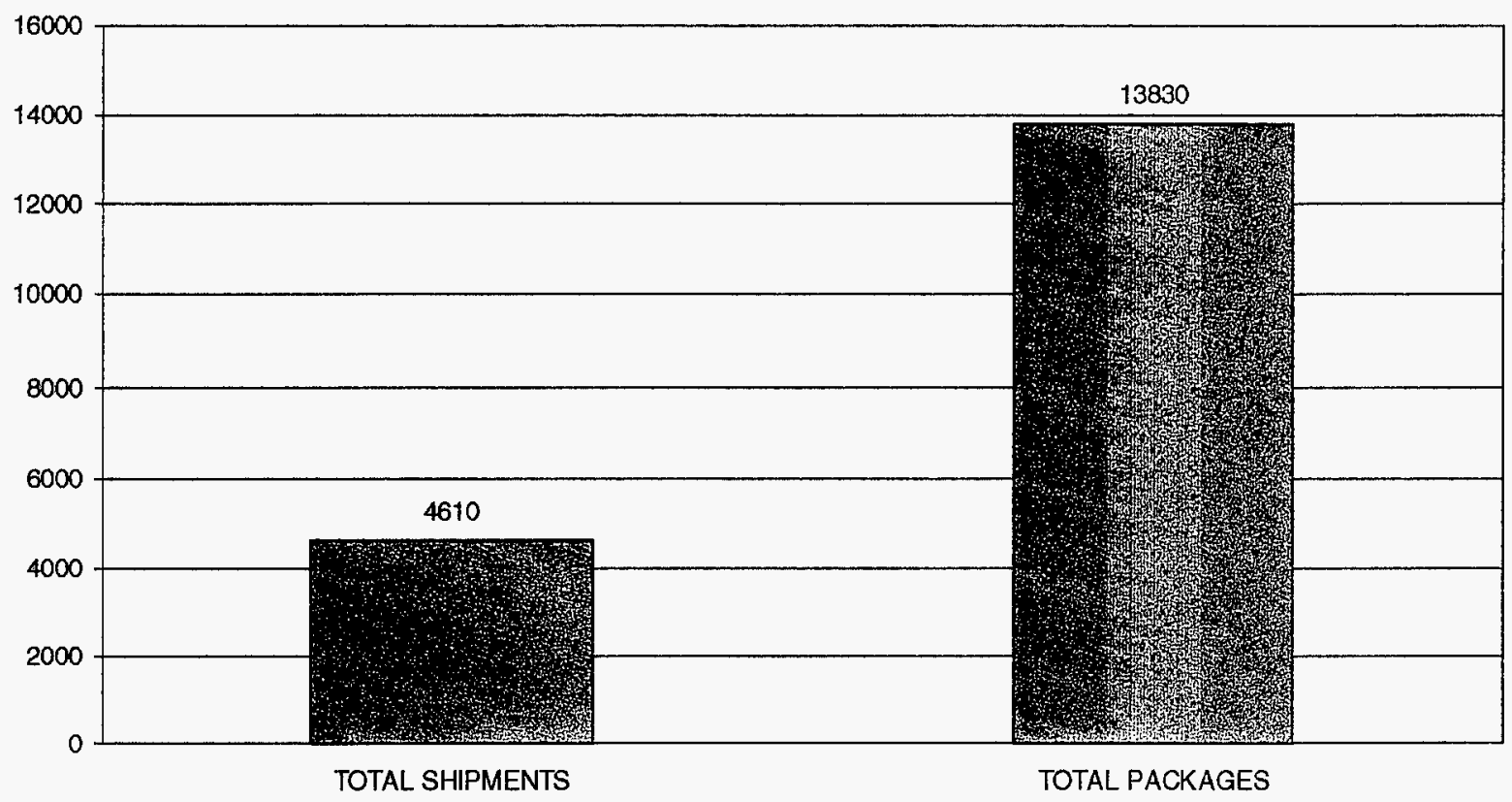

TRANSPORTATION COMPARISON CWWFA CHART:

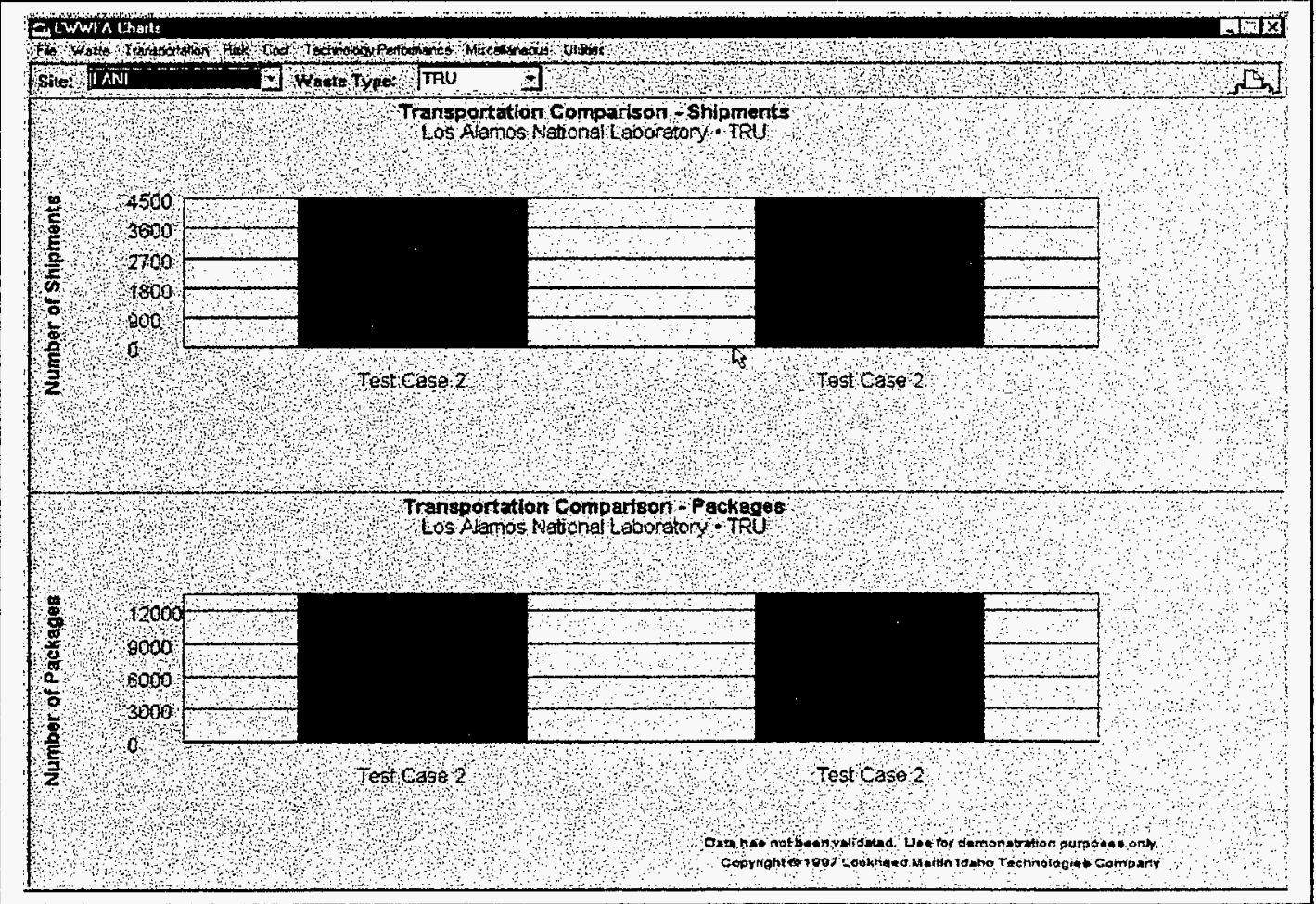


1.0 Waste Stream Information

\begin{tabular}{|l|l|}
\hline Originating Site & HASI \\
\hline Waste Stream ID \& Name & HASIWS1-CASE4 \\
\hline Originating Type & HLW \\
\hline Legacy volume (m3) & 45,000 \\
\hline
\end{tabular}

1.1 New Generation

\begin{tabular}{|l|l|l|}
\hline \multicolumn{1}{|c|}{ Start Year } & \multicolumn{1}{|c|}{ End Year } & \multicolumn{1}{c|}{ Annual Volume (m3) } \\
\hline NA & NA & NA \\
\hline & & \\
\hline & & \\
\hline & & \\
\hline
\end{tabular}

1.2 Shipping Route(s)

\begin{tabular}{|l|l|l|}
\hline \multicolumn{1}{|c|}{ Route ID } & \multicolumn{1}{|c|}{ Source Facility } & \multicolumn{1}{c|}{ Destination Facility } \\
\hline C4a-1 & Legacy-Hanf & SNLFAC1-CASE4 \\
\hline C4a-2 & Legacy-Hanf & HASIFAC1-CASE4 \\
\hline C4a-3 & SNLFAC1-CASE4 & NVTSFAC1-CASE4 \\
\hline C4a-4 & SNLFAC1-CASE4 & HASIFAC3-CASE4 \\
\hline C4a-5 & HASIFAC1-CASE4 & SNLFAC2-CASE4 \\
\hline C4a-6 & HASIFAC1-CASE4 & HASIFAC2-CASE4 \\
\hline
\end{tabular}

1.3 Shipping Schedule(s)

\begin{tabular}{|c|c|c|c|}
\hline Route ID: C4a-1 & \multicolumn{2}{|c|}{} & \multicolumn{1}{|c|}{ Waste Type } \\
\hline Start Year & \multicolumn{1}{|c|}{ End Year } & Annual Volume (m3) \\
\hline 1997 & 2010 & HLW & 1607 \\
\hline & & & \\
\hline & & & \\
\hline
\end{tabular}

1.3 Shipping Schedule(s) (continued)

\begin{tabular}{|c|c|c|c|}
\hline Route ID: C4a-2 & \multicolumn{2}{|c|}{} & \multicolumn{1}{|c|}{ Waste Type } \\
\hline Start Year & \multicolumn{1}{|c|}{ End Year } & Annual Volume (m3) \\
\hline 1997 & 2010 & HLW & 1607 \\
\hline & & & \\
\hline & & & \\
\hline
\end{tabular}

1.3 Shipping Schedule(s) (continued)

\begin{tabular}{|c|c|c|c|}
\hline Route ID: C4a-3 & \multicolumn{2}{|c|}{} & \multicolumn{1}{|c|}{ Annual Volume (m3) } \\
\hline Start Year & \multicolumn{1}{|c|}{ End Year } & Waste Type & 1125 \\
\hline & 2020 & LLW & \\
\hline & & & \\
\hline & & & \\
\hline
\end{tabular}

1.3 Shipping Schedule(s) (continued)

\begin{tabular}{|c|c|c|c|}
\hline Route ID: C4a-4 & & & \\
\hline Start Year & End Year & Waste Type & Annual Volume (m3) \\
\hline 2021 & 2030 & LLW & 1125 \\
\hline & & & \\
\hline & & & \\
\hline
\end{tabular}


CASE: Case $4 \mathrm{a}$

INITIAL PARAMETERS \& RESULTS REPORT

DATE: $11 / 13 / 97$

Evaluator: K B Oswald

1.3 Shipping Schedule(s) (continued)

\begin{tabular}{|c|c|c|c|}
\hline Route ID: C4a-5 & \multicolumn{2}{|c|}{ Waste Type } & \multicolumn{1}{|c|}{ Annual Volume (m3) } \\
\hline Start Year & \multicolumn{1}{|c|}{ End Year } & 2025 \\
\hline & 2020 & TRU & \\
\hline & & & \\
\hline & & & \\
\hline
\end{tabular}

1.3 Shipping Schedule(s) (continued)

\begin{tabular}{|c|c|c|c|}
\hline Route ID: $\mathrm{C} 4 \mathrm{a}-6$ & & & \\
\hline Start Year & End Year & Waste Type & Annual Volume (m3) \\
\hline 2021 & 2030 & TRU & 225 \\
\hline & & & \\
\hline & 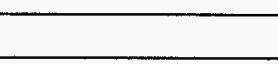 & 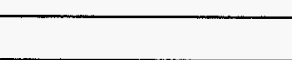 & \\
\hline
\end{tabular}

2.0 Facility Information

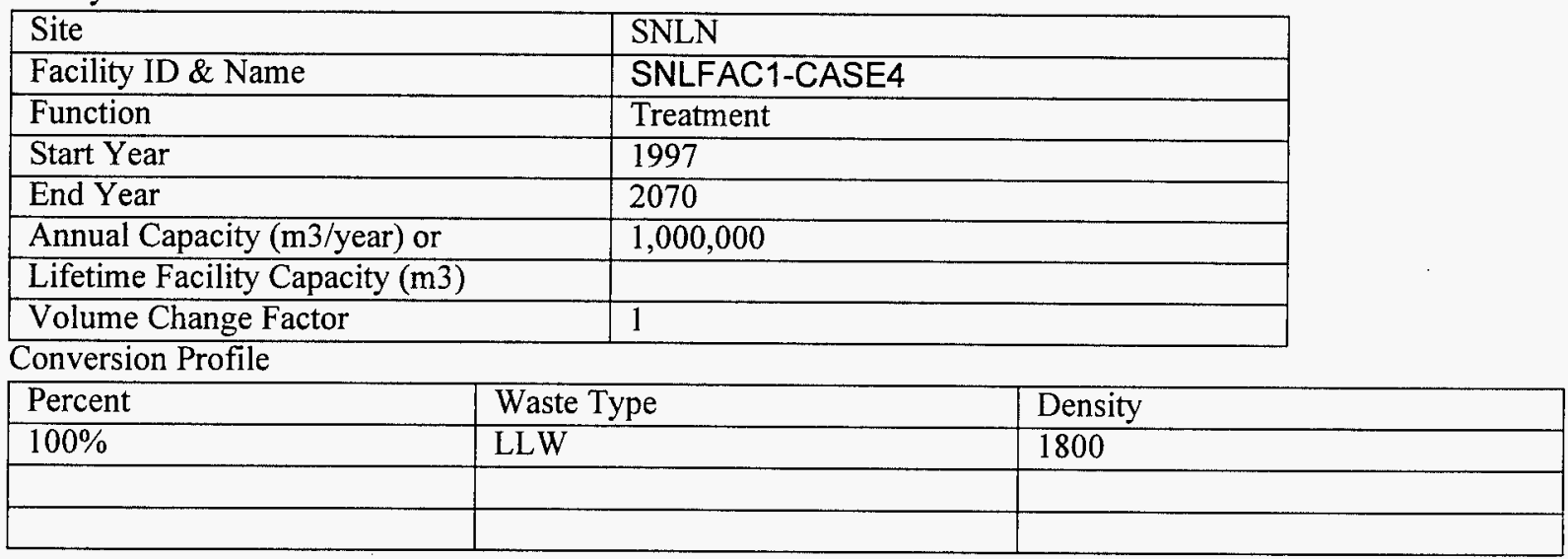

2.0 Facility Information

\begin{tabular}{|l|l|}
\hline Site & HASI \\
\hline Facility ID \& Name & HASIFAC1-CASE4 \\
\hline Function & Treatment \\
\hline Start Year & 1997 \\
\hline End Year & 2070 \\
\hline Annual Capacity (m3/year) or & $1,000,000$ \\
\hline Lifetime Facility Capacity (m3) & \\
\hline Volume Change Factor & 1 \\
\hline \\
\begin{tabular}{|l|l|l|}
\hline Ponversion Profile & Waste Type & Density \\
\hline 100 & TRU & 1800 \\
\hline & & \\
\hline
\end{tabular}
\end{tabular}

2.0 Facility Information

\begin{tabular}{|l|l|}
\hline Site & NVTS \\
\hline Facility ID \& Name & NVTSFAC1-CASE4 \\
\hline Function & Disposal \\
\hline Start Year & 1997 \\
\hline End Year & 2070 \\
\hline Annual Capacity (m3/year) or & $1,000,000$ \\
\hline Lifetime Facility Capacity (m3) & \\
\hline Volume Change Factor & 1 \\
\hline \\
\begin{tabular}{|l|l|l|}
\hline Ponversion Profile & Waste Type & Density \\
\hline NA & NA & NA \\
\hline
\end{tabular} \\
\hline
\end{tabular}




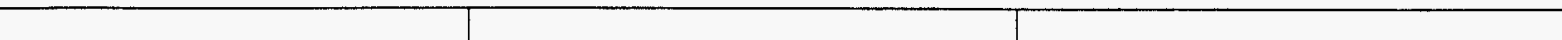

2.0 Facility Information

\begin{tabular}{|l|l|}
\hline Site & HASI \\
\hline Facility ID \& Name & HASIFAC3-CASE4 \\
\hline Function & Disposal \\
\hline Start Year & 1997 \\
\hline End Year & 2070 \\
\hline Annual Capacity (m3/year) or & $1,000,000$ \\
\hline Lifetime Facility Capacity (m3) & \\
\hline Volume Change Factor & 1 \\
\hline
\end{tabular}

Conversion Profile

\begin{tabular}{|l|l|l|}
\hline Percent & Waste Type & Density \\
\hline NA & NA & NA \\
\hline & & \\
\hline
\end{tabular}

2.0 Facility Information

\begin{tabular}{|l|l|}
\hline Site & SNLN \\
\hline Facility ID \& Name & SNLFAC2-CASE4 \\
\hline Function & Disposal \\
\hline Start Year & 1997 \\
\hline End Year & 2070 \\
\hline Annual Capacity (m3/year) or & $1,000,000$ \\
\hline Lifetime Facility Capacity (m3) & \\
\hline Volume Change Factor & 1 \\
\hline
\end{tabular}

Conversion Profile

\begin{tabular}{|l|l|l|}
\hline Percent & Waste Type & Density \\
\hline NA & NA & NA \\
\hline & & \\
\hline
\end{tabular}

2.0 Facility Information

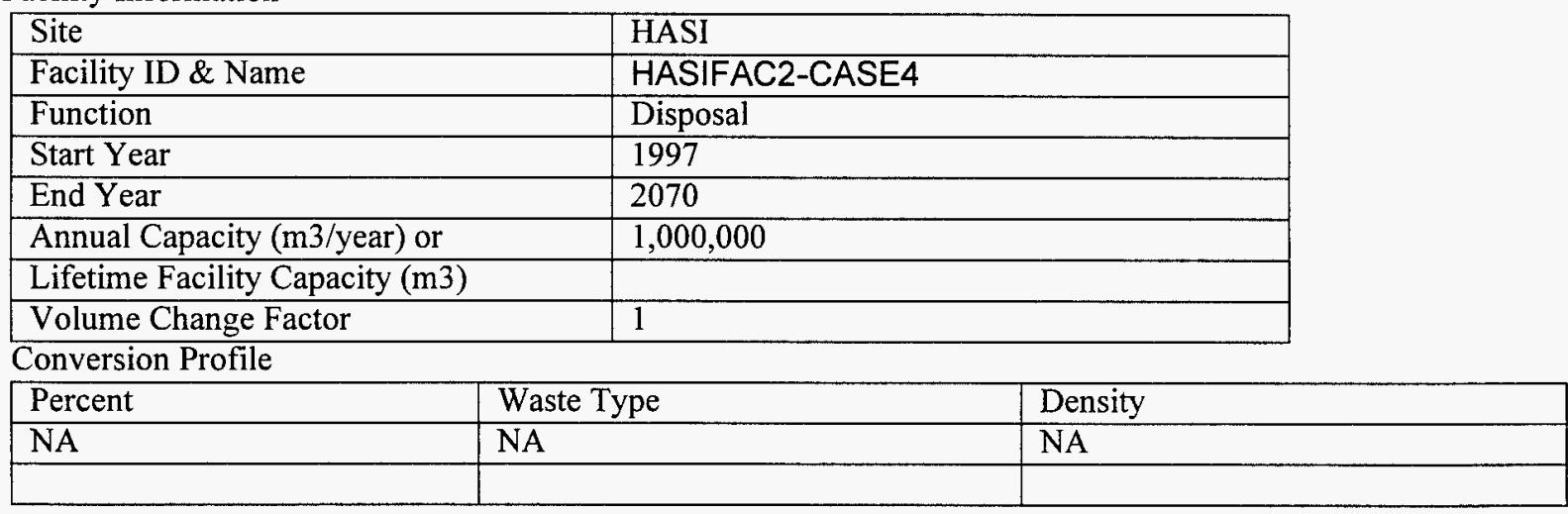


WASTE DISPOSITION - EXCEL CHART (HASI, HLW):

\section{Waste Disposition}

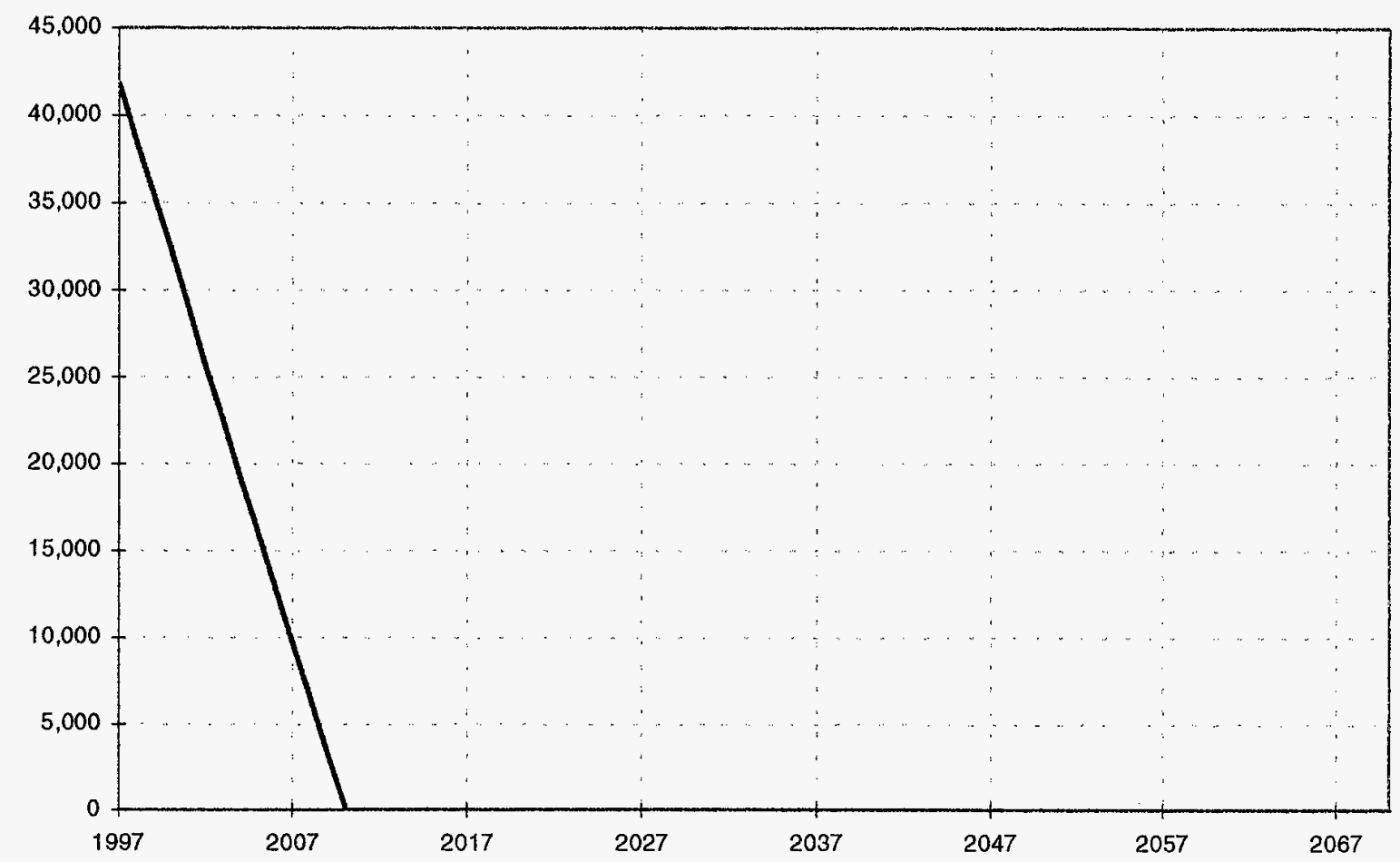

\section{WASTE DISPOSITION - CWWFA CHART:}

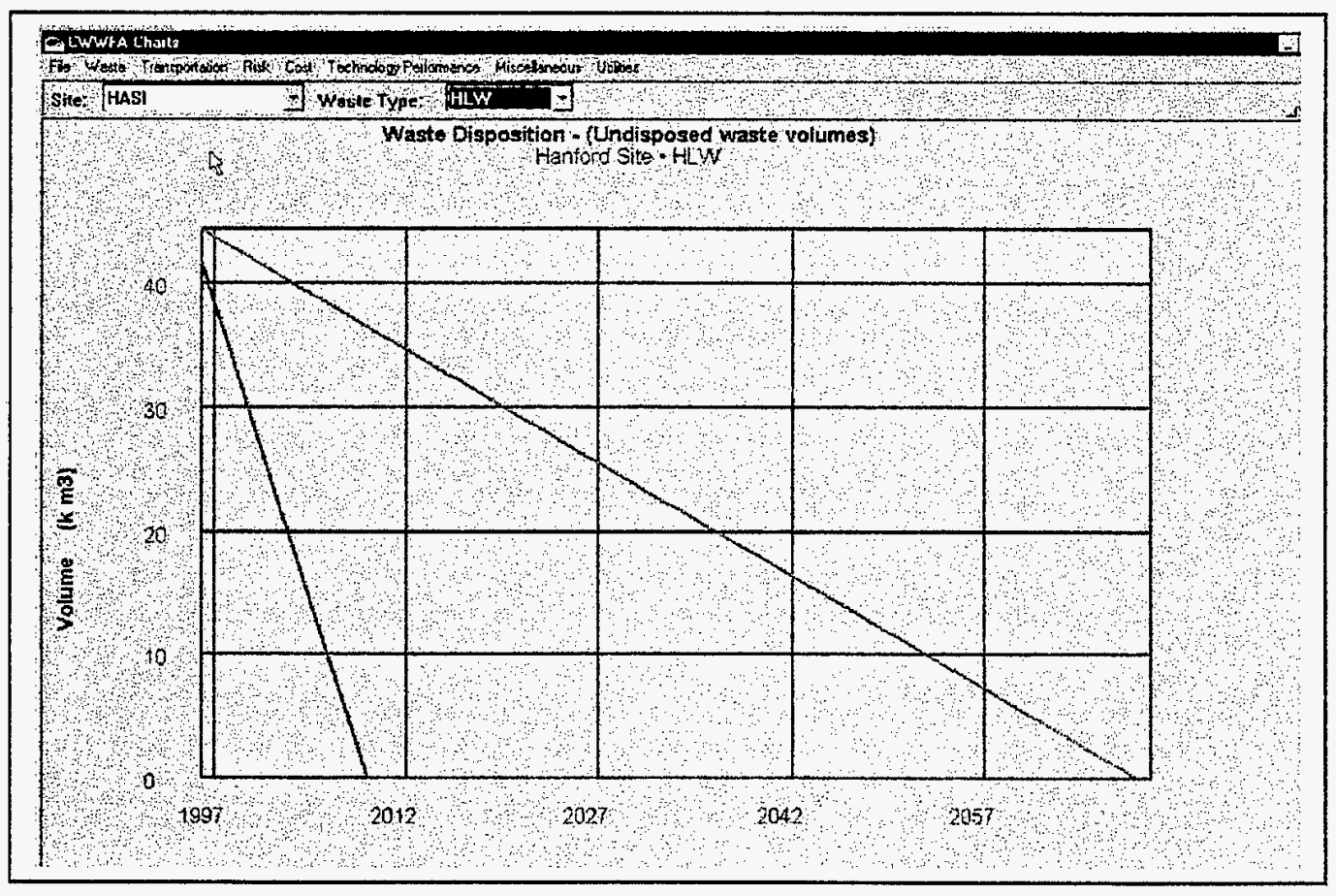




\section{FACILITY CAPACITY EXCEL CHART (NVTS, NVTSFAC1-CASE4):}

\section{Facility Capacity Detail}

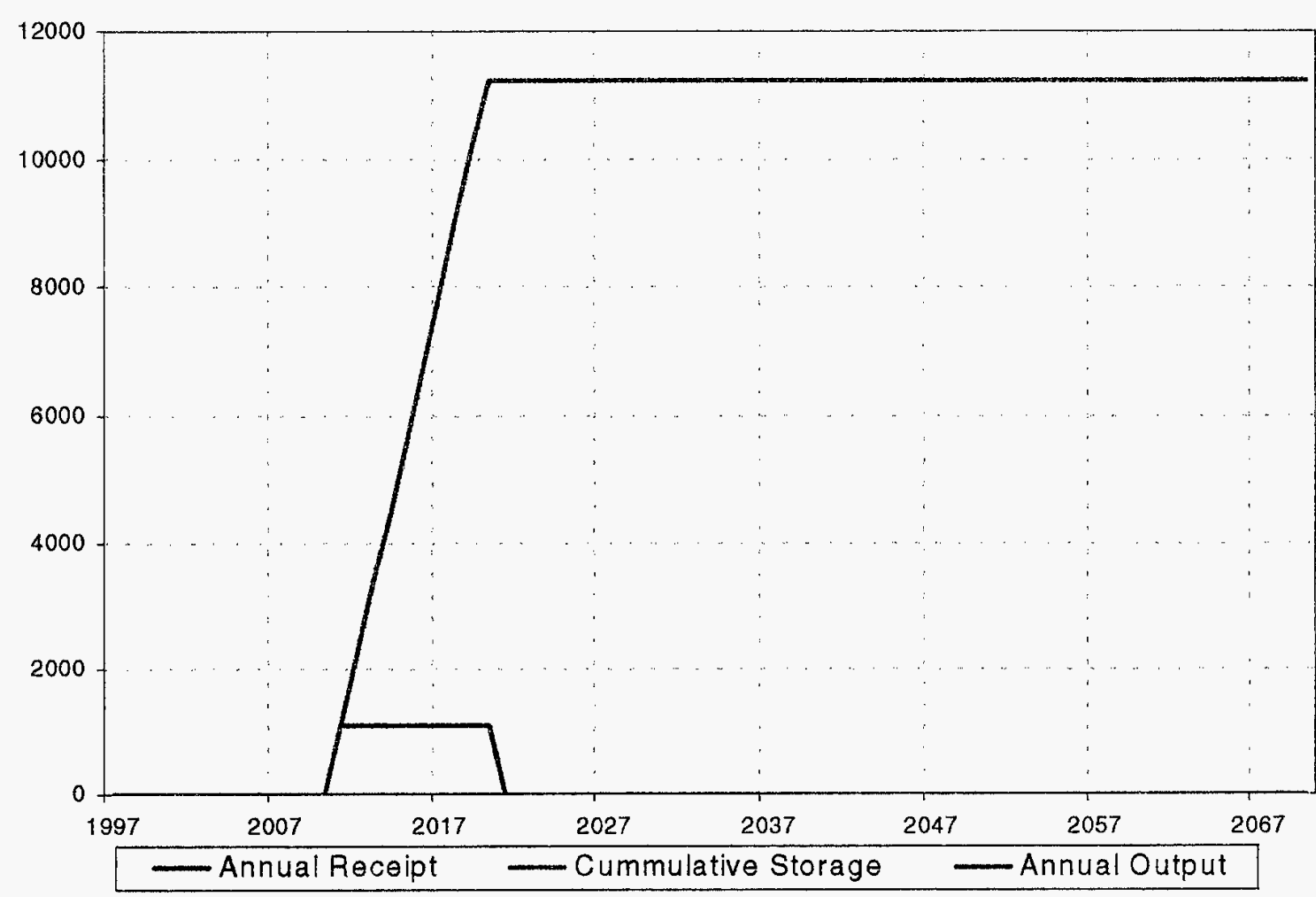

\section{FACILITY CAPACITY CWWFA CHART:}

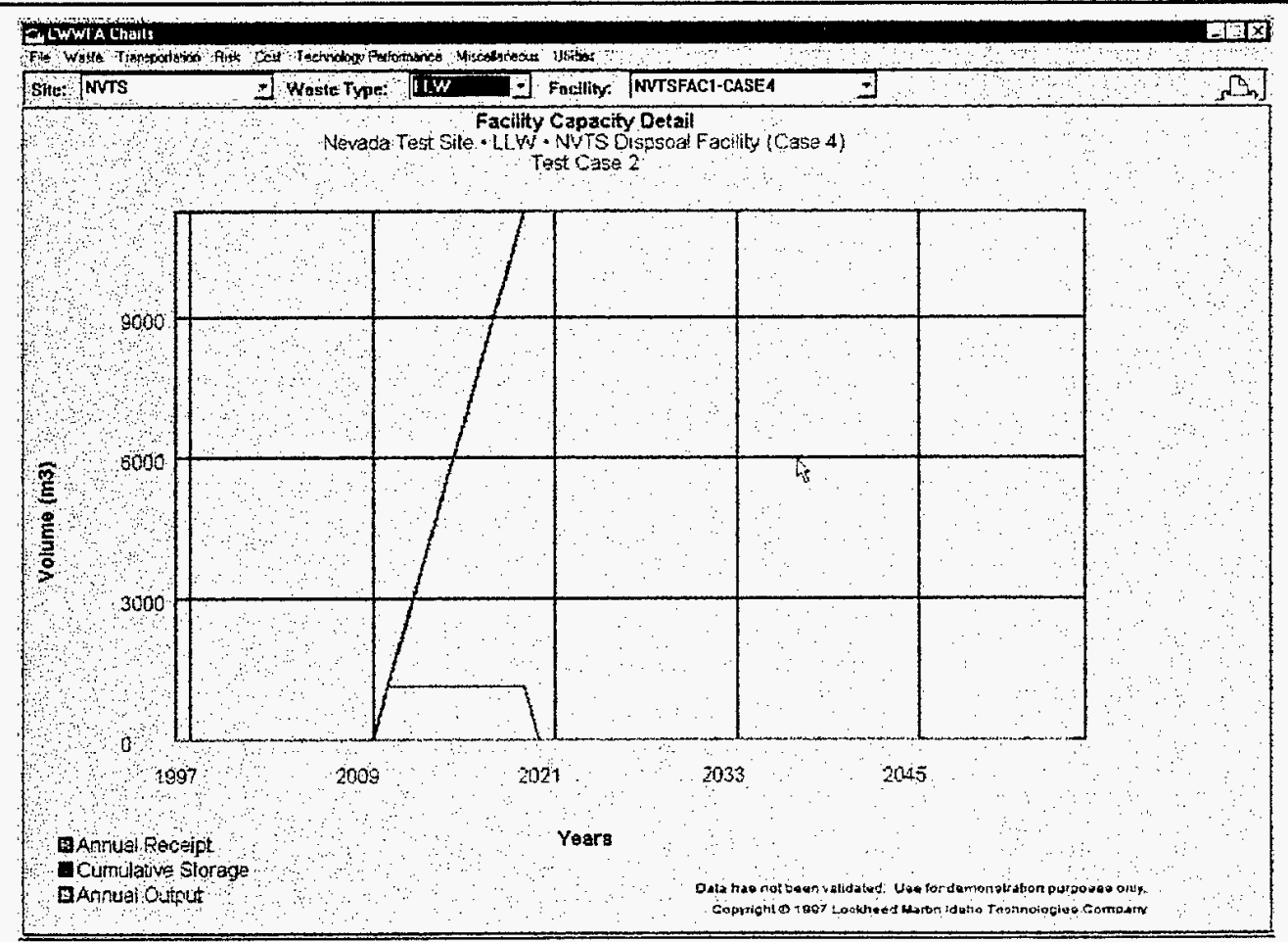


WASTE STREAM DETAIL EXCEL CHART (HASIWS1-CASE4):

\section{Waste Stream Detail}

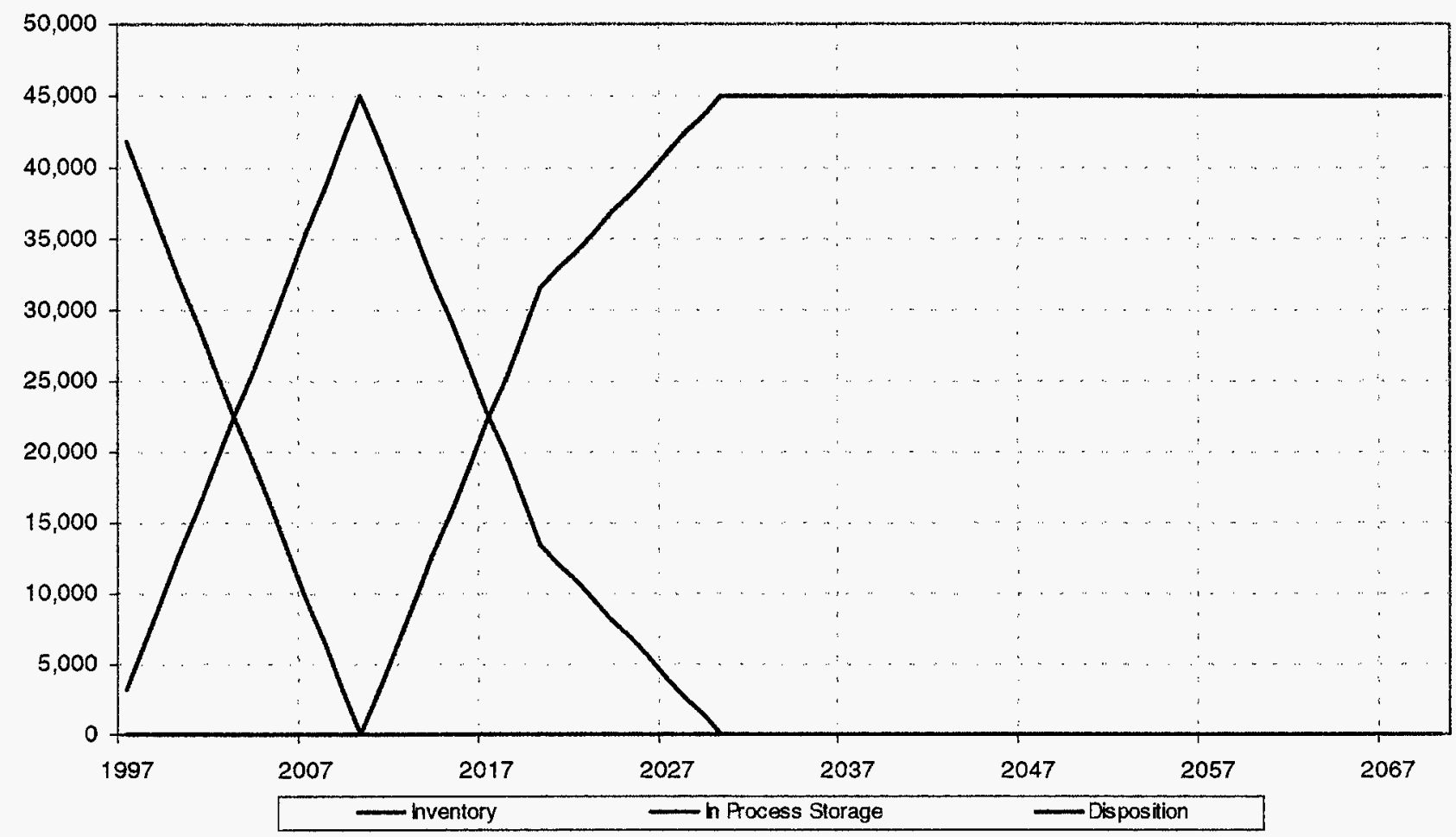

WASTE STREAM DETAIL CWWFA CHART:

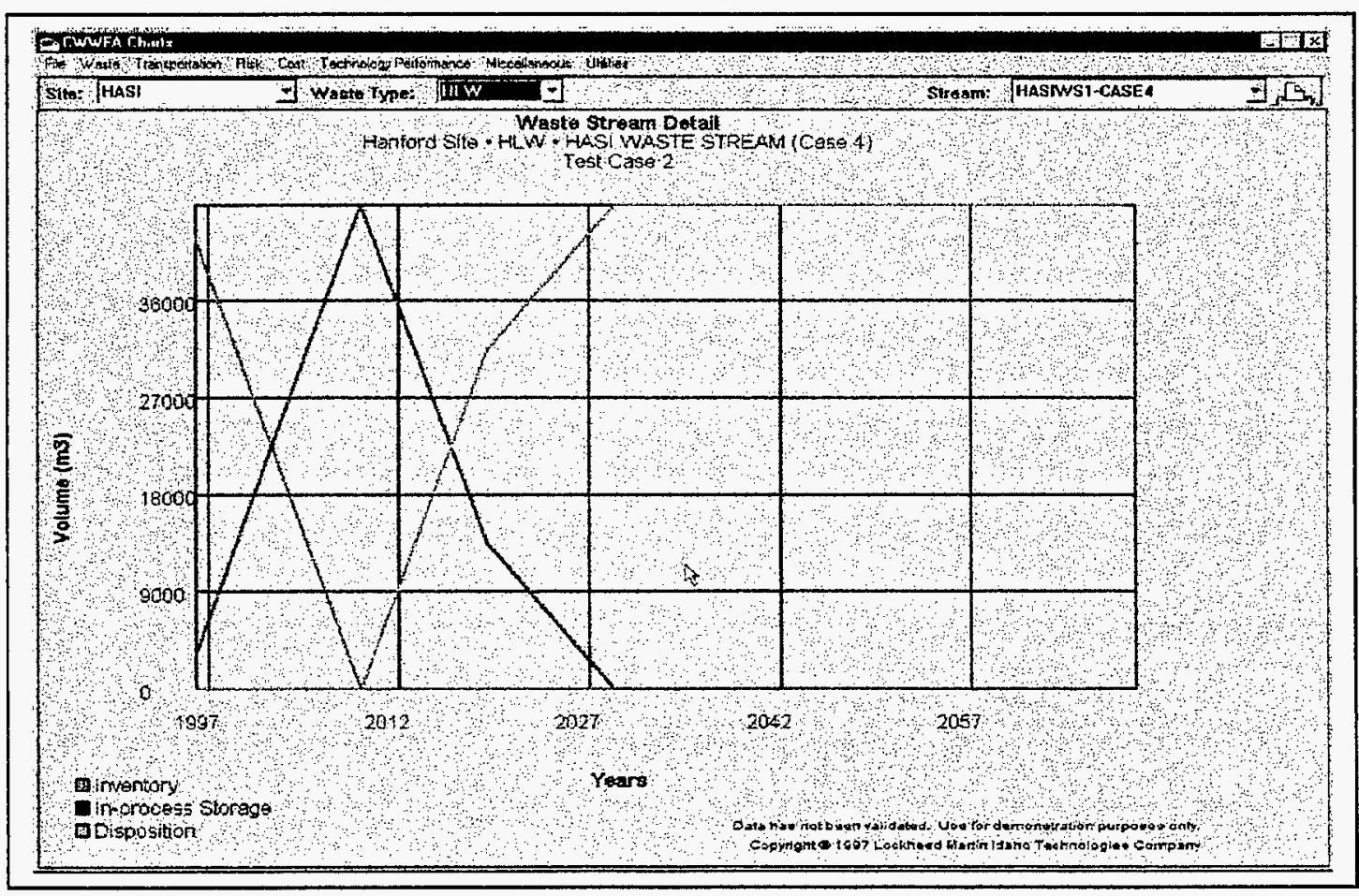


SHIPMENTS RECEIVED EXCEL CHART (SNLN, TRU):

\section{Shipments Received}

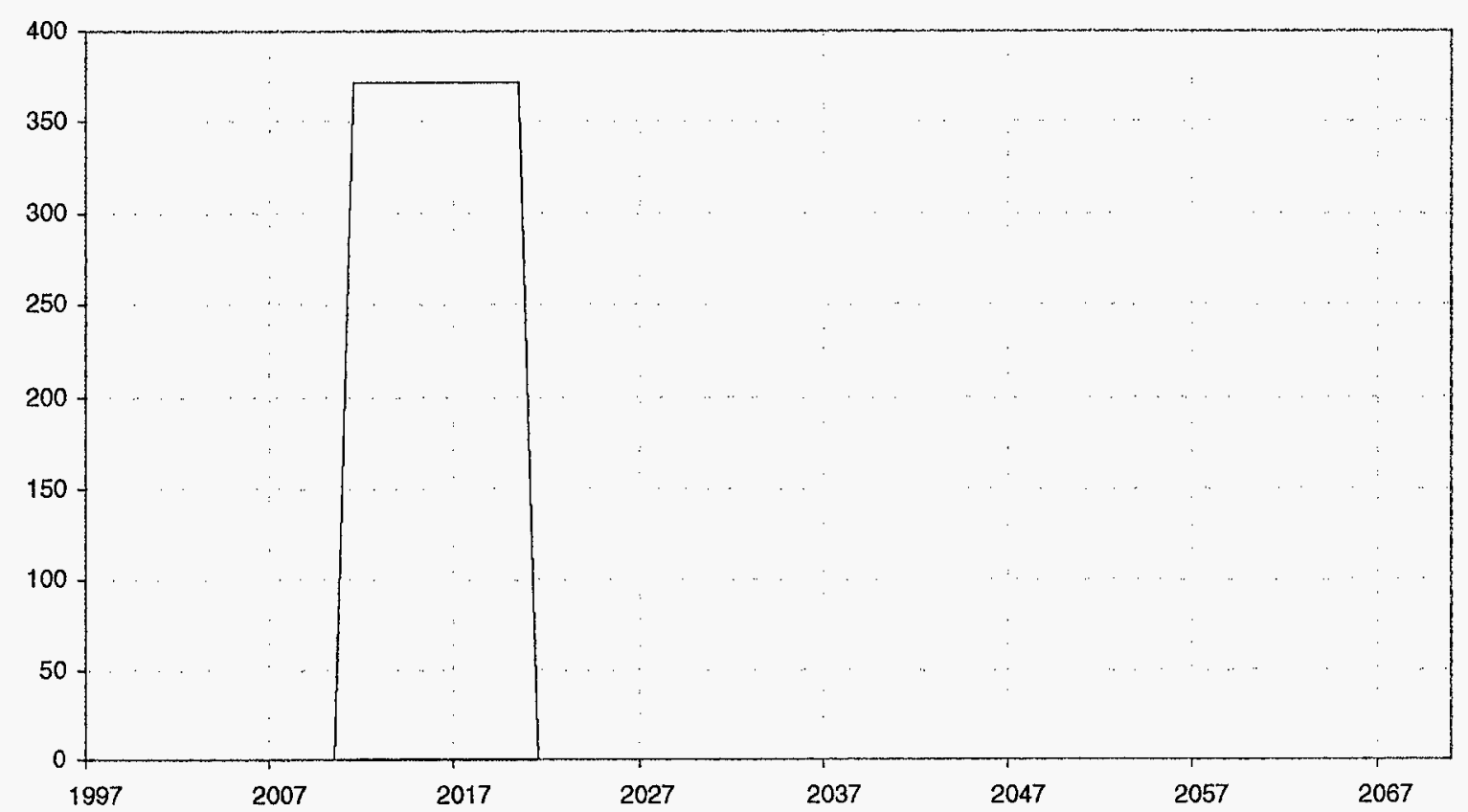

SHIPMENTS RECEIVED CWWFA CHART:

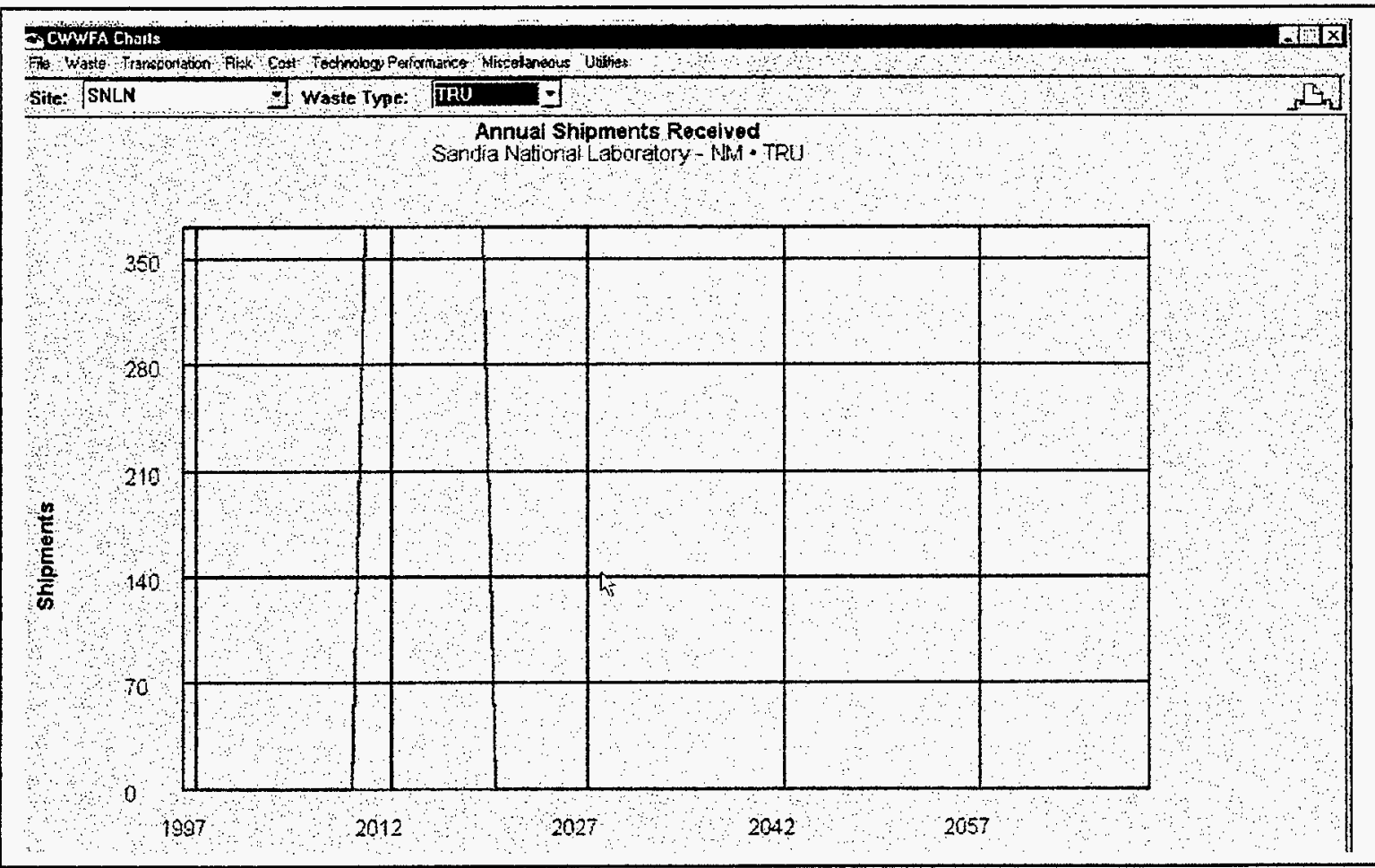


INITIAL PARAMETERS \& RESULTS REPORT
CASE: Case $4 a$
DATE: $11 / 13 / 97$
Evaluator: K B Oswald

SHIPMENTS SENT EXCEL CHART (SNLN):

\section{Shipments Sent}

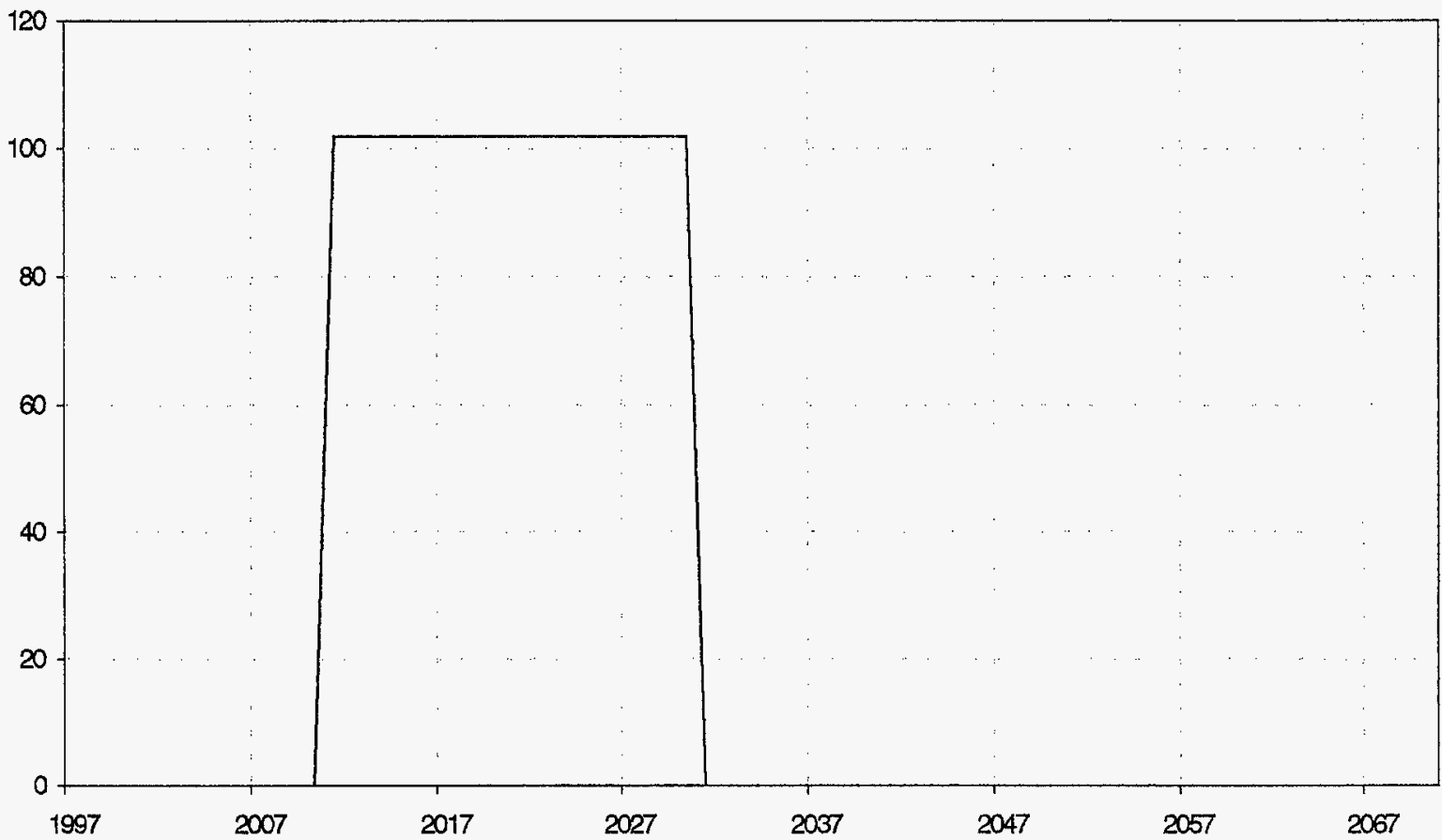

\section{SHIPMENTS SENT CWWFA CHART:}

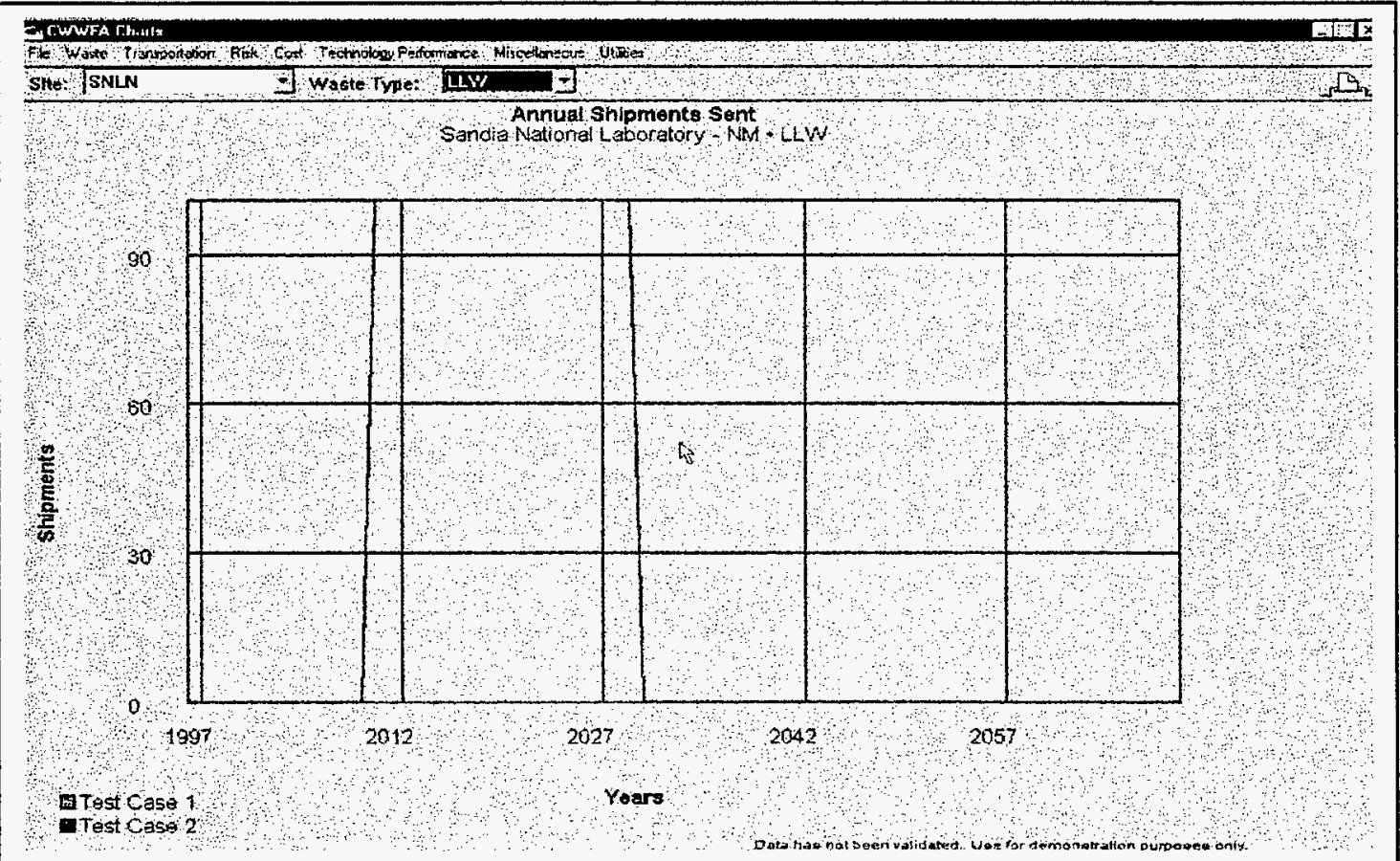


INITIAL PARAMETERS \& RESULTS REPORT

CASE: Case $4 a$

DATE: $11 / 13 / 97$

Evaluator: K B Oswald

TRANSPORTATION COMPARISON EXCEL CHART (SNLN, LLW):

TRANSPORTAION COMPARISON

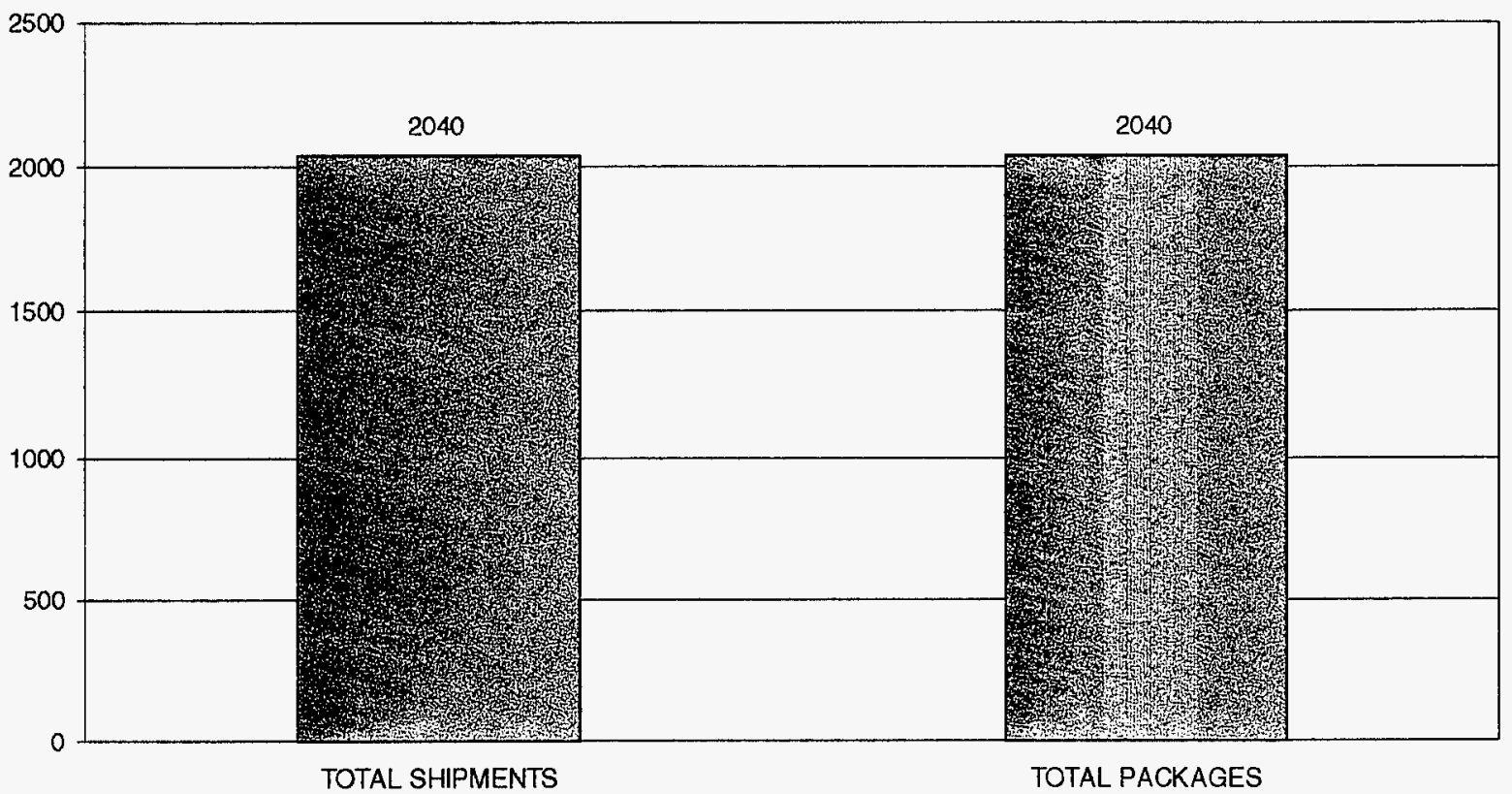

TRANSPORTATION COMPARISON CWWFA CHART:

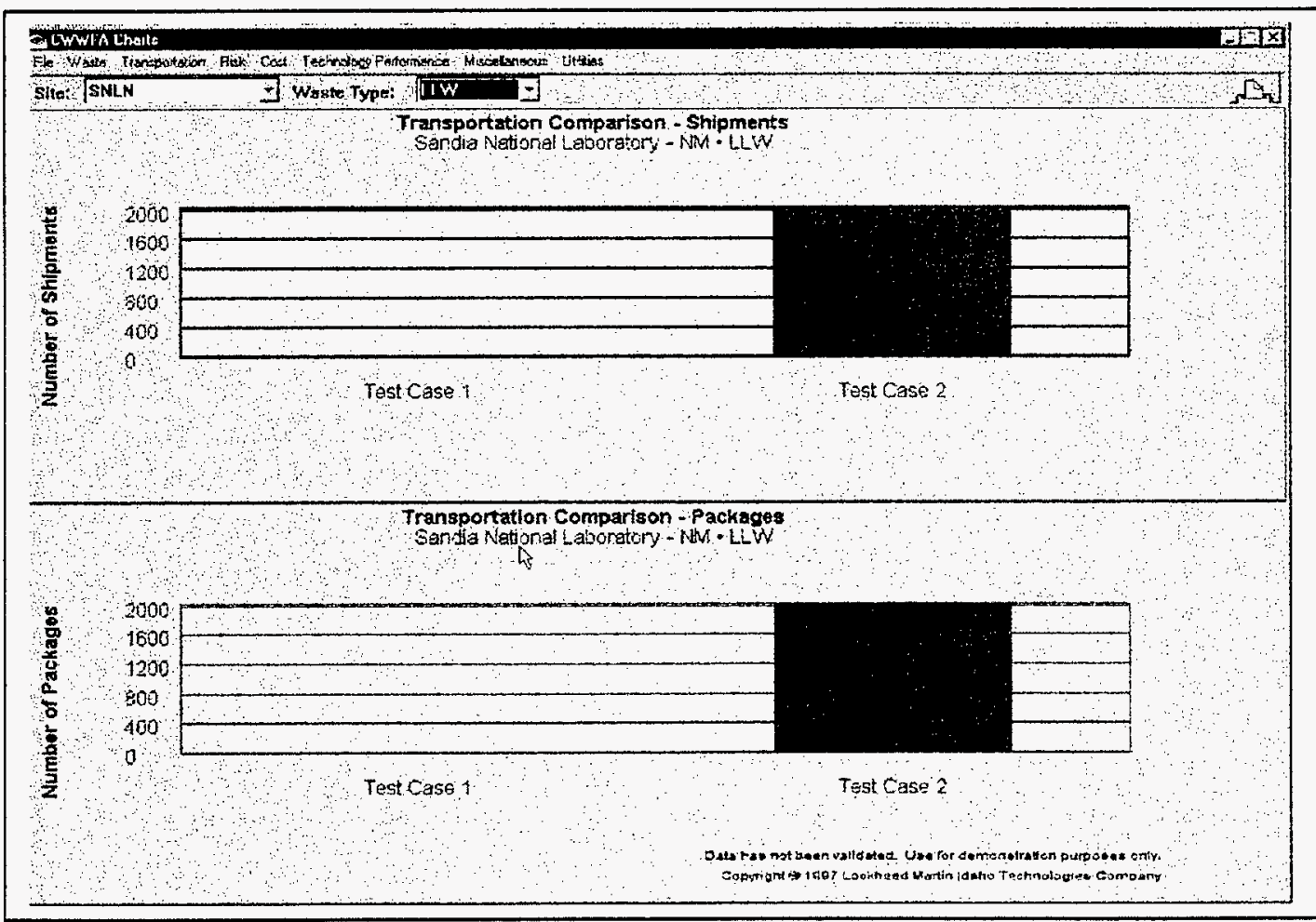


CASE: Case $4 b$

INITIAL PARAMETERS \& RESULTS REPORT

DATE: $11 / 13 / 97$

Evaluator: K B Oswald

1.0 Waste Stream Information

\begin{tabular}{|l|l|}
\hline Originating Site & HASI \\
\hline Waste Stream ID \& Name & HASIWS1-CASE4 \\
\hline Originating Type & HLW \\
\hline Legacy volume (m3) & 45,000 \\
\hline
\end{tabular}

1.1 New Generation

\begin{tabular}{|l|l|l|}
\hline \multicolumn{1}{|c|}{ Start Year } & \multicolumn{1}{|c|}{ End Year } & Annual Volume (m3) \\
\hline NA & NA & NA \\
\hline & & \\
\hline & & \\
\hline & & \\
\hline
\end{tabular}

1.2 Shipping Route(s)

\begin{tabular}{|l|l|l|}
\hline \multicolumn{1}{|c|}{ Route ID } & \multicolumn{1}{|c|}{ Source Facility } & \multicolumn{1}{c|}{ Destination Facility } \\
\hline C4a-1 & Legacy-Hanf & SNLFAC1-CASE4 \\
\hline C4a-2 & Legacy-Hanf & HASIFAC1-CASE4 \\
\hline C4a-3 & SNLFAC1-CASE4 & NVTSFAC1-CASE4 \\
\hline C4a-4 & SNLFAC1-CASE4 & HASIFAC3-CASE4 \\
\hline C4a-5 & HASIFAC1-CASE4 & SNLFAC2-CASE4 \\
\hline C4a-6 & HASIFAC1-CASE4 & HASIFAC2-CASE4 \\
\hline
\end{tabular}

1.3 Shipping Schedule(s)

\begin{tabular}{|c|c|c|c|}
\hline Route ID: C4a-1 & \multicolumn{2}{|c|}{ Waste Type } & Annual Volume (m3) \\
\hline Start Year & \multicolumn{1}{|c|}{ End Year } & HLW & 1607 \\
\hline 1997 & 2010 & & \\
\hline & & & \\
\hline & & & \\
\hline
\end{tabular}

1.3 Shipping Schedule(s) (continued)

\begin{tabular}{|c|l|l|l|}
\hline Route ID: C4a-2 & \multicolumn{2}{|c|}{} & \multicolumn{1}{|c|}{ Wnnual Volume (m3) } \\
\hline Start Year & \multicolumn{1}{|c|}{ End Year } & HLW & 1607 \\
\hline 1997 & 2010 & & \\
\hline & & & \\
\hline & & & \\
\hline
\end{tabular}

1.3 Shipping Schedule(s) (continued)

\begin{tabular}{|c|c|c|c|}
\hline Route ID: C4a-3 & \multicolumn{2}{|c|}{ Waste Type } & Annual Volume (m3) \\
\hline Start Year & \multicolumn{1}{|c|}{ End Year } & 1125 \\
\hline 2011 & 2020 & LLW & \\
\hline & & & \\
\hline & & & \\
\hline
\end{tabular}

1.3 Shipping Schedule(s) (continued)

\begin{tabular}{|c|c|c|c|}
\hline Route ID: C4a-4 & \multicolumn{2}{|c|}{ Waste Type } & Annual Volume (m3) \\
\hline Start Year & \multicolumn{1}{|c|}{ End Year } & HLW & 1125 \\
\hline & 2030 & & \\
\hline & & & \\
\hline & & & \\
\hline
\end{tabular}


1.3 Shipping Schedule(s) (continued)

\begin{tabular}{|c|c|c|c|}
\hline Route ID: C4a-5 & \multicolumn{2}{|c|}{ Waste Type } & \multicolumn{1}{|c|}{ Annual Volume (m3) } \\
\hline Start Year & \multicolumn{1}{|c|}{ End Year } & HLW & 2025 \\
\hline & 2020 & & \\
\hline & & & \\
\hline & & & \\
\hline
\end{tabular}

1.3 Shipping Schedule(s) (continued)

\begin{tabular}{|c|c|c|c|}
\hline Route ID: C4a-6 & \multicolumn{2}{|c|}{ Waste Type } & \multicolumn{1}{|c|}{ Annual Volume (m3) } \\
\hline Start Year & \multicolumn{1}{|c|}{ End Year } & LLW & 225 \\
\hline & 2030 & & \\
\hline & & & \\
\hline & & & \\
\hline
\end{tabular}

2.0 Facility Information

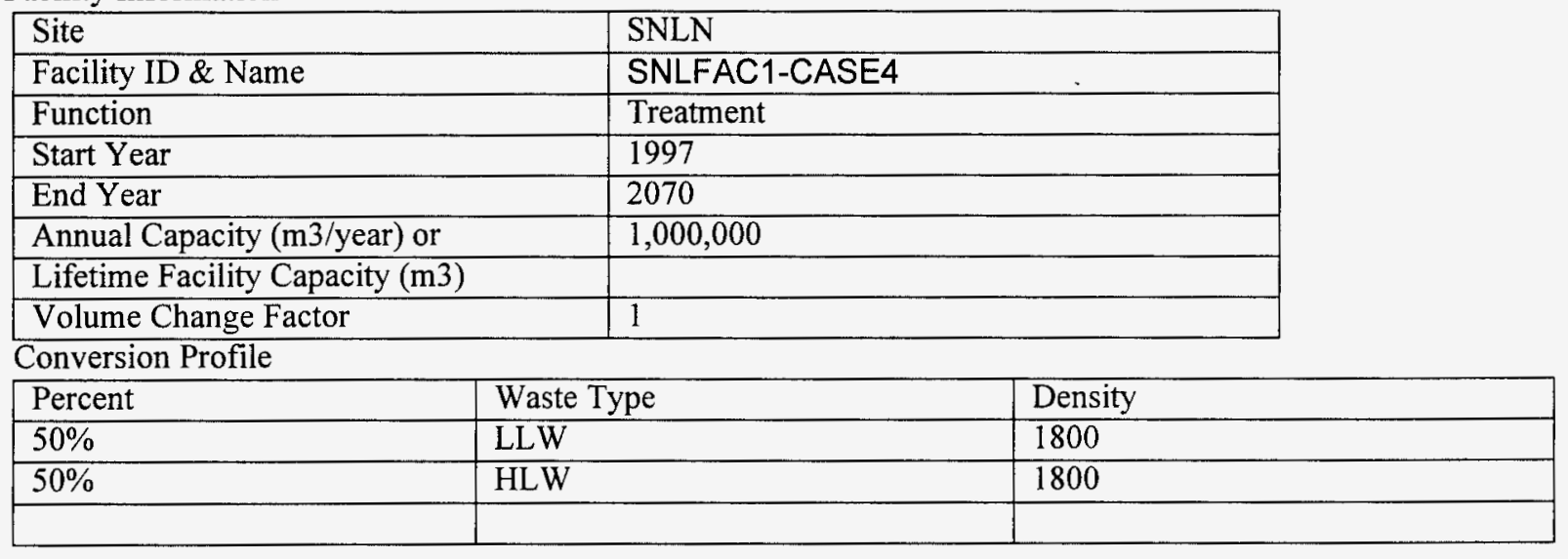

2.0 Facility Information

\begin{tabular}{|l|l|}
\hline Site & HASI \\
\hline Facility ID \& Name & HASIFAC1-CASE4 \\
\hline Function & Treatment \\
\hline Start Year & 1997 \\
\hline End Year & 2070 \\
\hline Annual Capacity (m3/year) or & $1,000,000$ \\
\hline Lifetime Facility Capacity (m3) & \\
\hline Volume Change Factor & 1 \\
\hline \\
\begin{tabular}{|l|l|l|}
\hline Ponversion Profile & Waste Type & Density \\
\hline 90 & HLW & 1800 \\
\hline 10 & LLW & 1800 \\
\hline
\end{tabular}
\end{tabular}

2.0 Facility Information

\begin{tabular}{|l|l|}
\hline Site & NVTS \\
\hline Facility ID \& Name & NVTSFAC1-CASE4 \\
\hline Function & Disposal \\
\hline Start Year & 1997 \\
\hline End Year & 2070 \\
\hline Annual Capacity (m3/year) or & $1,000,000$ \\
\hline Lifetime Facility Capacity (m3) & \\
\hline Volume Change Factor & 1 \\
\hline
\end{tabular}

Conversion Profile
\begin{tabular}{|l|l|l|}
\hline Percent & Waste Type & Density \\
\hline NA & NA & NA \\
\hline
\end{tabular}


2.0 Facility Information

\begin{tabular}{|l|l|}
\hline Site & HASI \\
\hline Facility ID \& Name & HASIFAC3-CASE4 \\
\hline Function & Disposal \\
\hline Start Year & 1997 \\
\hline End Year & 2070 \\
\hline Annual Capacity (m3/year) or & $1,000,000$ \\
\hline Lifetime Facility Capacity (m3) & \\
\hline Volume Change Factor & 1 \\
\hline
\end{tabular}

Conversion Profile

\begin{tabular}{|l|l|l|}
\hline Percent & Waste Type & Density \\
\hline NA & NA & NA \\
\hline & & \\
\hline
\end{tabular}

2.0 Facility Information

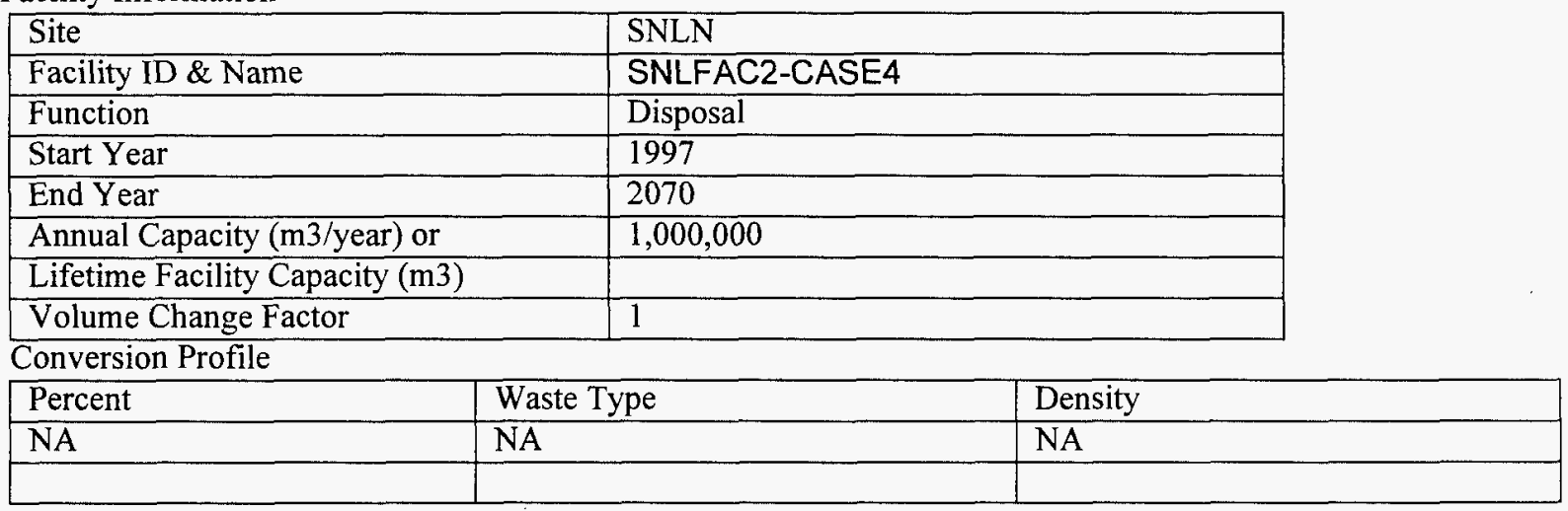

2.0 Facility Information

\begin{tabular}{|c|c|c|c|}
\hline & \multicolumn{2}{|l|}{ HASI } \\
\hline Facility ID \& Name & & \multicolumn{2}{|l|}{ HASIFAC2-CASE4 } \\
\hline \multicolumn{2}{|l|}{ Function } & \multicolumn{2}{|l|}{ Disposal } \\
\hline \multicolumn{2}{|l|}{ Start Year } & \multicolumn{2}{|l|}{1997} \\
\hline \multicolumn{2}{|l|}{ End Year } & \multicolumn{2}{|l|}{2070} \\
\hline \multicolumn{2}{|l|}{ Annual Capacity (m3/year) or } & $1,000,000$ & \\
\hline \multicolumn{4}{|l|}{ Lifetime Facility Capacity (m3) } \\
\hline \multicolumn{4}{|l|}{\begin{tabular}{|l|} 
Volume Change Factor \\
\end{tabular}} \\
\hline \multicolumn{4}{|l|}{ Conversion Profile } \\
\hline Percent & Waste Type & & Density \\
\hline NA & NA & & NA \\
\hline
\end{tabular}


WASTE DISPOSITION - EXCEL CHART (HASI, HLW):

\section{Waste Disposition}

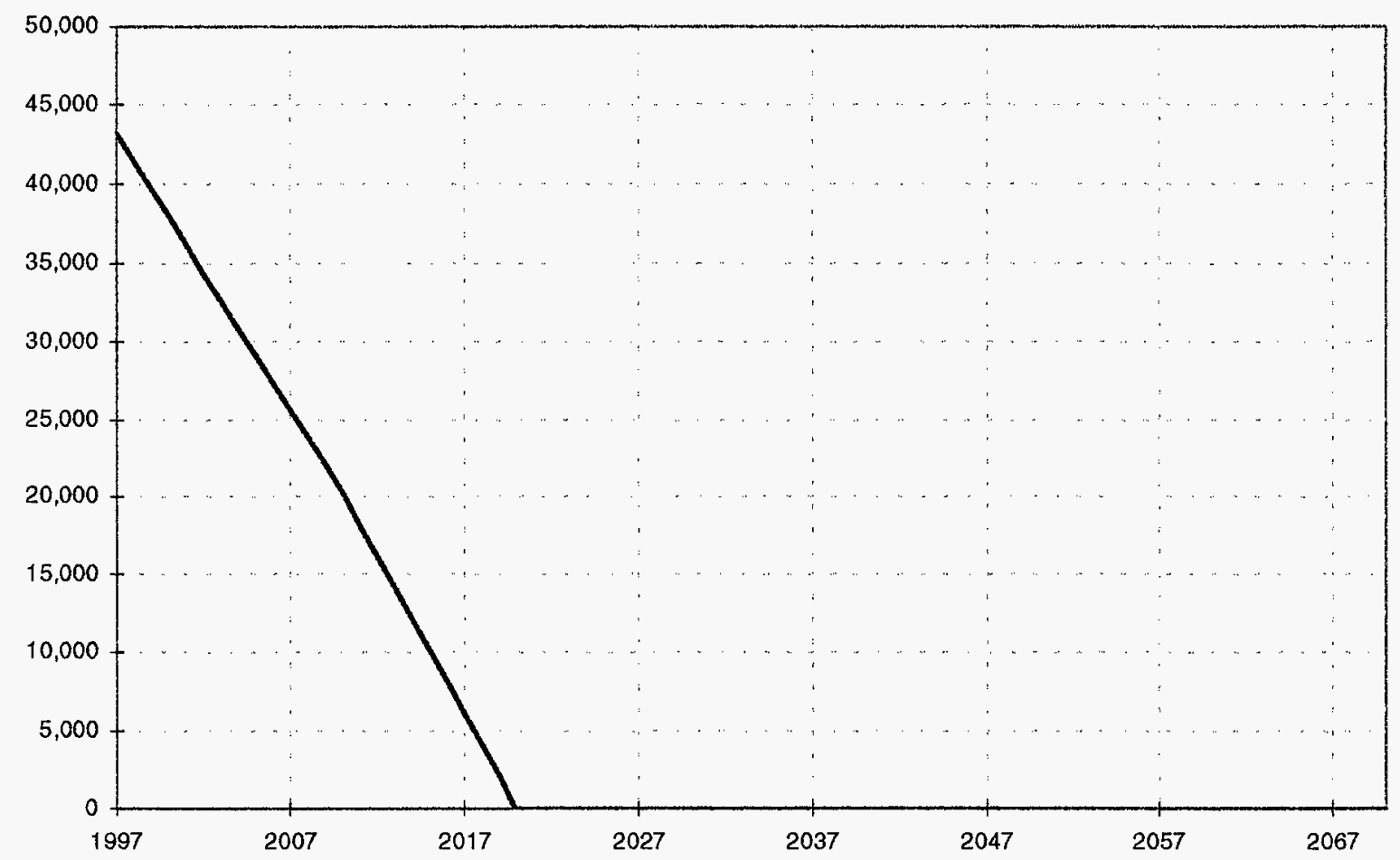

\section{WASTE DISPOSITION - CWWFA CHART:}

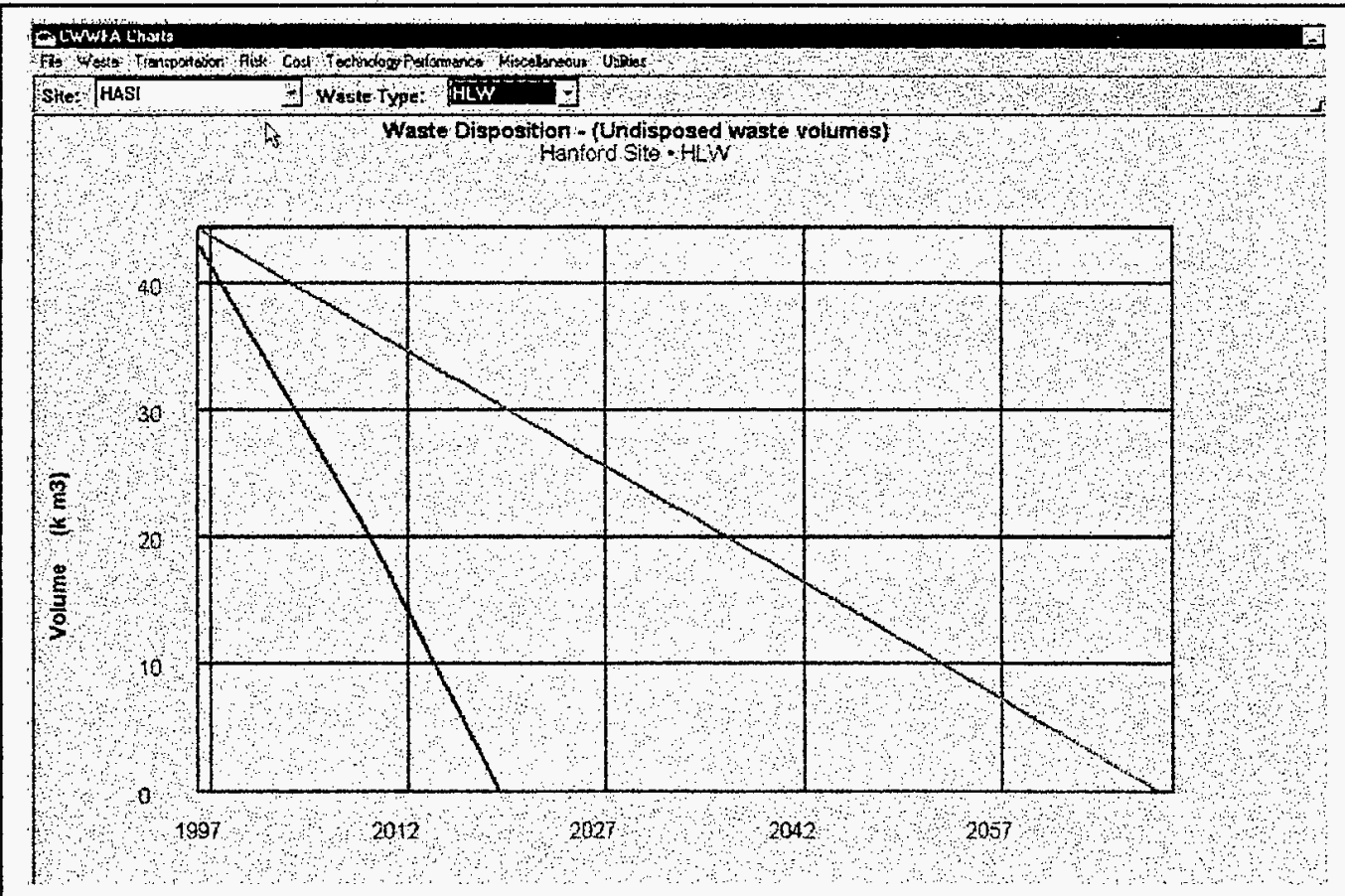


INITIAL PARAMETERS \& RESULTS REPORT

CASE: Case $4 \mathrm{~b}$

DATE: $11 / 13 / 97$

Evaluator: K B Oswald

FACILITY CAPACITY EXCEL CHART (HASI, NVTSFAC1-CASE4):

\section{Facility Capacity Detail}

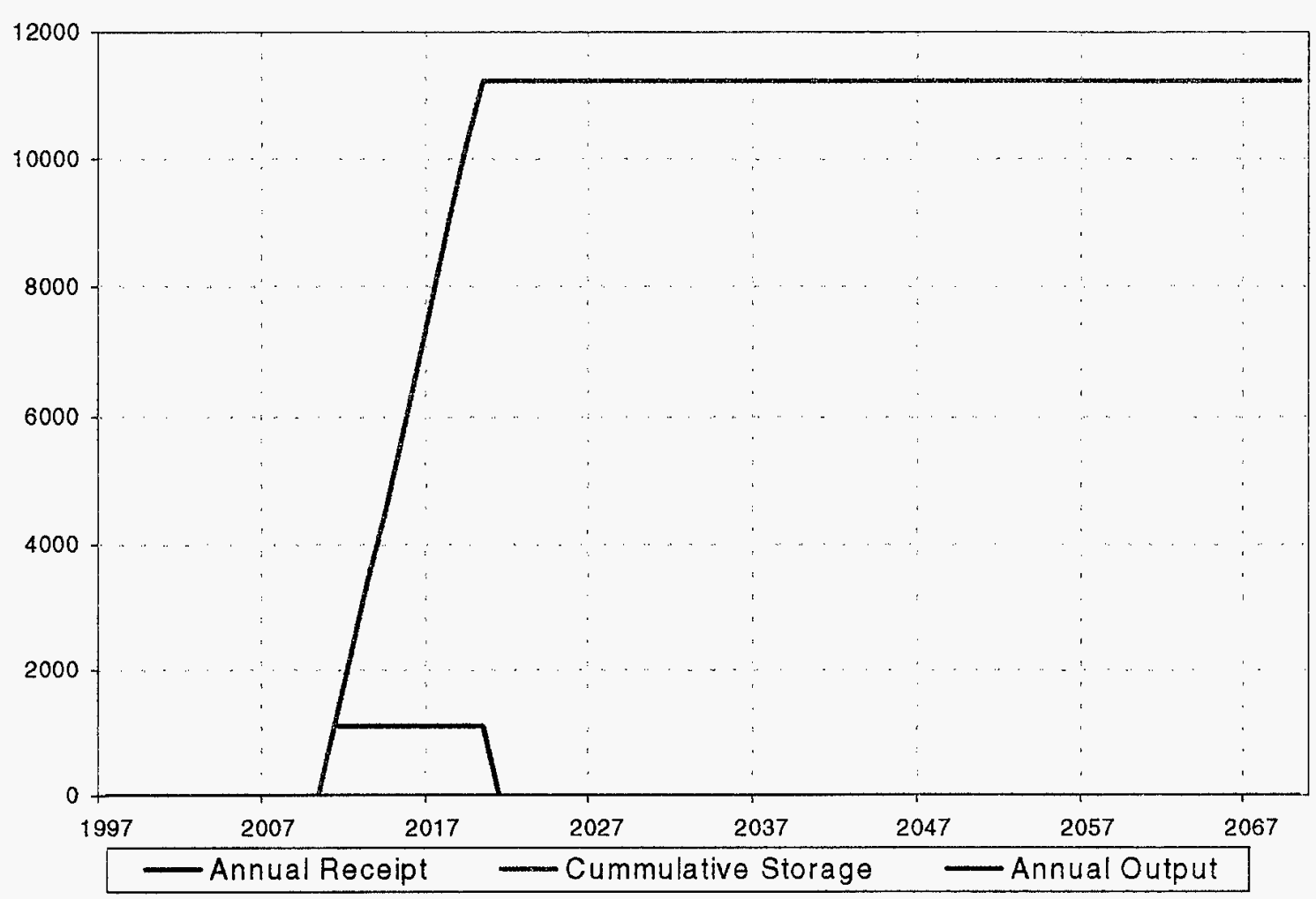

FACILITY CAPACITY CWWFA CHART:

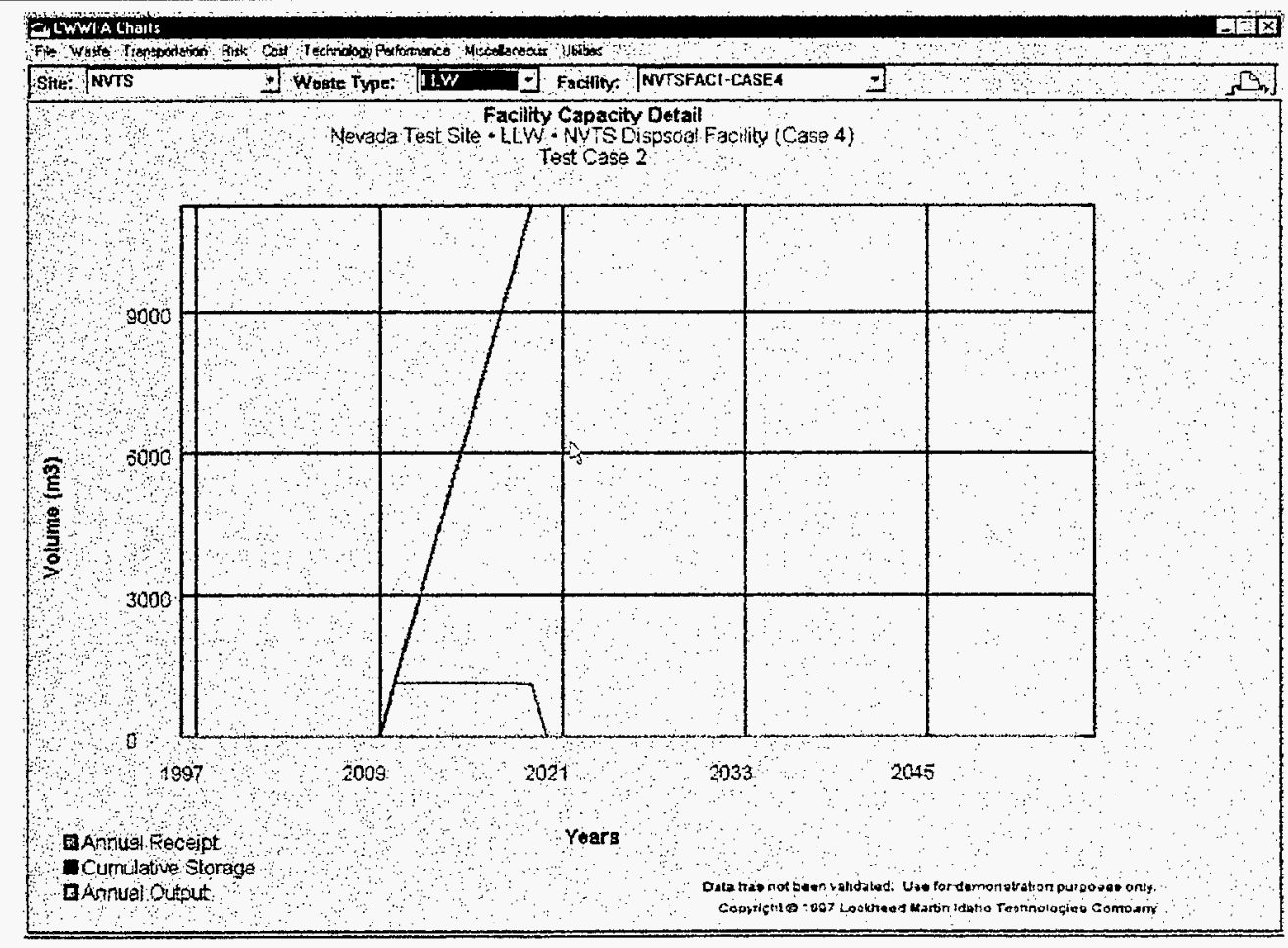


WASTE STREAM DETAIL EXCEL CHART (HASIWS1-CASE4):

\section{Waste Stream Detail}

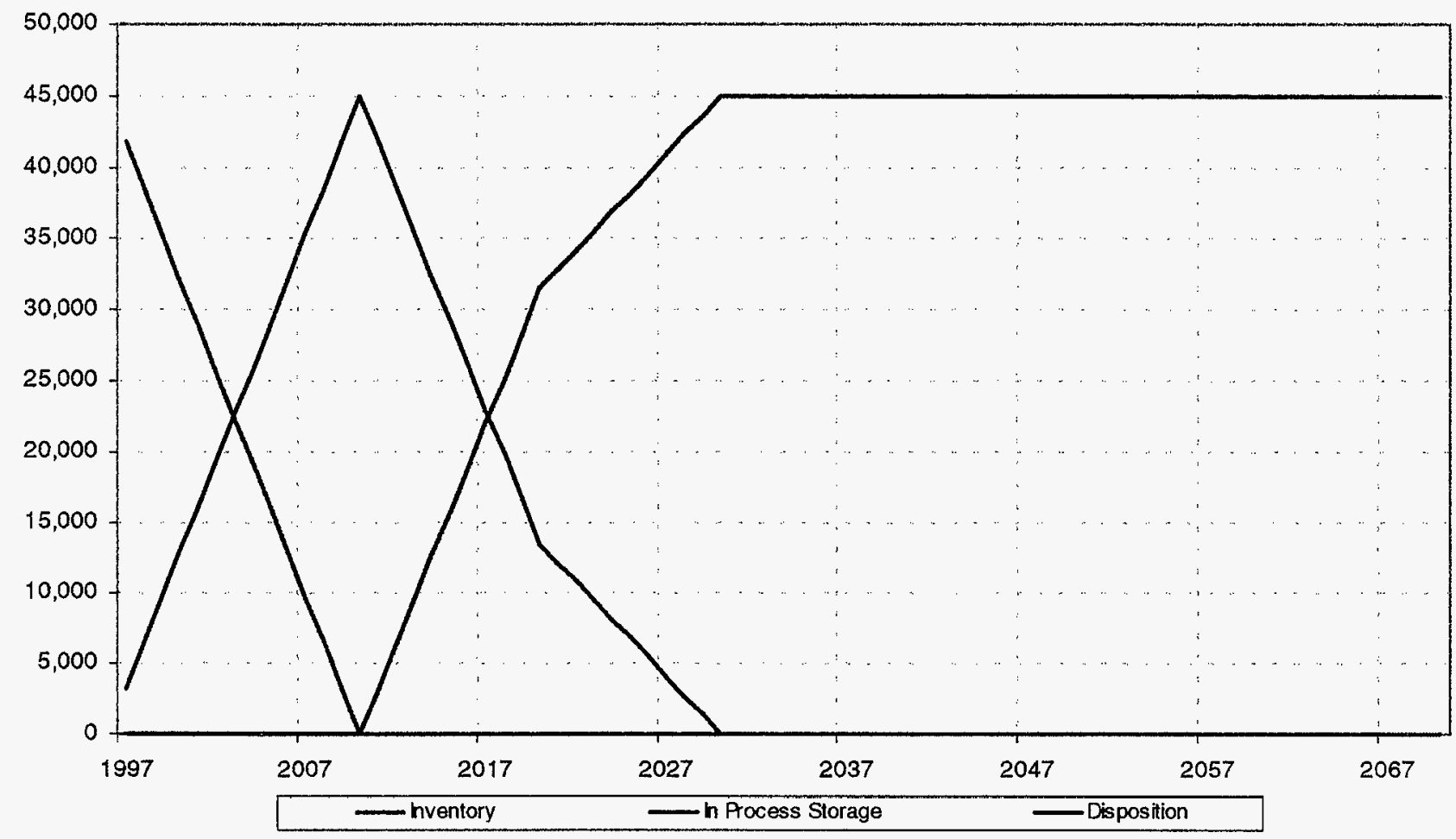

WASTE STREAM DETAIL CWWFA CHART:

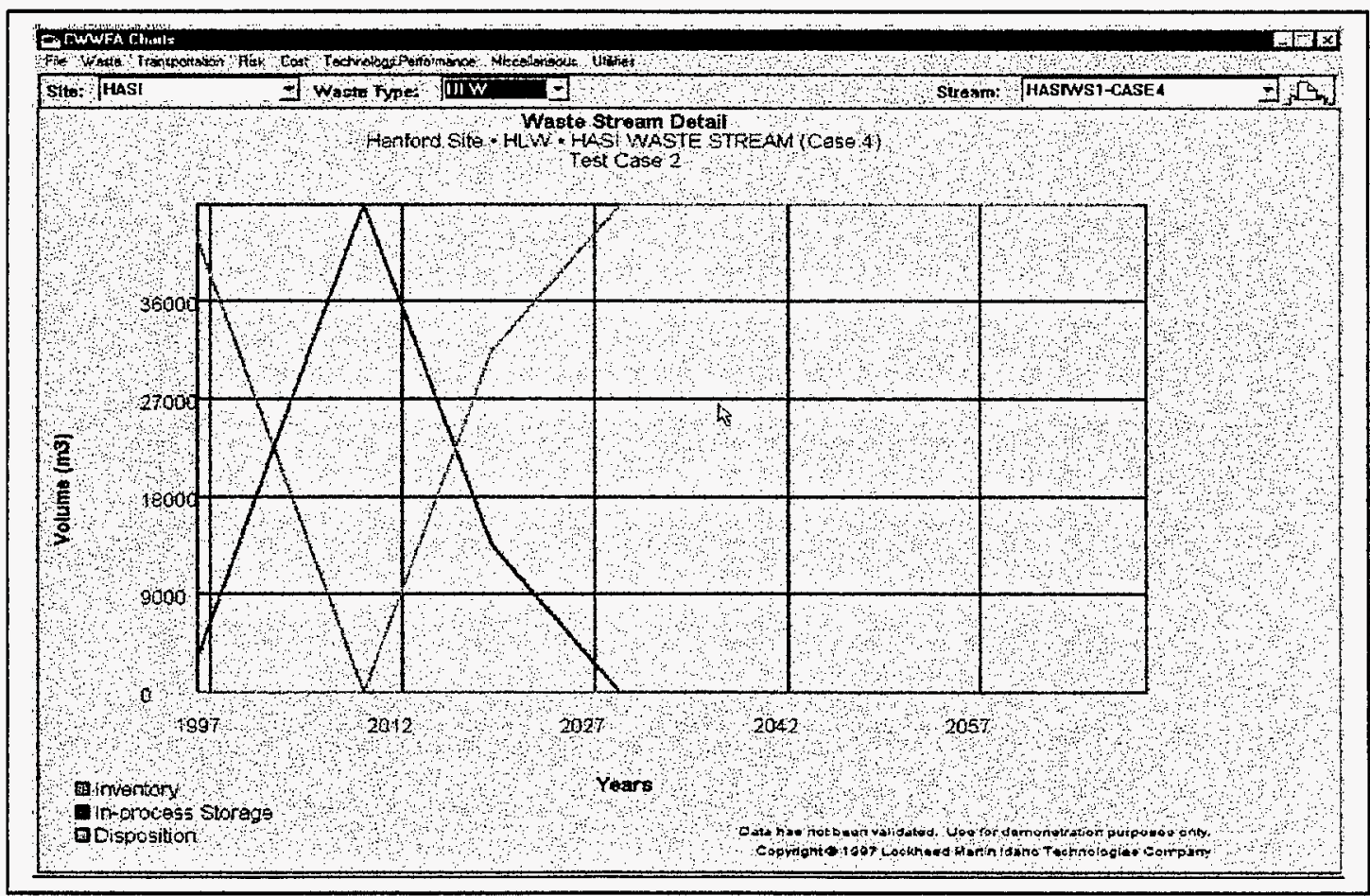




\section{Shipments Received}

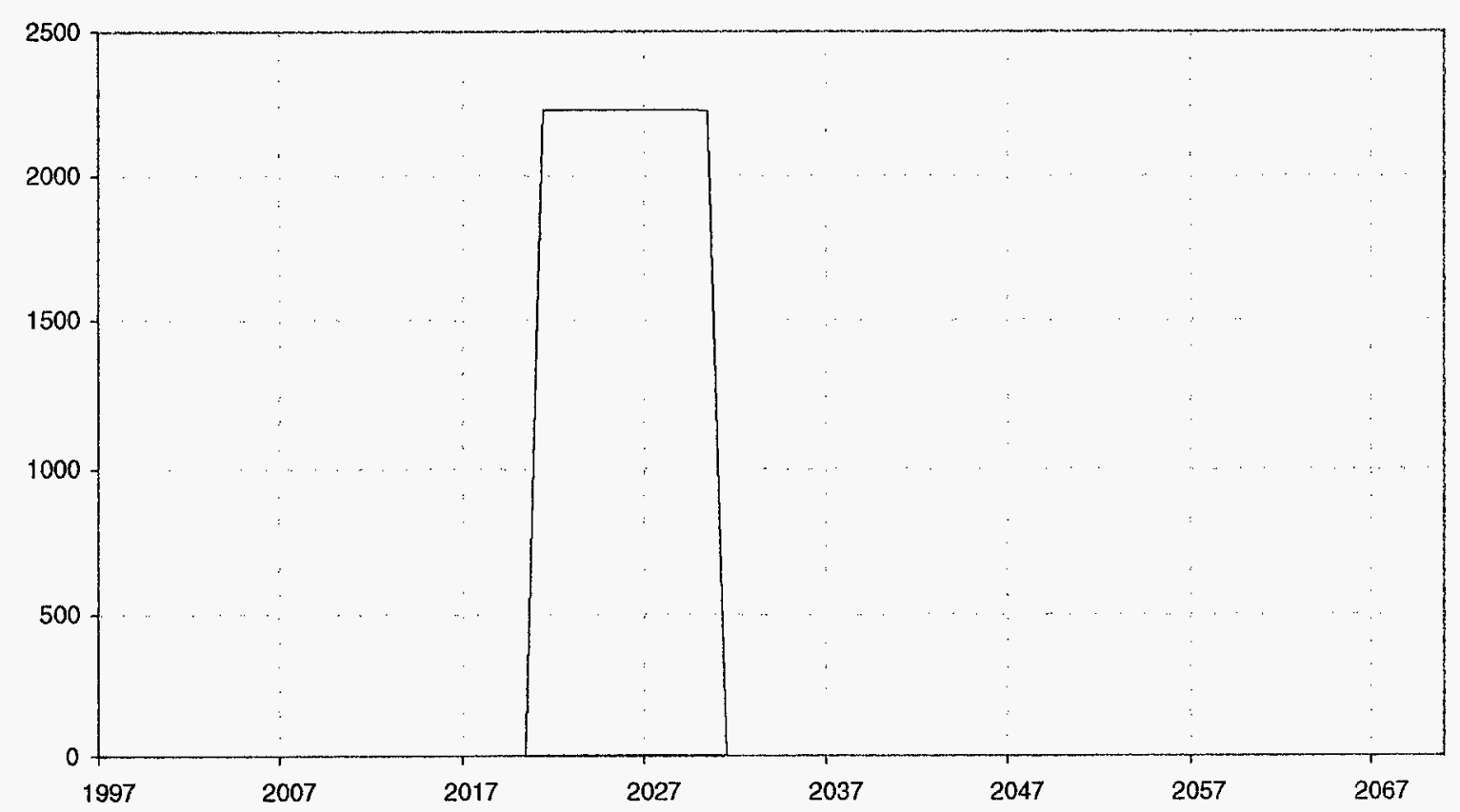

\section{SHIPMENTS RECEIVED CWWFA CHART:}

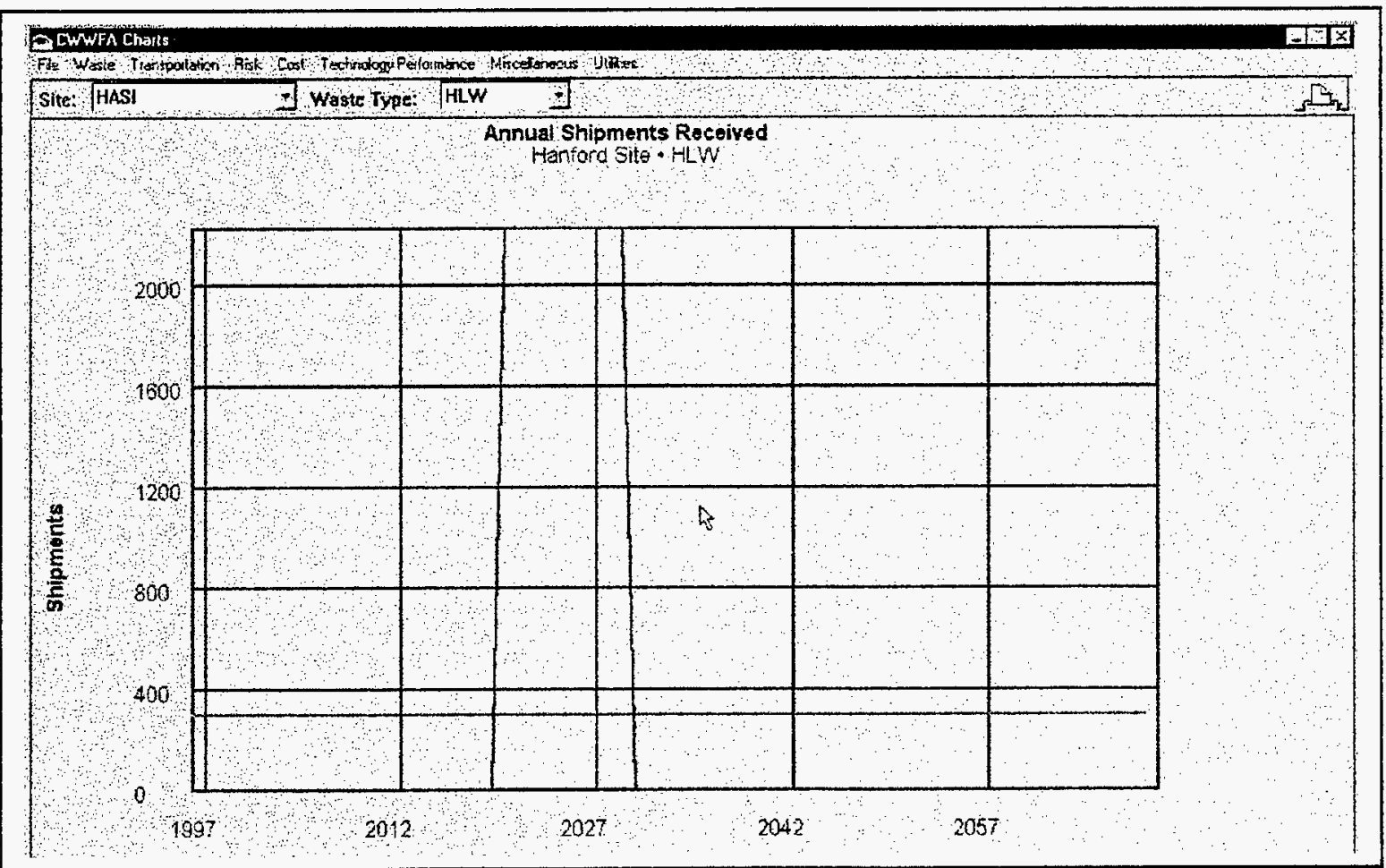




\section{Shipments Sent}

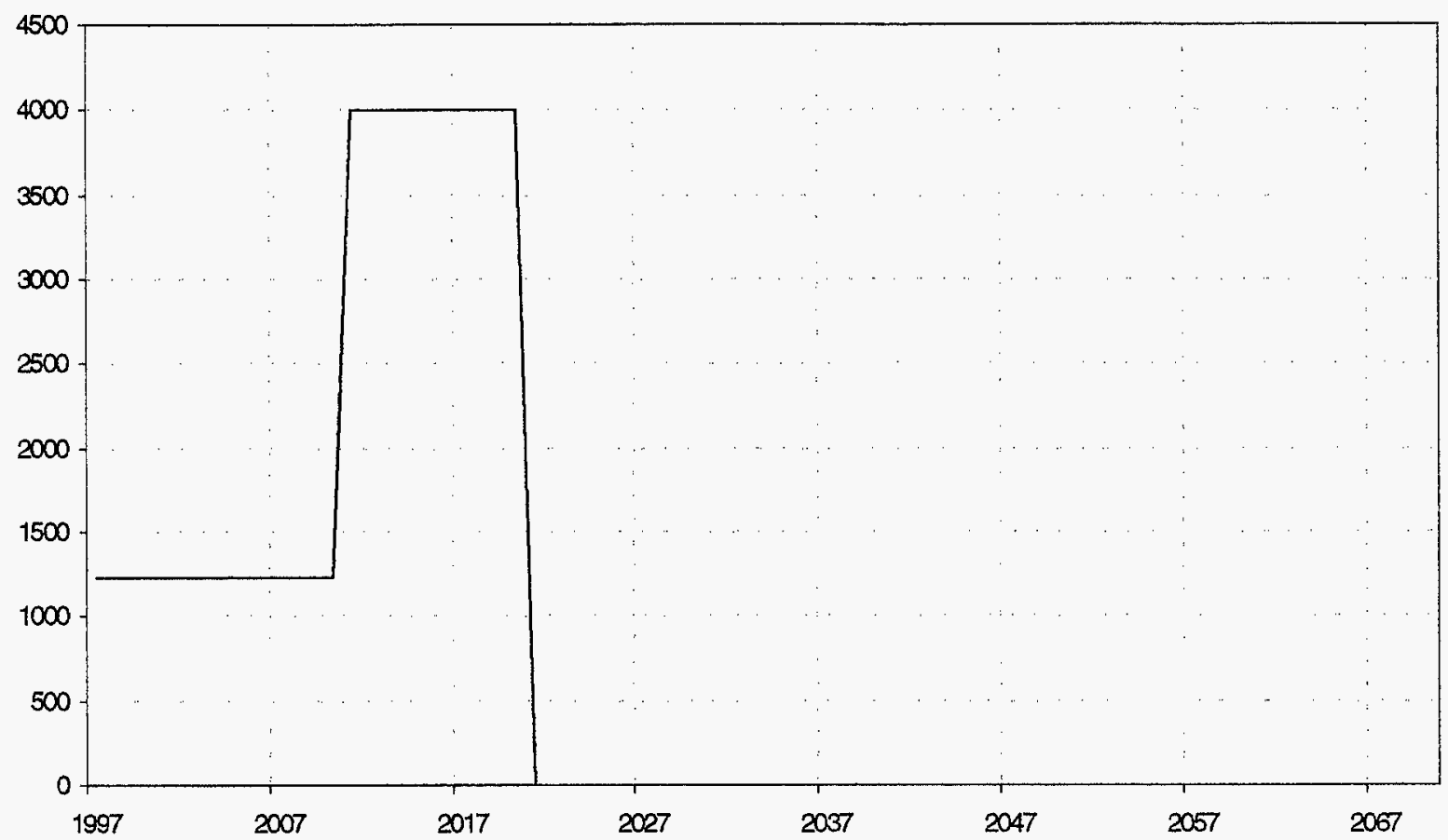

\section{SHIPMENTS SENT CWWFA CHART:}

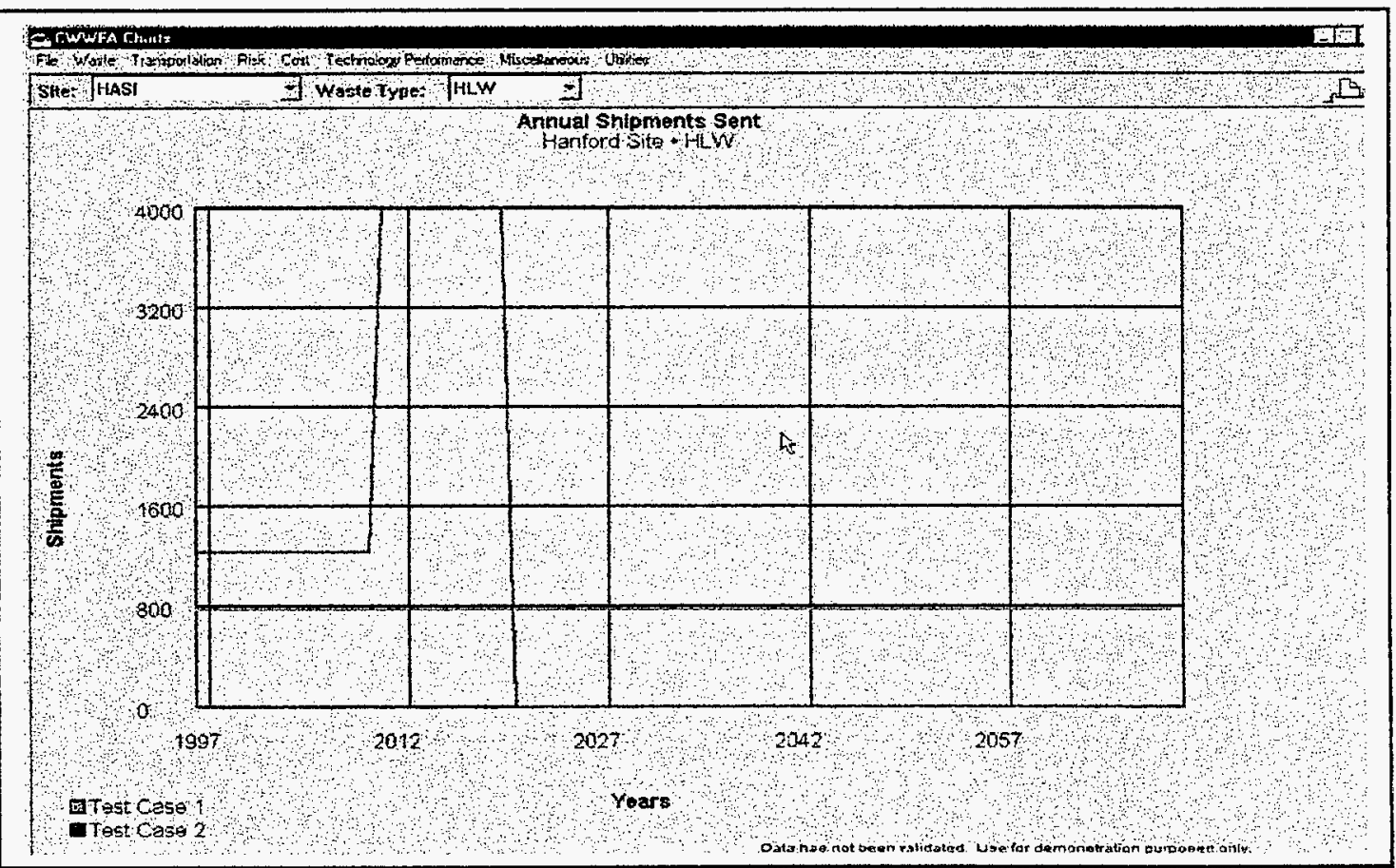


TRANSPORTAION COMPARISON

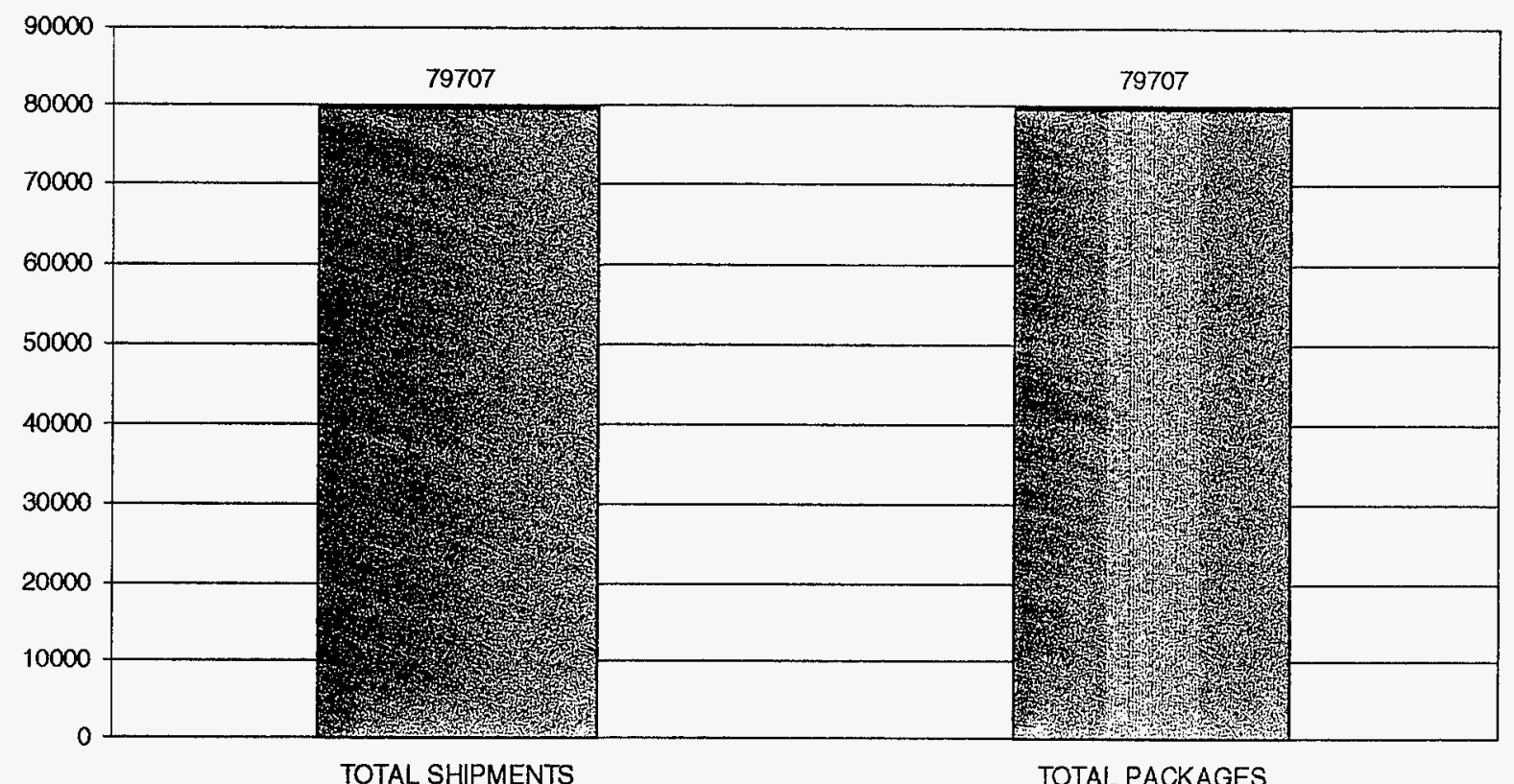

TRANSPORTATION COMPARISON CWWFA CHART:

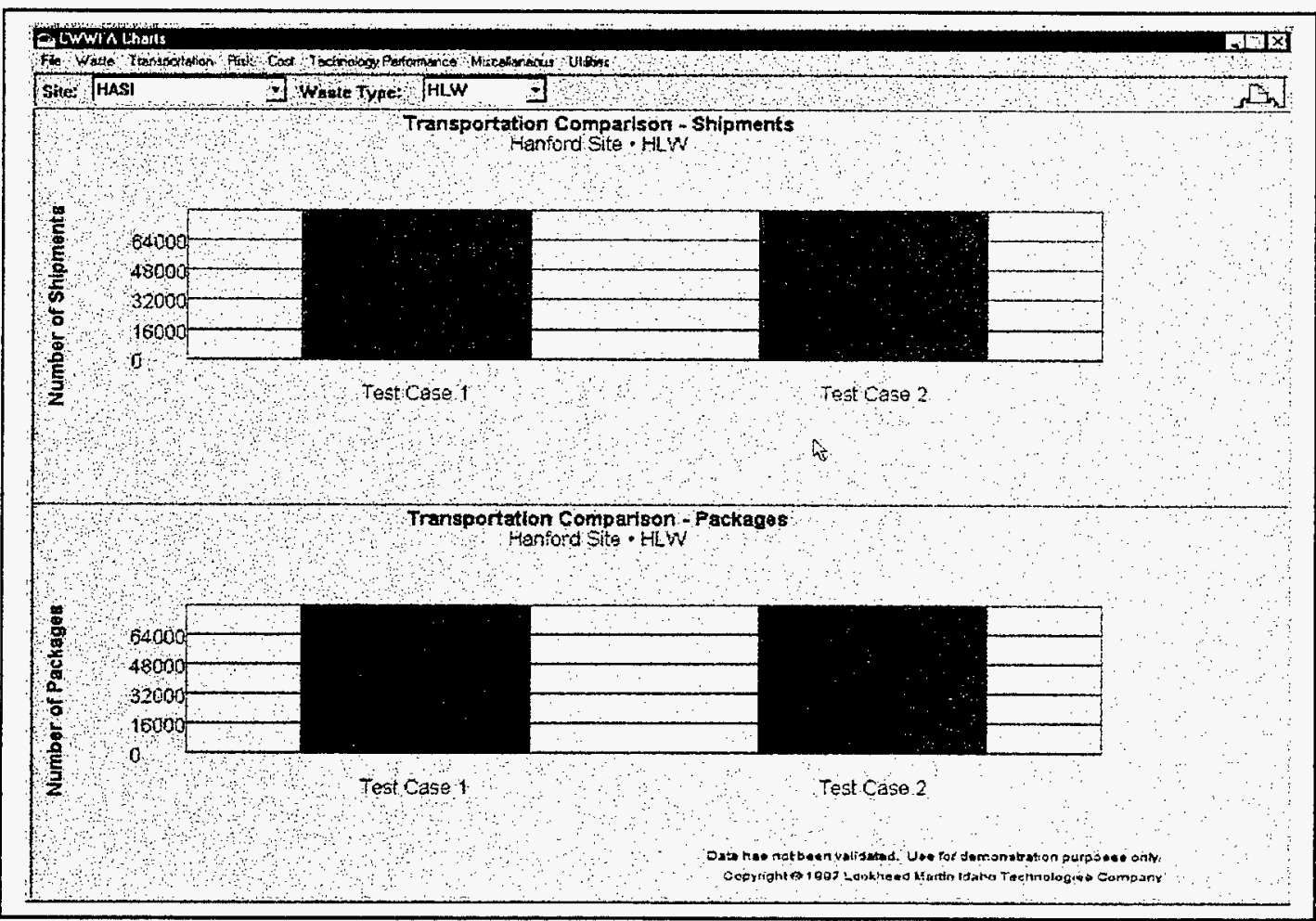


1.0 Waste Stream Information

\begin{tabular}{|l|l|}
\hline Originating Site & HASI \\
\hline Waste Stream ID \& Name & HASIWS1-CASE4 \\
\hline Originating Type & HLW \\
\hline Legacy volume (m3) & 45,000 \\
\hline
\end{tabular}

1.1 New Generation

\begin{tabular}{|l|l|l|}
\hline \multicolumn{1}{|c|}{ Start Year } & \multicolumn{1}{|c|}{ End Year } & \multicolumn{1}{c|}{ Annual Volume (m3) } \\
\hline NA & NA & NA \\
\hline & & \\
\hline & & \\
\hline & & \\
\hline
\end{tabular}

1.2 Shipping Route(s)

\begin{tabular}{|l|l|l|}
\hline \multicolumn{1}{|c|}{ Route ID } & \multicolumn{1}{|c|}{ Source Facility } & \multicolumn{1}{c|}{ Destination Facility } \\
\hline C4a-1 & Legacy-Hanf & SNLFAC1-CASE4 \\
\hline C4a-2 & Legacy-Hanf & HASIFAC1-CASE4 \\
\hline C4a-3 & SNLFAC1-CASE4 & NVTSFAC1-CASE4 \\
\hline C4a-4 & SNLFAC1-CASE4 & HASIFAC3-CASE4 \\
\hline C4a-5 & HASIFAC1-CASE4 & SNLFAC2-CASE4 \\
\hline C4a-6 & HASIFAC1-CASE4 & HASIFAC2-CASE4 \\
\hline
\end{tabular}

1.3 Shipping Schedule(s)

\begin{tabular}{|c|c|l|l|}
\hline Route ID: C4a-1 & \multicolumn{2}{|c|}{ Waste Type } & Annual Volume (m3) \\
\hline Start Year & \multicolumn{1}{|c|}{ End Year } & HLW & 1000 \\
\hline 1997 & 2010 & HLW & 1700 \\
\hline & 2015 & & \\
\hline & & & \\
\hline
\end{tabular}

1.3 Shipping Schedule(s) (continued)

\begin{tabular}{|c|l|l|l|}
\hline Route ID: C4a-2 & \multicolumn{2}{|c|}{} & \multicolumn{1}{|c|}{ Waste Type } \\
\hline Start Year & \multicolumn{1}{|c|}{ End Year } & \multicolumn{1}{|c|}{ Annual Volume (m3) } \\
\hline 1997 & 2010 & HLW & 1000 \\
\hline 2011 & 2015 & HLW & 1700 \\
\hline & & & \\
\hline & & & \\
\hline
\end{tabular}

1.3 Shipping Schedule(s) (continued)

\begin{tabular}{|c|c|c|c|}
\hline Route ID: $\mathrm{C} 4 \mathrm{a}-3$ & . & & \\
\hline Start Year & End Year & Waste Type & Annual Volume (m3) \\
\hline 2011 & 2020 & $\mathrm{LLW}$ & 1000 \\
\hline 2021 & 2025 & LLW & 250 \\
\hline
\end{tabular}

1.3 Shipping Schedule(s) (continued)

\begin{tabular}{|c|c|c|c|}
\hline Route ID: C4a-4 & \multicolumn{1}{|c|}{ Waste Type } & Annual Volume (m3) \\
\hline Start Year & \multicolumn{1}{|c|}{ End Year } & LLW & 1125 \\
\hline & 2030 & & \\
\hline & & & \\
\hline & & & \\
\hline
\end{tabular}


1.3 Shipping Schedule(s) (continued)

\begin{tabular}{|l|l|l|l|}
\hline Route ID: C4a-5 & \multicolumn{1}{|c|}{ Wnd Year } & \multicolumn{1}{|c|}{ Waste Type } & Annual Volume (m3) \\
\hline \multicolumn{1}{|c|}{ Start Year } & \multicolumn{1}{|c|}{ End } & 1000 \\
\hline 2016 & 2015 & TRU & 3050 \\
\hline & 2020 & TRU & \\
\hline
\end{tabular}

1.3 Shipping Schedule(s) (continued)

\begin{tabular}{|c|c|c|c|}
\hline Route ID: C4a-6 & \multicolumn{2}{|c|}{} & \multicolumn{1}{|c|}{ Waste Type } \\
\hline Start Year & \multicolumn{1}{|c|}{ End Year } & Annual Volume (m3) \\
\hline 2021 & 2030 & TRU & 225 \\
\hline & & & \\
\hline & & & \\
\hline
\end{tabular}

2.0 Facility Information

\begin{tabular}{|l|l|}
\hline Site & SNLN \\
\hline Facility ID \& Name & SNLFAC1-CASE4 \\
\hline Function & Treatment \\
\hline Start Year & 1997 \\
\hline End Year & 2070 \\
\hline Annual Capacity (m3/year) or & $1,000,000$ \\
\hline Lifetime Facility Capacity (m3) & \\
\hline Volume Change Factor & 1 \\
\hline
\end{tabular}

Conversion Profile

\begin{tabular}{|l|l|l|}
\hline Percent & Waste Type & Density \\
\hline $100 \%$ & LLW & 1800 \\
\hline & & \\
\hline & & \\
\hline
\end{tabular}

2.0 Facility Information

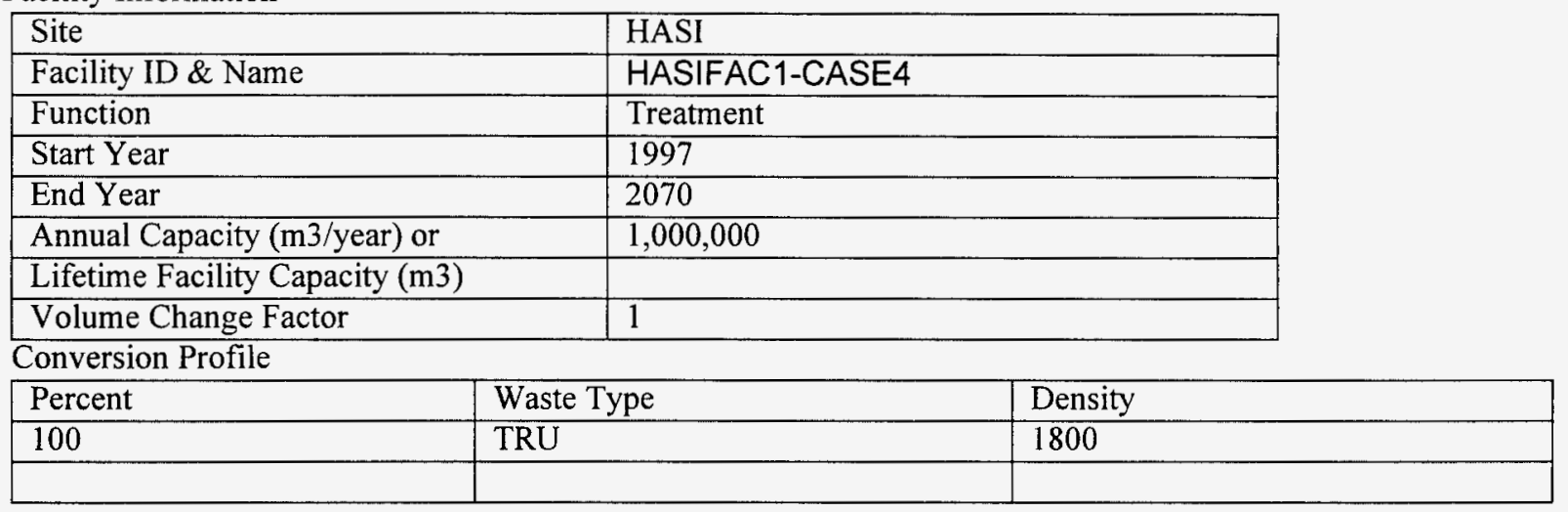

2.0 Facility Information

\begin{tabular}{|l|l|}
\hline Site & NVTS \\
\hline Facility ID \& Name & NVTSFAC1-CASE4 \\
\hline Function & Disposal \\
\hline Start Year & 1997 \\
\hline End Year & 2070 \\
\hline Annual Capacity (m3/year) or & $1,000,000$ \\
\hline Lifetime Facility Capacity (m3) & \\
\hline Volume Change Factor & 1 \\
\hline \\
\begin{tabular}{ll|l|}
\hline Conversion Profile & & \\
\hline Percent & Waste Type & Density \\
\hline NA & NA & NA \\
\hline
\end{tabular}
\end{tabular}


\begin{tabular}{|l|l|}
\hline & \\
\hline
\end{tabular}

2.0 Facility Information

\begin{tabular}{|l|l|}
\hline Site & HASI \\
\hline Facility ID \& Name & HASIFAC3-CASE4 \\
\hline Function & Disposal \\
\hline Start Year & 1997 \\
\hline End Year & 2070 \\
\hline Annual Capacity (m3/year) or & $1,000,000$ \\
\hline Lifetime Facility Capacity (m3) & \\
\hline Volume Change Factor & 1 \\
\hline
\end{tabular}

Conversion Profile

\begin{tabular}{|l|l|l|}
\hline Percent & Waste Type & Density \\
\hline NA & NA & NA \\
\hline & & \\
\hline
\end{tabular}

2.0 Facility Information

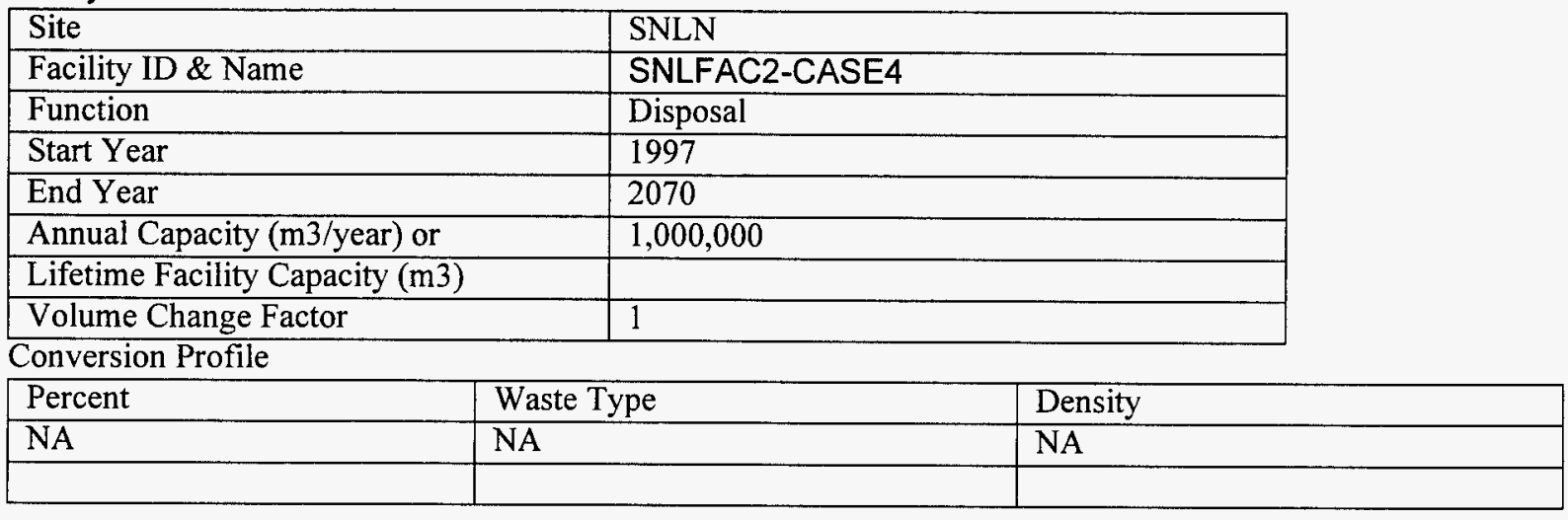

2.0 Facility Information

\begin{tabular}{|l|l|}
\hline Site & HASI \\
\hline Facility ID \& Name & HASIFAC2-CASE4 \\
\hline Function & Disposal \\
\hline Start Year & 1997 \\
\hline End Year & 2070 \\
\hline Annual Capacity (m3/year) or & $1,000,000$ \\
\hline Lifetime Facility Capacity (m3) & \\
\hline Volume Change Factor & 1 \\
\hline
\end{tabular}

Conversion Profile

\begin{tabular}{|l|l|l|}
\hline Percent & Waste Type & Density \\
\hline NA & NA & NA \\
\hline & & \\
\hline
\end{tabular}


CASE: Case $4 c$

DATE: $11 / 13 / 97$

Evaluator: K B Oswald

WASTE DISPOSITION - EXCEL CHART (HASI, HLW):

\section{Waste Disposition}

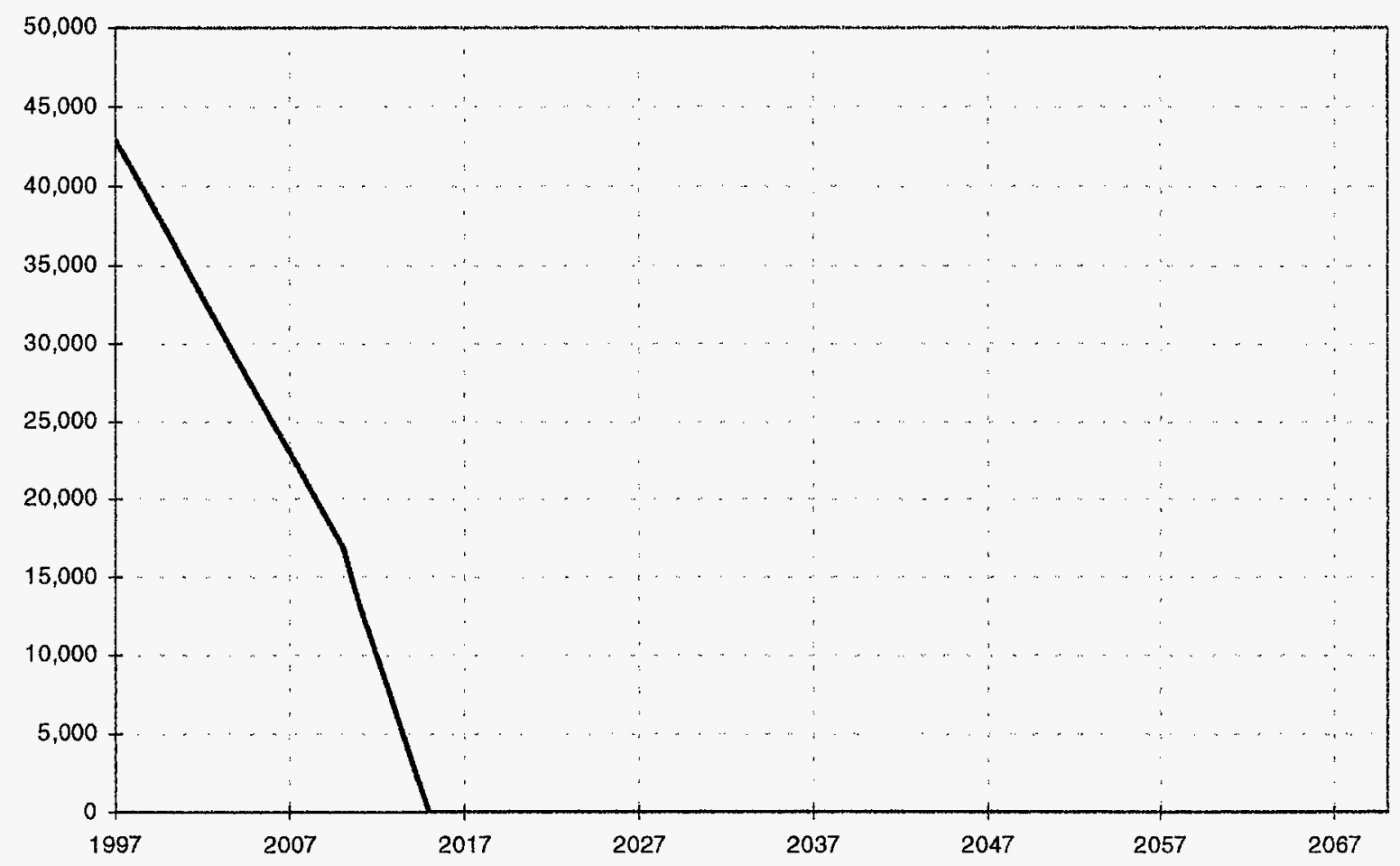

WASTE DISPOSITION - CWWFA CHART:

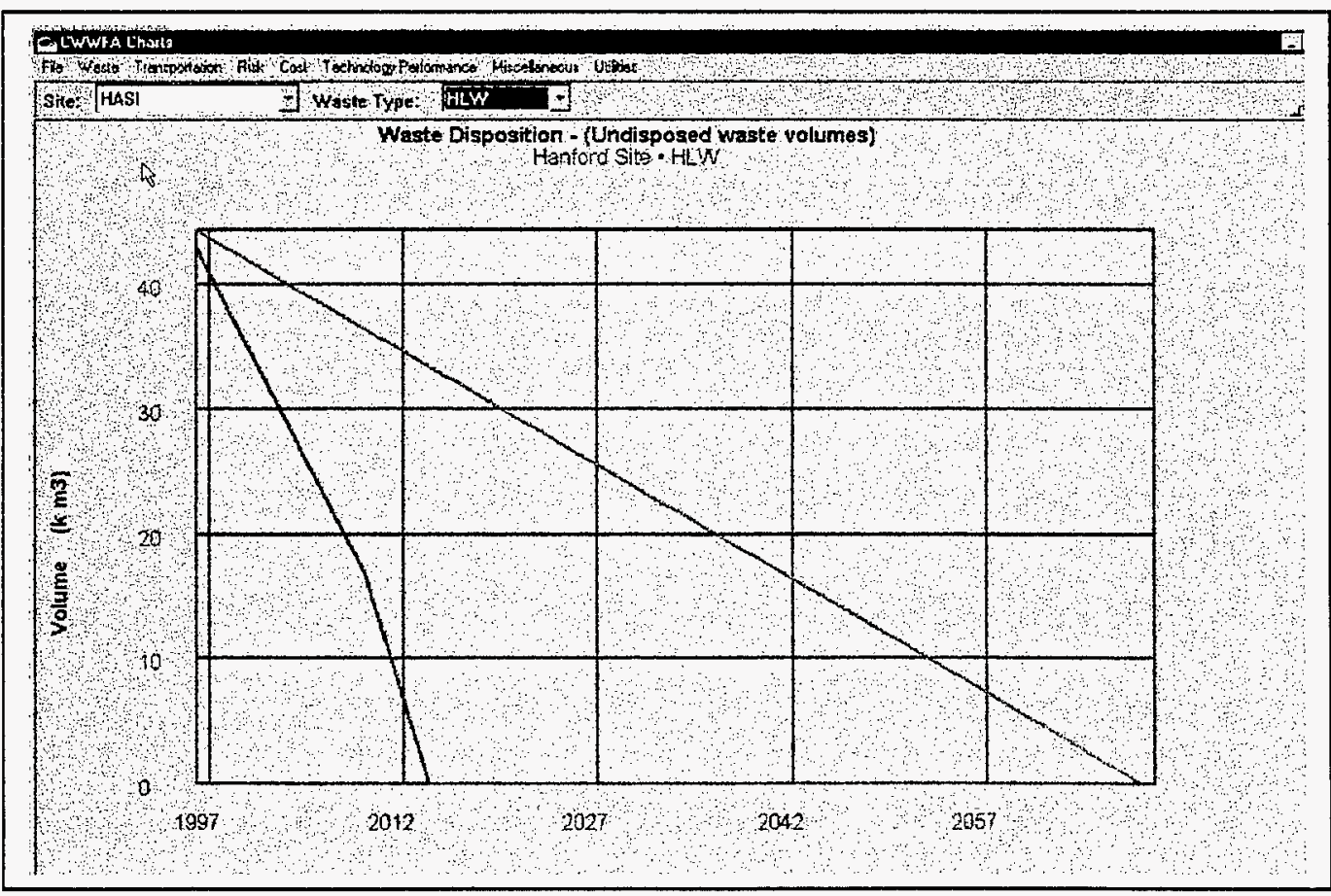


FACILITY CAPACITY EXCEL CHART (NVTS, NVTSFAC1-CASE4):

\section{Facility Capacity Detail}

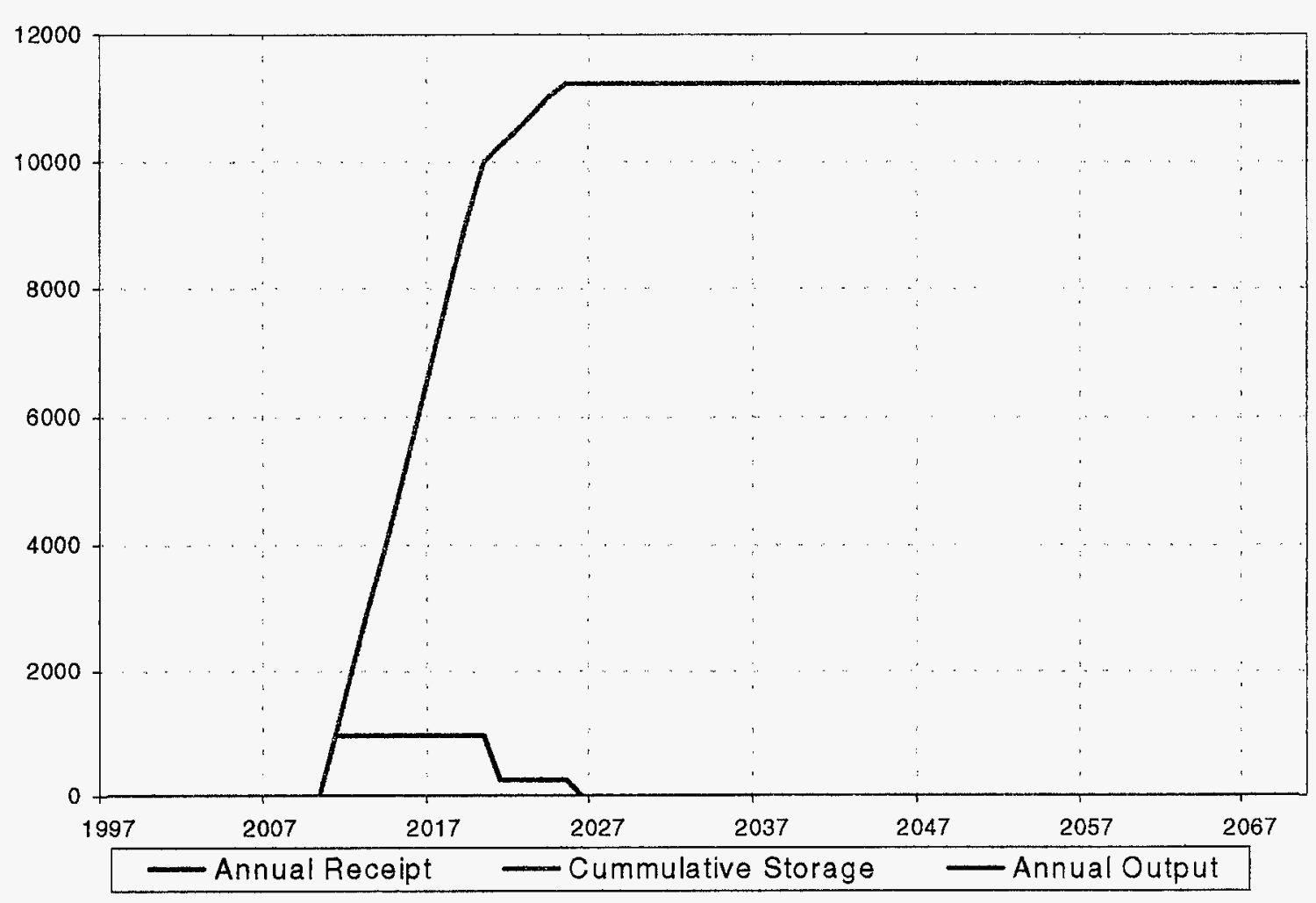

\section{FACILITY CAPACITY CWWFA CHART:}

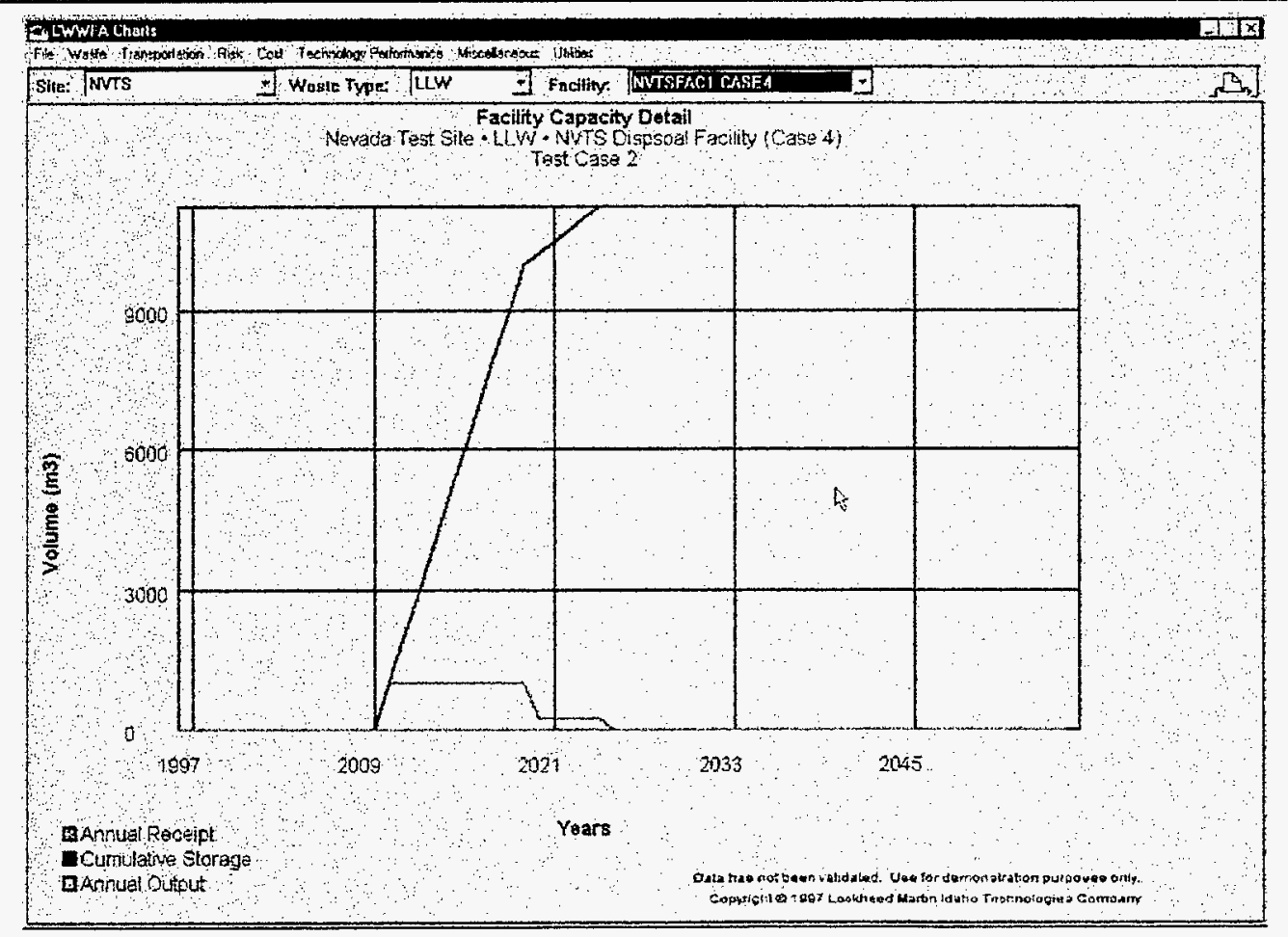


WASTE STREAM DETAIL EXCEL CHART (HASIWS1-CASE4):

\section{Waste Stream Detail}

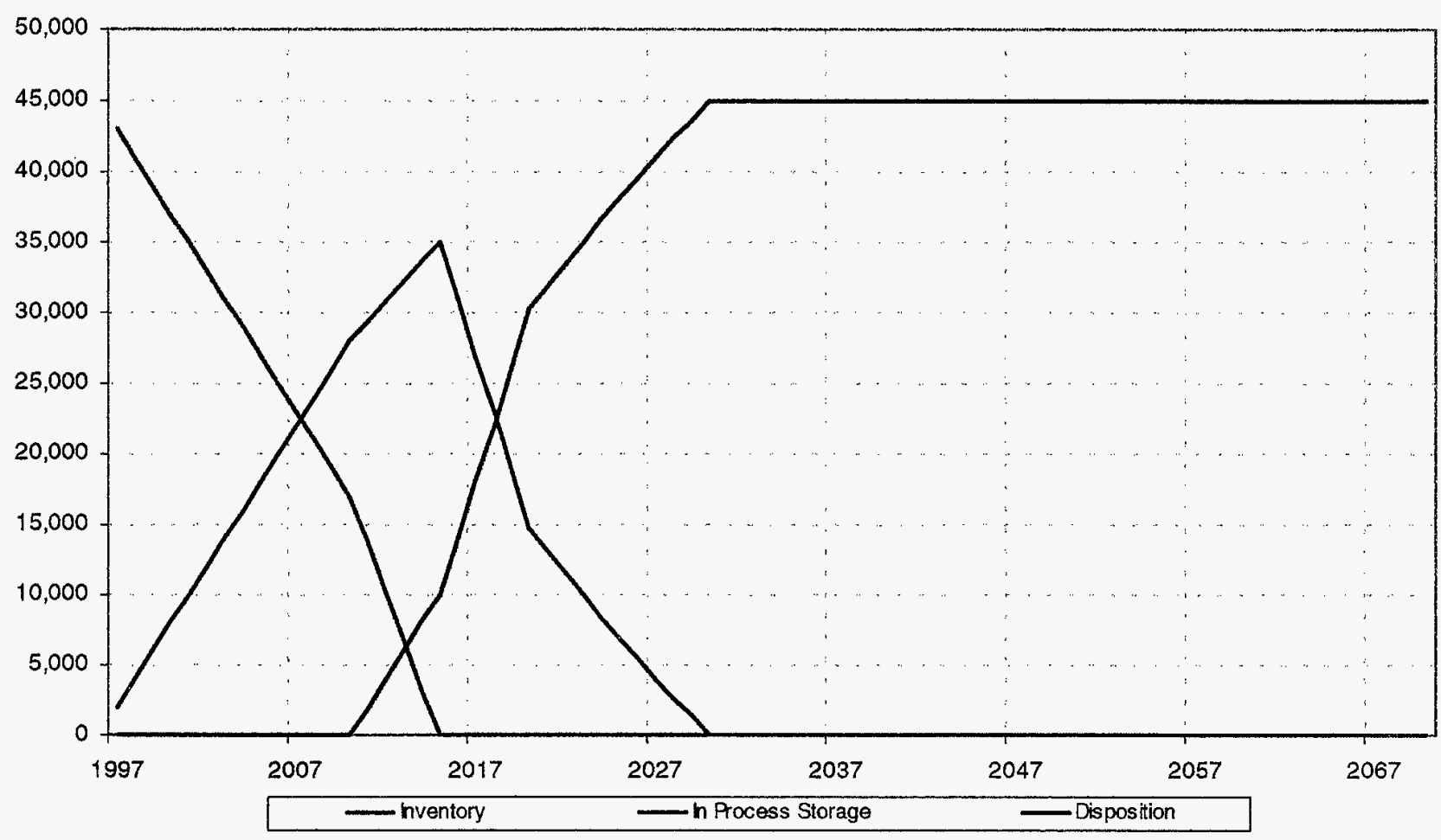

WASTE STREAM DETAIL CWWFA CHART:

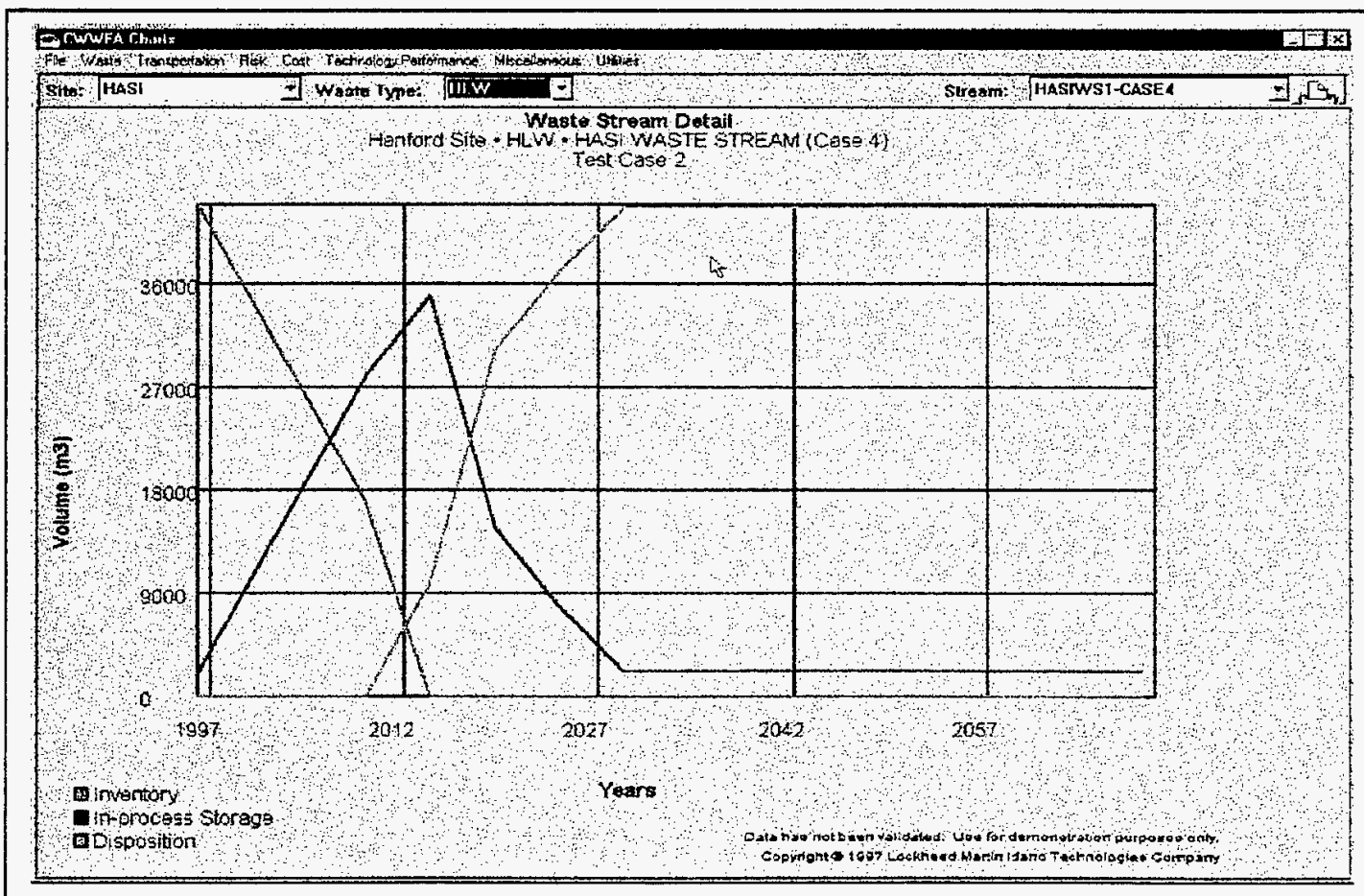


SHIPMENTS RECEIVED EXCEL CHART (HASI):

\section{Shipments Received}

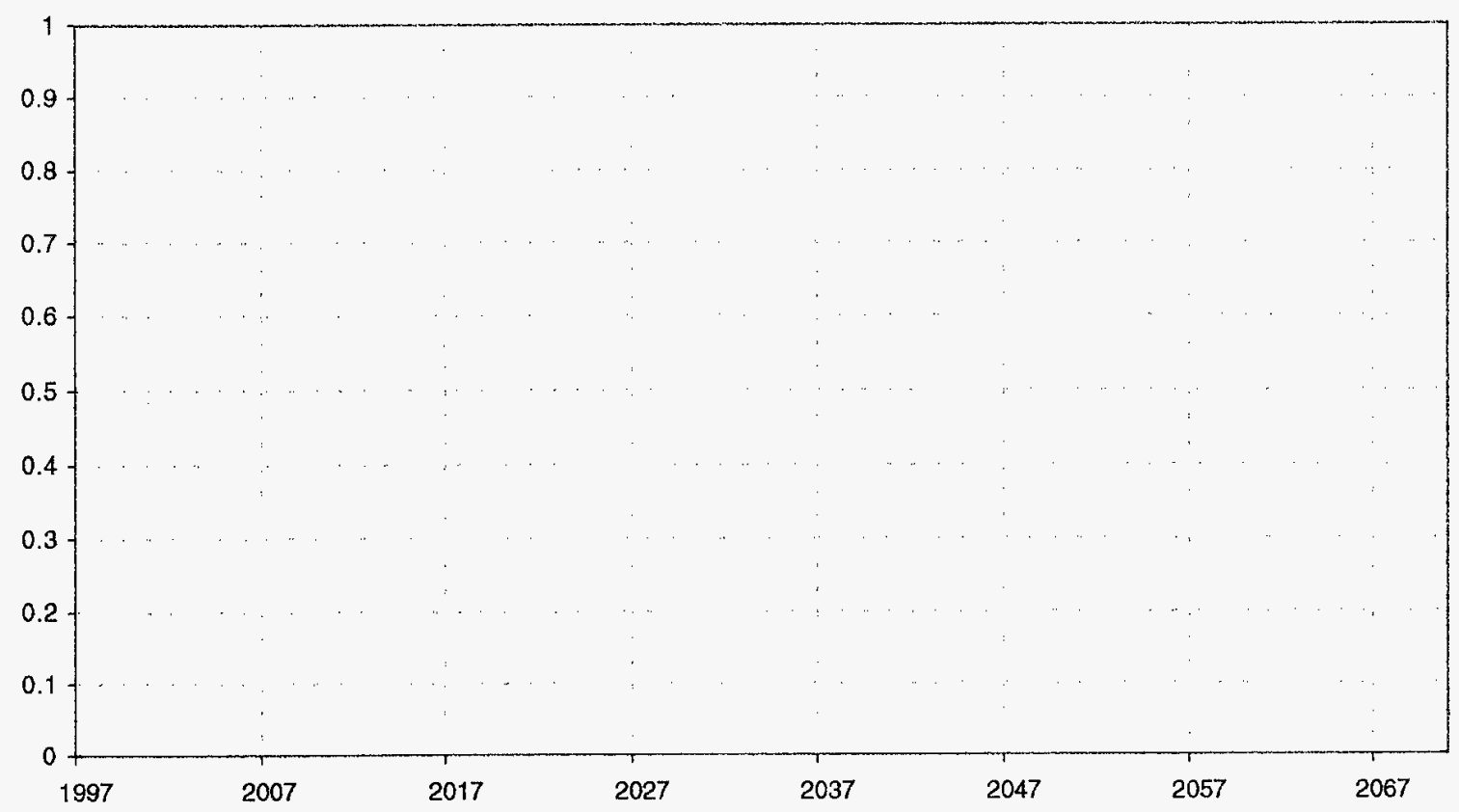

\section{SHIPMENTS RECEIVED CWWFA CHART:}

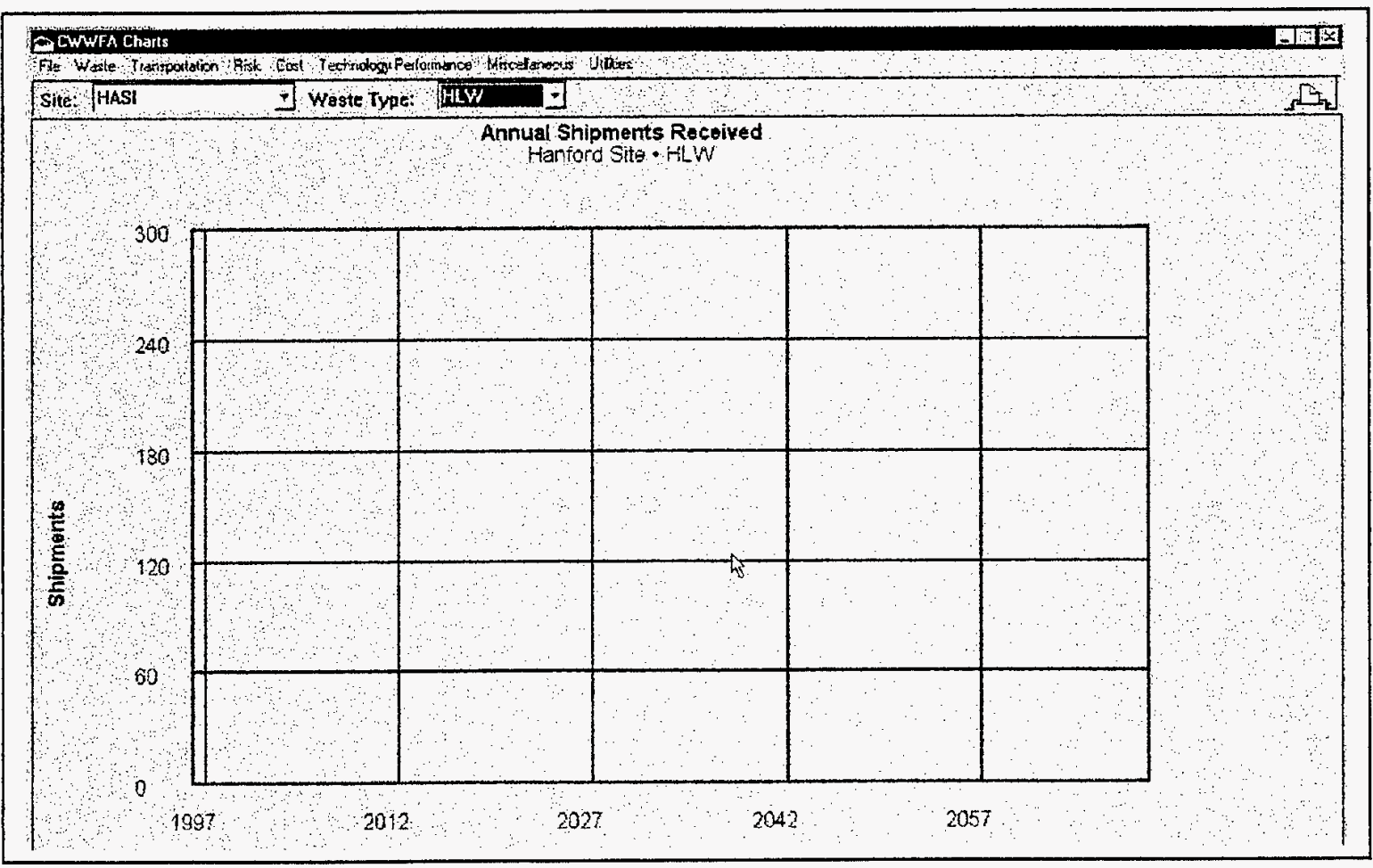


CASE: Case $4 c$

DATE: $11 / 13 / 97$

Evaluator: K B Oswald

SHIPMENTS SENT EXCEL CHART (HASI):

\section{Shipments Sent}

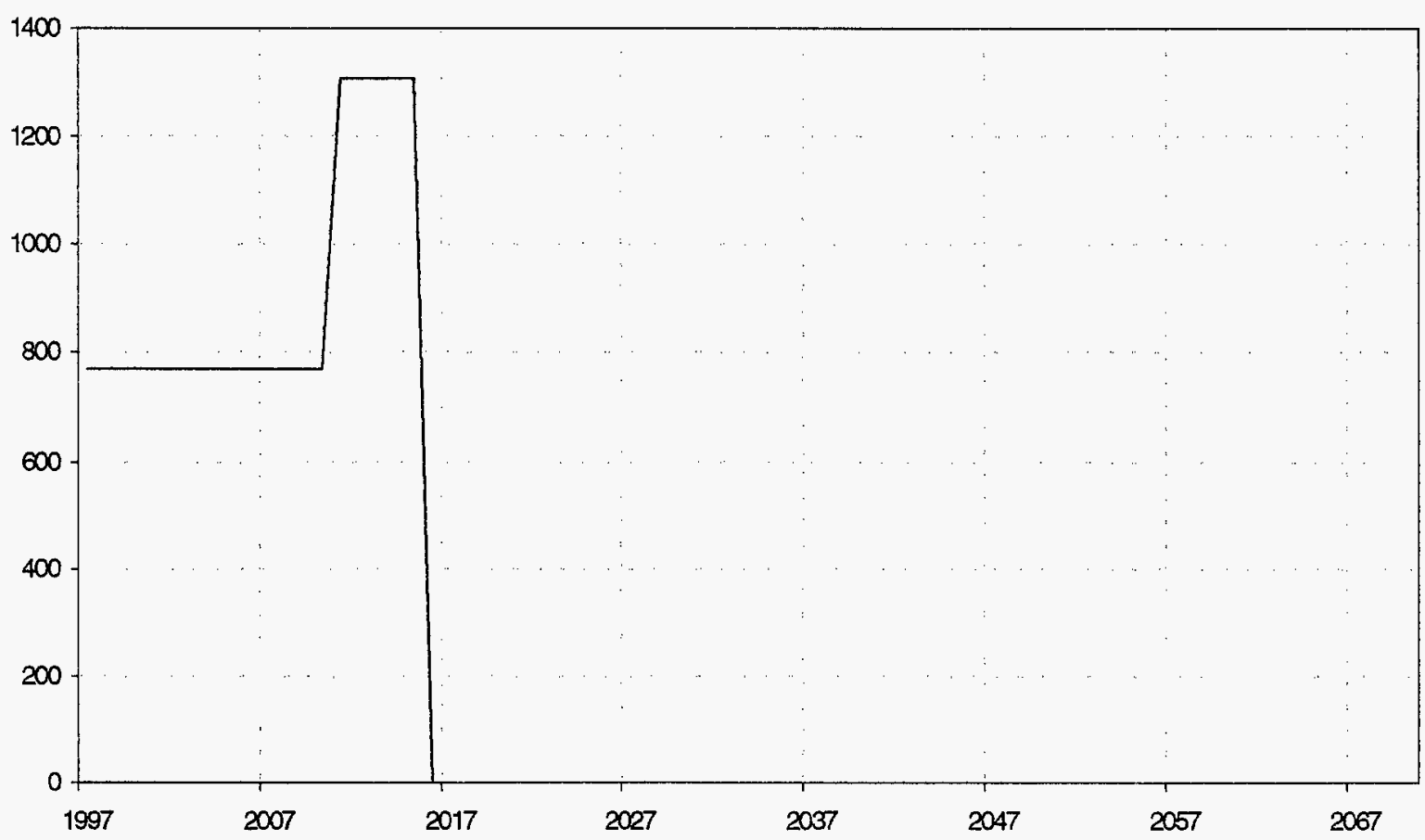

\section{SHIPMENTS SENT CWWFA CHART:}

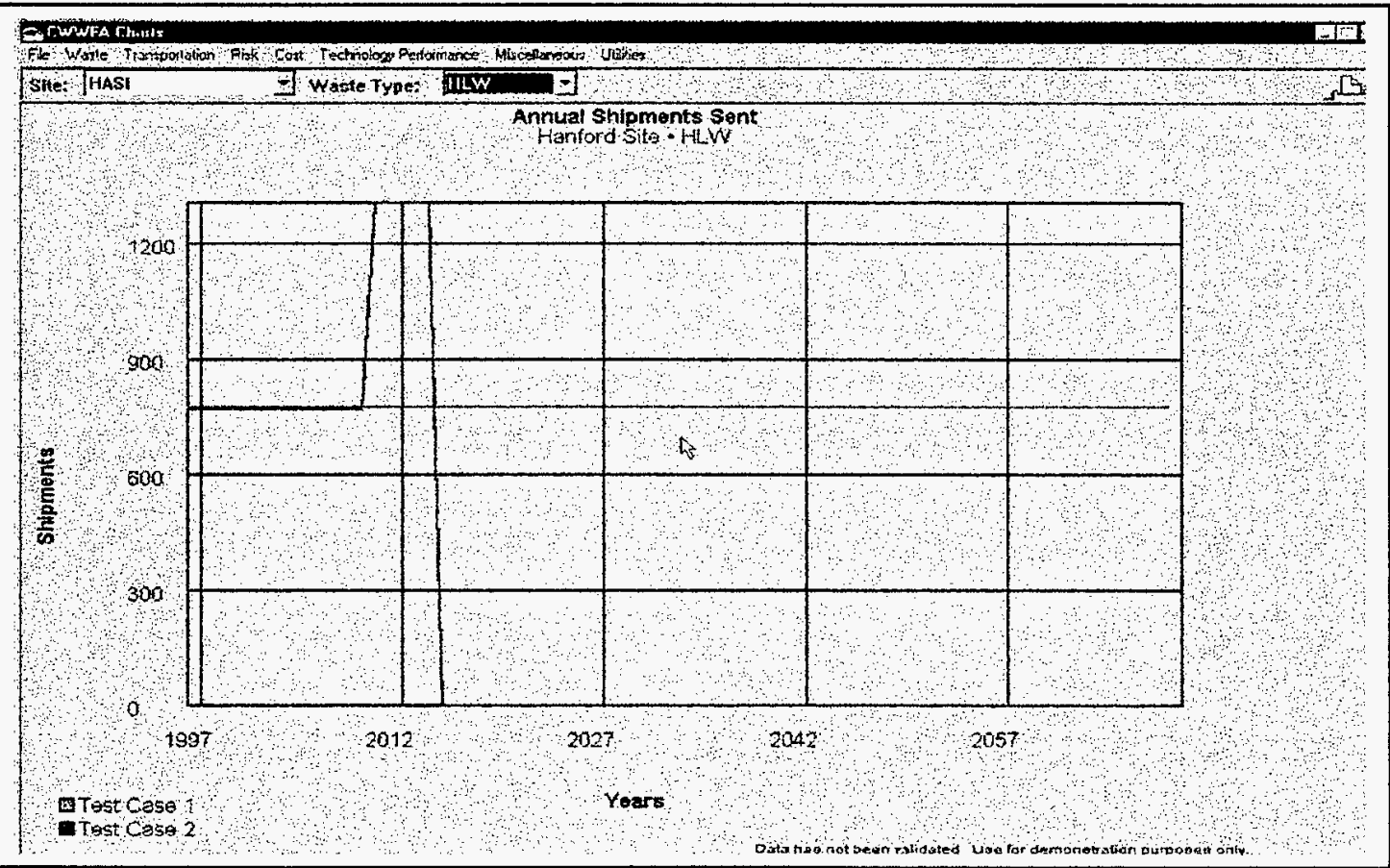




\section{TRANSPORTAION COMPARISON}

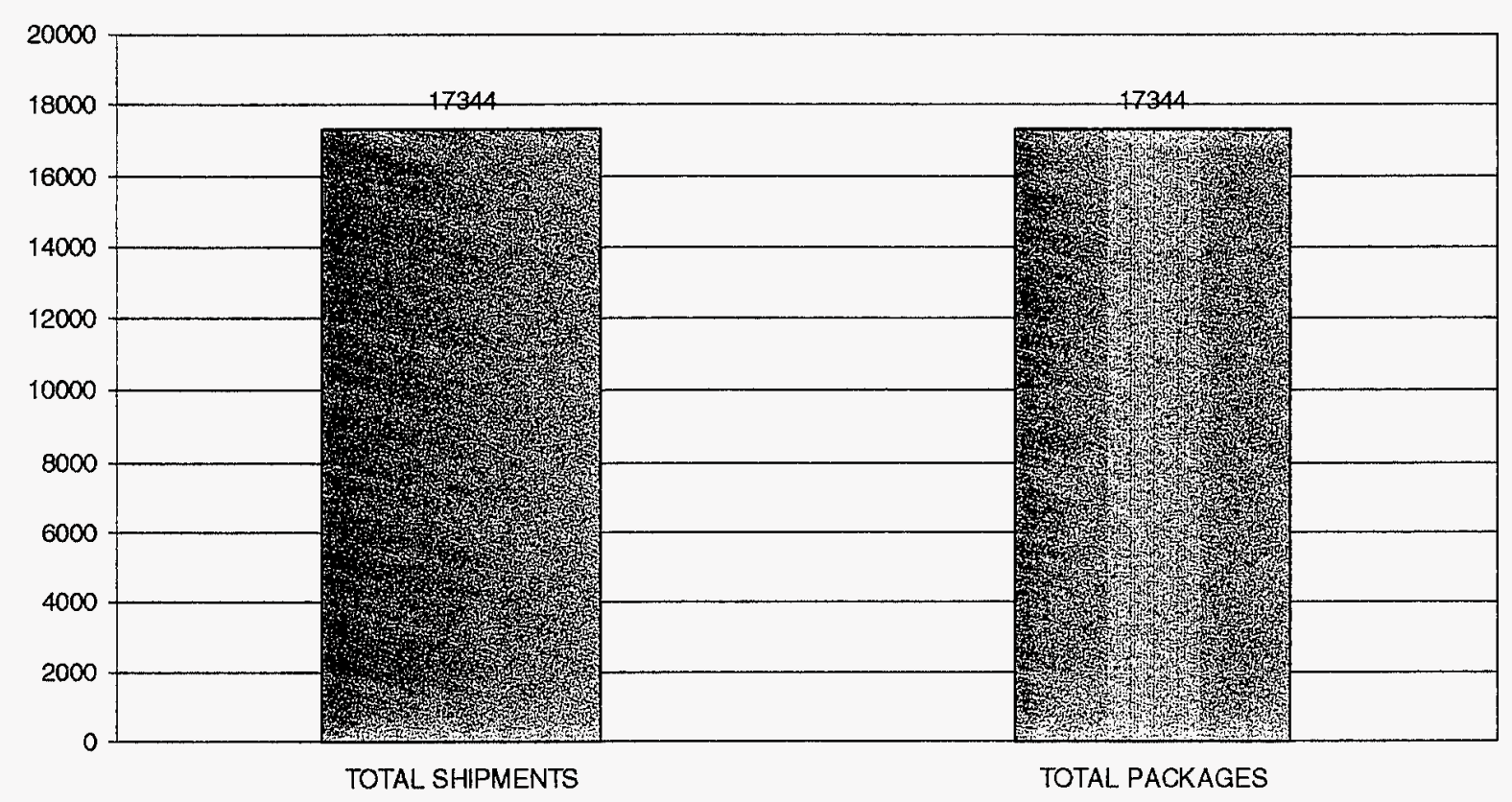

TRANSPORTATION COMPARISON CWWFA CHART:

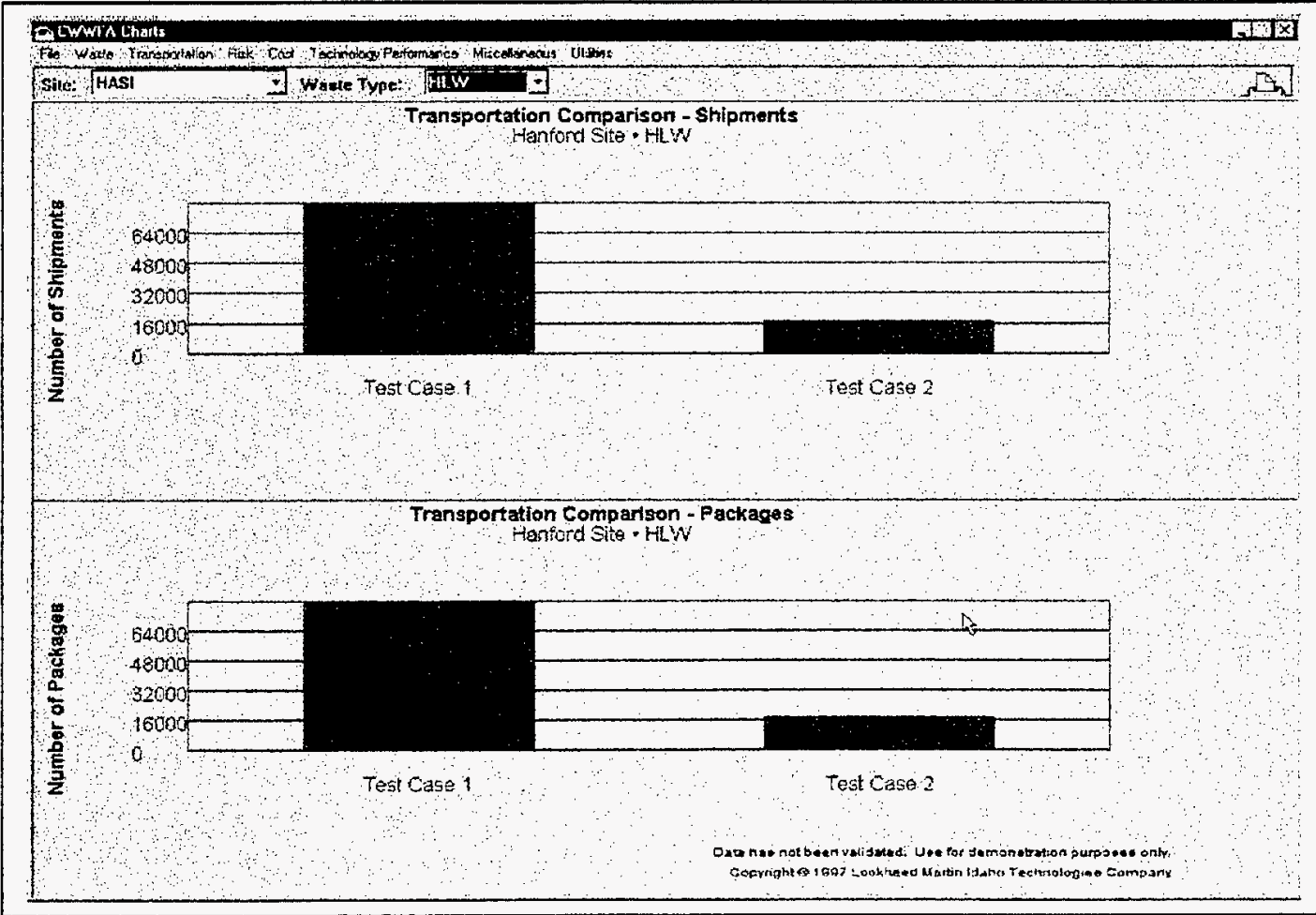


1.0 Waste Stream Information

\begin{tabular}{|l|l|}
\hline Originating Site & HASI \\
\hline Waste Stream ID \& Name & HASIWS1-CASE4 \\
\hline Originating Type & HLW \\
\hline Legacy volume (m3) & 45,000 \\
\hline
\end{tabular}

1.1 New Generation

\begin{tabular}{|l|l|l|}
\hline \multicolumn{1}{|c|}{ Start Year } & \multicolumn{1}{|c|}{ End Year } & Annual Volume (m3) \\
\hline 1997 & 2005 & 100 \\
\hline 2006 & 2010 & 200 \\
\hline & & \\
\hline & & \\
\hline
\end{tabular}

1.2 Shipping Route(s)

\begin{tabular}{|l|l|l|}
\hline \multicolumn{1}{|c|}{ Route ID } & \multicolumn{1}{|c|}{ Source Facility } & \multicolumn{1}{|c|}{ Destination Facility } \\
\hline C4a-1 & Legacy-Hanf & SNLFAC1-CASE4 \\
\hline C4a-2 & Legacy-Hanf & HASIFAC1-CASE4 \\
\hline C4a-3 & SNLFAC1-CASE4 & NVTSFAC1-CASE4 \\
\hline C4a-4 & SNLFAC1-CASE4 & HASIFAC3-CASE4 \\
\hline C4a-5 & HASIFAC1-CASE4 & SNLFAC2-CASE4 \\
\hline C4a-6 & HASIFAC1-CASE4 & HASIFAC2-CASE4 \\
\hline
\end{tabular}

1.3 Shipping Schedule(s)

\begin{tabular}{|c|c|c|c|}
\hline \multicolumn{4}{|l|}{ Route ID: C4a-l } \\
\hline Start Year & End Year & Waste Type & Annual Volume (m3) \\
\hline 1997 & 2010 & HLW & 1000 \\
\hline 2011 & 2015 & HLW & 1700 \\
\hline & & & \\
\hline
\end{tabular}

1.3 Shipping Schedule(s) (continued)

\begin{tabular}{|c|l|l|l|}
\hline Route ID: C4a-2 & \multicolumn{2}{|c|}{ Waste Type } & \multicolumn{1}{c|}{ Annual Volume (m3) } \\
\hline Start Year & \multicolumn{1}{|c|}{ End Year } & 1000 \\
\hline 1997 & 2010 & HLW & 1700 \\
\hline & 2015 & HLW & \\
\hline & & & \\
\hline
\end{tabular}

1.3 Shipping Schedule(s) (continued)

\begin{tabular}{|c|c|c|c|}
\hline \multicolumn{4}{|l|}{ Route ID: C4a-3 } \\
\hline Start Year & End Year & Waste Type & Annual Volume (m3) \\
\hline 2011 & 2020 & HLW & 1000 \\
\hline 2021 & 2025 & HLW & 250 \\
\hline & & & \\
\hline
\end{tabular}

1.3 Shipping Schedule(s) (continued)

\begin{tabular}{|c|c|c|c|}
\hline Route ID: C4a-4 & \multicolumn{2}{|c|}{ Waste Type } & Annual Volume (m3) \\
\hline Start Year & \multicolumn{1}{|c|}{ End Year } & LLW & 1125 \\
\hline & 2030 & & \\
\hline & & & \\
\hline & & & \\
\hline
\end{tabular}




\section{INITIAL PARAMETERS \& RESULTS REPORT}

CASE: Case $4 \mathrm{~d}$

DATE: $11 / 13 / 97$

Evaluator: K B Oswald

1.3 Shipping Schedule(s) (continued)

\begin{tabular}{|c|c|l|c|}
\hline Route ID: C4a-5 & \multicolumn{2}{|c|}{ Waste Type } & Annual Volume (m3) \\
\hline Start Year & \multicolumn{1}{|c|}{ End Year } & \multicolumn{1}{|c|}{ W } & 1000 \\
\hline 2011 & 2015 & HLW & 3050 \\
\hline 2016 & 2020 & HLW & \\
\hline & & & \\
\hline
\end{tabular}

1.3 Shipping Schedule(s) (continued)

\begin{tabular}{|c|c|c|c|}
\hline Route ID: C4a-6 & \multicolumn{2}{|c|}{ Waste Type } & \multicolumn{1}{c|}{ Annual Volume (m3) } \\
\hline Start Year & \multicolumn{1}{|c|}{ End Year } & LLW & 225 \\
\hline & 2030 & & \\
\hline & & & \\
\hline & & & \\
\hline
\end{tabular}

2.0 Facility Information

\begin{tabular}{|l|l|}
\hline Site & SNLN \\
\hline Facility ID \& Name & SNLFAC1-CASE4 \\
\hline Function & Treatment \\
\hline Start Year & 1997 \\
\hline End Year & 2070 \\
\hline Annual Capacity (m3/year) or & $1,000,000$ \\
\hline Lifetime Facility Capacity (m3) & \\
\hline Volume Change Factor & 1 \\
\hline
\end{tabular}

Conversion Profile

\begin{tabular}{|l|l|l|}
\hline Percent & Waste Type & Density \\
\hline $50 \%$ & LLW & 1800 \\
\hline $50 \%$ & HLW & 1800 \\
\hline & & \\
\hline
\end{tabular}

2.0 Facility Information

\begin{tabular}{|l|l|}
\hline Site & HASI \\
\hline Facility ID \& Name & HASIFAC1-CASE4 \\
\hline Function & Treatment \\
\hline Start Year & 1997 \\
\hline End Year & 2070 \\
\hline Annual Capacity (m3/year) or & $1,000,000$ \\
\hline Lifetime Facility Capacity (m3) & \\
\hline Volume Change Factor & 1 \\
\hline
\end{tabular}

Conversion Profile

\begin{tabular}{|l|l|l|}
\hline Percent & Waste Type & Density \\
\hline 90 & HLW & 1800 \\
\hline 10 & LLW & 1800 \\
\hline
\end{tabular}

2.0 Facility Information

\begin{tabular}{|l|l|}
\hline Site & NVTS \\
\hline Facility ID \& Name & NVTSFAC1-CASE4 \\
\hline Function & Disposal \\
\hline Start Year & 1997 \\
\hline End Year & 2070 \\
\hline Annual Capacity (m3/year) or & $1,000,000$ \\
\hline Lifetime Facility Capacity (m3) & \\
\hline Volume Change Factor & 1 \\
\hline
\end{tabular}

Conversion Profile

\begin{tabular}{|l|l|l|}
\hline Percent & Waste Type & Density \\
\hline NA & NA & NA \\
\hline
\end{tabular}




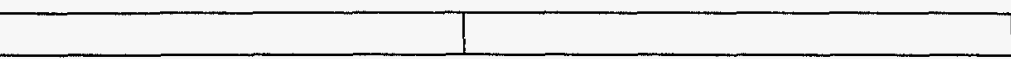

2.0 Facility Information

\begin{tabular}{|c|c|c|c|}
\hline Site & & HASI & \\
\hline Facility ID \& Name & & HASIFAC3-CASE4 & \\
\hline Function & & Disposal & \\
\hline Start Year & & 1997 & \\
\hline End Year & & 2070 & \\
\hline Annual Capacity (m3/year) or & & $1,000,000$ & \\
\hline Lifetime Facility Capacity (m3 & & & \\
\hline Volume Change Factor & 1 & 1 & \\
\hline Conversion Profile & & & \\
\hline Percent & Waste Typ & & Density \\
\hline $\mathrm{NA}$ & NA & & NA \\
\hline
\end{tabular}

2.0 Facility Information

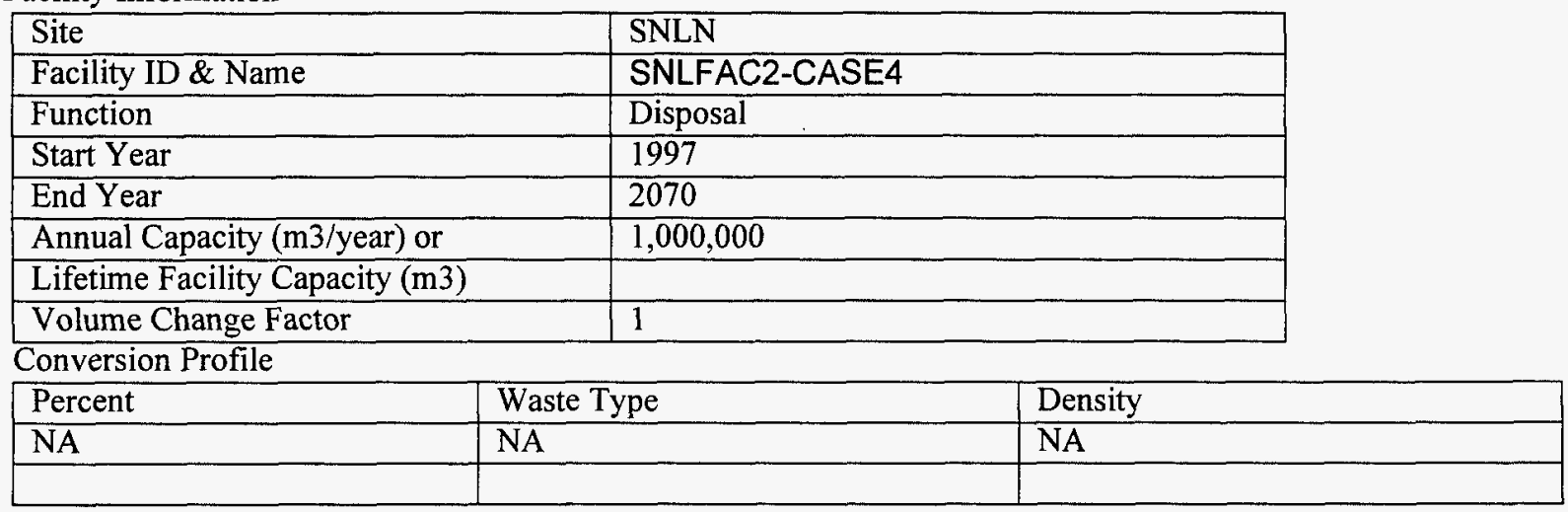

2.0 Facility Information

\begin{tabular}{|c|c|c|c|}
\hline \multirow{2}{*}{\multicolumn{2}{|c|}{ Site }} & \multirow{2}{*}{\multicolumn{2}{|c|}{ HASI }} \\
\hline & & & \\
\hline \multicolumn{2}{|l|}{ Facility ID \& Name } & \multicolumn{2}{|l|}{ HASIFAC2-CASE4 } \\
\hline \multicolumn{2}{|l|}{ Function } & \multicolumn{2}{|c|}{ Disposal } \\
\hline \multicolumn{2}{|l|}{ Start Year } & \multicolumn{2}{|c|}{1997} \\
\hline \multicolumn{2}{|l|}{ End Year } & \multicolumn{2}{|c|}{2070} \\
\hline \multicolumn{2}{|l|}{ Annual Capacity (m3/year) or } & \multicolumn{2}{|c|}{$1,000,000$} \\
\hline \multicolumn{4}{|l|}{ Lifetime Facility Capacity (m3) } \\
\hline Volume Change Factor & & \multicolumn{2}{|c|}{1} \\
\hline \multicolumn{4}{|l|}{ Conversion Profile } \\
\hline Percent & Waste Typ & & Density \\
\hline NA & NA & & $\mathrm{NA}$ \\
\hline
\end{tabular}


WASTE DISPOSITION - EXCEL CHART (HASI, HLW):

\section{Waste Disposition}

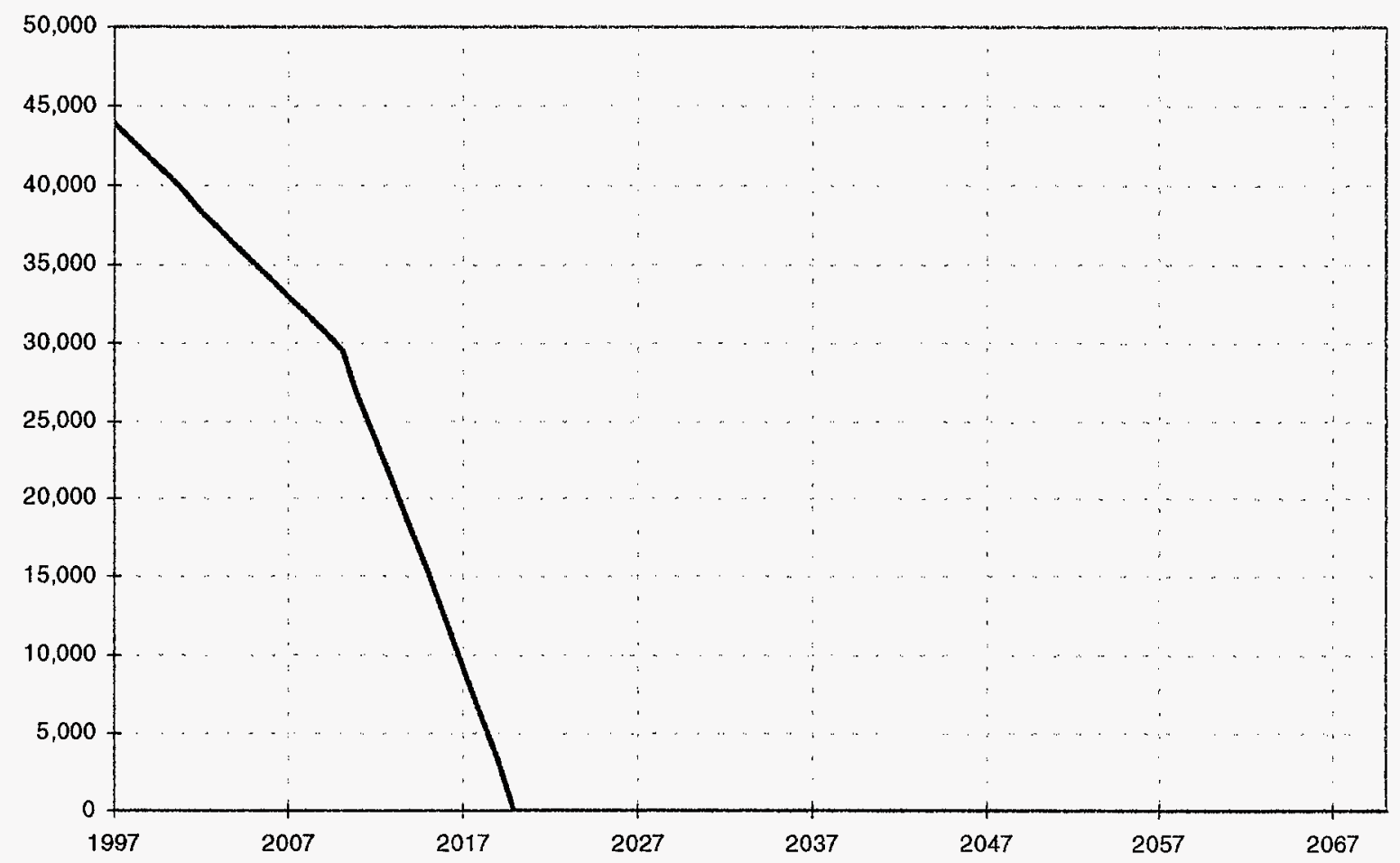

WASTE DISPOSITION - CWWFA CHART:

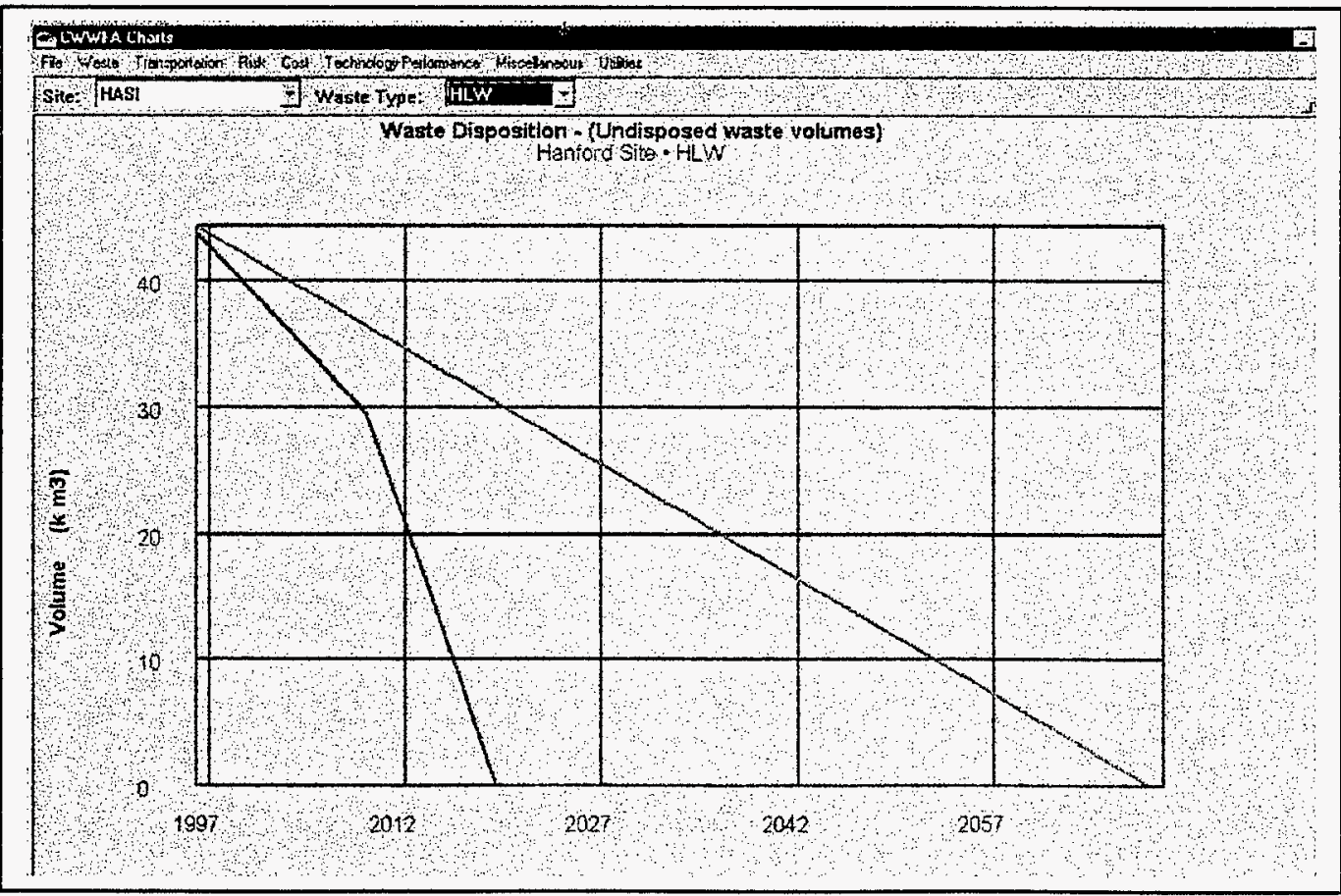




\section{FACILITY CAPACITY EXCEL CHART (NVTS, NVTSFAC1-CASE4):}

\section{Facility Capacity Detail}

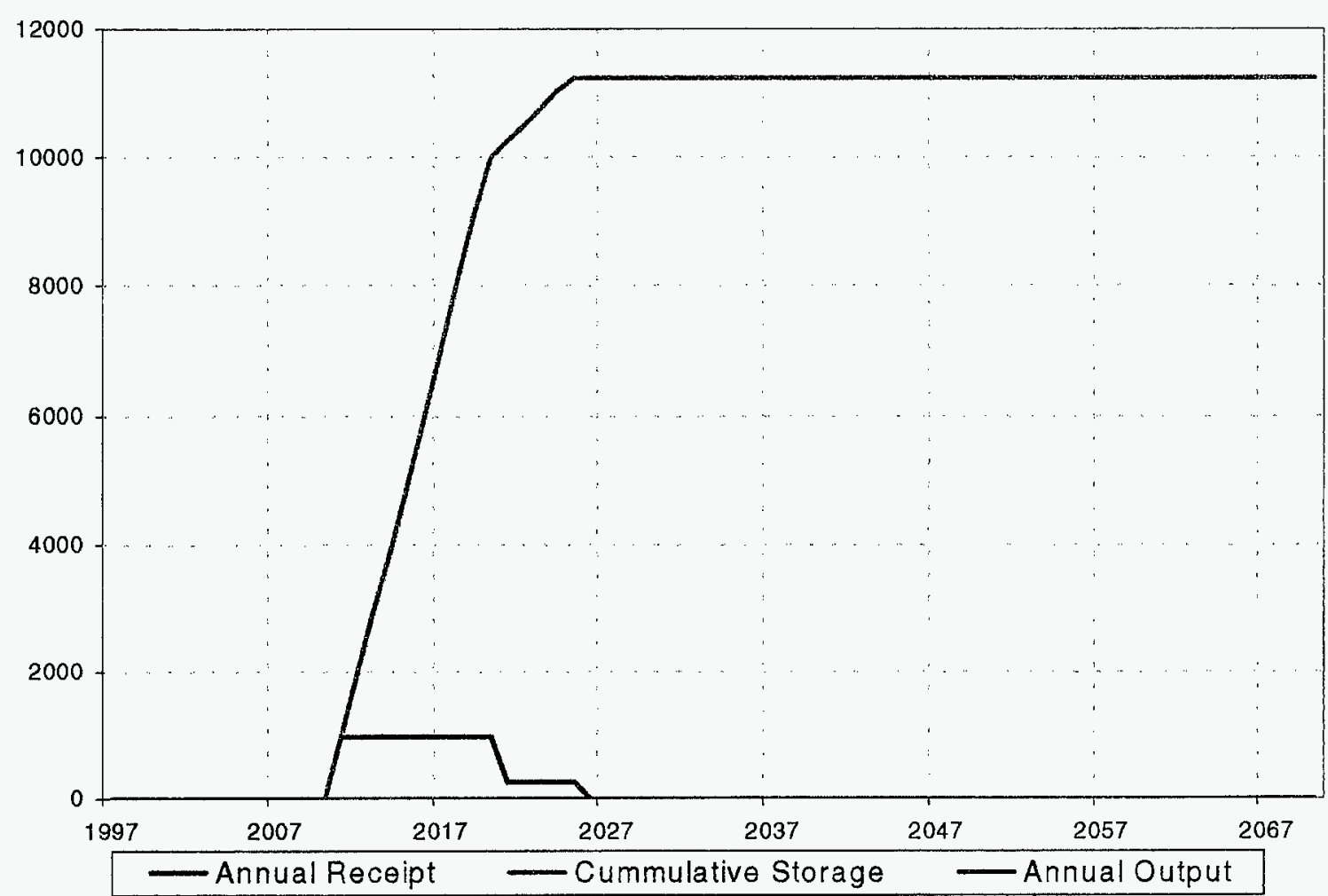

\section{FACILITY CAPACITY CWWFA CHART:}

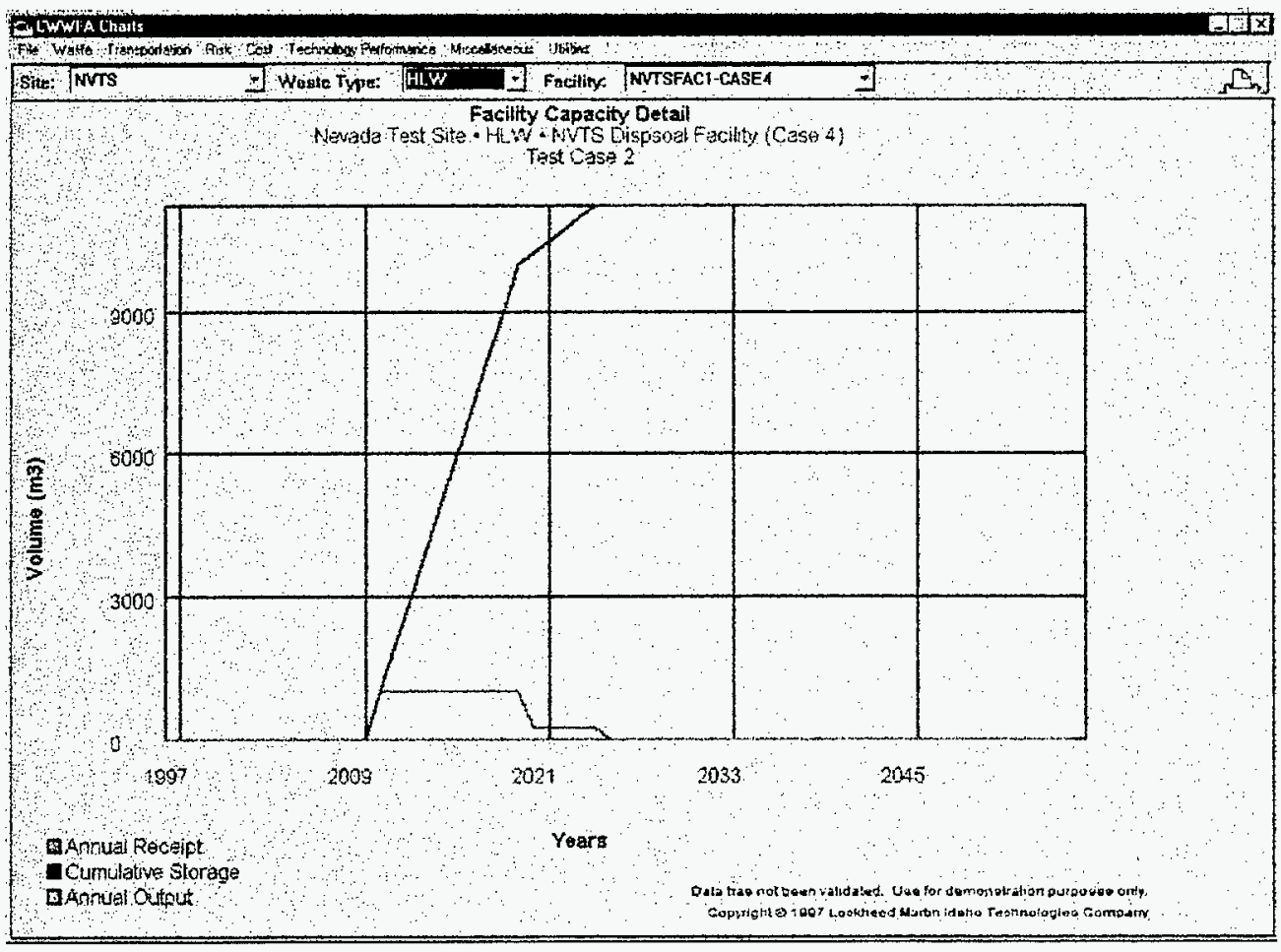


WASTE STREAM DETAIL EXCEL CHART (HASIWS1-CASE4):

\section{Waste Stream Detail}

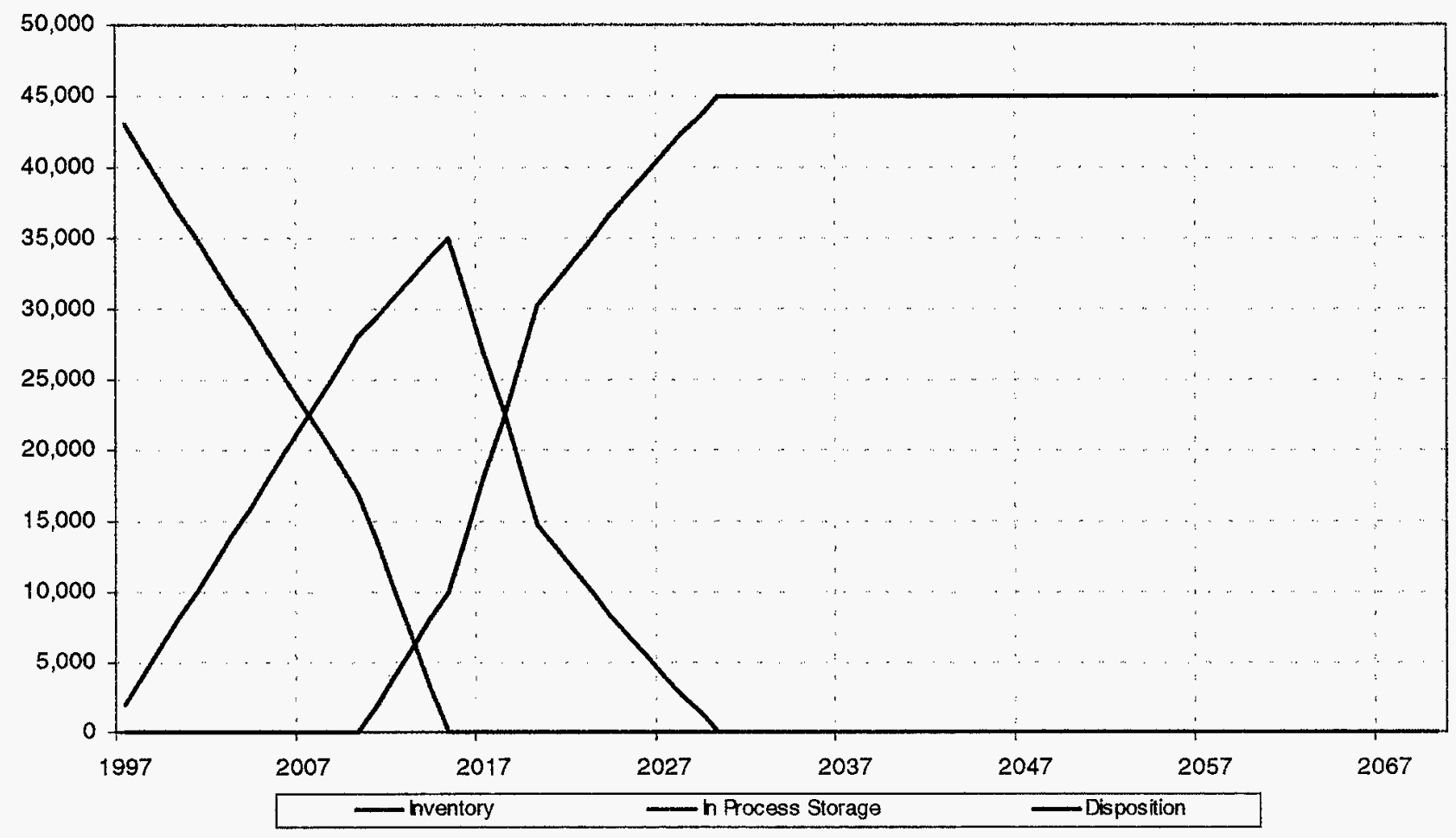

\section{WASTE STREAM DETAIL CWWFA CHART:}

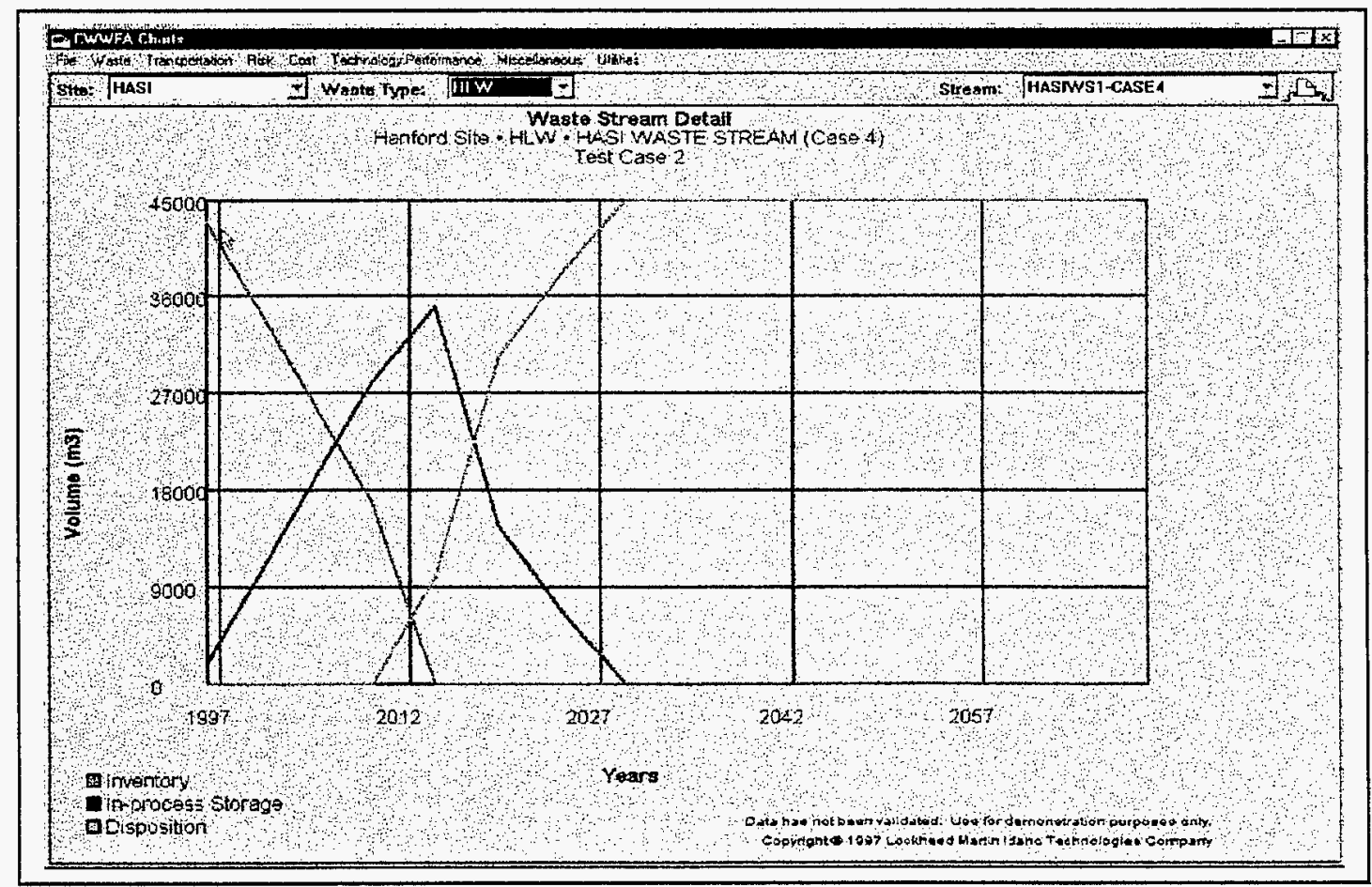


SHIPMENTS RECEIVED EXCEL CHART (HASI, HLW):

\section{Shipments Received}

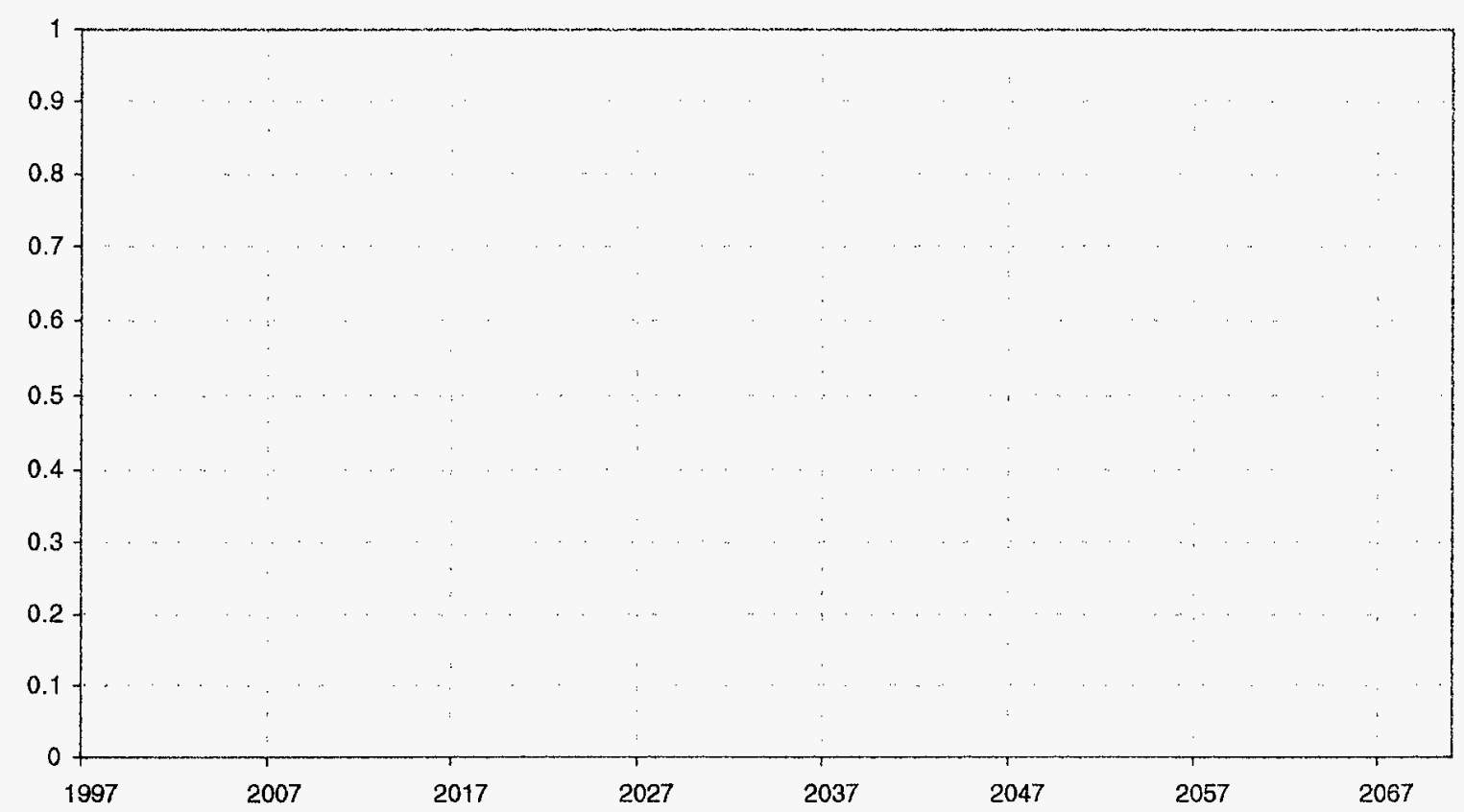

\section{SHIPMENTS RECEIVED CWWFA CHART:}

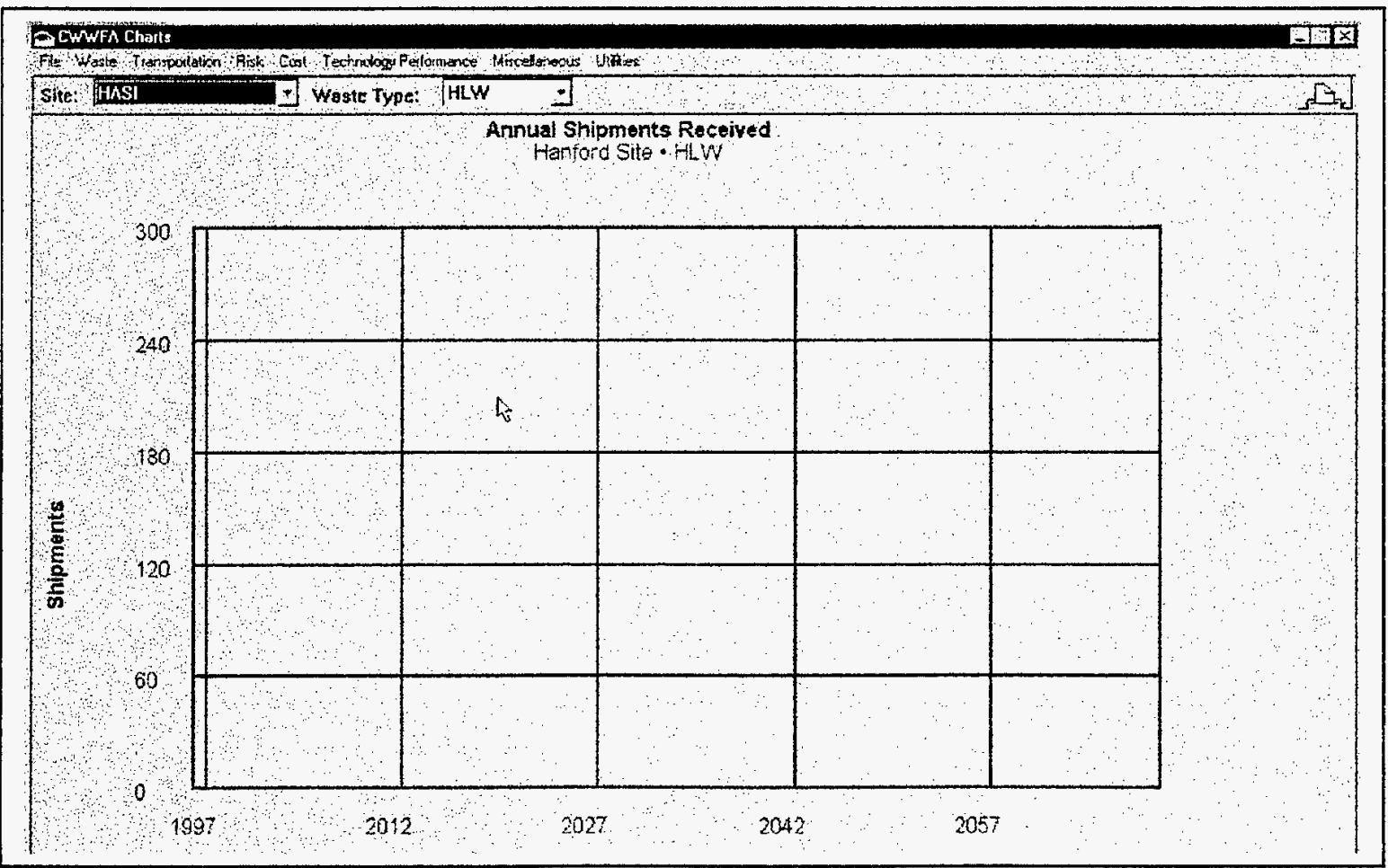




\section{Shipments Sent}

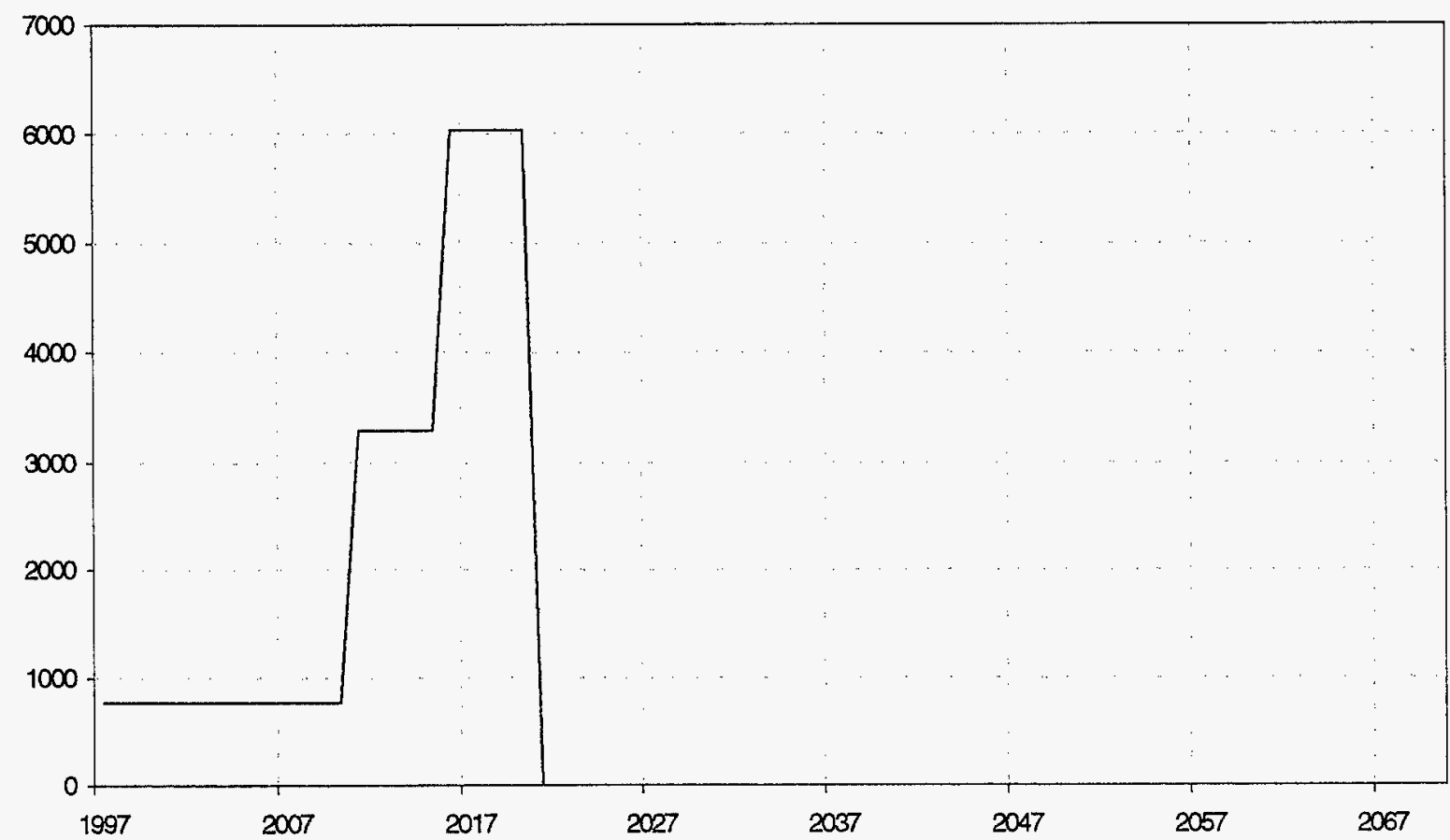

\section{SHIPMENTS SENT CWWFA CHART:}

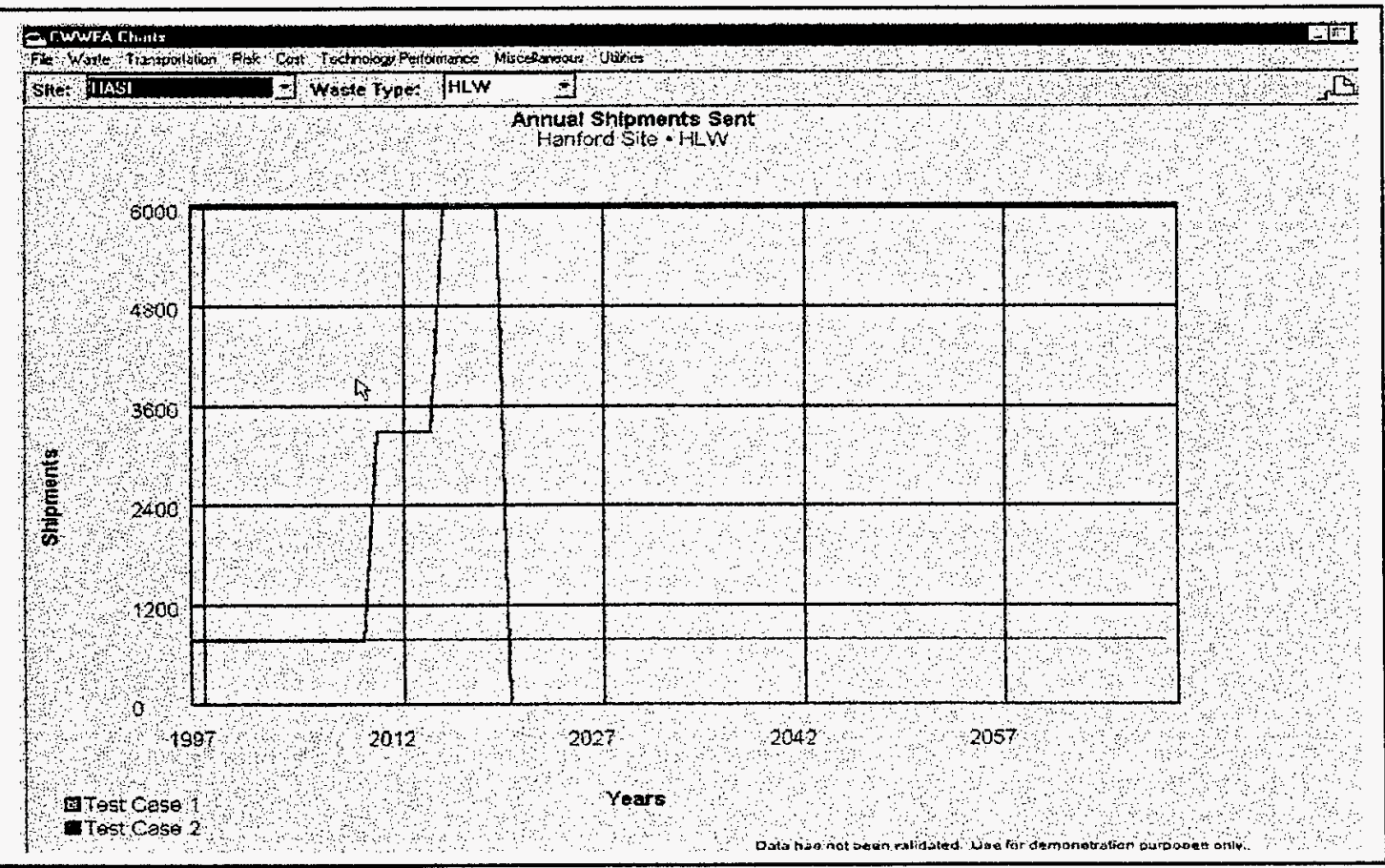



CASE: Case $4 \mathrm{~d}$
DATE: $11 / 13 / 97$
Evaluator: K B Oswald

TRANSPORTATION COMPARISON EXCEL CHART (HASI, HLW):

TRANSPORTAION COMPARISON

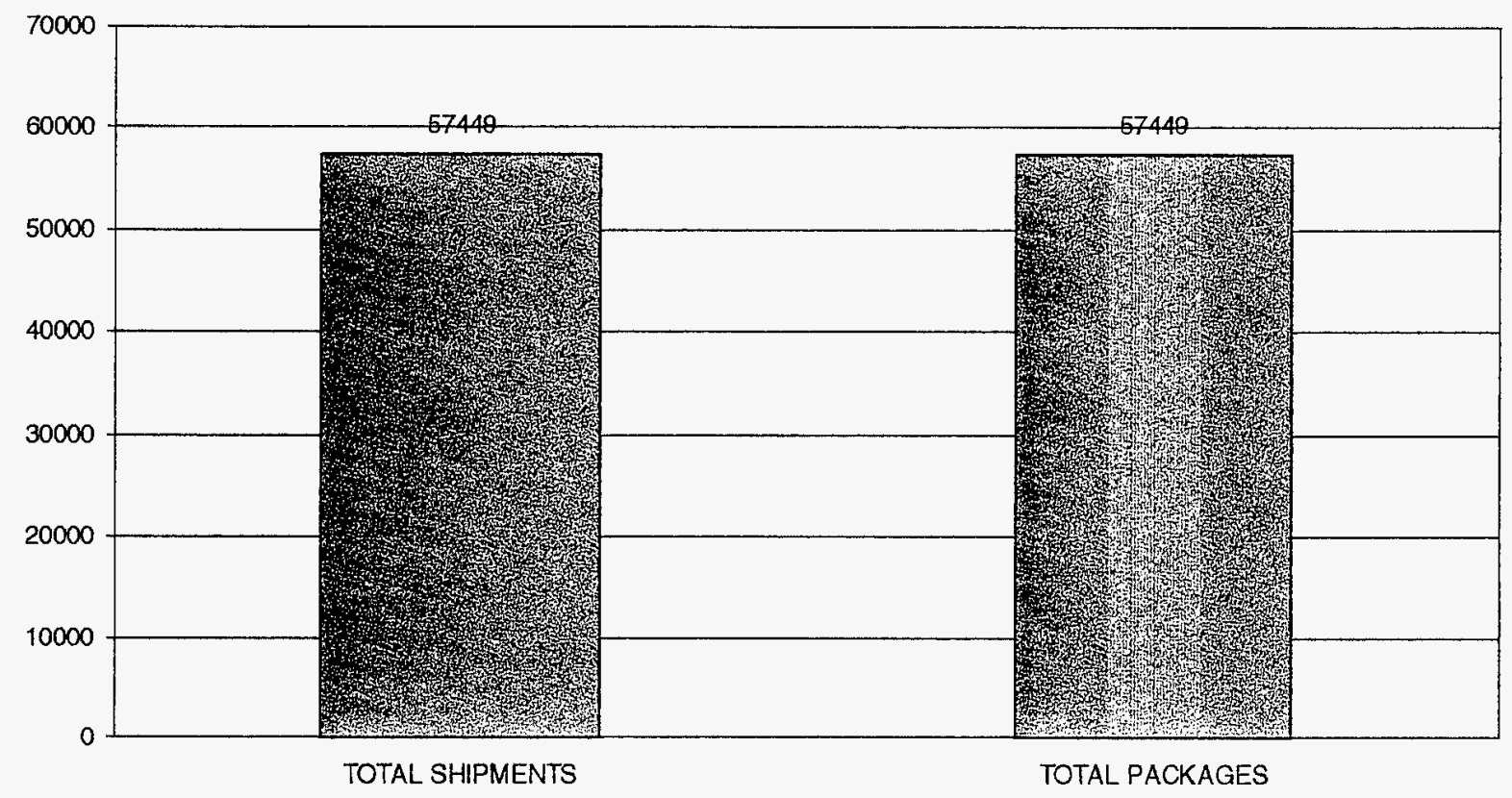

TRANSPORTATION COMPARISON CWWFA CHART:

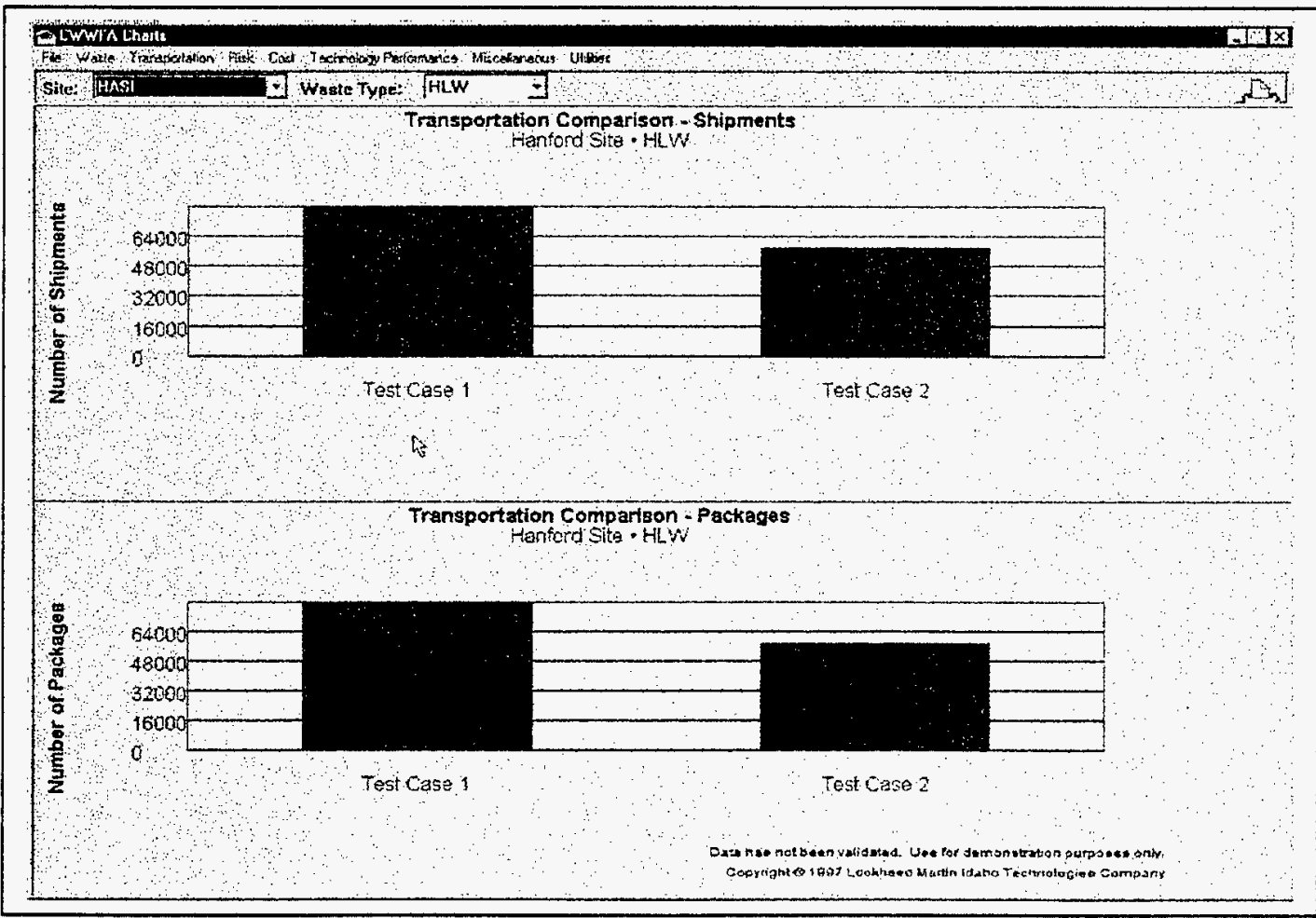


CASE: Case 5a

INITIAL PARAMETERS \& RESULTS REPORT

DATE: $11 / 13 / 97$

Evaluator: K B Oswald

1.0 Waste Stream Information

\begin{tabular}{|l|l|}
\hline Originating Site & INEEL \\
\hline Waste Stream ID \& Name & INELWS1-Case5 (Originating Type - MLLW) \\
\hline Legacy volume (m3) & 25,000 \\
\hline
\end{tabular}

1.1 New Generation

\begin{tabular}{|l|l|l|}
\hline \multicolumn{1}{|c|}{ Start Year } & \multicolumn{1}{|c|}{ End Year } & Annual Volume (m3) \\
\hline NA & NA & NA \\
\hline & & \\
\hline & & \\
\hline & & \\
\hline
\end{tabular}

1.2 Shipping Route(s)

\begin{tabular}{|l|l|l|}
\hline \multicolumn{1}{|c|}{ Route ID } & \multicolumn{1}{|c|}{ Source Facility } & Destination Facility \\
\hline C5a-1 & Legacy-INEEL & INELFAC1-CASE5 \\
\hline C5a-2 & INELFAC1-CASE5 & INELFAC2-CASE5 \\
\hline & & \\
\hline & & \\
\hline & & \\
\hline & & \\
\hline
\end{tabular}

1.3 Shipping Schedule(s)

\begin{tabular}{|c|c|l|c|}
\hline Route ID: C5a-1 & \multicolumn{2}{|c|}{} \\
\cline { 1 - 2 } Start Year & \multicolumn{1}{|c|}{ End Year } & \multicolumn{1}{c}{ Waste Type } & Annual Volume (m3) \\
\hline 1997 & 2070 & MLLW & 338 \\
\hline & & & \\
\hline & & & \\
\hline & & & \\
\hline
\end{tabular}

1.3 Shipping Schedule(s) (continued)

\begin{tabular}{|c|c|l|c|}
\hline Route ID: C5a-2 & \multicolumn{2}{|c|}{} & \multicolumn{1}{|c|}{ Waste Type } \\
\hline Start Year & \multicolumn{1}{|c|}{ End Year } & Annual Volume (m3) \\
\hline 1997 & 2070 & & 338 \\
\hline & & & \\
\hline & & & \\
\hline
\end{tabular}

1.0 Waste Stream Information

\begin{tabular}{|l|l|}
\hline Originating Site & FEMP \\
\hline Waste Stream ID \& Name & FEMPWS1-Case5 (Originating Type-MLLW) \\
\hline Legacy volume (m3) & 30,000 \\
\hline
\end{tabular}

1.1 New Generation

\begin{tabular}{|l|l|l|}
\hline \multicolumn{1}{|c|}{ Start Year } & \multicolumn{1}{|c|}{ End Year } & Annual Volume (m3) \\
\hline NA & NA & NA \\
\hline & & \\
\hline & & \\
\hline & & \\
\hline
\end{tabular}

1.2 Shipping Route(s)

\begin{tabular}{|l|l|c|}
\hline \multicolumn{1}{|c|}{ Route ID } & \multicolumn{1}{|c|}{ Source Facility } & Destination Facility \\
\hline C5a-3 & Legacy-FN & INELFAC1-CASE5 \\
\hline C5a-4 & INELFAC1-CASE5 & INELFAC2-CASE5 \\
\hline & & \\
\hline
\end{tabular}

1.3 Shipping Schedule(s)

\begin{tabular}{|c|c|l|l|}
\hline Route ID: C5a-3 & \multicolumn{2}{|c|}{} & \multicolumn{1}{|c|}{ Waste Type } \\
\hline Start Year & \multicolumn{1}{|c|}{ End Year } & Annual Volume (m3) \\
\hline 1997 & 2070 & MLLW & 405 \\
\hline & & & \\
\hline & & & \\
\hline
\end{tabular}


1.3 Shipping Schedule(s) (continued)

\begin{tabular}{|c|l|l|l|}
\hline Route ID: C5a-4 & \multicolumn{2}{|c|}{} & \multicolumn{1}{|c|}{ Waste Type } \\
\hline Start Year & \multicolumn{1}{|c|}{ End Year } & Annual Volume (m3) \\
\hline 1997 & 2070 & MLLW & \\
\hline & & & \\
\hline & & & \\
\hline & & & \\
\hline
\end{tabular}

1.0 Waste Stream Information

\begin{tabular}{|l|l|}
\hline Originating Site & OAKR \\
\hline Waste Stream ID \& Name & OAKRWS1-Case5 (Originating Type-MLLW) \\
\hline Legacy volume (m3) & 35,000 \\
\hline
\end{tabular}

1.1 New Generation

\begin{tabular}{|l|l|l|}
\hline \multicolumn{1}{|c|}{ Start Year } & \multicolumn{1}{|c|}{ End Year } & Annual Volume (m3) \\
\hline NA & NA & NA \\
\hline & & \\
\hline & & \\
\hline & & \\
\hline
\end{tabular}

1.2 Shipping Route(s)

\begin{tabular}{|l|l|l|}
\hline \multicolumn{1}{|c|}{ Route ID } & \multicolumn{1}{|c|}{ Source Facility } & Destination Facility \\
\hline C5a-5 & Legacy-OR & INELFAC1-CASE5 \\
\hline C5a-6 & INELFAC1-CASE5 & INELFAC2-CASE5 \\
\hline & & \\
\hline & & \\
\hline & & \\
\hline & & \\
\hline
\end{tabular}

1.3 Shipping Schedule(s)

\begin{tabular}{|c|l|l|l|}
\hline Route ID: C5a-5 & \multicolumn{2}{|c|}{} & \multicolumn{1}{|c|}{ Waste Type } \\
\hline Start Year & \multicolumn{1}{|c|}{ End Year } & 473 \\
\hline 1997 & 2070 & MLLW & \\
\hline & & & \\
\hline & & & \\
\hline
\end{tabular}

1.3 Shipping Schedule(s) (continued)

\begin{tabular}{|c|l|l|l|}
\hline Route ID: C5a-6 & \multicolumn{2}{|c|}{} \\
\cline { 1 - 3 } Start Year & \multicolumn{1}{|c|}{ End Year } & \multicolumn{1}{c}{ Waste Type } & Annual Volume (m3) \\
\hline 1997 & 2070 & MLLW & 473 \\
\hline & & & \\
\hline & & & \\
\hline
\end{tabular}

1.0 Waste Stream Information

\begin{tabular}{|l|l|}
\hline Originating Site & OAKR \\
\hline Waste Stream ID \& Name & OAKRWS2-Case5 (Originating Type-MLLW) \\
\hline Legacy volume (m3) & 40,000 \\
\hline
\end{tabular}

1.1 New Generation

\begin{tabular}{|l|l|l|}
\hline \multicolumn{1}{|c|}{ Start Year } & \multicolumn{1}{|c|}{ End Year } & Annual Volume (m3) \\
\hline NA & NA & NA \\
\hline & & \\
\hline & & \\
\hline & & \\
\hline
\end{tabular}


CASE: Case $5 \mathrm{a}$

DATE: $11 / 13 / 97$

Evaluator: K B Oswald

1.2 Shipping Route(s)

\begin{tabular}{|c|c|c|}
\hline \multicolumn{1}{|c|}{ Route ID } & Source Facility & Destination Facility \\
\hline C5a-7 & Legacy-OR & INELFAC2-CASE5 \\
\hline & & \\
\hline & & \\
\hline & & \\
\hline & & \\
\hline & & \\
\hline
\end{tabular}

1.3 Shipping Schedule(s)

\begin{tabular}{|c|l|l|l|}
\hline Route ID: C5a-7 & \multicolumn{2}{|c|}{ Waste Type } & Annual Volume (m3) \\
\hline Start Year & \multicolumn{1}{|c|}{ End Year } & WLLW & \\
\hline 1997 & 2070 & & \\
\hline & & & \\
\hline & & & \\
\hline & & & \\
\hline
\end{tabular}

2.0 Facility Information

\begin{tabular}{|l|l|}
\hline Site & INEEL \\
\hline Facility ID \& Name & INELFAC1-CASE5 \\
\hline Function & Treatment \\
\hline Start Year & 1997 \\
\hline End Year & 2070 \\
\hline Annual Capacity (m3/year) or & $1,000,000$ \\
\hline Lifetime Facility Capacity (m3) & \\
\hline Volume Change Factor & 1 \\
\hline
\end{tabular}

Conversion Profile

\begin{tabular}{|l|l|l|}
\hline Percent & Waste Type & Density \\
\hline $100 \%$ & MLLW & 1198 \\
\hline & & \\
\hline & & \\
\hline
\end{tabular}

2.0 Facility Information

\begin{tabular}{|l|l|}
\hline Site & INEEL \\
\hline Facility ID \& Name & INELFAC2-CASE5 \\
\hline Function & Disposal \\
\hline Start Year & 1997 \\
\hline End Year & 2070 \\
\hline Annual Capacity (m3/year) or & $1,000,000$ \\
\hline Lifetime Facility Capacity (m3) & \\
\hline Volume Change Factor & 1 \\
\hline
\end{tabular}

Conversion Profile

\begin{tabular}{|l|l|l|}
\hline Percent & Waste Type & Density \\
\hline NA & NA & NA \\
\hline & & \\
\hline
\end{tabular}


CASE: Case $5 \mathrm{a}$

DATE: $11 / 13 / 97$

Evaluator: K B Oswald

WASTE DISPOSITION - EXCEL CHART (INEEL, MLLW):

\section{Waste Disposition}

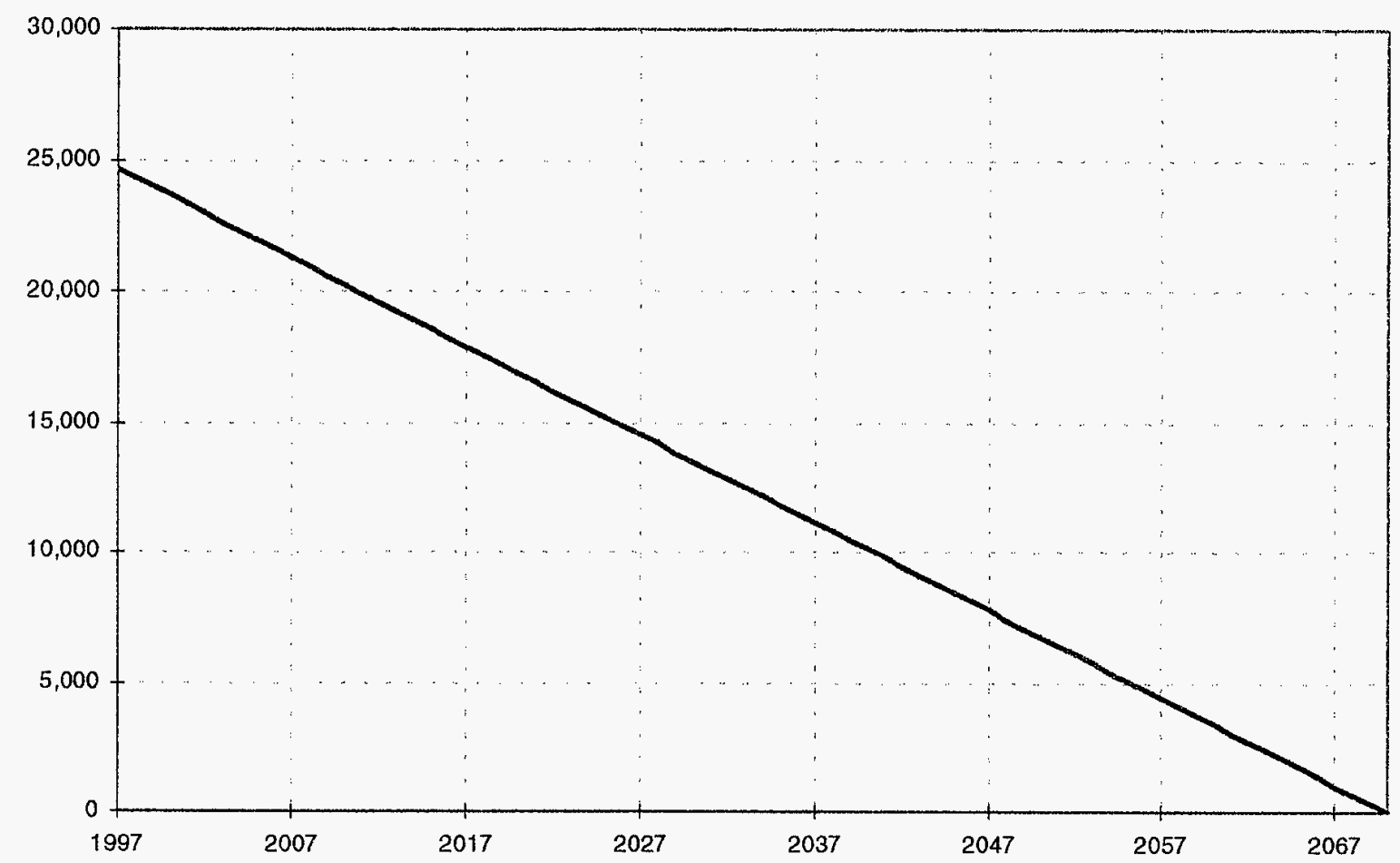

WASTE DISPOSITION - CWWFA CHART:

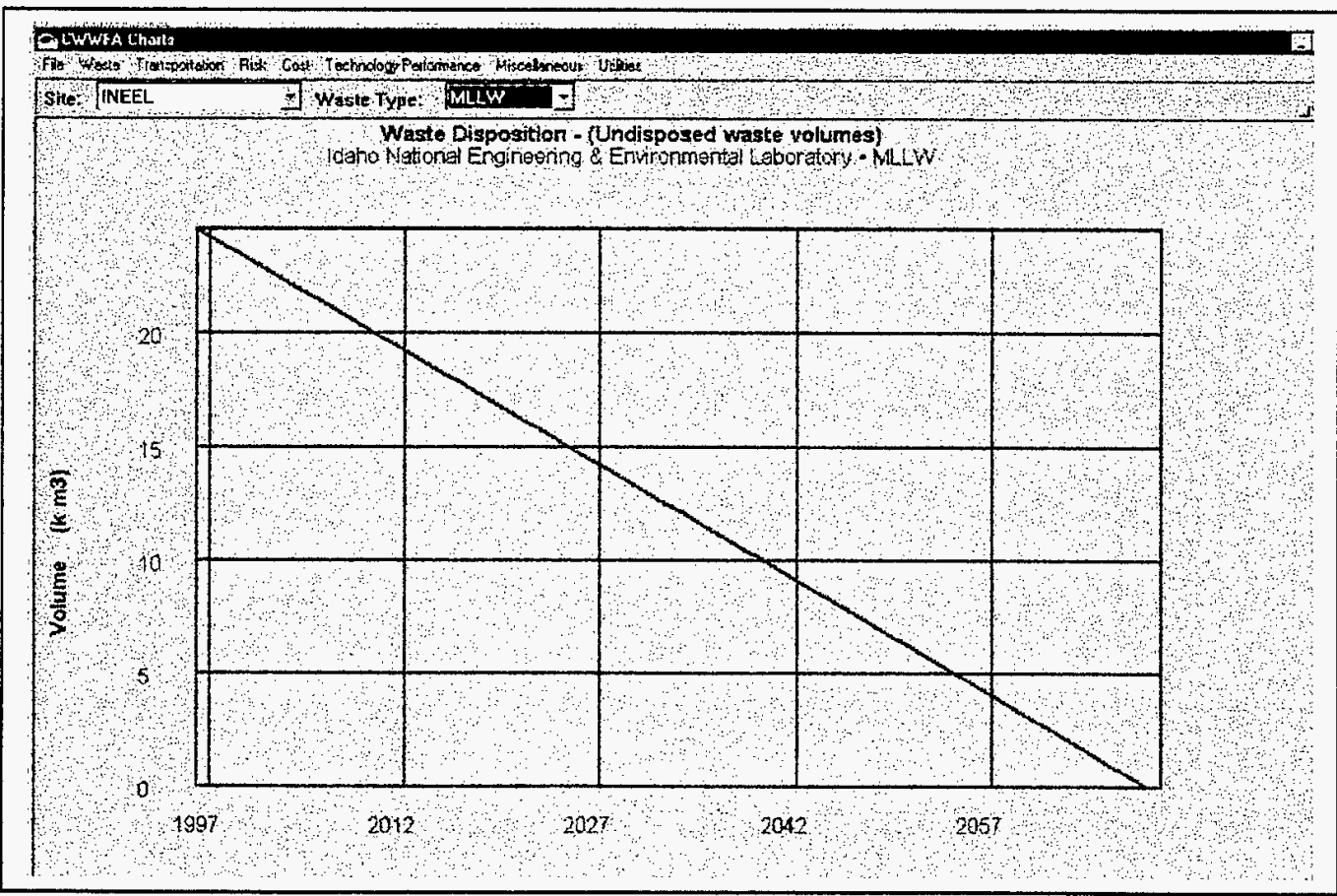




\section{FACILITY CAPACITY DETAIL EXCEL CHART:}

\section{Facility Capacity Detail}

$$
\text { INELFAC11-ASE5 }
$$

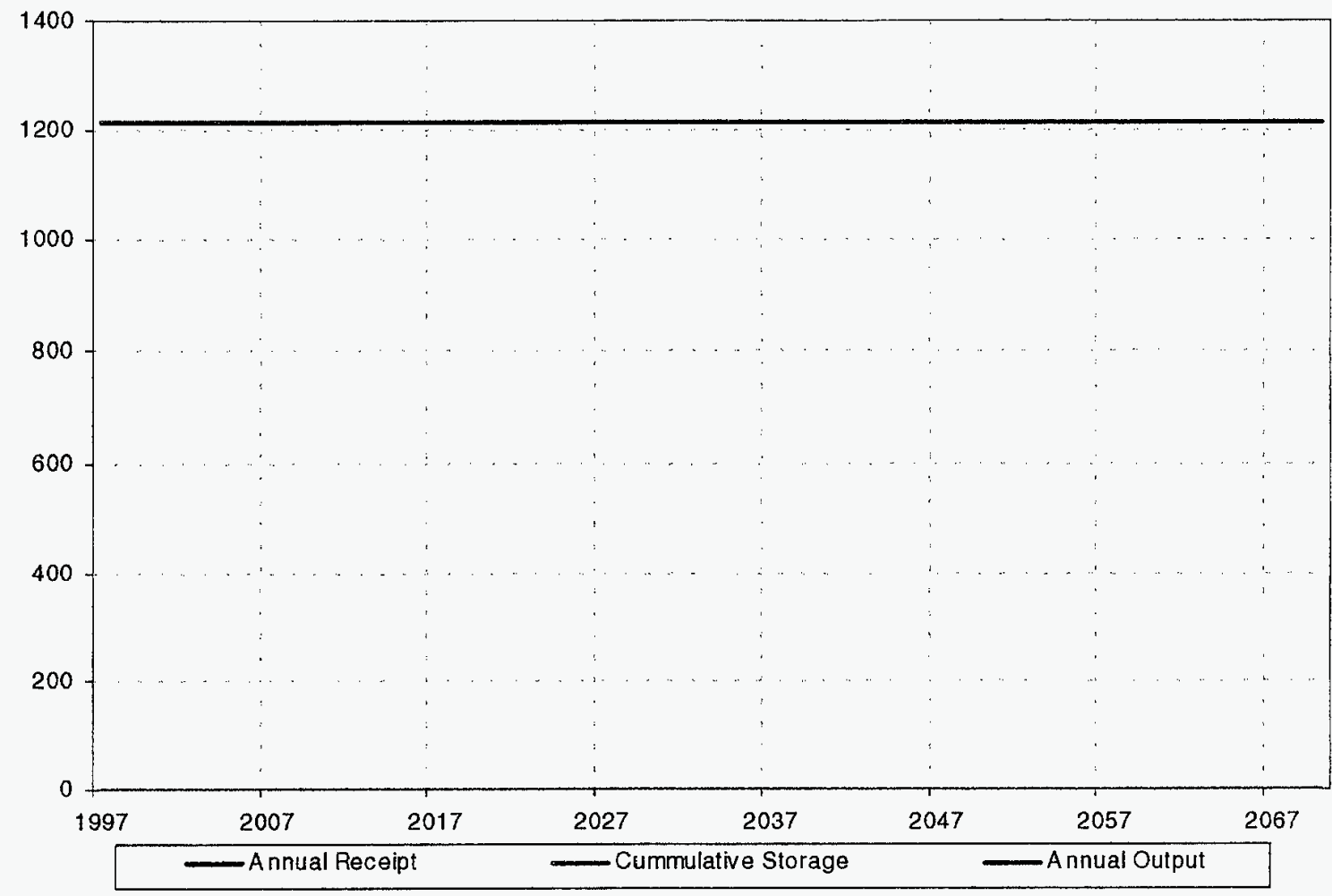

\section{FACILITY CAPACITY CWWFA CHART:}

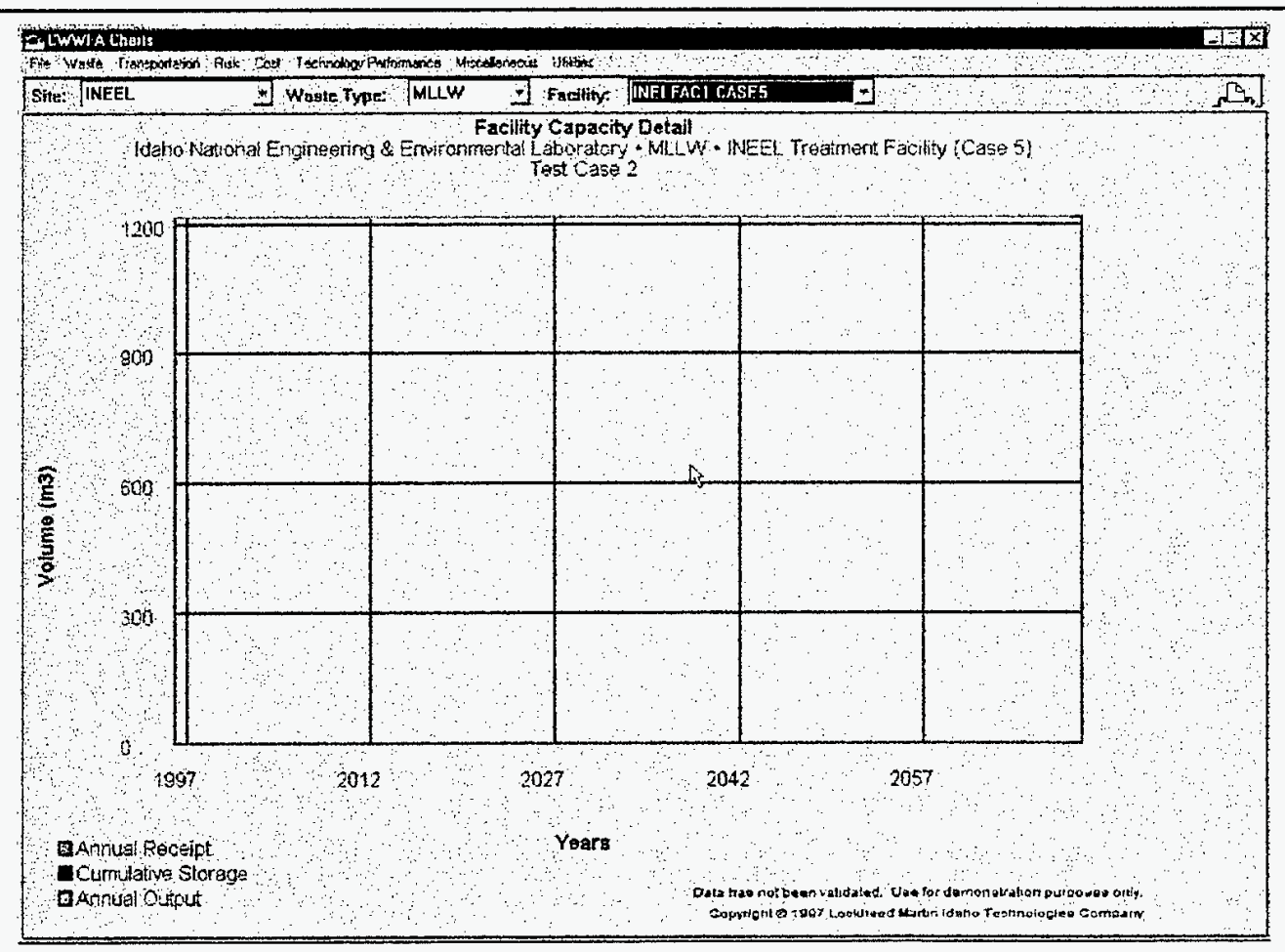


FACILITY CAPACITY DETAIL EXCEL CHART:

\section{Facility Capacity Detail} INELFAC2-CASE5

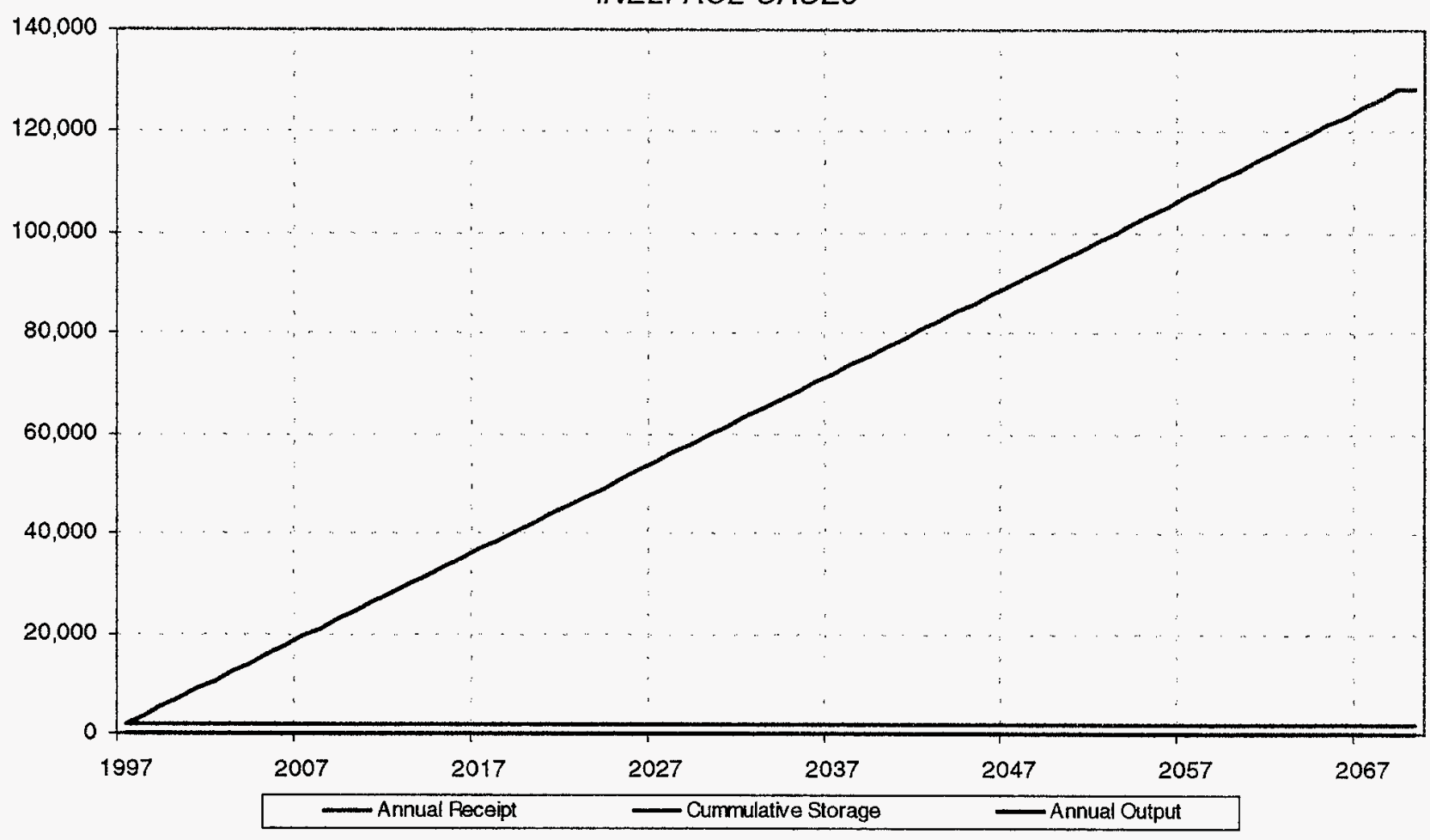

FACILITY CAPACITY DETAIL CWWFA CHART:

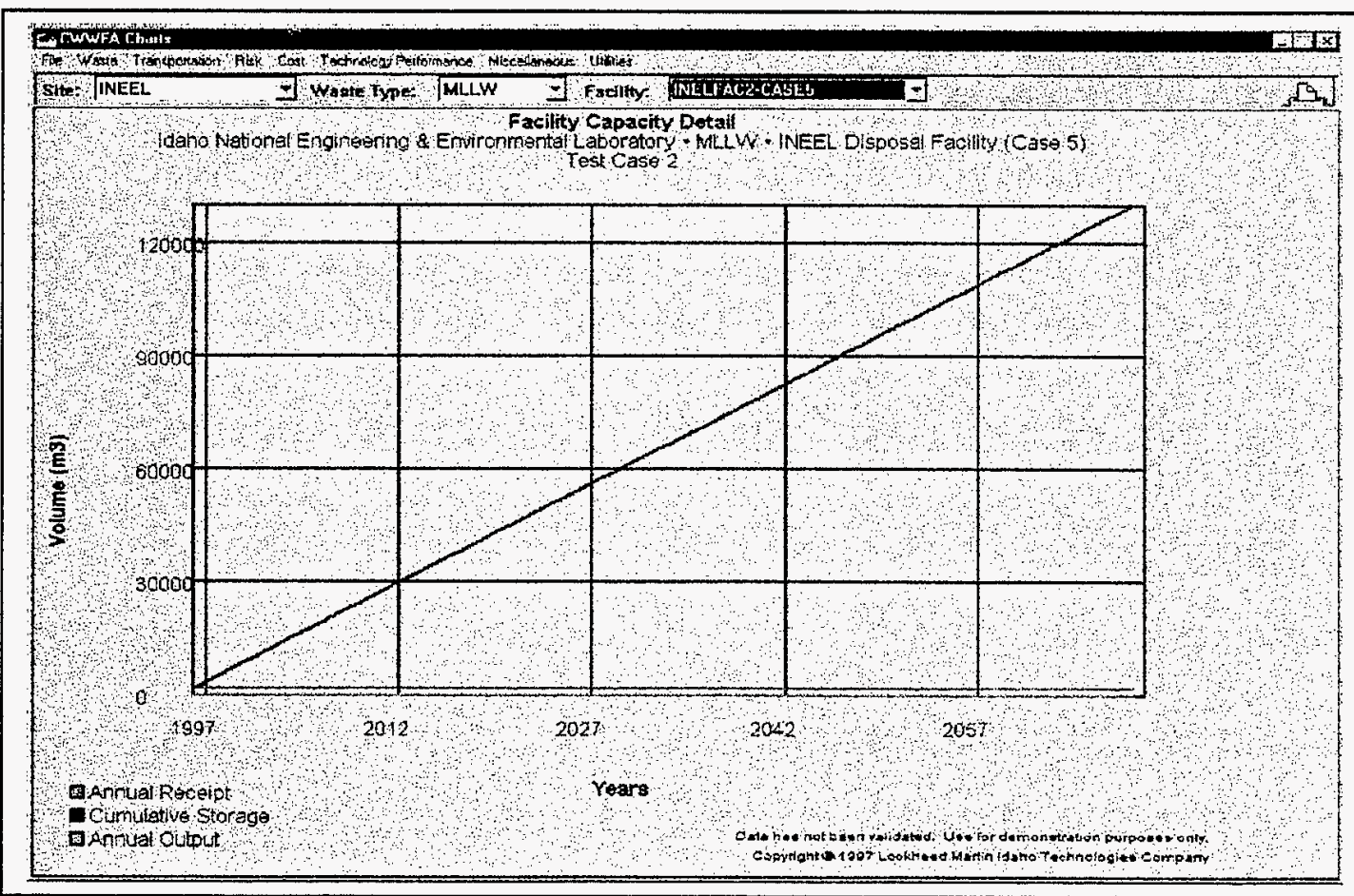




\section{Shipments Received}

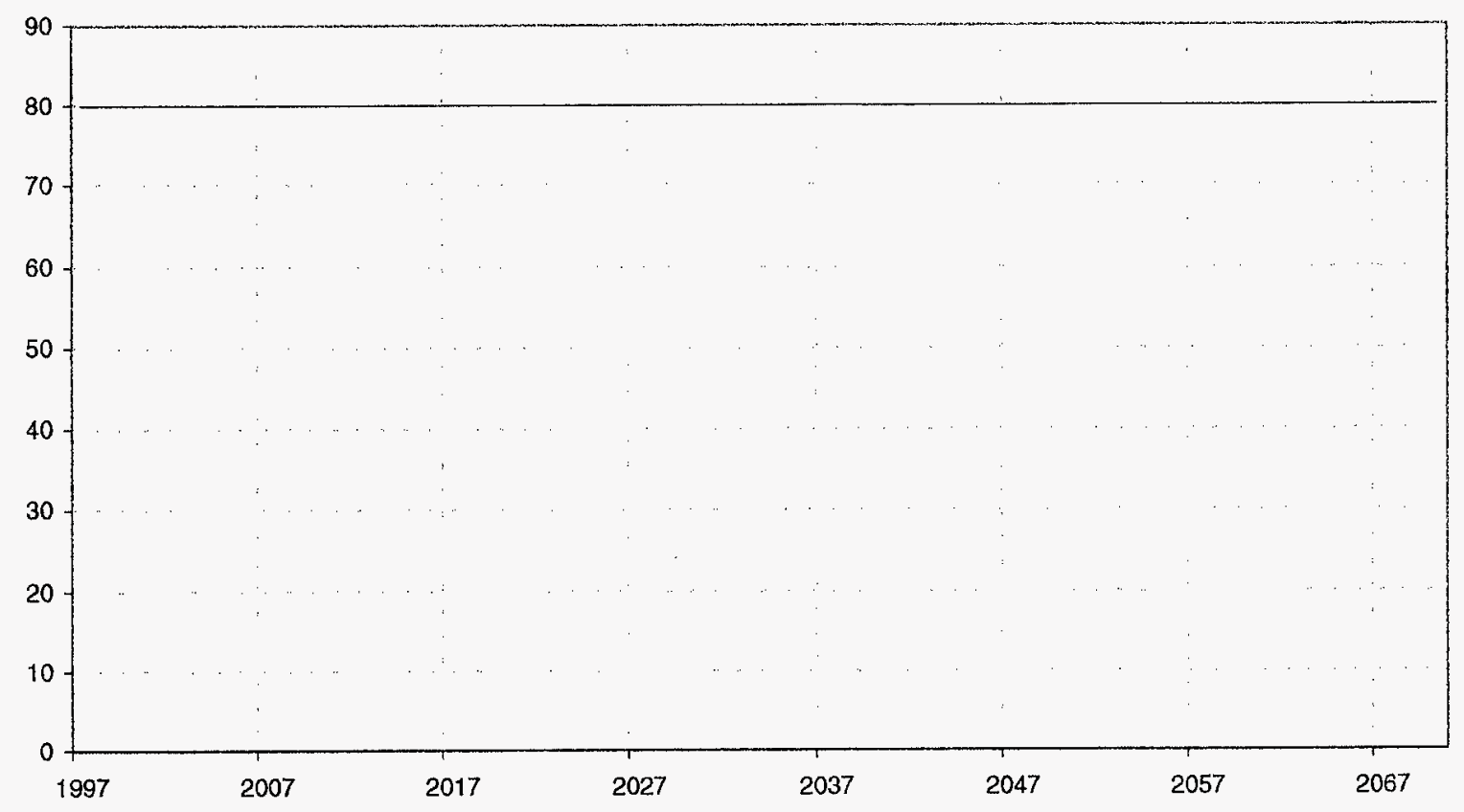

\section{SHIPMENTS RECEIVED CWWFA CHART:}

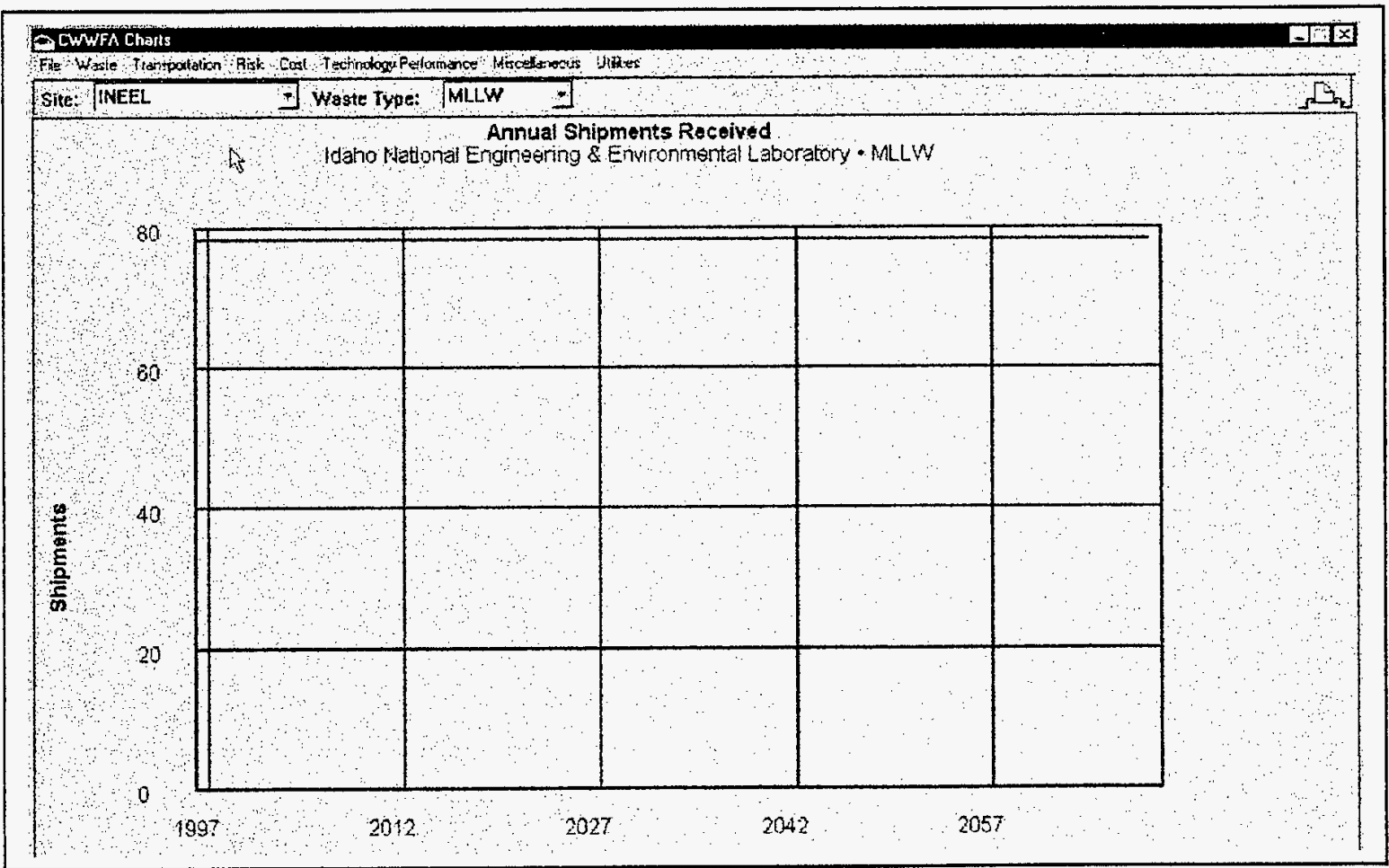


SHIPMENTS SENT EXCEL CHART (INEEL):

\section{Shipments Sent}

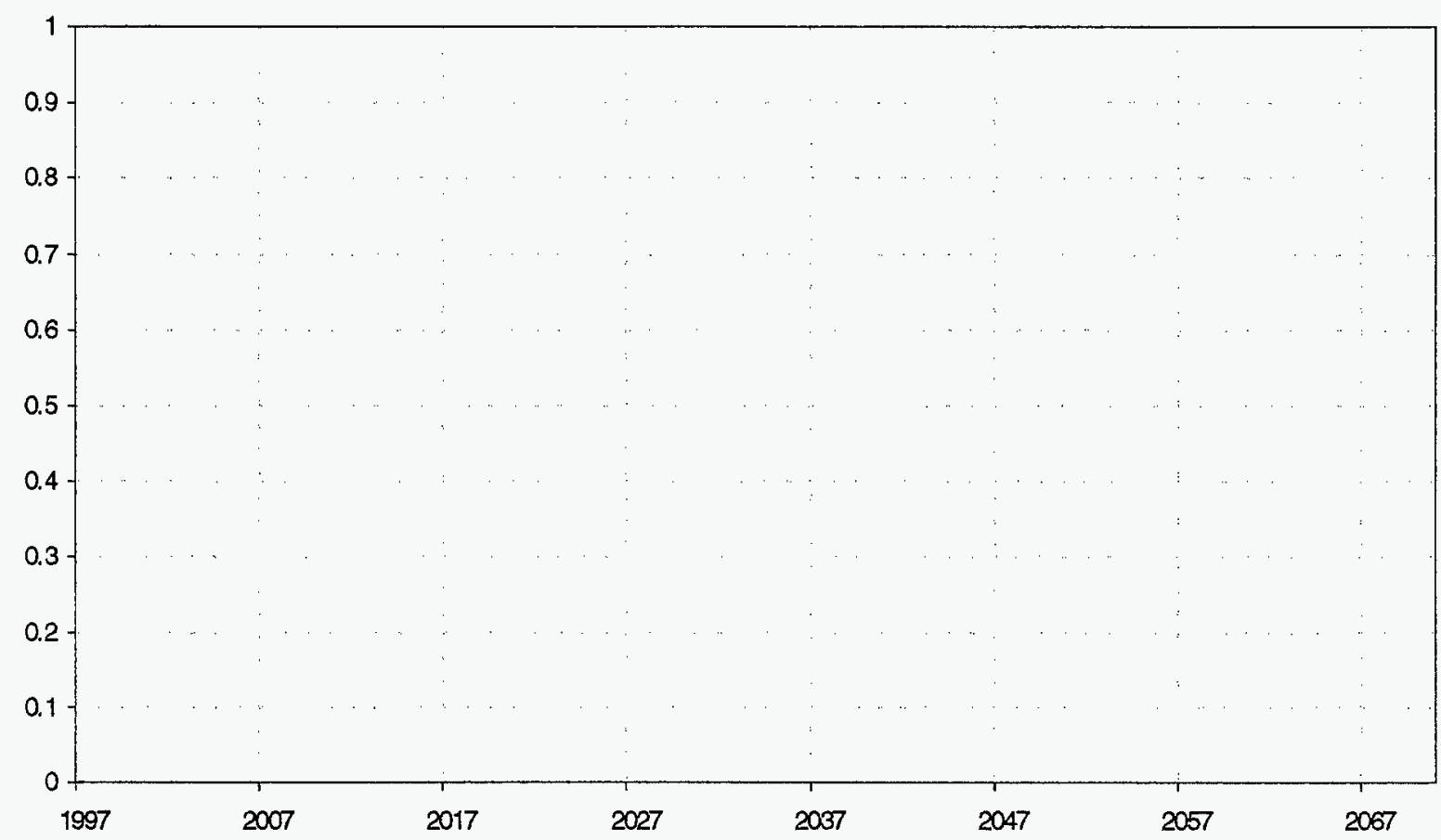

\section{SHIPMENTS SENT CWWFA CHART:}

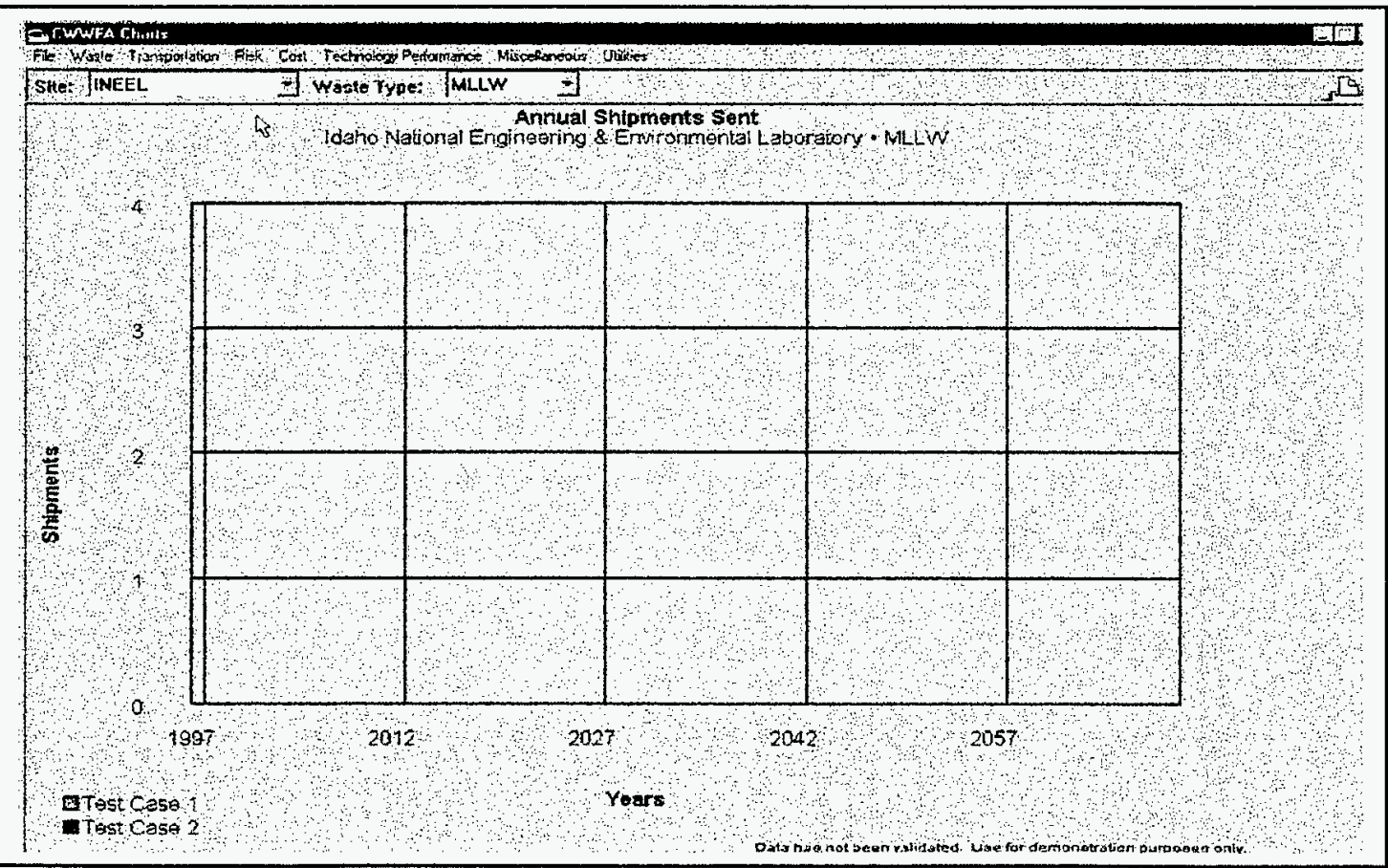


TRANSPORTAION COMPARISON

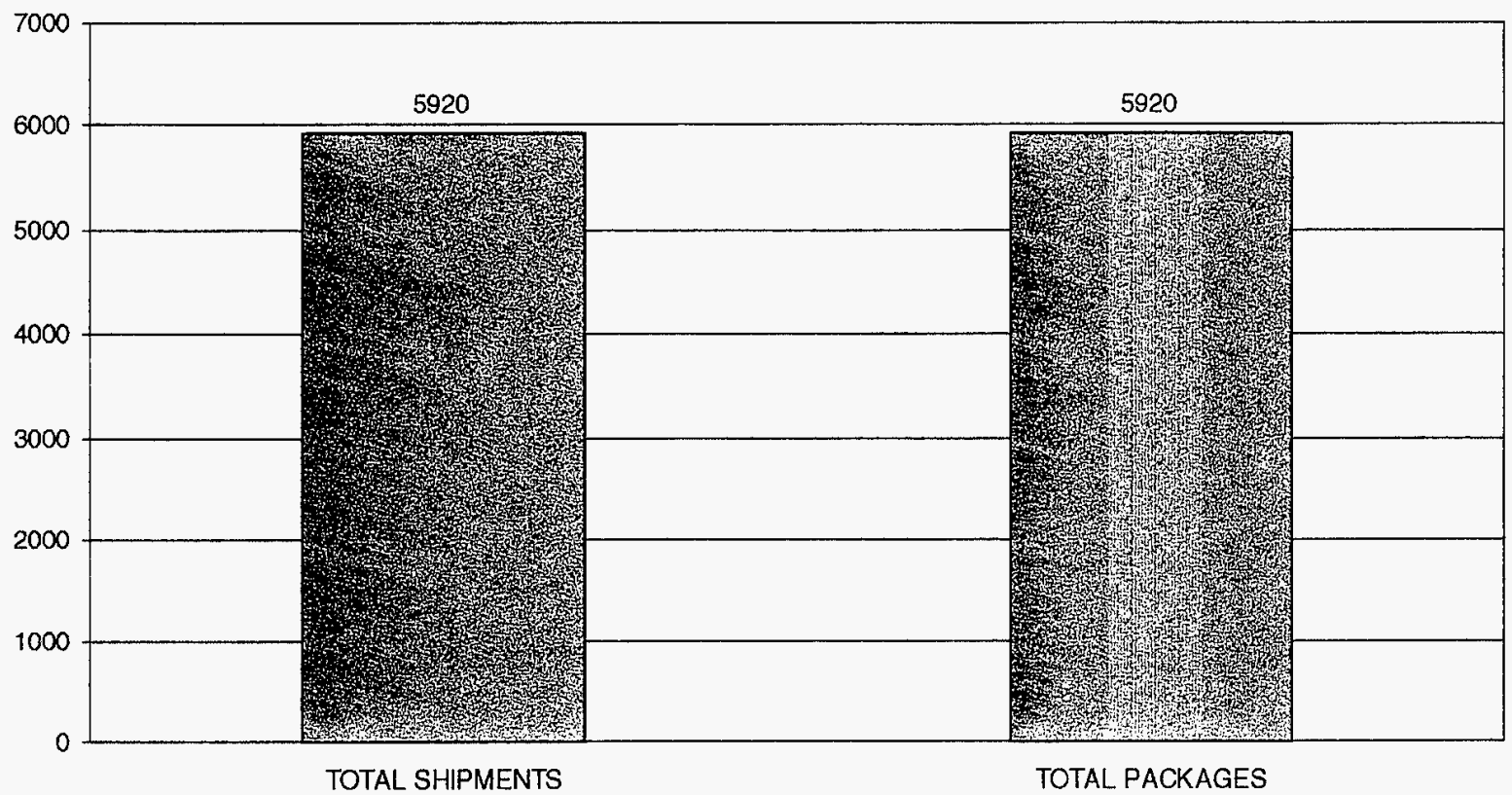

TRANSPORTATION COMPARISON CWWFA CHART:

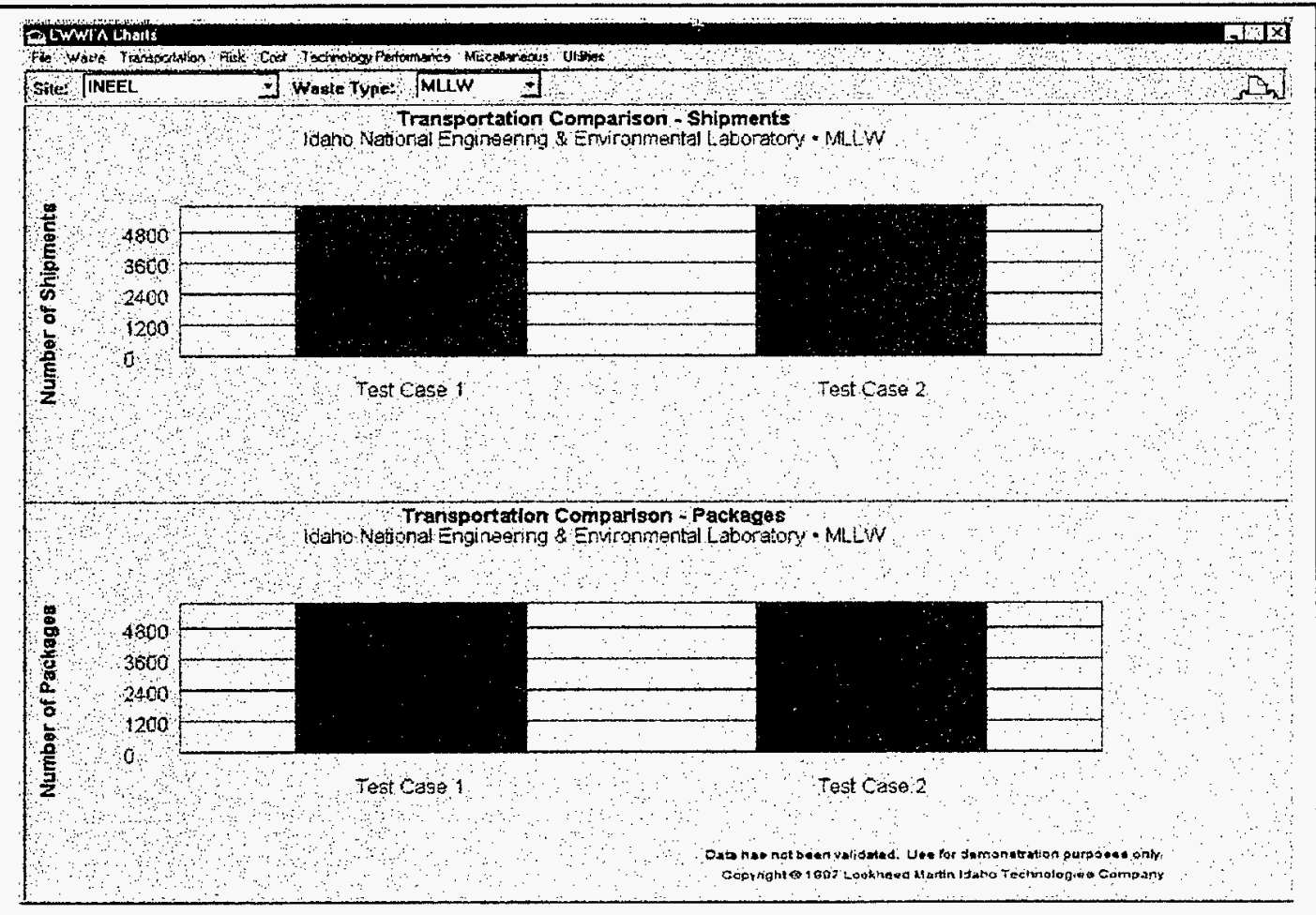




\section{INITIAL PARAMETERS \& RESULTS REPORT}

CASE: Case $5 b$

DATE: $11 / 13 / 97$

Evaluator: K B Oswald

1.0 Waste Stream Information

\begin{tabular}{|l|l|}
\hline Originating Site & INEEL \\
\hline Waste Stream ID \& Name & INELWS1-Case5 (Originating Type-MLLW) \\
\hline Legacy volume (m3) & 25,000 \\
\hline
\end{tabular}

1.1 New Generation

\begin{tabular}{|l|l|l|}
\hline \multicolumn{1}{|c|}{ Start Year } & \multicolumn{1}{|c|}{ End Year } & \multicolumn{1}{c|}{ Annual Volume (m3) } \\
\hline NA & NA & NA \\
\hline & & \\
\hline & & \\
\hline & & \\
\hline
\end{tabular}

1.2 Shipping Route(s)

\begin{tabular}{|l|l|l|}
\hline \multicolumn{1}{|c|}{ Route ID } & \multicolumn{1}{|c|}{ Source Facility } & \multicolumn{1}{c|}{ Destination Facility } \\
\hline C5a-1 & Legacy-INEEL & INELFAC1-CASE5 \\
\hline C5a-2 & INELFAC1-CASE5 & INELFAC2-CASE5 \\
\hline & & \\
\hline & & \\
\hline & & \\
\hline & & \\
\hline
\end{tabular}

1.3 Shipping Schedule(s)

\begin{tabular}{|c|c|c|c|}
\hline Route ID: C5a-1 & & & \\
\hline Start Year & End Year & Waste Type & Annual Volume (m3) \\
\hline 1997 & 2070 & MLLW & 338 \\
\hline & & & \\
\hline & & & \\
\hline
\end{tabular}

1.3 Shipping Schedule(s) (continued)

\begin{tabular}{|c|c|c|c|}
\hline Route ID: C5a-2 & & & \\
\hline Start Year & End Year & Waste Type & Annual Volume (m3) \\
\hline 1997 & 2070 & MLLW & 338 \\
\hline & & & \\
\hline & & & \\
\hline
\end{tabular}

1.0 Waste Stream Information

\begin{tabular}{|l|l|}
\hline Originating Site & FEMP \\
\hline Waste Stream ID \& Name & FEMPWS1-Case5 (Originating Type-MLLW) \\
\hline Legacy volume $(\mathrm{m} 3)$ & 30,000 \\
\hline
\end{tabular}

1.1 New Generation

\begin{tabular}{|l|l|l|}
\hline \multicolumn{1}{|c|}{ Start Year } & \multicolumn{1}{|c|}{ End Year } & Annual Volume (m3) \\
\hline NA & NA & NA \\
\hline & & \\
\hline & & \\
\hline
\end{tabular}

1.2 Shipping Route(s)

\begin{tabular}{|l|l|l|}
\hline \multicolumn{1}{|c|}{ Route ID } & \multicolumn{1}{|c|}{ Source Facility } & Destination Facility \\
\hline C5a-3 & Legacy-FN & INELFAC1-CASE5 \\
\hline C5a-4 & INELFAC1-CASE5 & INELFAC2-CASE5 \\
\hline & & \\
\hline
\end{tabular}

1.3 Shipping Schedule(s)

\begin{tabular}{|c|c|l|l|}
\hline Route ID: C5a-3 & \multicolumn{2}{|c|}{ Waste Type } & \multicolumn{1}{|c|}{ Annual Volume (m3) } \\
\hline Start Year & \multicolumn{1}{|c|}{ End Year } & MLLW & 405 \\
\hline 1997 & 2070 & & \\
\hline & & & \\
\hline & & & \\
\hline
\end{tabular}


1.3 Shipping Schedule(s) (continued)

\begin{tabular}{|c|c|c|c|}
\hline Route ID: C5a-4 & & & \\
\hline Start Year & End Year & Waste Type & Annual Volume (m3) \\
\hline 1997 & 2070 & MLLW & 405 \\
\hline & & & \\
\hline & & & \\
\hline
\end{tabular}

1.0 Waste Stream Information

\begin{tabular}{|l|l|}
\hline Originating Site & OAKR \\
\hline Waste Stream ID \& Name & OAKRWS1-Case5 (Originating Type-MLLW) \\
\hline Legacy volume (m3) & 35,000 \\
\hline
\end{tabular}

1.1 New Generation

\begin{tabular}{|l|l|l|}
\hline \multicolumn{1}{|c|}{ Start Year } & \multicolumn{1}{|c|}{ End Year } & Annual Volume (m3) \\
\hline NA & NA & NA \\
\hline & & \\
\hline & & \\
\hline & & \\
\hline
\end{tabular}

1.2 Shipping Route(s)

\begin{tabular}{|l|l|l|}
\hline \multicolumn{1}{|c|}{ Route ID } & \multicolumn{1}{|c|}{ Source Facility } & Destination Facility \\
\hline C5a-5 & Legacy-OR & INELFAC1-CASE5 \\
\hline C5a-6 & INELFAC1-CASE5 & INELFAC2-CASE5 \\
\hline & & \\
\hline & & \\
\hline & & \\
\hline & & \\
\hline
\end{tabular}

1.3 Shipping Schedule(s)

\begin{tabular}{|c|c|l|l|}
\hline Route ID: C5a-5 & \multicolumn{2}{|c|}{ Waste Type } & \multicolumn{1}{|c|}{ Annual Volume (m3) } \\
\hline Start Year & \multicolumn{1}{|c|}{ End Year } & MLLW & \\
\hline 1997 & 2070 & & \\
\hline & & & \\
\hline & & & \\
\hline & & & \\
\hline
\end{tabular}

1.3 Shipping Schedule(s) (continued)

\begin{tabular}{|c|c|c|c|}
\hline \multicolumn{4}{|l|}{ Route ID: C5a-6 } \\
\hline Start Year & End Year & Waste Type & Annual Volume (m3) \\
\hline 1997 & 2070 & MLLW & 473 \\
\hline & & & \\
\hline & & & \\
\hline
\end{tabular}

1.0 Waste Stream Information

\begin{tabular}{|l|l|}
\hline Originating Site & OAKR \\
\hline Waste Stream ID \& Name & OAKRWS2-Case5 (Originating Type-MLLW) \\
\hline Legacy volume $(\mathrm{m} 3)$ & 40,000 \\
\hline
\end{tabular}

1.1 New Generation

\begin{tabular}{|l|l|l|}
\hline \multicolumn{1}{|c|}{ Start Year } & \multicolumn{1}{|c|}{ End Year } & Annual Volume (m3) \\
\hline NA & NA & NA \\
\hline & & \\
\hline & & \\
\hline & & \\
\hline
\end{tabular}


INITIAL PARAMETERS \& RESULTS REPORT

CASE: Case $5 b$

DATE: $11 / 13 / 97$

Evaluator: K B Oswald

1.2 Shipping Route(s)

\begin{tabular}{|l|l|l|}
\hline \multicolumn{1}{|c|}{ Route ID } & Source Facility & Destination Facility \\
\hline C5a-7 & Legacy-OR & INELFAC2-CASE5 \\
\hline & & \\
\hline & & \\
\hline & & \\
\hline & & \\
\hline & & \\
\hline
\end{tabular}

1.3 Shipping Schedule(s)

\begin{tabular}{|c|c|c|c|}
\hline Route ID: C5a-7 & \multicolumn{2}{|c|}{ Waste Type } & \multicolumn{1}{|c|}{ Annual Volume (m3) } \\
\hline Start Year & \multicolumn{1}{|c|}{ End Year } & MLLW & \\
\hline 1997 & 2070 & & \\
\hline & & & \\
\hline & & & \\
\hline & & & \\
\hline
\end{tabular}

2.0 Facility Information

\begin{tabular}{|l|l|}
\hline Site & INEEL \\
\hline Facility ID \& Name & INELFAC1-CASE5 \\
\hline Function & Treatment \\
\hline Start Year & 1997 \\
\hline End Year & 2070 \\
\hline Annual Capacity (m3/year) or & 1000 \\
\hline Lifetime Facility Capacity (m3; & \\
\hline Volume Change Factor & 1 \\
\hline
\end{tabular}

Conversion Profile

\begin{tabular}{|l|l|l|}
\hline Percent & Waste Type & Density \\
\hline $100 \%$ & MLLW & 1800 \\
\hline & & \\
\hline & & \\
\hline
\end{tabular}

2.0 Facility Information

\begin{tabular}{|l|l|}
\hline Site & INEEL \\
\hline Facility ID \& Name & INELFAC2-CASE5 \\
\hline Function & Disposal \\
\hline Start Year & 1997 \\
\hline End Year & 2070 \\
\hline Annual Capacity (m3/year) or & 1250 \\
\hline Lifetime Facility Capacity (m3) & 37500 \\
\hline Volume Change Factor & 1 \\
\hline
\end{tabular}

Conversion Profile

\begin{tabular}{|l|l|l|}
\hline Percent & Waste Type & Density \\
\hline NA & NA & NA \\
\hline & & \\
\hline
\end{tabular}


WASTE DISPOSITION - EXCEL CHART (INEEL, MLLW):

\section{Waste Disposition}

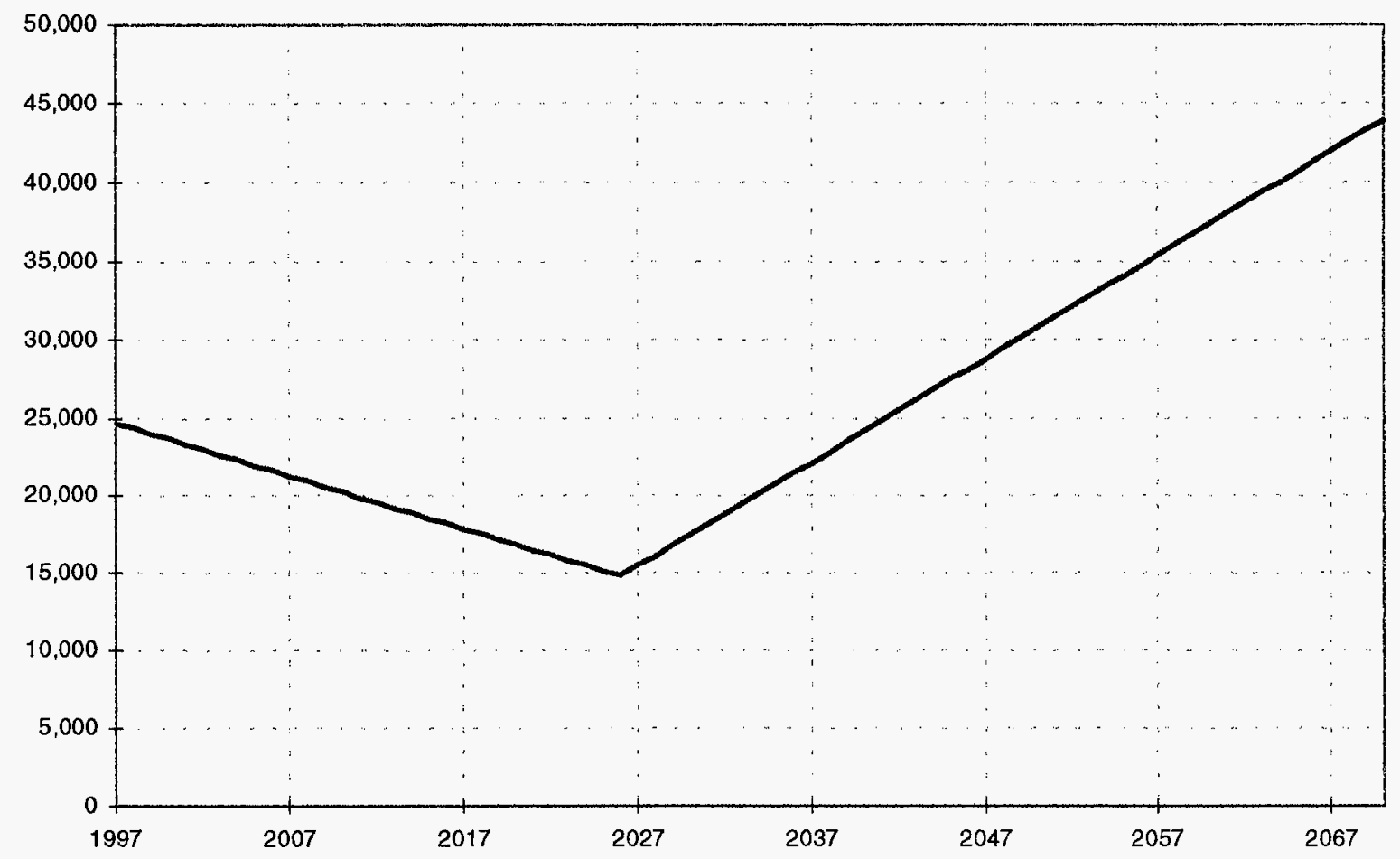

\section{WASTE DISPOSITION - CWWFA CHART:}

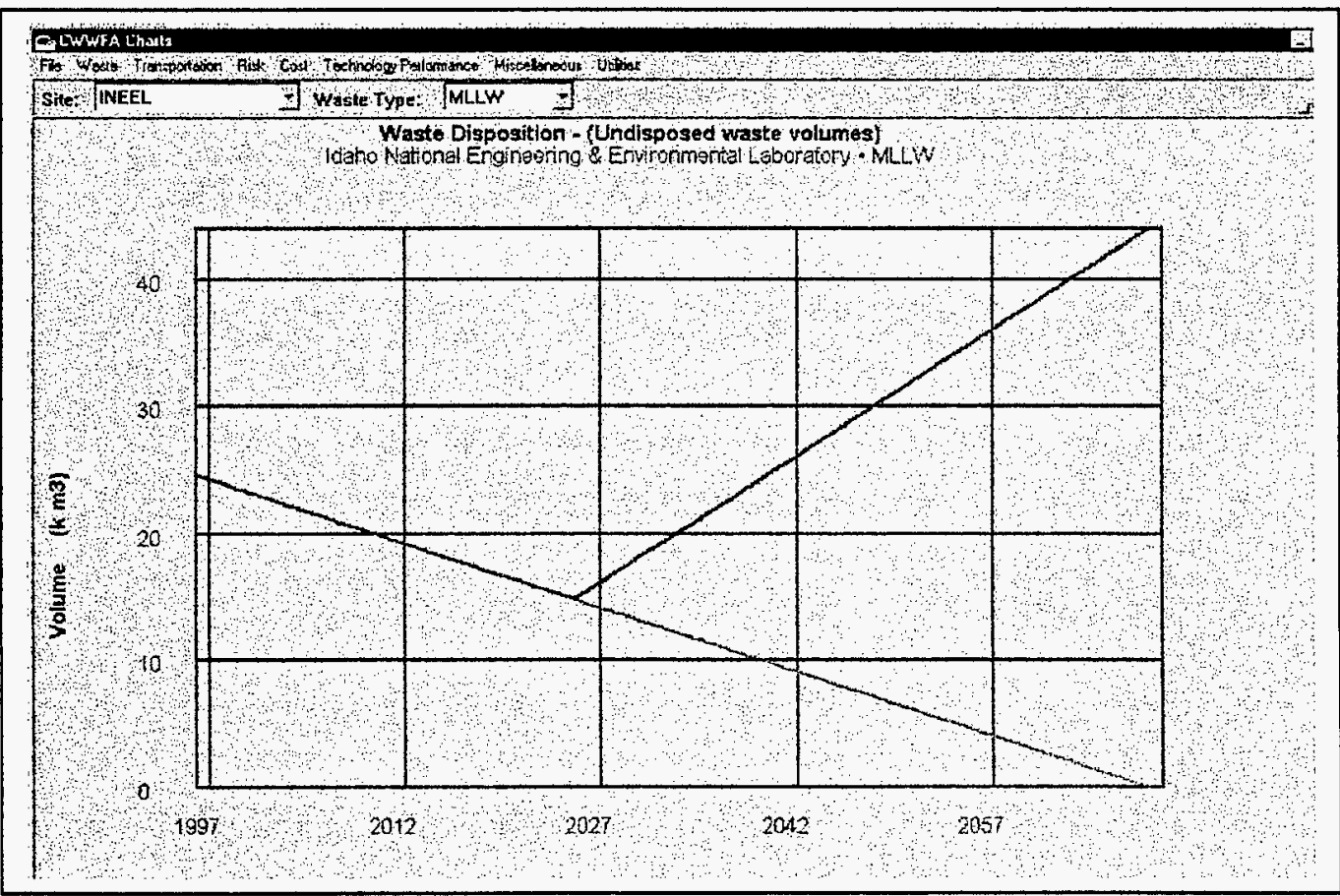


FACILITY CAPACITY EXCEL CHART (INEEL, INELFAC1-CASE5):

Facility Capacity Detail

INELFAC11-ASE5

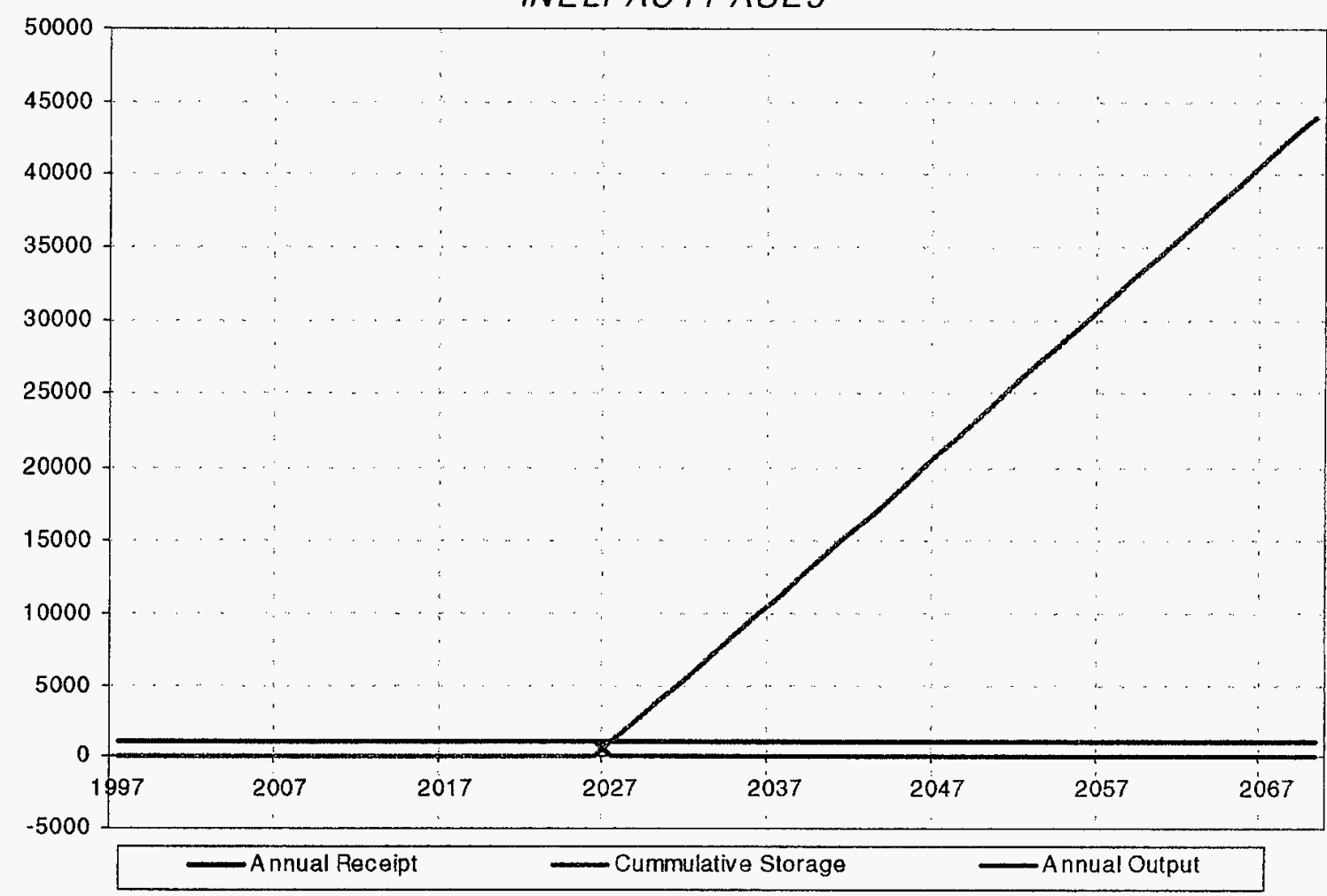

\section{FACILITY CAPACITY CWWFA CHART:}

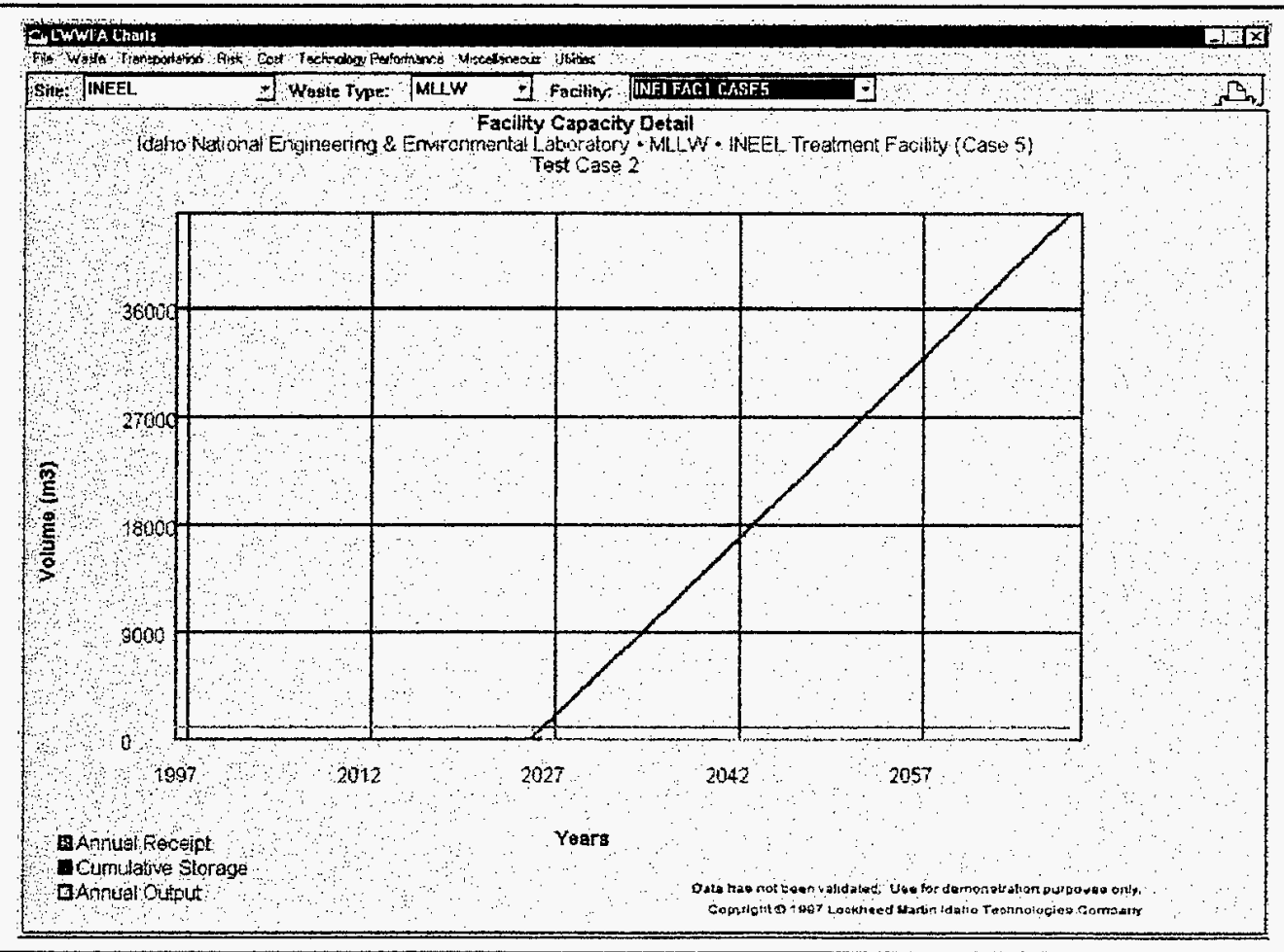


FACILITY CAPACITY DETAIL EXCEL CHART:

\section{Facility Capacity Detail} INELFAC2-CASE5

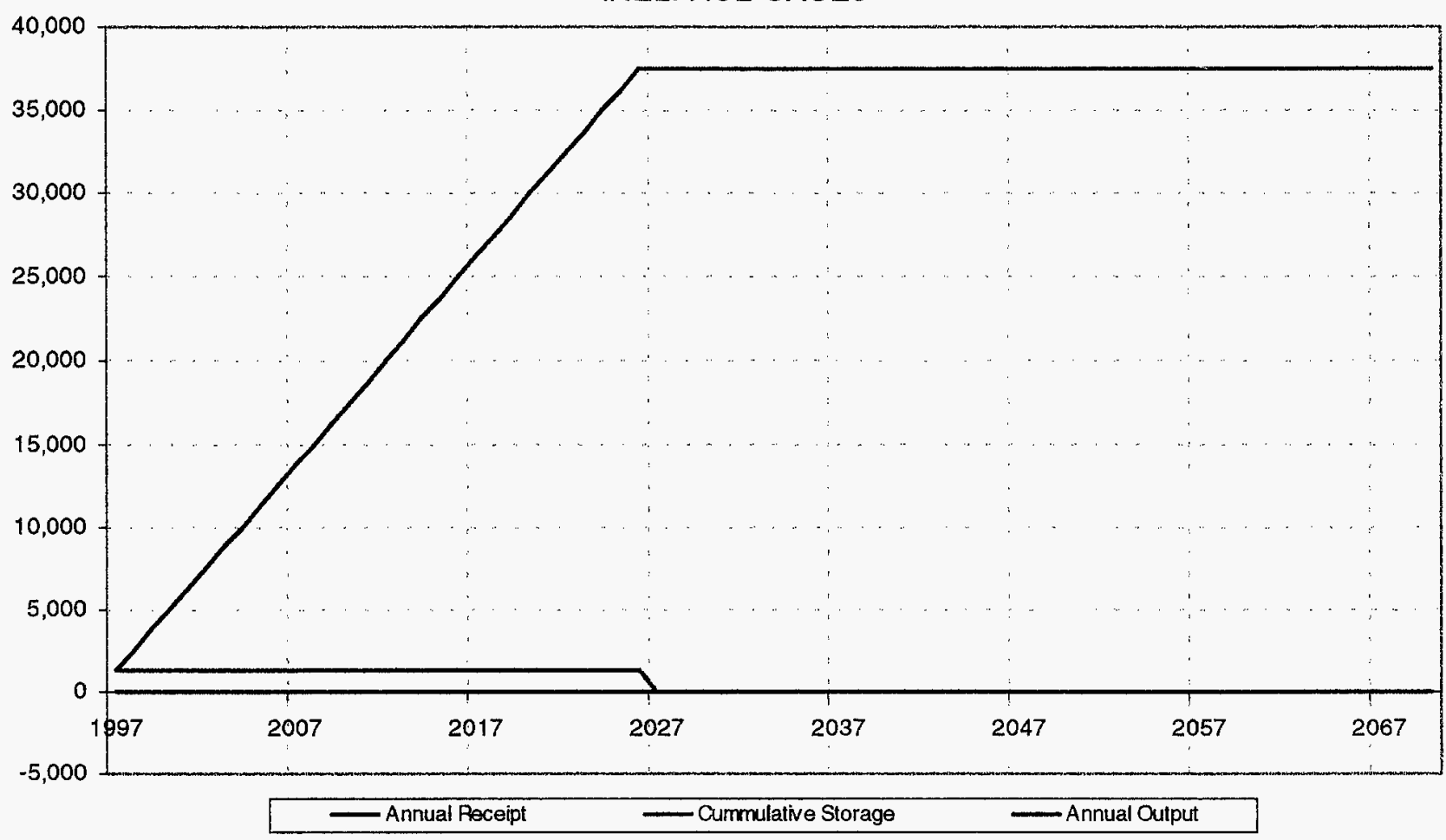

\section{FACILITY CAPACITY DETAIL CWWFA CHART:}

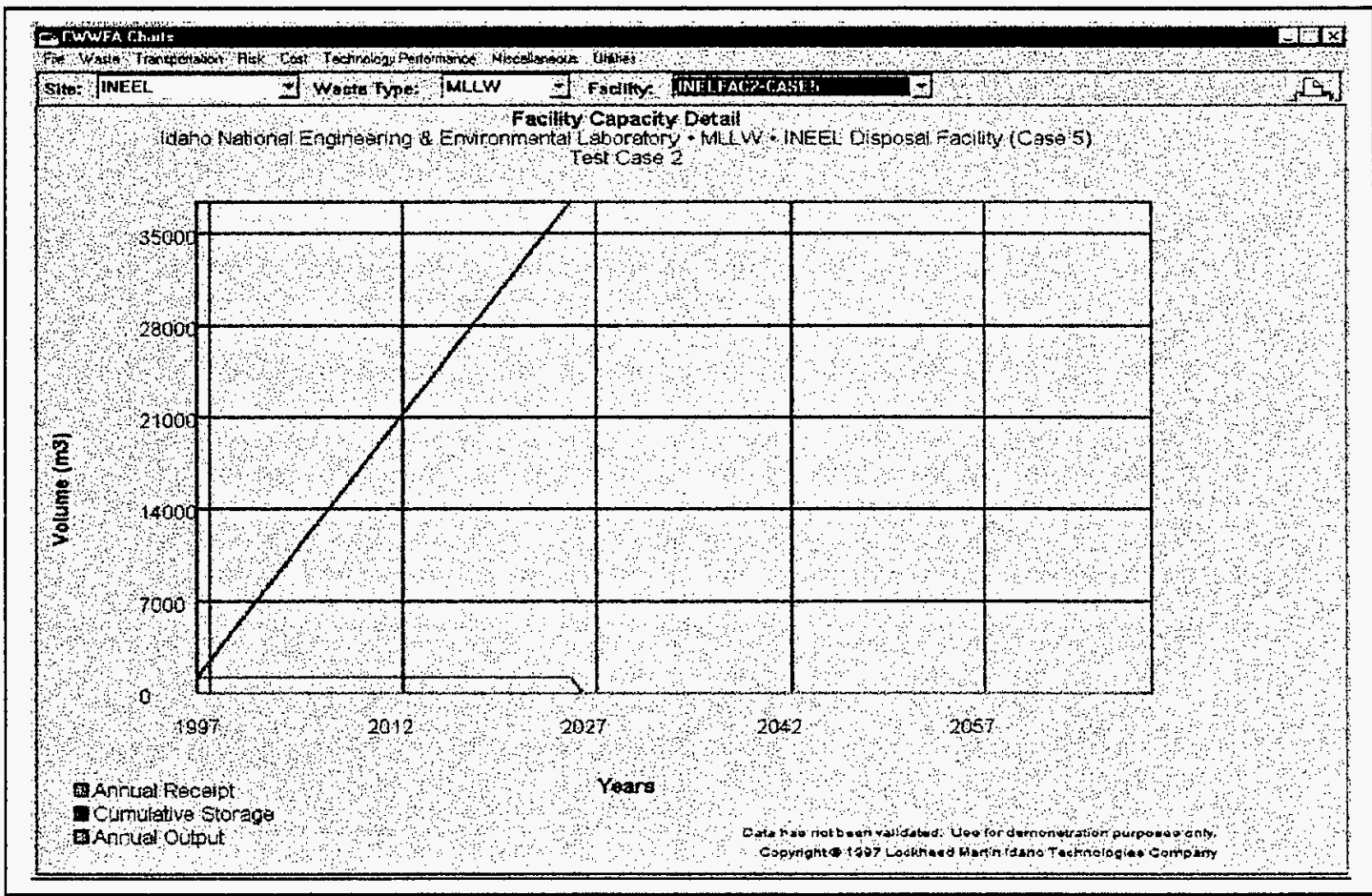


CASE: Case $5 \mathrm{~b}$

DATE: $11 / 13 / 97$

Evaluator: K B Oswald

SHIPMENTS RECEIVED EXCEL CHART (INEEL):

\section{Shipments Received}

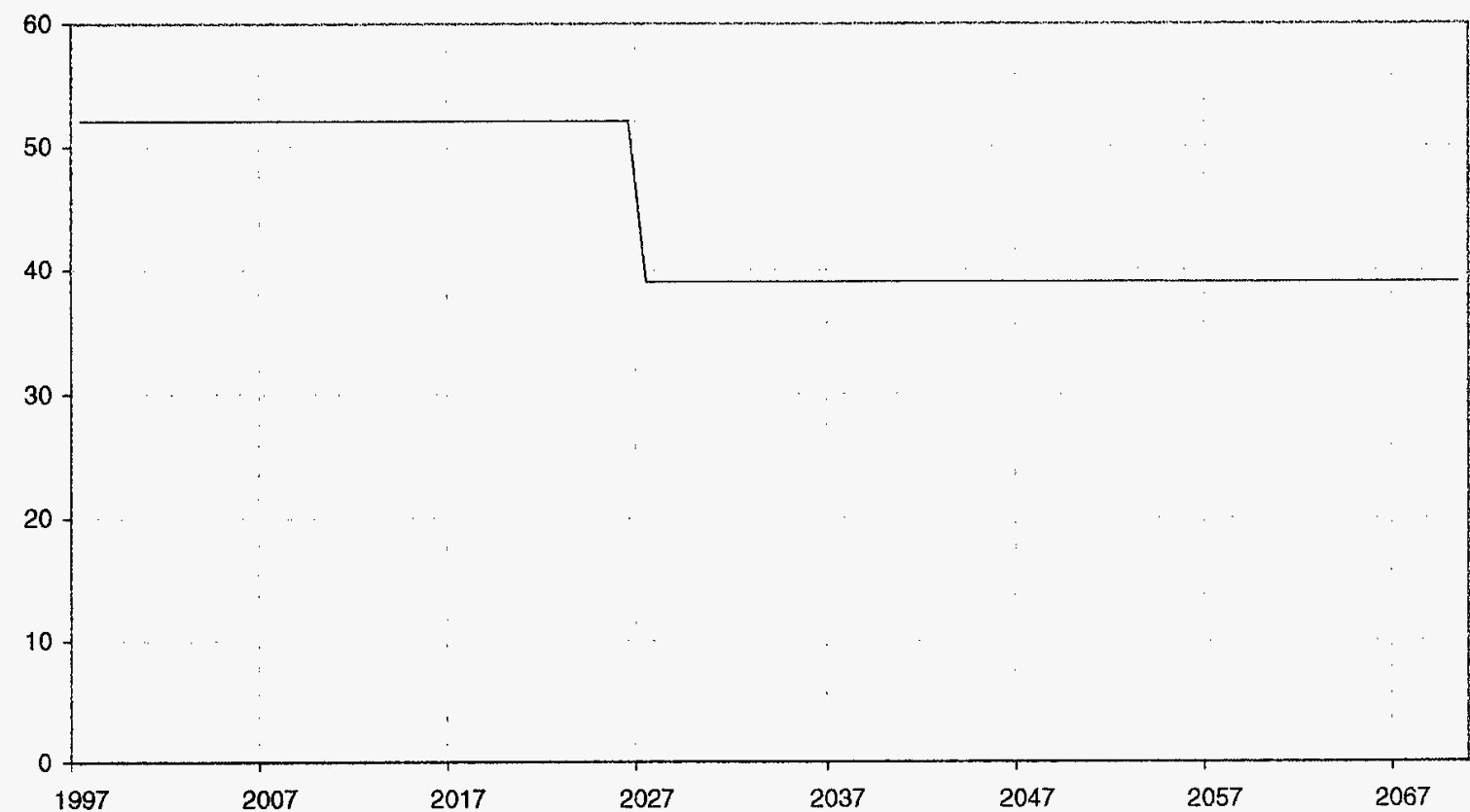

\section{SHIPMENTS RECEIVED CWWFA CHART:}

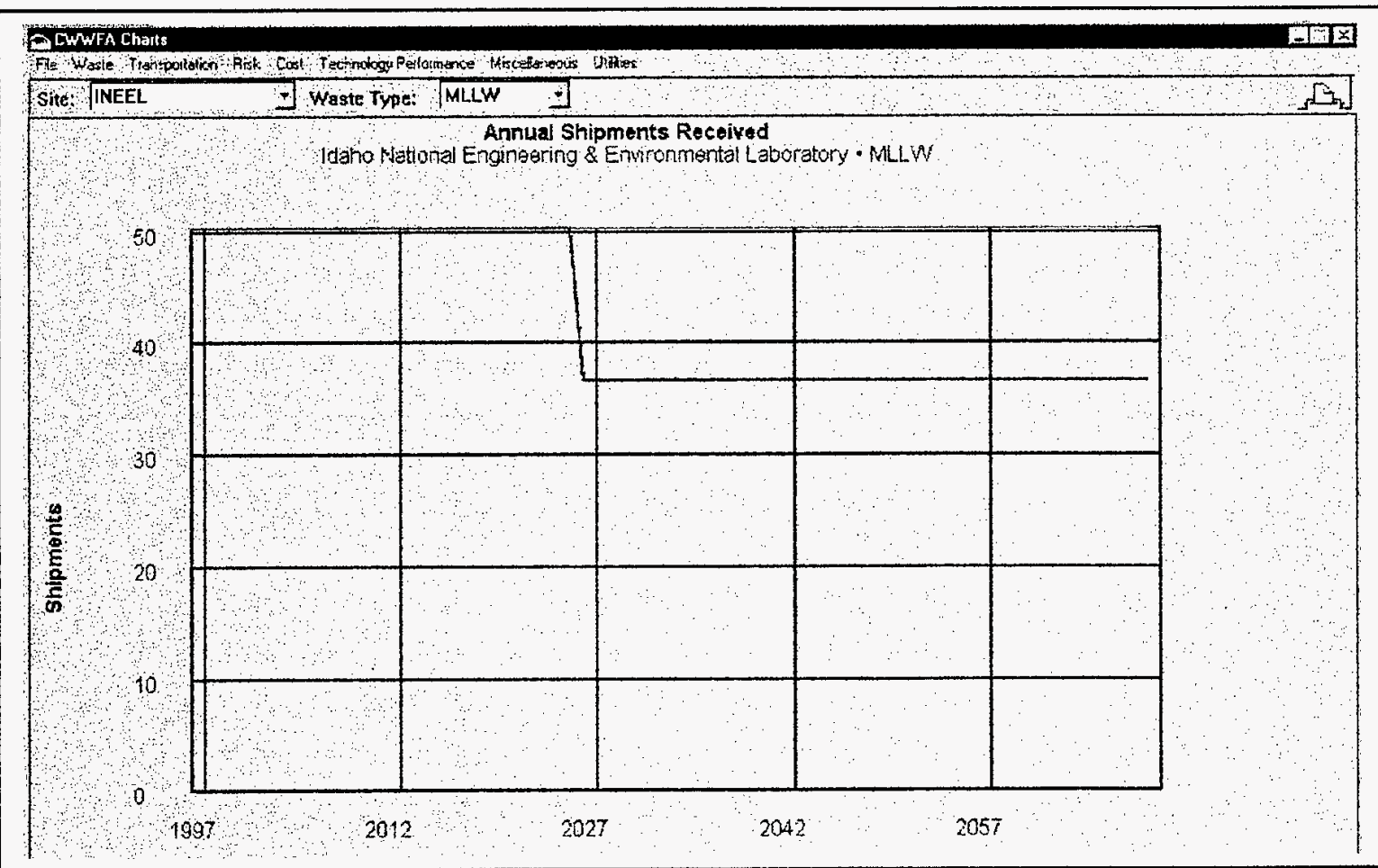


SHIPMENTS SENT EXCEL CHART (INEEL):

\section{Shipments Sent}

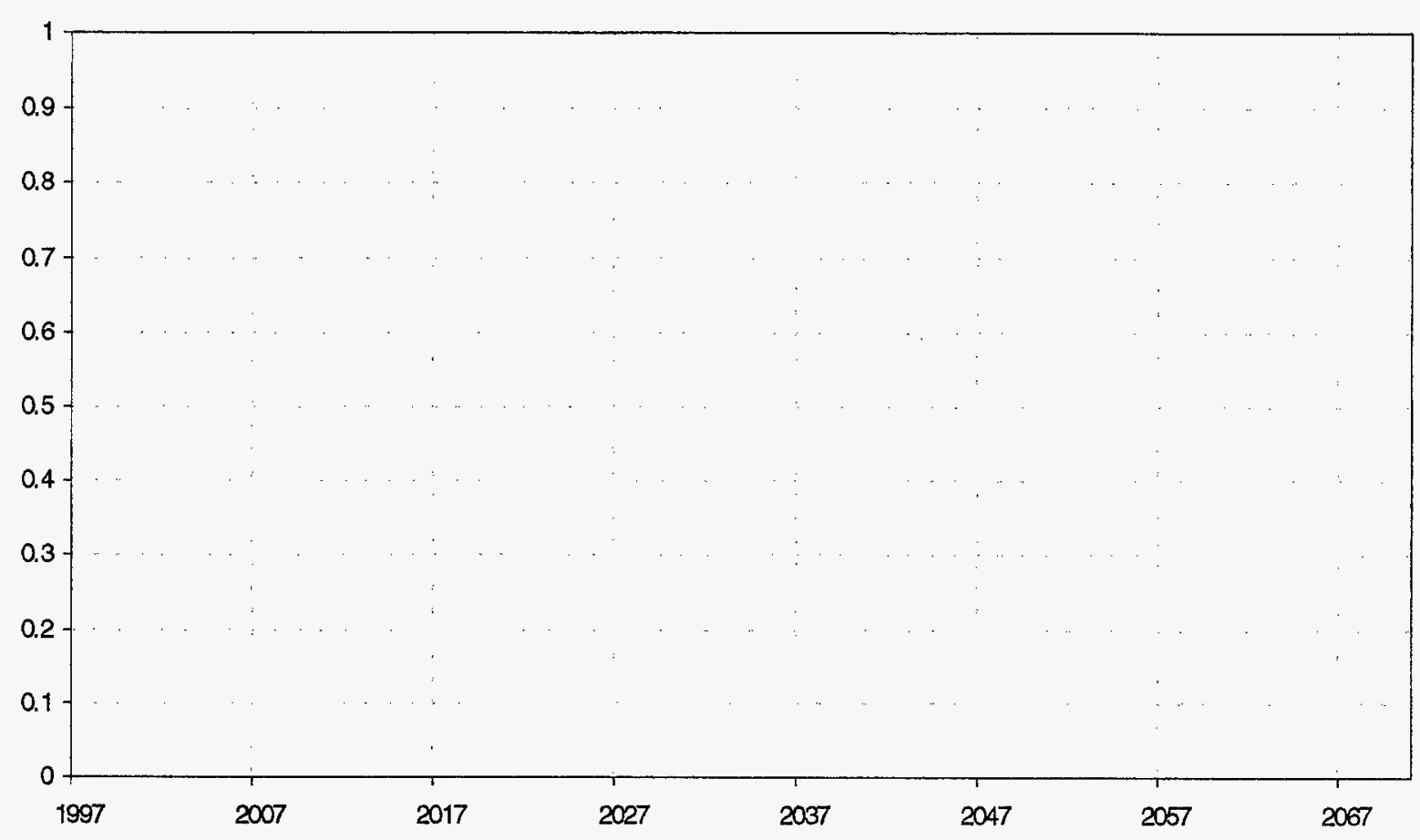

\section{SHIPMENTS SENT CWWFA CHART:}

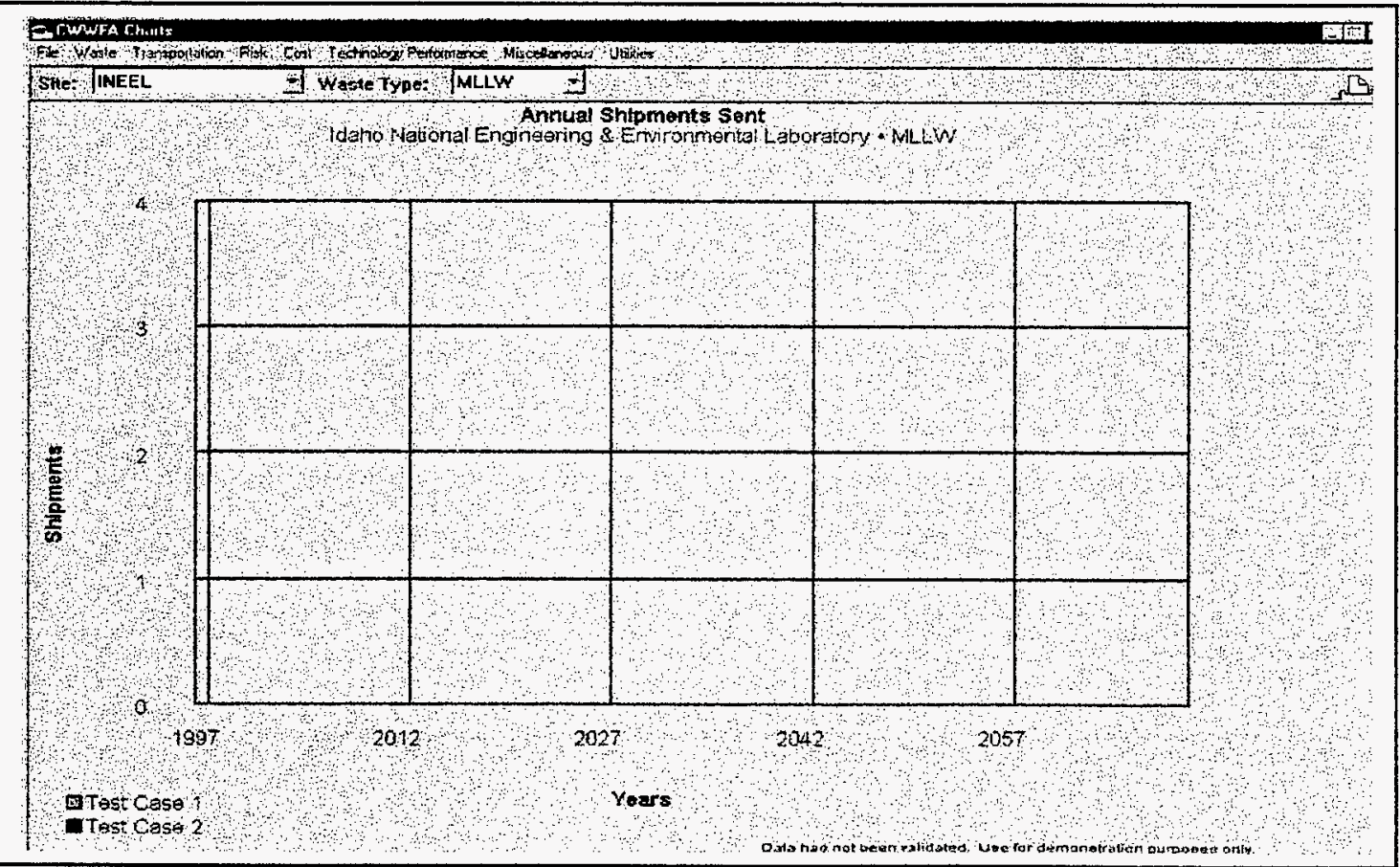


TRANSPORTATION COMPARISON EXCEL CHART (INEEL):

TRANSPORTAION COMPARISON

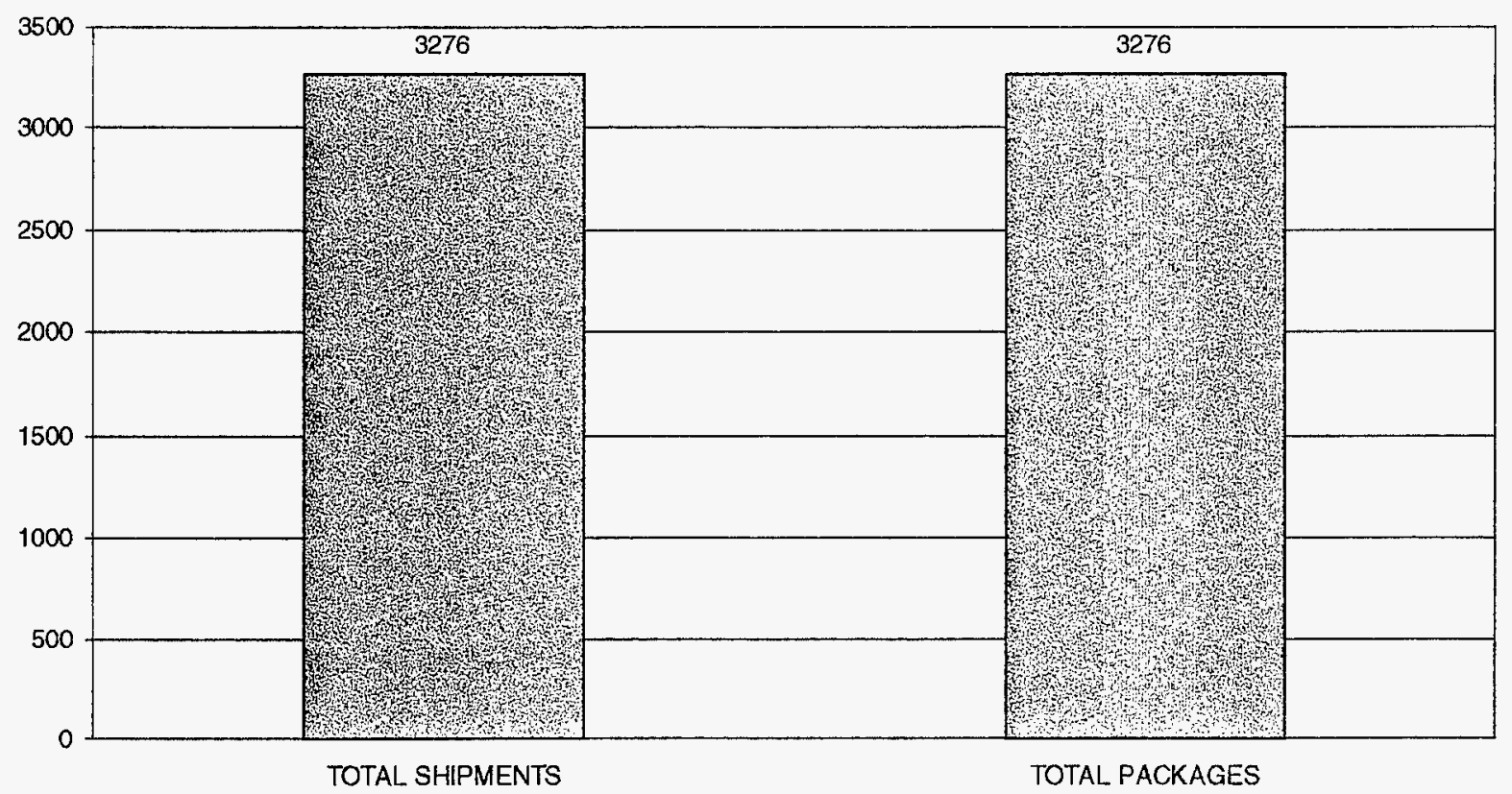

TRANSPORTATION COMPARISON CWWFA CHART:

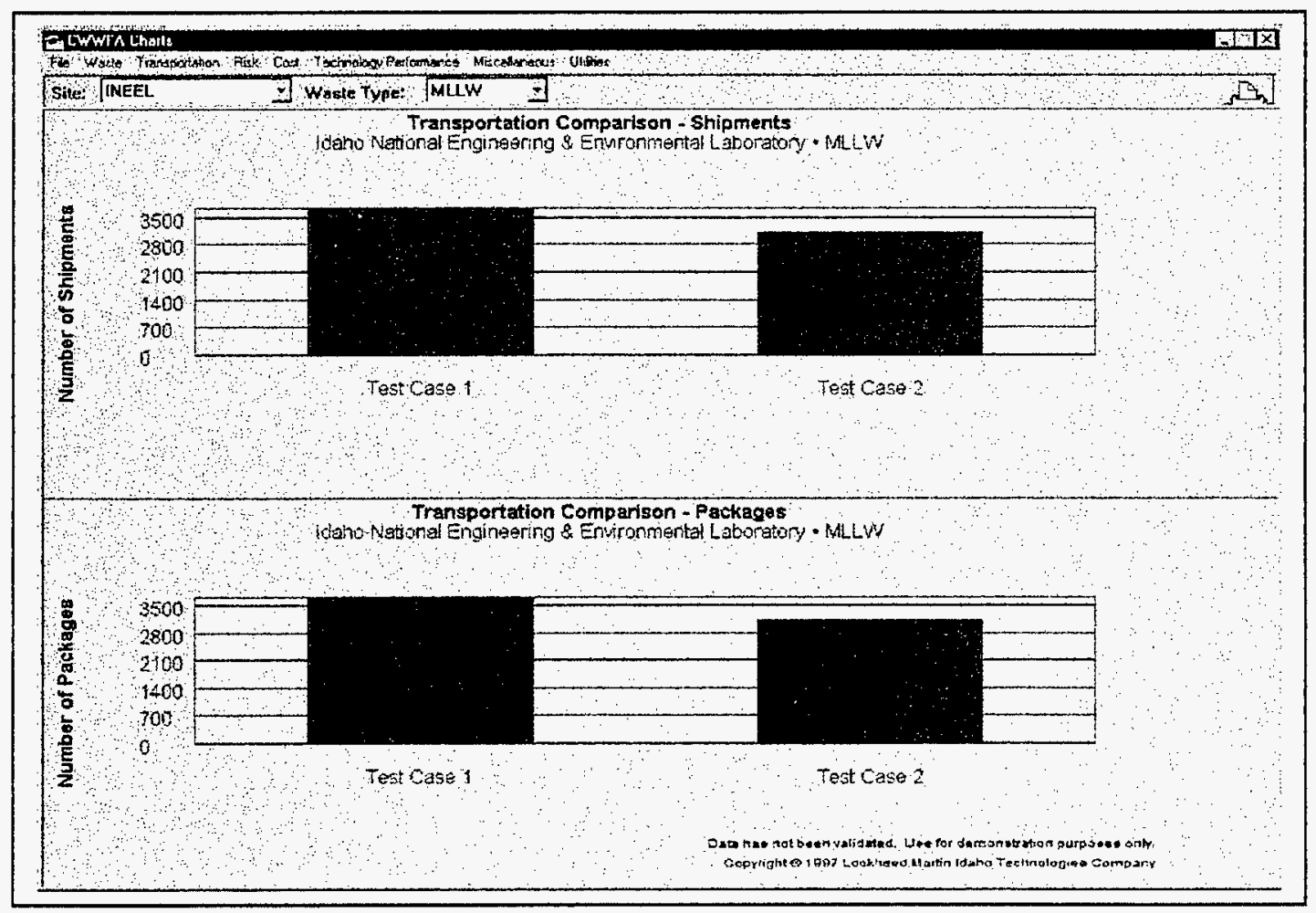


1.0 Waste Stream Information

\begin{tabular}{|l|l|}
\hline Originating Site & INEEL \\
\hline Waste Stream ID \& Name & INELWS1-Case5 (Originating Type - MLLW) \\
\hline Legacy volume $(\mathrm{m} 3)$ & 25,000 \\
\hline
\end{tabular}

1.1 New Generation

\begin{tabular}{|l|l|l|}
\hline \multicolumn{1}{|c|}{ Start Year } & \multicolumn{1}{|c|}{ End Year } & Annual Volume (m3) \\
\hline NA & NA & NA \\
\hline & & \\
\hline & & \\
\hline & & \\
\hline
\end{tabular}

1.2 Shipping Route(s)

\begin{tabular}{|l|l|c|}
\hline \multicolumn{1}{|c|}{ Route ID } & \multicolumn{1}{|c|}{ Source Facility } & Destination Facility \\
\hline C5a-1 & Legacy-INEEL & INELFAC1-CASE5 \\
\hline C5a-2 & INELFAC1-CASE5 & INELFAC2-CASE5 \\
\hline & & \\
\hline & & \\
\hline & & \\
\hline & & \\
\hline
\end{tabular}

1.3 Shipping Schedule(s)

\begin{tabular}{|c|c|c|c|}
\hline Route ID: C5a-1 & & & \\
\hline Start Year & End Year & Waste Type & Annual Volume (m3) \\
\hline 1997 & 2070 & MLLW & 338 \\
\hline & & & \\
\hline & & & \\
\hline
\end{tabular}

1.3 Shipping Schedule(s) (continued)

\begin{tabular}{|c|c|l|l|}
\hline Route ID: C5a-2 & \multicolumn{2}{|c|}{ Waste Type } & \multicolumn{1}{|c|}{ Annual Volume (m3) } \\
\hline Start Year & \multicolumn{1}{|c|}{ End Year } & 338 \\
\hline 1997 & 2070 & MLLW & \\
\hline & & & \\
\hline & & & \\
\hline
\end{tabular}

1.0 Waste Stream Information

\begin{tabular}{|l|l|}
\hline Originating Site & FEMP \\
\hline Waste Stream ID \& Name & FEMPWS1-Case5 (Originating Type-MLLW) \\
\hline Legacy volume (m3) & 30,000 \\
\hline
\end{tabular}

1.1 New Generation

\begin{tabular}{|l|l|l|}
\hline \multicolumn{1}{|c|}{ Start Year } & \multicolumn{1}{|c|}{ End Year } & Annual Volume (m3) \\
\hline NA & NA & NA \\
\hline & & \\
\hline & & \\
\hline
\end{tabular}

1.2 Shipping Route(s)

\begin{tabular}{|l|l|l|}
\hline \multicolumn{1}{|c|}{ Route ID } & \multicolumn{1}{|c|}{ Source Facility } & Destination Facility \\
\hline C5a-3 & Legacy-FN & INELFAC1-CASE5 \\
\hline C5a-4 & INELFAC1-CASE5 & INELFAC2-CASE5 \\
\hline & & \\
\hline
\end{tabular}

1.3 Shipping Schedule(s)

\begin{tabular}{|c|c|c|c|}
\hline \multicolumn{4}{|l|}{ Route ID: C5a-3 } \\
\hline Start Year & End Year & Waste Type & Annual Volume (m3) \\
\hline 1997 & 2070 & MLLW & 405 \\
\hline & & & \\
\hline & & & \\
\hline
\end{tabular}


1.3 Shipping Schedule(s) (continued)

\begin{tabular}{|c|c|c|c|}
\hline Route ID: C5a-4 & & & \\
\hline Start Year & End Year & Waste Type & Annual Volume (m3) \\
\hline 1997 & 2070 & MLLW & 405 \\
\hline & & & \\
\hline & & & \\
\hline
\end{tabular}

1.0 Waste Stream Information

\begin{tabular}{|l|l|}
\hline Originating Site & OAKR \\
\hline Waste Stream ID \& Name & OAKRWS1-Case5 (Originating Type - MLLW) \\
\hline Legacy volume (m3) & 35,000 \\
\hline
\end{tabular}

1.1 New Generation

\begin{tabular}{|l|l|l|}
\hline \multicolumn{1}{|c|}{ Start Year } & \multicolumn{1}{|c|}{ End Year } & Annual Volume (m3) \\
\hline NA & NA & NA \\
\hline & & \\
\hline & & \\
\hline
\end{tabular}

1.2 Shipping Route(s)

\begin{tabular}{|l|l|l|}
\hline \multicolumn{1}{|c|}{ Route ID } & \multicolumn{1}{|c|}{ Source Facility } & \multicolumn{1}{c|}{ Destination Facility } \\
\hline C5a-5 & Legacy-OR & INELFAC1-CASE5 \\
\hline C5a-6 & INELFAC1-CASE5 & INELFAC2-CASE5 \\
\hline & & \\
\hline & & \\
\hline & & \\
\hline & & \\
\hline
\end{tabular}

1.3 Shipping Schedule(s)

\begin{tabular}{|c|c|l|c|}
\hline Route ID: C5a-5 & \multicolumn{2}{|c|}{} & \multicolumn{1}{|c|}{ Waste Type } \\
\hline Start Year & \multicolumn{1}{|c|}{ End Year } & Annual Volume (m3) \\
\hline 1997 & 2070 & MLLW & 473 \\
\hline & & & \\
\hline & & & \\
\hline
\end{tabular}

1.3 Shipping Schedule(s) (continued)

\begin{tabular}{|c|c|c|c|}
\hline \multicolumn{4}{|l|}{ Route ID: C5a-6 } \\
\hline Start Year & End Year & Waste Type & Annual Volume (m3) \\
\hline 1997 & 2070 & MLLW & 473 \\
\hline & & & \\
\hline & & & \\
\hline
\end{tabular}

1.0 Waste Stream Information

\begin{tabular}{|l|l|}
\hline Originating Site & OAKR \\
\hline Waste Stream ID \& Name & OAKRWS2-Case5 (Originating Type - MLLW) \\
\hline Legacy volume (m3) & 40,000 \\
\hline
\end{tabular}

1.1 New Generation

\begin{tabular}{|l|l|l|}
\hline \multicolumn{1}{|c|}{ Start Year } & \multicolumn{1}{|c|}{ End Year } & Annual Volume (m3) \\
\hline NA & NA & NA \\
\hline & & \\
\hline & & \\
\hline & & \\
\hline
\end{tabular}


1.2 Shipping Route(s)

\begin{tabular}{|l|l|l|}
\hline \multicolumn{1}{|c|}{ Route ID } & Source Facility & Destination Facility \\
\hline C5a-7 & Legacy-OR & INELFAC2-CASE5 \\
\hline & & \\
\hline & & \\
\hline & & \\
\hline & & \\
\hline & & \\
\hline
\end{tabular}

1.3 Shipping Schedule(s)

\begin{tabular}{|c|l|l|l|}
\hline Route ID: C5a-7 & \multicolumn{2}{|c|}{} & \multicolumn{1}{|c|}{ Waste Type } \\
\hline Start Year & \multicolumn{1}{|c|}{ End Year } & Annual Volume (m3) \\
\hline 1997 & 2070 & MLLW & 608 \\
\hline & & & \\
\hline & & & \\
\hline & & & \\
\hline
\end{tabular}

2.0 Facility Information

\begin{tabular}{|l|l|}
\hline Site & INEEL \\
\hline Facility ID \& Name & INELFAC1-CASE5 \\
\hline Function & Treatment \\
\hline Start Year & 1997 \\
\hline End Year & 2020 \\
\hline Annual Capacity (m3/year) or & $1,000,000$ \\
\hline Lifetime Facility Capacity (m3) & \\
\hline Volume Change Factor & 1 \\
\hline
\end{tabular}

Conversion Profile

\begin{tabular}{|l|l|l|}
\hline Percent & Waste Type & Density \\
\hline $100 \%$ & MLLW & 1198 \\
\hline & & \\
\hline & & \\
\hline
\end{tabular}

2.0 Facility Information

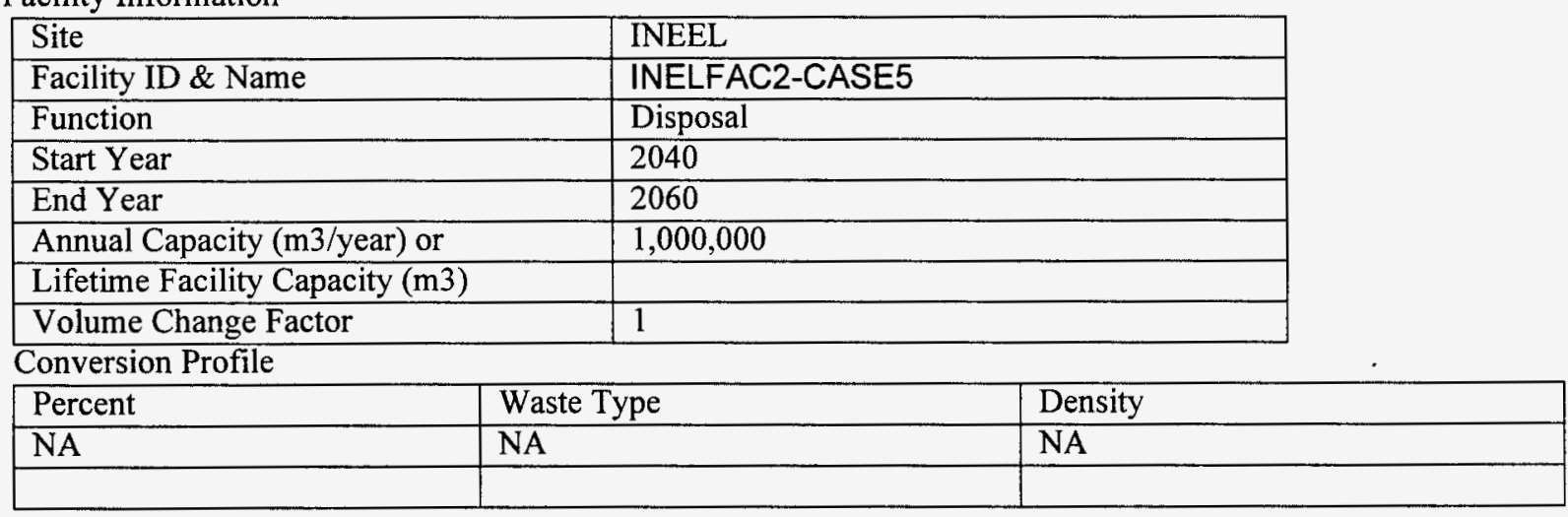


WASTE DISPOSITION - EXCEL CHART (INEEL, MLLW):

\section{Waste Disposition}

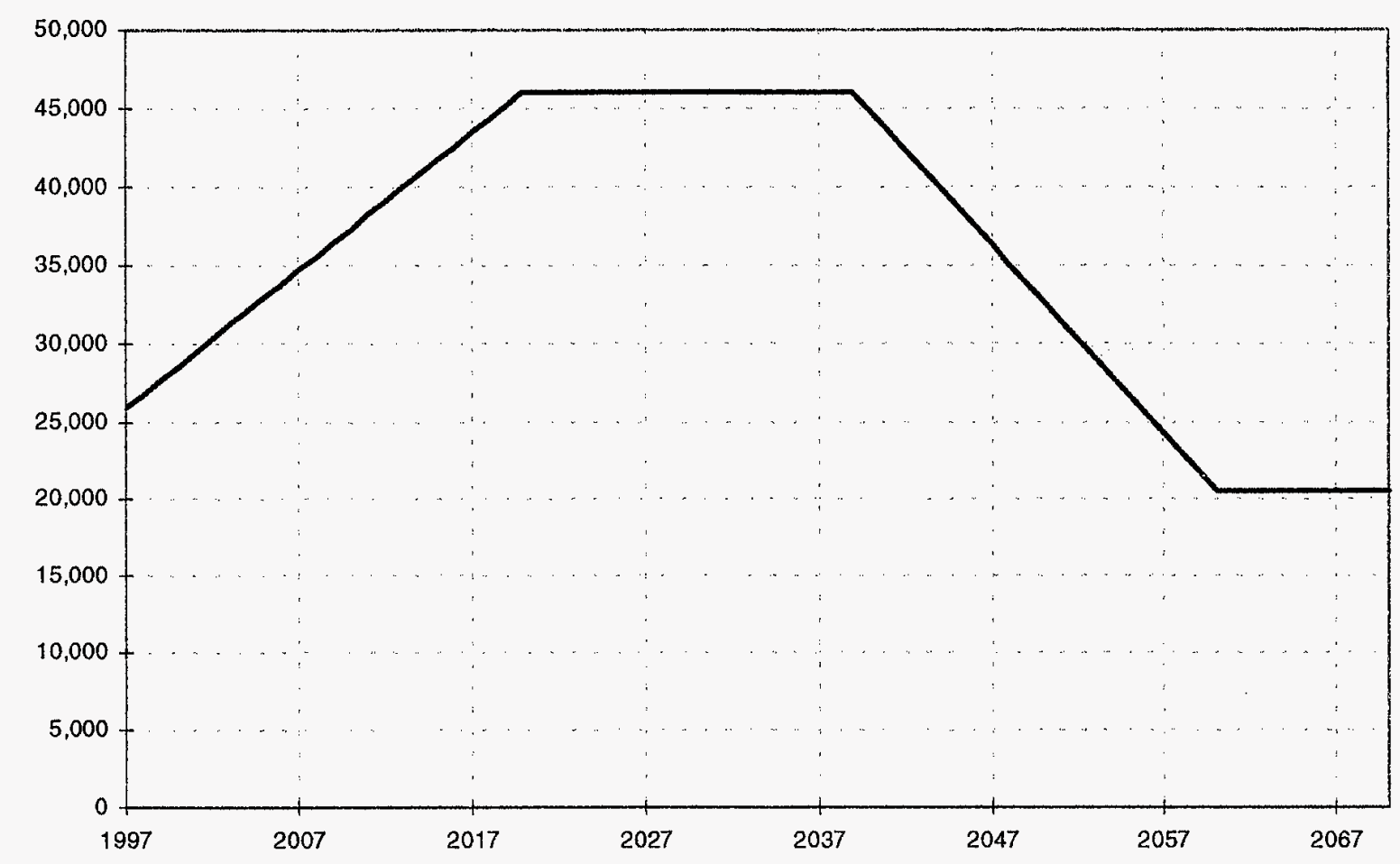

WASTE DISPOSITION - CWWFA CHART:

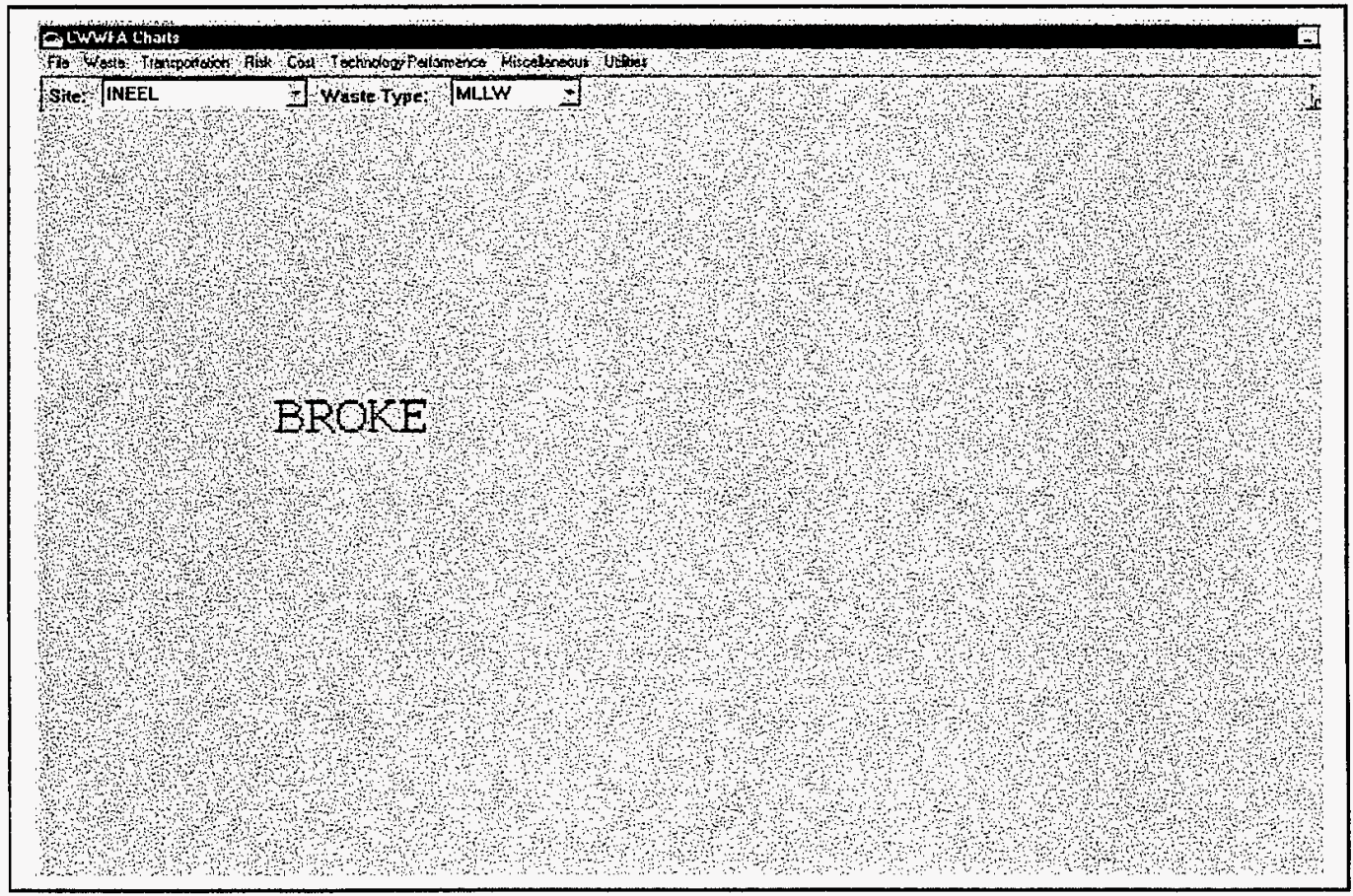


FACILITY CAPACITY EXCEL CHART (INEEL, INELFAC1-CASE5):

Facility Capacity Detail

INELFAC1-CASE5

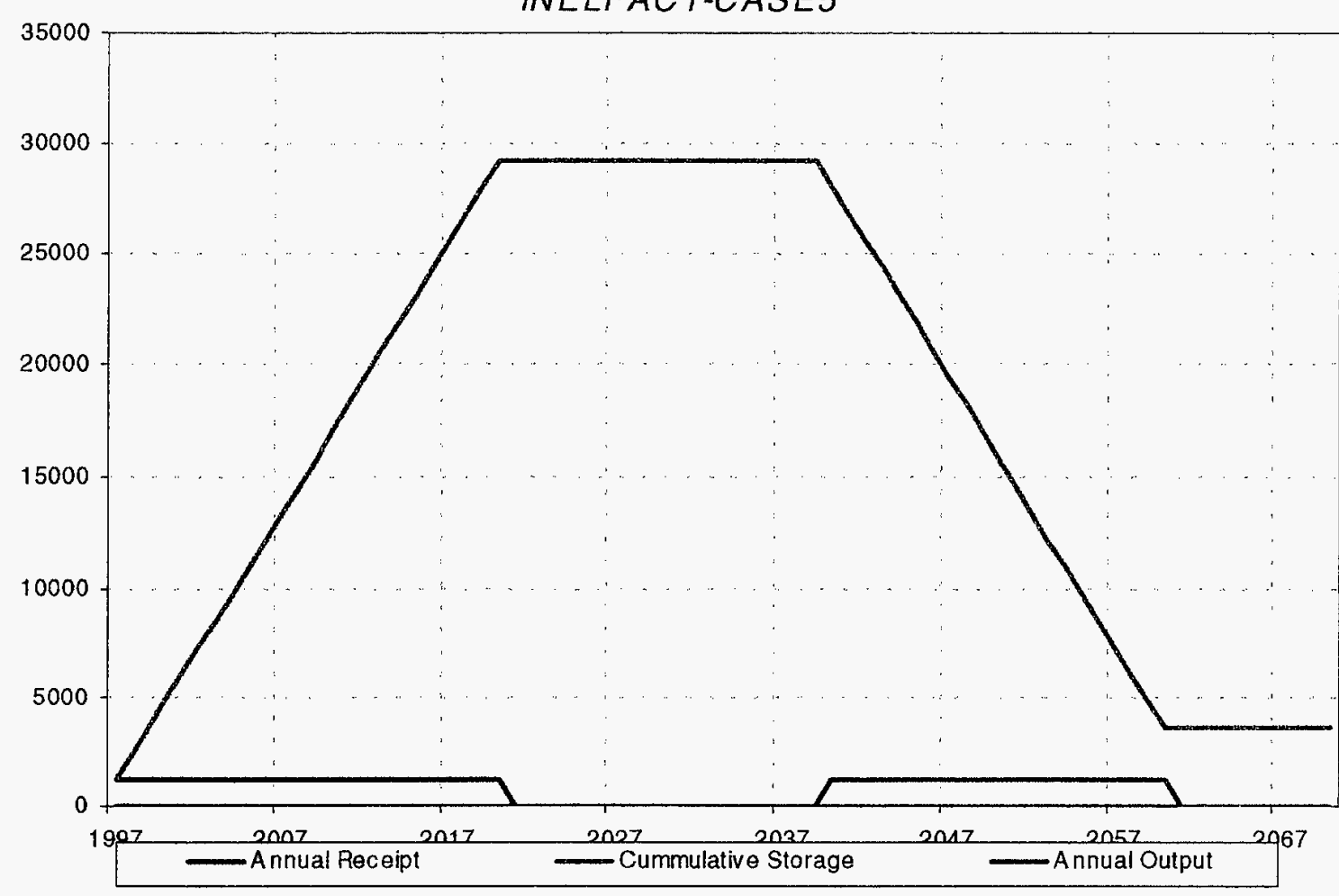

FACILITY CAPACITY CWWFA CHART:

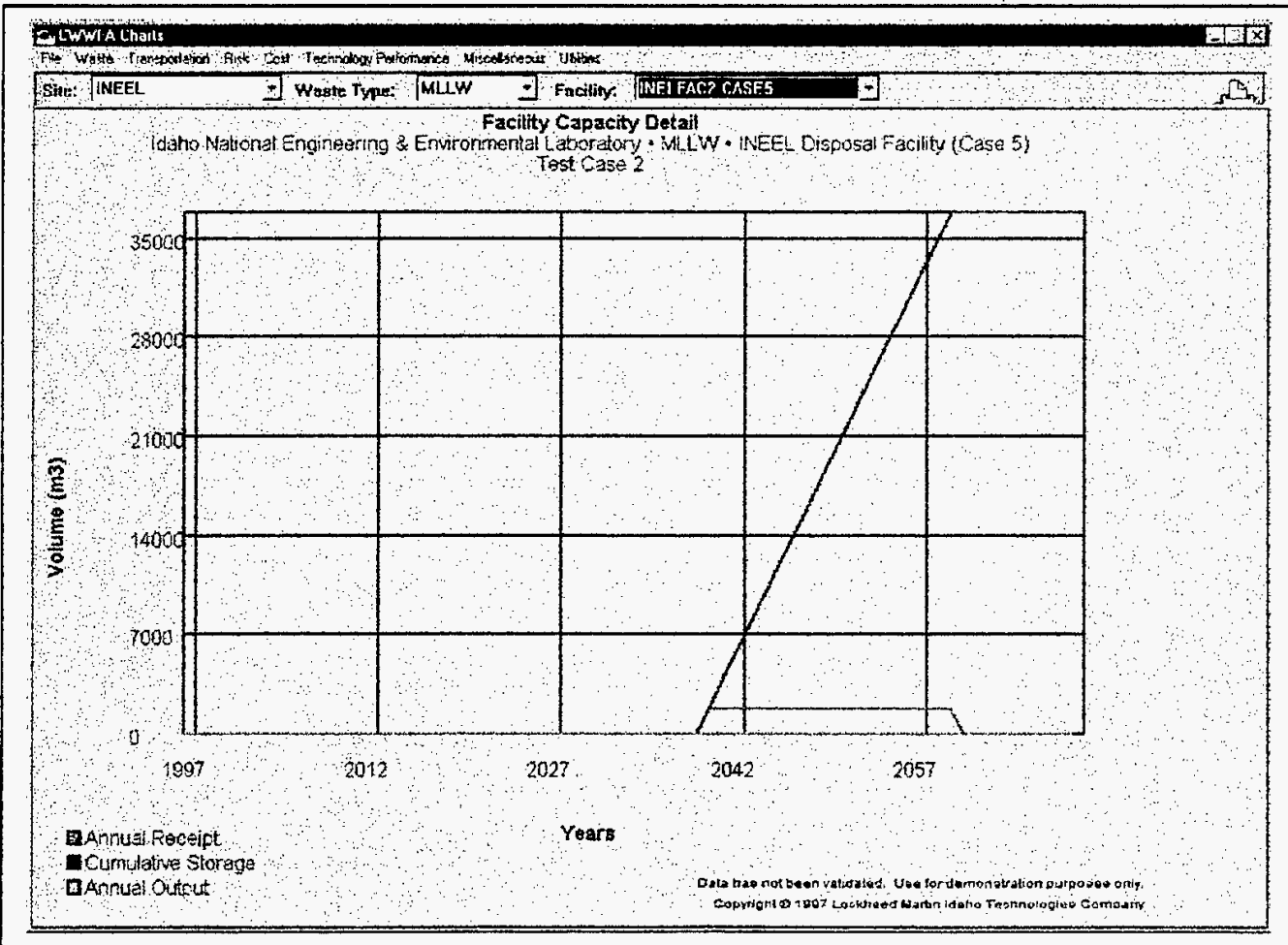


CASE: Case 5c

DATE: $11 / 13 / 97$

Evaluator: K B Oswald

WASTE STREAM DETAIL EXCEL CHART:

\section{Facility Capacity Detail}

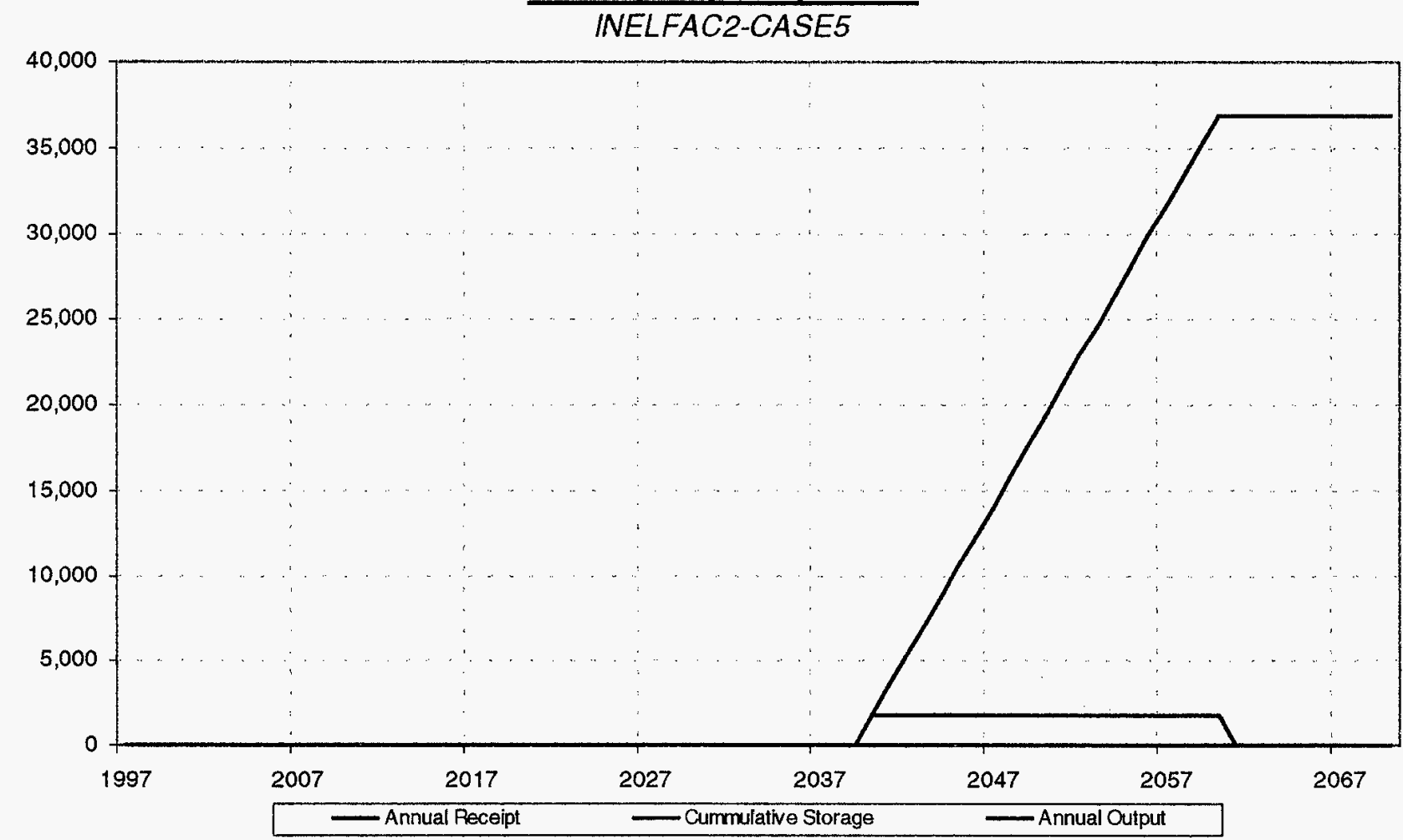

WASTE STREAM DETAIL CWWFA CHART:

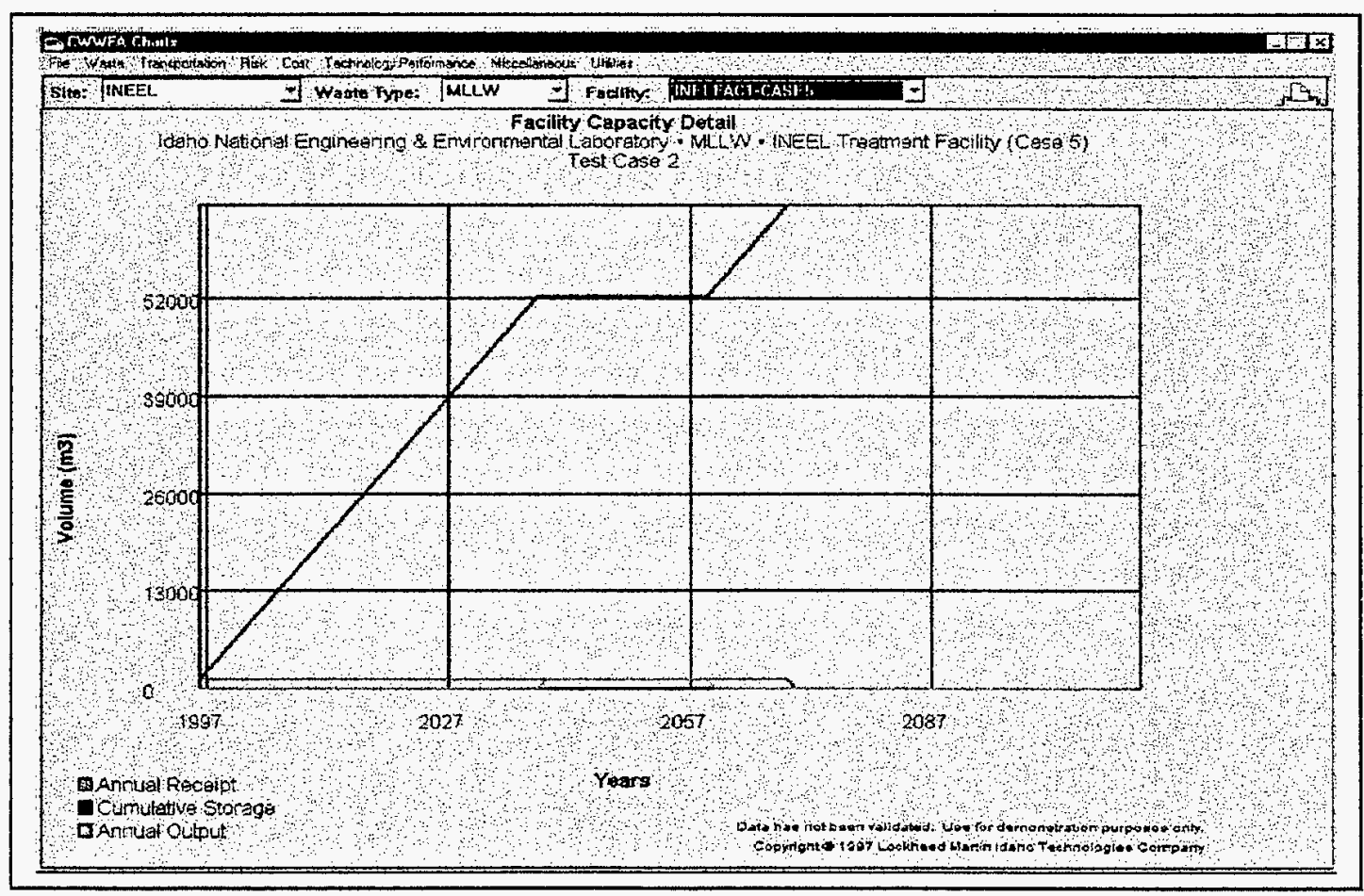


CASE: Case $5 \mathrm{c}$

INITIAL PARAMETERS \& RESULTS REPORT

SHIPMENTS RECEIVED EXCEL CHART (INEEL):

\section{Shipments Received}

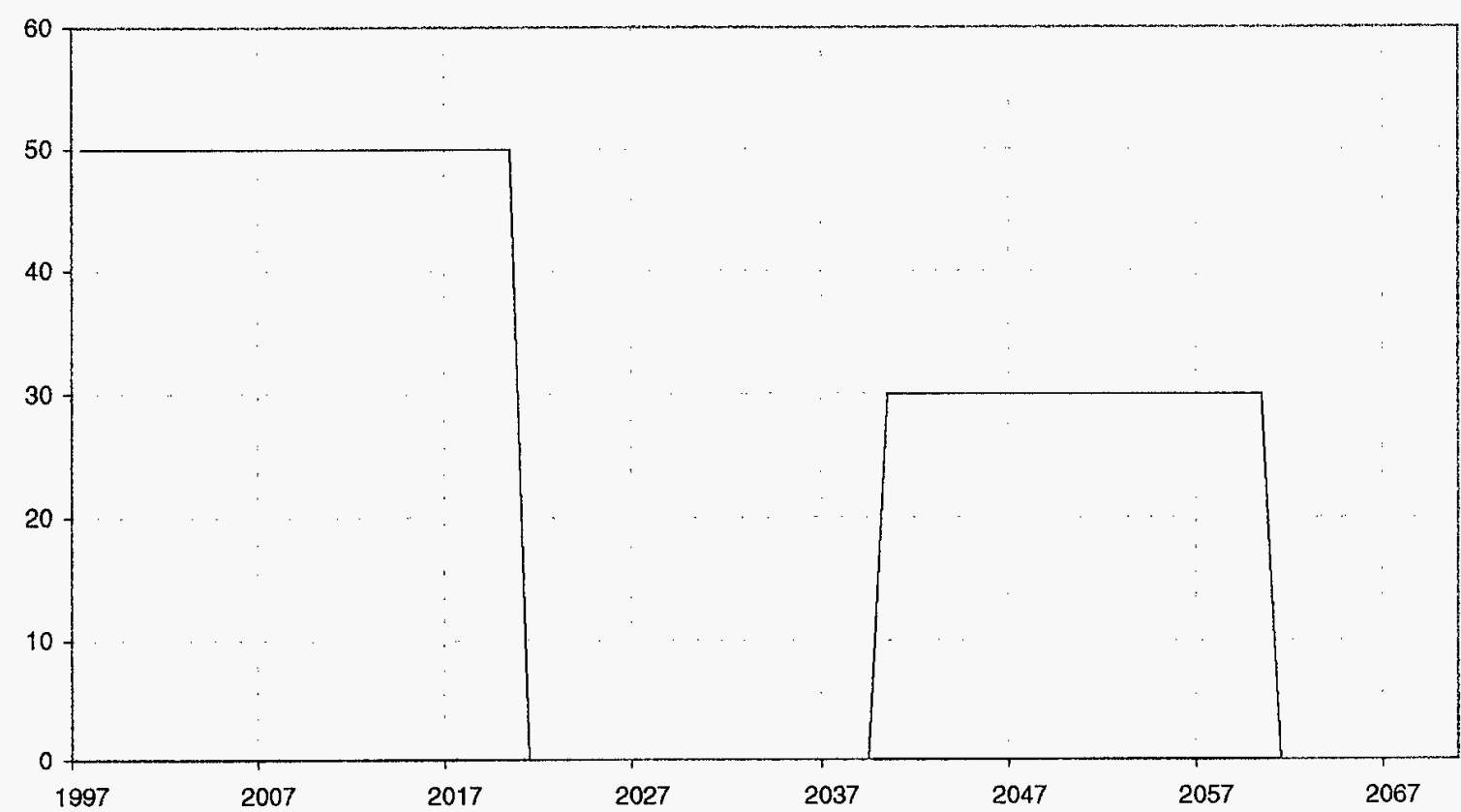

SHIPMENTS RECEIVED CWWFA CHART:

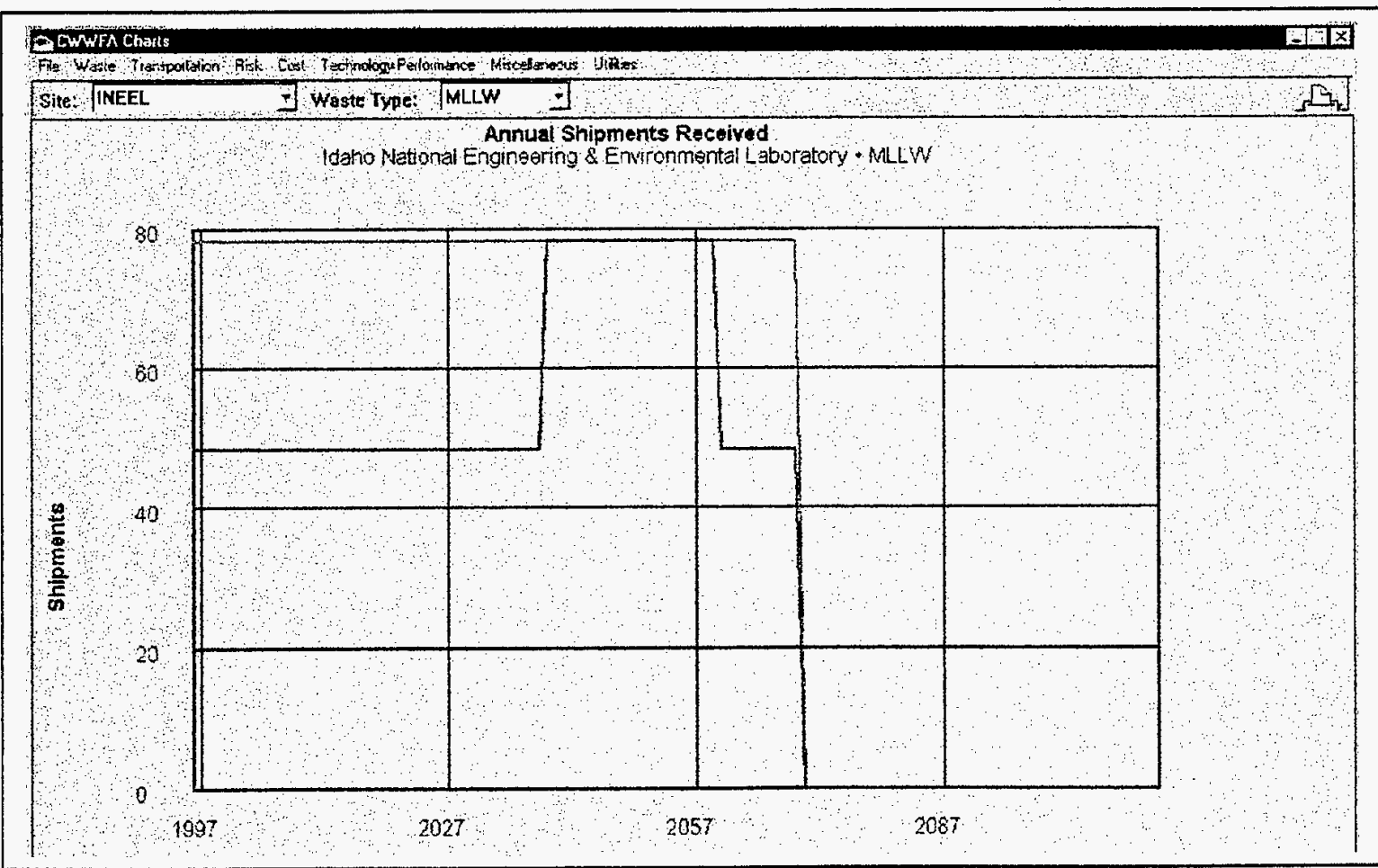


CASE: Case $5 \mathrm{c}$

DATE: $11 / 13 / 97$

Evaluator: K B Oswald

SHIPMENTS SENT EXCEL CHART (INEEL):

\section{Shipments Sent}

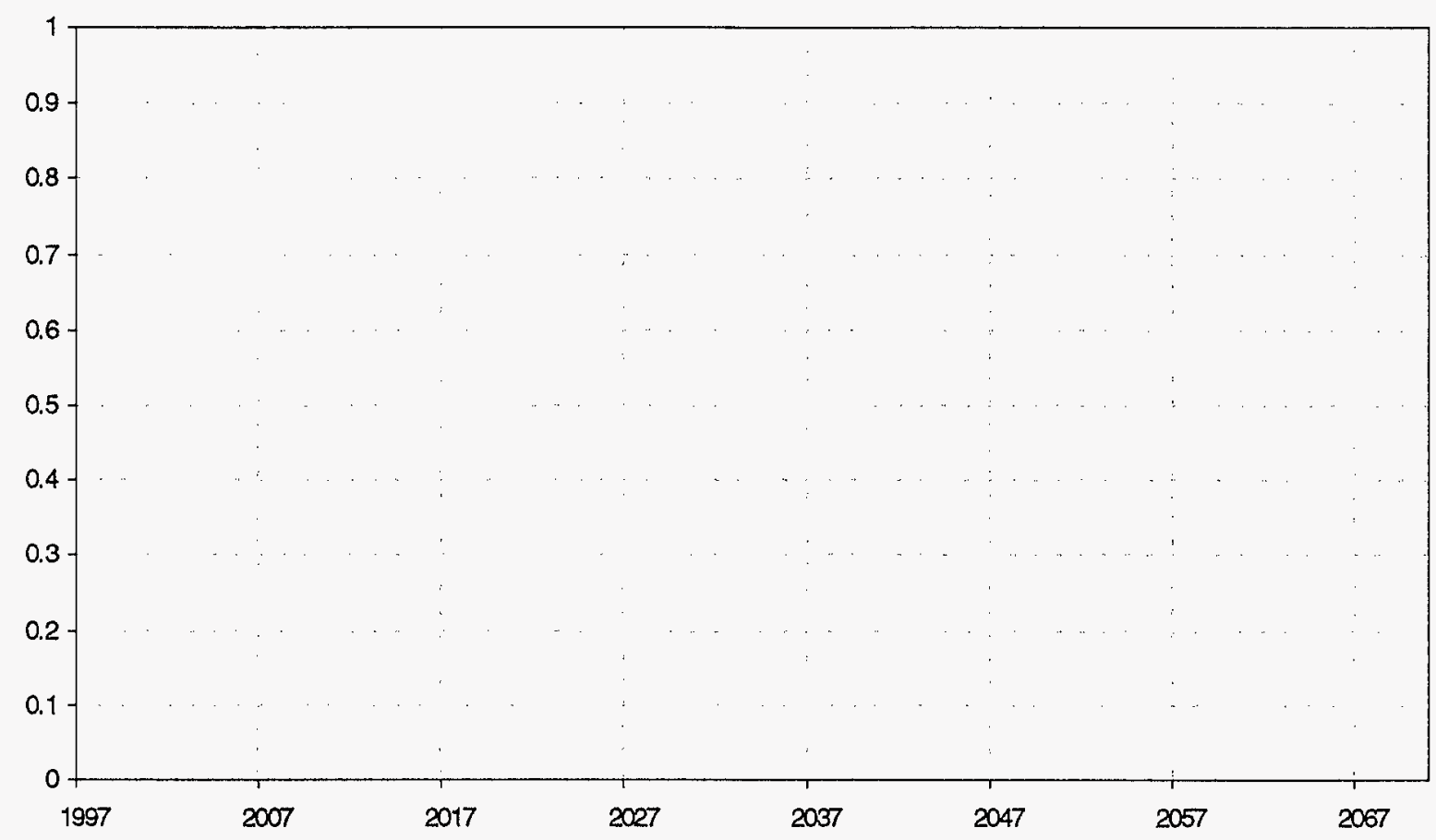

\section{SHIPMENTS SENT CWWFA CHART:}

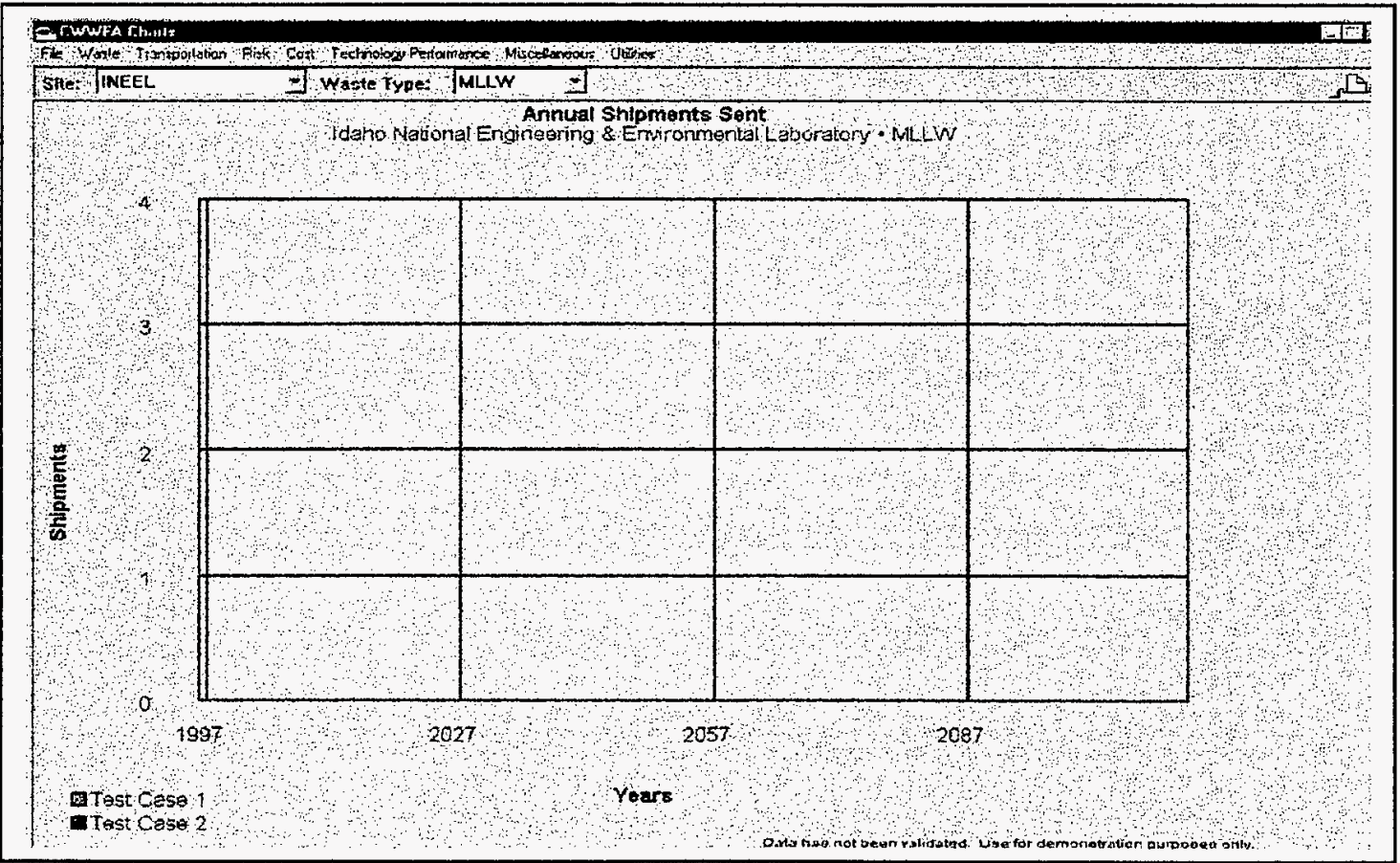




\section{TRANSPORTAION COMPARISON}

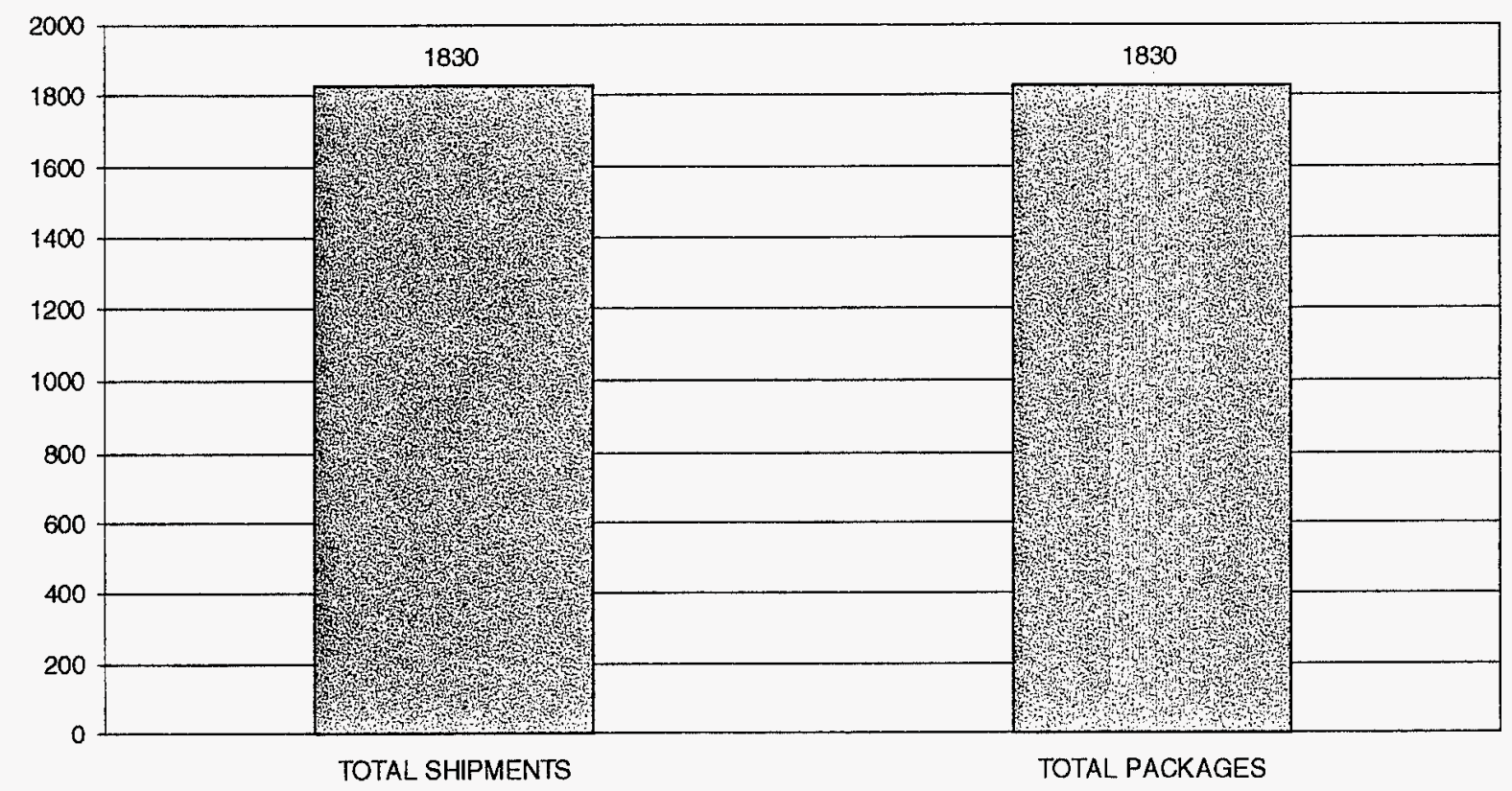

\section{TRANSPORTATION COMPARISON CWWFA CHART:}

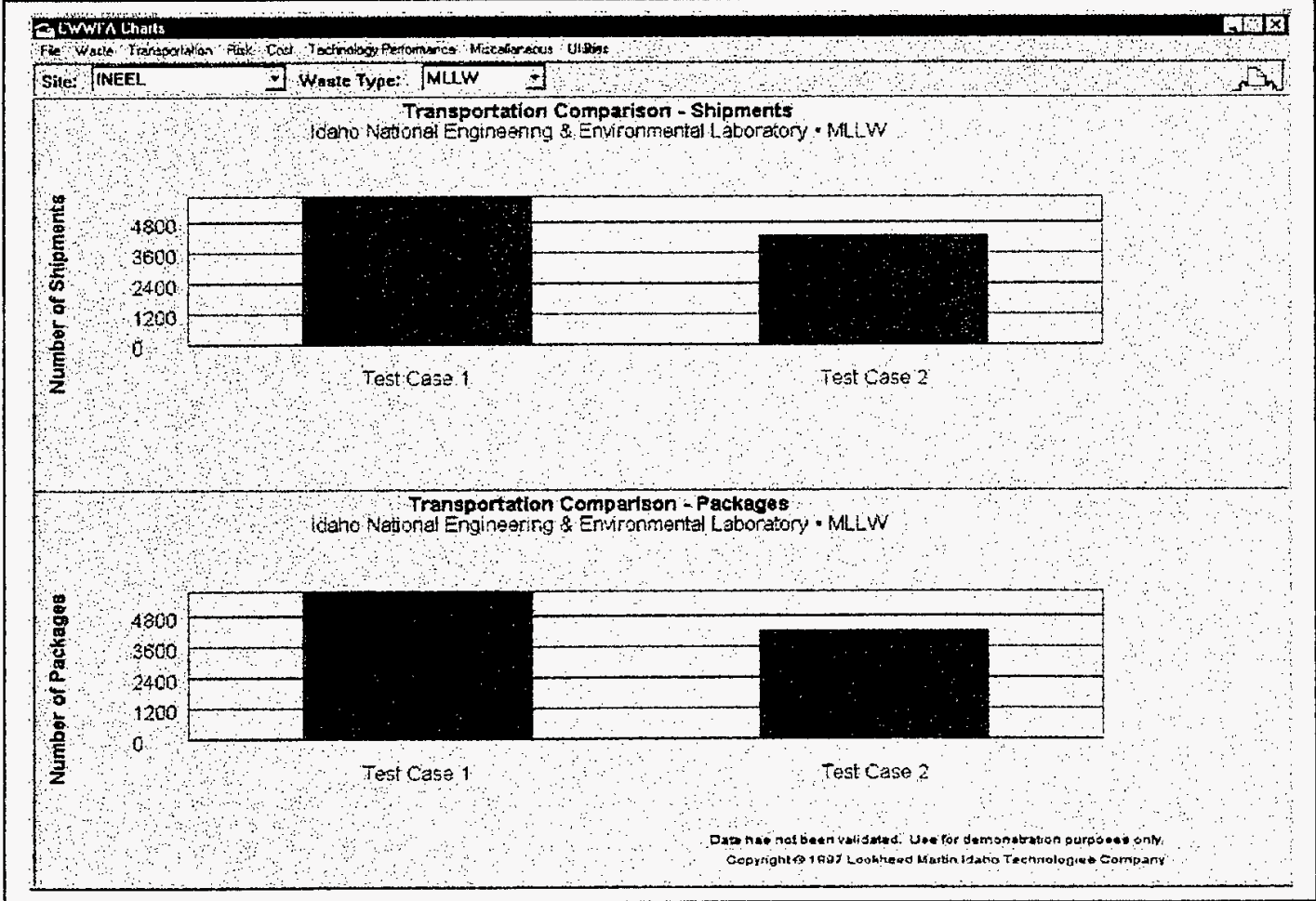




\section{TEST PROCEDURE - TP3.1.2 \\ Mortgage Reduction Performance}

\section{Purpose}

This test procedure has been designed to verify the CWWFA mortgage reduction results. The mortgage reduction performance refers to the reduction in total cost liability from waste being treated and disposed of as it moved through the system. This procedure will satisfy requirements 3.1.2.1 and 3.1.2.2 as referenced in Appendix A - Requirements Verification Matrix.

\section{Objective}

There are several objectives associated with this test procedure. The first objective is to evaluate for relative changes in cost (e.g., waste flow through the system) based on changes on waste quantities. The second objective includes a test on user ability to input cost data directly into the model using an input screen. The third objective is to evaluate the unit cost rates (e.g., fixed and variable costs) for waste processing and transportation.

\section{Test Method}

Method 1. - This test method is designed to verify the $1^{\text {st }}$ and $2^{\text {nd }}$ objectives. Two cases will be simulated based on the alternative case configuration. Test data are shown in Table 1. The test data are designed to show waste flow through a facility (i.e., HLWSWF) at varying quantities. Since the same algorithm is used for all waste types, only HLW waste will be tested for compliance. Tester will input the test data directly into the model using the CWWFA input screen. After the simulation, an assessment of the impact will be conducted by examining the CWWFA mortgage liability reduction and transportation cost charts.

\begin{tabular}{|l|l|l|ll|}
\hline \multicolumn{4}{|c|}{ Table 1. } \\
\hline \multicolumn{1}{|c|}{ Site } & \multicolumn{1}{|c|}{ Facility } & Quantity $\left(\mathbf{m}^{\mathbf{3}}\right)$ & \multicolumn{1}{c|}{ Output Chart } \\
\hline Hanford & HLW-SWF & 100,000 & 1) & Mortgage liability reduction \\
& & & 2) & Transportation cost \\
\cline { 4 - 5 } & & 166,000 & 1) & Mortgage liability reduction \\
& & & 2) & Transportation cost \\
\hline
\end{tabular}

Method 2 - This method is designed to verify the unit cost rates (e.g., disposal, treatment, storage, and shipment) and its usage in the model source codes. The first verification is conducted by evaluating the following sources for the unit cost rates.

1. INEEL Letter to File, Calculation of Cost Credits for the Complex-Wide Waste Flow Analysis Model - RPH-02-97, April 1997. 
2. Feizollahi, F. H. and D. E. Shropshire, 1944a, Interim Report: Waste Management Facilities Cost Information for Transportation of Radioactive and Hazardous Materials, INEL-95/0300, Rev. 1, (formerly EGG-WM-10877, Rev. 1), June.

For the source code evaluation, the unit cost and transportation rate tables will be examined for accuracy (e.g., tables are properly chained to calling routines within the source program).

\section{Reporting}

TP - $\quad$ Test Procedure

REQD - Requirement

$\mathrm{P} / \mathrm{F}$ - Pass/Fail

\begin{tabular}{|c|c|c|c|}
\hline TP & REQD & Result/Comment & $\mathbf{P} / \mathbf{F}$ \\
\hline $\begin{array}{l}\text { TP3.1.2 } \\
\text { Method } 1 \\
1^{\text {st }} \text { obj } \\
\text { Method } 2 \\
3^{\text {rd }} \text { obj }\end{array}$ & 3.1 .2 .1 & $\begin{array}{l}\text { Results from testing (shown on attached output charts) } \\
\text { show a visible change in mortgage reduction and } \\
\text { transportation costs as waste quantities are changed. This } \\
\text { change is consistent with the expected behavior of the } \\
\text { model. This test satisfied this requirement. } \\
\text { Overall, unit costs and component costs (i.e., disposal, } \\
\text { treatment, and storage) are consistent with the source } \\
\text { document \#1 listed above. There is a minor deficiency, } \\
\text { listed below, that needs to be corrected. } \\
\text { 1. on unit cost, LLW storage cost should be } \\
\quad \$ 350.00 \text { versus } \$ 2,000.00 \\
\text { Note: On mortgage rates, INEEL TRU treatment and } \\
\text { storage rates are reversed from what is listed in the source } \\
\text { document. However, this evaluation indicates that the } \\
\text { inputs are correct in the model database and the source } \\
\text { document is in error. } \\
\text { Transportation unit costs were compared against source } \\
\# 2 \text { and no deficiency was found. }\end{array}$ & $\begin{array}{l}\text { Pass - } \\
\text { minor } \\
\text { correction } \\
\text { needed }\end{array}$ \\
\hline $\begin{array}{l}\text { TP3.1.2 } \\
\text { Method } 1 \\
2^{\text {nd }} \text { obj }\end{array}$ & 3.1 .2 .2 & $\begin{array}{l}\text { There is not an available user input screen to enter cost } \\
\text { data into the system. Cost data had to be loaded into the } \\
\text { database via programming. }\end{array}$ & Fail \\
\hline
\end{tabular}


TP 3.1 .2

METHOD 1

$10 / 21 / 97$

CHART\#1

@ $166,000 \mathrm{~m}^{3}$

HASI

Waste Type: HLW

Mortgage Liability Reduction

Hanford Site - HLW

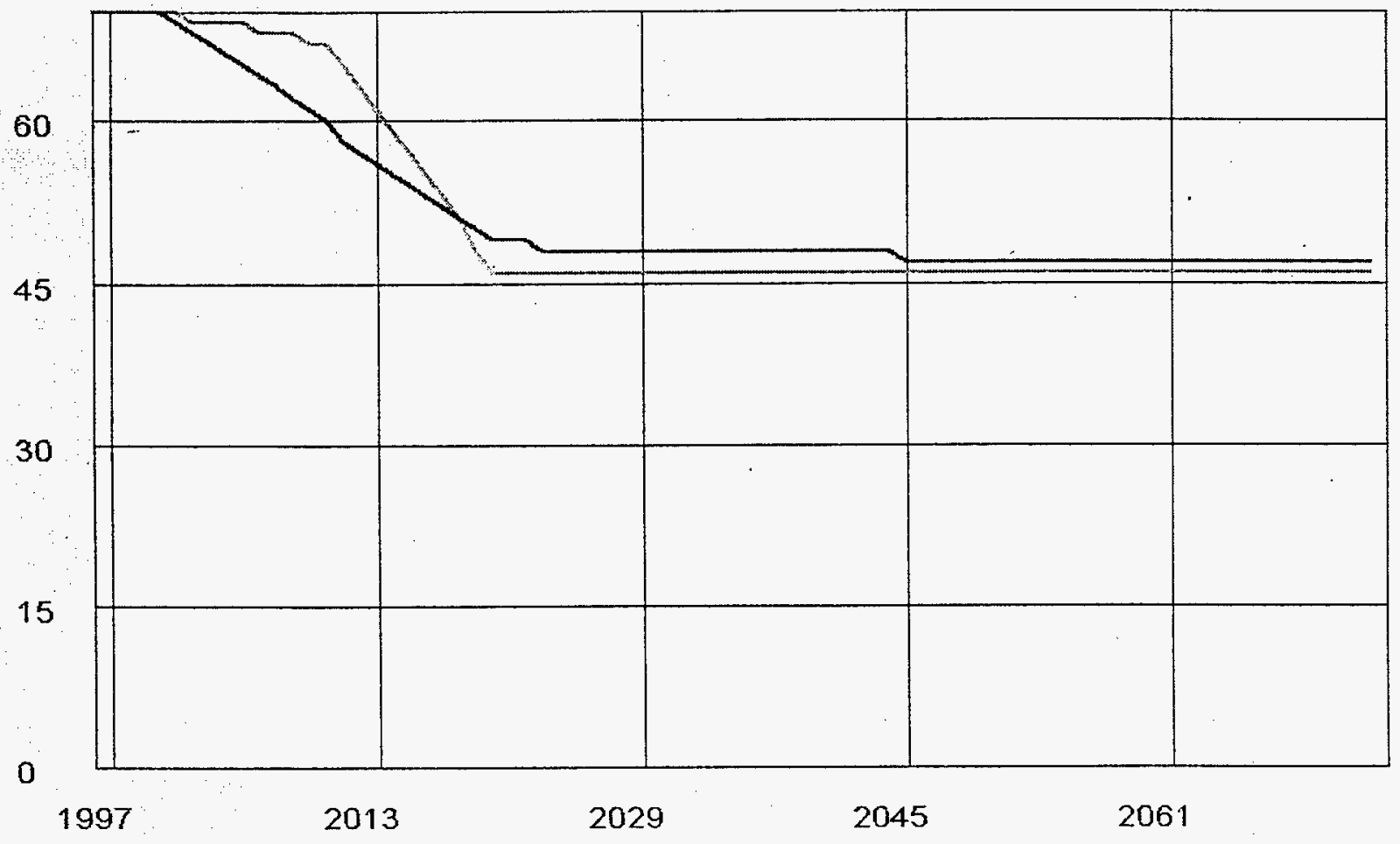

Years

Elternative Case wo-ER w-WWN

Data has not been validated. Use for demonstration purposes only. Copyright 61087 Lockheed Martin Idaho Technologies Company 
TP 3.1 .2

METHOD 1

$10 / 21 / 97$

CHART\#2

@ $100,000 \mathrm{~m}^{3}$

Site: HASI

Martgage Liability Reduction

Hanford Site $\cdot$ HLW

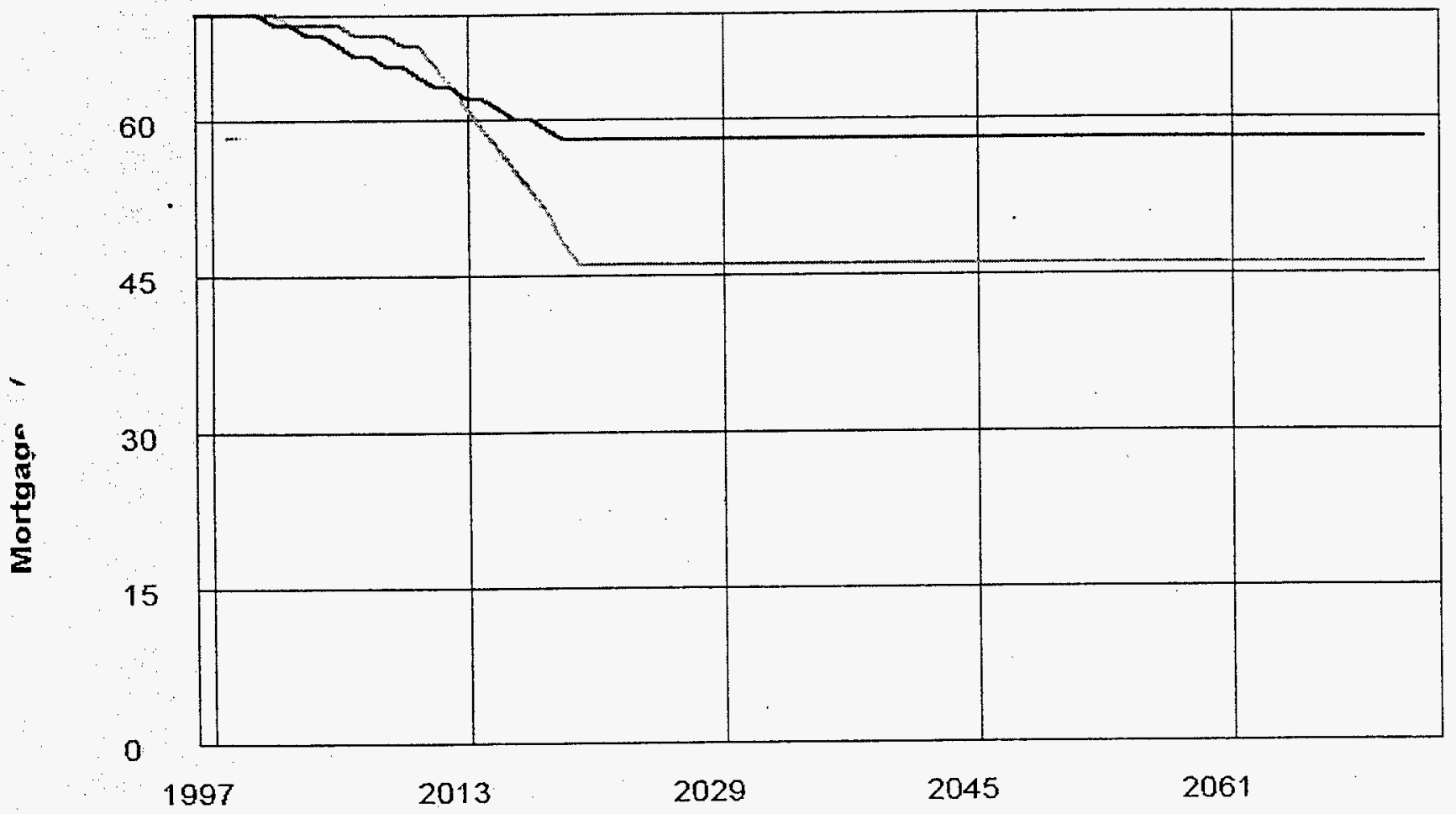

DBase Case wo-ER w-WMN

Years

Alternative Case wo-ER w-WMN

Data has not been validated. Use for demonstration purposes only. Copyright 18997 Lockheed Martin Idaho Technologies Company 
TP 3.1.2

METHOD 1

$10 / 21 / 97$

CHART\#3

@166,000 m $\mathrm{m}^{3}$

te:

Waste Type: HLW

Transportation Cost

Hanford Site - HLW

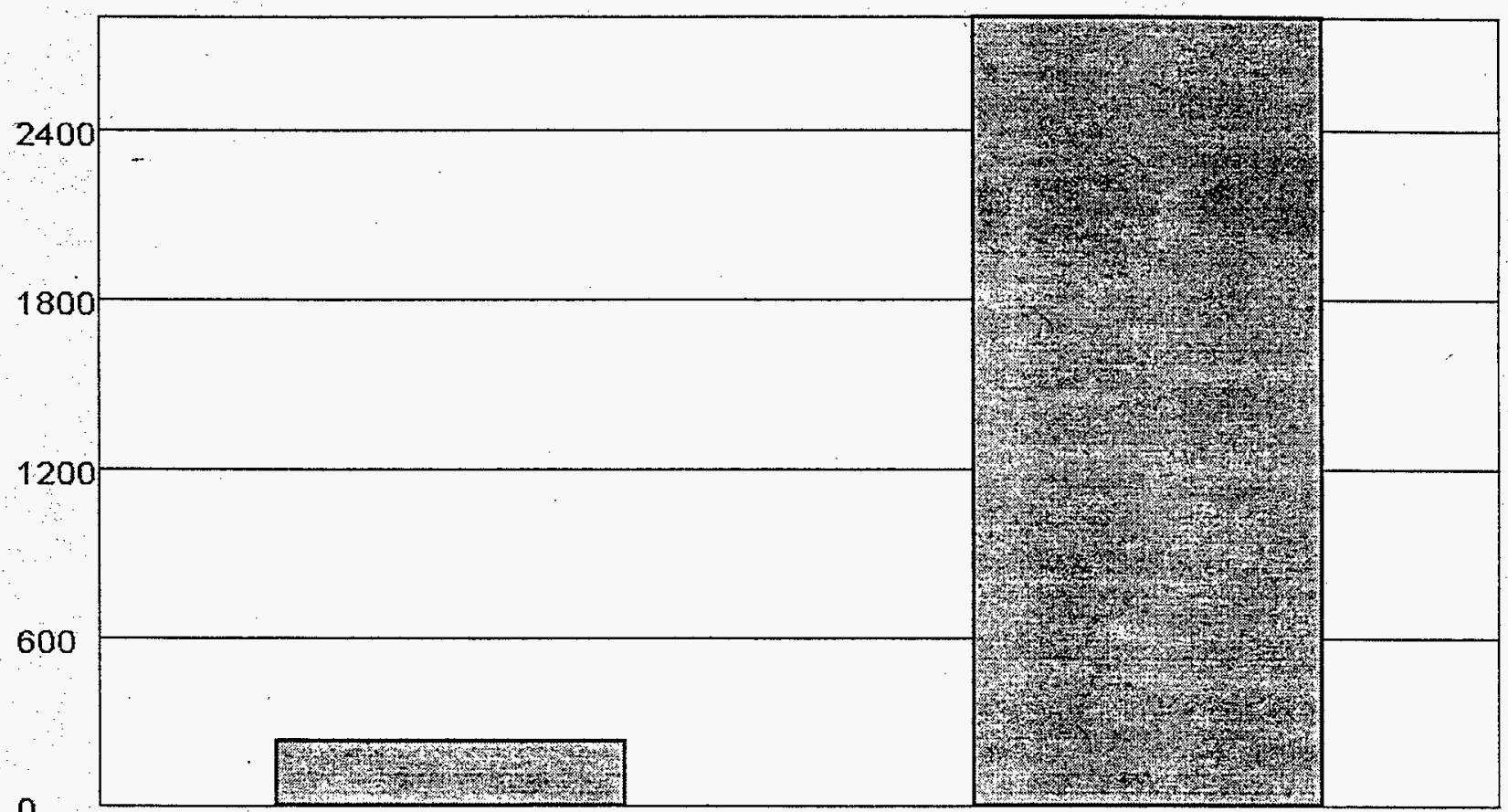

Base Case wo-ER w-WW

Alternative Case wo-ER w-WMN

Data has not been validated. Use for demonstration purposes only. Copyright 1997 Lockheed Martin Idaho Technologies Company 
TP 3.1.2

METHOD 1

$10 / 21 / 97$

CHART\#4

@100,000 $\mathrm{m}^{3}$

\section{Site:[HASI T Waste Type: $\mathrm{HLW}$}

Transportation Cost

Hanford Site - HLW

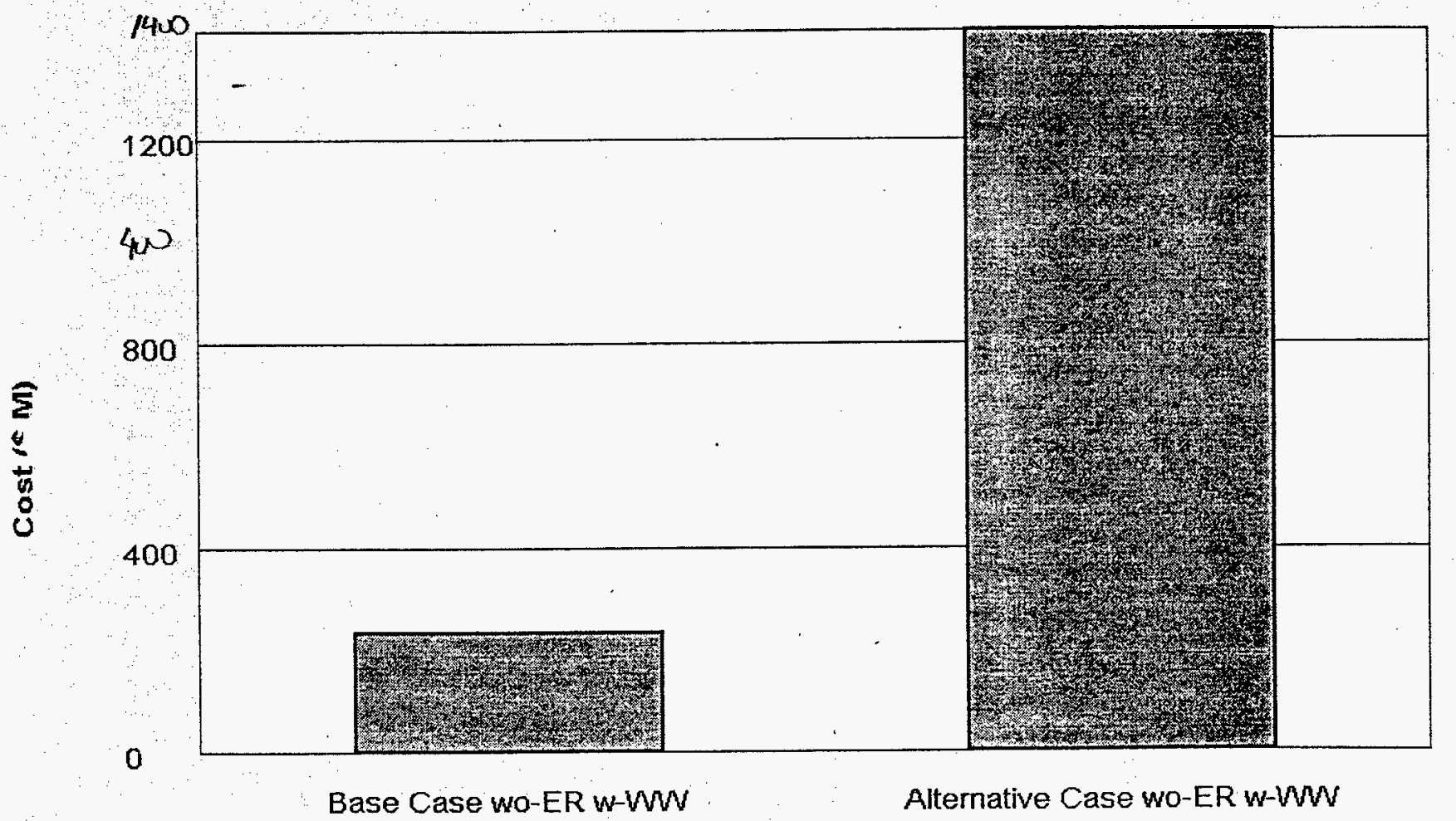


| type |

Jataset datasetAtCurrentVersion complex sites do:

[:site | site facilities do:

[:fac |

$$
\begin{aligned}
& \text { "TRU Cost" } \\
& \text { type := Type new. }
\end{aligned}
$$

type unitCostRates disposalCostRate nominal: 0 .

type unitCostRates treatmentCostRate nominal: 5500 .

type unitCostRates storageCostRate nominal: 700 .

fac costTypes at: \#TRU put: type.

"MLLW Cost"

type := Type new.

type unitCostRates disposalCostRate nominal: 2000.

type unitCostRates treatmentCostRate nominal: 14000 . type unitCostRates storageCostRate nominal: 500 . fac costTypes at: \#MLLW put: type.

"LLW Cost"

type := Type new.

type unitCostRates disposalCostRate nominal: $2000 . \quad$ (\$35)

type unitCostRates treatmentCostRate nominal: 3000 .

type unitCostRates storageCóstRate nominal: 2000.

fac costTypes at: \#LLW put: type.

"HLW Cost"

type := Type new.

type unitCostRates disposalCostRate nominal: 0 .

type unitCostRates treatmentCostRate nominal: $24000 . \checkmark$

type unitCostRates storageCostRate nominal: 450. fac costTypes at: \#HLW put: type. 
populateMortgageRates

"populate mortgageRates"

' type 1

Jataset datasetAtCurrentVersion complex sites do:

[:site $\mid$ site name $=$ 'HASI' ifTrue: $[\checkmark$ ok

"TRU Mortgage Liability"

type := Type new.

type mortgageRates disposalRate: 0

type mortgageRates treatmentRate ${ }^{100,500,000}$.

type mortgageRates storageRate: $58,300,000$.

site mortgageTypes at: \#TRU put: type.

"MLLW Mortgage Liability"

type := Type new.

type mortgageRates disposalRate: 17,100000 .

type mortgageRates treatmentRate: 217700000 .

type mortgageRates storageRate: 112600,000 :

site mortgage Types at: \#MLLW put: type.

"LLW Mortgage Liability"

type := Type new.

type mortgageRates disposalRate: 579700000 .

type mortgageRates treatmentRate: 210300000 .

type mortgageRates storageRate: 0 .

site mortgageTypes at: \#LLW put: type.

"HLW Mortgage Liability"

type := Type new.

type mortgageRates disposalRate: 0 .

type mortgageRates treatmentRate: $2656,300,000$.

type mortgageRates storageRate: 1,607,600,000.

]. site mortgageTypes at: \#HLW put: type.]

"populate mortgageRates"

Dataset datasetAtCurrentVersion complex sites do:

[:site | site name = 'WVDP' ifTrue:[ $\checkmark$

"LLW Mortgage Liability"

type := Type new.

type mortgageRates disposalRate: 20593000.

type mortgageRates treatmentRate: 0 .

type mortgageRates storageRate: 0 .

site mortgageTypes at: \#LLW put: type.

"HLW Mortgage Liability"

type := Type new.

type mortgageRates disposalRate: 0 . 
type mortgageRates treatmentRate: 615601000 .

type mortgageRates storageRate: 0 .

1.

site mortgageTypes at: \#HLW put: type.]

"populate mortgageRates"

Dataset datasetAtCurrentVersion complex sites do:

[:site | site name = 'INEEL' ifT rue:[

"TRU Mortgage Liability"

type := Type new.

type mortgageRates disposalRate: 0 .

type mortgageRates treatmentRate: 762,740000 .

type mortgageRates storageRate: 331500000 .

site mortgageTypes at: \#TRU put: type.

"MLLW Mortgage Liability"

type := Type new.

type mortgageRates disposalRate: 0 .

-type mortgageRates treatmentRate: 207400000.

type mortgageRates storageRate: 15580000.

site mortgageTypes at: \#MLLW put: type.

"LLW Mortgage Liability"

type := Type new.

type mortgageRates disposalRate: 155660000 .

type mortgageRates treatmentRate: 89270000 .

type mortgageRates storageRate: 0 .

site mortgageTypes at: \#LLW put: type.

"HLW Mortgage Liability"

type := Type new.

type mortgageRates disposalRate: 0 .

type mortgageRates treatmentRate: $5465,440,000$.

type mortgageRates storageRate: $170,900,000$.

]. site mortgageTypes at: \#HLW put: type.]

"populate mortgageRates"

Dataset datasetAtCurrentVersion complex sites do:

[:site / site name = 'OAKR' ifTrue:[

"TRU Mortgage Liability"

type := Type new.

type mortgageRates disposalRate: 0 .

type mortgageRates treatmentRate: 344400,000 .

type mortgageRates storageRate: 19600000 .

site mortgageTypes at: \#TRU put: type. 
"MLLW Mortgage Liability"

type := Type new.

type mortgageRates disposalRate: 50200000 .

type mortgageRates treatmentRate: 602200000 .

type mortgageRates storageRate: 94000000 .

site mortgageTypes at: \#MLLW put: type.

"LLW Mortgage Liability"

type := Type new.

type mortgageRates disposalRate: 86300000 .

type mortgageRates treatmentRate: 303200000 .

type mortgageRates storageRate: 86200000 .

site mortgageTypes at: \#LLW put: type.]

].

"populate mortgageRates"

Dataset datasetAtCurrentVersion complex sites do:

[:site | site name = 'SARS' ifTrue:[ $\checkmark$

"TRU Mortgage Liability"

type := Type new.

type mortgageRates disposalRate: $12,363,000$.

type mortgageRates treatmentRate: $834,373,000$.

type mortgageRates storageRate: 348459000 .

site mortgageTypes at: \#TRU put: type.

"MLLW Mortgage Liability"

type := Type new.

type mortgageRates disposalRate: 0 .

type mortgageRates treatmentRate: 146477000 .

type mortgageRates storageRate: 80261000 .

site mortgageTypes at: \#MLLW put: type.

"LLW Mortgage Liability"

type := Type new.

type mortgageRates disposalRate: 511754000 .

type mortgageRates treatmentRate: 626031000 .

type mortgageRates storageRate: 12125000 .

site mortgageTypes at: \#LLW put: type.

"HLW Mortgage Liability"

type := Type new.

type mortgageRates disposalRate: 0 .

type mortgageRates treatmentRate: $6,255,888,000$.

type mortgageRates storageRate: 2299539000.

]. site mortgageTypes at: \#HLW put: type.]

"populate mortgageRates" 
Dataset datasetAtCurrentVersion complex sites do:

[:site | site name = 'NVTS' ifTrue:[

"TRU Mortgage Liability"

type := Type new.

type mortgageRates disposalRate: 0 .

type mortgageRates treatmentRate: 0 .

type mortgageRates storageRate: 15300000 .

site mortgageTypes at: \#TRU put: type.

"MLLW Mortgage Liability"

type := Type new.

type mortgageRates disposalRate: 10000000.

type mortgageRates treatmentRate: 5200000 .

type mortgageRates storageRate: 0 .

].

site mortgageTypes at: \#MLLW put: type.]

"populate mortgageRates"

Dataset datasetAtCurrentVersion complex sites do:

[:site | site name = 'LANL' ifTrue:[

"TRU Mortgage Liability"

type := Type new.

type mortgageRates disposalRate: 0 .

type mortgageRates treatmentRate: 131768000 .

type mortgageRates storageRate: 84455000 .

site mortgageTypes at: \#TRU put: type.

"MLLW Mortgage Liability"

type := Type new.

type mortgageRates disposalRate: 99384000 .

type mortgageRates treatmentRate: 19423000.

type mortgageRates storageRate: 39321000 .

site mortgageTypes at: \#MLLW put: type.

"LLW Mortgage Liability"

type := Type new.

type mortgageRates disposalRate: 97476000 .

type mortgageRates treatmentRate: 86120000 .

type mortgageRates storageRate: 19904000.

site mortgageTypes at: \#LLW put: type.]

].

"populate mortgageRates"

Dataset datasetAtCurrentVersion complex sites do:

[:site | site name = 'FEMP' ifTrue:[ 


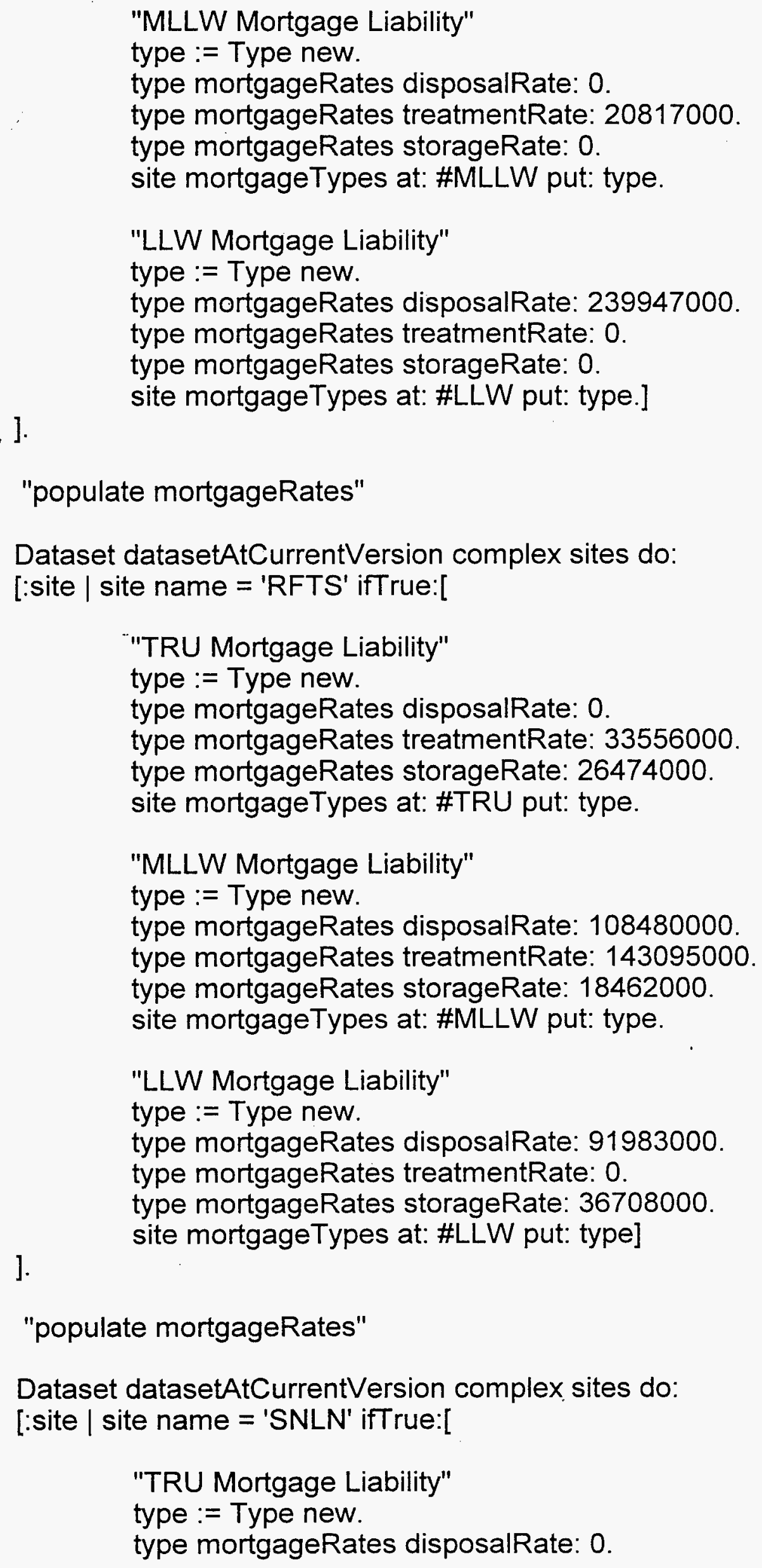

"populate mortgageRates"

Dataset datasetAtCurrentVersion complex sites do:

[:site | site name = 'RFTS' ifTrue:[

"populate mortgageRates"

Dataset datasetAtCurrentVersion complex sites do:

[:site | site name = 'SNLN' ifTrue:[

"TRU Mortgage Liability" type := Type new.

type mortgageRates disposalRate: 0 . 
type mortgageRates treatmentRate: 0 .

type mortgageRates storageRate: 0 .

site mortgageTypes at: \#TRU put: type.

"MLLW Mortgage Liability"

type := Type new.

type mortgageRates disposalRate: 0 .

type mortgageRates treatmentRate: 0 .

type mortgageRates storageRate: 0 .

site mortgageTypes at: \#MLLW put: type.

"LLW Mortgage Liability"

type := Type new.

type mortgageRates disposalRate: 0 .

type mortgageRates treatmentRate: 0 .

type mortgageRates storageRate: 0 .

].

site mortgageTypes at: \#LLW put: type.] 
row := TransportationConstants new.

row type: 'HLW'.

row mode: 'Road'.

row fixedCost: 8110 .

row packagesPerShipment: 1 .

row massCapacity: 909.

row volumeCapacity: 20.

self addRow: row.

row : = TransportationConstants new.

row type: 'HLW'.

row mode: 'Rail'.

row fixedCost: 1800

row packagesPerShipment: 1 .

row massCapacity: 14545.

row volumeCapacity: 424 .

self addRow: row.

row : = TransportationConstants new.

row type: 'TRU'.

row mode: 'Road'.

row fixedCost: 4630

row unitCost: 10.87.

row packagesPerShipment: 3.

row mass Capacity: 9818.

row volumeCapacity: 8.68 .

w: row.

row : = TransportationConstants new.

row type: 'TRU'.

row fixedCost: 9260 .

row unitCost: $\mathbf{3 3 . 9 5}$.

row packagesPershipment: 6 .

row mass Capacity: 19636.

rolf volumecapacity! 17.37.

row := TransportationConstants new.

row type: 'MLLW.'

row fixedCost: 880 .

row unitCost: 4.95 .

row unitCost: 4.95.

row packagespershipment:

row voluchacit 18.13.

self addRow: row.

row : = TransportationConstants new.

row type: 'MLW'.

row mode: Ralle

row unitCost: 2250 .

row packagesPershi

row 3 .

row masscapacity: 54545 .

row volumeCapacity:

self addRow: row.

row := TransportationConstants new.

row type: 'LLW'. 
- row flxedCost: 880

row unitCost: 4.98 .

row packagespershipment:

row masscapacity! 20000.

row volumeCapacity: 18.13 .

self addRow: row.

row := TransportationConstants new.

row type: 'LLW',

row mode: 'Rail'.

row fixedCost: 2250

row unitCost: 5.73 .

row packagespershipment: 3.

row masscapacity. 54545 .

dRow: row. 


\section{TEST PROCEDURE - TP3.1.3 \\ Health and Safety}

\section{Purpose}

This test procedure has been designed to validate the CWWFA risk results. The risk incorporated into the CWWFA used the Simplified Risk Model developed by LMITCO. ${ }^{1}$ This procedure will satisfy requirements 3.1.3.1, 3.1.3.2, and 3.1.3.3 as referenced in Appendix A - Requirements Verification Matrix.

\section{Objective}

The baseline Ten Year Plan (TYP) risk information is grounded on the risk analysis performed by Steve Eide for the Environmental Management Integration (EMI) program. The objective is to utilize static summary risk output from the Eide's study to validate the CWWFA risk results.

\section{Test Method}

Method 1. - Site risk result evaluation. Isolate CWWFA risk results at Hanford for HLW waste and compare to the Treatment, Storage, Disposal (TSD) graph provided by Steve Eide.

Method 2. - Processing facility evaluation. Isolate a processing facility at Hanford (Sludge, Wash facility), material stream (HAN01), take out the time component by shipping all the waste in one year, ignore interim storage risk produced by CWWFA, and compare to the facility risk sheet results and TSD graph provided by Steve Eide.

${ }^{1}$ R. G. Peatross, S.A. Eide, Users Guide for the Simplified Risk Model (SRM), INEL-96/0397, October 1996. 


\section{Reporting}

TP - Test Procedure

REQD - Requirement

$\mathrm{P} / \mathrm{F}$ - Pass/Fail

\begin{tabular}{|c|c|c|c|}
\hline$\overline{\mathbf{T P}}$ & REQD & Result/Comment & $\mathbf{P} / \mathbf{F}$ \\
\hline \begin{tabular}{|l|} 
TP3.1.3 \\
Method 1
\end{tabular} & \begin{tabular}{|l|}
3.1 .3 .1 \\
3.1 .3 .2 \\
3.1 .3 .3
\end{tabular} & $\begin{array}{l}\text { The total relative risk result from the Eide TSD graph is } \\
\text { approximately } 1,173 \text { for Hanford HLW. The CWWFA risk result } \\
\text { is } 143.02 \text {. There is a difference of } 1,029.98 \text {. } \\
\text { Comments: There are two facilities in CWWFA that are not } \\
\text { calculating risk. The risk numbers are not loaded for the facilities. }\end{array}$ & Fail \\
\hline $\begin{array}{l}\text { TP3.1.3 } \\
\text { Method 2 }\end{array}$ & \begin{tabular}{|l|}
3.1 .3 .1 \\
3.1 .3 .2 \\
3.1 .3 .3
\end{tabular} & $\begin{array}{l}\text { The total relative risk from Eide TSD graph for the sludge, wash } \\
\text { facility is approximately } 141 . \text { The CWWFA risk result is } 48.1 \text {. } \\
\text { There is a difference of } 92.9 \text {. } \\
\text { Comments: In further investigation discovered that Eide results } \\
\text { had two sets of facility constants for the } 4 \times 5 a-F a c \text { term. This is } \\
\text { because the Eide had more information on the HAN01 material } \\
\text { stream then was provided in the EMI disposition maps. This } \\
\text { information is not displayed on the disposition map and therefore } \\
\text { not included in the CWWFA data set. When comparing sludge, } \\
\text { wash phase } 1 \text { sheet (from Eide's results) that had the same } \\
\text { constants that were loaded into CWFA, the results were } 1 \text { factor } \\
\text { higher in CWWFA (exactly } 10 \text { times Steve's results for each } \\
\text { term). Will need to find out why (probably just have one term } \\
\text { which is an order of magnitude high). Will need to validate the } \\
\text { facility constants. } \\
\text { Alternatives - support multiple risk facility constants, average the } \\
\text { values, or take the set of facility constants that have the greater } \\
\text { risk (Eide's recommendation). } \\
\text { Other Findings } \\
\text { Eide does not have temporary storage risk in his calculations. } \\
\text { There is a temporary risk storage calculation in CWWFA, which } \\
\text { was implemented per direction from Eide. Not sure how to } \\
\text { validate these results? } \\
\text { Eide includes chemical constants as part of his risk calculations. } \\
\text { There is no chemical data risk in CWWFA. } \\
\text { In processing facilities after risk is calculated and the conversion } \\
\text { profile applies the actinide and nonactinide percentages the }\end{array}$ & Fail \\
\hline
\end{tabular}




\begin{tabular}{|c|c|c|}
\hline & & $\begin{array}{l}\text { buckets that are sent to storage (with the new actinide and } \\
\text { nonactinide percentages) do not contain destination information. } \\
\text { The nonactinide and actinide percentages are derrived based on the } \\
\text { assumption of where the waste will be sent. The end result is that } \\
\text { this causes the risk for various facility to be erroneous. This only } \\
\text { occurs where the processing facility has waste splits. A solution } \\
\text { would be to create a destination "facilityld" instances variable for } \\
\text { the Bucket, and Conversion Split Classes. Populate the facilityld } \\
\text { variable in the Conversion Split Class and when the Conversion } \\
\text { Splits are applied in Treatment assign the destination facilityld to } \\
\text { the Bucket facilityId. Subsequently, when the buckets are gathered } \\
\text { in storage for shipment the destination facility can be validated. } \\
\text { Therefore, the percentages of actinides and nonactinides are sent to } \\
\text { the correct destination and the risk will be compariable to Steve } \\
\text { Eides' risk. } \\
\text { Need to add the constant factors for the handling risk to the total } \\
\text { disposal risk calculations for disposal facilities. } \\
\text { Transportation risk needs to be validated. } \\
\text { In summary the CWWFA calculates risk differently then Eide's } \\
\text { risk results. The more CWWFA deviates from Eide's process of } \\
\text { calculating risk numbers the more difficult the validation effort } \\
\text { becomes because we can no longer use Eide's risk results as a } \\
\text { baseline. A corrective action is to follow a static risk calculation } \\
\text { (exactly like Eide) or in order to support a dynamic model like } \\
\text { CWWFA to change our approach to risk (risk based on waste type } \\
\text { or some other simplified approach). }\end{array}$ \\
\hline
\end{tabular}




\section{Purpose}

This test procedure has been designed to verify the CWWFA facility operational performance results. The facility operational performance refers to optimal utilization of treatment, storage, or disposal facilities, and identification of redundant capacities. This procedure will satisfy requirements 3.1.4.1 as referenced in Appendix A - Requirements Verification Matrix.

\section{Objective}

The objective of this procedure is to verify that CWWFA supports determination of facility utilization and redundant capacities by analysis.

\section{Test Method}

This test method relies on an analysis approach to make a determination of facility utilization. Table 1 represents varying sites, waste types, and facilities that are chosen for the analysis. After the simulation, an assessment of the impact will be conducted by examining the CWWFA facility capacity detail charts.

\begin{tabular}{|l|l|l|}
\hline \multicolumn{1}{|c|}{ Table1. Site } & \multicolumn{1}{|c|}{ Waste Type } & \multicolumn{1}{c|}{ Facility } \\
\hline Hanford & HLW & HLW vitrification \\
\hline INEEL & TRU & INEL2.1-T \\
\hline SRS & LLW & Legacy-SRS \\
\hline
\end{tabular}




\section{Reporting}

TP - Test Procedure

REQD - Requirement

P/F - Pass/Fail

\begin{tabular}{|c|c|c|c|}
\hline TP & REQD & Result/Comment & $\mathbf{P} / \mathbf{F}$ \\
\hline TP3.1.4 & 3.1 .4 .1 & $\begin{array}{l}\text { This requirement has four parts that need to be } \\
\text { addressed. The following discussion will be tailored to } \\
\text { address each part respectively. } \\
\text { Measure utilization at facilities } \\
\text { The attached output charts (charts \#1-3) demonstrated } \\
\text { the ability of the model to characterize the utilization } \\
\text { capacity at different facilities. An analysis of annual } \\
\text { receipt, storage limit, and annual output can be used to } \\
\text { measure utilization at different facilities. This part } \\
\text { complies with the requirement. } \\
\text { Compare maximum capacities that are utilized } \\
\text { It is not possible to compare maximum utilization unless } \\
\text { the analyst has information regarding the facility } \\
\text { maximum capacity. The current output charts are scaled } \\
\text { to the waste volume that flows through the facility and } \\
\text { not necessarily scaled to its maximum capacity. } \\
\text { Therefore a comparison on maximum utilization cannot } \\
\text { be made. This is a deficiency. } \\
\text { Provide identification of redundant capabilities } \\
\text { including existing DOE facilities and private sector } \\
\text { capabilities. } \\
\text { The model does not have current capabilities to identify } \\
\text { redundancy (or to determine if the redundancy is good } \\
\text { or bad) at the facility level, at other DOE facilities, or } \\
\text { the private sector. This requirement is unclear. } \\
\text { Recommend that this requirement be renegotiated for } \\
\text { removal with the customer. This is a major deficiency. } \\
\text { Identify applications for use of excess capacity } \\
\text { This requirement is beyond the realm of this model. } \\
\text { This is a simulation tool. This requirement is unclear. } \\
\text { Recommend that this requirement be renegotiated for } \\
\text { removal with the customer. This is a major deficiency. }\end{array}$ & Fail \\
\hline
\end{tabular}


TP 3.1.4

$10 / 22 / 97$

CHART\#1

e:

Waste Type:HLW Facllity: HANF-HLVF-2

Facility Capacity Detail

Hanford Site $\cdot H L W \cdot H L W$ Vitrification

Alternative Case wo-ER WWW

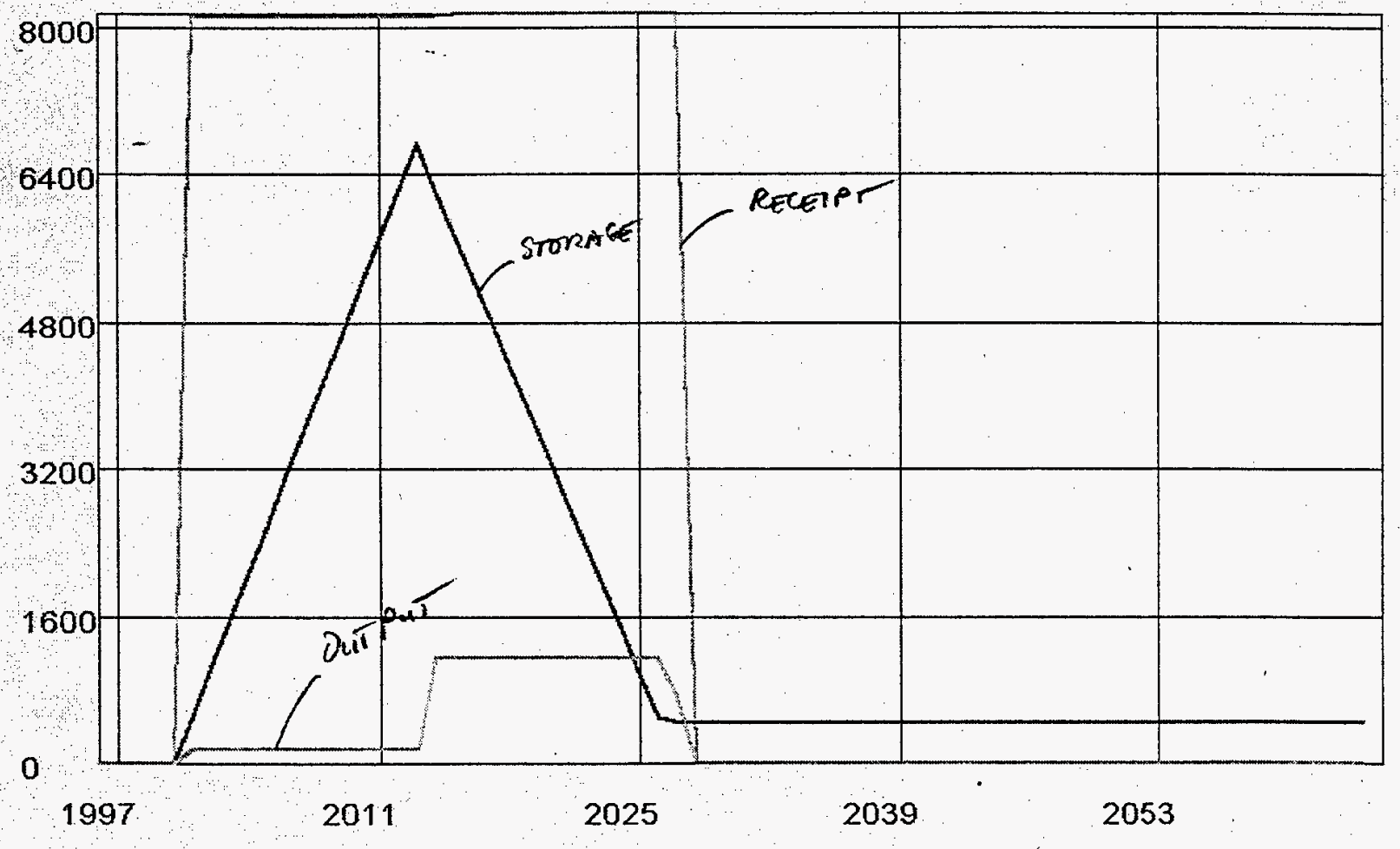

Q Annual Recelpt

Years

- Cumulative Storage

DAnnual Output

Data has not been validated. Use for demonstration purposes only. Copyright \& 1997 Lockheed Martin Idaho Technologies Company 
TP 3.1 .4

10/22/97

CHART\#2

Site: INEEL

Waste Type:TRU

Facility: INEL2.1-T

Facility Capacity Detail

Idaho National Engineering \& Environmental Laboratory - TRU • SWEPP Characterization Alternative Case wo-ER W-WMN

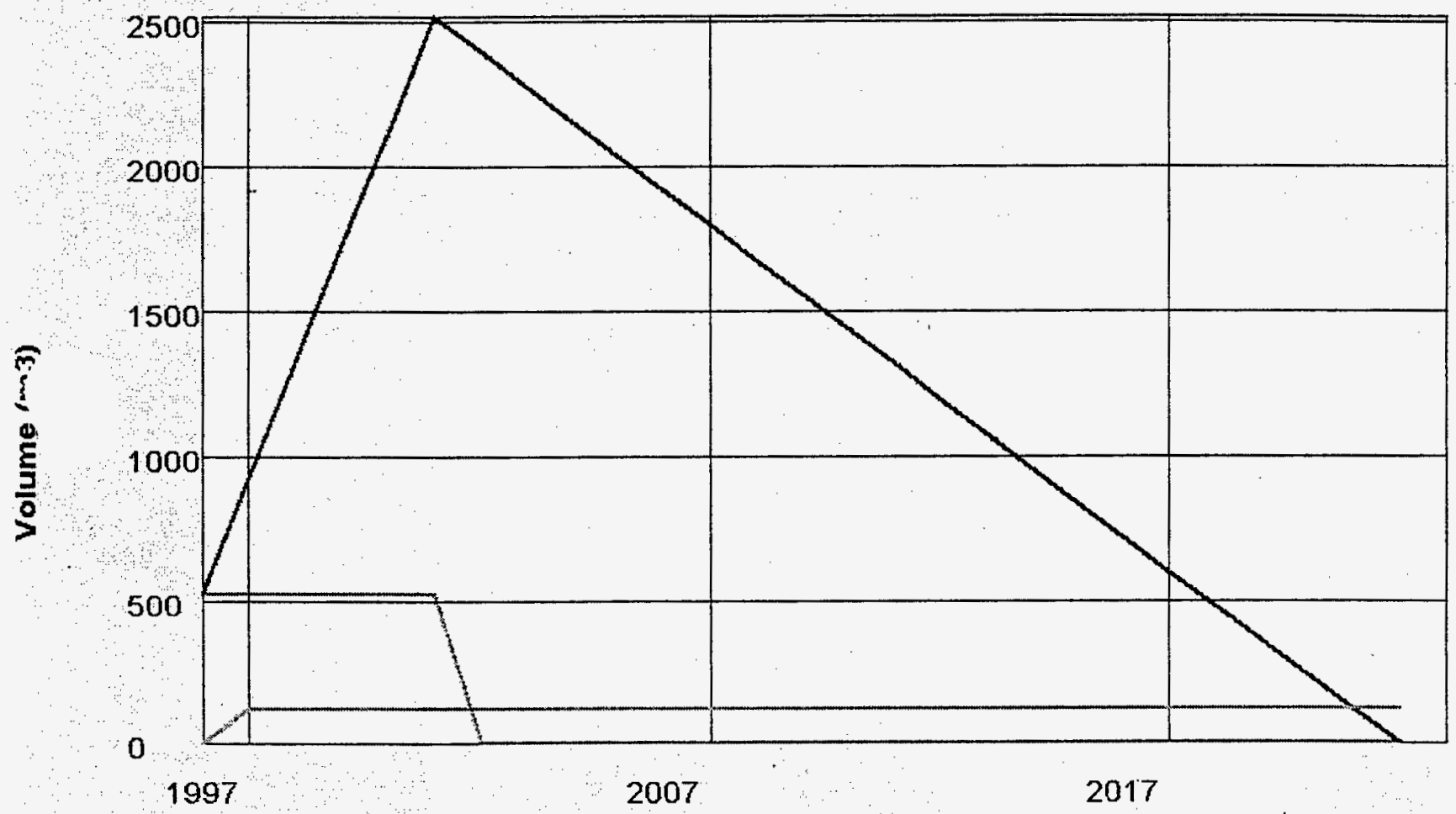

■Annual Receipt

Years

Cumulative Storage

GAnnual Output

Data has not been validated. Use for demonstration purposes anty. Copyright 1907 Lookheed Martin idaho Technologies Compamy 
TP 3.1 .4

10/22/97

CHART\#3

Facility Capacity Detail

Savannah River Site - LLW - Legacy Facility

Alternative Case wo-ER w-WW

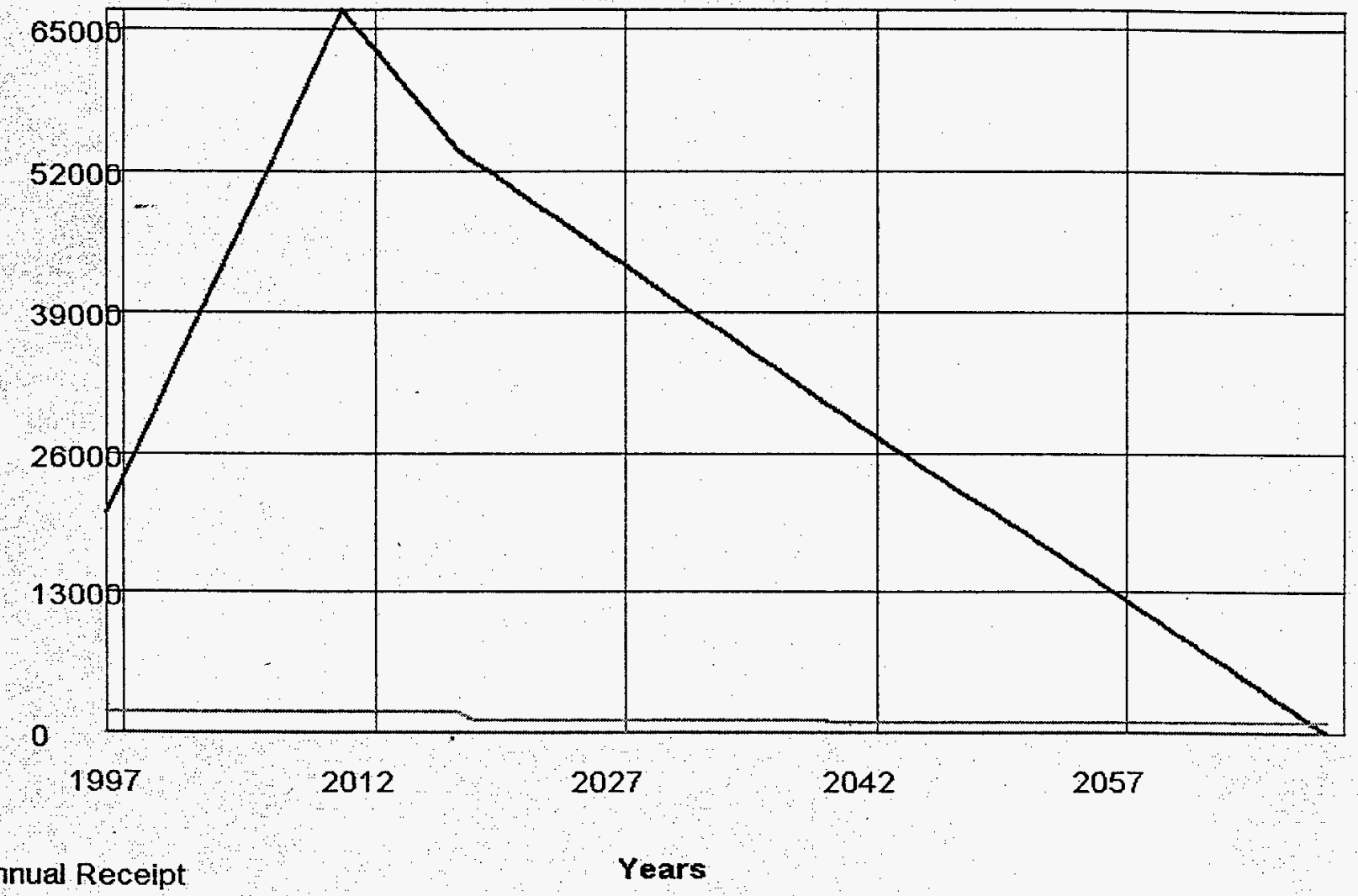

\section{Cumulative Storage}

Gannual Output

Data has not been validated. Use for demonstration pürposes only. Copyright 1997 Lockheed Martin Idaho Technologles Compary 


\section{Test Procedures - TP3.1.6}

Equity Analysis

\section{Purpose}

This test procedure is designed to test requirement 3.1.6.1 to incorporate generic equity analysis capability using existing stakeholder data. Requirement 3.1.6.2 refers to future enhancements of the model planned for FY98 and not currently present in the model.

\section{Objective}

The objectives are to confirm requirement 3.1.6.1 has been met by the following:

1. Calculations are correct compared to the expected results from the algorithm, and

2. Stakeholder positions changes with changes to off-site conditions.

\section{Test Method}

Method 1 - Calculations were tested by manually calculating equity positions and comparing the results to the model. The base case without wastewater or ER was compared to the alternative case with the same conditions.

Eight test cases were developed. Tests were developed by a random combination of sites, stakeholders, weighting, objectives and thresholds. All tests were based on High Level waste calculations. The possible value selections are as follows:

Sites

1. Hanford

1. INEEL

2. Savana River

3. Oak Ridge

4. WIPP

5. Yucca Mt.

6. West Valley

7. Mound

8. Rocky Flats

9. Las Alamos

10. Sandia

11. Fernald
Stakeholders

1. State government

2. Tribal government

3. Local Government

4. Environmental groups

5. Civic and volunteer organizations

6. General public

7. Community leaders

8. Site administrators 
Weighting combinations

1. Site volume $=10 \%$, Complex Volume $=75 \%$, Transport $=15 \%$

2. Site volume $=30$, Complex Volume $=35 \%$, Transport $=35 \%$

3. Site volume $=75 \%$, Complex Volume $=15 \%$, Transport $=10 \%$

Influence objective (one for each influence) Threshold Value (one for each influence)

1. Increase

2. Decrease
1. Lowest

2. Mean

3. Highest

Method 2 -The purpose of this test was to ensure the model is reflecting complex wide interactions. Calculations were tested by observing stakeholders positions at a site before and after changes are made to conditions at another site. The base case without waste water or ER was compared to the alternative case with the same conditions. The hypothesis is that stakeholder positions at one site will change as the result of waste processing conditions at another site.

The test conditions:

1. Environmental stakeholders at Hanford and INEEL placed $100 \%$ of their weight on the site volume influence.

2. State government stakeholders at Oak Ridge placed $100 \%$ of their weight on Interstate Transportation.

3. The shipping schedules transferring HLW from Hanford and INEEL to Oak Ridge were altered to stop all shipping of HLW to Oak Ridge.

\section{Reporting}

TP - Test Procedure

REQD - Requirement

$\mathrm{P} / \mathrm{F}$ - Pass/Fail

\begin{tabular}{|l|l|l|l|}
\hline \multicolumn{1}{|c|}{ TP } & REQD & \multicolumn{1}{|c|}{ Result/Comment } & \multicolumn{1}{|c|}{ P/F } \\
\hline $\begin{array}{l}\text { TP3.1.6 } \\
\text { Method 1 }\end{array}$ & 3.1 .6 .1 & $\begin{array}{l}\text { Five of the eight test conditions ran as designed with he } \\
\text { exception of the Mass value for transportation in Figure } \\
\text { 1. This error appears to be present on all stakeholders }\end{array}$ & $\begin{array}{l}\text { Pass - } \\
\text { Minor } \\
\text { correction }\end{array}$ \\
\hline
\end{tabular}




\begin{tabular}{|l|l|l|l|}
\hline Method 2 & $\begin{array}{l}\text { at Oak Ridge, but does not effect any other sites or } \\
\text { stakeholders. See test conditions and results below. } \\
\text { Three of the test conditions did not run because } \\
\text { stakeholder threshold values have not been included in } \\
\text { the model for these sites. }\end{array}$ & \\
\hline TP3.1.6 & 3.1 .6 .2 & $\begin{array}{l}\text { Not applicable. Capability is not scheduled for } \\
\text { inclusion in the model at this time. }\end{array}$ & N/A \\
\hline
\end{tabular}


Test Conditions and Results of Test Method 1

\begin{tabular}{|c|c|c|c|c|c|c|c|c|}
\hline \multirow[t]{2}{*}{ INFLUENCE } & \multicolumn{3}{|c|}{ STAKEHOLDER VALUES } & \multicolumn{5}{|c|}{ CALCULATED VALUES } \\
\hline & WEIGHT & OBJECTIVE & $\begin{array}{l}\text { SIGNIFICANT } \\
\text { DEVIATION }\end{array}$ & DEVIATION & $\begin{array}{r}\text { MEASU } \\
\text { expected }\end{array}$ & $\begin{array}{l}\text { JRE } \\
\text { model }\end{array}$ & $\begin{array}{r}\text { MAS } \\
\text { expected }\end{array}$ & model \\
\hline Site Volume & 10 & Decrease & 535 & 74581 & -3 & -3 & -30 & -30 \\
\hline $\begin{array}{l}\text { Complex Volume } \\
\text { Intersite }\end{array}$ & 75 & Increase & 105530 & 2203035 & 3 & 3 & 225 & 225 \\
\hline Transportation & 15 & Decrease & 11280 & -61932 & 3 & 3 & 45 & 15 \\
\hline TOTAL & 100 & & & & & & & \\
\hline
\end{tabular}

Figure 1

Tribal Stakeholders at Oak Ridge

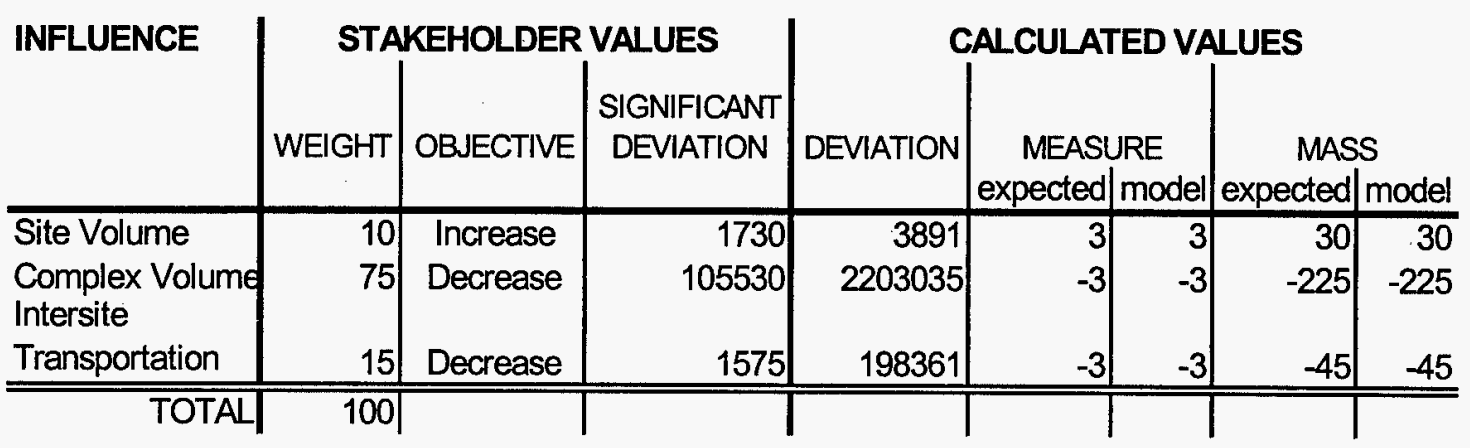

Figure 2

Environmental Stakeholders at Fernald

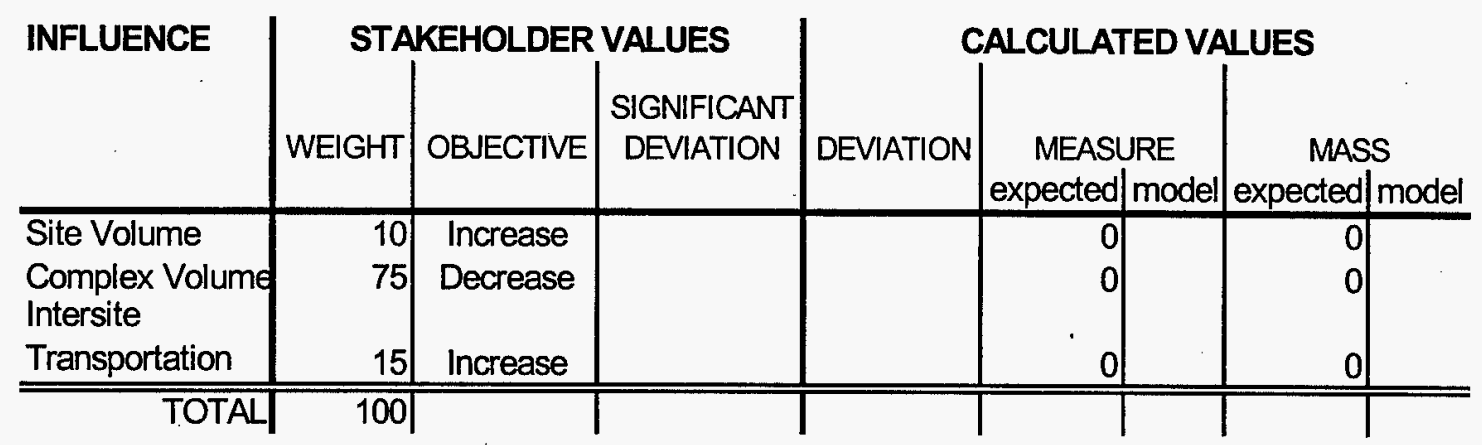

Figure 3

Site Administrator Stakeholders at Yucca Mt. 


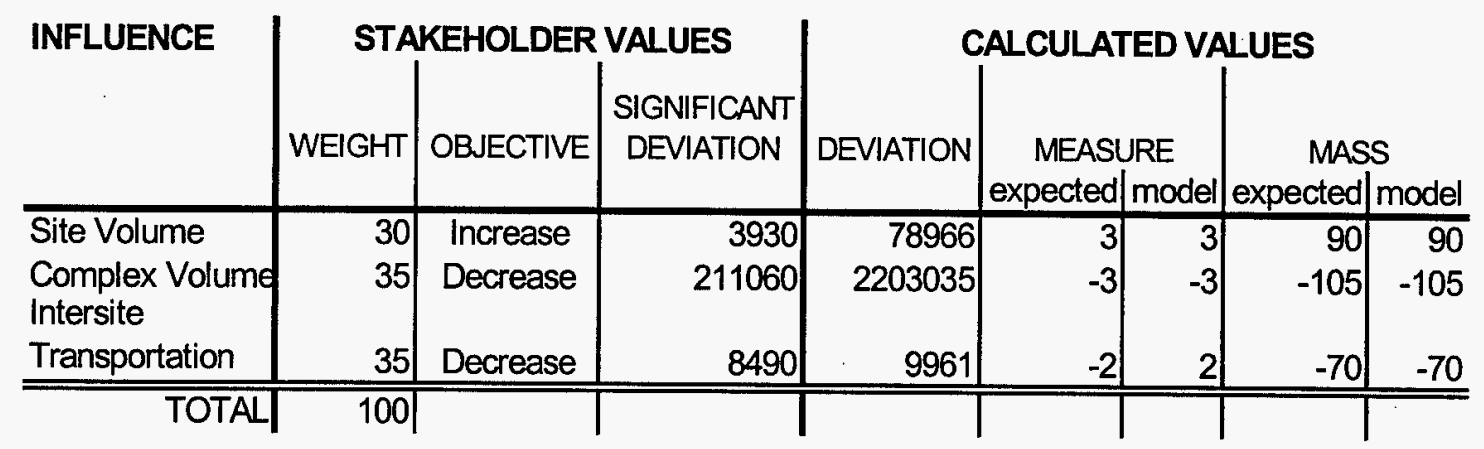

Figure 4

State Government stakeholders at INEEL

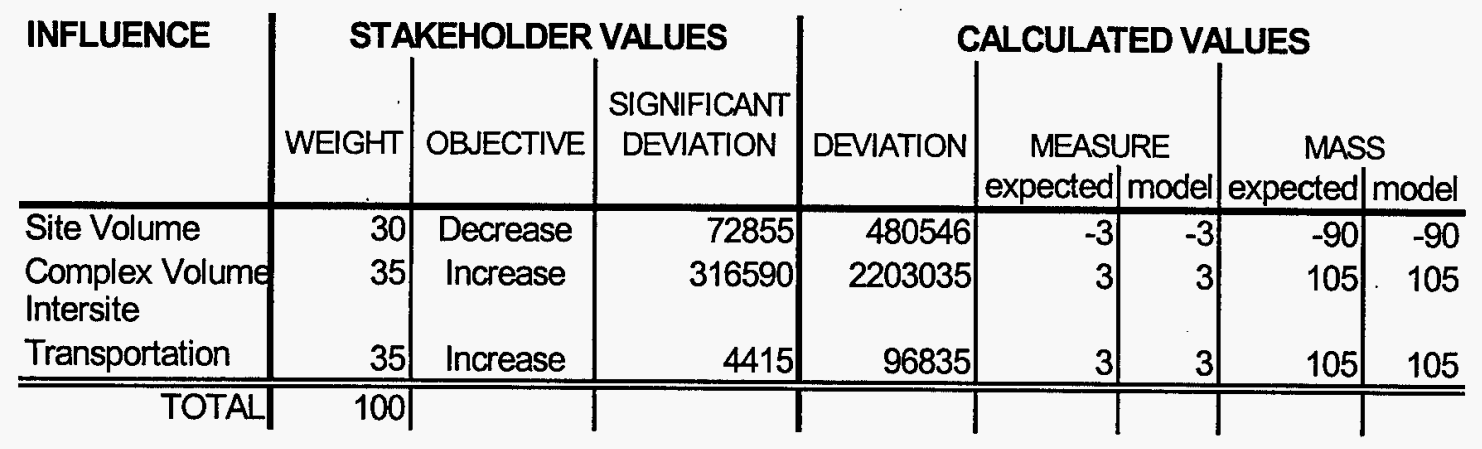

Figure 5

Tribal Government Stakeholders at Hanford

\begin{tabular}{|c|c|c|c|c|c|c|c|c|}
\hline \multirow[t]{2}{*}{ INFLUENCE } & \multicolumn{3}{|c|}{ STAKEHOLDER VALUES } & \multicolumn{5}{|c|}{ CALCULATED VALUES } \\
\hline & WEIGHT & OBJECTIVE & $\begin{array}{l}\text { SIGNIFICANT } \\
\text { DEVIATION }\end{array}$ & DEVIATION & $\begin{array}{r}\text { MEASI } \\
\text { expected }\end{array}$ & $\begin{array}{l}\text { URE } \\
\text { |model }\end{array}$ & $\begin{array}{r}\text { MAS } \\
\text { expected }\end{array}$ & $\begin{array}{l}\mathrm{S} \\
\text { model }\end{array}$ \\
\hline Site Volume & 30 & Increase & 112770 & 741220 & 3 & 3 & 90 & 90 \\
\hline $\begin{array}{l}\text { Complex Volumg } \\
\text { Intersite }\end{array}$ & 35 & Increase & 105530 & 2203035 & 3 & 3 & 105 & 105 \\
\hline Transportation & 35 & Decrease & 14295 & -210 & 1 & 1 & 35 & 35 \\
\hline TOTAL & 100 & & & & & & & \\
\hline
\end{tabular}

Figure 6

General Public Stakeholders at Savannah River 


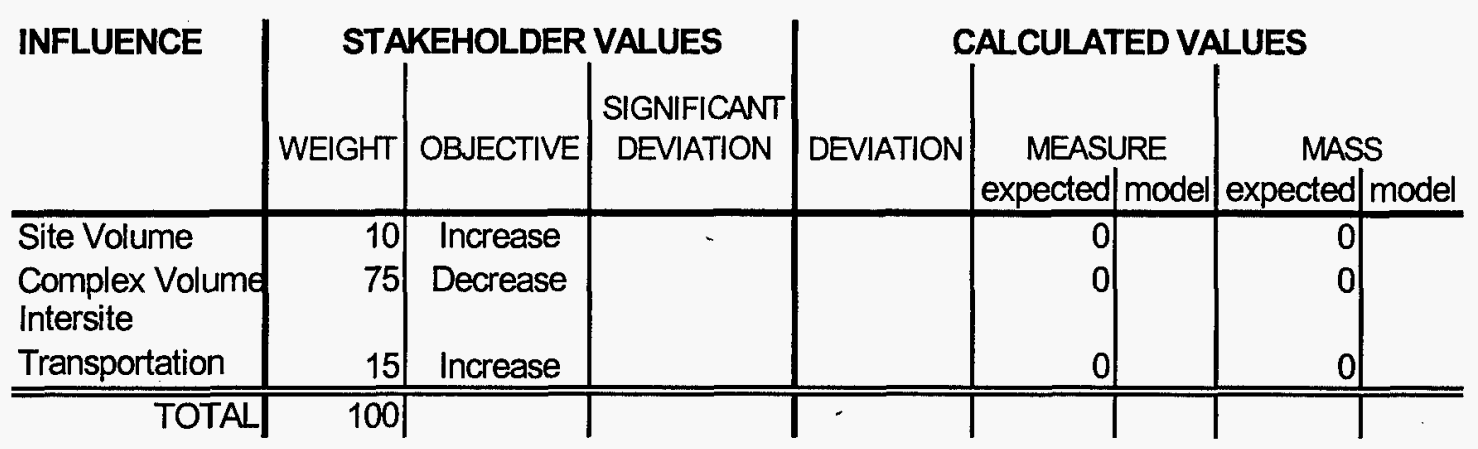

Figure 7

Site Administrator Stakeholders at Mound

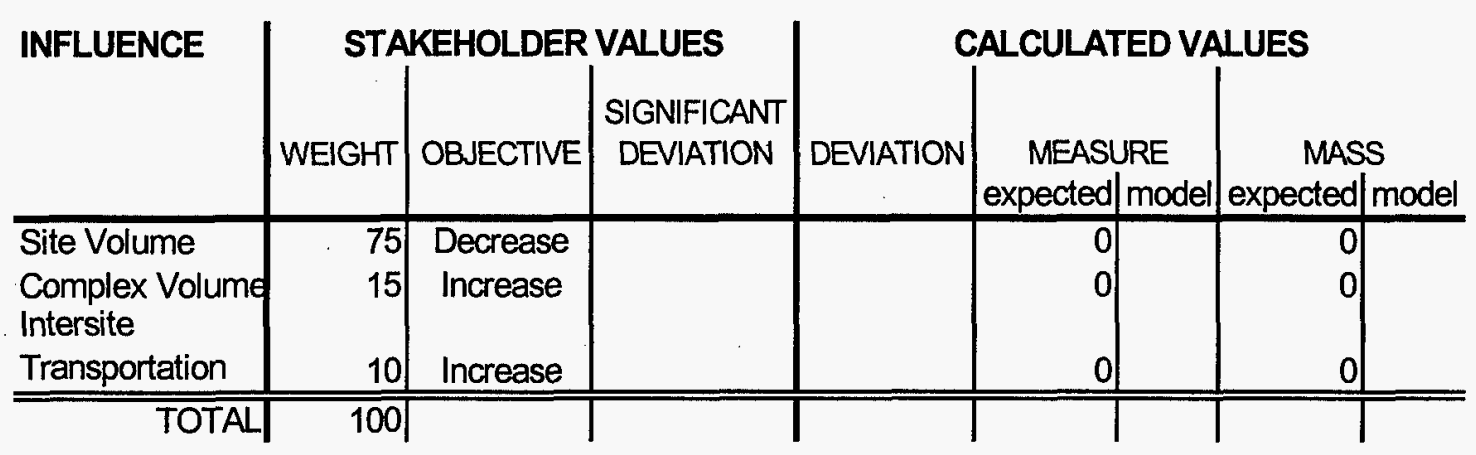

Figure 8

General Public Stakeholders at WIPP

Test Conditions and Results of test Method 2

\begin{tabular}{|c|c|c|c|c|c|c|}
\hline & \multicolumn{6}{|c|}{ Stakeholder Positions (score) \& Influence Value } \\
\hline & \multicolumn{2}{|c|}{ Hanford Waste Volume } & \multicolumn{2}{|c|}{ INEEL Waste Volume } & \multicolumn{2}{|c|}{$\begin{array}{c}\text { Oak Ridge } \\
\text { Transportation }\end{array}$} \\
\hline & $\begin{array}{r}\text { Stakehold } \\
\text { er Score }\end{array}$ & $\begin{array}{c}\begin{array}{c}\text { Influence } \\
\text { Value }\end{array} \\
\end{array}$ & $\begin{array}{r}\text { Stakehold } \\
\text { er Score }\end{array}$ & $\begin{array}{c}\text { Influence } \\
\text { Value }\end{array}$ & $\begin{array}{r}\text { Stakehold } \\
\text { er Score }\end{array}$ & $\begin{array}{c}\text { Influence } \\
\text { Value }\end{array}$ \\
\hline $\begin{array}{l}\text { Before } \\
\text { Change }\end{array}$ & -300 & 480,546 & -300 & 78,967 & 300 & $-61,932$ \\
\hline $\begin{array}{l}\text { After } \\
\text { Change }\end{array}$ & -300 & 498,064 & -300 & 79,330 & 300 & $-75,904$ \\
\hline
\end{tabular}

The model did demonstrate the complex interactions by decreasing the waste transported to Oak Ridge (see influence values). However, the significant deviations at the sites involved were not sensitive enough to reflect a change in the stakeholder positions as the influence values changed. 


\section{TEST PROCEDURE - TP3.1.7 \\ Technology Performance}

\section{Purpose}

This test procedure has been designed to verify the technology performance assessment capability of CWWFA. Technology performance assessment refers to ability to analyze a new technology system or facility that can be substituted (e.g., rerouting of waste flow into a new facility) into the disposition path or to define a new specification (e.g., volume reduction) for existing facility. This procedure will satisfy requirements 3.1.7.1 and 3.1.7.2 as referenced in Appendix A - Requirements Verification Matrix.

\section{Objective}

There are two objectives associated with this procedure. The first objective is to verify that CWWFA provides for assessment of new system capabilities through rerouting of waste flow into a new facility. The second objective verifies the effect of changing specification through a volume reduction.

\section{Test Method}

Method 1 - This test method demonstrates a rerouting of waste from one facility (e.g., treatment or storage) to another within CWWFA. The rerouting of waste shows the impact on a facility behavior (i.e., increased waste volume and storage impacts). After the simulation, an assessment of the impacts will be conducted by examining the CWWFA facility capacity detail and waste stream detail charts. Table 1 and $1 \mathrm{~A}$ provide the new shipping routes and schedules for this test. The CWWFA input screen will be used to input the new routing data.

\begin{tabular}{|l|l|l|l|l|l|}
\hline \multicolumn{3}{|l}{ Table 1. - Shipping Route } & $\begin{array}{l}\text { Transport } \\
\text { Package }\end{array}$ & $\begin{array}{l}\text { Total } \\
\text { Volume }\end{array}$ \\
\hline ID & $\begin{array}{l}\text { Source } \\
\text { Facility }\end{array}$ & Facility & $\begin{array}{l}\text { Transport } \\
\text { Mode }\end{array}$ & Intrasite & 93.0 \\
\hline 1 & Legacy-LANL & LANL1.5.9 & Intrasite & Intrasite & 93.0 \\
\hline 2 & LANL1.5.9 & $\begin{array}{l}\text { LANL- } \\
\text { TRU-2 }\end{array}$ & Intrasite & .4 \\
\hline
\end{tabular}

\begin{tabular}{|l|l|l|l|l|}
\hline \multicolumn{3}{|c|}{ Table 1A. - Shipping Schedule } & \multicolumn{2}{c|}{ LANL/TRU/Waste Stream LANL2.4 } \\
\hline ID & Start Year & End Year & Waste Type & Annual Volume \\
\hline 1 & 1997 & 2004 & TRU & 12 \\
\hline 2 & 1997 & 2004 & TRU & 12 \\
\hline
\end{tabular}

Method 2 -This method demonstrates ability to define technology performance specifications through volume reduction at a facility. Table 1 and $1 \mathrm{~A}$ Are used to demonstrate this test with a change in the annual volume shipping schedule from $12 \mathrm{~m}^{3} /$ year to $6 \mathrm{~m}^{3}$ /year. After the simulation, an assessment of the impacts will be conducted by examining the CWWFA facility capacity detail charts. 
Reporting

TP - $\quad$ Test Procedure

REQD - Requirement

P/F - Pass/Fail

\begin{tabular}{|l|l|l|l|}
\hline \multicolumn{1}{|c|}{ TP } & REQD & \multicolumn{1}{|c|}{ Result/Comment } & P/F \\
\hline $\begin{array}{l}\text { TP3.1.7 } \\
\text { Method 1 }\end{array}$ & 3.1.7.1 & $\begin{array}{l}\text { Charts \#1- 4 verify that the model is behaving as } \\
\text { expected. There is an increase in annual receipt to } \\
\text { the affected facilities (LAN1.5.9-T and LANL- } \\
\text { TRU-2). Storage also shows an impact due to } \\
\text { facility capacity constraint. Chart \#5 shows the } \\
\text { impact to the entire waste stream. See charts } \\
\text { below. } \\
\text { As part of the testing requirement, the CWWFA } \\
\text { input screen was used to input the changed data. } \\
\text { However, the input screen failed to allow the user } \\
\text { to input new routes or shipping schedules. The data } \\
\text { was finally inputted into the model database via } \\
\text { programming. This is a minor deficiency. }\end{array}$ & $\begin{array}{l}\text { Pass - } \\
\text { minor } \\
\text { correction }\end{array}$ \\
\hline $\begin{array}{l}\text { TP3.1.7 } \\
\text { Method 2 }\end{array}$ & 3.1 .7 .2 & $\begin{array}{l}\text { Chart \#6 verifies that the model behaves as } \\
\text { expected due to a volume reduction through the } \\
\text { facility. Storage has increased possibly because of } \\
\text { unintended impact to the disposition schedule. }\end{array}$ & Pass \\
\hline
\end{tabular}


4

3

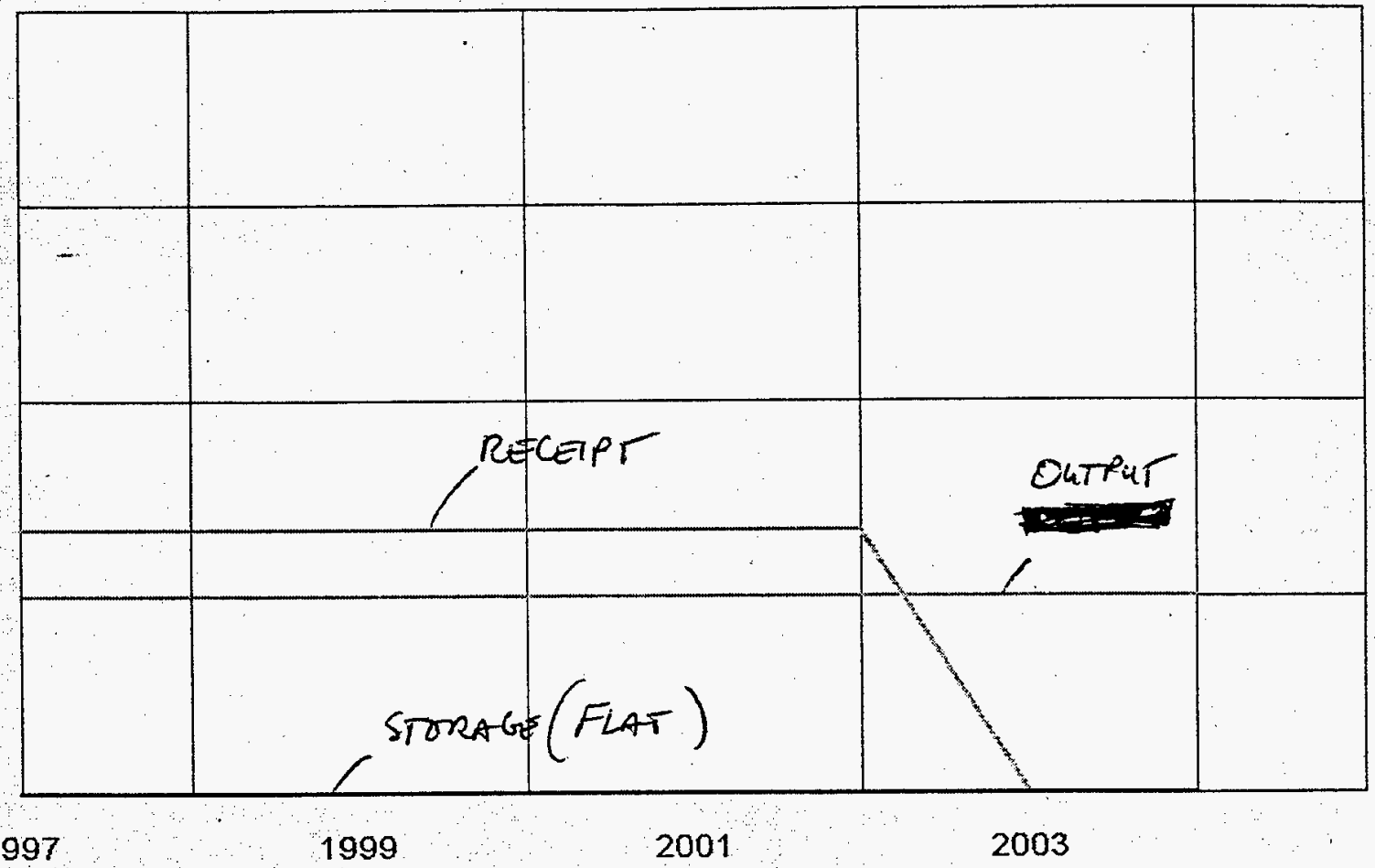


TP 3.1.7

METHOD 1

10/23/97

CHART\#2

Q $12 \mathrm{~m}^{3}$

\begin{tabular}{|c|c|c|}
\hline Site: $\longdiv { \text { LANL } }$ & Waste Type:TTRU & 7 Facility: LANL1.5.9-T \\
\hline
\end{tabular}

Los Alamos National Laboratory - TRU - Mobile RTR

Alternative Case w-ER wo-WM

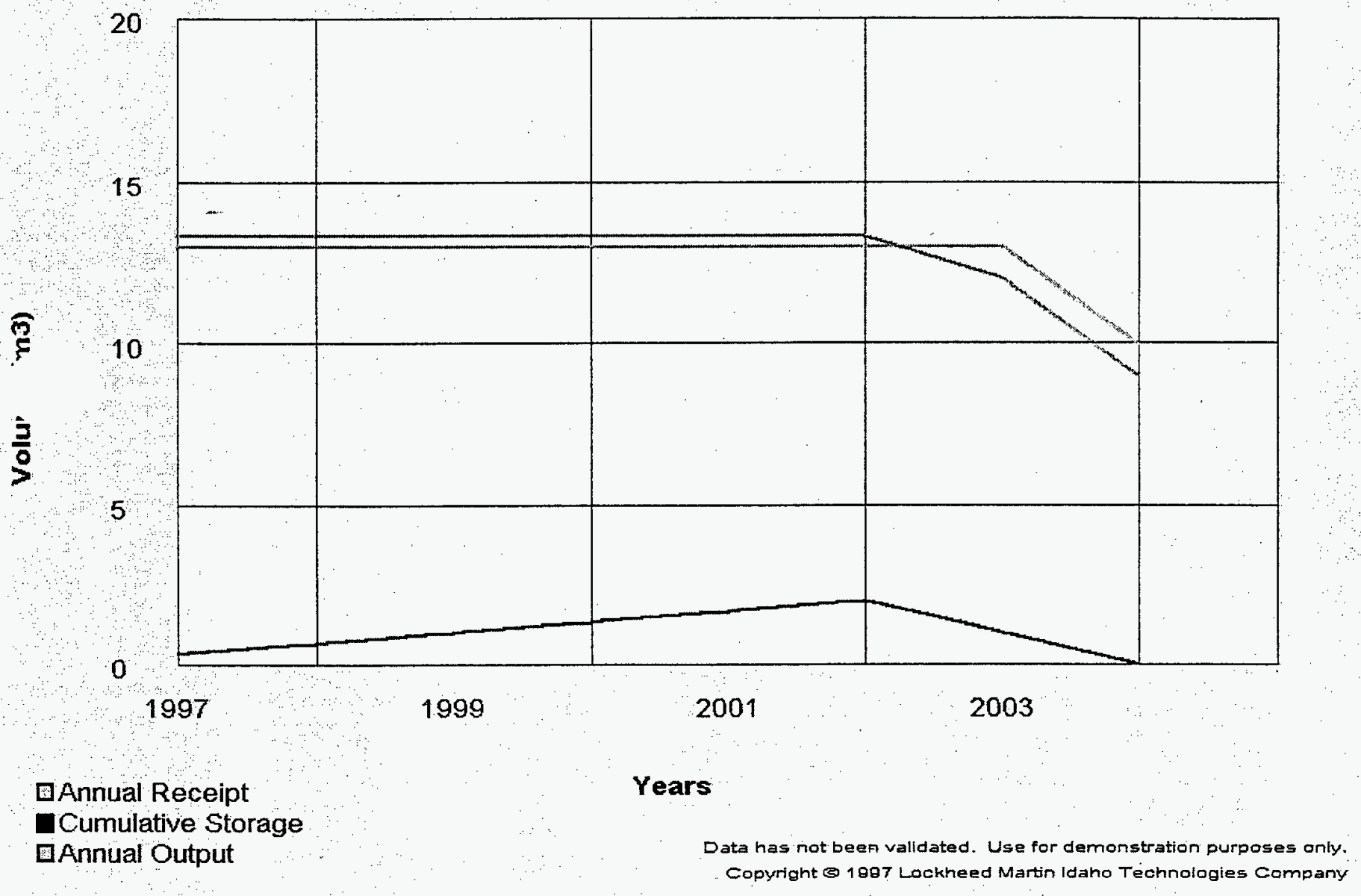


TP 3.1 .7

METHOD 1

$10 / 23 / 97$

CHART\#3

Los Alamos National Laboratory - TRU - TBD Mobile Size Reduction/Decon Alternative Case w-ER wo-WW

4

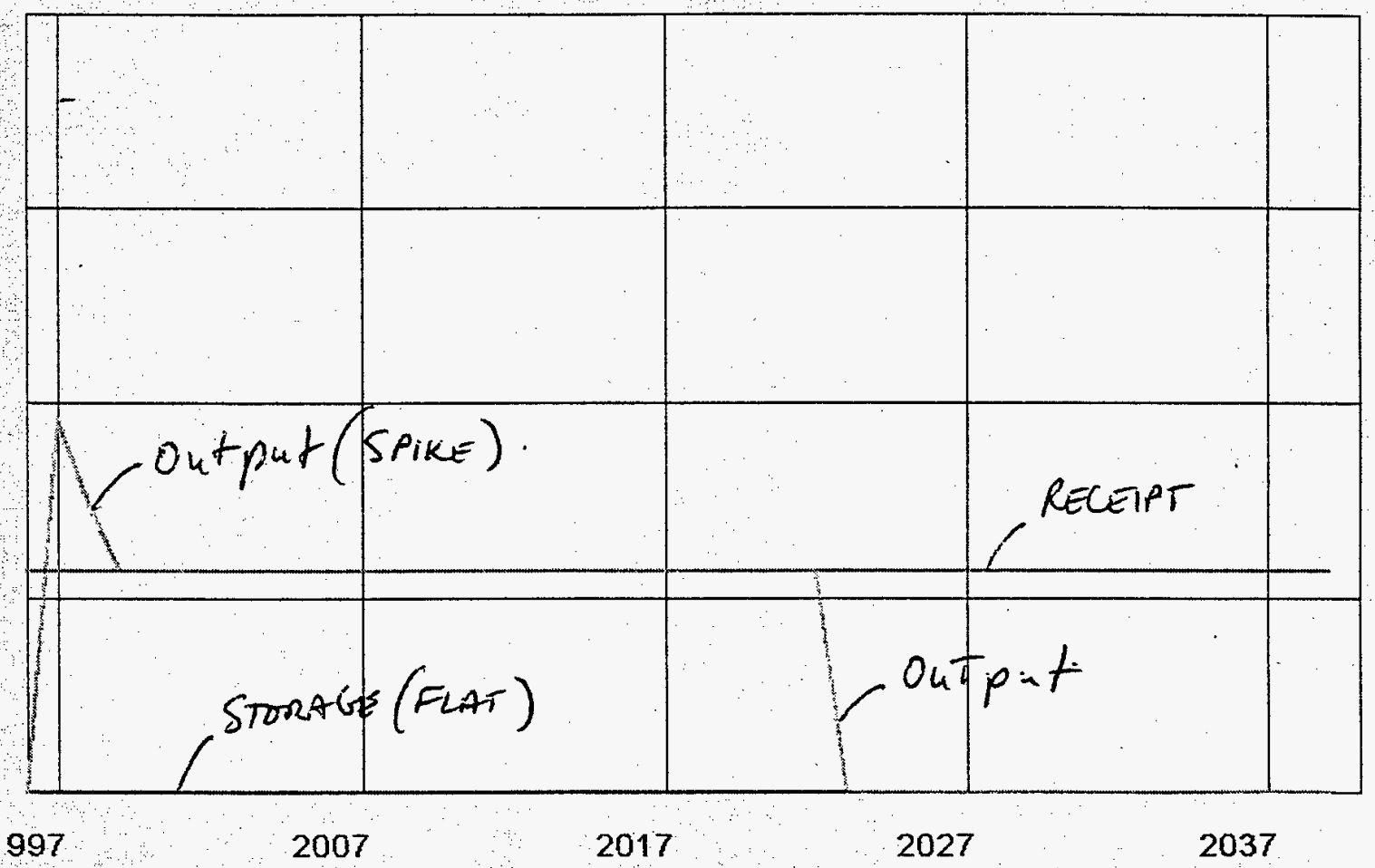


TP 3.1.7

METHOD 1

10/23/97

CHART\#4

(c) $12 \mathrm{~m}^{3}$

Site: LANL Waste Type:TRU

Facility: LANL-TRU-2

Los Alamos National Laboratory - TRU - TBD Mobile Size Reduction/Decon

Alternative Case w-ER wo-WW

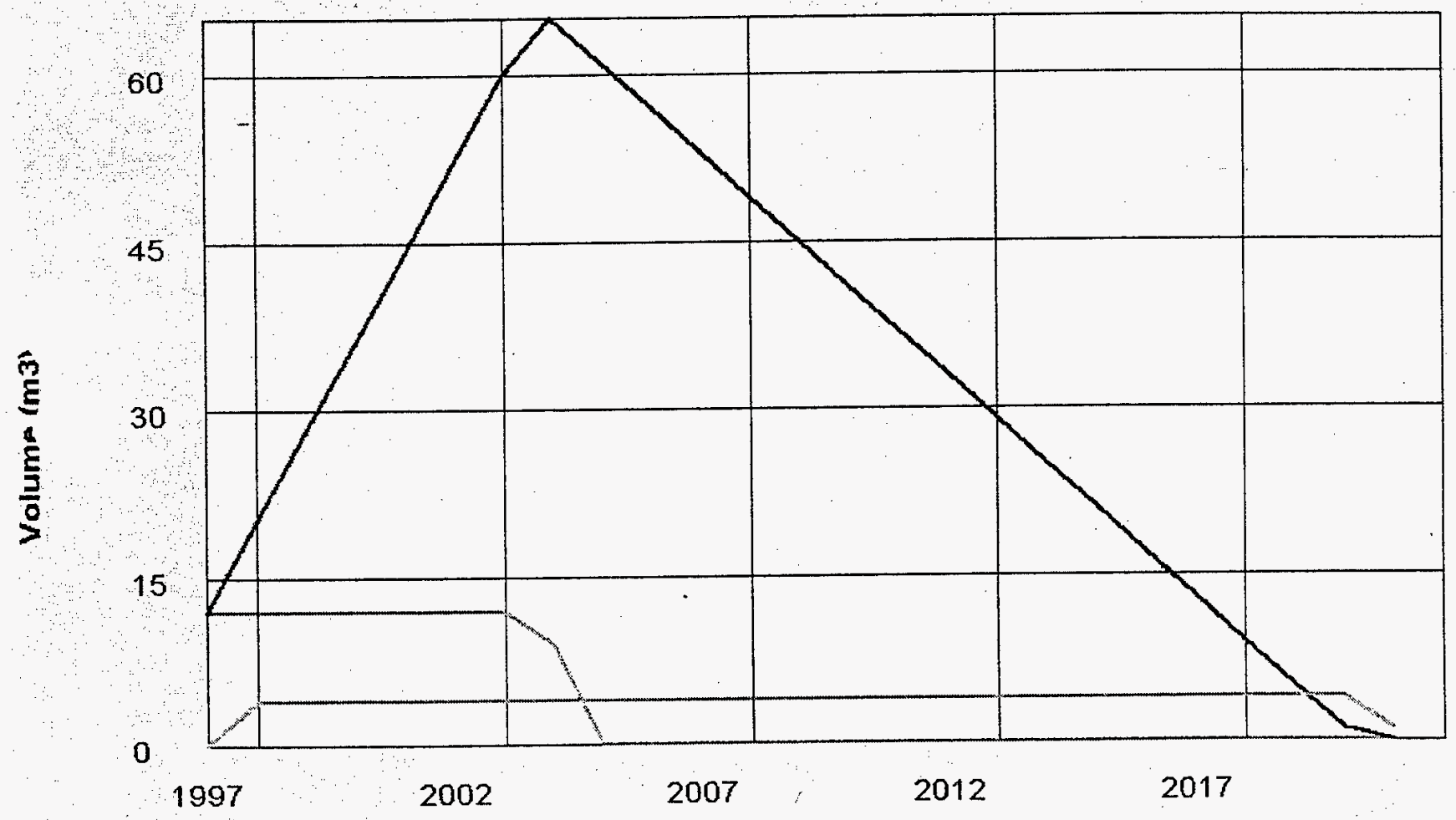

Annual Receipt

Years

Cumulative Storage

annual Output

Data has not been validated. Usa for demonstration purposes only: Copyright \& 1897 Lockheed Martin ldaho Technologles Company 
TP 3.1.7

METHOD 1

$10 / 23 / 97$

CHART\#5

ROLIE WASTE FLOW

\section{WANL}

Waste Type: TRU

\section{Waste Stream Detail}

Stream: LANL2.4

Los Alamos National Laboratory - TRU - RH TRU

Alternative Case w-ER wo-WW

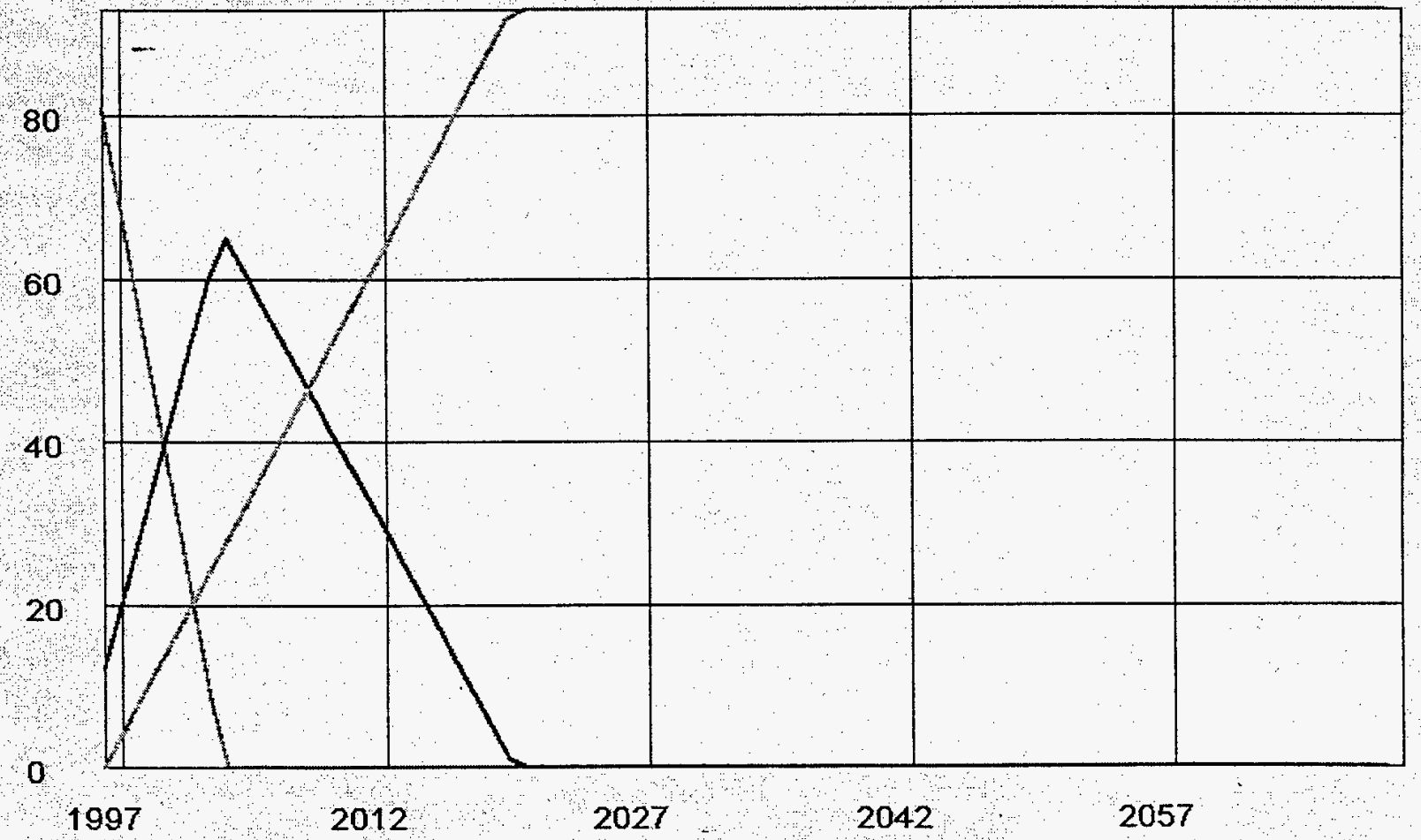

Rinventory

Years

Vin-process Storage

EDisposition

Data has not been validated Use far demonstration purposes only 
TP 3.1.7

METHOD 2

$10 / 23 / 97$

CHART\#6

Site: LANL Waste Type: TRU 9 Facility: LANL-TRU-2

Facility Capacity Detail

Los Alamos National Laboratory - TRU - TBD Mobile Size Reduction/Decon Alternative Case w-ER wo-WW

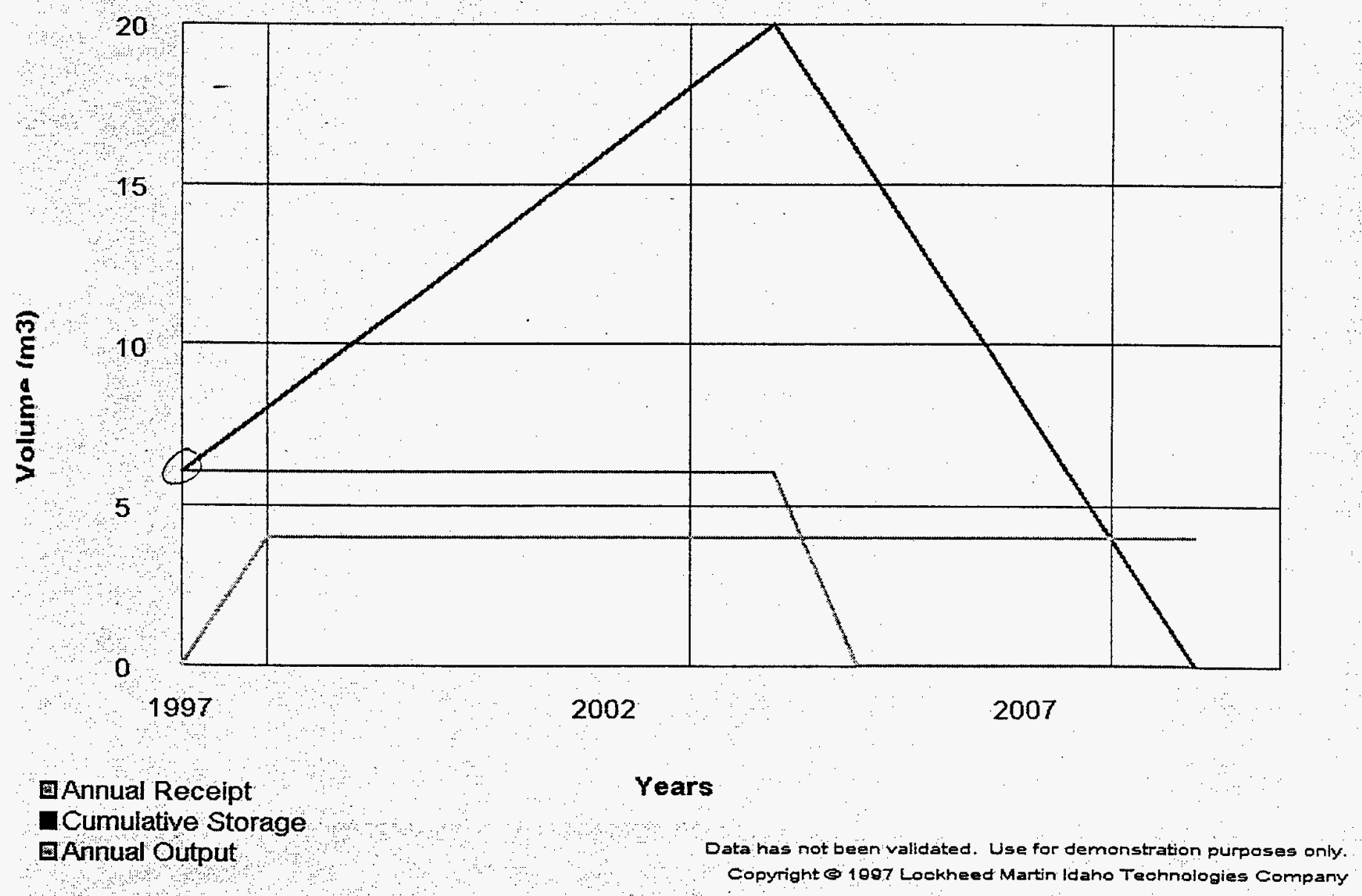




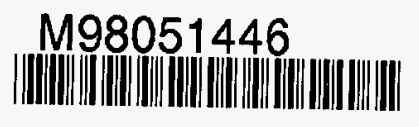

Report Number (14) $/ N E E L / E X T \ldots-97-0 / 337$

Publ. Date (11)

Sponsor Code (18)

$$
\frac{199711}{\text { DOE/ENA,XF }}
$$

UC Category (19) 